\title{
Multi sensor validation and error characteristics of arctic satellite sea surface temperature observations
}

Høyer, Jacob L.; Karagali, loanna; Tonbo, Rasmus; Dybkjær, Gorm

Published in:

Proceedings

Publication date:

2011

Document Version

Publisher's PDF, also known as Version of record

Link back to DTU Orbit

Citation (APA):

Høyer, J. L., Karagali, I., Tonbo, R., \& Dybkjær, G. (2011). Multi sensor validation and error characteristics of arctic satellite sea surface temperature observations. In Proceedings (pp. 221-221). GHRSST.

\section{General rights}

Copyright and moral rights for the publications made accessible in the public portal are retained by the authors and/or other copyright owners and it is a condition of accessing publications that users recognise and abide by the legal requirements associated with these rights.

- Users may download and print one copy of any publication from the public portal for the purpose of private study or research.

- You may not further distribute the material or use it for any profit-making activity or commercial gain

- You may freely distribute the URL identifying the publication in the public portal

If you believe that this document breaches copyright please contact us providing details, and we will remove access to the work immediately and investigate your claim. 


\section{PROCEEDINGS OF THE GHRSST XII SCIENCE TEAM MEETING}

John Mclntyre Conference Centre, Edinburgh $27^{\text {th }}$ June - $1^{\text {st }}$ July 2011

Version September $26^{\text {th }}, 2011$

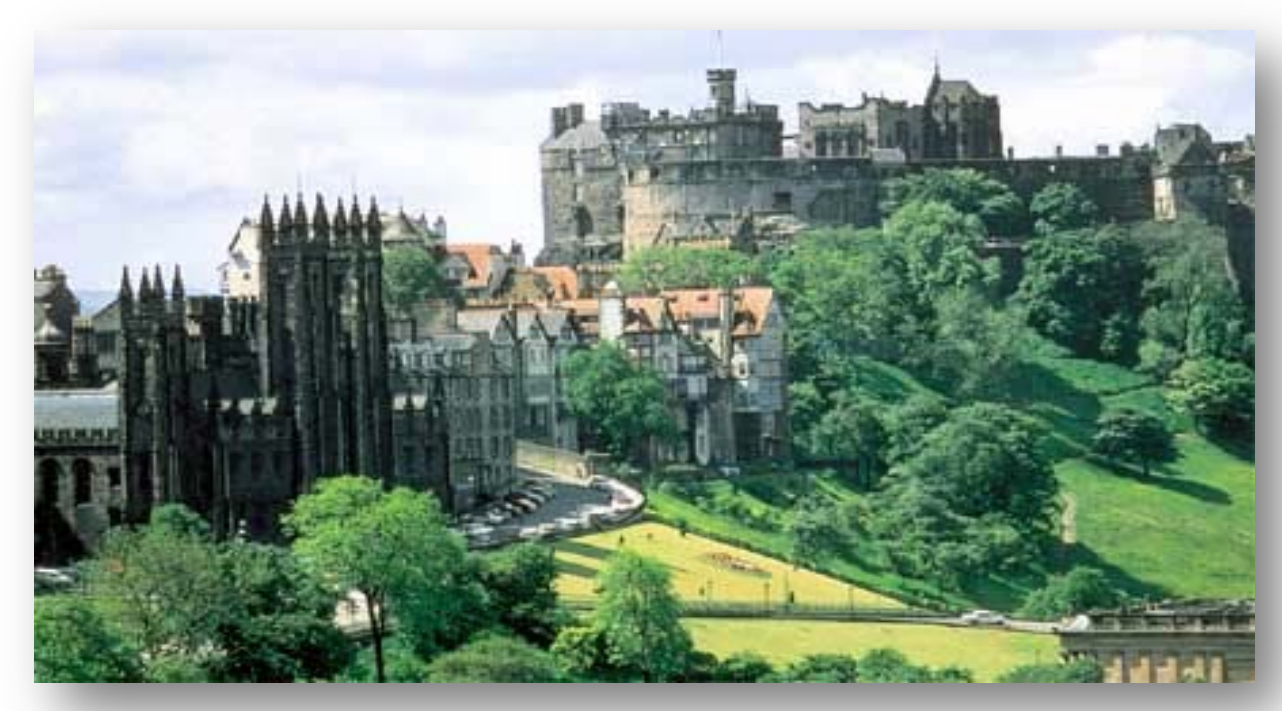

Meeting sponsored by: 
GHRSST Project Office

\title{
Copyright 2011@ GHRSST
}

This copyright notice applies only to the overall collection of papers: authors retain their individual rights and should be contacted directly for permission to use their material separately. Editorial correspondence and requests for permission to publish, reproduce or translate this publication in part or in whole should be addressed to the GHRSST Project Office. The papers included comprise the proceedings of the meeting and reflect the authors opinions and are published as presented. Their inclusion in this publication does not necessarily constitute endorsement by GHRSST or the co-organisers.

\section{GHRSST \\ International \\ Project Office}

National Centre for Earth Observation
Dept of Meteorology, The University of Reading
Earley Gate Bldg 58, Reading RG6 6BB, UK

ghrsst-po@nceo.ac.uk

Tel +44 (0) 1183785579 Fax +44 (0) 1183785576

\author{
www.ghrsst.org
}




\section{CONTENTS}

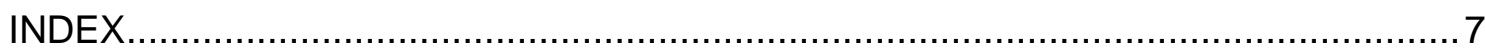

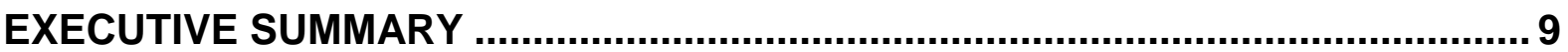

CHAPTER 1: AGENDA WITH LINKS TO DOCUMENTS .................................... 13

AGENDA FOR GHRSST XII WITH LINKS TO DOCUMENTS ................................. 14

\section{CHAPTER 2: MEETING SUMMARY}

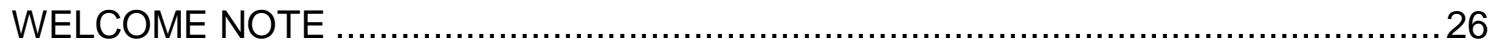

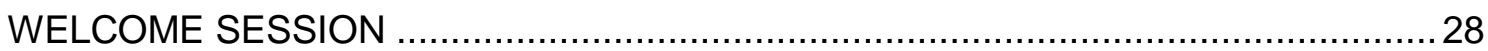

SESSION 1: GHRSST COMPONENTS AND MAJOR PROJECTS …...................... 30

SESSION 2.1: SST USE IN ATMOSPHERE AND OCEAN APPLICATIONS ............... 39

SESSION 2.2: CLIMATE AND MARINE BIOLOGY APPLICATIONS ......................... 41

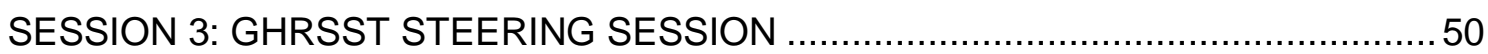

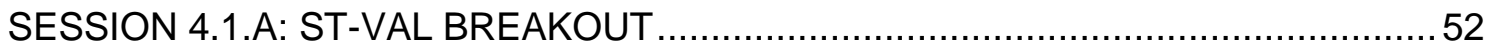

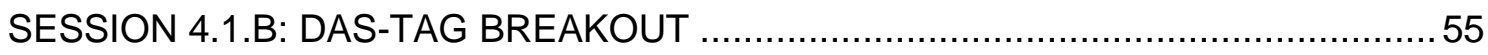

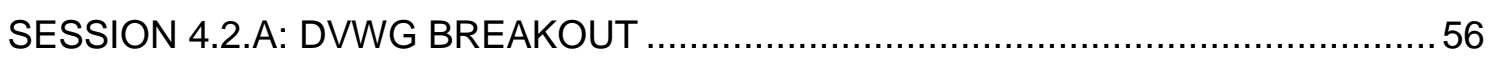

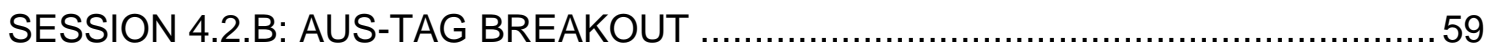

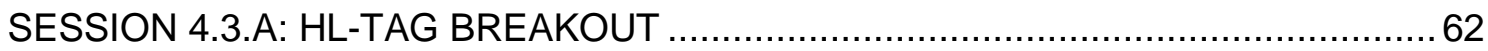

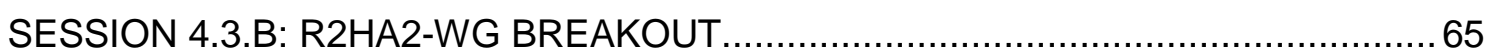

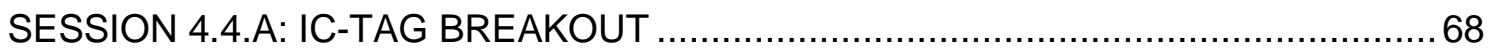

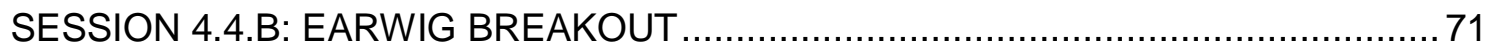

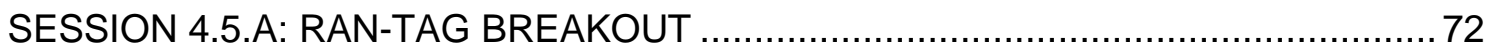

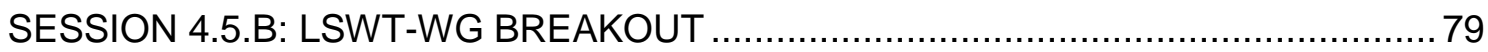

SESSION 5: SUMMARY AND CONCLUSION OF BREAKOUTS …........................ 81

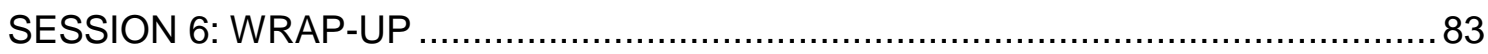

CHAPTER 3: SCIENTIFIC ABSTRACTS AND REPORTS

R05 - REPORT FROM THE GHRSST PROJECT OFFICE (GPO): 2010/11 .............. 86

R07 - REPORT FROM AUSTRALIA - BLUELINK AND IMOS ................................ 90

R08 - REPORT FROM THE NAVOCEANO RDAC …...................................... 106

R09 - REPORT FROM MULTI-SENSOR IMPROVED SEA-SURFACE TEMPERATURE (MISST) FOR IOOS .................................................... 108

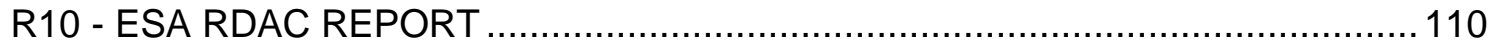

R11 - REPORT FROM MYOCEAN RDAC ................................................. 111

R12 - REPORT FROM EUMETSAT AND OSI SAF ......................................... 115

R13 - REPORT FROM JAPAN (JAXA, JMA AND TOHOKU UNIVERSITY).............. 122

R14 - REPORT FROM THE GLOBAL DATA ASSEMBLY CENTER (GDAC)

AND FROM THE DATA ASSEMBLY AND SYSTEMS TECHNICAL

ADVISORY GROUP (DAS-TAG) 
R15 - REPORT FROM THE REANALYSIS TECHNICAL ADVISORY GROUP (RAN-TAG) AND THE LONG TERM STEWARDSHIP AND REANALYSIS FACILITY (LTSRF)

R16 - REPORT FROM THE INTER-COMPARISON TECHNICAL ADVISORY GROUP (IC-TAG)

R18 - REPORT FROM THE SATELLITE SEA SURFACE TEMPERATURE VALIDATION WORKING GROUP (ST-VAL WG)

R20 - REPORT FROM THE DIURNAL VARIABILITY WORKING GROUP (DVWG)

R21 - REPORT FROM THE APPLICATIONS AND USERS SERVICES TECHNICAL ADVISORY GROUP (AUS-TAG)

R22 - REPORT FROM THE GHRSST HIGH LATITUDE - TECHNICAL ADVISORY GROUP

R23 - REPORT FROM THE ESTIMATION AND RETRIEVALS WORKING GROUP TO THE $12^{\text {TH }}$ GHRSST SCIENCE TEAM MEETING

NIGHTTIME-ONLY OPTIMUM INTERPOLATION SST FIELDS

AUSTRALIAN BUREAU OF METEOROLOGY SST REQUIREMENTS FOR NWP AND ATMOSPHERE-OCEAN COUPLED MODELS

CLASSIFICATION OF SEA-ICE IN HIGH LATITUDE RETRIEVALS FROM AATSR

GLOBAL 1-KM SEA SURFACE TEMPERATURE (G1SST) FOR RESEARCH AND APPLICATIONS

COUPLED OCEAN-ATMOSPHERE INTERACTION AT OCEANIC MESOSCALES

WHAT'S IN A GHRSST L4 OR L2P FILE?

SOURCES OF ERROR IN SATELLITE-DERIVED FLUXES. 198

THE IMPACT OF DIURNAL WARMING ON SST FRONTS 198

GLOBAL SEA SURFACE TEMPERATURE RETREIVAL USING AN OPTIMAL ESTIMATION ALGORITHM

RECENT ADDITIONS TO THE SST QUALITY MONITOR (SQUAM) 200

THE GLOBAL MONITORING FOR ENVIRONMENT AND SECURITY (GMES) SENTINEL-3 MISSION 202

SEA SURFACE TEMPERATURE FROM THE ALONG-TRACK SCANNING RADIOMETER REPROCESSING FOR CLIMATE PROJECT. 204

OSTIA REANALYSIS: A HIGH RESOLUTION SST AND SEA-ICE REANALYSIS 208

OPTIMAL ESTIMATION OF SST FROM AVHRR 1991-2010 208

APPLYING BARNE'S TECHNIQUE FOR DAILY SST (AVHRR AND TRMM / MICROWAVE) IMAGES COMPOSITION

MULTI-SENSOR IMPROVED SEA-SURFACE TEMPERATURE (MISST) FOR IOOS

CONSISTENCY OF SEA SURFACE TEMPERATURE ANALYSES IN DEPICTING ENSO BEHAVIOR 
OCEAN HEAT BUDGET FOR THE FLORIDA REEF TRACT: THE ROLE OF SMALL-SCALE PROCESSES IN MAINTAINING PERSISTENT COASTAL SEA TEMPERATURE GRADIENTS.

COMPARISON OF SATELLITE SST PRODUCTS WITH IN SITU SKIN SST MEASUREMENTS

NASA ACTIVITIES AND PERSPECTIVES.

MULTI SENSOR VALIDATION AND ERROR CHARACTERISTICS OF ARCTIC SATELLITE SEA SURFACE TEMPERATURE OBSERVATIONS

JOINT RETRIEVALS OF AEROSOL AND SST FROM AATSR

ET-OOFS REQUIREMENTS

REQUIREMENTS ON SST FOR NWP, AIR-SEA INTERACTION AND

OCEAN MODELLING

OCEAN WAVE EFFECTS ON THE DAILY CYCLE IN SST

THE ROLE OF SMALL-SCALE AND SHORT-TERM SST VARIABILITY IN THE ERROR OF GRIDDED OBSERVATIONS

DIURNAL WARMING IN THE NORTHERN EUROPEAN SHELF SEAS:

OBSERVATIONS VS. MODELLING.

ANALYSES OF SATELLITE AND IN SITU SST FOR CLIMATE APPLICATIONS

STUDY ON PRODUCING THE MULTI-SENSOR SST WITH HIGH RESOULTION OVER THE EAST ASIA AT KMA/NIMR

MERMAID: PROPOSED EXTENSION TO FURTHER SENSORS / PRODUCTS INCLUDING THE (A)ATSR SERIES AND SENTINEL-3 OPTICAL INSTRUMENTS.

A POSSIBLE CONNECTION BETWEEN EL NINO AND SST ALGORITHM ERRORS IN THE TROPICAL ATLANTIC IN SPRING 2010.

OPERATIONAL USE OF NWP MODEL OUTPUTS IN SATELLITE SST CALCULATIONS

INCREASING AMPLITUDE OF EL NIŇO IN THE CENTRAL EQUATORIAL PACIFIC AND RECORD WARMING IN THE SOUTH PACIFIC AND WESTERN ANTARCTICA

RELATING SEA SURFACE TEMPERATURE TO WATER AND CARBON DIOXIDE FLUXES

GLOBAL LAKE SURFACE WATER TEMPERATURES FROM ATSR

NOAA SEA SURFACE TEMPERATURE PRODUCTS GEOSTATIONARY SST L2P AND BLENDED SST L4 PRODUCTS.

THE DBCP-GHRSST PILOT PROJECT

SEA SURFACE TEMPERATURES FROM SATELLITE OBSERVATIONS AS CLIMATE DATA RECORDS

THE EUROPEAN SPACE AGENCY CLIMATE CHANGE INITIATIVE FOR SEA SURFACE TEMPERATURE. 265

DEFINITIONS OF SEA-SURFACE TEMPERATURE. 266 
NEW APPROACHES TO THE INFRARED ATMOSPHERIC CORRECTION ALGORITHM.

SEVIRI SST AND SHIPBOARD SKIN SST COMPARISONS: INITIAL RESULTS

DIURNAL VARIABILITY IN SHALLOW COASTAL WATERS. 269

THE CALIBRATION OF THE BORADBAND INFRARED SENSORS ONBOARD NOAA SATELLITES 270

EUMETSAT SUPPORT TO GHRSST

DEVELOPMENT AND EVALUATION OF SEA SURFACE TEMPERATURE ALGORITHMS FOR GOES-R ABI USING MSG SEVIRI AS A PROXY 278

MEDSPIRATION LATEST DEVELOPMENTS 279

SST PROFILES RECORDED BY UN-PUMPED NEAR-SURFACE ARGO MEASUREMENTS 280

USER REQUIREMENTS FOR SST REANALYSIS. 283

ESTIMATING AND PRESENTING UNCERTAINTIES IN AN HISTORICAL SEA-SURFACE TEMPERATURE ANALYSIS. 292

OBJECTIVE DETERMINATION OF FEATURE RESOLUTION IN AN SST ANALYSIS

THE IMPACT OF NEW SOURCES OF SATELLITE DATA ON THE OSTIA SYSTEM. 298

GHRSST WINDSAT AND AMSR-E VERSION 7 SSTS 299

USING GHRSST DATA CONSTRAINTS TO ESTIMATE THE CIRCULATION AND CLIMATE OF THE OCEAN

\section{APPENDICES}

APPENDIX 1 - PARTICIPANTS LIST 302

APPENDIX 2 - SCIENCE TEAM 2011/12 


\section{INDEX}

Arino, Olivier 279

Armstrong, Edward

$9,131,171$

Autret, Emmanuelle. ..279

Banzon, Patria Viva. 179

Barker, Kathryn 234

Barras, Vaughan 180

Beggs, Helen $.9,90,180$

Berruti, B 202

Berry, David I 230

Bingham, Andrew 131

Blouch, Pierre. 263

Brandon, Tess B 137

Brix, Holger 300

Buck, Justin 280

Bulgin, Claire 185

Buongiorno, A 202

Buongiorno-Nardelli, Bruno 111

Carboni, Elisa 199,222

Casey, Kenneth $9,108,137,213$

Cayula, Jean-François 106

Chao, Yi. 193

Chelton, Dudley. 194

Chin, Toshio Mike 171, 195

Clayson, Carol Anne 198

Corlett, Gary 161

Cornillon, Peter 108, 198, 213

Cox, Caroline 199,222

Dash, Prasanjit .257

Dash,Prasanjit .200

Dendamrongvit, Sareewan. .266

Donlon, Craig 9, 202

Dybkjær, Gorm .221

Eastwood, Steinar. .115

EMBURY, OWEN.................................204

Fanton D'Andon, Odile ...............................234

Ferraria, M-H.....................................202

Fiedler, Emma........................................208

Filipiak, Mark ......................................208

Finch, Chris .......................................... 131

França, Gutemberg Borges.
Frerick, J 202

Gentemann, Chelle 108, 213, 299

Gierach, Michelle M 214

Good, Simon A. 283

Goryl, P 202

Grainger, Don 199, 222

Gramer, Lewis J.... 215

Guan, Lei 217

Hacker, Peter 220

Harris, Andrew $178,257,270$

Heiberg, Hanne 111

Hendee, James C 215

Hoeyer, Jacob L 221,225

Hosoda, Kotaro 122

Houghton, Nigel 110

Høyer, Jacob 111, 175

Huang, Haiyan 199, 222

Huang, Thomas 131

Ignatov, Alexander $155,200,278$

Imaoka, Keiji 122

Ishizaki, Shiro 122,222

Janssen, Peter A E M 223

Kachi, Misako. 122

Kaiser-Weiss, Andrea

$9,86,280$

Kaplan, Alexey

9,224

Karagali, Ioanna

221, 225

Kawamura, Hiroshi

122,232

Kennedy, John J ................................. 292

Kent, Chris .......................................... 234

Kent, Elizabeth C ................................. 230

Kihai, Yury......................................... 278

Kim, Mi-Ja ........................................ 231

Kim, Youngmi...................................... 231

Klein, U ............................................. 202

Kubat, Miroslav ........................................ 266

Kurihara, Yukio ..................................... 122

Laur, H .............................................. 202

Lavender, Samantha............................. 234

Layden, Aisling.......................................... 243

Le Borgne, Pierre..............115, 198, 240, 268 
GHRSST XII -

Proceedings, Edinburgh

GHRSST Project Office

Lee, Tong

214, 241

Legendre, Gérard .......................................240

Lerebourg, Christophe ...............................234

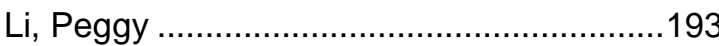

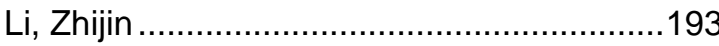

Lindstrom, Eric ...........................................220

Liu, W. Timothy .............................................242

MacCallum, Stuart N ...................................243

Majewski, Leon ...............................................90

Mariano, Arthur J......................................215

Marsouin, Anne .240

Martin, Matthew

9, 111, 155, 208, 298

Maturi, Eileen Maria ....................................257

Mavrocordatos, C .........................................202

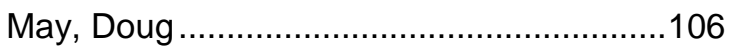

Mazeran, Constant .......................................234

McKenzie, Bruce ..........................................106

McPhaden, Micheal...................................241

Meldrum, David .............................................263

Menmenlis, Dimitris .....................................300

Merchant, Christopher

$9,185,204,208,243,264,265$

Minnett, Peter J

$9,266,268,269$

Mittaz, Jonathan

257,270

Murakami, Hiroshi ..........................................122

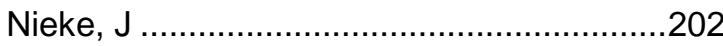

Nightingale, Tim ............................................199

O'Carroll, Anne....................................115, 277

Oliveira, Antônio do Nascimento..................209

Ou, Mi-Lim .................................................231

Paltoglou, George ...........................................90

Péré, Sonia..................................................240

Petrenko, Boris..............................................278

Piollé, Jean-François............................111, 279

Potash, Robert ............................................257

Poulsen, Caroline .................................199, 222

Poulter, David................................................111

Quinn, Sarah ............................................280
Issue: Final

Date: September 27, 2011

Rayner, Nick A.

283, 292

Rebhan, H..................................................202

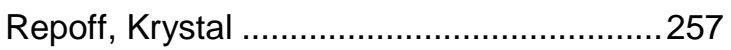

Reynolds, Richard W ........................179, 233

Roberts-Jones, Jonah........................208, 298

Roquet, Hervé ...........................111, 240, 268

Ruiv, Bianca Couto ...................................... 209

Sakaida, Futoki ............................................122

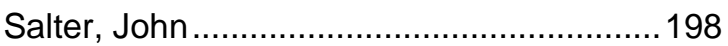

Sandery, Paul ............................................... 180

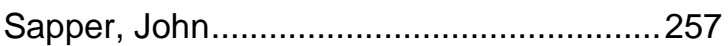

Saunby, Michael ........................................292

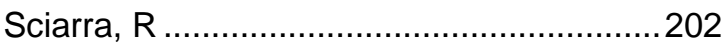

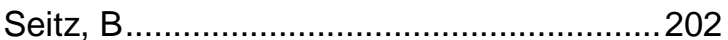

Shabanov, Nikolay .......................................278

Shimada, Udai ......................................... 122

Siddans, Richard.................................199, 222

Smith, Andrew............................................222

Smith, Deborah K.........................................299

Steinle, Peter ................................................. 180

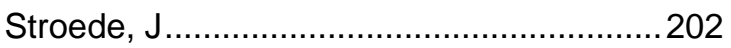

Tang, Benyang.......................................... 193

Teng, Weicheng .............................................. 217

Thomas, Gareth ...............................199, 222

Thompson, Charles...................................... 131

Tonbo, Rasmus............................................221

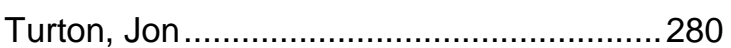

Vazquez, Jorge .........................131, 171, 300

Verein, Ruslan ............................................90

Vieira Paes, Rosa Cristhyna de Oliveira.....209

Wick, Gary A ................................................ 166

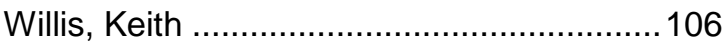

Xie, Xiaosu ................................................ 242

Yoshioka, Noriya........................................ 122

Zhang, Kailin ................................................. 217

Zhong, Aihong .............................................90

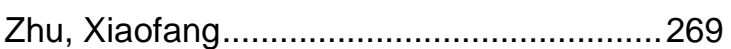




\section{EXECUTIVE SUMMARY}

\section{Andrea Kaiser-Weiss ${ }^{1}$, Peter $\mathrm{J} \mathrm{Minnett}^{2}$, Helen Beggs ${ }^{3}$, Ed Armstrong ${ }^{4}$, Kenneth Casey $^{5}$, Chris Merchant ${ }^{6}$, Alexey Kaplan ${ }^{7}$, Matthew Martin ${ }^{8}$, Craig Donlon ${ }^{9}$}

(1) NCEO, University of Reading, UK, Email: ghrsst-po@nceo.ac.uk,

(2) Meteorology \& Physical Oceanography, University of Miami (USA), Email:

pminnett@rsmas.miami.edu

(3) Centre for Australian Weather and Climate Research, Bureau of Meteorology, Melbourne (Australia), Email: H.Beggs@bom.gov.au

(4) Jet Propulsion Laboratory, California Institute of Technology, 4800 Oak Grove Dr, Pasadena, CA (USA), Email: Edward.m.armstrong@jpl.nasa.gov

(5) NOAA/NODC, Silver Spring MD, (USA), Email: Kenneth.Casey@noaa.gov

(6) University of Edinburgh, Edinburgh, EH7 3JN (UK), Email : c.merchant@ed.ac.uk

(7) Lamont-Doherty Earth Observatory of Columbia University, Palisades, NY 10964 (USA), Email : alexeyk@ldeo.columbia.edu

(8) UK Met Office, Exeter, (UK), Email : Matthew.Martin@metoffice.gov.uk

(9) ESA/ESTEC, Keplerlaan 1, 2201 AZ, Noordwijk (The Netherlands),

ESA/ESRIN, via Galileo Galilei, Frascati, Rom, (Italy), Email: craig.donlon@esa.int

The Science Team chair, Craig Donlon, welcomed all participants and reminded the Science Team of the GHRSST aim to provide the best quality SST for short, medium and long-term applications - and on which we can measure the success of GHRSST. The GHRSST phase-1 Pilot Project is complete, the phase-2 international Regional/Global Task Sharing system is built and operating, and GHRSST is now in phase-3: operational delivery of a wealth of SST products and developing Climate Data Records.

Progress was presented for the major GHRSST components, and the Space Agencies pointed out their priorities and expressed their continuing support of GHRSST. For instance:

- The GHRSST Project Office, funded by ESA and now hosted at the University of Reading, continues to support the GHRSST Science Team for a $10^{\text {th }}$ year.

- The Global Data Assembly Centre (GDAC) which is hosted at NASA JPL Physical Oceanography DAAC (PO.DAAC) now interfaces to 12 Regional Data Assembly Centres (RDACs), and ingests $\sim 8000$ files per day ( $-35 \mathrm{~GB})$. The PO.DAAC and currently holds a total volume of 202 TB. A new web-portal, metadata database, data mining, subsetting and visualisation tools and a GHRSST forum have been established in this year.

- The Long Term Stewardship and Reanalysis Facility (LTSRF) is hosted at NOAA NODC; its operations are in constant progress with automatic daily acquisition from the GDAC and archiving. Current archive holdings are over 28 TB (1981-2010). Progress with reanalysis is being made at both the individual sensor level (L2, L3) and with merged L4 products. One of the tasks for $2011 / 2012$ is to develop the SST Climate Variable Data Processing Framework.

- The GHRSST Multi-Product Ensemble system (GMPE) carried out a gradient inter-comparison and assessed the suitability of the ensemble spread as an error estimate. The GMPE median is shown to be more accurate than any individual L4 product in the initial comparison against the independent near-surface Argo data. With the SST Quality Monitor (SQUAM), one L3 and 13 L4 GHRSST products have been cross-compared and validated against in-situ data with the iQuam system.

Progress at the various contributing RDACs has been achieved through 
continuing operational processing of SST data streams from multiple, complementary satellite sensors and additionally by providing new or improved data. For example:

- New L3 data are now in production by ESA Medspiration and the Australian BOM.

- Real-time and reprocessed $S_{S T} T_{\text {skin }}$ from AVHRR and MTSAT is being provided by $A B O M$.

- A new processing chain using physical retrievals accounting for atmospheric moisture and Saharan dust in geostationary products has been implemented at the OSI-SAF.

- Navoceano selects the best quality data from the GDAC, and adds error estimates to be used for Navy Coupled Ocean Data Assimilation (NCODA).

- EUMETSAT RDAC is now providing very high data quality from IASI (within the error bounds of the matched buoys).

- MyOcean stressed as its main achievement from the last year their OSTIA SST Reanalysis.

- The ESA RDAC is processing all AATSR on a purely operational basis.

- RSS reprocessed the entire AMSR-E data set and is producing a Windsat data set; the MISST RDAC will proceed according to availability of funds.

- The Japanese GHRSST server resumed distributing AMSR-E within a week after the earthquake in March, which also caused a wall of the test building to collapse causing contamination of the clean room and the proto-flight AMSR-2 model. No serious damage was suffered, and after repairing the test facilities and cleaning of the instruments, all the components were tested and found to work nominally. The launch is still scheduled for the Japan Fiscal Year 2011 (late 2011 to early 2012).

All RDACs have either started or are planning to move to the revised GHRSST data Processing specification (GDS2) that was completed and agreed through international consensus in 2011. There are continuing discussions within various RDACs about multi-resolution products and how the resulting uncertainties should be estimated and communicated.

The use of available in-situ data for validation and calibration was discussed and Helen Beggs pointed out that for regions sparse in buoy observations, the IMOS ships of opportunity (SOOP) SST provide an alternative, calibrated, in-situ data set for validation. David Meldrum gave a presentation on the DBCP Pilot Project releasing upgraded drifting buoys based on GHRSST specifications. Further evaluation and financial commitment to DBCP buoy upgrades are required through a joint DBCP-GHRSST pilot project.

During the Users and Science Symposium on the second day of the meeting, it was clear that SST continues to be of great interest to the numerical weather prediction community, where SST serves as an indispensable boundary condition. There is increasing interest in lake surface temperatures and in ocean mesoscale SST patterns and their feedbacks on winds that can in turn affect rain rate and cyclone intensity. More scientific understanding is needed to link $\mathrm{SST}_{\text {skin }}$ and the foundation temperature ( $S S T_{\text {fnd }}$ ), i.e., in understanding diurnal variability. More explicit reporting of information content and uncertainties for each analysed SST value is needed for subsequent optimal applications. A recently observed ENSO influence on the satellite SST errors in the Atlantic Ocean was highlighted, and the research issues associated with estimating long-term SST climate records were discussed by several speakers. The approaches to uncertainty and validations were reviewed by Chris Merchant, who called for validation of both the SST and their uncertainty estimates. Merchant discussed a decomposition of uncertainty estimates into uncorrelated (random), synoptically correlated (pseudo- 
random, which can be simulated) and large-scale correlated components (systematic); examples will be developed and tested as part of the ESA CCI SST project.

A discussion was held about GHRSST needing to capture better the user requirements from the operational community, the climate change community and other users. A wide range of user requirements with respect to uncertainty characterisation can be served by GHRSST. In particular, the working groups ST-VAL, IC-TAG, DVWG and EARWiG are addressing various aspects of uncertainty estimation required by users. Craig Donlon called for coordination of user feedback and urged the GHRSST Science Team to take steps to share user feedback. Peter Minnett pointed out the importance of user requirements in the design of next generation satellite instruments.

Peter Minnett led a discussion on clarification of the current GHRSST definitions. A need for an additional piece of information on wavelengths together with the GHRSST definition on SST skin, and the need to educate the users was identified.

On Tuesday evening, the GHRSST Advisory Council met with a focus on how GHRSST and CEOS VC-SST are best interfaced. It reviewed the ST Chair election procedure, which was declared to have been well-conducted, and endorsed the election of the new ST chair.

Ten Breakout sessions were held by the GHRSST sub-groups:

ST-VAL, the Satellite Sea Surface Temperature Validation Working Group, discussed the value of ship measurements, the QC of Drifter Data, the DBCP Pilot Project concerning upgraded drifters, use of Argo near surface data, and the ST-VAL workplan for the next years.

DAS-TAG, the Data Assembly and Systems Technical Advisory Group, reviewed the GDS2 revisions, the GDS2 transition timeline, possible netCDF4 translation from netCDF3, GDS2 format compliance checker and the advantages of netCDF-4 vs. NetCDF-3.

DVWG, the Diurnal Variability Working Group, discussed diurnal warming estimates derived from satellite data, insitu data and modelling, as well as effects of waves, wind and advection in diurnal variability. Pierre Le Borgne proposed a SEVIRI SST skin $_{\text {hourly analysis in delayed }}$ mode. Helen Beggs described the Tropical Warm Pool Diurnal Variability (TWP+) data set and research plans. She invited for an inter-sessional meeting of the DVWG and other GHRSST working groups on two themes of particular relevance to Australia scientists: researching DV over the Tropical Warm Pool (kick-starting the full exploitation of the TWP+ data set) and SST retrieval and validation over the Southern Ocean.

AUS-TAG, the Applications and User Services Technical Advisory Group, addressed the Users Manual, a "onepager" concept for first-time SST users, a dashboard concept for the RDACs and several new data discovery and access tools.

HL-TAG, the High Latitude Technical Advisory Group, discussed efforts to improve cloud and ice masking. Users are calling for the highest possible resolution of sea ice products, ideally for one data set where all ice data have been merged and different resolutions are reflected in the uncertainties. Future work includes developing algorithms for high latitude SST and IST, reanalysis products, lake ice products, and validation with in-situ measurements.

R2HA2, the Rescue and Reprocessing of Historical AVHRR Archives Working Group, met for the first time. It agreed on the next steps to identify and locate historical archives (pre-2000) of AVHRR HRPT and LAC data, and to copy them to a central location. R2HA2 will define in the next year a common L1P format for storing these data.

IC-TAG, the Inter-Comparison Technical Advisory Group, added a new objective to 
GHRSST XII -

Proceedings, Edinburgh

GHRSST Project Office

its Terms of References: "to assess and improve the specification of error in the L4 analyses". This issue is under active discussion in the group, in addition to ongoing inter-comparison work. A three-part paper on the GMPE, L4-SQUAM and HRDDS systems, which form the basis of inter-comparison in the IC-TAG, is in preparation for submission to a special issue of Deep Sea Research II.

RAN-TAG, the Reanalysis Technical Advisory Group reviewed the current status and future plans of reanalysis activities, and defined and discussed the implementation of a Data Processing Framework for the SST Essential Climate Variable. Twenty-seven projects from the international SST reanalysis community were summarized. The quality metrics and standards (scientific, engineering and data management) of several high-level programs (GCOS, ESA CCI, NOAA CDR) are planned to be considered, together with community-established metrics. Further, the group called for an established, traceable, reliable network of in situ radiometers as reference measurement standard. The group thanked Ken Casey for his dedicated and energetic work to build and develop the RAN-TAG over the last 10 years. Chris Merchant will take over as new Chair.

EARWiG, the Estimation and Retrievals Working Group discussed the open issues related to the retrievals (especially for a $0.05 \mathrm{~K} / \mathrm{dec}$ ade stability requirement). Several new experimental approaches to the process of SST retrieval boosted fresh thinking about how more value/quality can be squeezed out from the data. The group welcomed the multi-sensor match-up dataset being built within the ESA $\mathrm{CCl}$, presented by Chris Merchant, as this sort of development was called for since the GHRSST VIII ST Meeting (Melbourne, 2007). Another priority of EARWiG is the calibration of the instruments.

LWST, the Lake Surface Water Temperature Working Group met for the first time. The Met-Offices have interest in
Issue: Final

Date: September 27, 2011

improvements of Lake Surface Water temperatures. Stuart MacCallum presented ARC Lake temperatures. Emma Fiedler tested the use of LWSTs in the OSTIA system. The lake definitions, retrieval issues, in-situ validation and error estimates as well as improved cloud masks needs addressing in the next years.

Finally, the Actions resulting from GHRSST XII were reviewed and approved. Several new Science Team members were proposed for election (Misako Kachi, Shiro Ishizaki, Viva Banzon, Bill Emery, Lei Guan, Gary Corlett, Jon Mittaz, Tim Liu). Echoing the sentiments of the entire Science Team, David Llewellyn-Jones thanked Craig Donlon for his more than 10 years of dedicated work as GHRSST Founding Chair. Craig Donlon wished GHRSST and its Science Team well under the new ST Chair, Peter Minnett, who thanked Craig Donlon for his leadership of GHRSST for over a decade and gave some perspectives of the New ST Chair. Peter expressed the debt that we all owe Craig for his tireless dedication to establishing GHRSST as a well-organized group that has become a model for how international groups can cooperate to achieve mutual aims. GHRSST is recognized by many as an example to be followed for how Climate Data Records can be derived from satellite data.

The next venue in 2012 will be in Tokyo, where GHRSST XIII will be hosted by JAXA in collaboration with JMA.

All public presentations, reference and background documents can be accessed via the web-site:

https://www.ghrsst.org/documents/q/categ ory/ghrsst-science-team-meetings/ghrsstxii-edinburgh/. 


\section{AGENDA FOR GHRSST XII WITH LINKS TO DOCUMENTS}

\begin{tabular}{|ll|}
\hline Sunday $26^{\text {th }}$ June 2011 \\
\hline 14:00 & $\begin{array}{l}\text { GHRSST informal meeting point at the entrance of the National Gallery, The Mound } \\
\text { (off Princes Street), Edinburgh EH2 2EL. }\end{array}$ \\
\hline 18:00 & $\begin{array}{l}\text { Informal dinner for those who are interested in going out in Edinburgh and catching } \\
\text { up on the SST gossip. Meet at } 6 \mathrm{pm} \text { at Ten Hill Place Hotel, } 10 \text { Hill Place, Edinburgh } \\
\text { EH8 9DS, Tel: 0131 662 2080, http://www.tenhillplace.com }\end{array}$ \\
\hline
\end{tabular}

\begin{tabular}{|c|c|c|}
\hline \multicolumn{3}{|c|}{ Monday, 27th June 2011 - Plenary Session Prestonfield Room } \\
\hline Time & Item & Speaker \\
\hline 08:00 & Registration and Coffee & \\
\hline 08:45 & Welcome and logistics & Chris Merchant \\
\hline 09:00 & Welcome address from University of Edinburgh & Prof Tudhope/Merchant \\
\hline 09:15 & Welcome from the ST chair, aim of the meeting & Craig Donlon \\
\hline 09:30 & $\begin{array}{l}\text { Review of outstanding actions from the Action list R3 } \\
\text { (please be sure to provide updates on progress to } \\
\text { GPO prior to the meeting to save time) }\end{array}$ & Craig Donlon \\
\hline & $\begin{array}{c}\text { Session 1: } \\
\text { GHRSST Components and Major Projects }\end{array}$ & $\begin{array}{l}\text { Chair: Craig Donlon } \\
\text { Rapporteur: Ken Casey }\end{array}$ \\
\hline 10:00 & Progress at the GHRSST Project Office & Andrea Kaiser-Weiss \\
\hline 10:20 & Progress at the Medspiration RDAC & Jean-François Piollé \\
\hline 10:40 & Progress at the Australian BoM RDAC & Helen Beggs \\
\hline $11: 00$ & Tea/Coffee Break & \\
\hline $11: 20$ & Progress at the MISST RDAC & $\begin{array}{l}\text { Peter Cornillon } \\
\text { for Chelle Gentemann }\end{array}$ \\
\hline $11: 40$ & Progress at the OSI-SAF RDAC & Pierre LeBorgne \\
\hline $12: 00$ & Progress at the Navoceano RDAC & Bruce McKenzie \\
\hline $12: 20$ & $\begin{array}{l}\text { Progress at the JAXA RDAC support to GHRSST } \\
\text { (GCOM, AMSR-E and other) }\end{array}$ & Misako Kachi \\
\hline $12: 40$ & Progress at the ESA AATSR RDAC & Nigel Houghton \\
\hline 13:00 & Lunch & \\
\hline $14: 00$ & Progress at the EUMETSAT IASI RDAC & Anne O'Carroll \\
\hline $14: 20$ & Progress at the MyOcean RDAC & Hervé Roquet \\
\hline $14: 40$ & Progress with GMPE and SQUAM systems & $\begin{array}{l}\text { Matthew Martin and } \\
\text { Alexander Ianatov }\end{array}$ \\
\hline $15: 00$ & Progress at GDAC & Ed Armstrong \\
\hline $15: 20$ & Tea/Coffee Break - Deadline for posters to be han & d in at meeting reception \\
\hline $15: 40$ & Progress at LTSRF & Kenneth Casey \\
\hline $16: 00$ & Space Agency support & $\begin{array}{l}\text { Chair: Craig Donlon } \\
\text { Rapporteur: Ken Casey }\end{array}$ \\
\hline 16:00 & NASA support to GHRSST & Peter Hacker \\
\hline $16: 20$ & ESA support to GHRSST & Oliver Arino \\
\hline $16: 40$ & EUMETSAT support to GHRSST & Anne O'Carroll \\
\hline $\begin{array}{l}17: 00- \\
17: 20\end{array}$ & Open Discussion rising key issues to address & $\begin{array}{l}\text { Moderator: ST chair elect } \\
\text { Peter Minnett }\end{array}$ \\
\hline
\end{tabular}




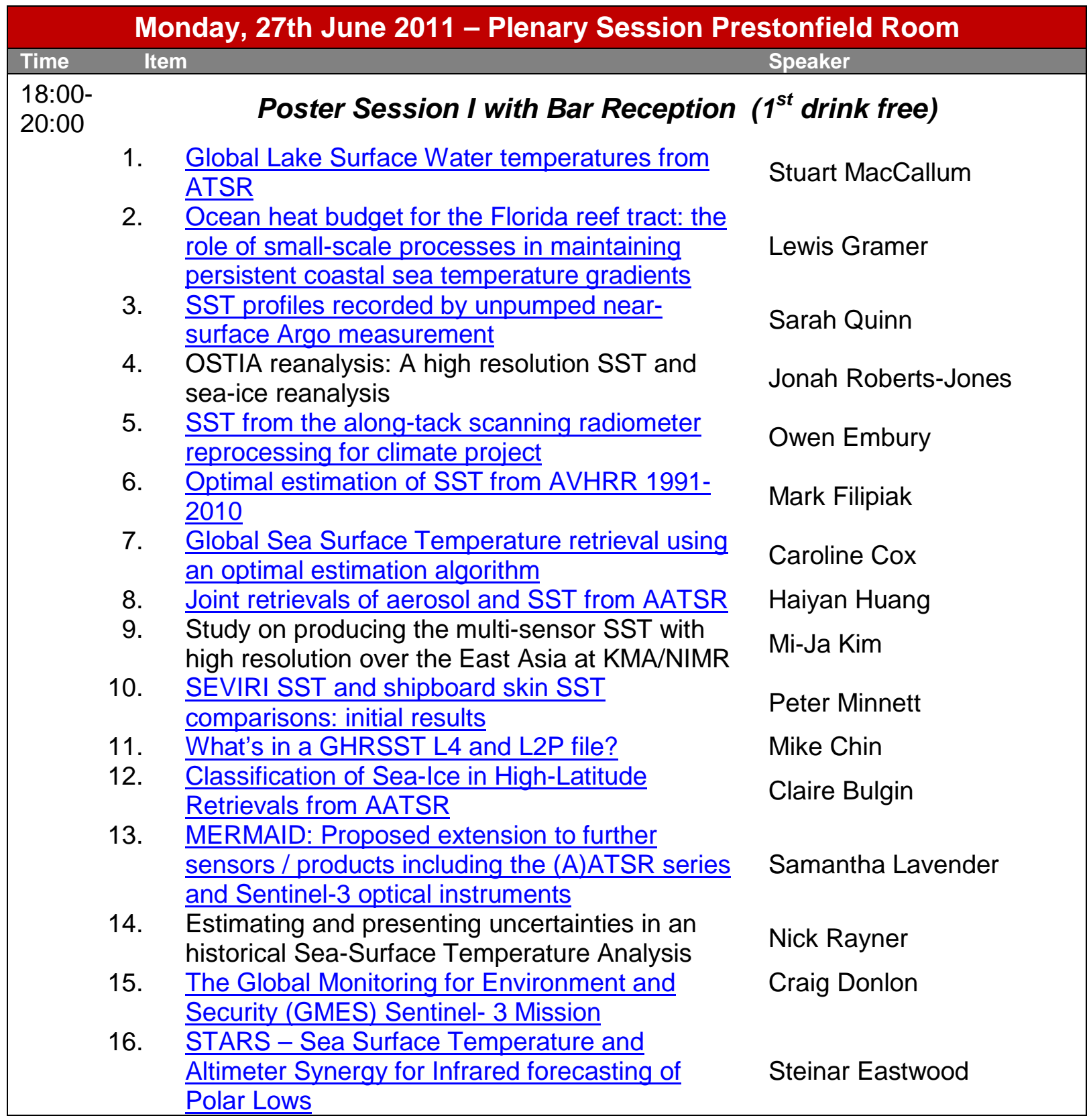




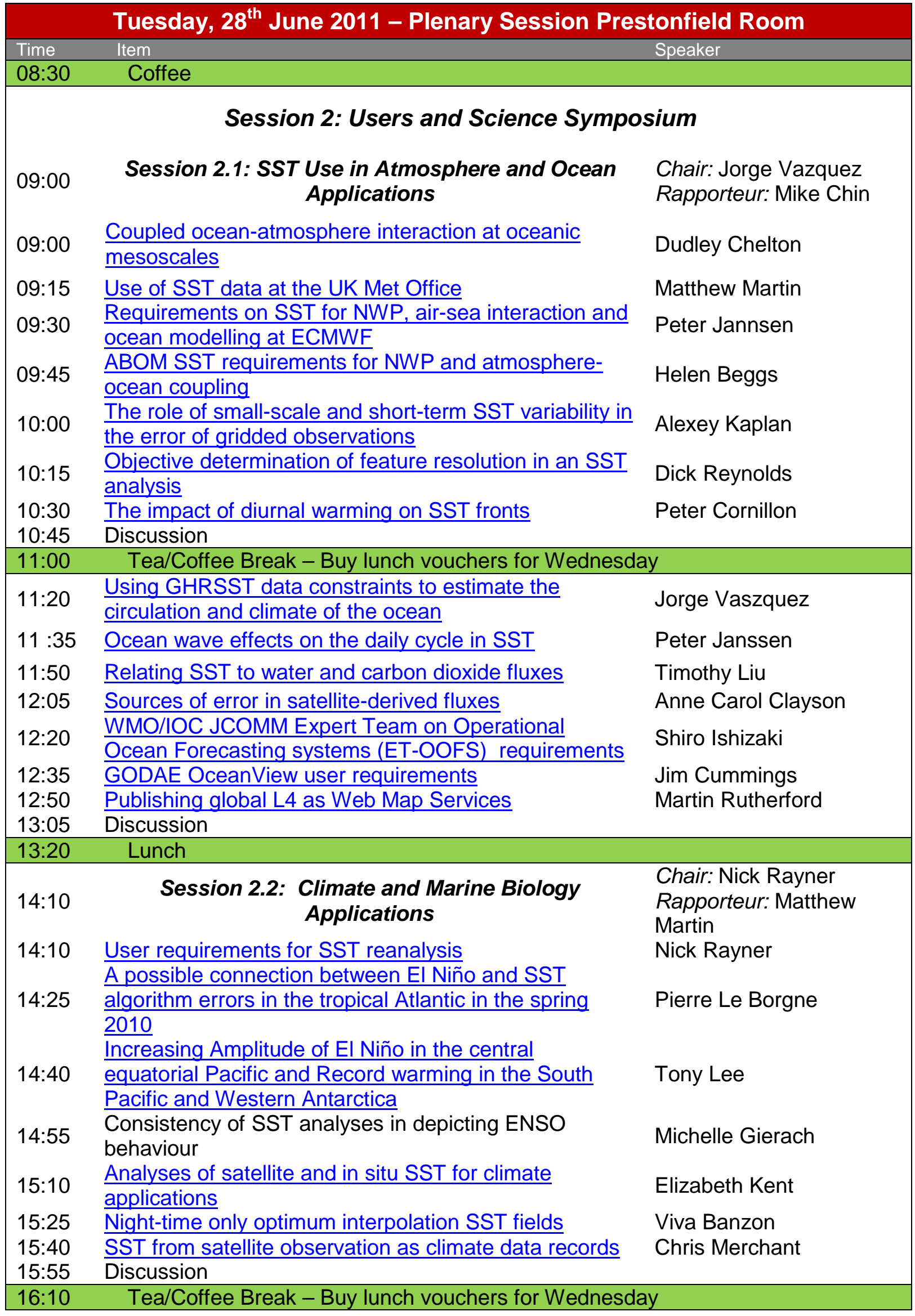


GHRSST Project Office

\section{Tuesday, 28 ${ }^{\text {th }}$ June 2011 - Plenary Session Prestonfield Room}

\begin{tabular}{lll} 
Time Item & Speaker \\
\hline $16: 30$ & GHRSST
\end{tabular}

16:30 GHRSST WINDSAT and AMSR-E version 7 SST Deborah Smith

16:45 Global 1-km Sea Surface Temperature (G1SST) for $\quad$ Yi Chao

17:00 IOCCG SST requirements Samantha Lavender

17:15 Use of SST in the ESA Storm surge project (eSurge) Jacob Hoeyer

17:30- Discussion

17:45 180 Posters Session II

20:00 For posters list and links please see Poster Session I on Monday

18:00- Advisory Council Session - Prestonfield Room 


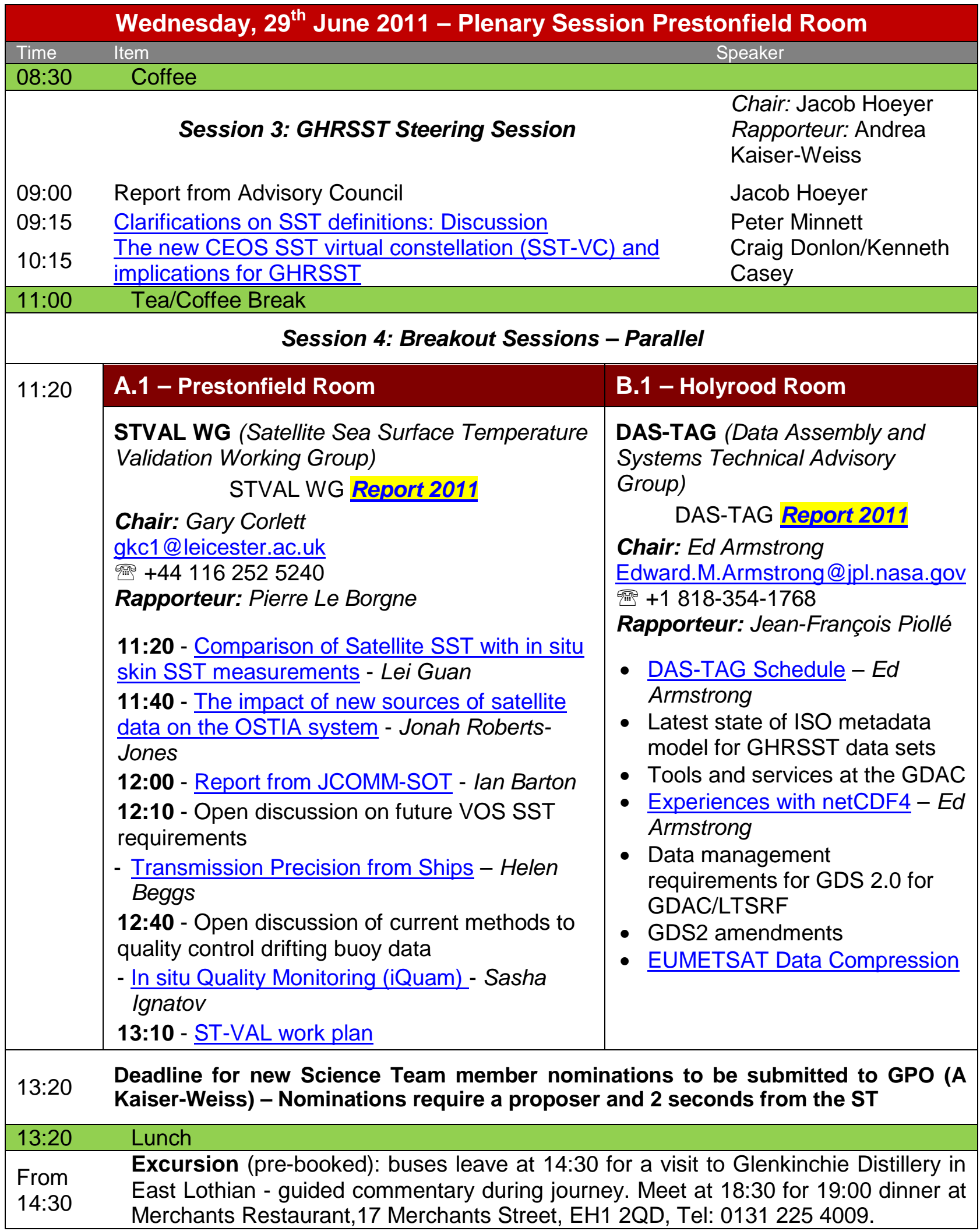




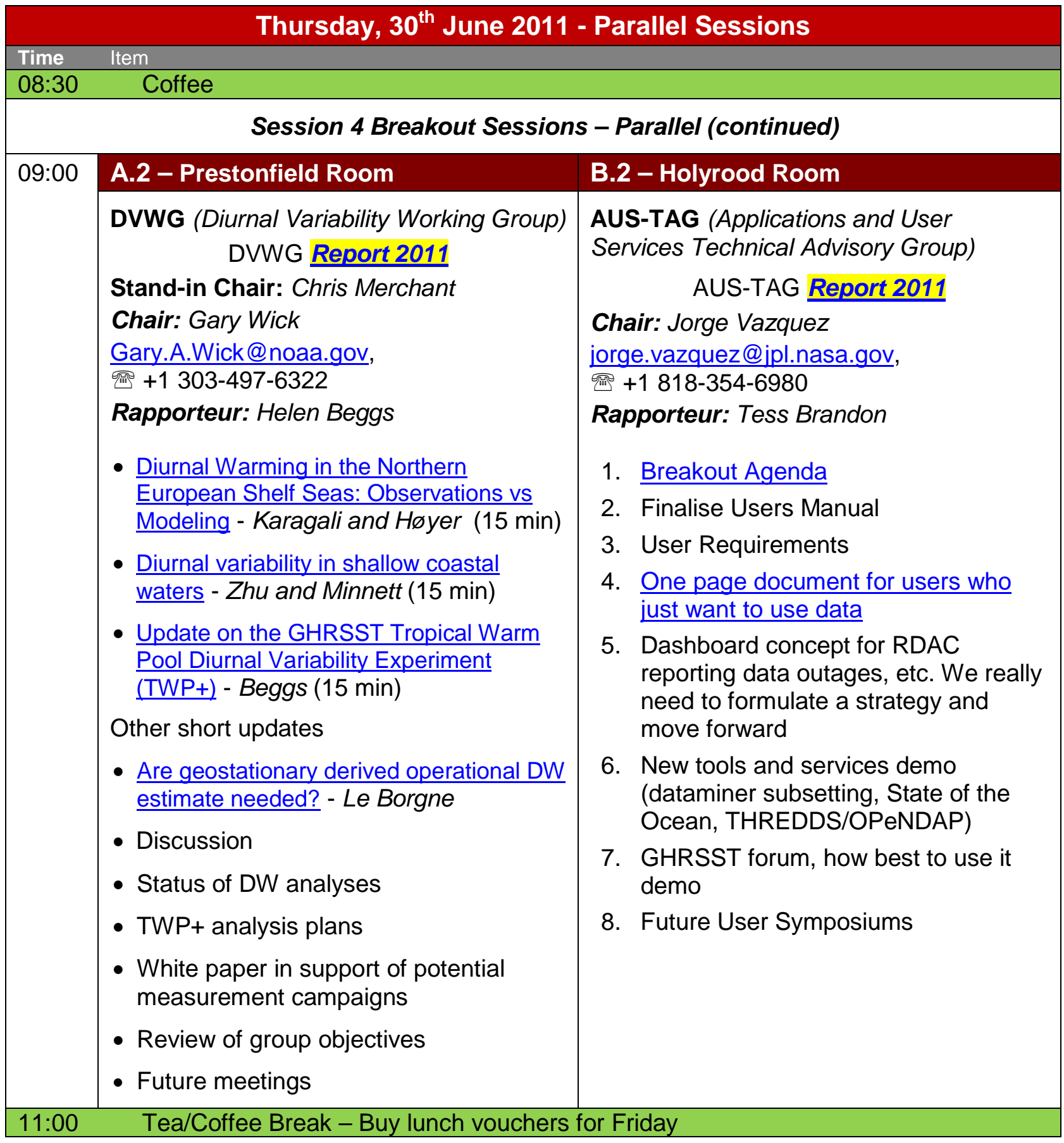




\begin{tabular}{|c|c|c|}
\hline \multicolumn{3}{|c|}{ Thursday, 30 ${ }^{\text {th }}$ June 2011 - Parallel Sessions } \\
\hline Time & Item & \\
\hline \multicolumn{3}{|c|}{ Session 4 Breakout Sessions - Parallel (continued) } \\
\hline $11: 20$ & A.3 - Prestonfield Room & B.3 - Holyrood Room \\
\hline & 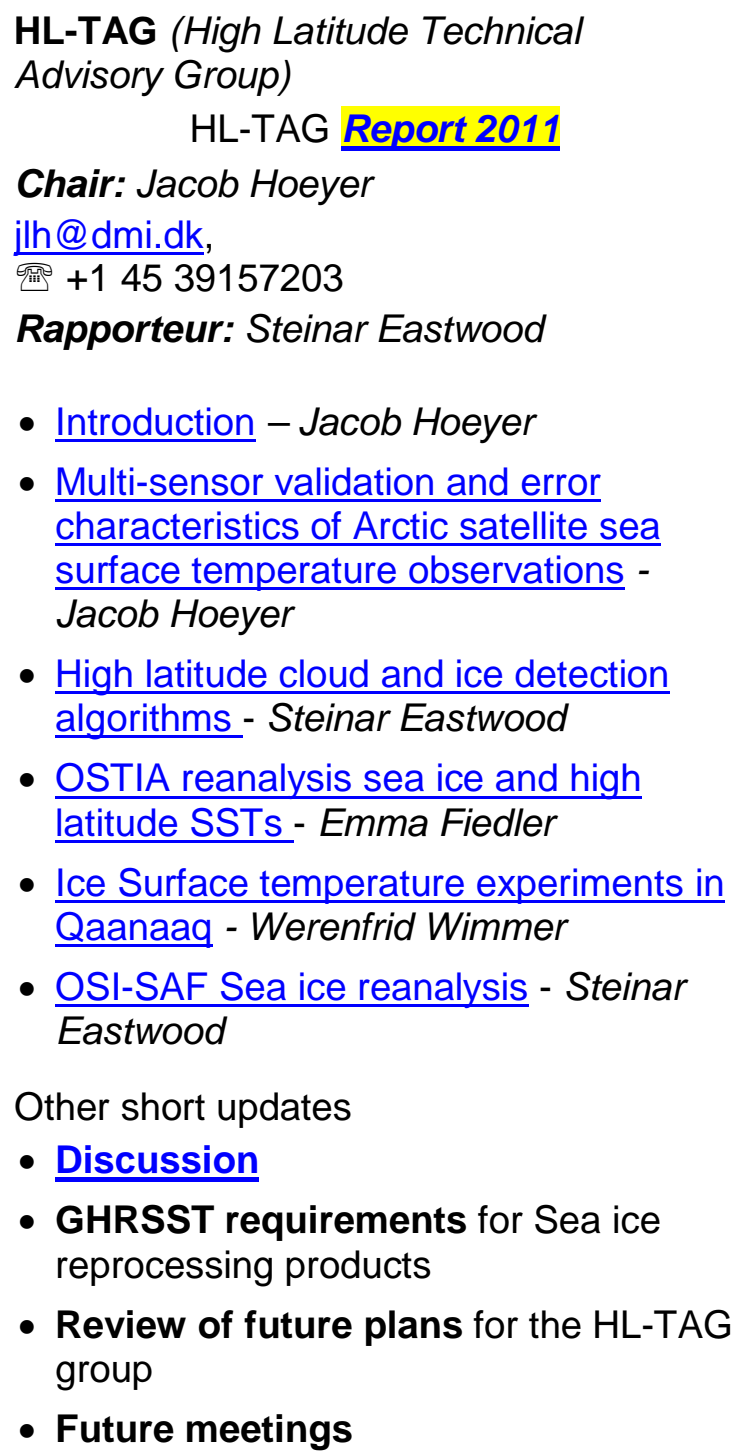 & $\begin{array}{l}\text { R2HA2 -WG (Rescue \& Reprocessing of } \\
\text { Historical AVHRR Archives Working } \\
\text { Group) } \\
\text { Chair: Peter Cornillon } \\
\text { pcornillon@gso.uri.edu } \\
\text { Rapporteur: Tess Brandon } \\
\text { Activites and plans }\end{array}$ \\
\hline $13: 20$ & Lunch & \\
\hline
\end{tabular}




\section{Thursday, $30^{\text {th }}$ June 2011 - Parallel Sessions}

Session 4 Breakout Sessions - Parallel (continued)

$14: 10$

$16: 10$

\section{A.4 - Prestonfield Room}

IC-TAG (The Inter-Comparison Technical Advisory Group)

\section{IC-TAG Report 2011}

Chair: Matt Martin

matthew.martin@metoffice.gov.uk

留 +44 (0)1392 886465

Rapporteur: Alexey Kaplan

\section{Objectives:}

1. To review L4 inter-comparison work over the past year in the form of presentations.

2. To review progress with the DSR-II paper on GMPE and L4-SQUAM.

3. To discuss the issue of analysis error estimation, particularly in the presence of incomplete data where there may be artificial gradients.

4. To make recommendations for improved L4 inter-comparison work over the coming year.

Detailed Breakout Agenda see:

https://www.ghrsst.org/files/download.php?m=doc uments\&f=110606144928-

ICTAGbreakoutagenda.doc

14:10 - 14:15 : Introduction, Overview and objectives - Matt Martin

14:15 - 15:10: Presentations on L4 work (15 minutes each)

- DDS - Dave Poulter

- Applying Barne's technique for daily SST (AVHRR and TRMM/ Microwave) Images Composition - Gutemberg Borges França et al.

- NOAA Sea Surface Temperature products geostationary SST L2P and blended SST L4 products - Eileen Maturi et al

- (5 min) High Resolution components of L4 products vs L2P - Michael Chin

- Add. Contributions (each 5 min or less)

- $\underline{4} 4$ error description

15:10 - 15:30: Progress with DSR-II

Intercomparison papers on GMPE and L4-

SQUAM - Matt Martin and Sasha Ignatov

\section{B.4 - Holyrood Room}

EARWiG (Estimation and Retrievals Working Group)

\section{EARWiG Report 2011}

Chair: Andy Harris

Andy.Harris@noaa.gov, 臸 +1 301-763-8102 x169

Rapporteur: Chris Merchant

- Introduction - Andy Harris

- Operational use of NWP model outputs in satellite SST calculations - Pierre LeBorgne et al

- New approaches to the infrared Atmospheric Correction algorithm - Minnett et al

- Development and Evaluation of Sea surface Temperature Algorithms for GOES-R ABI using MSG SEVIRI as a proxy Petrenko et al

- The calibration of the Broadband Infrared sensors onboard NOAA satellites

- - Mittaz and Harris

Invitation to participate in Algorithm Selection exercise with Climate Change Initiative for SST - Chris Merchant

- ORAC-Caroline Cox 


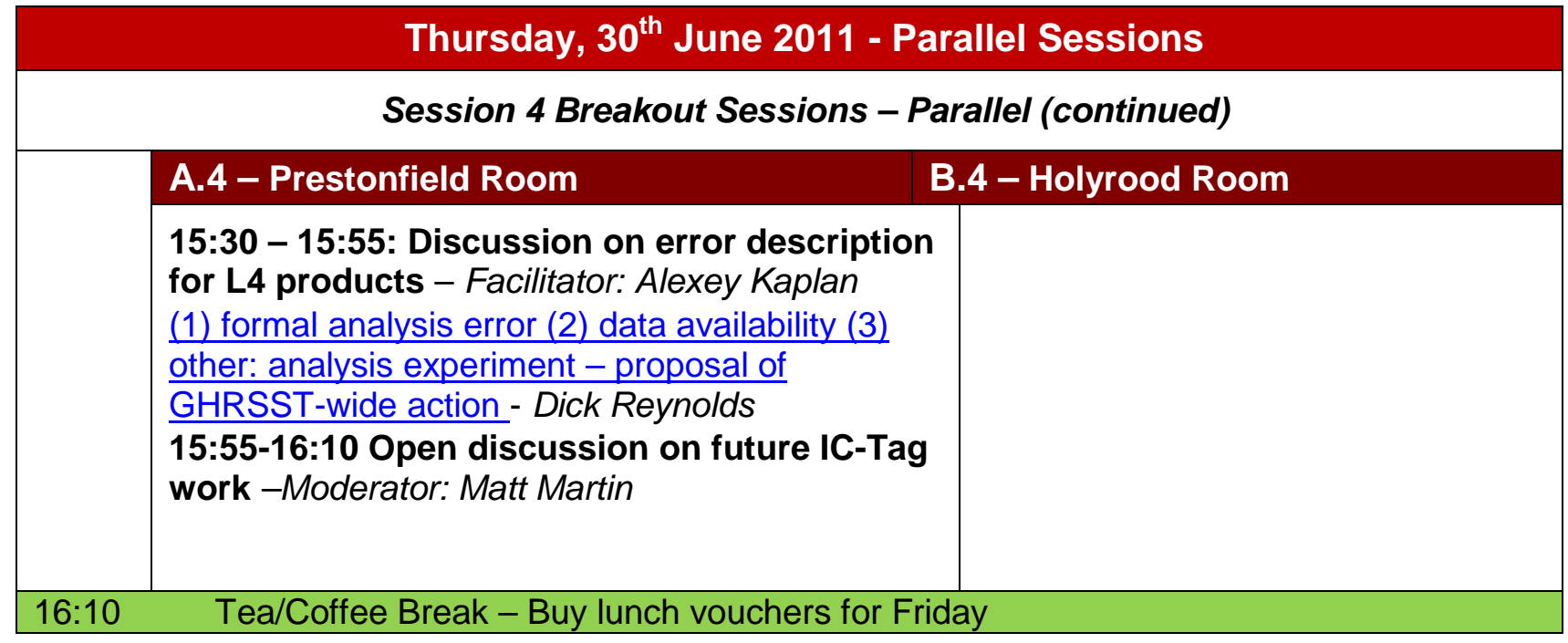




\section{Thursday, 30 $0^{\text {th }}$ June 2011 - Parallel Sessions}

\section{Session 4 Breakout Sessions - Parallel (continued)}

\begin{tabular}{|c|c|c|}
\hline \multirow[t]{2}{*}{ 16:30- } & A.5 - Prestonfield Room & B.5 - Holyrood Room \\
\hline &  & 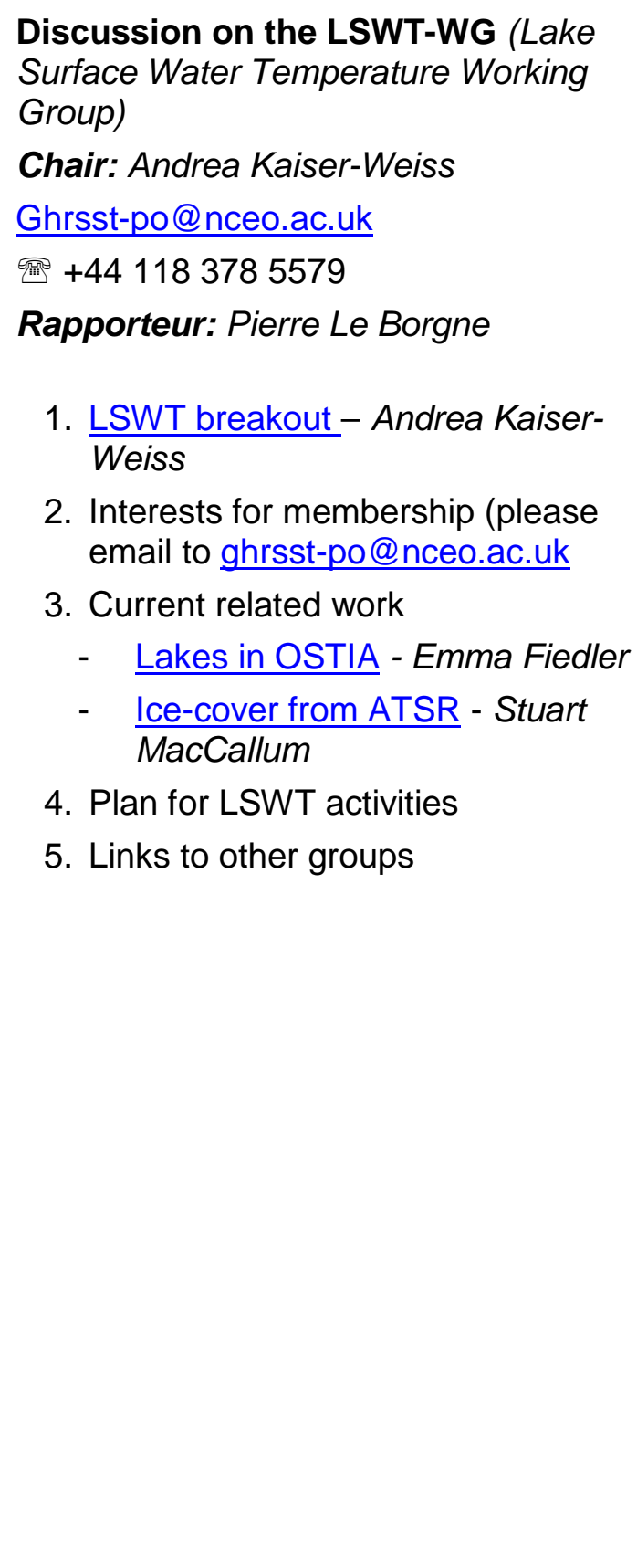 \\
\hline
\end{tabular}




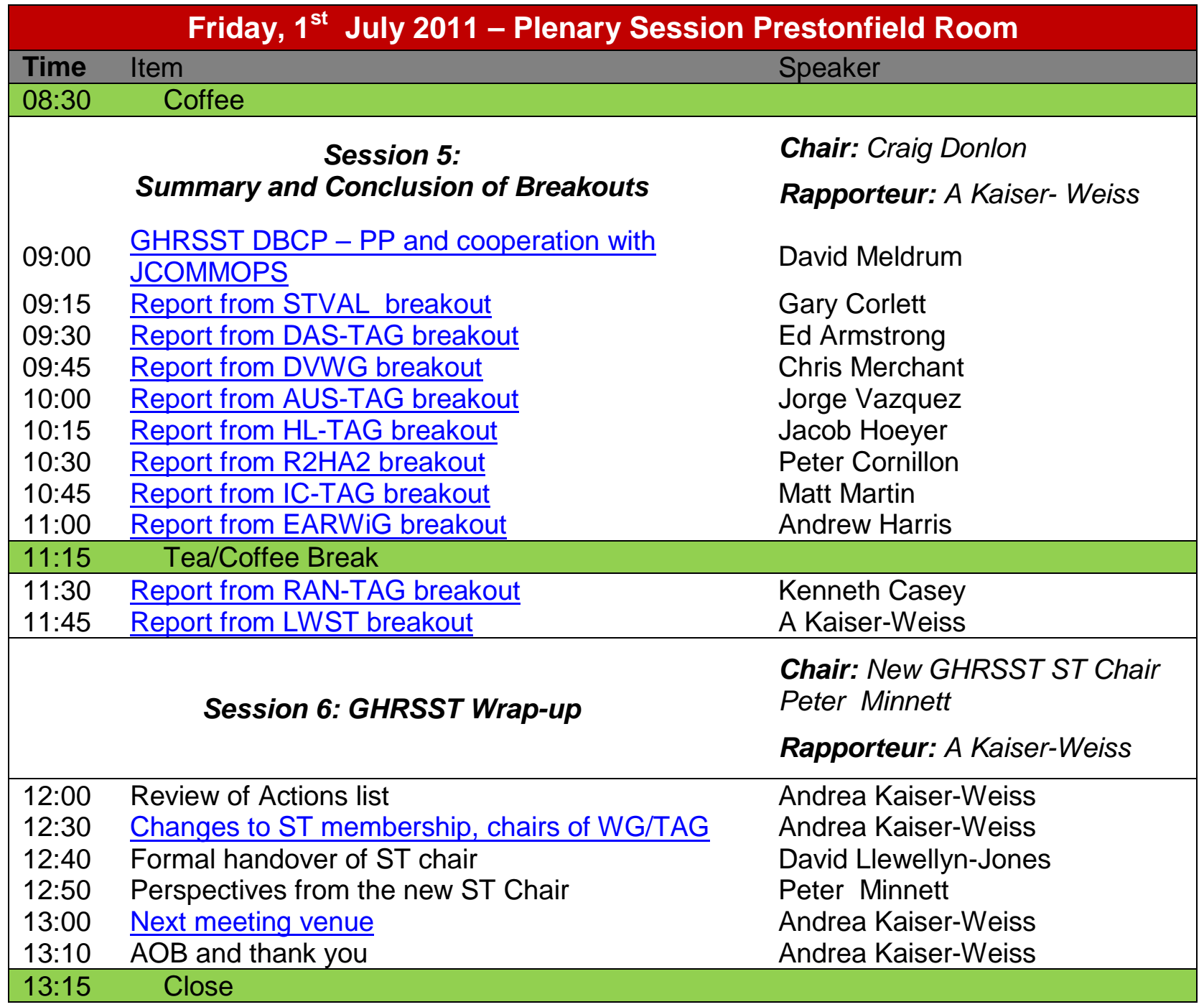


GHRSST XII -

Proceedings, Edinburgh

GHRSST Project Office
Issue: Final

Date: September 27, 2011

\section{CHAPTER 2: MEETING SUMMARY}




\section{WELCOME NOTE}

Hello:

....and welcome to Edinburgh, Scotland and the $12^{\text {th }}$ GHRSST Science Team meeting! Following the successful transition to the GDS-v2.0 earlier this year GHRSST is now focussing on critical scientific elements to ensure that high quality SST products and services are maintained for the benefit of users. As part of this work, a number of important activities have been initiated including the development of the GHRSST Development and Implementation Plan (GDIP), an updated survey of GHRSST User Requirements captured in a GHRSST User Requirement Document (URD), development of an appropriate framework within GHRSST to help coordinate the production of SST Climate Data Records and progress towards an initial GHRSST calibration and validation plan. These activities provide a framework in which GHRSST can focus its work but of course, these activities, while coordinated by the GPO, are only as successful as the inputs and discussions of the GHRSST Science Team! Our G12 meeting provides an important opportunity to critically review GHRSST activities and to maintain international consensus on the way forward for the future benefit of the stakeholders that have invested in the project and the users that depend on GHRSST products and services.

On a separate front, the future of GHRSST is becoming clearer as we continue to work closely with the Committee on Earth Observations Satellites (CEOS). The maturity of GHRSST calls for a formal relationship with CEOS, which coordinates the activities of Agencies for the sustained cost-effective collection of the satellite measurements on which GHRSST bases its work. We have developed our relationship with CEOS over the past 24 months through detailed discussions at many agencies and have concluded that, building on the success of GHRSST, a
CEOS virtual constellation for SST (SSTVC) should be established with GHRSST providing the implementation mechanism. This development provides a means for GHRSST to develop a deeper relationship with CEOS agency stakeholders.

At the G12 meeting we shall use breakout groups to focus the attention of world expertise within the GHRSST TAG and WG to review and develop their own work plans for the coming year. As you will know form your own experience, breakouts can be difficult to report and the teams should try and support breakout chairs and their Rapporteur with sufficient information to provide a report fit for the Workshop proceedings. This format has worked exceptionally well during previous GHRSST workshops and please come prepared with slides, questions and practical solutions that can be incorporated into GHRSST plans and specifications. As members of the international Science Team of GHRSST we all have an obligation to serve the RDAC and GDAC projects with a clear roadmap, based on our collective scientific judgment and consensus opinion to guide and nurture a globally integrated and sustainable high resolution SST operational system for the benefit of all.

After 10 years as the GHRSST Science Team Chair, I will be stepping down from this position at the G12 meeting (but remain a member of the GHRSST Science Team). Together with Ken Casey, I will continue to work with GHRSST through the CEOS SST-VC linkages that are now in preparation which is much more in tune with my current position at ESA. I also believe that change is good: as GHRSST marches forwards it is time to allow others to exercise their creativity and vision to lead GHRSST on. I would like say, from the bottom of my heart, thank you to the GHRSST Science Team, who have "made it happen".

You have all been brilliant and long may you remain so in the coming years as 
GHRSST XII -

Proceedings, Edinburgh

GHRSST Project Office

GHRSST continues to serve both users and producers of SST products and services. "Simply the Best" remains our challenge scientifically, operationally and practically. I have total faith in all of you to carry the GHRSST baton forwards and to continue the excellent work, results and outcomes of GHRSST into the future. Again, I thank you for all you have done and for the support you have provided "for better and for worse" over the past 10 years! To the new GHRSST chair: I wish you all the success for the future and can only say it will be a wonderful experience working with such a talented group as we have within GHRSST.

On behalf of the GHRSST Science Team I would like to take this opportunity to thank Chris Merchant and the University of Edinburgh team for all of their help and support in Hosting and preparing this workshop. Thanks also to all the sponsors
Issue: Final

Date: September 27, 2011

and you, the participants, who make these important events possible.

I also want to thank each of you for your continued contributions, support and dedication to GHRSST and I look forward to meeting you all once again in Edinburgh for a productive and stimulating workshop.

\section{Craig Donlon}

(Chair of the GHRSST Science Team) 


\section{WELCOME SESSION}

Chris Merchant welcomed the GHRSST XII participants and provided quick summary of the GHRSST related research and activities in Edinburgh, for instance the ATSR Reprocessing for Climate has been completed, and the current ESA CCI on SST is lead from Edinburgh.

The official welcome address was given by Prof Sandy Tudhope from the University of Edinburgh, who gave an introduction to the history of science and learning in Scotland, and stressed the presence of University of Edinburgh in climate and paleoclimate research. The School of geosciences was formed in 2002 and comprises now 400 researchers in geosciences which is the largest of its sort in the UK. He wished the assembly well in its work here.

Craig Donlon, the outgoing ST chair, welcomed the participants and reminded all on the aim of the meeting. He thanked the sponsors, University of Edinburgh, Chris Merchant, and the attendees. He reviewed the aims of GHRSST - best quality SST for short, medium, and long term applications He pointed out that the GHRSST strategy has not had to change fundamentally since the beginning, and that there are additions and alterations on the course. He reviewed the GHRSST organization and stressed the strong, vibrant Science Team remains the key. Two areas were stressed as needing some work:

- Lake Temperatures

- Configuration of the Advisory Council now that the CEOS Virtual Constellation (VC) for SST is progressing

The GHRSST Data Specification GDS2 was highlighted as a key recent accomplishment Also highlighted was the progress in the R/GTS:

o GHRSST data in wide use

o Uncertainties are being used but are a challenge and need improvements

o Endorse SST VC o GHRSST is "alive and adapting"

Donlon stressed the resources flowing into GHRSST, e.g. MISST-2, $\mathrm{CCl}$, the GPO, etc. His suggestions for the future included:

o Sentinel-3, 2-satellite system with a vast quantity of L1b data

o NPOESS Preparation Project (NPP)

o The Science Team to work with the GHRSST Project Office (GPO), help with the web presence and to provide input

- CEOS VC Implementation and connection to CEOS Working Groups (e.g., WG Climate <-> RAN-TAG)

o Revise and update the GHRSST Development and Implementation Plan (GDIP)

o Stay connected with the users - keep them involved. Listen and talk to them. Find ways to get their feedback

o Keep updating the GDS2

o Explore opportunities through VIIRS and Sentinel-3 for $\mathrm{Cal} / \mathrm{Val}$

o Consider GHRSST Technical Report series, like that of IOCCG. Perhaps one could be on $\mathrm{Cal} / \mathrm{Val}$

- Explore the idea of an SST ECV Data Processing Framework - new products, working with GCOS SST WG, etc.

o Seek better links to agencies in China, India, South Korea, Argentina, possibly through CEOS VC-SST.

Concluding, Donlon asked "Where are We Today?", and explained that the Phase-1 (Pilot Project) is complete, the Phase-2 (R/GTS System) is complete, and we are now in Phase-3: (Climate Data Records) which is well underway.

The Aims and Objectives of this meeting were summarized as following:

o Review status of R/GTS

o Review User Requirements

o Review GDS2 updates

o Help the new Chair and the GPO 
GHRSST XII -

Proceedings, Edinburgh

GHRSST Project Office

- Agree on future of GHRSST

o GDIP, Users, Science
Issue: Final

Date: September 27, 2011

o Enjoy yourself and remember you are the community that makes it happen!

Finally, Donlon reviewed the outstanding actions from the Action list R3. 


\section{SESSION 1: GHRSST COMPONENTS AND MAJOR PROJECTS}

\section{Chair: Craig Donlon'; Rapporteur: Kenneth Casey ${ }^{2}$}

(1) ESA/ESTEC, Keplerlaan 1, 2201 AZ, Noodwijk, The Netherlands,

Email: craig.donlon@esa.int

(2) NOAA/NODC, Silver Spring MD, USA, Email: Kenneth.Casey@noaa.gov

Progress at the GHRSST Project Office: Andrea Kaiser-Weiss

1. Reviewed the GPO and personal pet interests as well (Data assimilation, Argo)

2. Highlight role of GPO in overall GHRSST structure - direction from Advisory Council and Science Team

3. Reviewed the Statement of Working governing the GPO - 9 tasks from web page to GDS to User Requirements to Capacity Building

4. New GHRSST brochures (can be mailed)

5. Letters of Support can be mailed out

6. Reviewed the GHRSST web site

7. Reviewed GPO aims and required actions

o Web page/communications input

o User requirements input from ST and G-12 participants

o Several others...

8. Reviewed GHRSST12 aims

o Monday: record progress

o Tuesday: Collect User Requirements

o Wednesday: GHRSST strategy

o Thursday: Breakouts

o Friday: review

Progress at the Medspiration RDAC: Jean-François Piollé

1. Medspiration-2 is the follow on to Medspiration

2. Has new objectives

o Continue to deliver quality products

- Advanced services for SST

\section{- Support specific user needs}

3. Reviewed the ODYSSEA processing system and products

o Did a complete 2005-2009 reprocessing of the historical Medspiration products

o Developed a western tropical Atlantic ODYSSEA product to support SMOS, a South African regional product, and Great Barrier Reef product

o Lots of improvements, enabled since Sept 2010

o Showed details of the processing chain

o Many "sub products" are also generated and made available... native and adjusted SST in L3C and L3S

4. Implemented in situ validation datasets

o Put into daily netCDF-4 files in "MyOcean" format. Simpler than Argo, one depth

o Can ftp from IFREMER, including ones without global in the file name. 1. Supporting a generic matchup database framework http://www.ifremer.fr/matchupdb/i hm/exp/MDBInterface.swf and can do daily FTP updates in netCDF-4

5. Performs a routine monitoring of single sensor L3 data streams

6. Reprocessing for 2005-today to be released at end of 2011

o Downloaded data from the LTSRF

o Converted to netCDF-4 (about 10 $\mathrm{Tb}$ in total) 
o Stresses need for an open processing capability to be associated with the LTSRF and other large archives

o A large effort at CERSAT using cloud computing put 150 TB online, improved reprocessing speeds and access through thinks like OPeNDAP... http://www.ifremer.fr/opendap/cer dap1/ghrsst//2p/contents.html

7. New files will be sent to GDAC/LTSRF

8. Action: discuss possibilities of open processing system associated with the archive... ACTION G12/01: How should this work and how should it be set up on GHRSST web outreach sites? (Piolle, Armstrong, Casey)

\section{Progress at the Australian BoM RDAC: Helen Beggs}

1. Reporting on BLUElink and IMOS

2. Making HRPT AVHRR in L2P, L3U, L3C, and L3S GDS2 format.

3. Also making L3U for MTSAT-1R reprocessing back to 2006

4. GAMSSA and RAMSSA still in production. GAMSSA in GMPE and SQUAM

5. GAMSSA_skin and RAMSSA_skin will be presented tomorrow

6. L3S products still in development, but L2P, L3C, and L3U are available at ftp://aodaac2cbr.act.csiro.au/imos/ghrsst

7. By Dec 2011, GDS v2.0 L2P/L3U/L3C/L3S back to 1992 to GHRSST GDAC and IMOS for AVHRR HRPT

8. For MTSAT-1R/2, Real-time and reprocessed MTSAT-2 SSTskin hourly, 0.05 degree L3U files back to July 2010 to IMOS

9. Three-way comparisons conducted between MTSAT-1R SSTfnd, HRPT AVHRR, and Drifting Buoys

10. Working to improve the quality and quantity of in situ SST in the
Australian region through the IMOS Ship of Opportunity SST

o 13 vessels are putting real time data onto the GTS, available from http://imos.aodn.org.au/webportal and on GTS

- Errors are comparable to drifting buoys except for two Ferries which use engine intakes

Australia now putting Trackob format data onto the GTS

o Action: Advertise higher precision data availability in Trackob format on GHRSST web site.

11. With support from BLUElink and IMOS BoM aims to provide following netCDF 4 GDS v2.0 format files to GHRSST GDAC:

o Dec 2011: Reprocessed HRPT AVHRR SSTskin L2P, L3U, L3C and L3S from Australian and Antarctic groundstations back to 1992

o Dec 2011: Operational daily, global and regional SSTfnd L4 analyses (RAMSSA and GAMSSA) - currently in GDS v1.7 format

o Jun 2013: Real-time HRPT AVHRR SSTskin L2P, L3U and L3C from Casey and Davis Antarctic stations

Progress at the MISST RDAC: Peter Cornillon for Chelle Gentemann

1. NOPP MISST-2 "MISST for IOOS" partially funded last week (NOAA IOOS Program funding provided)

2. Reviewed the overall set of objectives, all subject to the final budget allocations

3. NASA SST Science Team Meeting, 24 November 2011, Coconut Grove FL

4. SST cluster at WCRP meeting in Boulder in October

5. HRPT back to 1982 on East Coast US, 1990 to present for West Coast US 
GHRSST XII -

Proceedings, Edinburgh

GHRSST Project Office

Progress at the OSI-SAF RDAC: Pierre Le Borgne

1. Current products from Geostationary

o Regular - hdf, grid, netCDF (not L2P)

o Medspiration - GDS v1.7 L2P

o Experimental - 1 hour, netCDF (not L2)

2. New Geostationary

o Starting June 2011 (or soon)

- 0.05 degree resolution hourly, MSG, GOES-E, in GDS2.0 L2P

o Saharan Dust Index - night and day

3. Present situation from Polar

o Regular

1. METOP granules (global, swath projection, $1 \mathrm{~km}$ resolution, netcdf gds v1.7)

2. METOP $12 \mathrm{~h}$ (global, regular grid, $0.05^{\circ}$ resolution, netcdf gds v1.7)

3. METOP and NOAA-19 NAR $12 \mathrm{~h}$ (European Seas, stereopolar, $2 \mathrm{~km}$ resolution, netcdf gds v1.7)

4. NOAA-18, NOAA-19 and METOP 12h, imbedded in the MAP product (see above)

o Experimental

1. NOAA-18, NOAA-19 and METOP 12h (European High Latitudes, stereopolar $10 \mathrm{~km}$ resolution)

4. Reviewed OSI-SAF research and development activities, frontal work, optimal estimation, diurnal warming

5. Change Calendar:

o June 2011: Availability of new OSI SAF products

1. MSG in netcdf GDSv2

2. GOES in netcdf GDSv2

3. Atlantic High Latitudes (AHL) in HDF 5

o February 2012: end of « old» OSI-SAF geostationary products

o March 2012: AHL in netcdf GDSv2
Issue: Final

Date: September 27, 2011

o No later than mid 2012: all OSISAF products in netcdf GDSv2

\section{Progress at the Navoceano RDAC:} Bruce McKenzie

1. $\mathrm{L} 2 \mathrm{P}$

o NOAA-18 global $9 \mathrm{~km}$, NOAA-19 global $9 \mathrm{~km}$, NOAA-19 regional $2.2 \mathrm{~km}$, Metop-A global $9 \mathrm{~km}$

o Including the brightness temperatures in the L2P files. Sea ice and wind speed are added by GDAC.

2. L4 K10 analysis - no in situ data goes into this. No changes since past talks.

o RMS of $0.63 \mathrm{~K}$ and bias of $-0.20 \mathrm{~K}$

3. Use a lot of L2P data from GDAC: AATSR, MSG, AMSRE orbital, MODIS Aqua... then generate a matchup database for each. Consistent error statistics are generated for each (independent of what was provided in the L2P, except for orbital AMSRE).

4. These L2P files are stripped down to just the best quality, the consistent errors are added, then are sent as flat binary through OCNQC to the Navy Coupled Ocean Data Assimilation (NCODA) system for use in regional Navy Coastal Ocean Models (NCOM) and the Hybrid Coordinate Ocean Model (HYCOM)

5. Showed the statistics they generate for each of the products

6. Looked at L2P data availability gaps of 10 hours or more - hoping to work with GDAC and its providers to reduce those

7. Concerns about the timeliness of delivery to and from the GDAC were discussed

8. Reviewed MISST-2 Year 1 activities, pending funding

o Switch L2P and L4 to GDS 2.0

9. Reviewed the NPP schedule - Launch 25 October 2011, VIIRS available Launch+42 days, SDRs validated at Launch plus 13 months 
10. Testing NPP readiness using the proxy dataset (MODIS data reformatted into NPP HDF5)

11. Hope to have GDS V2 NPP VIIRS by July 2012, pending funding - likely 2 $\mathrm{km}$ resolution, processed using their own algorithms not the IDPS SSTs

Progress at the JAXA RDAC support to GHRSST (GCOM, AMSR-E and other): Misako Kachi

1. Provided status of AMSR-E on Aqua

2. Provided status of the AMSR-2 instrument for GCOM-W.

o Wall of the test building collapsed in the earthquake, contaminating the clean room. Proto-flight model was contaminated, but not serious damage was suffered. Remaining test delayed while the building was repaired.

o Now, after cleaning, all the components were tested and found to work normally!

o A recovery schedule has been created.

- Launch still scheduled for Japan Fiscal Year 2011 (late 2011 to early 2012)

3. Gave status of the GCOM-W ground system

o Professor Taikan Oki from Univ. of Tokyo is the lead of the science team

o Standard product similar to AMSR-E.

o Research product will be defined after selection of 3 RA PIs

o Near real time product will be distributed via sftp

4. Standard products to be distributed via http and sftp. Launch plus one year is when general users will be able to access the data, after cal/val initial phase

5. Research products to be sent from EORC

(http://sharaku.eorc.jaxa.jp/GCOM W/ ), including browse images and cal/val monitoring pages
6. GCOM-W Data Distribution Service:

o In preparation for AMSR2, AMSR and AMSR-E standard products (and real-time products for special users).

- Enable to search by three ways

1. choose by categories and/or geophysical parameters

2. choose by looking explanation of products

3. choose by name of satellites and/or sensors

o User friendly interface

o Format transfer of product files from HDF5(4) to

1. GeoTIFF, TIFF

2. netCDF (netCDF 4 classic model,CF-1.4)

3. HDF5 (for AMSR/AMSR-E, whose original format is HDF4)

- Available by sftp for heavy users

7. GCOM-C1 is progressing through tests of its SGLI sensor

o SGLI has a $250 \mathrm{~m}$ resolution mode

o Launch is scheduled around 2014

8. Status of GHRSST JAXA Server

o Password protected ftp server. Please send request to ghrsst@jaxa.jp, then I will send you back access information.

o Currently distributes AMSR-E near-real-time data in GDS 1.6.

o Operation was stopped during electric outages in Tsukuba area caused by the earthquake in March, but resumed within a week.

9. Transfer to GDS 2.0

o Have been working on update of GDS format version to 2.0. Modification of program is almost completed.

o Can start to distribute new format AMSR-E SST in L2P and L3C via server soon. 
o New fields in GDS 2.0 dt_analysis (OISST), wind_speed and sea_ice_fraction (AMSR-E L2 SSW and SIC)

10. Considering how to make TRMM/VIRS in GDS2 format as well. Currently waiting for ECMWF data to include as wind_speed field. ECMWF has approved the request for the winds but has not established the transfer yet.

11. Future Plans -provided an extensive set of activities out to 2016

Progress at the ESA AATSR RDAC: Nigel Houghton

1. Purely operational processing. No science in this center.

2. ATSR-1 and 2 Archives, and the AATSR archive, all in ENVISAT format

o Approx. 40 TB via ftp and HTTP

3. L2P services

o ATSR-1, ATSR-2, and AATSR historical products available by ftp and media

4. Lots of missing data with reason

5. Beginning to deal with long term preservation aspects

6. Matchup Database provided for whole series as an ESA product

7. ESA L2P is version 1.5

8. Reviewed the Near Real Time L2P AATSR process - sent on to PO.DAAC

9. Presented the NRT L2P performance - L2P only takes 10 minutes to generate once received, but

10. The Archive L2P (GDS 1.7 in netCDF3) can be given in LTO-4 tapes. About 1 TB.

11. Looking into conversion to GDS2, netCDF-4 - planned for October 2011

12. Improvements to UK-MM-PAC and ground systems underway.

Progress at the EUMETSAT IASI RDAC: Anne O'Carroll
1. Reviewed the Metop-Infrared Atmospheric Sounding Interferometer (IASI) instrument on Metop

2. Spectral range 3.62 to $\mathbf{1 5 . 5}$ microns, $2112 \mathrm{~km}$ swath width, and resolution of $12 \mathrm{~km}$ at nadir.

3. Doing a L2P core product since March

- Demonstration product available from 24/03/2011

o 'Core' L2P, but includes ECMWF wind-speed

o Skin SSTs, GDS2.0 version

o Product available from EUMETSAT EO Portal in nearreal time (rolling archive)

o https://eoportal.eumetsat.int/user Mgmt/login.faces

o Two registrations necessary initially, then $\mathrm{ftp}$ access available

o All data archived in UMARF http://archive.eumetsat.int/umarf

o OSI-SAF likely to provide 'full' L2P product including validation MMD in 2012

o EUM.MET.DOC.10.0472 Validation of IASI L2Pcore Sea Surface Temperature (www.eumetsat.int)

4. Matchup statistics at night (bias of $0.31 \mathrm{~K}$, stdv $=0.32$ ) and day (bias $=-$ $0.15 \mathrm{~K}$ and $\mathrm{stdv}=0.33 \mathrm{~K}$ ) reviewed

5. Monthly 3-way statistics generated: Overall global errors:

o $\quad$ IASI $=0.27 \mathrm{~K}$

o Metop-A AVHRR $=0.14 \mathrm{~K}$

o drifting buoy $=0.20 \mathrm{~K}$

6. Show complementary benefit to AVHRR

7. Future planned upgrades to IASI L2 PPF:

o New cloud detection method based on artificial neural networks (end 2011)

o Address slight angular dependency

o Inclusion of band 3 (shorter wavelengths) in retrieval at nighttime 
o Use of OSI-SAF sea ice edge information

o Detection of dust layers for flagging and possibly correction

8. Metop-B planned for launch 02 April 2012

Progress at the MyOcean RDAC: Hervé Roquet

1. http://www.myocean.eu.org/

2. 7 MyOcean regions (global, arctic, Baltic, NWS, IBI, Med Sea, Black Sea)

3. Five MyOcean Production Centres - 5 TACs (Thematic Assembly Centres), 1 global and 6 regional MFCs (Monitoring and Forecasting Centers)

4. SST TAC partners: UKMO, M-F/CMS, IFREMER, NOCS, CNR-ISAC, DMI, and Met.no

5. After registering, you can get access to the data through FTP and an OPeNDAP/TDS service

6. Big Achievements:

o Operational acceptance of SST TAC NRT production, as part of MyOcean V1 system (December 2010)

o Production, validation and delivery of a 23 year (1985-2007) global SST re-analysis, based on the OSTIA analysis system

o Implementation of a routine quality monitoring of input L2P products based on IFREMER MDBs

o Implementation of common metrics for the quality monitoring and validation of all SST TAC near real-time $L 3 / L 4$ products

o Both displayed at http://projets.ifremer.fr/cersat/Dat a/Quality-control/MyOcean-SSTQC/Control-Validation-MyOcean

7. SST TAC is strongly relying on the GDAC for near real time access to L2P SST products so recent reorganization at PO.DAAC was challenging. Would have liked more notice and parallel access.

8. Future Plans:

o Horizontal resolution increase of some regional L4 products (Baltic Sea, Arctic)

o New operational products (regional L3S product over Mediterranean and Black Sea, global ODYSSEA L4 product)

o Transition to GDS V2

1. Need for detailed schedule of the GDS V2 transition from all $\mathrm{L} 2 \mathrm{P}$ producers, minimum of one month overlap highly desirable

2. Need for detailed information, available a long time in advance, on the GDS V2 transition at PODAAC FTP server level

o Use of new input L2P products : S3-A/SLSTR, NPP/VIIRS...

o Development of an operational Ice Surface Temperature analysis

o Increase in the time sampling of L3/L4 products (diurnal variability)

o Production of several SST reanalyses at global and regional scales

9. MyOcean-2 proposal has been accepted by the European Commission, negotiation phase will end in September 2011

Progress with GMPE and SQUAM systems: Matthew Martin and Alexander Ignatov

1. GHRSST Multi-Product Ensemble (GMPE) system runs daily, completing by 1500 UTC

2. Includes (via ftp) various L4 analyses from:

- CMC (Meteorological Service of Canada) * GAMSSA (Bureau of Meteorology, Australia) * FNMOC (Fleet Numerical Meteorology and Oceanography Centre, USA) * MGDSST (Japan 
Meteorological Agency, Japan) * NAVO K10 (Naval Oceanographic Office, USA) * NOAA AVHRR OI (National Oceanic and Atmospheric Administration, USA) * NOAA AVHRR/AMSR OI (National Oceanic and Atmospheric Administration, USA) * OSTIA (Met Office, UK) * RSS MW (Remote Sensing Systems, USA) * RSS MW+IR (Remote Sensing Systems, USA)

3. 94 Users Registered at MyOcean

4. Web Map Service available: http://data.ncof.co.uk:8080/ncWMS/go diva2.html

5. Summary Description Template (2 pages) for the L4 products was developed

6. Received descriptions from RTG, RSS (MW/IR), OISST (AVHRR, AVHRR/AMSR), JMA, GAMSSA, RAMSSA, OSTIA, CMC, available at https://www.ghrsst.org/ghrsst-

science/science-team-groups/ictag/ic-tag-14-summary-information/

7. Compared GMPE to Argo - median seems unbiased. Showed the results.

8. Also looked at horizontal gradients between input products and the GMPE median.

9. Evaluated whether the ensemble standard deviation is a good estimate of the error. Compared to monthly standard deviation in 10 degree bins of the GMPE median-minus-Argo. Found reasonable correlation. Slope of 0.36 implies that the spread generally underestimates the actual error in the GMPE median by that fraction.

10. Comparisons of GMPE with the independent Argo data showed the GMPE median to be more accurate than any of the contributing L4 analyses (globally and regionally). Standard deviation of $0.40 \mathrm{~K}$ globally. Done on a monthly basis (about 20th of the month) for the previous month.
11. Review of SQUAM was then provided by Sasha Ignatov

12. L2, L3, and L4 subsystems in the SQUAM system

13. Pathfinder V5.0 is the only $L 3$ in SQUAM so far. $13 \mathrm{~L} 4 \mathrm{~s}$ are in.

14. Showed some example diagnostics from the L4-SQUAM

15. Adding more Geostationary next year is a key focus as is adding in VIIRS as soon as it becomes available.

16. In future years, AATSR, AMSR-E would be added if possible. Also, in situ validation back to 1980 (currently only 1991 onward)

\section{Progress at GDAC: Ed Armstrong}

1. DMAS system is now fully operational for all GHRSST data streams

2. Reviewed the Data Management and Archive System architecture

3. Working with LTSRF, now serving up $100,000,000$ files! By comparison, the OBPG has served up 66 million files since 1992 (check that year)

4. Showed accesses by continent... more from Asia than Europe.

5. Covered the new PO.DAAC web site, all based on Drupal

6. New web site at http://podaac.jpl.nasa.gov

7. Updating all of the metadata... for example, the titles are of varying quality in the various metadata record

8. Granule export to NASA ECHO continues

9. Metrics harvesting of usage stats to be implemented on a routine basis

10. ISO 19115-2 metadata work continues - including work to expose inventories via OpenSearch

11. Showed the DataMiner (subsetter using OPeNDAP) and State of the Ocean (GoogleEarth)

12. Deploying the TDS later this year

13. POET has become "newPOET"

14. A new GHRSST forum is now available 
http://podaac.nasa.jpl.gov/forum/foru $\underline{\mathrm{m}} 4$

15. Improvements to L2P filling

16. Concern: GDS2 preparation and validation of datasets being done solely by GDAC

\section{Progress at LTSRF: Kenneth Casey}

The LTSRF operations are in constant progress with automatic daily acquisition and archive from GDAC, all data are made available online FTP, HTTP, and OPeNDAP, and WCS, WMS. All data searchable via NODC Ocean Archive System and through extensive metadata bases- both FGDC and original DIF, and FAQ/TXT/etc.

Current Archive Holdings are over 28 TB, 1981 - 2010, which are 59,982 accessions (RDAC/Sensor/Level/Date) resulting in more than 1,352,901 files:

L2P for AMSR-E, AVHRR-16, AVHRR-17, AVHRR-18 (GAC and LAC/HRPT) , MODIS Aqua and Terra, SEVIRI, AATSR, TMI, GOES-11, GOES-12, AVHRRMetop, AVHRR-18 HRPT NAR, AVHRR19 HRPT

L4 for OSTIA and EUR/MED analysis AND NCDC Daily OI (AVHRR-only and AVHRR+AMSRE), ODYSSEA Global, ODYSSEA Galapagos, ODYSSEA NorthWestern European Shelves, REMSS MW+IR OI, DMI North Sea Baltic, ABOM RAMSSA and GAMSSA, NAVO K-10

A live access server allows regional selection, maps, Hoevmoller plots, line plots, scatter plots.

- Active Archive efforts were made with:

- Browse/KML Graphic "Exerciser"

- Pathfinder V5.2

- CEOS SST-VC

- GCOS Intercomparisons

- Anomalous submissions - $60 \%$ resolved

In summary, it was another good year for the LTSRF and GHRSST Reanalysis.
Progress was made on both individual sensor reprocessing efforts (L2, L3) and on merged L4 products. Inputs to GCOS and CEOS-VC were given. Now in the Reanalysis Breakout session (Thursday) the stage for 2011-2012 will be set with the SST ECV Data Processing Framework.

NASA support to GHRSST: Peter Hacker

1. All missions except CLAREO and DESTINI are on track - those two are slipping to meet reduced budgets

2. Reviewed the ways in which NASA supports GHRSST - GDAC, NOPP, RDAC activity, Physical Oceanography program, new and ongoing satellite missions

3. NASA supports work focusing on establishing and validating the CDR quality of VIIRS SST.

4. Two MEaSUREs projects: MUR and HRPT reprocessing

5. NASA SST Science Team

6. Supporter of the new NOPP MISST-2 project

7. How GHRSST ST members can help:

o Deliver scientific breakthroughs and well-cited publications.

o Keep NASA and the rest of the Science Team informed of scientific breakthroughs and publications.

o Actively attend and support Science Team meetings.

o Respond as needed to requests from the Project Scientist and Team Leader for scientific and technical input.

o Enhance Interagency and international coordination

8. ROSES11 will focus on research, not product development - supporting use of GHRSST data for innovative physical oceanography research

9. SPURS 2012 Field Campaign reviewed this big program to study salinity processes, and how the modified Argo floats being put into use their could be useful with their 
high-res $(5 \mathrm{~cm})$ surface profile capabiltiy

10. NASA Issues and Perspectives:

o What does NASA bring to GHRSST

o Is there more that NASA can bring to GHRSST Science Team activities?

o Desirability of NASA's change of emphasis to RESEARCH in the future.

o Recognition that many multisensor activities are ongoing to optimize the utility of individual parameters (a broader arena or redefinition of CDRs).

o How do SST groups interface with a broader parameter focus in the future (salinity, density, upper ocean processes groups, etc.)?

ESA support to GHRSST: Oliver Arino

1. ESA Support to GHRSST summarized

2. 2003-2008: UK Met Office Project Office (Donlon)

3. 2010-2013: Univ. of Reading Project Office (Kaiser-Weiss)

4. DUE Medspiration

5. Climate Change Initiative (CCl) working under the international coordination of UNFCCC, GCOS, CEOS, individual partnet space agencies, GMES partners...

6. $10 \mathrm{ECV}$ teams were formed under $\mathrm{CCl}$, including one on SST (sea level and ocean color are the other ocean projects)

7. Described the Sentinel-3 configuration

8. Introduced the idea of a GlobCurrent project, including use of SST and ocean color patterns to determine surface currents. 5-9 March 2012, in IFREMER, Brest France. SAR, HFRadar, ADCP, etc...

EUMETSAT support to GHRSST: Anne O'Carroll

1. Reviewed the existing and future missions supported by EUMETSAT

o Metop A and B

o EPS-Second Generation under development

o New sensor under development, METimage

o Work on IASI taking place now

o Sentinel-3 in the future

o Future MTG missions (total of five)

2. Support the SAFs (Satellite Application Facilities)

3. Supporting CDR work 


\title{
SESSION 2.1: SST USE IN ATMOSPHERE AND OCEAN APPLICATIONS
}

\author{
Chair: Jorge Vazquez; Rapporteur: Mike Chin \\ Jet Propulsion Laboratory/California Institute of Technology, 2800 Oak Grove, \\ Pasadena CA 91109, USA, \\ Emails: Jorge.vazquez@jpl.nasa.gov and mike.chin@jpl.nasa.gov
}

In the session for "SST Use in Atmosphere and Ocean Applications" in the Users and Science Symposium, the following fourteen talks were presented:

1. Dudley Chelton: Couple oceanatmosphere interaction at oceanic mesoscales.

2. Matt Martin: Use of SST data at the UK Met Office.

3. Peter Jannsen: Requirements on SST for NWP, air-sea interaction and ocean modelling at ECMWF.

4. Helen Beggs: ABOM SST requirements for NWP and atmosphere ocean coupling.

5. Alexey Kaplan: The role of smallscale and short-term SST variability in the error of gridded observations.

6. Dick Reynolds: Objective determination of feature resolution in an SST analysis.

7. Peter Cornillon: The impact of diurnal warming on SST fronts.

8. Jorge Vazquez: Using GHRSST data constraints to estimate the circulation and climate of the ocean.

9. Peter Janssen: Ocean wave effects on the daily cycle in SST.

10. Tim Liu: Relating SST to water and carbon dioxide fluxes.

11. Anne Carol Clayson: Sources of error in satellite-derived fluxes.

12. Shiro Ishizaki: WMO/IOC JCOMM Expert Team on Operational Ocean Forecasting systems (ET-OOFS) requirements.

13. Jim Cummings: GODAE OceanView user requirements.

14. Martin Rutherford: Publishing global L4 as Web Map Services.

While these talks cover a wide range of topics, a general and brief summary is provided below.

SST continues to receive strong interests from the numerical weather prediction community $[2,3,4]$, where SST serves as an indispensable bottom boundary condition for the tropospheric dynamic models. Recent interests include: (i) lake surface temperature, including the Great Lakes of North America and the Caspian Sea, and (ii) ocean meso-scale SST pattern. The former can affect the regional weather especially for those lakes with large enough surface areas. The latter is believed to be important because of the SST feedback on small-scale wind patterns [1]. For example, the SST gradients (e.g., fronts) can cause atmospheric convergence or divergence above them, altering the small-scale wind patterns which can in turn affect the rain rate and cyclone intensity. Analysis based on high resolution satellite SST data can provide information on both lake surface temperature and SST gradients. However, there are following caveats: (i) the "foundation" or "bulk" SST values do not always translate well into the "skin" SST values needed for estimating the latent heat flux especially due to regional and seasonal dependence [10]; (ii) there are systematic voids in high-resolution SST data coverage which is again regionally and seasonally dependent [6]. The former calls for more scientific understanding and modeling of near-surface and air-sea interface dynamics, while the latter calls for a more explicit reporting of the sensor type and coverage for each analyzed SST value. Moreover, the diurnal warming can affect the SST gradients by masking them or creating fictitious SST gradients [7]. Understanding of diurnal variability of SST is thus important from this perspective as 
GHRSST Project Office

well. Wave breaking along with buoyancy can play a significant role in diurnal variation of SST even under low wind conditions [9].

Forecasting of ocean parameters including SST is also drawing interests [2,4], with an idea that dynamically forecast SST would allow a better estimate of latent heat flux used by the atmospheric forecast model than persisting the present SST values. Two GODAE follow-on programs support ocean forecasting activities: Expert Team Operational Ocean Forecasting (ETOOFS) and OceanView. ET-OOFS [12] has a stronger operational emphasis among the two. ET-OOFS uses a 0.1 by 0.1 horizontal degrees resolution ocean model with three layers assigned in the top 5 vertical meters. AVHRR and AMSRE SST data are assimilated using a 3DVar technique into the ET-OOFS model.
OceanView [13], on the other hand, has research emphasis that addresses estimation of both real-time SST and SST climate record. For assimilation of SST data, the choice of the assimilation method and its operating parameters can lead to significant differences in the vertical profiles of the model variables [8].

Issues associated with estimating longterm SST climate record have been raised by several speakers. A recurring problem is the lack of long-term stability in the SST data due to sensor variation and other sources of uncertainty [11]. Correlations among the SST data should be accounted for correctly in order to address the effects of small-scale and short-term variabilities on the long-term SST estimates [5].

Finally, a geographic information system (GIS) for visualization and distribution of the SST data has been reported [14]. 


\title{
SESSION 2.2: CLIMATE AND MARINE BIOLOGY APPLICATIONS
}

\author{
Chair: Nick Rayner ${ }^{1}$; Rapporteur: Matthew Martin ${ }^{2}$
}

(1) Met Office, Hadley Centre, Exeter, UK, Email: nick.rayner@metoffice.gov.uk

(2) Met Office, Fitzroy Rd, Exeter, UK, Email: matthew.martin@metoffice.gov.uk

A series of presentations were given which are summarised below. A discussion was then held on user requirements which is also summarised.

Nick Rayner. User requirements for SST reanalysis as part of the ESA SST $\mathrm{CCl}$ project.

Several hundred climate scientists were asked for SST requirements. Responses were received from over 100 scientists who had varied requirements. Some common themes were drawn out of these and used to inform product specifications. More details are in a document which is available from the project web-site (http://www.esa-sst-cci.org). Threshold (minimum), breakthrough (improvements) and objective (the ultimate aim) requirements were collected in order to make sure the data produced by the product are useful. Key aspects of the requirements presented were: multidecadal records, temporal resolution, frequency of updates, levels of drift, uncertainty estimates. Responses were analysed by data level, assuming $2 / 3$ respondents were satisfied.

- Multi-decadal aspects: Threshold requirements had varied results. Breakthrough and objective requirements show a need for greater than 30 year record of SST. One reason is to observe oscillations such as the Atlantic Multi-Decadal Oscillation (Knight et al.).

- Temporal resolution: Threshold requirements were for monthly and daily values. Breakthrough requirements were for some daily, some higher resolution data. Objective requirement was for subdaily resolution (eg. Minobe et al showed influence of Gulf Stream on the troposphere where high resolution (spatial and temporal) SSTs have large impact).

- Frequency of updates: Even at threshold, a reasonable number of users require updates within a day (e.g. Met Office GloSea4 seasonal forecast system runs twice a day in order to provide ensemble information for forecasts). Need compatible data for hindcasts and seasonal forecasts.

- Levels of drift: Threshold requirements were for a few hundredths of a degree drift. This needs to be done in order to monitor the small changes in climate monitoring.

- Representation of uncertainty: Should include full error budget (e.g. Kennedy et al. 2011) which is needed for detection and attribution studies. E.g. Ensemble of surface temperature data-sets are used to assess robustness of attribution of changes to various forcings.

Summary: These are challenging requirements. Other users need simpler and lower resolution information. Need to be flexible in design of reanalysis products to satisfy diverse user needs.

Llewellyn-Jones: Very useful for GCOS requirements. Will this be put forward to GCOS?

Rayner: Craig Donlon has represented these requirements at GCOS.

Donlon: Encourages everyone producing SST reanalyses to look at the document.

Pierre LeBorgne. Impact of El Nino on tropical Atlantic errors in spring 2010.

Large errors were observed in tropical Atlantic SSTs in spring 2010 in data from Meteosat 9 (day and night data) and 
Metop. Negative biases are in the data at $10-20 \mathrm{~N}$ in the Atlantic which also affects the GOES13 data. Errors (bias and standard deviation) were particularly large in April 2010, when looking at comparisons to buoy data, using MetOp and MSG data, and confirmed by NAVOCEANO results.

To study the reason for this, they studied errors in MSG data, and GOES-E data. A positive anomaly in tropical Atlantic developed in Spring 2010 which was underestimated by MSG but still seen in MSG and MetOp. Is this linked to EI Nino? They looked at data in the periods 2003, 2005, 2007, 2010. Positive anomalies in the Atlantic were only seen in 2005 and 2010. Both these periods correspond to negative anomalies in MSG data. Why should these MSG errors be linked to El Nino?

Observed and predicted errors in April 2010 were much larger than the previous year. Which atmospheric conditions cause these errors? Differences between 2009 and 2010 showed: large positive anomalies also seen in OSTIA; ECMWF winds are much lower; surface humidity is also higher. These features are consistent with a reduction in the trade wind speeds which lead to a northward displacement of the ITCZ. Total column water vapour (TCWV) increased in 2010 in the region. Humidity profile also increased along $25 \mathrm{~W}$ compared to 2009. MSG errors are sensitive to humidity profile errors which is the underlying cause link of errors in SST measurements to El Nino.

Summary: positive SST anomalies in the tropical Atlantic were seen in 2005 and 2010 and were related to the end of El Nino periods. Atmospheric humidity profiles change after El Nino. They plan to update the MSG data processing to cope with anomalous atmospheric profiles.

Barton: He has also seen a similar issue with anomalous water vapour profiles.

Janssen: There was an upgrade to atmospheric system in 2010 (resolution) which may have affected the total column water vapour in the ECMWF model.
Tony Lee. El Nino amplitude in the tropical Pacific and S Pacific and W Antarctica.

Warming in south-central Pacific (SCP), central-equatorial Pacific (CEP) and western Antarctica (WA) was seen in the Reynolds $1 / 4$ degree analysis. El Nino has recently been much stronger in the CEP than the eastern Pacific which is associated with a SCP warm anomaly. These anomalies are related through the Walker and Hadley circulations.

The Nino3 or Nino3.4 boxes are generally used to diagnose El Ninos and these are mainly related to the eastern Pacific. Nino4 is suitable for diagnosing central Pacific El Nino (CPEN). The data shows that Nino4 amplitudes have been increasing whereas Nino3 are decreasing (although not so statistically significant) over the last $\sim 30$ years. Also there is increasing frequency in the CPEN with roughly a factor of two increase.

Could this be caused by global warming? Trends in neutral or La Nina years are fairly small but trend in El Nino years much larger (0.24 C per decade). So averaging tropical SSTs could be misleading. It seems that larger El Ninos are seen rather than just a general global warming.

El Nino affects a change in the pressure pattern in the south Pacific. Recently (2010) there's been a massive affect on S Pacific SSTs which is also seen in the Argo data record. Causes include: reduced latent heat loss; ocean processes (meridional heat advection); reduced vertical mixing; reduction in MLD. Anticyclone over central $S$ Pacific also affects Antarctica SSTs and ice there due to diverted warm air from mid-latitudes.

Michelle Gierach. Consistency of SST analyses depicting ENSO behaviour.

Presented an investigation of intensity and frequency of central Pacific El Ninos (CPEN), following on from Tony Lee's talk. Investigated how the various SST products represent these over the past 3 
GHRSST XII -

Proceedings, Edinburgh

GHRSST Project Office

decades. The products compared were: OISST 0.25 AVHRR + in situ; OISST 1 deg AVHRR + in situ; ERSST 2 deg in situ only; HadISST1 1 deg AVHRR + in situ.

Calculated 3-month smoothed SST anomalies in Nino4 region, referenced to their own climatologies. Generally they agree quite well over that period. However, average maximum difference in peak amplitude and time of CPEN do vary between the products by up to $0.17 \mathrm{deg}$ and 1.7 months respectively. La Ninas have larger time differences between the analyses. 1984-5 and 2007-8 CPENs had 17 month difference in time of peak amplitude and 0.39deg.

Each product has different multi-decadal trends in CPEN amplitude. The GHRSST (Reynolds 0.25 degree daily) product has larger amplitude of 2009-10 El Nino which is probably mainly due to different spatial smoothing applied in the objective analyses studied. So small-scale variability needs to be retained in order to represent the peak values better, even for low frequency variability assessments. If they apply a spatial smoothing to the GHRSST (Reynolds) product, the amplitudes are reduced to be similar to the other products. Other factors could be: relative weighting of data; large-scale bias correction; data sources used. These other factors are currently being investigated.

Summary: The study highlights the importance of the analysis method in characterising ENSO characteristics and to the reliability of using historical reconstructions of SST to infer lowfrequency changes in ENSO behaviour.

Chelton: Would be useful to look at the OSTIA reanalysis to see if this issue is also there.

Kaplan: Results could be very sensitive to the latest El Nino amplitude.

Liz Kent. Analysis of satellite and in situ SST for climate applications.

GCOS view of observing system contains three types of observations: global
Issue: Final

Date: September 27, 2011

reference network (highly detailed and accurate observations at a few locations); global baseline network (large, but still limited, number of global locations); comprehensive networks (regional, national and international data to fill in the gaps $\rightarrow$ satellite data included in here). Looked at the various components of the SST observing system for climate and categorised them in terms of temporal and spatial coverage, stability, quality and whether they could be used as a reference data-set.

Reference/validation data possibilities:

- OceanSITES: only 20 high quality sites. Untested as reference standard.

- Research vessels: sparse data, moving platforms hard to use, untested as reference standard.

- Drifting buoys: Reverdin 2010, some buoys are biased by $>0.1 \mathrm{C}$ on deployment and others showed trends of $>0.1 \mathrm{C} /$ year. Inter-drifter biases had standard deviation of $\sim 0.3 \mathrm{C}$ (Kennedy et al.). Probably not reference standard.

- Moored buoys: ARC used operational moored buoys to assess stability. Neede extensive QC to get a subset of buoys which were suitable as a reference (used ARC data to do this). After QC, the moored buoys were suitable for assessing regional changes in the satellite time-series. For instance, small differences in the coastal region of USA could be detected when ECMWF fields were upgraded. Can only do this in the tropics and some coastal regions and had to remove annual cycle to do the analysis. So these data are ok but need a lot of work to get a reference data-set.

The ICOADS in situ archive is often used for validation exercises. Not all research or OceanSITES data are available in ICOADS, and high quality data are not always easily identifiable. ICOADS Value Added Data-set (IVADS) project has been funded to do data adjustments, improved data characterisation and QC which has just been funded. 
Can we make better SST climate datasets?

- Dual view satellite data can be used to assess errors in the in situ data, while in situ data are used to biasadjust IR and MW SSTs.

- How much effort should be put into high accuracy in a very few locations?

- Should traceability to standards in the marine environment be aimed for?

- How should this sort of sparse data be used in climate data-sets?

- How should skin/sub-skin/bulk SSTs be accounted for when comparing networks?

- What other variables are required to do this?

- Can observing system adequacy assessments be used and converted into a network design?

Hacker: Can Argo data be used as a reference for these various data-sets and connected historically to WOCE data?

Llewellyn-Jones: Why is ATSR categorised as only possibly a reference? LK: GCOS traceability to standards is important which isn't clear for satellite data.

Llewellyn-Jones: Radiometers on ships could be used as a reference if they were put more regularly on ships.

Viva Banzon. Night-time only optimally interpolated SST fields.

Looking at definition of climate data records (CDRs) and whether AVHRR SSTs meet that definition.

CDR is defined as a time-series of measurements of sufficient length, consistency, continuity to determine climate variability and change. Need $\sim 30$ years of data for this and AVHRR fits this, but requires large amount of calibration work. Reynolds SST is being used to assess climate models in IPCC.

The fundamental CDR (FCDR) comes from radiances (which require intercalibration between sensors); thematic CDRs are geophysical variables which should be derived from the FCDRs. At present, in situ data is used to "anchor" SSTs from the 1980s. In the 1990s intercalibration among satellites is also possible although there are problems, e.g. due to the Mount Pinatubo eruption.

Using the daily $1 / 4$ gridded gap-free analysis from NCDC, looked at AVHRRonly analysis from 1981. Currently the day-time and night-time data are both used in the analysis. This is being used to develop new algorithms for Pathfinder data. A test version of OISST with nighttime only data has been produced and compared to the day-night version. The day-night OISST is similar to the nighttime version, but there is a small impact of day-time data. The plan is therefore to use only night-time in situ observations with night-time pass satellite data using the same methodology as daily OI. A constant bias correction of ships is carried out as well as bias correction of AVHRR. Similar patterns are seen in night-time and daily OI. Both products show a seasonal bias.

So should they just produce a night-time analysis? What artefacts are seen in the night-time-day-time analyses, calculated as monthly averages? Generally the daytime is warmer as expected. Saw an artefact in the ocean near South Africa. This was an isolated event which was smeared out in the bias correction. The analysis appears to be sensitive to cloud contamination.

Looked at number of days with $>30 \%$ coverage of satellite data. Cloudy areas, e.g. in the Antarctic are not accurate due to lack of data which needs to be communicated to the users. One issue with this is that using night-time only data means less data are going in. There is a need for better cloud screening of the L2 and L3 data. List of producers of L2 and L3 data to discuss these issues with cloud screening aspects. Will become available as part of the STVAL group.

Comment: Mittaz is working on L0 and L1 calibration for FCDR aspects which he will be presenting in EARWiG. 
GHRSST XII -

Proceedings, Edinburgh

GHRSST Project Office

Chris Merchant. SSTs from satellites as Climate Data Records.

Investigating the nature of errors and uncertainties. Three propositions:

- Systematic and random decomposition is inadequate for SST because of partially correlated errors. Affects average for climate applications and treatment of SSTs for assimilation.

- Spatio-temporal correlations in SST errors come straight from the physics and retrieval process.

- Developers of SST CDRs should develop and deliver physically based uncertainty information.

Approaches to uncertainty and validation:

- Validation gives the uncertainty estimate, e.g. mean discrepancy is bias, standard deviation is random uncertainty.

- Validate both the SST and their uncertainty estimates. Estimate the uncertainty directly from physics and retrieval method and assess it using independent data.

Limitation of SSES:

- For most uncertainty levels there is a reasonable relationship with the actual error.

- Once the uncertainty is smaller, it is more difficult to measure/validate because the validation data-sets have errors of similar magnitudes.

- For accurate SST retrievals, SSES errors are overestimate the actual errors.

Can predict the uncertainties, and these agree with other error estimates (e.g. from OSTIA), showing example from LeBorgne. The spatial patterns of uncertainty change and are correlated with errors in the atmospheric state.

In ARC and ESA SST $\mathrm{CCl}$ projects the uncertainties are decomposed into: uncorrelated (random), synoptically correlated (pseudo random, and can be simulated), large-scale correlated components (systematic). Other aspects, not taken into account currently, include
Issue: Final

Date: September 27, 2011

contaminants (e.g. high aerosol events), other sampling effects and stability errors.

This approach was prototyped in ARC. Pixel noise is propagated through the algorithm and to the cell level. Sampling uncertainty is represented by accounting for the amount of a cell which has been observed. Algorithmic uncertainties are also taken into account.

Validated this approach by:

- Compared to drifting buoys (and removed impact of buoy errors) to the predicted uncertainty.

- Reasonable correlation between the error and uncertainty although slightly underestimated.

- Bias calculated between ARC and buoys compared to ARC uncertainty. Good estimates for most uncertainty ranges.

- Chi square statistic measures the goodness of fit of actual error and estimated uncertainty. ARC provides a 1.7 value which implies and underestimation of uncertainty in ARC.

In SST $\mathrm{CCl}$ project: 3 uncertainty components will be attributed individually: uncorrelated, synoptically correlated and large-scale correlated.

Questions to the Science Team:

- What uncertainty information should be supplied?

- Where should this work go within GHRSST: EARWig or STVAL.

- SST CCl product spec extends GDSv2.0 in regard to uncertainty estimates: comments?

Donlon: Cloud detection errors - how should these be reported?

Smith (for Gentemann). Remote sensing systems AMSR-E and WindSat V07 SSTs.

Entire AMSR-E data-set has been reprocessed and produced in GDS1.6 format. Changes compared to the previous version include:

- GHRSST ancillary fields included: wind, vapour, cloud, rain. 
- Full L2P produced including dsst from AMSR-minus-Reynolds and OSTIA (OSI-SAF) sea-ice concentration.

3 major changes in the algorithm:

- Water vapour continuum absorption model was re-derived.

- Clear-sky bias in cloud water was removed.

- The beam-filling correction in the rain algorithm was modified.

Impact of these changes on the new version of the data are:

- Increased water vapour values.

- Changed range of cloud water values.

- Increased global mean rain rates (by about 16\%).

- New emissivity model.

- New post-hoc correction for SST.

A diurnal cycle correction was made to the Reynolds SSTs which are used in the algorithm. Post-hoc correction to SST is small at wind-speeds smaller than $15 \mathrm{~m} / \mathrm{s}$. Drifting buoys comparison for day and night showed generally good agreement with buoys but bias at high SSTs and high winds. This could be due to rain contamination. Night-time low-wind SSTs are significantly improved which is thought to be due to the diurnal correction to the Reynolds SSTs.

Diurnal warming in SSTs are significantly higher in the new AMSR-E values compared to V05. This was expected because of changes in the emissivity. They were validated from June 2002 June 2011 compared to fixed and drifting buoys. Standard deviation errors are $0.52 \mathrm{~K}$ against drifting buoys and higher for fixed buoys. There are larger errors off the east coast of north America.

Windsat was launched in Jan 2003 and data was made available from Feb 2003. It was designed to measure wind speed and direction but SST is available. Data are available through RSS from Feb 2003 to December 2010, although not available in real-time yet. The files include SST, wind speed, water vapour, cloud water, rain rate. More information on this data is available from www.remss.com/windsat.
Windsat SSTs have standard deviation of $0.52 \mathrm{~K}$ compared to drifting buoys with small bias $(0.02 \mathrm{~K})$, and slightly higher for fixed buoys. No huge areas of bias.

Reynolds: The high SST and high wind speed errors are worrying. Answer: RSS would like to improve the rain contamination flags.

Yi Chao. Global 1-km SST analysis for research and applications.

The production of the analysis was motivated by developing real-time coastal models for the California coastal observing system with 3DVar data assimilation. There were requests to do this for other regions so it was decided to do it globally for SST using a 2DVar version. The main users are IOOS, as well as regional and coastal users who need real-time $(<24$ hours) $1-\mathrm{km}$ data, comparable to the resolution of other coastal observations (e.g. HF radar). The analyses are meant for use in data assimilation and real-time forecasting and validation/visualisation, also for less sophisticated users.

Would like to be able to access all L2p data from GDAC and for distributing the G1SST data. This analysis is also involved in SQUAM and GMPE. www.sst.jp.nasa.gov displays images of the product.

Input data include: TMI, AMSR-E, GOES, MTSAT, SEVIRI, METOP AVHRR, MODIS, AATSR, NOAA AVHRR as well as the HRPT data from BoM. The spatial coverage of the data is about $82 \%$ at $25 \mathrm{~km}$, and about $50 \%$ for IR data.

The system uses a 2DVar blending algorithm, with persistence of the analysis from the previous day as a first guess. The error covariances vary with latitude/longitude and model the variations of correlations along and across the coast. Files which contain only the $1 \mathrm{~km}$ data are provided as well as the full gap-free analysis product.

Reserve 20\% in situ data for independent validation on a daily basis. Biases are approximately 0.16 and RMS errors are 
about 0.6K. Comparisons with geostationary data show large $(0.44 \mathrm{~K})$ bias too cold.

Future work: Look at issues with geostationary data; will try to reduce the bias to take account of diurnal warming; implementing a multi-scale 2DVar (with $25 \mathrm{~km}, 5 \mathrm{~km}$ and $1 \mathrm{~km}$ scales); retrospective analysis (before Sep 2008). It is also planned to implement a bias correction.

\section{Sam Lavender (on behalf of David} Antoine). IOCCG SST requirements.

IOCCG is endorsed by CEOS and is made up of space agencies, scientists (who sit on it for only 3 years) and managers. Annual meetings are held to discuss progress and strategy. Working groups produce reports. The group represents open-ocean, coastal and inland waters. Scientists are on IOCCG to do specific tasks, e.g. produce a specific report. These can be downloaded from www.ioccg.prg/reports ioccg.html.

Synergy between OCR (ocean colour radiometry) and SST sensors:

- Top of the atmosphere (TOA) radiances are available from both types of sensors, e.g. OLCl and SLSTR on Sentinel-3. Uses bands from both sensors to do atmospheric correction (currently only over land).

Requirements to enable more OCR/SST collaborations include: band centres and bandwidth as close as possible, pixel collocation, easy combination of the 2 products (e.g. formats). Joint users of OCR and SST include: qualitative approaches looking at patterns; indirect use; quantitative use. Examples of some joint work are: radar backscatter in SST and OCR, diurnal variability in both colour and SST, primary production modelling (temperature is a driving factor in primary productivity); assimilation of both SST and ocean colour.

Relevant satellite missions for SST/OCR studies include: MERISIAATSR, OLCI/SLSTR, MODIS, VIIRS/AVHRR.
Common SST/OCR requirements for the data production are:

- Common gridding logic for regional and global products.

- Long-term stability for climate-related studies.

- Same spatial resolution for OCR and SST.

- Temporal resolution (which generally depends on the application).

There should be more discussions between SST and OCR communities.

Donlon: Collocation of radiances from $\mathrm{OLCl}$ and SLSTR data will be available from Sentinel-3 in the L1c files.

Jacob Hoyer. Use of SST in the ESA storm surge project (eSurge).

eSurge is an ESA Data User Element (DUE) project designed to encourage the establishment of long-term relationships between user communities and Earth Observations.

A Storm Surge user consultation meeting was held in 2009 to define user requirements for the project including defining the priority EO information, services and areas of interest. They identified a number of champion users to provide guidance to the project.

The main user requirements were:

- Coastal altimeter products.

- SAR, passive MW and optical satellite products

- Historical archive data products derived from satellite, in situ data and models.

- Near-real time (NRT) service of products

- Training and documentation of the products.

- Impact assessment studies to show benefit of using the data.

The project will last for 30 months and started 2 weeks ago. It is using a large number of ESA and other satellite products. Phase-1 aims to prepare and qualify the eSurge system. Phase-2 aims to provide services and run impact assessments, and to hold training courses 
GHRSST XII -

Proceedings, Edinburgh

GHRSST Project Office

and user consultation meetings. Specific areas of interest are: European seas, Indian Ocean, Yellow Sea, and possibly other areas.

All input data will be processed to agreed format(s), archived and documented with a web portal for the EO data and images. This will be done as a NRT serviced in Phase 2. Input data is planned to be: altimeter, satellite SST data (OSTIA reanalysis data to start with and then higher resolution regional products), scatterometer, SAR, optical data, NWP and NOP model data, storm surge model outputs, water level in situ data.

The main project partners are: Logica (management), NOCS (altimetry and some modelling), DMI (data assimilation and merged altimeter and tide gauge products), CMRC (design of portal and training), KNMI (providing NWP data).

Another complimentary project is called eSurge Venice (specific to Venice area). More information is available from www.storm-surge.info.

Anyone interested to be a user or interested in providing input should contact Jacob.

After the presentations a discussion was held which is summarised below.

Vazquez: How should user requirements
be gathered from the various
presentations?

Donlon: The user requirements should be collated from the presentations during the day and then presented to the AUS-TAG and updated based on discussions.

Arino: Previously, requirements have been collected from the operational community and climate change community by groups such as ESA and NASA. GHRSST should capture "other" users.

McKenzie: NAVOCEANO can provide some user requirements to GHRSST and they are able to respond to user requirements if funding and time permits.
Issue: Final

Date: September 27, 2011

Llewellyn-Jones: Some user requirements are not feasible, and they therefore need to be peer reviewed.

Casey: Instruments will not be able to be changed in the long-term. Users always want as much as possible. Really the products will be defined by what is technically feasible.

Rayner: Uncertainty estimates are within our control though so these types of requirements can be addressed.

Merchant: Some users may have specific user requirements, and it would be useful to get feedback on existing products. (Some feedback on existing data sets is available in the SST_cci URD at http://www.esa-sst-cci.org.)

Donlon: Feedback needs to be shared and responded to in a coordinated way.

Minnett: There is an important role for user requirements in the design of the next generation of satellite instruments, but the requirements do need to be within reach. They should also be written in such a way as to support instrument development.

Lee: GHRSST should revisit GCOS and JCOMM requirements. They should own the requirements.

Rayner and Donlon: This was done for GCOS as part of the ESA SST CCl requirements.

Cornillon: There are many different user communities. We need to decide which communities to address. Some users want very simple data without lots of other data, other users want a lot of ancillary information.

Vazquez: Encourages people to use the GHRSST forum for this type of discussion.

Donlon: Suggests setting up some chatroom sessions at appointed times and on particular topics.

Merchant: LeBorgne's talk highlighted large scale anomalies in retrieval errors which are issues for detecting climate anomalies. Need to get a grip on those sort of errors. 
GHRSST XII -

Proceedings, Edinburgh

GHRSST Project Office

Kaplan: In IC-TAG want to improve error estimates. Does the user survey help with these decisions?

Rayner: Requirements on uncertainty
Issue: Final

Date: September 27, 2011

estimates were mainly for simple information, but some users do require more complicated information. We should accommodate both. 


\title{
SESSION 3: GHRSST STEERING SESSION
}

\author{
Chair: Jacob Hoeyer ${ }^{1}$; Rapporteur: Andrea Kaiser-Weiss ${ }^{2}$
}

(1) Centre For Ocean \& Ice, Danish Meteorological Institute, Lyngbyvej 100, Copenhagen $\varnothing$, 2100, Denmark, Email: Jlh@Dmi.Dk

(2) NCEO, University Of Reading, Department Of Meteorology, Reading, Uk, Email: Ghrsst-Po@Nceo.Ac.Uk

\begin{abstract}
Jacob Hoeyer (out-going AC Chair) reported from the Advisory Council meeting. All Actions from the last meeting have been closed. The Advisory Council endorsed the decision by the Science Team for Peter Minnett to take over as the ST Chair. The AC congratulated the incoming Chair and welcomed him to the post. The Advisory Council also endorsed the process saying it was a clean and democratic proposal, to be followed in future, and allow more time for the election process. The recommendations of the Council are that: no vice-chair or co-chair will be appointed, the term will be for five years with a one year overlap, and that reelection will also be possible. The voting for the new ST Chair will be at the start of the $5^{\text {th }}$ year of the incumbent's tenure, so that the new ST Chair can become involved. Also the incumbent can stand for re-election if they wish. The AC reinforced the relationship between the $A C$ and the CEOS virtual constellation as a positive step forward. The AC recommended that the GHRSST Development and Implementation Plan (GDIP) should cover 2012-2022. The AC recommended that for transition to GDS2.0 at least 1 month overlap should be allowed (i.e., 2 parallel datasets produced). The AC recognised the workload the transition implies for GDAC and urged DAS-TAG to organise a shared code for GDS2.0 compliance checking. The AC requested DAS-TAG to consider the geolocation issue pointed out by a study of EUMETSAT. AC requested that the ST-VAL TAG responds to input from Helen Beggs about GHRSST user requirements on SOT, JCOMM and ESURFMAR. Pierre Le Borgne raised the issue of a common tool to remap L2P.
\end{abstract}

Misako Kachi (JAXA) was elected as the new Chair of the AC. The AC thanked the outgoing Chair for his efforts.

Peter Minnett lead a discussion on clarification of the current GHRSST definitions. Aim is to have definitions as accurate and concise as possible. When SSTskin is recorded, wavelength need to be given. Not easy if the retrieval uses different wavelengths, here several wavelengths need to be specified. A need for an additional piece of information on wavelengths together with the GHRSST definition on SSTskin, and the need to educate the users was identified. SSTfoundation was confirmed to be useful to have for applications in NWP. Also, they have been found most consistent. The current definition of SST foundation at the GHRSST web-page was found fit for purpose. Andy Harris asked for a better cartoon on the web-site.

Craig Donlon explained how GHRSST will serve as CEOS VC-SST. The CEOS SST-VC will provide a formal connection to GHRSST stakeholders. It will increase the international visibility of the GHRSST success, will lead to better agency participation, better "buy in".

CEOS will not exercise control about what GHRSST is doing.

Following match-up in structure of groups:

\begin{tabular}{|l|l|}
\hline $\begin{array}{l}\text { CEOS VC } \\
\text { group }\end{array}$ & $\begin{array}{l}\text { Respective GHRSST } \\
\text { sub-group }\end{array}$ \\
\hline WGISS & DAS-TAG \\
\hline WGCV & ST-VAL \\
\hline WGCLIM & RAN-TAG \\
\hline WG Edu & AUS-TAG \\
\hline WGCV & IC-TAG \\
\hline
\end{tabular}


GHRSST Project Office

CEOS VC-SST proposed objectives:

1. Develop and improve satellite SST Essential Climate Variable (e.g., CEOS WG-Climate)

2. Improve user feedback to CEOS Agencies

3. Minimise duplication of existing activities

4. Development and optimization of the SST constellation: maximise synergy benefits

5. Develop and implement metrics for SST services, products and users (feedback statistics to CEOS)

6. Coordinate consensus reference documents

7. Encourage timely access to products

8. Improve EO SST calibration, intercalibration and validation (WGCV QA4EO implementation)

9. Develop training activities for satellite SST practitioners (WGEdu)

Liaise with the other virtual constellations
We propose to implement the SST-VC building on the existing Group for High Resolution SST (GHRSST) framework. Using this approach, the CEOS SST-VC has instant access to:

- A baseline SST virtual constellation system of systems

- Internationally agreed SST products, services and user outreach services

- Consensus technical documentation for the constellation

- A functional coordination mechanism active at the international level

CEOS will interface to GHRSST activities using the GHRSST Stakeholder Advisory Council. The benefits of GHRSST serving as CEOS VC-SST are that with this the CEOS SST activities are strengthened. Craig Donlon requested CEOS Agencies to nominate representatives to GHRSST Advisory Council. 


\section{SESSION 4.1.A: ST-VAL BREAKOUT}

\section{Chair: Gary Corlett ${ }^{1}$; Rapporteur: Pierre Le Borgne ${ }^{2}$}

(1) University of Leicester, UK, Email: gkc1@le.ac.uk

(2) Centre de Météorologie Spatiale, Météo-France, Lannion, France, Email: Pierre.LeBorgne@meteo.fr

Lei Guan gave a presentation on an ISAR deployment on the Dong Fang Hong 2, which operates in South China, East China, and Yellow Seas. The instrument is the fifth ISAR radiometer built by NOCS and it was a participant radiometer in the 2010 CEOS IR radiometer intercomparison at NPL. Initial instrument problems with the rain gauge were overcome and the instrument has now been operating autonomously since November 2010. Initial results are very promising in such tough areas to validate satellite data. A mechanism for formal data exchange is required.

Jonah Roberts-Jones presented the impact of updated SEVIRI and GOES-E on OSTIA. There is a clear qualitative impact but little quantative impact from both datasets. He raised the question whether we can provide correlation scales for SSES? This will be a topic for the MyOcean meeting planned for early November.

lan Barton reported from JCOMM Ship Observations Team (SOT) VI and Observations Coordination Group (OCG) IV and summarised recommendations from GHRSST to both meetings and subsequent actions on GHRSST. The topic of SSTs from ships that participate in the International Ocean Carbon coordination Program (IOCCP) was briefly discussed and further work to look at the exact locations of this data is required.

\section{Ship Measurements}

It is clear that GHRSST data can be used to better understand the different biases in the various ship measurement methods. Thus GHRSST is to lead and report to SOT/OCG at their next meetings, and also maybe directly to the ship operators.
Current sources of ship match-ups to GHRSST data include:

- CMS match-up dataset

- iQuam

- Met Office Hadley Centre comparisons to ARC

- MyOcean (MDB/Met Office stats)

ST-VAL will coordinate feedback to SOT/OCG and Helen Beggs to act as primary liaison to SOT. Consideration will be given to using a central resource such as the GDAC to distribute results and findings to VOS providers.

The OCG requested advice from GHRSST on:

1. Mounting of radiometers on ships (Werenfrid Wimmer to lead)

2. Procedures for improved contact measurements (Helen Beggs to lead)

ST-VAL will coordinate best practice technical documents on the above two topics.

The SOT requested advice on future data transmission resolution for ship measurements:

- $0.01^{\circ}$ resolution in SST is endorsed by GHRSST

- GHRSST also noted the position reporting resolution was $0.01^{\circ}$ for ships without automated weather stations (AWS) and $0.001^{\circ}$ for ships with AWS

- Liz Kent noted $10 \%$ resolution for cloud cover contradicts the existing climate record, which is in octal resolution

- Helen Beggs to report back to SOT (Henry Kleta and Pierre Blouch) 
- Helen also asked about GHRSST requirements for XBTs? None forthcoming as data not yet exploited.

\section{QC of Drifter Data}

Drifting buoys are currently main reference dataset for GHRSST, used for common reference for SSES. Initial discussions at the Joint Working Group meeting in Boulder had shown that GHRSST was making some progress towards implementing common methods but was still some way of completion. A general discussion on how best to proceed took place.

Two main options were considered:

- A single source of in situ data used by all L2P providers has many disadvantages, not least operational agencies having to rely on third party data

- A consistent blacklist is the preferred option as current methods are somewhat arbitrary.

Sasha Ignatov presented iQuam tool http://www.star.nesdis.noaa.gov/sod/sst/iq uam/.

It was proposed:

- CMS to synthesise blacklists (Pierre Le Borgne to lead)

- ST-VAL to produce best practice technical document for drifting buoy QC (Sasha Ignatov to lead)

It was also suggested to use ICOADS for climate data set validation for improved Metadata.

\section{DBCP Pilot Project}

Many updated drifters now deployed. A list is kept on GHRSST website https://www.ghrsst.org/ghrsstscience/science-team-groups/stvalwg/dbcp-ghrsst-pilot-project/

ST-VAL will make an initial assessment for next DBCP meeting in September 2011 (Gary Corlett), need a volunteer to report to the DBCP (Geneva). Post session note: David Meldrum agreed to do this is no one else from ST-VAL could attend.
Many L2P providers requested the data be made available via the GHRSST website as they do not have direct access to the BUFR encoded raw data. Gary Corlett will discuss this possibility with Pierre Blouch to see what can be done.

\section{Use of Argo within GHRSST:}

- GHRSST has need for independent global validation measurements

- For retrievals, skin to depth models (including diurnal variability)

- High accuracy Argo data offers such capability

- Closest to surface measurement (3-5 m)

- Near surface profiles (un-pumped and second sensor)

- Argo is a recent addition to the GHRSST portfolio

- Not yet used for retrievals, validation etc.

- Relatively small number of daily near-surface measurements provided by current Argo network ( 300 per day)

- Significant benefit to GHRSST to reserve Argo data for independent validation and improvement of nearsurface models for now

- Revisit at future science team meetings as scientific knowledge improves and Argo network expands

The near-surface measurements from Argo profiling floats provide a valuable, very accurate new data set that has yet to be fully exploited within GHRSST. As such the ST-VAL group has requested that the GHRSST Science Team recommends that Argo data be reserved within GHRSST for independent validation of retrievals, products, models and analyses, and that the use of Argo data within GHRSST shall be reviewed at each future GHRSST Science Team meeting.

\section{ST-VAL Workplan 2011-2016:}

The GHRSST Project Office had asked all work groups to prepare a work plan for the 
period 2011 to 2016 prior to the meeting. The GHRSST Advisory Council subsequently requested this be changed to a plan for 2012 to 2022 following their meeting during GHRSST XII. The ST-VAL chair presented the outline of the ST-VAL work plan, which will be prepared around a set of agreed tasks. The tasks are:

- SSES

- Adoption of 'Common Principles'

- Improvement of validation methods

- Definition of uncertainties

- QC of drifter data

- DBCP pilot project

- Continued evaluation of Argo

- For independent validation

- Continued interaction with in situ providers

- Requirements for SOT data

- Maybe even a coordinated network?!

- Argo near-surface profiles

- Thermistor chains

- Exploitation of forthcoming campaigns

- For example SPURS

- See report from Boulder in ST-VAL pre-meeting report

- Calibration

- In collaboration with EaRWiG
- Next generation SSES (uncertainties)

- Ideally derived from first principles

- Needed for climate

- $\quad$ SST Reference network

- Composition and capability

- Work with RAN-TAG

- Documentation (a lot required, particularly for the website)

Next steps:

- Prepare content outline and writing tasks (Gary Corlett)

- Add content (ST-VAL)

- Iterate with Science Team Chair and Project Office

- Work plan should contain list of resources and funded activities as requested by Craig Donlon.

Website: The ST-VAL web pages on the GHRSST website are still missing SSES descriptions from L2P providers. Gary Corlett agreed to provide a template that other L2P providers could follow.

New ST-VAL members: Helen Beggs (BoM), Craig Donlon (ESA), Lei Guan (OUQ) and Anne Marsouin (CMS) have all joined ST-VAL. 


\section{SESSION 4.1.B: DAS-TAG BREAKOUT}

\section{Chair: Ed Armstrong ${ }^{1}$; Rapporteur: Jean-François Piollé ${ }^{2}$}

(1) Jet Propulsion Laboratory, California Institute of Technology, 2800 Oak Grove, Pasadena CA 91109, Email: Edward.M.Armstrong@jpl.nasa.gov

(2) Ifremer/CERSAT, 29 Plouzané, France, Email : jfpiolle@iremer.fr

GDS 2 review: 30 odd responses/concerns raised by reviewers

Many editorial issues. Consensus reached that recommended fixes implemented as is. Issue of enumeration list/ bitwise flags reviewed. Should we have mixed list/bitwise flagging structure? Consensus was separation of these two flag methods. Examples will reflect this. A provider can still if desired combine the two without breaking CF or GDS 2.

GDS 2 document modifications: Andrea will handle the minor editorial changes, Ed will handle revisions to masking and geolocation description/CDL revisions.

The DAS-TAG strongly recommends netCDF4 data model adoption. However, some RDACs (EUR-MyOcean, UKMO) are contractual obliged to continue netCDF3 data products in the near future. Some concerns on the netCDF4 support by older third party software.

A netCDF4 format/CF compliance checker is needed. Despite the chair's concern not enough traction to develop a format/compliance checker. Ed will modify a GDS 1 ' $C$ ' reader for format checking and minimal compliance by Sept 2011, the program to be shared within GHRSST community.

Review of survey results conducted by the LTSRF on the GDS2 transition schedule:

- At least 38 existing GDS 1.7 GHRSST datasets will be migrated to GDS 2

- Unknown, but at least 15+ new datasets will be introduced in GDS 2

- The adoption will occur at varying scales over next 6-12 months

- 30 days overlap of dataset GDS 1/2 versions

- GDAC will ingest both versions for at least 30 days

DAS-TAG recommend end of 2012 as date of adoption (GDS 2/netCDF4) for all GHRSST RDACs with caveats for those with contractual obligations to netCDF3.

DAS-TAG Members: Ed Armstrong (Chair), Jean Francois Piolle (Co-Chair), Kenneth Casey, Tess Brandon Craig Donlon, Dave Foley, Jean-Francois Piolle, Chelle Gentemann, David Poulter, Gary Wick, Jorge Vazquez, Ted Habermann, Leon Majewski, Eileen Maturi. 


\title{
SESSION 4.2.A: DVWG BREAKOUT
}

\author{
Chair: Chris Merchant ${ }^{1}$; Rapporteur: Helen Beggs ${ }^{2}$
}

(1) School of GeoSciences, University of Edinburgh, UK, Email: c.merchant@ed.ac.uk (2) Centre for Australian Weather and Climate Research, Bureau of Meteorology, Melbourne, Australia, Email: H.Beggs@bom.gov.au

Chris Merchant stood in as chair of the Diurnal Variability Working Group Breakout Session since Gary Wick was unable to attend. There were two science presentations followed by a status update and discussion of the Tropical Warm Pool Diurnal Variability Experiment (TWP+). These discussions were followed by a presentation on a data-driven diurnal variation (DV) analysis, discussion on a measurement campaign white paper, DVWG priority list and an inter-sessional meeting of the DVWG in conjunction with other GHRSST working groups.

Ionna Karagali: "Diurnal Warming in the Northern European Shelf Seas: Observations and Modeling"

Ionna described how SEVIRI SSTskin data were used to produce DV analyses in the North/Baltic seas. They observed DV anomalies mainly between May and August of duration generally $<3$ hours. Some anomalies exceeded $2 \mathrm{~K}$. The majority of the $>2 \mathrm{~K}$ cases occur in the Baltic and east side of the Greenland Sea. They used a statistical model from Filipiak et al. 2010 (FMKLB) and a prognostic scheme from Zeng and Beljaars (2005) and compared these two DV models with the SEVIRI-based DV analysis. With the Filipiak scheme, the DW event peaks were not as high as those obtained for the SEVIRI DV analysis. The same was observed for the Zeng - Beljaars model but the ZB model DW peaks were slightly higher. Both models spatially overestimated diurnal warming. They observed low correlation between SEVIRI DSST, surface winds, $\mathrm{Kd}(490)$ and net heat flux although correct trends were present. Overall, DV modeling was difficult point for point but they had some success in representing distributions of DV amplitude/incidence.
Peter Minnett: "Diurnal Variability in Shallow Coastal Waters"

The aim of the study was to look at diurnal warming (DW) in shallow water in coral reef areas, motivated by a desire to study the DV contribution to coral bleaching events. The investigators at the University of Miami took surface DW estimates over coral reefs and attempted to extrapolate these down to coral depths. The two coral reef regions studied were in the Caribbean and Great Barrier Reef. Within the Great Barrier Reef with tidal currents of up to 1.3 $\mathrm{m} / \mathrm{s}$ between reefs they observed a surprising positive correlation between tidal strength and DV amplitudes. The hypotheses that will be investigated are sticky water, shallower water depths during spring tides and the advective importation of high DV water. "Sticky water" relates to the phenomenon that under strong tidal currents the bottom friction is so high that currents go around the reef instead of over.

Their conclusions were that wind affects DW amplitude in shallower water depths and waves might be an important mechanism for mixing. The effect on the DW of the bottom albedo is very small except in very shallow waters. A new proposal will investigate the advective component of the DW.

\section{Helen Beggs: "GHRSST Tropical Warm Pool Diurnal Variability Project (TWP+)"}

The TWP+ data status and project plan were presented. Details can be found in section 8 of the Report to GHRSST12 from Australia submitted to the proceedings of the $12^{\text {th }}$ GHRSST Science Team Meeting. The TWP+ data set includes gridded, netCDF format, SST observations, SST analyses and NWP hourly forecast fields for DV modeling and verification. The project plan is 
systematic, including data set assessment, model comparisons and impact assessments. The feedback from the session was positive with the following suggestions for additional data sets to be included in TWP+:

- Sea state (eg. GlobWave or ERAinterim data sets)

- RAMA array mooring meteorological data (for NWP and DV model validation)

- Advection (current) data (eg. from BLUElink ocean analyses)

- Satellite humidity and temperature profile data (possibly available from the BoM data assimilated into the ACCESS-R NWP model)

- Campaign data (Cummings) if the experiment is extended to 2011

The project plan does not currently include a data-driven DV analysis, which is another opportunity/priority.

Additional DV models that could be included in TWP+:

- Uni of Edinburgh statistical Look-up Table model (Filipiak)

- Met Office FOAM ocean model SST1m at sub-daily time-scales (Martin)

- Reparameterised Kantha-Clayson model including sea state (Clayson)

Comments on the TWP+ experiment design included that the 24 hour data cycle (in each file) should begin at the start of the day (dawn) not 0 UTC.

\section{Pierre Le Borgne: "Are geostationary derived operational DW estimate needed?"}

Pierre Le Borgne compared SEVIRI DSST with drifting buoy derived DW estimates. He found that the mean DV cycle in the SEVIRI data led the buoys by a few hours in cases where $1.5 \mathrm{~K}>\mathrm{DW}<2.5 \mathrm{~K}$ although the DW amplitudes were almost equal. However, for $\mathrm{DW}>2.5 \mathrm{~K}$ the drifting buoys measured larger DW amplitudes than SEVIRI. The comparative amplitudes may be affected by stratification, direct buoy heating, diurnal skin effect, late-afternoon convection (cloud contamination of SEVIRI data), satellite retrieval (in)sensitivity or horizontal resolution issues.

There was considerable discussion regarding the possible causes of the SEVIRI-observed DW commencing several hours earlier than the drifting buoy DW for DW below $2.5 \mathrm{~K}$, including:

- Time lag for heat to transfer down through the water column to drifting buoy depths $(-20 \mathrm{~cm})$

- Stratification of the water column

- Skin layer

The consensus was that the data-driven approach is a useful complement to assimilation and modeling. Pierre Le Borgne proposes to make the SEVIRI SSTskin hourly analysis routinely available in delayed mode to maximize the quality of the interpolation between the data fields.

Currently the SEVIRI data-driven analysis (DVA) is the only data-driven DVA. The DV Working Group would like to encourage other experiments in DVA production. Possible future DV analyses could be:

- OSTIA data-driven DV analysis (Merchant)

- Zeng-Beljaars SSTskin analyses (Cummings)

- Ocean colour information

- Global DSST from GOES and MTSAT series satellites?

Craig Donlon pointed out that one observes over the Tropics strong, deep convection in the afternoon and that DV events are important for prediction of convection events for aircraft.

\section{Whitepaper in support of potential measurement campaigns}

The DVWG is aiming for a white paper on requirements for measurement campaigns by November 2011 . It was proposed that Gary Wick will coordinate the white paper by email. Immediate comments from the working group are sought. 
GHRSST Project Office

\section{Review of group objectives}

The following priority list from the Boulder DVWG meeting was approved:

1. Production of data-driven SSTskin analyses

2. Continued model validation

3. Consensus approaches on reporting DW for L4 products

4. Web presence.

\section{Action on Gary Wick to update DV web page.}

\section{Proposal for a GHRSST Joint Working Group Meeting}

There was a high level of interest in an inter-sessional meeting with working days on DV, in conjunction with other working groups. After discussion with the chairs of five of the GHRSST working groups/TAGs (EARWiG, ST-VAL, DV-WG, HL-TAG and IC-TAG), Helen Beggs and Chris Merchant put the following proposal for a GHRSST Joint Working Group Meeting to the GHRSST Science Team on the final day of the $12^{\text {th }}$ GHRSST Science Team Meeting:

Venue: Bureau of Meteorology Head Office, Melbourne, Australia

\section{Participating Working Groups: EARWIG, ST-VAL, DV-WG, HL-TAG, IC- TAG}

Themes: (i) TWP+ Diurnal Variability Project, (ii) SST retrievals and validation south of $50^{\circ} \mathrm{S}$
Proposed dates: 5 days either commencing 27 February 2012 or 5 March 2012

Funding: Bureau of Meteorology to cover all lunches, teas and ice-breaker

Registration: Excursion and conference dinner ( AUD\$180)

\section{Schedule:}

- Mon: TWP+ presentations + discussion. Icebreaker.

- Tue: Southern Ocean SST presentations + discussion

- Wed: Parallel working day on both themes. Excursion in late afternoon to see sub-Antarctic penguins and conference dinner.

- Thur - Fri a.m.: Parallel working days on both themes

- Fri p.m.: Working day outcome presentations

Action on Helen Beggs to survey GHRSST Science Team and Working Group members for possible attendance and preferred dates. Note that since the $12^{\text {th }}$ GHRSST ST Meeting Helen has booked two conference rooms for both of the proposed weeks in Feb/Mar 2012, following the AGU Ocean Sciences Meeting (20-24 Feb 2012). 


\title{
SESSION 4.2.B: AUS-TAG BREAKOUT
}

\author{
Chair: Jorge Vazquez ${ }^{1}$; Rapporteur: Tess B. Brandon ${ }^{2}$ \\ Jet Propulsion Laboratory, NASA, US, Email: Jorge.Vazquez@jpl.nasa.gov \\ National Oceanographic Data Center, NOAA, US, Email: tess.brandon@noaa.gov
}

\section{Introduction}

The goals of this Applications and User Services (AUS-TAG) breakout session were to address and discuss several issues relevant to user services, including the Users Manual, a "one-pager" concept for first-time SST users, a dashboard concept for RDAC reporting, and several new data discovery and access tools.

\section{Users Manual}

The TAG Chair provided an update on the Users Manual, declaring it to be in good shape and in need of a final edit by a single person for consistency and flow. A volunteer for this task was identified and the Chair will coordinate with this person to accomplish this final edit. The Users Manual is already publicly available, but after this final edit it will be declared operational and announced to the user community via GHRSST forums and RSS feeds.

\section{User requirements and services}

The group discussed the need for a comprehensive (if possible) list of user requirements of GHRSST SST products. An action was given to the group to create such a list based on the user presentations given during the second day of this meeting, as well as from the 128page report of the ESA $\mathrm{CCl}$ User Requirements Development exercise.

In general, the group agreed that according to the access statistics, a large majority of GHRSST users are able to navigate the various discovery and access mechanisms to get what they need; however, there is definitely room for improvement. The group discussed the need for an integrated user services system to track user requests, problems and activity, including downloads.
Currently user access statistics are provided annually by the GDAC and the LTSRF, but such statistics would be valuable to obtain from individual RDACs as well. Many of these RDACs already employ internal systems to track user requests, so an ideal solution would leverage these existing capabilities to create an integrated system that could be managed by the GDAC and/or LTSRF. To that end, the GHRSST Project Coordinator took an action to develop a survey of existing RDAC user services capabilities. One potential tool to use as the central collation point of these systems is the collection of new GHRSST forums hosted by the PO.DAAC.

\section{Dashboard for RDACs}

As one potential improvement to transparency and clarity for users, the group discussed the concept of a status dashboard for RDACs. This dashboard would be operationally updated according to several metrics to report data outages, processing errors and updates, reprocessing, etc. The dashboard would use a "red-yellow-green" stoplight paradigm to indicate operating status, which would be published to the front page of the GHRSST Program website. In general, the dashboard discussion elucidated the need for better communication between the RDACs and the GDAC, especially regarding issues of temporary data outages and updates to data. No actions were given as a result of this discussion.

\section{One-pager for users}

Mike Chin presented on his work developing a one-pager for first-time SST users. The goal of this effort is to increase the GHRSST user base beyond traditional science/SST users. The work has evolved 
from a literal "one-pager" to a series of web pages that direct the user who a) is in a hurry and b) may not have the computing resources GHRSST members usually enjoy. These web pages are organized into five main topics that introduce users to GHRSST and show them how to download and read the data: 1) What GHRSST offers - including data descriptions of L2P, L3 and L4 product categories; 2) Where the data are - GDAC and LTSRF; 3) Which data set to download - promoting user awareness of issues such as SST type, resolution, and temporal and spatial coverage; 4) How to download the products - featuring subsetting via OPeNDAP, introduction to data volumes, etc.; and 5) How to read a file - including template codes in C, Fortran, Matlab and IDL for both GDS1.6 and 2.0.

The group stressed the need for browse graphics to show examples of types of data sets and sensors (e.g. AVHRR vs. ATSR vs. geostationary) as a part of these web pages, perhaps rolled into a description of the data. The group also discussed the design of a hierarchical set of web pages that would allow the user to prioritize search parameters (e.g. length of time series, resolution, spatial coverage) in order to arrive at the most appropriate available GHRSST product for their specific application.

The AUS-TAG for feedback, and ultimately hosted on the GHRSST Program Office website.

\section{Data discovery $\&$ access tools}

Several new data discovery, visualization, and access tools, both operational and experimental, were demonstrated live by the PO.DAAC. These included "State of the Ocean" (http://podaactools.jpl.nasa.gov/soto/), the DataMiner (http://podaac-

tools.jpl.nasa.gov/dataminer/aegina/src/da taminer.php), and the NASA MUR L4 analysis project page (http://mur.jpl.nasa.gov/).
The State of the Ocean tool is a visualization tool for many PO.DAAC data sets that allows the user to browse rolling 5 -day averages of the most recent nearreal-time data. It functions much like Google Earth, allowing the user to manipulate layer transparency to do qualitative analysis of multiple data sets.

The DataMiner is a discovery and access tool that allows a user to specify search parameters and plot the search results on a map, then click to download selected data. Some aspects of the DataMiner still need work, and the search and plot functionality of the tool took longer than would be practical in operational use. The DataMiner is designed to retrieve data from both the GDAC and the LTSRF depending on data date, keeping the access point consistent for the user. A potential improvement to dramatically increase performance would be to use pre-generated browse images to plot results, rather than uncompressing and subsetting data sets on the fly.

Finally, the project page for JPL's MUR L4 SST product was demonstrated. It uses out-of-the-box freeware to visualize the global $1 \mathrm{~km}$ analysis at full resolution, and was shown as another example of the various ways data visualization can be handled for GHRSST data.

\section{Summary of action items}

Action: Provide a last edit of the Users Manual for consistency and flow, by September 2011

Responsible Party: Eileen Maturi (Jorge Vazquez agrees to remind Eileen frequently)

Action: Develop and execute a questionnaire for all RDACs about existing user services capabilities and activities

Responsible Party: GHRSST Program Coordinator and AUS-TAG

Action: Schedule and set up a "GHRSST forum date" with a preordained topic so that the Science Team and users can check out the various forums and capabilities 
GHRSST XII -

Proceedings, Edinburgh

GHRSST Project Office

Responsible Party: Jorge Vazquez

Action: Set up metrics/operations dashboard for RDACs to post to www.ghrsst.org

Responsible Party: AUS-TAG

Action: Develop draft web page "onepager" for first time SST users and send to AUS-TAG and GHRSST Project Coordinator for review

Responsible Party: Mike Chin

Action: Create a list of user requirements based on the user presentations given during this meeting, as well as from the 128-page documented generated from the ESA CCl User Requirements Development exercise.

Responsible Party: AUS-TAG
Issue: Final

Date: September 27, 2011

Action: Ensure that each RDAC is represented by at least one member on both the AUS-TAG and DAS-TAG

Responsible Party: AUS-TAG Chair, DAS-TAG Chair

Action: Update the existing "read" software templates provided by the PO.DAAC to read GDS2.0 data (September 2011)

Responsible Party: Ed Armstrong, Mike Chin

Action: Visit and test various data discovery and access tools provided by the PO.DAAC - Data Miner, State of the Ocean, MUR project page - and provide feedback to the PO.DAAC

Responsible Party: AUS-TAG, GHRSST Science Team 


\section{SESSION 4.3.A: HL-TAG BREAKOUT}

\section{Chair: Jacob Hoeyer ${ }^{1}$; Rapporteur: Steiner Eastwood ${ }^{2}$}

(1) Danish Meteorological Institute, Email : jlh@dmi.dk

(2) Norwegian Meteorological Institute, Email : s.eastwood@met.no

\section{Introduction}

The 2 hour high latitude TAG breakout meeting was divided into two parts, one part with presentations on progress made within the group and another part with discussion and work out of the development plans for the future work.

The agenda for the breakout is given below:

\section{Presentations}

- OSTIA reanalysis sea ice and high latitude SSTs - Jonah R. Jones

- Multi-sensor validation and error characteristics of Arctic satellite sea surface temperature observations Jacob Høyer

- High latitude cloud and ice detection algorithms - Steinar Eastwood

- Ice surface temperature experiments in Qaanaaq - Werenfrid Wimmer

- OSI-SAF Sea ice reanalysis update Steinar Eastwood

- Other short updates

Discussion

- GHRSST requirements for Sea ice reprocessing products

- Review of future plans for the HL-TAG group

- Future meetings

\section{Progress during the last year}

Some highlights and keywords from the presentations are listed below, for the complete presentations, please see the http://www.ghsst.org webpage.

Jonah R. Jones: OSTIA reanalysis sea ice and HL SST

- Consistency between SSTs and sea ice better in $\mathrm{NH}$ than $\mathrm{SH}$
- Could be sea ice extent issue or SST issue, perhaps combination of both

- Shorten correlation length scales in high latitudes so SST information is not spread too far under ice, change relaxation timescales

- Next reanalysis as part of ESA SST $\mathrm{CCl}$ project

Jacob L. Høyer: Validation of L2P in the Arctic

- Most reliable satellite products: NAVOGAC and AATSR

- Spatial scales of satellite errors: 300$500 \mathrm{~km}$, temporal scales of a few days.

- Correlation of errors: significant correlation between IR products, low correlation between AMSRE and IR products.

Steinar Eastwood: High Latitude Cloud and Sea Ice Detection for SST retrieval

- Bayesian approach to mask ice/clouds in the Arctic have been developed in OSI SAF AVHRR and works well at day time

- Effort in ESA SST CCl project to improve cloud and ice masking at high latitudes, including night time

- First test show that AVHRR method works on AATSR when PDFs are shifted

- Suggest that the HL TAG shares training data for cloud and ice detection

Steinar Eastwood: OSI SAF reprocessing sea ice concentration data set

- Next version to be released July/August 2011 (v1.1)

- Covers 1978-10.2009

- Fixed bug in uncertainty estimates 
- Extended uncertainty estimates by splitting its different components

- Smearing uncertainty

- Algorithm uncertainty

- Inclusion of Caspian Sea

Fred Wimmer: Qaasitex

Observed parameters during field campaign to Qaanaaq in Northwestern Greenland

- Contact Temperature

o Air/Snow Temperature

o Thermistor Chains

- Infrared

o ISAR

o IR - Campbell Scientific

- Microwave

o DTU - 32GHz, $16 \mathrm{GHz}$

- CTD

- ADCP

- Ice thickness
o Classic
o SAMS conductivity
o Laser altimeter (Twin Otter)

\section{Discussion}

- Input to Sea ice providers (NSIDC):

- We would like the highest possible resolution of the Sea ice products.

- Climate records should be constructed, based upon one type of sensors if not included in uncertainty estimate.

- Action: To look at the users needs and provide feedback to NSIDC.

- Action: to look at the resolution issue, and users need.

Discussion with Nick Rayner about what HadISST wants. Ideally, they would like one data set where all ice data have been merged, to provide the best possible resolution, as long as all differences caused by different satellite resolution and instrument are reflected in the uncertainties. BUT, as long as this is not guarantied, it is better to split high resolution and low resolution products.

\section{Projects with HL related work:}

- ESA Climate Change Initiative (CCl) project on SST. High latitude SST development and testing, Marginal Ice Zone SST and Improved high latitude cloud and ice detection (UoE, DMI + Met.No)

- OSI-SAF: the next 5 years will develop the ice surface temperature algorithms for the Metop satellites (DMI+Met.no).

- Greenland Climate research centre: Focus upon Greenland, L4 SST reanalysis, IST field experiments (DMI)

- MyOcean 2: Level 4 IST analysis, Arctic + Baltic SST L4 (DMI+ Met.no)

- MyOcean R\&D project : Multisensor bias adjustment methods in the Arctic ocean for use in L4. (DMI, CMS + Met.no.

- Research Network in UK A research network for surface temperature in the UK has recently been funded (UoE,Leicester, UK-Metoffice). The activities in the second year of the project will focus upon quantifying surface temperature across the Arctic.

- IMOS: Algorithm development and validation activities to reprocess and improve the $1 \mathrm{~km}$ HRPT observations (BOM).

- NAARCOS: Thermistor string buoys DMI + SAMS

- NSIDC Sea ice reprocessing: A sea ice reprocessing will be carried out (NSIDC)

- ESA CCI on Sea ice : Call is out now (Met.no + DMI)

- UpTempo: Buoys and gliders in Beaufort Sea (Mike Steele, APL)

- US NAVO: 5 year program on more open water in Chucki Sea and effect on retreating ice Navy

\section{Future planned work within HL- TAG}

- SST:

- Develop and validate several retrieval algorithms for the high latitudes 
GHRSST Project Office

- Validated performance in Marginal Ice zone.

- Improve on cloud and ice detection

- L4 Arctic multisensor bias adjustment method tested.

- IST

- Improve operational 1 km L2 MetopA IST, develop Metop-B ist

- Combine MW and IR using modelling results.

- Develop L4 IST product

- Need for quality controlled validation data

- Sea Ice

- NSIDC Sea ice reprocessing available

- Updated OSI-SAF reanalysis

- Lake Sea Ice products

- Intercomparisons of Sea ice products

- In situ
- Radiometer for high latitude field work (SST and IST).

- Ice mass balance buoys (with floating device)

- Wave glider.

- IMOS: new observation techniques

\section{Conclusions:}

- HL-TAG is doing well

- Good progress in several areas

- Several projects have just started to look at high latitude issues

- Joint meeting with other working groups in Melbourne

7. Actions:

- Jacob to look at the resolution issue, and users needs and give feed back to NSIDC on the SST requirements for the Sea Ice reanalysis products

- Jacob: To work with the other working group chairs to organize an intersession joint science meeting in Melbourne in Feb-March, 2012. 


\section{SESSION 4.3.B: R2HA2-WG BREAKOUT}

\section{Chair: Peter Cornillon'; Rapporteur: Tess Brandon ${ }^{2}$}

University of Rhode Island, US, Email: pcornillon@me.com

National Oceanographic Data Center, NOAA, US, Email: tess.brandon@noaa.gov

\section{Introduction}

The Rescue and Reprocessing of Historical AVHRR Archives (R2HA2) Working Group met for the first time as an official GHRSST Working Group. Goals of the meeting were to (i) define the membership and leadership of the working group; (ii) review and update the Terms of Reference of the working group, including its objectives and means of accomplishing those objectives, as first laid out at GHRSSTX; and (iii) establish a path forward. The objectives and methods of the working group as laid out in this report were arrived at by discussion and consensus during the breakout session.

\section{R2HA2 membership}

The following attendees of the breakout session agreed to become official members of the R2HA2 Working Group:

- Peter Cornillon (Chair)

- Ed Armstrong

- Ken Casey

- Eileen Maturi

- Jon Mittaz

- Hervé Roquet (Vice-Chair)

Of course, membership is not restricted to these individuals and it was agreed that a message would be sent by the working group chair to the GHRSST community inviting those with an interest to join.

\section{Group objectives}

The objectives of the working group are to:

1. Identify historical archives $^{1}$ of AVHRR HRPT and LAC data.

\footnotetext{
${ }^{1}$ The focus here will be on pre-2000 archives of HRPT and LAC data since a global $1 \mathrm{~km}$ archive of MODIS data exists post-2000. However, post-2000 AHVRR HRPT and LAC data will be accepted if provided, they will simply not be the focus.
}

2. Copy these archives to a central data repository.

3. Convert these data to a consistent $\mathrm{L}^{2} \mathrm{P}^{2}$ format in netCDF4.

4. Reprocess these data in a consistent manner to GDS2.0 L2P and serve them via the GHRSST Regional/ Global Task Sharing Framework (R/GTS).

\section{Necessary steps}

In order to accomplish its objectives, the working group will:

1. Identify and locate historical archives (pre-2000) of AVHRR HRPT and LAC data.

2. Copy data from historical archives to a central location.

3. Identify a central assembly center(s) (CAC).

4. Define a format (L1P) in which the data are to be stored.

5. Define if/how contributions are to be stitched together at the CAC.

6. Determine how to handle navigation information.

7. Identify where the reprocessing is to be performed.

8. Define the SST algorithm to be used for reprocessing.

9. Determine how to perform the navigation.

\section{Path forward \\ Short term (1-2 years)}

In the coming year, the group intends to accomplish three primary goals. The first is to establish the group formally by collecting a list of official members. The Chair will contact interested parties in order to accomplish this goal.

The second goal is to identify historical archives of AVHRR HRPT and LAC data,

\footnotetext{
${ }^{2}$ L1P is a level 1 format that will defined by the R2HA2 explicitly for AVHRR HRPT and LAC data.
} 
and to begin to copy those data to a central location. The group agreed that because a global $1 \mathrm{~km}$ archive exists for the post-2000 period, its priority objective is to rescue and reprocess pre-2000 data. A list of known historical archives was generated at the GHRSSTX meeting, and working group members will add to this list as necessary. The Chair will contact parties identified for each historical archive, ask if they are willing to make their data part of this effort, and if so, work with those parties to copy their data to a central location. Though ultimately the working group intends to identify a Central Assembly Center (CAC) where the data will be collected and converted to a common format, identifying that CAC is not a priority initially.

The third goal for the next one to two years is to define a common output format, to which all the historical data will be converted. The group agreed that the scientific format for this should be netCDF4, and that it should be called L1P. Several questions arose related to the output format, including if and how to stitch the data together, how to handle navigation information, and how to handle calibration coefficients. The group discussed these issues in some detail, but ultimately selected a subgroup to resolve them and define the output format. This subgroup consists of Hervé Roquet, who will lead the subgroup, Jon Mittaz and Ed Armstrong, and intends to look at the GDS2.0 Technical Specifications document as a starting-place for defining the L1P products. It was suggested that this subgroup invite Bob Evans of UMiami and George Paltoglou of the Australian Bureau of Meteorology to participate in the their activities. Both have extensive experience with regard to issues related to level 1 data but were not at the meeting or in this breakout session.

\section{Longer-term considerations}

After identifying, copying, and reformatting a significant collection of historical AVHRR HRPT and LAC data, the group intends to reprocess this collection using an existing/vetted algorithm and generate a consistent L2P product in GDS2.0 format, to be served via the GHRSST R/GTS. This will provide a way to check the validity of the original conversion to L1P, and will give users confidence in the group's efforts, as well as a point of comparison for other products.

Reprocessing within the GHRSST framework will also facilitate archive of both the L2P products and the lower-level L1P products at the NOAA National Oceanographic Data Center (NODC), as the GHRSST Long Term Stewardship and Reanalysis Facility, has a standing agreement to archive GHRSST products. The group also discussed the archival of the original recovered historical data (preconversion to L1P). Though this issue will most likely not be addressed in the next couple of years, the group acknowledged that at a minimum, the NOAA Archive would likely act as the deep archive for these data, and will pursue any required negotiation with NOAA (most likely through the NODC) to that end. In addition, to facilitate archival in the future, communication with potential data providers should explicitly state the group's intent to archive and distribute openly all data recovered. The volume of the recovered archives is estimated to be less than, but on the order of 100 TB. In line with archive considerations, the group discussed the need for thorough metadata, documentation and documented code throughout this process. Finally, the group discussed potential synergies with existing reprocessing and data rescue efforts. One of these efforts is led by George Paltoglou of the Australian Bureau of Meteorology. The group will reach out to George in an effort to coordinate. The NOAA Climate Data Record Program is funding work to reprocess historical AVHRR GAC data, and could potentially provide a source of funding to the R2HA2 working group after the group has performed a proof of concept by producing L1P products from the historical archives. The group acknowledged that these L1P products would represent a success in and of themselves, and be useful to other climate 
GHRSST XII -

Proceedings, Edinburgh

GHRSST Project Office

applications such as cloud, ice and land product generation. Meanwhile, the group will enhance its visibility through existing connections such as the Data Archiving and Access Requirements Working Group in the U.S.

\section{Summary of action items}

Action: Contact attendees of 2009 GHRSSTX AVHRR HRPT meeting in Santa Rosa and ask if they are interested in official membership in the R2HA2 working group

Responsible Party: Peter Cornillon

Action: Send NODC Submission Information Form to Peter Cornillon

Responsible Party: Ken Casey

Action: Write a draft plan - "here's what we want to do; here's how" - to include in communication with potential data providers

Responsible Party: Peter Cornillon

Action: Go through list of potential data providers from 2009 meeting report; contact and try to start acquiring the data
Issue: Final

Date: September 27, 2011

Responsible Party: Peter Cornillon

Action: Define L1P output format for R2HA2 effort, as described in this report

Responsible Party: Hervé Roquet, Jon Mittaz, Ed Armstrong

Action: Contact George Paltoglou of the Australian Bureau of Meteorology to invite him to participate on the L1P definition subgroup.

Responsible Party: Jon Mittaz

Action: Contact Bob Evans of the University of Miami to invite him to participate on the L1P definition subgroup.

Responsible Party: Hervé Roquet

Action: Contact George Paltoglou of the Australian Bureau of Meteorology to discuss coordination of R2HA2 effort

Responsible Party: Peter Cornillon

Action: Provide Peter Cornillon with list of AVHRR HRPT/LAC archives (ongoing)

Responsible Party: R2HA2 WG 


\section{SESSION 4.4.A: IC-TAG BREAKOUT}

\section{Chair: Matthew Martin'; Rapporteur: Alexey Kaplan ${ }^{2}$}

(1) Met Office, Fitzroy Rd, Exeter, Uk, Email: Matthew.Martin@Metoffice.Gov.Uk

(2) Lamont-Doherty Earth Observatory Of Columbia University, Palisades Ny 10964, Us, Email: Alexeyk@Ldeo.Columbia.Edu

\section{Introduction}

In the beginning of the breakout session Matt Martin (Chair) reviewed IC-TAG's Terms of Reference and moved to add an item "To assess and improve the specification of error in the L4 analyses" that became one of the de facto focuses of group activities. There were no objections to this proposal.

Then Chair reviewed current group membership (there was no changes) and objectives of the session:

1. To review L4 inter-comparison work over the past year in the form of presentations.

2. To review progress with the DSR-II paper on GMPE and L4-SQUAM.

3. To discuss the issue of analysis error estimation, particularly in the presence of incomplete data where there may be artificial gradients.

4. To make recommendations for L4 inter-comparison work over the coming year.

The agenda for this breakout session was pre-planned towards these objectives; the audience proposed no modifications to the agenda at this time.

The breakout agenda consisted of 4 individual scientific presentations, updates on the status of the 3-part DSR-II paper in preparation by the IC-TAG members, and a discussion of sensible ways to communicate error in the L4 products. Individual presentations included reports on the progress in terms of the relevant to the IC-TAG goals increased functionality, planned new data products, growing collaboration, and new scientific results of the intercomparison of the established products.

\section{Presentations}

In particular, David Poulter described the new functionality of the DDS system that allows users to produce and to receive by email customized plots from the GlobWave diagnostic system. He also discussed (not-finalized) plans to make this functionality available for SST data in the DDS.

G.B.Franca reported on the general activities of REMO network in Brazil (REMO is composed of Petrobras, Brazilian Navy, and four Brazilian public universities) and in particular on their work on producing interpolated SST fields. REMO's main goals are: (1) to develop an assimilative ocean forecast system for the Brazilian continental shelf and slope regions; and (2) to help environmental authorities in case of oil disasters. Their area of interest is so-called METAREA $V$ (area in the Atlantic Ocean from Brazilian coast to the 20 degree West), and daily SST analyses are produced for this area on the basis of AVHRR and TMI data using Barnes interpolation technique. Results are validated using PIRATA buoys; comparison with OSTIA results showed a good agreement as well. Daily SST time series are ready to be transferred to GHRSST in the NetCDF format (start date is September 1, 2002) for the inter-comparison purposes. Their work in progress and the near-future goals include: to implement a physical retrieval SST model (based on the Optimal Estimation); to include SST from others satellites sensors; to carry on continuously the SST validation; to improve spatial resolution of the present SST product; and to start cooperation with the GHRSST team.

Eileen Maturi reported on the recent progress and near-future plans for new NOAA operational L2P and blended L3 
and L4 SST products (which she described in detail) from geostationary satellites that they will be providing as GHRSST data sets. There are many improvements whose implementation is currently ongoing: improved accuracy due to the implementation of physical retrieval methodology with radiance bias correction, increased number of levels in the CRTM, and improved Bayesian cloud mask; improved thermal IR calibration, and improved coverage (Electro-L).

Michael Chin presented his preliminary results of the intercomparison of a considerable selection of the $\mathrm{L} 2 \mathrm{P}$ and $\mathrm{L} 4$ products in terms of their wavenumber spectra. He showed intriguing if not fully understood and interpreted results that showed MODIS L2P data having straight power spectrum line with a slope between -1.5 and -2 in the wavelength range roughly 2-1000km, while other L2P products (AVHRR and AMSRE) and L4 products (NCDC, OSTIA, GAMSSA, K10, REMSS, and MUR) were turning down at various wavelength in this interval. IC-TAG moved to encourage M.Chin to pursue this line of research and to achieve complete understanding of the spectral properties of these GHRSST products.

\section{Discussion of IC-TAG papers for publication in Deep Sea Research II}

M.Martin and A.Ignatov, leading authors of the two parts of the DSR-II paper in preparation reported on the status of their papers. Martin has described the structure of the paper (which followed closely the presentation on the GMPE system he gave earlier at the plenary session) and described immediate goals and (very) near deadlines in finishing the preparation of this paper. One of the major advances reported in his paper is the use of ARGO near-surface measurements, as independent data, for validating $L 4$ analyses used in the GMPE system; he received a request to make sure that indeed no analyses discussed in this context use ARGO data. In the course of the work on this paper important IC-TAG action item has been advanced: a unified descriptions for L4 analyses have been designed; descriptions in this format were collected and made publicly available for RTG, RSS (MW/IR), OISST (AVHRR, AVHRR/AMSR), JMA, GAMSSA, RAMSSA, OSTIA, and CMC. Such descriptions are still outstanding for FNMOC, G1SST, NAVO, K10, POES/GOES (the product itself is being worked on now), and ODYSSEA.

A.Ignatov described the progress with the 2nd DSR-II paper which is describing the SQUAM system. He described the planned structure of the paper and the concept of the SQUAM system; discussed possible additional topics to cover. He explained that the goal of this paper is to describe new unctionality provided to users by SQUAM, rather than to discuss the actual intercomparison of the SST products; he explained that the QC of the in situ data is done using another of their system, iQUAM, which he described earlier at the plenary session and which feeds its QC output into SQUAM. He also explained the treatment of daily vs nondaily data in SQUAM.

\section{Discussion of analysis error issues}

The last general item of the breakout session was a discussion of error description for L4 products, meant to move IC-TAG closer to the fulfilling the action item (G10-14): "Define and agree on the most sensible error estimate for L4 data products and provide inputs to GHRSST and L4 producers." The discussion was based on Nov 2010 email discussion between a few IC-TAG members whose relevant excerpts and a summary of possible approaches were made available to all meeting participants prior to this meeting. The options considered were roughly divided into three groups: (1) Representing L4 error using formal error estimates; (2) Communicating uncertainty in the L4 products using data coverage information; and (3) Other approaches (e.g. re-evaluating or redoing L4 products rather than just dealing with their error). In the course of this discussion, participants only restated and affirmed (sometimes heatedly) their 
GHRSST Project Office

positions that were expressed by them earlier.

Clearly, further progress will require experiments with various proposed approaches and clarification of their comparative practical values through these experiments and further analyses. One of the first action items should be the inspection of the current content of the analysis_error variables in the existing L4 products. This effort will be helped by Johan Roberts-Jones, who volunteered to add the functionality to visualize these variables (error fields in the included L4 products) to the GMPE system. Craig
Donlon suggested to pursue the reviewing of different options for L4 error specification by writing a white paper on the subject.

\section{Future work}

In the view of presented results and main discussions that took place at the breakout session, the main goals of IC-TAG for the next year should be: (1) Complete DSR-II papers; (2) Performing the L4 product error inter-comparisons and clarifying relative values of various approaches to their reporting. 


\section{SESSION 4.4.B: EARWIG BREAKOUT}

\section{Chair: Andy Harris ${ }^{1}$; Rapporteur: Chris Merchant ${ }^{2}$}

(1) NOAA-CICS, University of Maryland, Cooperative Institute for Climate Studies, College Park, Maryland, USA, Email:Andy.Harris@noaa.gov

(2) University of Edinburgh, Edinburgh, EH7 3JN (UK), Email : c.merchant@ed.ac.uk

Andy Harris gave an introduction to EARWiG. Pierre LeBorgne showed the operational use of NWP model outputs in satellite SST. Peter Minnett presented new approaches to the infrared Atmospheric Correction algorithm( genetic algorithm, withheld validation, regression trees). Petrenko talked about Development and Evaluation of SST Algorithms for GOES-R ABI using MSG SEVIRI as a proxy. Jon Mittaz presented the calibration of the Broadband Infrared sensors onboard NOAA satellites.

Chris Merchant invited to participate in Algorithm Selection exercise with Climate Change Initiative. Description of algorithm, including notify use of in situ No resources, just data are on offer. Selection set available 1 Nov, submit results 30 Nov! Participants will gain algorithm feedback, co-authorship, contribution to significant SST dataset.

Caroline Cox introduced the Oxford RAL Aerosol and Cloud retrieval algorithm recently extended to SST.

A discussion has held on EARWiG direction and relation to NASA SST and ERNESST.

EARWiG should give best practise recommendation to data providers:

- Instrument calibration

- Cloud detection (IR)

- The inverse problem

- Effects of aerosols (IR)
- Side-lobe \& precipitation contamination (MW)

- Lake surface temperature

- Uncertainty estimates for the above

GHRSST should try to put pressure on satellite data calibrators to make improvements of instrument calibration a high priority and point out the ability of SST to help show up poor calibration

\section{EARWiG priorities:}

SST retrieval is not a "done deal":

- Especially when considering $<0.05 \mathrm{~K} /$ decade stability requirement

- Simulations show that the main biases in current products are due to algorithmic limitations

- Despite this, it is hard to get algorithm/product improvement funded

Calibration is another priority of EARWiG.

Feedback should be given to providers, CEOS CalVal, Virtual Constellation

SST retrieval is a live issue (lots of new things going on). EARWiG should lead the way (ERNESST meetings are very informal and problem-specific) with:

- Best practice recommendations

- Multi-sensor Matchup Dataset

- Themed workshop (joint-WG) in Melbourne, March 2012 


\title{
SESSION 4.5.A: RAN-TAG BREAKOUT
}

\author{
Chair: Kenneth S. Casey ${ }^{1}$, Rapporteur: Tess B. Brandon ${ }^{1}$, Nick Rayner ${ }^{2}$
}

(1) National Oceanographic Data Center, NOAA, (U.S.A.),

Emails: Kenneth.Casey@noaa.gov and Tess.Brandon@noaa.gov

(2) Met Office Hadley Centre, (U.K.), Email: nick.rayner@metoffice.gov.uk

\section{Introduction}

The three main goals of the Reanalysis Technical Advisory Group (RAN-TAG) breakout session were to (i) review the current status and future plans of reanalysis activities; (ii) define and discuss implementation of a Data Processing Framework (DPF) for the SST Essential Climate Variable (EVC); and (iii) identify the priority actions for the coming years.

\section{Status Report}

The first goal of the RAN-TAG breakout session was to collect and publish an authoritative status report, documenting the current state and future plans of the international SST reanalysis community. Twenty-seven projects were summarized in an easy-to-understand and consistent format; these summaries will be made available on the GHRSST and Long Term Stewardship and Reanalysis Facility (LTSRF) websites following the meeting, as the 2011 GHRSST Reanalysis International Status Report. These activities are reviewed in brief below.

\section{Historical and in situ Activities}

- AVHRR FCDR (Mittaz, NOAA/CICS): Working on better calibration for the entire L1b series; data to be available in 2-3 years. RAN-TAG consensus that this is an effort both the RAN-TAG and GHRSST should be heavily involved in and support.

- HadSST3 (Rayner, UKMO): Data available from http://www.metoffice.gov.uk/hadobs.

Currently updating from 2007 to present; making use of new kind of graphic to capture stability, to be discussed for incorporation in the Data Processing Framework.
- ERSSTV3b (Banzon et al., NOAA/NCDC): Satellite data has been removed; Boyin Huang has fully taken over for Viva Banzon.

\section{Level 1 Activities}

- AVHRR HRPT/LAC (Cornillon, URI): Now an official working group within GHRSST (R2HA2) led by Peter Cornillon; critical activity as data sets age; working first to identify and collect various data sets available around the world.

- IMOS HRPT (Beggs, ABoM): Australian effort similar to R2HA2, involves stitching; used to feed L2P products; going to reprocess Casey \& Davis stations to include more Antarctic coverage.

- AVHRR GAC Optimal Estimation (Filipiak, Merchant, Univ. of Edinburgh): SSTs currently available for 1991-2010; looking forward, will correct brightness temperatures using buoys and ATSR (ESA SST-CCI), and extend the data back to 1978.

\section{Level 2 and 3 Activities}

- nOAA gOES E/W, MTSAT, MSG (Maturi, NOAA/OSPO): Seeking funding to reprocess; need to make algorithm improvements. Major effort needed to reprocess these data sets.

- IASI (O'Carroll, EUMETSAT): L2P core produced from March 2010 experimentally; demonstration product available from EUMETSAT in nearreal time from Marcy 2011; no current plans to reprocess.

- MODIS (Evans, RSMAS): Proposal submitted to NASA, no word yet; 
algorithm development and improvement underway.

- AVHRR Pathfinder (Casey, NODC): V5.2 currently processing; public review period completed in GDS2.0 format, completely GDS2.0 compliant except will not have error or pixel-bypixel time information; no current plans to generate $\mathrm{L} 2 \mathrm{P}$, but would like to in the coming year.

- NORMAP (Eastwood, Met.No): AVHRR FCDR effort for high latitudes; data may be available by end of 2012 .

- ATSR Reprocessing for Climate (Merchant, Univ. of Edinburgh): ARC data ready on DVD for demo; seeking feedback to feed into ESA $\mathrm{CCl}$; no plans yet to create an ARC GHRSST format. Operational version with simplified coefficients fed through GHRSST. Related, but different datasets: Full V1.5 ATSR-1, ATSR-2, AATSR record in GDS 1.7 format received by LTSRF on physical media; LTSRF to work with GDAC to ingest and archive.

- SEVIRI (Roquet, MeteoFrance): Plans to reprocess back to January 2004; is funded by EUMETSAT and ready for delivery after 2014.

\section{Level 4 Activities}

- Mediterranean and Black Sea Analysis (Nardelli, GOS/CNR): On hold while CNR focused on other priorities this past year.

- $\quad$ OSI SAF Sea Ice (Eastwood, Met.No): Used by PFV5.2 and 6; reprocessing all available passive microwave data (SMMR, SSM/I); might see Version 1.1 July/August this year; back to 1978.

- HadISST2 (Rayner, UKMO): New prototype available soon; will include ATSR Reanalysis for Climate (ARC) data by 2012 and then be updated monthly.

- MUR Phase I (Chin, NASA/JPL): Phase I 2002-present; trying to cover Aqua period as much as possible; Version 3 is under plan to optimize analysis scales; Terra period (back to 1999) intended.

- NOAA POES-GOES Blended (Maturi, NOAA/OSPO): Submitted a proposal to reprocess; improved error categorization; inclusion of microwave SST.

- DMI OI (Hoeyer, DMI): Preliminary release sometime this year, $4 \mathrm{~km}$ resolution, 1985 to present.

- ESA SST CCl (Merchant, Univ. of Edinburgh): Plan is to generate an SST CDR for 1991 to 2010 using ATSR + AVHRR; independent of in situ data.

- OSTIA (Roberts-Jones, UKMO): Paper currently in the works; reanalysis completed; no plan to provide full reanalysis to the GDAC, from the perspective of MyOcean.

- Nighttime-only Daily OISST (Banzon, NOAA/NCDC): Currently experimental, to see if it would be useful to produce regularly.

- MGDSST (Ishizaki, JMA): 1985-2007 available; SSTs for 1985-2004 analyzed using Pathfinder V5; reanalysis for 1981-1984 will use Pathfinder V5.2 and revise climatologies; compare with long-term in situ COBE analysis.

- Lamont-Doherty Kaplan Analysis (Kaplan, LDEO): Will provide analysis using common input QC'd data for GCOS SST intercomparison pilot project ASAP.

\section{Data Tools and Intercomparison Activities}

- GCOS SST Intercomparison Framework (Brandon, NODC): GCOS SST Intercomparison team generated a set of quality-controlled data to be used as a common input data set to test various analysis methods; currently two analyses have been run using this data (COBE and ERSST) and provided through the intercomparison framework; still need Kaplan and HadISST analyses. 


\section{SST ECV Data Processing Framework}

The second goal of the RAN-TAG breakout session was to discuss the establishment of a Data Processing Framework (DPF) for the SST Essential Climate Variable. GHRSST and the RANTAG are in a unique position to define and implement this DPF, since this group comprises many of the world's experts on SST Climate Data Records (CDR). Progress toward defining an international DPF for the SST ECV is now considered possible due to the advances made in the last ten years.

First, the group has already worked with the rest of GHRSST to establish the GDS2.0 and the GHRSST Regional/Global Task Sharing (R/GTS) Framework, which defines the flow of data from GHRSST RDACs to the GDAC and on to the LTSRF for long term archive and stewardship. It was noted that the R/GTS has made an enormous amount of data available to SST production centers all around the world in a consistent, easy to access and use format, enabling more accelerated SST climate product development than ever before.

Second, the effective international coordination developed by the RAN-TAG and the GCOS SST and Sea Ice Working Group was noted as a key, foundational component necessary to establish the SST ECV DPF.

Third, the RAN-TAG has already developed the conceptual framework, known as the SST ECV "cube", during the GHRSST Science Team Meeting in 2009. This conceptual framework, illustrated in Figure 1, clearly identifies the need not for a single SST ECV product, but rather a comprehensive suite of products needed to meet the wide range of climate SST user requirements. These requirements identify a range of SST ECV products, spanning SST product types, spatialtemporal resolutions, and processing level.

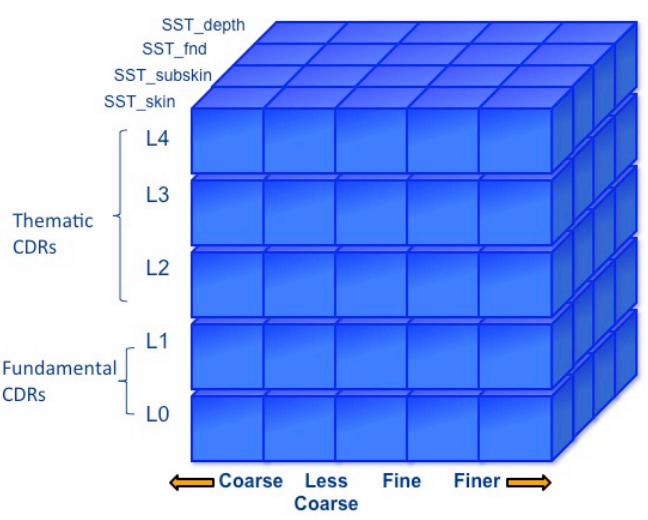

Figure 1: The SST Essential Climate Variable Conceptual Framework (established by GHRSST in 2009).

The work that remains is to build from that concept to an actual DPF. This includes developing processes and metrics on top of the GDS 2.0 format and R/GTS data management scheme, to define and document a clear framework for data providers who wish their data sets to be considered and evaluated as SST ECVs. Once defined and documented, the group must work to implement the DPF so that the vision and concept illustrated in Figure 1 can be achieved.

\section{What should be in the DPF?}

Several high-level programs have developed quality metrics and standards that should be considered in the creation of the SST ECV DPF. These include the GCOS Climate Monitoring Principles, the NOAA Climate Data Record Program Maturity Model, the ESA Climate Change Initiative $(\mathrm{CCl})$, and the draft SST $\mathrm{CCl}$ Climate Operations Model. Scientific data requirements and metrics include stability, uncertainty, accuracy and consistency. Engineering and data management metrics include software readiness, metadata maturity, documentation, product validation, public access, data delivery schedule, data utility, and operational updates. Using GHRSST expertise for SST science and data production, these requirements and metrics should be tailored to best fit the SST ECV. The SST CCl User Requirements Development exercise also 
provided a list of requirements from climate research users' perspectives.

In addition to measuring SST products against the requirements laid out by these high-level programs, the group discussed the need for community-established metrics. These should be accessible and not overly burdensome to calculate, thus enabling more groups to participate. Existing community-developed infrastructure should be leveraged. For example, data could be required to be produced in GDS 2.0 format, submitted through the GHRSST R/GTS, and incorporated into the GCOS SST Intercomparison Facility.

The group discussed the need for an established, traceable, reliable network of in situ radiometers to be used for data assessment. This kind of referable measurement standard is critical for proper validation of a Climate Data Record. The group emphasized that this network must be broader than individual satellite missions and validation projects, and must be maintained over the long term. One way to accomplish this might be to leverage existing programs by installing radiometers on platforms such as NOAA ships, volunteer observing ships, and the TAO array of moored buoys.

\section{To whom does the DPF apply?}

Through the various efforts of programs such as GCOS, ESA CCl, and the NOAA CDR Program, a set of overarching, programmatic requirements for the SST ECV has become available. These programs provide the opportunity for products in the SST ECV data cube to be traceable to internationally-recognized standards. The DPF as a whole can be applied to any data providers who wish their data sets to be considered and evaluated as SST ECVs. The framework should be used much in the way the GHRSST community is already used, to foster communication and identify potential synergies and opportunities for collaboration in generating the SST CDRs. Defining and implementing the DPF is part of GHRSST's natural maturation as an international organization and will establish the authoritative framework for all products seeking designation as an SST ECV. GHRSST has defined the standards for SST products, and now must define a way to designate a subset of those products worthy of the "SST ECV" title, in order to further build up and maintain reliability as an international community.

\section{How do we implement the DPF?}

Several topics were discussed that could lead up to the implementation of the DPF. One suggestion was that in order to accommodate more CDR efforts and maximize inclusivity, a slight step back from full-GDS 2.0 compliance might be allowed (e.g. a core GDS set of variables). This caused significant concern for most RAN-TAG members, who felt that the fundamental point of the DPF is to build additional requirements on top of the GDS and GHRSST R/GTS. Therefore, developing a more minimal set of requirements would go against the end goal of establishing robust requirements for the SST ECV. Indeed, if the group were to define a new class of GHRSST data sets within an updated GDS specifically for ECVs, that new set would most likely involve more extensive content, format accuracy, and precision requirements; additional metrics calculation; and requirements for inclusion in product intercomparison systems. Thus the idea of stepping back from full GDS 2.0 compliance was not generally supported, but the group acknowledged the need to carefully consider the issue when defining the DPF.

The group also briefly discussed applying the GDS format to in situ data sets so that they may become a more integrated part of the existing GHRSST data system and thus more formally part of the DPF. Some RAN-TAG members have experience applying the GDS conventions to in situ data, and the GHRSST team could help provide conversion code to in situ data providers to help them bring their data into the system. This experience has been applied to in situ datasets like ICOADS 
and HadSST in the context of the GCOS SST Intercomparison system.

Existing intercomparison tools, such as the GMPE and the HR-DDS, could also be leveraged as a means of assessing SST CDRs against each other and against reference data sets. Products could be incorporated into and compared with an extended version of the GMPE as a means of establishing community consensus on the strengths and weaknesses of any individual SST ECV. The HR-DDS would also benefit from including SST ECV products, though the actual benefit of the DDS to the ECV products themselves is not clear. Unlike 10 years ago, the community now has several intercomparison tools, together providing comprehensive assessment capabilities which can be used as references during the definition of the SST ECV DPF and possibly leveraged during its implementation.

In general, the group agreed that some care needs to be taken when developing metrics and requirements such that they are achievable by those who wish to contribute to the SST ECV. If metrics are too onerous, it will be difficult to insist they be computed for all candidate SST ECV products. In addition, the limitations of existing CDR efforts must be considered in terms of legal, resource, and technical restrictions. In other words, the group must be pragmatic, and perhaps develop the DPF in phases, increasing the robustness of the framework as it progresses.

\section{Next steps}

The group agreed that the best way forward would be to develop a draft framework, discuss it, and work to agree on it by the next Science Team meeting. The outgoing RAN-TAG Chair and incoming Chair agreed to coordinate this activity along with the Chair of the GCOS SST and Sea Ice Working Group and the new CEOS Virtual Constellation for SST (SST-VC). The GHRSST Program Office is funded to support the development of this document, and will be leveraged during this process. At the same time, a few specific tasks will be performed in support of the DPF draft. One task is to examine the NOAA Climate Data Record Program Maturity Index and tailor its elements to the SST ECV; this action will be performed by the new RAN-TAG Chair, Dr. Chris Merchant (see Section 5 below). Another action will be to perform an inventory of existing SST CDR efforts, the limitations and restrictions of those efforts, and their expected outputs. That action will be performed by the co-chair of the CEOS SST-VC, Dr. Craig Donlon. This entire effort will be done in coordination and conjunction with the CEOS SST-VC and the GCOS SST and Sea Ice Working Group, to ensure that longer-term, presatellite era data sets are also included and to ensure that satellite-era data sets integrate appropriately with these.

\section{Prioritized List of Actions}

The third goal of the breakout session was to develop a prioritized list of actions for the coming years. In preparation for the meeting, the Chair solicited input on priority actions from members. During the breakout session, the group reviewed this input and ranked the actions according to their usefulness ("Will accomplishing this action help your work?") by show of hands. While not strictly rigorous, this ranking method has proven effective over the last few years and can be conducted in a short amount of time during the breakout session. The following list shows the top priority actions for the RAN-TAG in the coming year:

1. Complete AVHRR Pathfinder Version 6 (GAC and HRPT/LAC)

2. Develop consolidated, calibrated, geolocated AVHRR GAC/LAC L1b archive

3. Determine how to assess and compare stability of SST CDRs

4. Generate bias and uncertainty estimates for each in situ SST measurement used to produce reanalysis products 
5. Improve bias correction of L4 products, particularly during the Pinatubo period

6. Perform ESA SST $\mathrm{CCl}$ reanalysis back to 1981

7. Define the ECV Data Processing Framework

8. Establish an in situ radiometer network

9. Perform reanalysis of in situ data sources into a common format

10. Assess product accuracy using consistent independent SST data sets such as Argo

11. Identify a subset of reference data for a reanalysis period

12. Provide (A)ATSR reprocessed data in GDS2.0 format

13. Complete Pathfinder V5.2 GAC for L2P, L3U and L3C data

14. Provide MODIS reprocessed data in GDS2.0 format

15. Generate an accurate, long-term, daily foundation SST analysis from infrared and microwave SST using the best available processing methods

16. Assess and provide feedback on ATSR Reprocessing for Climate (ARC) v1.0/v1.1 SST release

17. Provide guidance/recommendations through GHRSST, GDAC and LTSRF websites to non-expert SST users in choosing from an overwhelming variety of GHRSST products

18. Fix the calibration of AVHRR and AMSR-E to remove latitudinal and other biases before reprocessing of data sets

19. Quantify uncertainties and their covariance structure through a bottom-up approach, i.e. understanding the uncertainties inherent in each step of data production
20. Complete the GCOS SST Intercomparison pilot study

21. Explore NIST traceability for SST CDRs

22. Provide an interactive look-up table for all GHRSST products to help users prioritize their search parameters when choosing the most appropriate SST product for their application

\section{Terms of Reference and Membership}

An action was taken by the RAN-TAG Chair during GHRSST-XI to update the Terms of Reference (ToR). This action remains open, with the ToR currently dominated by old requirements and the outdated Concept of Operations. After reviewing the ToR in the past year, the Chair took an action to work with the new RAN-TAG chair to coordinate an update to and simplification of the group's ToR that will incorporate the latest GCOS/RANTAG climate data requirements. This update will be completed by the end of July of this year and distributed to the RAN-TAG for review.

The group also reviewed its membership. As a result of this review, Dr. Viva Banzon and Dr. George Paltoglou were added as new members; no current members stepped down from the RAN-TAG.

Finally, after nomination earlier in the meeting, the group considered and approved by consensus Dr. Chris Merchant to take over as Chair of the RAN-TAG from Dr. Kenneth S. Casey, effective at the close of GHRSST XII. The group thanked Dr. Casey for his dedicated and energetic work for the RAN-TAG over the last ten years. 
GHRSST Project Office

\section{Summary of Action Items}

\begin{tabular}{|c|c|c|c|c|c|}
\hline Action \# & Description & Tasked To & \multicolumn{2}{|c|}{ Deadline } & Status \\
\hline $\begin{array}{l}\text { G12-RANTAG- } \\
01\end{array}$ & $\begin{array}{l}\text { Revise RAN-TAG Terms of } \\
\text { Reference by end of July } 2011 \\
\text { and send back to RAN-TAG } \\
\text { members for discussion }\end{array}$ & $\begin{array}{l}\text { RAN-TAG } \\
\text { (Casey and } \\
\text { Merchant) }\end{array}$ & $\begin{array}{l}\text { July } \\
2011\end{array}$ & $31^{\text {st }}$ & $\begin{array}{l}\text { Draft } \\
\text { completed and } \\
\text { sent } \\
\text { Merchant for } \\
\text { review on } 10 \\
\text { July. }\end{array}$ \\
\hline $\begin{array}{l}\text { G12-RANTAG- } \\
02\end{array}$ & $\begin{array}{l}\text { Update RAN-TAG membership } \\
\text { list on GHRSST web site and } \\
\text { "group" list on GHRSST } \\
\text { system. }\end{array}$ & $\begin{array}{l}\text { RAN-TAG } \\
\text { (Casey) }\end{array}$ & $\begin{array}{l}\text { July } \\
2011\end{array}$ & $31^{\mathrm{st}}$ & $\begin{array}{l}\text { Updated web } \\
\text { site with list, } \\
\text { emailed GPO } \\
\text { on } 02 \text { July } \\
2011 \text { to ask } \\
\text { about "group" }\end{array}$ \\
\hline $\begin{array}{l}\text { G12-RANTAG- } \\
03\end{array}$ & $\begin{array}{l}\text { Send a list of upcoming } \\
\text { meetings relevant to the RAN- } \\
\text { TAG to Ken by the end of next } \\
\text { week for posting on GHRSST } \\
\text { web site }\end{array}$ & $\begin{array}{l}\text { RAN-TAG } \\
\text { (All } \\
\text { Members) }\end{array}$ & July $8^{\text {th }}$ & 2011 & $\begin{array}{lr}\text { Initial lists } \\
\text { received. } & \text { Need } \\
\text { to ask } & \text { GPO } \\
\text { how to add } \\
\text { calendar } \\
\text { entries. }\end{array}$ \\
\hline $\begin{array}{l}\text { G12-RANTAG- } \\
04\end{array}$ & $\begin{array}{l}\text { Develop first draft of a Data } \\
\text { Processing Framework as a } \\
\text { cooperative effort between the } \\
\text { RAN-TAG , GCOS SST/Sea } \\
\text { Ice Working Group, and CEOS } \\
\text { SST-VC }\end{array}$ & $\begin{array}{l}\text { RAN-TAG } \\
\text { (Casey, } \\
\text { Rayner, } \\
\text { Merchant) }\end{array}$ & $\begin{array}{l}\text { August } \\
2011\end{array}$ & $31^{\text {st }}$ & \\
\hline $\begin{array}{l}\text { G12-RANTAG- } \\
05\end{array}$ & $\begin{array}{l}\text { Collect and compile inventory } \\
\text { table of "who's doing what" for } \\
\text { GCOS ECV CDRs for input to } \\
\text { Data Processing Framework }\end{array}$ & $\begin{array}{l}\text { RAN-TAG } \\
\text { (Donlon) }\end{array}$ & $\begin{array}{l}\text { July } \\
2011\end{array}$ & $31^{\text {st }}$ & \\
\hline $\begin{array}{l}\text { G12-RANTAG- } \\
06\end{array}$ & $\begin{array}{l}\text { Translate NOAA Climate Data } \\
\text { Record Program Maturity } \\
\text { Model Table to SST ECV }\end{array}$ & $\begin{array}{l}\text { RAN-TAG } \\
\text { (Merchant) }\end{array}$ & $\begin{array}{l}\text { August } \\
2011\end{array}$ & $15^{\text {th }}$ & \\
\hline $\begin{array}{l}\text { G12-RANTAG- } \\
07\end{array}$ & $\begin{array}{l}\text { Consider a new name for the } \\
\text { RAN-TAG - link to G12- } \\
\text { RANTAG-01. }\end{array}$ & $\begin{array}{l}\text { RAN-TAG } \\
\text { (Merchant } \\
\text { and Casey) }\end{array}$ & $\begin{array}{l}\text { July } \\
2011\end{array}$ & $31^{\text {st }}$ & $\begin{array}{l}\text { Will propose } \\
\text { name "ECV- } \\
\text { TAG" in new } \\
\text { Terms } \\
\text { Reference }\end{array}$ \\
\hline $\begin{array}{l}\text { G12-RANTAG- } \\
08\end{array}$ & $\begin{array}{l}\text { Post } 2011 \text { International Status } \\
\text { Report to GHRSST and LTSRF } \\
\text { Web sites }\end{array}$ & $\begin{array}{l}\text { RANT-TAG } \\
\text { (Casey) }\end{array}$ & $\begin{array}{l}\text { July } \\
2011\end{array}$ & $31^{\text {st }}$ & \\
\hline
\end{tabular}




\section{SESSION 4.5.B: LSWT-WG BREAKOUT}

\section{Chair: Andrea Kaiser-Weiss ${ }^{1}$, Rapporteur: Pierre Le Borgne ${ }^{2}$}

(1) NCEO, University of Reading, Department of Meteorology, Reading, UK, Email: ghrsst-po@nceo.ac.uk

(2) Centre de Météorologie Spatiale, Météo-France, Lannion, France, Email: Pierre.LeBorgne@meteo.fr

Andrea Kaiser-Weiss gave an introduction why Lake Surface Water Temperatures (LSWT) are of interest:

1. Met-offices:

e.g. Weather warning,

fishermen information

NRT, daily, + ice

ECMWF: $10 \mathrm{~km}$

Matt: any improvements welcome

2. Climate scientists:

Long series, high stability,

Onset of melting and freezing

3. Other scientific users: see ARC Lake project

What has been done so far:

- ARC Lake

- Met-office project

- Calibration for satellites -> Simon Hook

- GHRSST : LWST under leadership of Bob Grumbine: land-sea mask

Following issues are expected and have been discussed to find ways to address:

Lake definition and respective lake mask (the latter might vary over time) are of high priority. Further issues to be addressed include:

Retrieval issues - because of varying height of different lakes, atmospheric effects, errors because of land contamination, small amount of in-situ data $\Rightarrow$ collaboration with EARWiG required

Cloud mask - is optimized for application over ocean

In-situ validation - ARC OE retrievals: applied case by case search (GTS)

Error estimates for LSWT.
LSWT needs to decide: What size of lakes can we reasonably provide a temperature for?

Ice information is wanted, too.

\section{Presentation by Stuart MacCallum}

Lake > 500 km2 closed (excludes rivers)

In situ on a case by case basis, except (Great lakes)

Results available at www.geos.ed.ac.uk/arclake

Impact on weather forecast; climate studies

Presentation by Matt Martin.

Large effect on NWP models (e.g. impact on Lake Victoria storm forecast)

Discrepancies between Bob Grumbines and Arc Lake masks

Need for lake ice info

(Ian Barton suggested Environment Canada)

Currently GHRSST has 3 sources of Lake masks: Bob Grumbine; ARC Lake project (contact Stuart MAcCallum) and from Pathfinder. Anyone interested can download the Pathfinder mask from ftp.nodc.noaa.gov

A need for a LWST consensus on a common Lake mask was expressed. It might be time varying? Pierre LeBorgne suggested to ask the Metoffices and OSISAF what they can realistically do. Agreement on a Lake mask will be one of the first actions of the group. 
GHRSST XII -

Proceedings, Edinburgh

GHRSST Project Office

\section{LWST Membership:}

Simon Hook (suggested chair), Stuart MacCallum, Emma Fiedler, Matt Martin, Chris Merchant, Pierre Le Borgne, Andy Harris, Owen Embury, Jorge Vasquez, Helen Beggs, Ken Casey, Mike Chin, Caroline Cox

\section{LWST Actions}

(to report at GHRSST XIII):
Issue: Final

Date: September 27, 2011

AC-G12-LWST-1: LSWT to organise itself, to agree on a draft ToR, to agree on a lake definition

AC-G12-LWST-2: LSWT to collect user requirements and ask interested users to join. 


\title{
SESSION 5: SUMMARY AND CONCLUSION OF BREAKOUTS
}

\author{
Chair: Craig Donlon ${ }^{1}$, Rapporteur:Andrea Kaiser-Weiss ${ }^{2}$
}

(1) ESA/ESTEC, Keplerlaan 1, 2201 AZ, Noodwijk, The Netherlands, Email: craig.donlon@esa.int

(2) NCEO, University of Reading, Department of Meteorology, Reading, UK, Email: ghrsst-po@nceo.ac.uk

David Meldrum updated on the Joint DBCP-GHRSST Pilot Project, which upgrades drifting buoys to be deployed (his presentations was shifted from Wednesday to Friday). He asked for GHRSST feedback on the usefulness of these new data to reduce the regional bias in satellite SST.

All the 10 GHRSST sub-groups had breakout sessions. The summaries and conclusions from these Breakout sessions have been presented in plenary on the last day of the meeting. See full Breakout session reports for comprehensive information. Below only a few highlights are given for each group.

ST-VAL, the Satellite Sea Surface Temperature Validation Working Group, discussed the value of ship measurements, the QC of Drifter Data, the DBCP Pilot Project concerning upgraded drifters, use of Argo near surface data, and the ST-VAL workplan for the next years.

DAS-TAG, the Data Assembly and Systems Technical Advisory Group, reviewed the GDS2 revisions, the GDS2 transition timeline, possible netCDF4 translation from netCDF3, GDS2 format compliance checker and the advantages of netCDF-4 vs. NetCDF-3.

DVWG,the Diurnal Variability Working Group, discussed diurnal warming derived from satellite data, in-situ data and modelled diurnal warming, as well as effects of waves, wind and advection in diurnal variability. Pierre LeBorgne proposed a SEVIRI SSTskin hourly analysis in delayed mode. However, the latter is shifted in time versus the buoy observations.
Helen Beggs suggested an inter-sessional meeting of the DVWG and other GHRSST working groups on the thematic issues of high latitudes.

AUS-TAG, the Applications and User Services Technical Advisory Group, addressed the Users Manual, a "onepager" concept for first-time SST users, a sashboard concept for the RDACs and several new data discovery and access tools.

HL-TAG, the High Latitude Technical Advisory Group, discussed efforts to improve cloud and ice masking. Users are calling for the highest possible resolution of sea ice products, ideally for one data set where all ice data have been merged and different resolutions are reflected in the uncertainties. Future work includes developing algorithms for high latitude SST and IST, reanalysis products, lake ice products, and validation with in-situ measurements.

R2HA2, the Rescue and Reprocessing of Historical AVHRR Archives Working Group, met for the first time. It agreed on the next steps to identify and locate historical archives (pre-2000) of AVHRR HRPT and LAC data, and to copy them to a central location. R2HA2 will define in the next year a common L1P format for storing these data.

IC-TAG, the Inter-Comparison Technical Advisory Group, added a new objective to its Terms of References: "to access and improve the specification of error in the L4 analysis". This issue is under active discussion in the group, in addition to ongoing inter-comparison work.

RAN-TAG, the Reanalysis Technical Advisory Group reviewed the current status and future plans of reanalysis 
GHRSST Project Office

activities, and defined and discussed the implementation of a Data Processing Framework for the SST Essential Climate Variable. Twenty-seven projects from the international SST reanalysis community were summarized. The quality metrics and standards (scientific, engineering and data management) of several high-level programs (GCOS, ESA CCI, NOAA CDR) are planned to be considered, together with community-established metrics. Further, the group called for an established, traceable, reliable network of in situ radiometers as reference measurement standard. The group thanked Ken Casey for his dedicated and energetic work to build and develop the RAN-TAG over the last 10 years. Chris Merchant will took over as new Chair.
EARWiG, the Estimation and Retrievals Working Group discussed the open issues related to the retrievals (especially for a $0.05 \mathrm{~K} /$ decade stability requirement). Another priority of EARWiG is the calibration of the instruments.

LWST, the Lake Surface Water Temperature Working Group met for the first time. The Met-Offices showed high interest in improvements of Lake Surface Water temperatures. Stuart MacCallum presented the ARC Lake temperatures, which Matt Martin tested for use in the OSTIA reanalysis. The lake definitions, retrieval issues, in-situ validation and error estimates as well as improved cloud masks needs addressing in the next years. 


\section{SESSION 6: WRAP-UP}

\section{Chair: Peter Minnett ${ }^{1}$, Rapporteur:Andrea Kaiser-Weiss ${ }^{2}$}

(1) METEOROLOGY \& Physical Oceanography, University of Miami, USA, Email: pminnett@rsmas.miami.edu

(2) NCEO, University of Reading, Department of Meteorology, Reading, UK, Email: ghrsst-po@nceo.ac.uk

\section{Review of Actions list}

The Actions resulting from GHRSST XII were reviewed and approved, they are listed at the GHRSST web-site and attached in the Appendix of this document.

Several new Science Team members were proposed for election:

Misako Kachi

Proposed by: Craig Donlon

Seconded by: Tim Nightingale

Seconded by: Ken Casey

Shiro Ishizaki

Proposed by: Ken Casey

Seconded by: Matt Martin

Seconded by: Craig Donlon

Viva Banzon

Proposed by Dick Reynolds

Seconded by Jorge Vazquez

Seconded by Peter Minnett

Bill Emery

Proposed by Sandra Castro

Seconded by Peter Minnett

Seconded by Gary Wick

Lei Guan

Proposed by David Llewellyn-Jones

Seconded by Peter Minnett

Seconded by Dave Poulter

\section{Gary Corlett}

Proposed by Pierre LeBorgne

Seconded by Craig Donlon

Seconded by Jacob Hoeyer

.... and many more

Jon Mittaz

Proposed by Andy Harris

Seconded by Chris Merchant
Seconded by Pierre LeBorgne

Tim Liu

Proposed by Peter Minnett

Seconded by Craig Donlon

Seconded by Jorge Vazquez

\section{ST Members stepping down:}

Andy Bingham

\section{Formal handover of ST chair}

David Llewellyn-Jones thanked the outgoing ST chair Craig Donlon for his more than 10 years of dedicated work as GHRSST Founding Chair. The Science Team recognised his outstanding commitment and acknowledged with gratitude his personal contribution to establishing GHRSST as the international forum for producers and users of Satellite Sea Surface Temperature data.

Craig Donlon wished GHRSST and its Science Team well under the new ST chair Peter Minnett.

Peter Minnett gave his perspectives as the new ST Chair. He expressed his gratitude to built upon the success of GHRSST, at the threshold of a new area with 3 new-generation SST sensors to be launched in the next several years.

$\mathrm{He}$ plans to broaden GHRSST by including more countries, to strengthen international collaboration, and to establish better mechanisms for feedback from the applications community. He will ensure a two-way flow of information and innovation between GHRSST and national space and meteorological agencies. He envisages galvanizing the GHRSST momentum into the exploitation of the new sensors and linking their measurements to 
GHRSST XII -

Proceedings, Edinburgh

GHRSST Project Office

historic ones for climate data record extensions.

Next venue: GHRSST XIII in 2012 in Tokyo, hosted by JAXA in collaboration with JMA
Issue: Final

Date: September 27, 2011

AOB and thank you: Andrea KaiserWeiss thanked Chris Merchant for the excellent local organisation and Emma Danby and Silvia Bragaglia-Pike for their preparations and support in the background. 


\section{R05 - REPORT FROM THE GHRSST PROJECT OFFICE (GPO): 2010/11}

\section{Andrea Kaiser-Weiss}

\section{NCEO, University of Reading, UK, Email: ghrsst-po@nceo.ac.uk}

The International Project Office for the Group for High Resolution Sea Surface Temperature (GHRSST) coordinates the operation and stimulates the development of GHRSST for a period of three consecutive years, serving GHRSST in the most appropriate manner and taking advice from the GHRSST Advisory Council, and consults the GHRSST Science Team. The details of the expected work are specified in the Statement of Work (SoW).

The project to provide the GHRSST Project Office (GPO) hosted at NCEO, University of Reading, kicked off on $2^{\text {nd }}$
August 2010. The appointed GHRSST Project Coordinator is Andrea KaiserWeiss (AKW).

Firstly, as the new GHRSST Project Coordinator, A. Kaiser-Weiss (AKW) started to work with the Science Team, each Task Group and Working Group by discussing their current scientific and technical issues, their needs for support (meetings, web-page, collaboration, discussions) and their future plans.

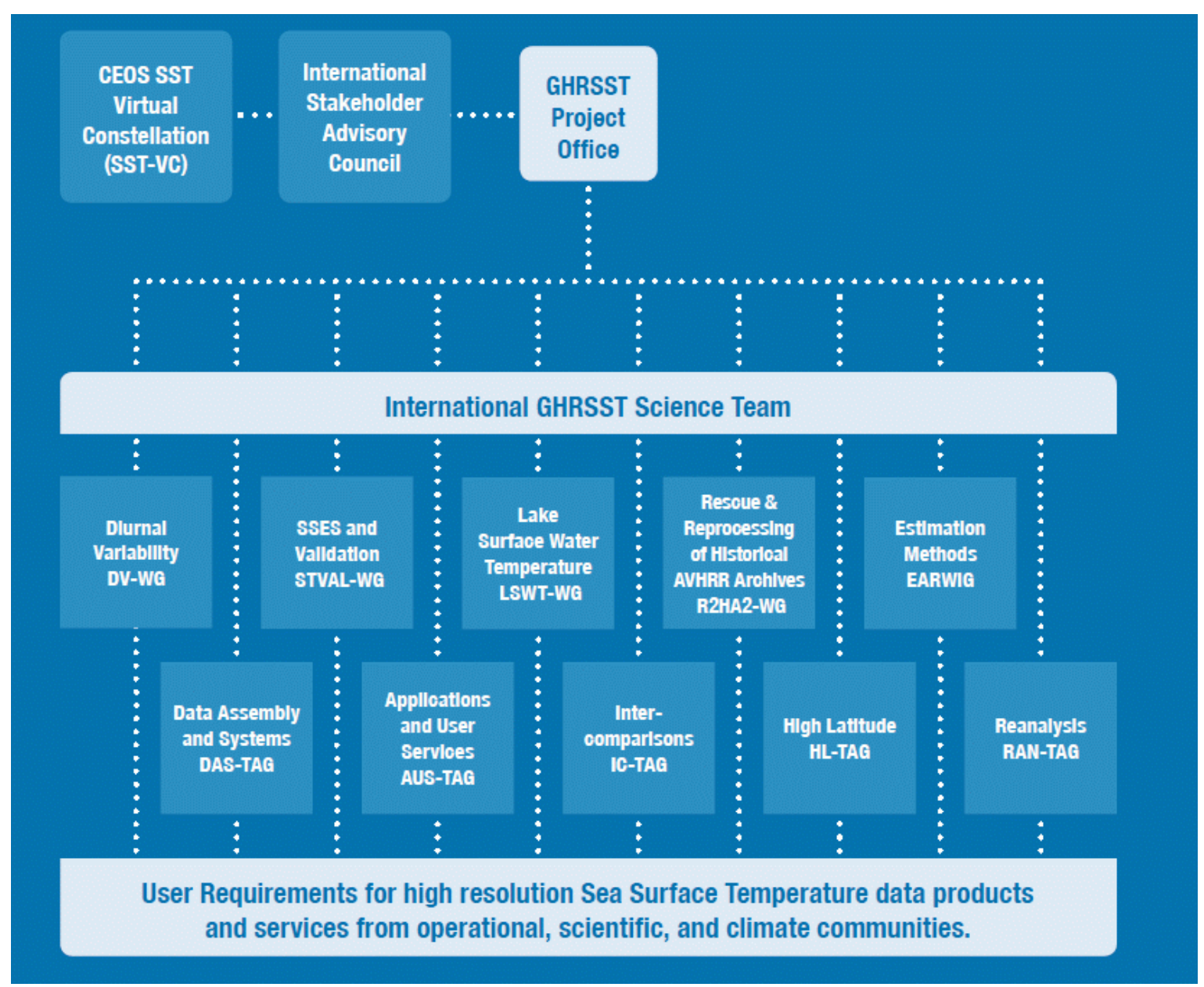

Fig. 1: GHRSST organization showing the GHRSST working groups (WGs) and technical advisory groups (TAGs) in 2010 and 2011.

The following activities to provide support are highlighted:
- EARWIG (Estimation and Retrievals Working Group). Encouragement was 
given to EARWIG to start activities and update the website.

- AUS-TAG (User support Technical Advisory Group): An initial workplan was developed, and collaboration with the Regional Data Assembly Centres (RDACs) was initiated.

- DAS-TAG (Data Assembly and Systems Technical Advisory group): The review of GDS2.0 (GHRSST Data Specification version 2.0) was managed. As a result, the community consensus format GDS2.0 was released on $1^{\text {st }}$ October as planned. Upcoming issues are discussed within DAS-TAG and AKW updates an GDS2 amendments tracker which published via web-page https://www.ghrsst.org/files/download. php?m=documents\&f=110506220330 ḠDS20Amendmentstracker280411.xI S.

- The Regional Data Assembly Centres (RDACs) asked for formal approval of their use of ECMWF wind fields; AKW collected the RDAC requirements, and organized the official approval.

- RAN-TAG (Reanalysis Technical Advisory Group). AKW organised the RAN-TAG review of the GCOS-107 (Systematic

Observation

Requirements for the Satellite-based Products for Climate).

- IC-TAG (Intercomparison Technical Advisory Group. A discussion about Level 4 data metrics was initiated and contributions collected for the benefit of the IC-TAG.

- STVAL-TAG (Satellite SST Validation Working Group) and DWWG (Diurnal Variability Working Group). For STVAL-TAG, the DBCP-Pilot project on upgrading drifting buoys was followed. Also for STVAL, and for the benefit of the Diurnal Variability Working Group (DWWG), analysis of near-surface Argo float data have been initialized. A Masters student has been recruited to work on those with joint supervision of GPO and the UK Met Office.

- DVISTVAL/HL groups (Diurnal Variability, Validation and High Latitude Groups) required and got support for organizing an internal meeting.

- GHRSST XI. AKW attended the GHRSST XI meeting in Lima before her contract started, and had a chance to meet many GHRSST participants there.

- $\mathrm{AKW}$ made several trips within the UK (Southampton, Exeter, Leicester, and Edinburgh) to build a working relationship with the GHRSST Team members, and abroad to meet up with the Japanese GHRSST community in Tokyo and the US GHRSST community when visiting the NASASST in Seattle (November 2010) and the joint working group meeting in Boulder (Feb/March 2011) where AKW caught up with scientific progress by participants and consolidated the results.

Secondly, a major focus was consolidating or building contacts with users, and also with stakeholder agencies.

- In Tokyo, AKW introduced herself to the key persons at GODAEOceanview and ET-OOFS (JCOMM Expert Team - Operational Ocean Forecasting Systems). A discussion on guiding ocean application users (Level 3 data assimilation) and a discussion on guiding NWP users (who wanted skin SST) spun off. Contacts with users were built up at the University of Reading Department of Meteorology, the UK Met Office (JCMM- Joint Centre for Mesoscale Modelling), ESSC (Environmental System Science Centre, NERC, UK) with respect to ocean reanalysis, ECMWF (seasonal forecasting, modelling group with special interest in both lake temperature and SST). 
- External representation included a special highlight presentation at the NCEO (the UK National Centre for Earth Observation) annual meeting, and at a meeting with the ESA Climate Office in Harwell, a Departmental Seminar for the Department of Meteorology in Reading, and presentations to a delegation of the Indian Space Agency. A Brazilian delegation visited GPO in January 2011.

- $\mathrm{AKW}$ participated in the DBCP (Data Buoy Cooperation Panel) and Argo meeting at Oban to pursue the DBCPGHRSST-Pilot Proposal, to meet JCOMM (Joint WMO-IOC Technical Commission for Oceanography and Marine Meteorology) representatives as well as to communicate the GHRSST needs for updated buoy measurements. AKW is involved in ongoing discussions about progress of the DBCP-GHRSST-PP and how long-term stability of buoy calibration might be achieved, and organized the GHRSST reply to the Argo nearsurface measurement requirements.
- AKW pursued discussions on how GHRSST could best serve as CEOSVC for SST, and in the ATSR SAG where future GHRSST contributions will be needed for validation planning.

- A project review was performed for NOPP (the US Oceanographic Partnership Program).

- AKW established contacts to the International Ocean Colour Coordinating Group (IOCCG) and participated at the $16^{\text {th }}$ annual IOCCG Committee meeting at Plymouth, 1517 February 2011.

- AKW GPO promoted GHRSST and discussed the needs of climate users in five informal meetings during 2010/2011 at the University of Reading with climate scientists.

In parallel, networking and promoting GHRSST continued by inviting GHRSST major stakeholders and users to sponsor GHRSST XII as well as to participate and to present.

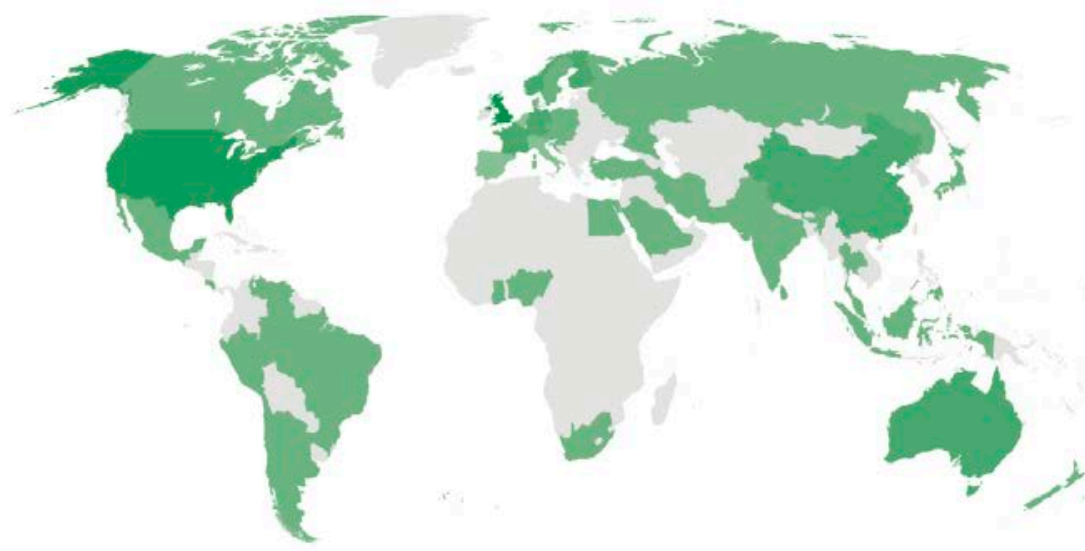

Fig. 2: Internet traffic to the GHRSST web-site in April to May 2011 indicate the global interest in the GHRSST activities.

The Proceedings for GHRSST XI were compiled:

https://www.ghrsst.org/files/download. php?m=documents\&f=110614151229ProceedingsFinal140611.pdf

The GHRSST web-site https://www.ghrsst.org had a major re- design and refresh based on a review where requirements of the Science Team were gathered and as a result a new home page was planned together with the migration to a new CMS system with bespoke features, such as blogs, dashboard and modernized news system and improved access to documents and 
publications. The SST definition page was improved following discussions with the Science Team. News items were released approximately 2-3 monthly, together with so far three quarterly Newsletters. Specific pages were created for meetings.

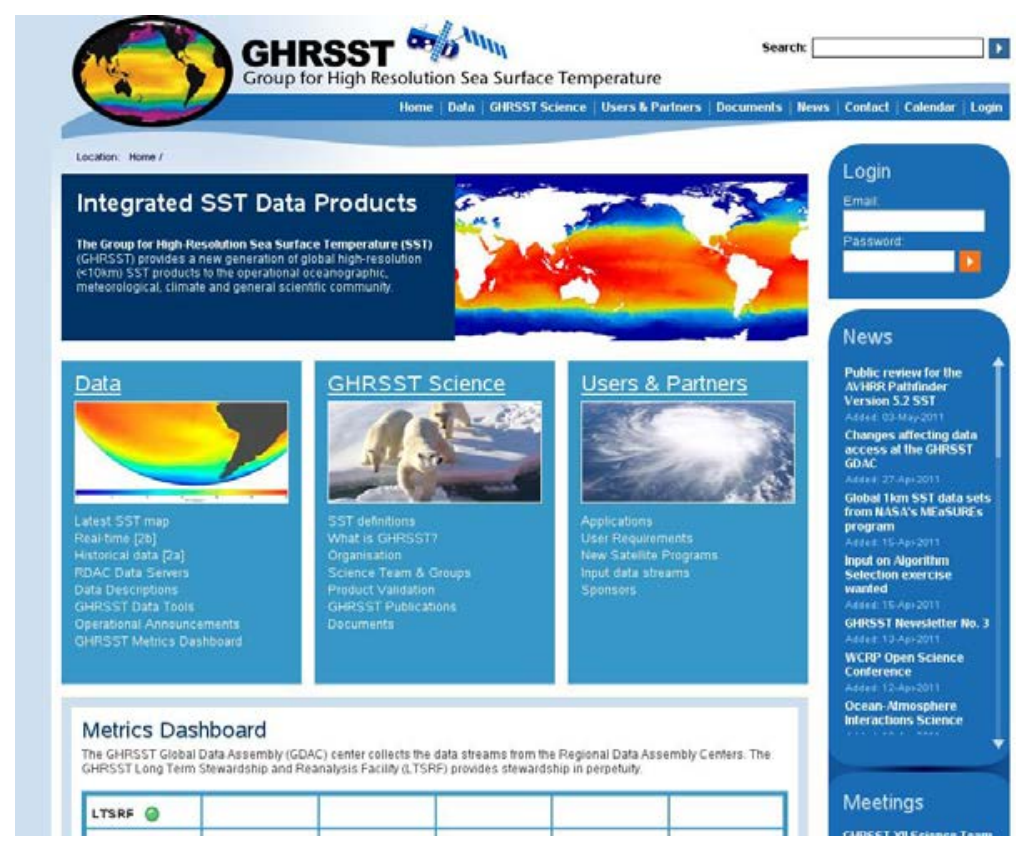

Fig. 3: The re-designed and re-structured GHRSST home page.

The GHRSST XII Science Team Meeting in Edinburgh was organized and the Scientific Agenda prepared with help of the Science Team Chair and AUS-TAG and considering the comments from the Science team. Sponsoring has been organized by $\mathrm{AKW}$ and more than 30 invitations for participation have been sent. Based upon the collected reports from the Data Assembly Centres and the GHRSST working groups and Technical Advisory groups, a GHRSST Development and Implementation Plan
(GDIP) has been drafted.

A GHRSST brochure has been designed using input from the Science Team and review from the major stakeholders.

The full report of the GHRSST project office with respect to the Statement of Work with references to further documents prepared by the GPO can be found here:

https://www.ghrsst.org/files/download. php? $m=$ documents \& $f=110519125956-$ D25AnnualReport17May.docx

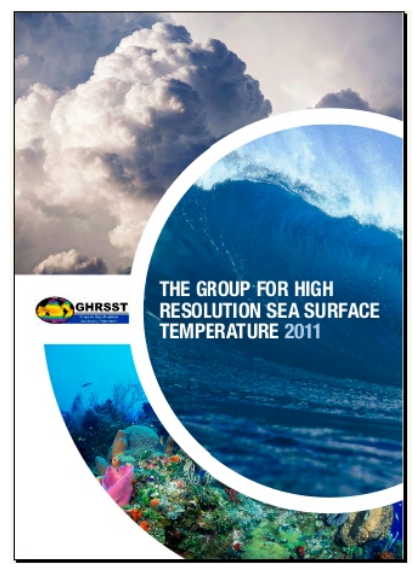

Fig 4: A GHRSST brochure aimed at interested scientists, program managers and the general public. 


\title{
R07 - REPORT FROM AUSTRALIA - BLUELINK AND IMOS
}

\author{
Helen Beggs $^{(1)}$, Leon Majewski ${ }^{(2)}$, George Paltoglou ${ }^{(1)}$, Ruslan Verein ${ }^{(1)}$ \\ and Aihong Zhong ${ }^{(2)}$
}

(1) Centre for Australian Weather and Climate Research, Bureau of Meteorology, Melbourne (Australia), Email: H.Beggs@bom.gov.au

(2) Bureau of Meteorology, Melbourne (Australia), Email: Leon.Majewski@bom.gov.au

\section{ABSTRACT}

Since the $11^{\text {th }}$ GHRSST-PP Science Team Meeting there have been a number of exciting new sea surface temperature (SST) products released by the Australian Bureau of Meteorology with support from the BLUElink Project and the Integrated Marine Observing System (IMOS). In addition to the operational regional and global SST analyses (RAMSSA and GAMSSA) contributed to the GHRSST Global Data Assembly Centre (GDAC) and the GHRSST Multi-Product Ensemble Project, the Bureau is also producing single sensor HRPT AVHRR SST in GDS V2.0 L2P/L3U/L3C formats which we intend to supply to the GDAC before December 2011. Other products routinely produced by the Bureau which may be of interest to the GHRSST community are the experimental regional and global skin SST analyses (RAMSSA_skin and GAMSSA_skin), reprocessed MTSAT-1R skin SST GDS v2.0 L3U files, operational 14-day "Mosaic" HRPT AVHRR SST composite product in GHRSST-L3 format and validation-quality, real-time SSTdepth data from thirteen ships of opportunity. This report summarises the advances made in the research and development of new SST products by BLUElink and IMOS from 1 June 2010 to 1 June 2011 and plans for activities up to the end of the IMOS and BLUElink III projects (June 2013).

\section{Introduction}

For the past eight years, the Australian Government, through the Australian Bureau of Meteorology (Bureau, http://www.bom.gov.au), Royal Australian Navy and CSIRO have contributed to
BLUElink> Ocean forecasting Australia (Brassington et al., 2007; http://www.bom.gov.au/bluelink), a project to deliver ocean forecasts for the Australian region. BLUElink includes ocean model, analysis and assimilation systems, and provides timely information and forecasts on oceans around Australia. Phases I and II of the project have completed and Phase III has commenced and will run until June 2013. Operational high resolution $\left(0.1^{\circ}\right.$ horizontal resolution) ocean analyses and forecasts are available as maps from http://www.bom.gov.au/bluelink and netCDF files from http://godae.bom.gov.au.

Commencing in 2007, the BLUElink support for the Group for High Resolution SST (GHRSST) has been strongly augmented by funding from the Integrated Marine Observing System (IMOS, http://www.imos.org.au), a nation-wide collaborative program designed to observe the oceans around Australia, running until June 2013.

The main BLUElink and IMOS contribution to GHRSST is through an Australian Regional Data Assembly Centre (RDAC) system based at the Bureau of Meteorology, delivering the following types of GHRSST data products:

- Locally received High Resolution Picture Transmission (HRPT) Advanced Very High Resolution Radiometer (AVHRR) SST L2P (geolocated, single swath), L3U (gridded, single swath), L3C (gridded, single sensor) and L3S (gridded, multiple sensor) files (Paltoglou et al., 2010) 
- L4 files from "RAMSSA", the operational, daily, $1 / 12^{\circ}$ resolution, SST analysis over the region $20^{\circ} \mathrm{N}$ to $70^{\circ} \mathrm{S}, 60^{\circ} \mathrm{E}$ to $170^{\circ} \mathrm{W}$ (Beggs et al., 2011a), and the operational, global, daily, $1 / 4^{\circ}$ resolution SST analysis system ("GAMSSA") (Zhong and Beggs, 2008; Beggs, 2008)

- MTSAT-1R and MTSAT-2 hourly, $1 / 20^{\circ}$ resolution, SST L3U (gridded, single scene) files

Other contributions include:

- High quality in situ SST available via the GTS in real time from vessels of the Australian Volunteer Observing Fleet (AVOF) fitted with Automatic Weather Stations and other ships of opportunity in the Australian region (Beggs et al., 2009a; Beggs et al., 2010a; Beggs et al., 2011b)

- High quality in situ meteorological and SST available via the IMOS ocean portal in near real-time from a Southern Ocean mooring (http://imos.org.au/sofs.html and Beggs et al., 2010b)

- NOAA/BoM collaboration on MTSAT$1 R$ SST calibration/validation and processing

- Regional hourly and Global 3-hourly skin SST analyses in a GHRSST L4like format ("RAMSSA_skin" and "GAMSSA_skin")

- Provision of satellite and numerical weather prediction (NWP) model data for the GHRSST Diurnal Variability Working Group study of SST diurnal variation models over the Western Pacific Tropical Warm Pool (TWP+)

\section{SST from Ships of Opportunity}

Typically, SST observations from volunteer observing ships (VOS) in the Australian region have been of uncertain accuracy. Until recently, SST observations from Australian research vessels have been difficult to access in a timely manner in consistent formats. Ship SST observations in the Australian region have therefore not been used for near real-time validation of satellite SST observations. From 2008, the IMOS Project has enabled accurate, quality controlled, SST data to be supplied in near real-time (within 24 hours) from VOS, passenger ferries and research vessels in the Australian region.

Table 1. Details of IMOS Ship SST Data Available Via the GTS and IMOS Ocean Portal

\begin{tabular}{|c|c|c|c|}
\hline Vessel & Callsign & $\begin{array}{c}\text { Data } \\
\text { Start }\end{array}$ & $\begin{array}{c}\text { SST } \\
\text { Sensor }\end{array}$ \\
\hline $\begin{array}{c}\text { RV Southern } \\
\text { Surveyor }\end{array}$ & VLHJ & $\begin{array}{c}4 \text { Feb } \\
2008\end{array}$ & SBE 3 \\
\hline RV L'Astrolabe & FHZI & $\begin{array}{c}30 \mathrm{Dec} \\
2008\end{array}$ & SBE 38 \\
\hline $\begin{array}{c}\text { RSV Aurora } \\
\text { Australis }\end{array}$ & VNAA & $\begin{array}{c}12 \text { Oct } \\
2008\end{array}$ & SBE 38 \\
\hline $\begin{array}{c}\text { PV SeaFlyte } \\
\text { Rottnest Is Ferry) }\end{array}$ & VHW5167 & $\begin{array}{c}30 \mathrm{Apr} \\
2008\end{array}$ & SBE 38 \\
\hline $\begin{array}{c}\text { PV Fantasea One } \\
\text { (Whitsunday Ferry) }\end{array}$ & VJQ7467 & $\begin{array}{c}5 \mathrm{Nov} \\
2008\end{array}$ & AD590 \\
\hline $\begin{array}{c}\text { PV Spirit of } \\
\text { Tasmania II } \\
\text { (Bass Strait Ferry) }\end{array}$ & VNSZ & $\begin{array}{c}10 \mathrm{Dec} \\
2008\end{array}$ & SBE 48 \\
\hline MV Portland & VNAH & $\begin{array}{c}20 \mathrm{Jun} \\
2009\end{array}$ & SBE 48 \\
\hline MV Stadacona & C6FS9 & $\begin{array}{c}10 \mathrm{Aug} \\
2009\end{array}$ & SBE 48 \\
\hline RV Solander & VMQ9273 & $\begin{array}{c}5 \mathrm{Dec} \\
2010\end{array}$ & SBE 38 \\
\hline MV Highland Chief & VROB & $\begin{array}{c}30 \mathrm{Sep} \\
2009\end{array}$ & SBE 48 \\
\hline MV Iron Yandi & VNVR & $\begin{array}{c}10 \mathrm{Feb} \\
2010\end{array}$ & SBE 48 \\
\hline RV Cape Ferguson & VNCF & $\begin{array}{c}5 \mathrm{Dec} \\
2010\end{array}$ & SBE 38 \\
\hline
\end{tabular}

As part of IMOS, the Bureau of Meteorology (Bureau) has instrumented six vessels of the Australian Volunteer Observing Fleet with hull-mounted temperature sensors (Sea Bird SBE 48), supplying high-quality bulk SST observations every hour. There are also two passenger ferries reporting one minute averaged SST measurements for CSIRO Marine and Atmospheric Research (Rottnest Island ferry) and the Australian Institute of Marine Science (Whitsunday Island to Hook Reef ferry). In addition, there are near real-time, one minute 
averaged SST and salinity data streams available from five research vessels (RV Southern Surveyor, RSV Aurora Australis, RV L'Astrolabe, RV Solander and RV Cape Ferguson). In total, thirteen vessels contribute near real-time data to IMOS (Table 1 and Figure 1).

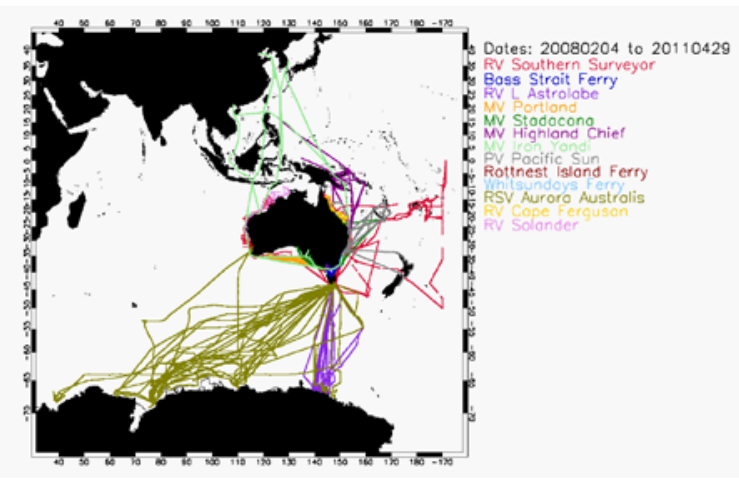

Figure 2. Locations of IMOS QC'd ship SST observations to 29 April 2011 from 13 vessels.

All SST data are quality assured (Beggs et al., 2009a) and placed in real-time on the Global Telecommunications System (GTS). The quality controlled (QC'd) SST data are also available in netCDF format with QC flags and metadata via the IMOS ocean data portal (http://imos.aodn.org.au/webportal) or directly from http://opendaptpac.arcs.org.au/thredds/dodsC/IMOS/SO OP/SOOP-SST/ and

http://opendap-

tpac.arcs.org.au/thredds/dodsC/IMOS/SO OP/SOOP-ASF/catalog.html.

Comparisons between AATSR, AVHRR, buoy and IMOS ship SST observations indicate that at least ten of the IMOS ship data streams have comparable errors to those obtained from drifting buoys (Section 4, Beggs et al., 2010a and Beggs et al., 2011b). In waters with little or no coverage by buoys, AVHRR SST calibration, validation and bias-correction will be improved by using IMOS ship SST observations in addition to available drifting buoy SST data.

The IMOS ship SST data has been used in real-time SST analysis systems (including RAMSSA and GAMSSA) and for validation of satellite SST, SST analyses and ocean models (Beggs et al., 2011b).

\section{Geostationary MTSAT-1R skin SST}

Geostationary satellites provide measurements of skin SST over the same scene every 15 to 60 minutes, particularly useful for the study of diurnal warming of the surface ocean. Since mid-2007, the Bureau has routinely generated SSTskin products from the Japanese geostationary satellite, MTSAT-1R, using the NOAAdeveloped Geostationary Satellite Derived Sea Surface Temperature Processing System (Maturi et al., 2008). The original version of the software (v1) installed at the Bureau in 2007 was modified to accept locally generated NWP fields and further modified to output GHRSST formatted, single scene L2P files. A match-up database system was developed to determine the difference between satellite retrievals and in situ measurements from drifting buoys. In May 2010 the Bureau's MTSAT-1R SST processing system was further upgraded to version 3 (v3) to incorporate a physical retrieval methodology and University of Edinburgh/NOAA Baysean cloud clearing, following a visit by Jon Mittaz and Andy Harris from NOAA/University of Maryland. During early 2011 the processing system was updated to version 4 (v4) to use regression against drifting buoy SST rather than physical retrieval to convert from brightness temperatures to SST.

Between June 2005 and June 2006 the Bureau received data from MTSAT-1R in HiRID format. In June 2006 the Bureau upgraded its satellite reception hardware to be capable of receiving MTSAT-1R data in HRIT format (10-bit). Results from the match-up database demonstrated that the HiRID data received by the Bureau was not of sufficient quality to obtain an accurate SSTskin retrieval due to the degraded signal. The standard deviation (when compared to drifting buoys) for daytime HRIT data with a quality level 5, using the 11 and $12 \mu \mathrm{m}$ channels, collected during 1 January to 30 April 
2009 was $0.7^{\circ} \mathrm{C}$ for the version 4 system. The corresponding standard deviation for night-time HRIT data, which also incorporates the $3.75 \mu \mathrm{m}$ channel, was $0.5^{\circ} \mathrm{C}$. The mean bias for both day and night SST retrievals was around $0.05^{\circ} \mathrm{C}$.

In December 2009 the Bureau's NWP system was upgraded to use the UK Unified Model. The upgrade has resulted in improved accuracy of the NWP forecasts along with increases in the vertical, spatial and temporal resolution of the NWP fields (Puri et al., 2010). These changes necessitated an upgrade of the MTSAT-1R system to handle the new ACCESS-G NWP output data format. By June 2011, v4 MTSAT-1R SSTskin 0.05 $x 0.05^{\circ}$ gridded, single scene L3U files (Figure 2) back to June 2006 are expected to be made available to Australian researchers via the IMOS Australian Ocean Distributed Archive and Access Centre (AO-DAAC - see http://imos.org.au/srs data.html) and the Bureau's OPeNDAP server (contact h.beggs@bom.gov.au for access). On 1 July 2010, MTSAT-1R HRIT transmission was replaced with MTSAT-2 data and the Bureau currently produces real-time experimental SSTskin L3U files from MTSAT-2. These MTSAT-2 files should be available via the IMOS AO-DAAC by December 2011.

(a)

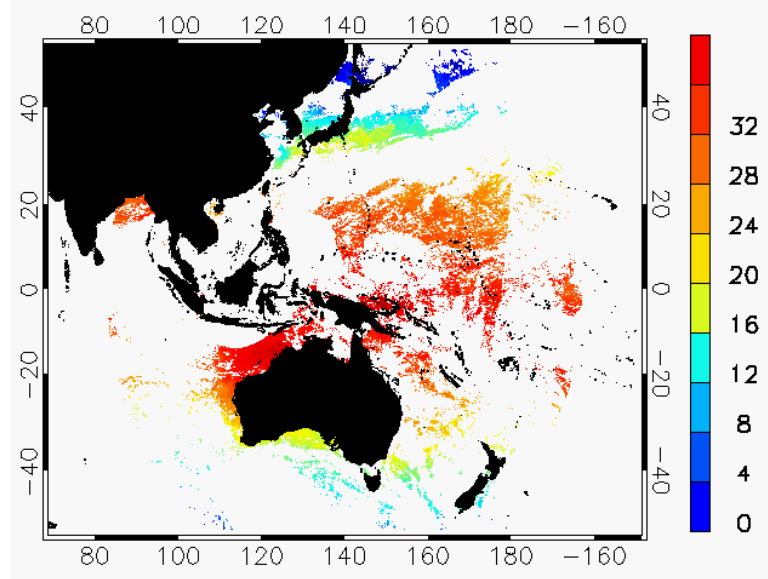

(b)

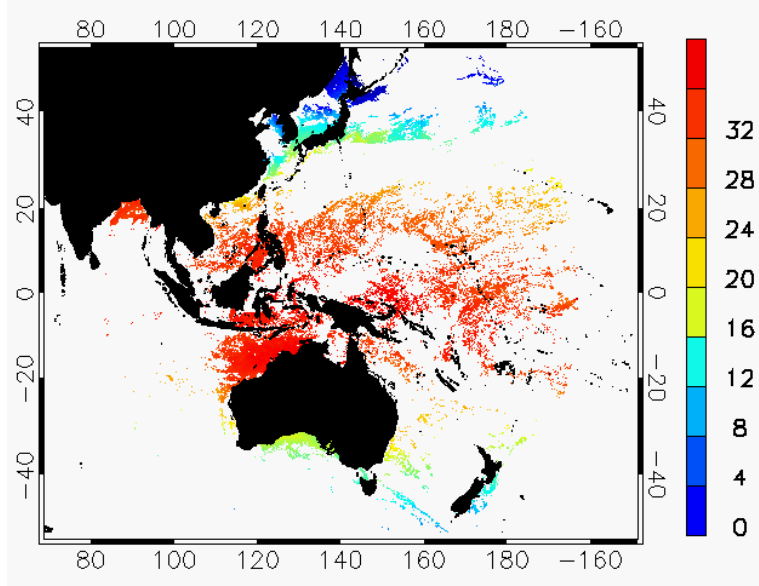

Figure 2. An example of the output from the v4 MTSAT-1R processing system of L3U SSTskin for (a) 0530 UT (day) and (b) 1630 UT (night) on 10 April 2009. SST is plotted for cloud-free pixels (quality level = 3 to 5 ).

\section{Locally Received AVHRR SST}

The highest resolution $(1.1 \mathrm{~km})$ data from AVHRR sensors on the NOAA polarorbiting meterological satellites can only be obtained through receiving direct broadcast HRPT data from the satellite as this data is not stored onboard. In Australia HRPT data is received by a consortium of agencies (Bureau of Meteorology, WASTAC, AIMS and CSIRO) at groundstations located in Darwin, Townsville, Melbourne, Hobart, Perth and Alice Springs and in Antarctica at Casey and Davis Stations. As part of the IMOS Project the Bureau of Meteorology, in collaboration with CSIRO Marine and Atmospheric Research, is stitching this raw data and producing realtime, HRPT AVHRR SSTskin data from operational NOAA polar-orbiting satellites in the GHRSST GDS v2.0 L2P, L3U, L3C and L3S formats (Casey et al., 2010). In addition to the $1.1 \mathrm{~km}$ resolution HRPT AVHRR SSTskin values and other mandatory fields, the L2P files contain bias and standard deviation estimates based on match-ups with in situ drifting buoy SST data from the GTS, and 3hourly forecasts of averaged $10 \mathrm{~m}$ winds from the Bureau's legacy GASP Global NWP model (Puri et al., 1998) up to 30 June 2010 and the ACCESS-G NWP model (Puri et al., 2010) after that date. 
GHRSST XII -

Proceedings, Edinburgh

GHRSST Project Office

Single sensor (one and three night/day) composite HRPT AVHRR SST files have been produced in GHRSST GDS v2.0 L3C format (Casey et al., 2010) over a cylindrical equidistant projection $\left(0.02^{\circ}\right.$ latitude $\times 0.02^{\circ}$ longitude (Figure 3 ). Multiple sensor (one and three night/day) $0.02^{\circ}$ latitude $\times 0.02^{\circ}$ longitude HRPT AVHRR SST files have been produced for testing but the optimal spatial and temporal resolution is under review. Existing raw, archived, high-resolution HRPT AVHRR data from all operational NOAA polar-orbiting satellites over the Australian region back to 1992 will be progressively reprocessed into SSTskin L2P, L3U, L3C and L3S and made available to GHRSST and IMOS by December 2011. Currently, HRPT AVHRR SSTskin GDS v2.0 L2P, L3U and L3C files from NOAA-15, 17, 18 and 19 (back to 2004) are available from the IMOS AO-DAAC and FTP server (ftp://aodaac2-

cbr.act.csiro.au/imos/GHRSST/). Maps of HRPT AVHRR L3C SSTskin are available from the IMOS Ocean Portal under Satellite Remote Sensing (http://imos.aodn.org.au/webportal/).

The new IMOS HRPT AVHRR L2P SSTs exhibit nearly half the error of the Bureau's pre-existing HRPT AVHRR level 2 SST data from NOAA-17 and NOAA-18 satellites, with standard deviations compared with drifting buoys during nighttime of 0.24 to $0.27^{\circ} \mathrm{C}$ for NOAA-17, 18 and 19 , and during daytime of 0.34 to $0.35^{\circ} \mathrm{C}$ (Paltoglou et al., 2010). This significant improvement in accuracy has been achieved by implementing new CLAVR-based cloud clearing algorithms, implementing new BT to SST transforms with new day-time terms including latitude and higher order, and using regional, QC'd drifting buoy SST observations for the regression. The SSTs at drifting buoy depths $(20-30 \mathrm{~cm})$ are converted to a skin SST at $\sim 10 \mu \mathrm{m}$ depth by subtracting $0.17^{\circ} \mathrm{C}$ to account for the cool skin. Table 2 gives the mean and standard deviation of quality level 5 IMOS nighttime, $1 \mathrm{~km}$ resolution, NOAA-18 AVHRR SST minus
Issue: Final

Date: September 27, 2011

SST data from IMOS and non-IMOS ships and drifting buoys over the region $70^{\circ} \mathrm{E}$ to $190^{\circ} \mathrm{E}, 20^{\circ} \mathrm{N}$ to $70^{\circ} \mathrm{S}$, during 1 December 2008 to 30 September 2010 (Beggs et al., 2011b). The data are considered matched if within \pm 2 hours and collocated within the same $\sim 1 \mathrm{~km}$ pixel.

(a)

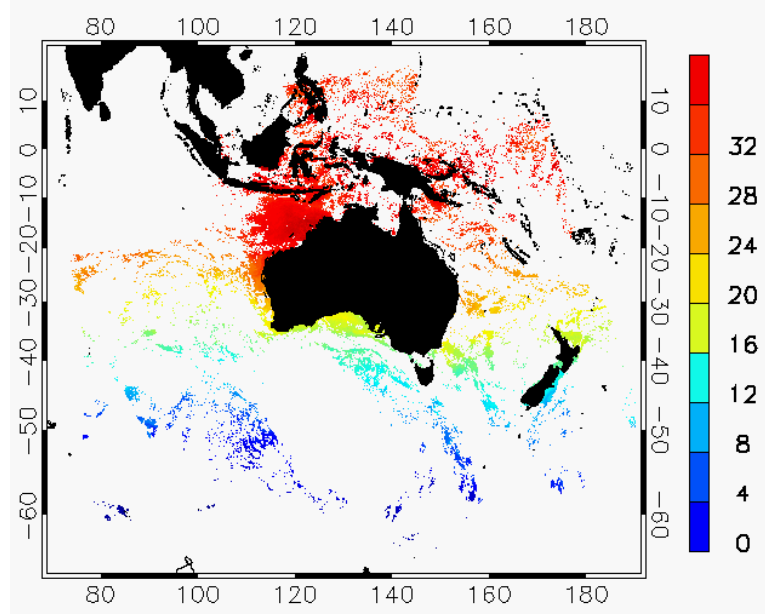

(b)

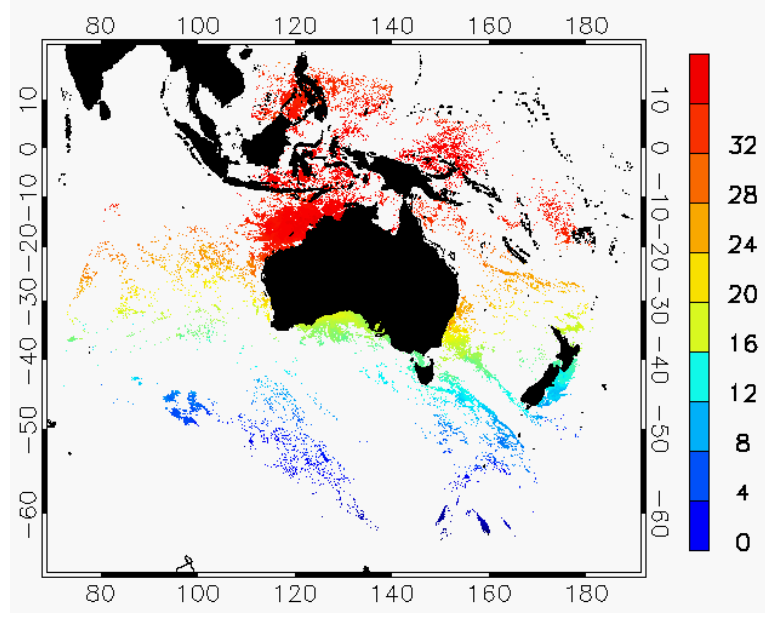

Figure 3. Example of (a) day ( 1330 LT) and (b) night ( $0130 \mathrm{LT}) 0.02^{\circ} \times 0.02^{\circ} \mathrm{L} 3 \mathrm{C}$ SSTskin from NOAA-18 HRPT AVHRR SST data for 10 April 2009. SST is plotted for cloud-free pixels (quality level = 3 to 5).

Multiple sensor, composite HRPT AVHRR SST files from all operational NOAA polarorbiters have also been produced in GHRSST-L3 netCDF format from the Bureau's legacy 14-day weighted mean, AVHRR Mosaic (Figure 4). The data has been reformatted to a cylindrical equidistant projection $\left(0.01^{\circ}\right.$ latitude $\mathrm{x}$ $0.01^{\circ}$ longitude) over the region $8^{\circ} \mathrm{S}$ to $48^{\circ} \mathrm{S}, 104^{\circ} \mathrm{E}$ to $165^{\circ} \mathrm{E}$, and is currently 
GHRSST XII -

Proceedings, Edinburgh

GHRSST Project Office

available back to 1 January 2001 from IMOS via http://imos.org.au/srs data.html.

Table 2. Mean and Standard Deviation of Nighttime AVHRR SST from NOAA-18 minus In Situ SST.

\begin{tabular}{|c|c|c|c|}
\hline $\begin{array}{c}\text { In Situ Data } \\
\text { Stream }\end{array}$ & $\begin{array}{c}\text { Number of } \\
\text { Matchups }\end{array}$ & $\begin{array}{c}\text { Mean } \\
\text { (K) }\end{array}$ & $\begin{array}{c}\text { Standard } \\
\text { Deviation } \\
\text { (K) }\end{array}$ \\
\hline $\begin{array}{c}\text { RV Southern } \\
\text { Surveyor }\end{array}$ & 146 & -0.01 & 0.22 \\
\hline RV L'Astrolabe & 29 & 0.07 & 0.23 \\
\hline $\begin{array}{c}\text { RSV Aurora } \\
\text { Australis }\end{array}$ & 107 & -0.07 & 0.24 \\
\hline PV SeaFlyte & 19 & -0.02 & 0.73 \\
\hline $\begin{array}{c}\text { PV Spirit of } \\
\text { Tasmania II }\end{array}$ & 686 & 0.03 & 0.30 \\
\hline MV Portland & 104 & 0.13 & 0.37 \\
\hline $\begin{array}{c}\text { MV Highland } \\
\text { Chief }\end{array}$ & 109 & -0.03 & 0.35 \\
\hline MV Stadacona & 308 & 0.09 & 0.43 \\
\hline MV Iron Yandi & 51 & -0.06 & 0.27 \\
\hline Non-IMOS Ships & 2277 & 0.01 & 1.64 \\
\hline Drifting Buoys & 6431 & 0.05 & 0.36 \\
\hline
\end{tabular}

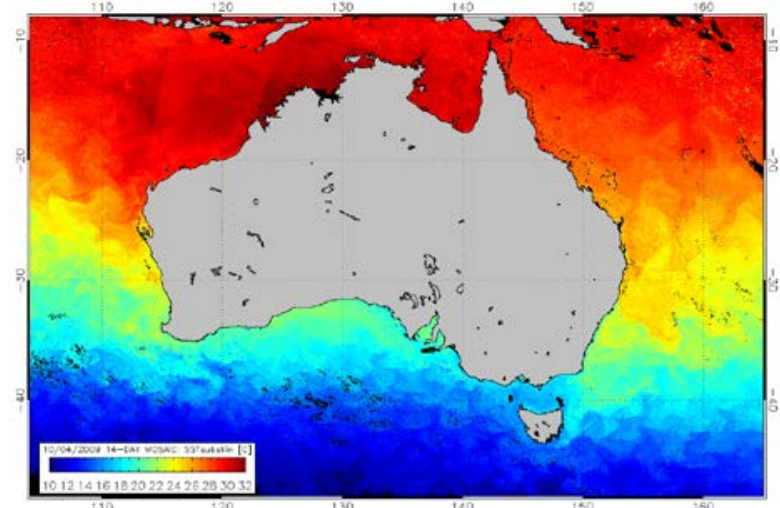

Figure 4. Example of the Bureau's legacy 14-day "Mosaic" SST produced from locally received 2009 NOAA-17 and NOAA-18 AVHRR data for the period 28 March to 10 April.

For further details on the new AVHRR L2P and single-sensor L3U and L3C products see Paltoglou et al. (2010), and for the legacy 14-day "Mosaic" L3P product see Rea (2004).

The HRPT AVHRR L2P products are being used in experimental and preoperational SST analysis systems (RAMSSA, GAMSSA, NASA JPL'S G1SST and Medspiration's Great Barrier Reef analysis). They are also being used in the GHRSST TWP+ experiment (see Section 8).
Issue: Final

Date: September 27, 2011

Future work for the period to June 2013 will include:

- Test calibration of HRPT AVHRR SST over the Southern Ocean by validating against new IMOS SST data (eg. ships, Argo, seals)

- Investigate improving the cloud and ice detection in AVHRR SST over the Southern Ocean

- By December 2011, provide reprocessed (back to 1992) HRPT AVHRR SSTskin L2P, L3U and L3C files incorporating Australian and Antarctic data via IMOS and the GHRSST GDAC - all ready providing real-time files from Australian ground stations via IMOS and Bureau OPeNDAP servers

- By December 2011, combine delayed mode Antarctic and Australian AVHRR L3U files to produce trial 1day, 3-day and 6-day, gridded, multisensor, L3S files over the RAMSSA domain $\left(60^{\circ} \mathrm{E}-190^{\circ} \mathrm{E}, 20^{\circ} \mathrm{N}-70^{\circ} \mathrm{S}\right)$ back to 2007

- By June 2013, provide real-time HRPT AVHRR SSTskin L2P, L3U and L3C files from Davis and Casey Antarctic stations

\section{RAMSSA - Regional Australian Multi-Sensor SST Analysis}

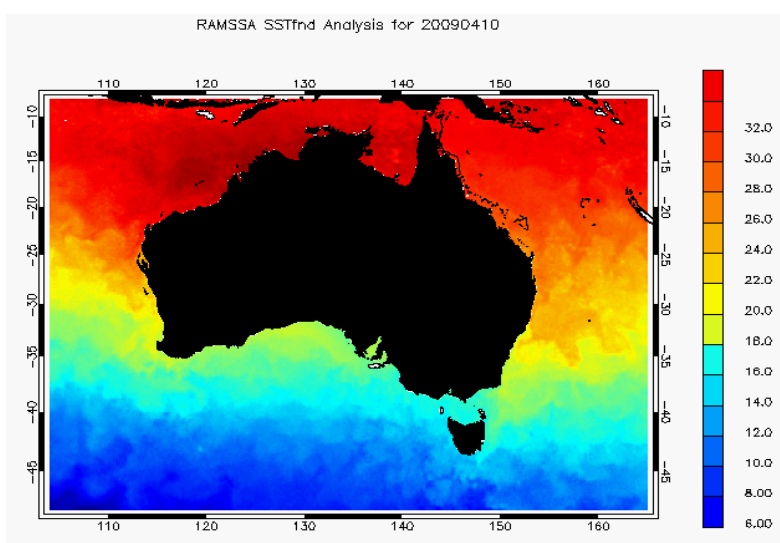

Figure 5. An example of the RAMSSA v1.2 daily regional $1 / 12^{\circ}$ resolution SST analysis for 10 April 2009, plotted over the 14-day Mosaic domain for comparison with Figure 4.

A real-time, high-resolution, Regional Australian Multi-Sensor Sea surface temperature $\underline{A}$ nalysis (RAMSSA) system 
GHRSST XII -

Proceedings, Edinburgh

GHRSST Project Office

has been developed at the Australian Bureau of Meteorology as part of the BLUElink Ocean Forecasting Australia project. The pre-existing operational, $1 / 4^{\circ}$ resolution, regional SST analysis system (Smith et al., 1999) has been modified to produce $1 / 12^{\circ}$ resolution, daily SST analyses over the Australian region $\left(20^{\circ} \mathrm{N}\right.$ $-70^{\circ} \mathrm{S}, 60^{\circ} \mathrm{E}-170^{\circ} \mathrm{W}$ ) (Figure 5).

The high-resolution analysis system combines SST data from infrared (AVHRR and AATSR) and microwave (AMSR-E) sensors on polar-orbiting satellites with in situ measurements to produce daily "foundation" SST estimates (SSTfnd), largely free of nocturnal cooling and diurnal warming effects. To produce foundation SST estimates, input data is filtered depending on the corresponding regional NWP surface wind speed and day/night. The method used to produce the pre-operational ("Gamma Test") and v1.0 RAMSSA products is described in detail in Beggs (2007). The RAMSSA v1.0 system became operational on 13 June 2007, was upgraded to v1.1 on 26 October 2007 (system modified to reduce "speckliness" in analyses), v1.2 on 10 June 2008 (incorporating the NAVOCEANO GHRSST GAC AVHRR L2P SST products), v1.3 on 9 April 2009 (incorporating the NAVOCEANO $1 / 120^{\circ}$ land/sea mask) and v1.4 on 1 September 2009 (replacing the LAPS NWP winds with those from ACCESS-R). See Beggs et al. (2011a) for details of the v1.1 to v1.4 methodology. Reprocessed RAMSSA v1.1 files are available on request back to 1 October 2006. By $\sim 0300$ UT each day, the operational analyses of the previous day's observations can be downloaded as GDS v1.7 netCDF L4 files from the GHRSST GDAC hosted by PO.DAAC (via ftp://podaac-

ftp.jpl.nasa.gov/allData/ghrsst/data/L4/AU S/ABOM/RAMSSA 09km/). Archived RAMSSA L4 files back to 12 June 2006 are available from http://godae.bom.gov.aul and back to 1 April 2008 from the GHRSST Long-Term Stewardship Facility at NODC (ftp://ftp.nodc.noaa.gov/pub/data.nodc/ghr sst/L4/AUS/ABOM/RAMSSA 09km/).
Issue: Final

Date: September 27, 2011

Over the period 1 October 2007 to 31 March 2008 and region $60^{\circ} \mathrm{E}$ to $180^{\circ} \mathrm{E}$, $20^{\circ} \mathrm{N}$ to $65^{\circ} \mathrm{S}$, the RAMSSA v1.1 $1 / 12^{\circ}$ SSTfnd analyses exhibited mean(Analysed SSTfnd(date) - Buoy SSTfnd(date+1)) of $0.03 \pm 0.42^{\circ} \mathrm{C}$, comparable with the Met Office $0.05^{\circ}$ resolution daily SSTfnd analysis, OSTIA $\left(-0.05 \pm 0.39^{\circ} \mathrm{C}\right)$. Over this same period, RAMSSA v1.1 agreed more closely with Ifremer's ODYSSEA and Met Office' OSTIA SSTfnd analyses than with other GHRSST microwave and infrared blended L4 analyses such as NCDC's AVHRR+AMSR-E SSTblend or Remote Sensing System's MW+IR SSTfnd analyses, with mean(RAMSSA SSTfnd ODYSSEA SSTfnd) of $-0.02 \pm 0.40^{\circ} \mathrm{C}$ and mean(RAMSSA SSTfnd - OSTIA SSTfnd) of $0.10 \pm 0.35^{\circ} \mathrm{C}$. The major differences between RAMSSA and these other foundation SST analyses relate to RAMSSA's method for creating superobservations and assigning weights to the various input data streams, and Ifremer and the Met Office analysis systems' bias-correction of all satellite input data using SST data from the AATSR. The lack of bias-correction of data input into RAMSSA has minimal effect north of $40^{\circ} \mathrm{S}$ where RAMSSA is on average within $\pm 0.07^{\circ} \mathrm{C}$ of other multi-sensor SST analyses. South of $40^{\circ} \mathrm{S}, \mathrm{RAMSSA}$ is on average $0.09^{\circ} \mathrm{C}$ to $0.25^{\circ} \mathrm{C}$ warmer than the four bias-corrected, GHRSST-L4 analyses studied, due to systematic biases over this region in the calibration of the satellite SST data streams used for RAMSSA (Beggs et al., 2011a).

The RAMSSA analyses are used in realtime as the boundary condition for the Bureau's regional numerical weather prediction models (ACCESS-R, ACCESS$A$ and ACCESS-C) and to validate the BLUElink operational ocean model (OceanMAPS) SST5m forecasts/ analyses. They are used experimentally in the regional skin SST analyses (Section 7) and the GHRSST TWP+ experiment (Section 8).

Future work on RAMSSA in 2011/2012 will include investigating the blending of 
GHRSST XII -

Proceedings, Edinburgh

GHRSST Project Office

satellite SST GHRSST L2P files available through Eumetsat (1 km ATS_NR_2P AATSR SSTskin) and IMOS (1 km HRPT AVHRR SSTskin and $4 \mathrm{~km}$ MTSAT-1R SSTskin).

\section{GAMSSA - Global Australian Multi-Sensor SST Analysis}

A real-time $\underline{\text { Global }}$ Australian Multi-Sensor Sea surface temperature Analysis (GAMSSA) system has been developed at the Australian Bureau of Meteorology as part of the BLUElink project. The operational, RAMSSA $1 / 12^{\circ}$ resolution, regional SST analysis system (Beggs, 2007; Beggs et al., 2011a) has been modified to produce $1 / 4^{\circ}$ resolution, daily global foundation SST analyses (Beggs, 2008; Zhong and Beggs, 2008) (Figure 6).

The GAMSSA system blends NAVOCEANO'S GAC $9.9 \mathrm{~km} \times 4.4 \mathrm{~km}$ resolution AVHRR L2P SST data (NOAA18, NOAA-19 and METOP-A), European Space Agency's $0.17^{\circ}$ AATSR skin SST Meteo Product (EnviSat), Remote Sensing System's $25 \mathrm{~km}$ resolution AMSR-E L2P sub-skin SSTs (Aqua) and in situ ship and buoy SSTs from the GTS. To produce foundation SST estimates, input data are filtered depending on the corresponding global NWP surface wind speed and day/night.

The GAMSSA v1.0 system started Alpha testing at the Bureau on 6 December 2007, Beta testing on 4 May 2008, and became operational on 2 October 2008. The system was upgraded to v1.1 on 9 April 2009 (incorporating the NAVOCEANO $1 / 120^{\circ}$ land/sea mask) and v1.2 on 1 September 2009 (replacing the GASP NWP winds with ACCESS-G winds). By 0330 UT each day, the operational analyses of the previous day's observations can be downloaded as GDS v1.7 L4 files from the GHRSST GDAC hosted by PO.DAAC (via ftp://podaacftp.jpl.nasa.gov/allData/ghrsst/data/L4/GL OB/ABOM/GAMSSA $28 \mathrm{~km} /$ ). Archived RAMSSA L4 files back to 23 July 2008 are available from http://godae.bom.gov.aul and back to 24 August 2008 from the GHRSST Long-Term Stewardship Facility
Issue: Final

Date: September 27, 2011

at

NODC

(ftp://ftp.nodc.noaa.gov/pub/data.nodc/ghr sst/L4/GLOB/ABOM/GAMSSA $28 \mathrm{~km} /$ ).

10 Apr 2009 Fine Global Foundation SST Analysis $\Delta=1.0^{\circ} \mathrm{C}$

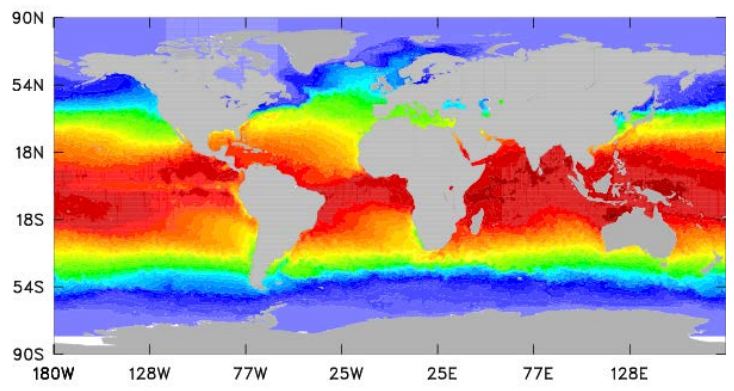

Figure 6. An example of the GAMSSA v1.1 daily global $1 / 4^{\circ}$ resolution SSTfnd analysis for 10 April 2009.

Over the period 20 May to 30 August 2008, the GAMSSA v1.0 $1 / 4^{\circ}$ SSTfnd analyses exhibited mean(Analysed SSTfnd (date) - Buoy SSTfnd (date+1)) of $-0.04 \pm 0.50^{\circ} \mathrm{C}$ globally, comparable with the Met Office $0.05^{\circ}$ resolution daily SSTfnd analysis, OSTIA, over the same region and period (OSTIA SSTfnd - Buoy SSTfnd $=-0.07 \pm 0.44^{\circ} \mathrm{C}$ ) and with lower error than NCDC's AVHRR+AMSR-E $0.25^{\circ}$ resolution daily SSTblend analysis (NCDC SSTfnd - Buoy SSTfnd $=-0.03 \pm$ $0.64^{\circ} \mathrm{C}$ ). GAMSSA v1.0 agreed more closely with Met Office' OSTIA SSTfnd analyses than with other GHRSST microwave and infrared blended L4 analyses (NCDC SSTblend and ODYSSEA SSTfnd) over this same period, with mean(GAMSSA SSTfnd OSTIA SSTfnd) of $0.07 \pm 0.46^{\circ} \mathrm{C}$.

The GAMSSA analyses have contributed to the GHRSST Multi-Product Ensemble (GMPE) and Analysis Intercomparison Project (http://ghrsstpp.metoffice.com/pages/latest analysis/ss $\mathrm{t}$ monitor/daily/ens/index.html) since 10 March 2009. Long-term and daily intercomparisons produced by the NOAA SST Quality Monitor (L4-SQUAM: http://www.star.nesdis.noaa.gov/sod/sst/s quam/L4/index.html) show that GAMSSA SSTfnd is between 0 and $0.5^{\circ} \mathrm{C}$ warmer 
GHRSST XII -

Proceedings, Edinburgh

GHRSST Project Office

than the GMPE daily SSTblend analysis over the Southern Ocean. For the period 1 June 2008 to 30 June 2009, the GAMSSA analyses were on average slightly warm compared to independent drifting buoys south of $40^{\circ} \mathrm{S}$, with mean(Analysed SSTfnd (date) - Buoy SSTfnd (date+1)) of $0.05 \pm 0.48^{\circ} \mathrm{C}$. It would therefore appear that the satellite observations over the Southern Ocean going into GAMSSA are overall warmer compared with those going into other analyses contributing to GMPE. The same AVHRR and AMSR-E SST data streams ingested into operational RAMSSA have been shown to be on average biased warm by between 0.0 and $0.3^{\circ} \mathrm{C}$ south of $40^{\circ} \mathrm{S}$ between $60^{\circ} \mathrm{E}$ and $170^{\circ} \mathrm{W}$ (Beggs et al., 2011a). Validation of satellite SST observations and analyses over the Southern Ocean will be a priority at the Bureau.

The GAMSSA analyses are used in realtime as the boundary condition for the Bureau's new global NWP model (ACCESS-G: Puri et al., 2010) based on the Met Office's Unified Model. They are also used to initialise the Bureau's seasonal forecast model (POAMA 2.0: http://poama.bom.gov.au).

Future work on GAMSSA in 2011/2012 will include testing the blending of new GHRSST L2P SST products (1 km AATSR SSTskin, $1 \mathrm{~km}$ HRPT AVHRR SSTskin and $4 \mathrm{~km}$ MTSAT-1R SSTskin).

\section{Global and Regional Skin SST Analyses (GAMSSA_skin and RAMSSA_skin)}

An experimental, regional, hourly, $1 / 12^{\circ}$ resolution, skin SST analysis ("RAMSSA_skin" - Figure 7(a) and 8(a)) and global, 3-hourly, 1/4 resolution, skin SST analysis ("GAMSSA_skin" - Figure 7(b) and 8(b)) have been developed at the Bureau of Meteorology as part of the BLUElink-II Project. Both skin analyses are formed by adding a simple, empirically based estimate of $\triangle S S T$ at that time to the daily RAMSSA SSTfnd or GAMSSA SSTfnd analysis. That is,
Issue: Final

Date: September 27, 2011

RAMSSA_skin SSTskin $=$ RAMSSA SSTfnd $+\Delta$ SST $-0.2^{\circ} \mathrm{C}$

and

GAMSSA_skin SSTskin = GAMSSA SSTfnd $+\Delta$ SST $-0.2^{\circ} \mathrm{C}$,

where $\Delta$ SST $=$ SSTsubskin - SSTfnd, calculated from a simple algorithm developed by Chelle Gentemann, based on geostationary SEVIRI SSTsubskin and AMSR-E surface wind data (Gentemann et al., 2003). No allowance is made in this empirical $\triangle$ SST model for cloud or net heat flux, although it accounts for the daily variation of incoming solar radiation by calculating the solar zenith angle and using the mean value of the solar constant for 1978 to 1998 calculated to 1366.22 $\mathrm{Wm}^{2}$

(see

http://remotesensing.oma.be/RadiometryP apers/article2.html). The maximum range of the $\triangle$ SST model is 0 to $3^{\circ} \mathrm{C}$.

(a)

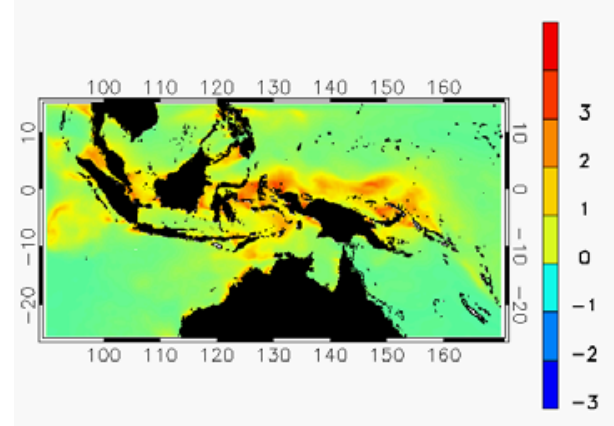

(b)

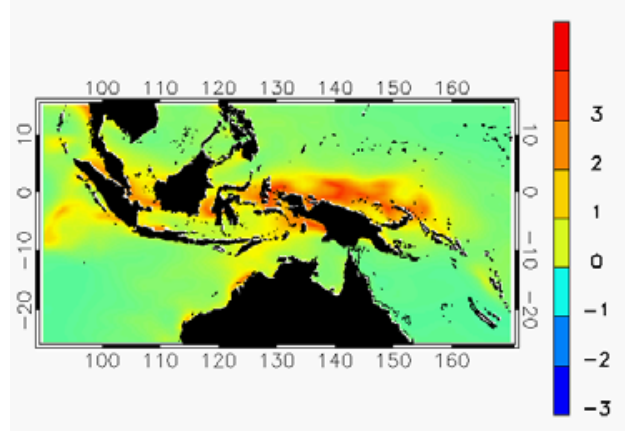

Figure 7. (a) RAMSSA skin SSTskin - RAMSSA SSTfnd and (b) GAMSSA_skin SSTskin GAMSSA SSTfnd analyses plotted over the region $25^{\circ} \mathrm{S}$ to $15^{\circ} \mathrm{N}, 90^{\circ} \mathrm{E}$ to $170^{\circ} \mathrm{E}$, for 10 April 2009 at 0600 UTC (1500 LT at $\left.135^{\circ} E\right)$. 
GHRSST XII -

Proceedings, Edinburgh

GHRSST Project Office

A constant $0.2^{\circ} \mathrm{C}$ is used to transform the SSTsubskin estimate to SSTskin following the SEVIRI skin to subskin constant $0.2^{\circ} \mathrm{C}$ adjustment (OSI-SAF Project Team, 2006). For RAMSSA_skin, the inputs to the $\Delta$ SST algorithm are mean hourly, $0.375^{\circ}$ resolution, $10 \mathrm{~m}$ winds from the Bureau's ACCESS-R regional NWP 24 hour forecasts (Puri et al., 2010). For GAMSSA_skin, the mean 3-hourly, $1.25^{\circ}$ Ion $\times 0.833^{\circ}$ lat resolution, $10 \mathrm{~m}$ winds from the Bureau's ACCESS-G global NWP 24 hour forecasts are used (Puri et al., 2010).

(a)

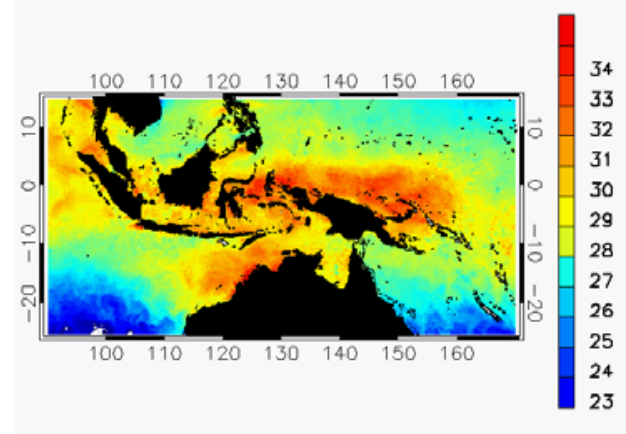

(b)

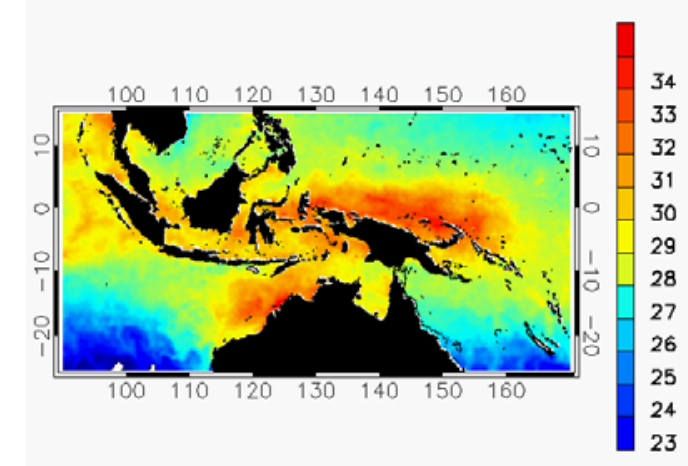

Figure 8. (a) RAMSSA_skin and (b)

GAMSSA_skin SSTskin analyses, plotted over the region $25^{\circ} \mathrm{S}$ to $15^{\circ} \mathrm{N}, 90^{\circ} \mathrm{E}$ to $170^{\circ} \mathrm{E}$, for 10 April 2009 at 0600 UTC $\left(\sim 1330\right.$ LT at $110^{\circ} \mathrm{E}$ and 1500 LT at $\left.135^{\circ} \mathrm{E}\right)$.

Both RAMSSA skin and GAMSSA skin have been validated against the $1 \mathrm{~km}$ resolution AATSR SSTskin L2P product available from GHRSST (http://ghrsst.nodc.noaa.gov/). For the period 1-31 January 2009, RAMSSA_skin - AATSR SSTskin $=0.14 \pm 0.38^{\circ} \mathrm{C}$. For the same period, GAMSSA_skin - AATSR
Issue: Final

Date: September 27, 2011

SSTskin $=0.10 \pm 0.38^{\circ} \mathrm{C}$. These are encouragingly low errors and indicate that under clear-sky conditions Chelle Gentemann's simple empirical model in conjunction with ACCESS-R and ACCESS-G forecast winds should be useful in predicting diurnal warming in all but the most extreme cases ( $\triangle$ SST > $3^{\circ} \mathrm{C}$ ).

Comparison with MODIS composite SSTskin and MTSAT-1R hourly SSTskin indicates that RAMSSA_skin analyses capture diurnal warming maxima quite effectively where there are clear skies but night-time (pre-dawn) minima are too warm by up to $+3^{\circ} \mathrm{C}$ (Beggs et al., 2009b), due to the equator crossing times of the satellites contributing SST data to the RAMSSA SSTfnd analyses being several hours before local sunrise, the "foundation" time. These polar orbiters (NOAA-17, NOAA-18, NOAA-19, METOP$A$, EnviSat and Aqua) have nighttime equator crossing times between $9-11$ pm and 1 - 2 am local time. Luckily, this should not adversely affect using RAMSSA_skin for quality control of satellite sounder observations assimilated into the Bureau's new NWP models as all satellites used for ACCESS NWP models have equator crossing times of around 10 pm and 1:30 am, not close to dawn.

It is anticipated that in 2011/12 the Bureau will test the efficacy of using the RAMSSA_skin and GAMSSA_skin analyses for quality control of satellite data assimilated into the ACCESS NWP models. RAMSSA skin will also be used to investigate whether using forecast SSTskin analyses, rather than using a persisted SSTfnd as the boundary condition, might improve the skill of NWP forecasts.

Hourly RAMSSA_skin analyses are available over the domain $65^{\circ} \mathrm{E}$ to $185^{\circ} \mathrm{E}$, $15^{\circ} \mathrm{N}$ to $65^{\circ} \mathrm{S}$, back to 1 October 2008 , in a GHRSST L4 format similar to RAMSSA (contact h.beggs@bom.gov.au for access). Likewise, the 3-hourly, global, GAMSSA_skin analyses are available in the same format back to 1 June 2008. 


\section{Tropical Warm Pool Diurnal Variability Experiment (TWP+)}

The Western Pacific Tropical Warm Pool (TWP) north of Australia is one of the most difficult areas of the ocean to measure sea surface temperature. This is due to a combination of high diurnal warming (under low wind speed, high insolation conditions exceeding $5^{\circ} \mathrm{C}$ over small spatial/time scales - Figure 9), frequent cloud cover reducing the amount of SST observations from infrared satellite sensors, and island chains reducing the spatial coverage of SST measurements from microwave satellite sensors.

During 2009 to 2011, the Bureau of Meteorology in collaboration with MétéoFrance compiled a data set of satellite SST data (from v4 MTSAT-1R, HRPT AVHRR and METOP-A), RAMSSA SSTfnd analyses and the Australian hourly, $0.375^{\circ}$ resolution, LAPS and ACCESS-R NWP forecasts over the "TWP+" region $\left(25^{\circ} \mathrm{S}\right.$ to $15^{\circ} \mathrm{N}, 90^{\circ} \mathrm{E}$ to $170^{\circ} \mathrm{E}$ ) for the periods 1 January to 30 April 2009 and 1 January to 30 April 2010.

(a)

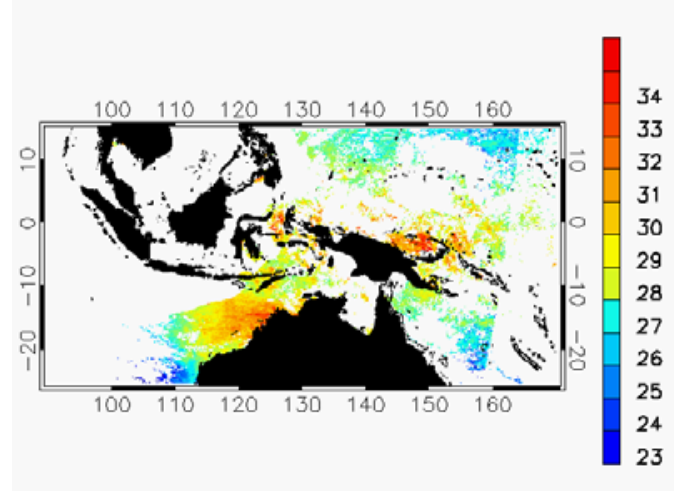

(b)

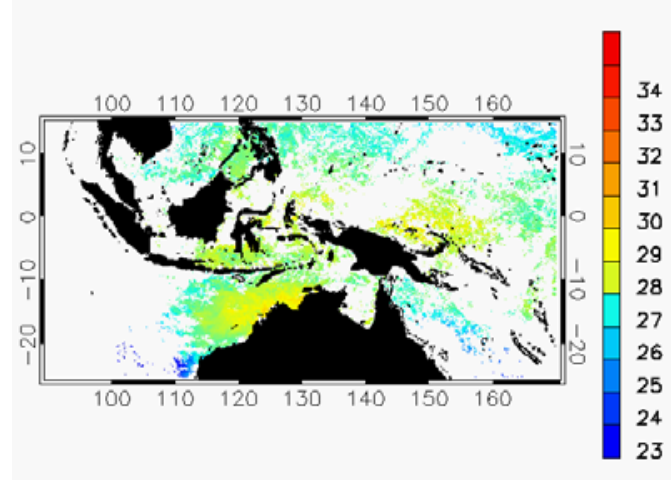

Figure 9. Composite maps of $0.05^{\circ}$ skin SST from MTSAT-1R for 10 April 2009 over the TWP+ domain during the (a) day (0630 UT) and (b) night (1630 UT) - supplied by the Bureau of Meteorology and the IMOS Project.

RAMSSA, METOP-A and MTSAT-1R SST products have been regridded onto a common grid over the TWP+ domain with resolution of $0.025^{\circ}$ for METOP-A and $0.05^{\circ}$ (MTSAT-1R and RAMSSA). The LAPS and ACCESS-R NWP surface wind and flux fields have been left on their original $0.375^{\circ}$ grid. This "TWP+" data set will be used by members of the GHRSST Diurnal Variability Working Group to test their diurnal warming models over the Western Pacific Tropical Warm Pool region. The TWP+ data set is available via the Bureau's OPeNDAP server. Contact h.beggs@bom.gov.au for access details.

The following studies are planned for the period 2011 - 2013 using the TWP+ data sets:

1. Compare LAPS and ACCESS-R surface fields to decide which NWP model and period (Jan - Apr 2009 or Jan - Apr 2010) to use for TWP+ work (Sandra Castro)

2. Assess TWP+ satellite SST products:

a. Compare MTSAT-1R SSTfnd, HRPT AVHRR SSTfnd and drifting buoy SSTfnd in a 3-way intercomparison (Leon Majewski)

b. Compare MTSAT-1R SSTskin, HRPT AVHRR SSTskin, METOPA SSTsubskin and AMSR-E 
SSTsubskin to AATSR SSTskin climate data record (Helen Beggs)

3. Using MTSAT-1R SSTskin and NWP winds, quantify frequency and extent of diurnal warming events (Helen Beggs, Leon Majewski, Gary Wick and Sandra Castro)

4. Using MTSAT-1R SSTskin assess 3 simple parameterisation diurnal variation (DV) models that use the TWP+ NWP surface fluxes as inputs

a. Gentemann et al (2003) as used in RAMSSA_skin (inputs: winds) (Helen Beggs and Sandra Castro)

b. Castro Look Up Table (inputs: winds, SW radiation) (Sandra Castro)

c. Clayson new parameterisation model (inputs: winds, SW radiation, $24 \mathrm{hr}$ precipitation) (Carol Ann Clayson)

5. Using MTSAT-1R SSTskin assess $\geq$ 4 full physical DV models that use TWP+ NWP surface fluxes as inputs for selected clear sky DW events

a. COARE (Gary Wick)

b. POSH v2 (Chelle Gentemann and Rachel Weihs)

c. Reduced Resolution KanthaClayson (Andy Harris - TBC)

d. Modified Kantha-Clayson (Gary Wick)

e. Others?

6. For the period 1 January - 30 April 2010, assess the BLUElink air-sea coupled model (CLAM) both with and without DV correction from SST at $2.5 \mathrm{~m}$ to skin using ACCESS-R surface fluxes (Paul Sandery, Helen Beggs)

7. For the period 1 January - 30 April 2010, assess the impact of a simple DV model (that uses ACCESS-R forecast fluxes as inputs) on assimilation of atmospheric profile data into ACCESS-R (Chris Tingwell, Helen Beggs, Peter Steinle)

8. For the period 1 January - 30 April 2010, assess the impact of using RAMSSA_skin SSTskin analyses rather than a persisted SSTfnd as the boundary condition for ACCESSR NWP forecasts (Vaughan Barras, Helen Beggs).

\section{Use of GHRSST L2P in BLUElink> Ocean Forecasting}

From mid-February 2010, GHRSST NAVOCEANO $9 \mathrm{~km} \times 4 \mathrm{~km}$ resolution, Global Area Coverage (GAC) AVHRR L2P files from NOAA-18 and METOP-A have been incorporated as an additional SST data stream assimilated into the BLUElink operational ocean model, OceanMAPS (Brassington et al., 2007). The GAC AVHRR L2P data complement the previously assimilated $25 \mathrm{~km}$ resolution AMSR-E SST data. Using GAC AVHRR together with AMSR-E improves spatial coverage, particularly within $75 \mathrm{~km}$ of coasts, with GAC AVHRR adding increased resolution (Andreu-Burillo et al., 2010). Assimilating both AMSR-E and AVHRR SSTfnd products results in OceanMAPS SST5m analyses closer to buoy observations compared with assimilating AMSR-E and AVHRR SST separately.

\section{Future Plans for BLUElink> and IMOS SST Products (2011-2013)}

As part of the next phase of the IMOS and BLUElink-III Projects (June 2011 - June 2013), the Bureau of Meteorology aims to:

- Provide reprocessed (back to 1992) HRPT AVHRR SSTskin L2P, L3U and L3C files incorporating Australian and Antarctic data via IMOS and the GHRSST GDAC (December 2011) all ready providing real-time files from Australian ground stations via IMOS and Bureau OPeNDAP servers

- Combine delayed mode Antarctic and Australian AVHRR L3U files to produce trial 1-day, 3-day and 6-day, gridded, multi-sensor, L3S files over the RAMSSA domain $\left(60^{\circ} \mathrm{E}-190^{\circ} \mathrm{E}\right.$, $\left.20^{\circ} \mathrm{N}-70^{\circ} \mathrm{S}\right)$ back to 2007 (December 2011)

- Provide real-time HRPT AVHRR SSTskin L2P, L3U and L3C files from 
Davis and Casey Antarctic stations (June 2013)

- Provide reprocessed (back to June 2006) hourly, $0.05^{\circ} \times 0.05^{\circ}$ gridded, MTSAT-1R SSTskin L3U files to IMOS (June 2011)

- Provide real-time and reprocessed hourly, $0.05^{\circ} \times 0.05^{\circ}$ gridded, MTSAT2 SSTskin L3U files to IMOS (December 2011)

- Equip two additional SOOP vessels with hull-contact temperature sensors and provide real-time, quality assured ship SST data streams from these vessels plus the New Zealand research vessel RV Tangaroa and CSIRO'S RV Linnaeus to the GTS and IMOS (June 2012)

- Upgrade operational RAMSSA and GAMSSA to incorporate new IMOS L2P data streams for enhanced accuracy/resolution (HRPT AVHRR and possibly MTSAT-2) and L2P data from AATSR (December 2011)

- Bureau researchers will test RAMSSA_skin SSTskin analyses for both the quality control of satellite sounder data being assimilated into ACCESS-R NWP analyses and as a boundary condition for ACCESS-R forecasts (June 2013)

- Bureau researchers will assess the impact of incorporating a DV model in the BLUElink coupled limited area model (CLAM) (June 2012).

\section{Main Users of BLUElink and IMOS SST Products}

The main users of the Bureau of Meteorology's BLUElink and IMOS SST products are:

- Bureau NWP systems

- Currently require accurate, daily, foundation SST analyses at the appropriate spatial resolution for the NWP boundary condition and for quality control of satellite sounder data assimilated into NWP

o May in future require accurate skin SST analyses produced using foundation SST analyses plus $\triangle$ SST using NWP forecast fluxes/winds as inputs both for a boundary condition and for quality control of satellite sounder data

- Bureau Ocean Prediction Systems

o Currently require accurate, foundation SST L2P files from both microwave and infrared satellite sensors for daily data assimilation

o Require accurate, timely in situ SST (eg. IMOS ship SST) and accurate, timely SSTfnd analyses for validation of SST(5m) analyses and forecasts

o In future require a fast, accurate diurnal variation model to convert from forecast SST $(2.5 \mathrm{~m})$ to SSTskin

- Seasonal Forecasters

o Currently require accurate, global, daily foundation SST at the appropriate spatial resolution for coupled model initialisation

- Weather forecasters

o Require SST analyses and forecasts at as high resolution as possible for sea fog prediction with updates several times per day

- Royal Australian Navy

o Require accurate, foundation SST L2P files from both microwave and infrared satellite sensors for daily data assimilation into daily global relocatable ocean model at $\leq 0.05^{\circ}$ resolution

- Australian Researchers/Education

o Modelers require accurate L2P or L3U files at a range of resolutions

o Biologists generally require gapfree SSTfnd analyses or SSTskin L3C composites at as high resolution as possible, going back in time as far as possible

- Recreational users (eg. fishers, boaters, surfers, divers, swimmers, yachtees)

- Commercial users (fishing, ocean transport, oil/gas drilling, coastal engineering)

- Search and Rescue 
- Government agencies (police, environmental protection, coastal area management)

- Hazardous spill management

- Aquaculture

Unless otherwise stated above, in general the main users of the Bureau's SST products require visual maps of SST realtime analyses and forecasts at as high spatial resolution as possible with the capability of selecting an area/time. The production of accurate SSTskin products at hourly, $4 \mathrm{~km}$ resolution from MTSAT-1R and MTSAT-2 and twice-daily, $1 \mathrm{~km}$ resolution from the NOAA polar-orbiting satellites will facilitate the production of higher spatial resolution SST analyses at the Bureau. They will also assist the validation of diurnal warming models over the Australian region, necessary for the production of sub-daily skin SST analyses and forecasts.

\section{Acknowledgments}

The work was supported by both the BLUElink> Ocean Forecasting Australia Project (a joint project between the Royal Australian Navy, CSIRO Marine and Atmospheric Research and the Australian Bureau of Meteorology) and the Integrated Marine Observing System (an initiative of the Australian Government being conducted as part of the National Collaborative Research Infrastructure Strategy and the Super Science Initiative).

\section{References}

\subsection{Links to Web Pages, OPeNDAP and FTP Servers}

Bureau of Meteorology Operational SST Analysis Web Page:

http://www.bom.gov.au/marine/sst.shtml

$\begin{array}{llr}\text { BLUElink Ocean } & \text { Forecasting } & \text { Australia } \\ \text { Project } & \text { Web } & \text { Site: } \\ \text { http://www.bom.gov.au/bluelink/ } & \end{array}$

BLUElink SST Products Research Web Page:

http://www.cawcr.gov.au/projects/SST/SS T external.html $\begin{array}{ll}\begin{array}{l}\text { Bureau of Meteorology } \\ \text { GODAENDAP }\end{array} & \text { Server: } \\ \text { http://godae.bom.gov.au } & \end{array}$

Bureau of Meteorology Seasonal Forecast Model (POAMA) Web Site: http://poama.bom.gov.au.

Bureau of Meteorology Web Site: http://www.bom.gov.au

Group for High Resolution SST (GHRSST) Web Site: http://www.ghrsst.org

GHRSST Global Data Assembly Centre Web Page: http://ghrsst.jpl.nasa.gov

GHRSST Long-Term Stewardship Facility at NODC Web Site: http://ghrsst.nodc.noaa.gov/

GHRSST Multi-Product Ensemble (GMPE) and Analysis Intercomparison Project Web Page: http://ghrsstpp.metoffice.com/pages/latest analysis/ss t monitor/daily/ens/index.html

Integrated Marine Observing System (IMOS) Web Site: http://www.imos.org.au

IMOS GHRSST SST Products Web Page: http://imos.org.au/sstproducts.html

IMOS Ocean Data Portal: http://imos.aodn.org.au/webportal

IMOS Remote Sensing Data Web Page: http://imos.org.au/srs data.html

IMOS Remote Sensing FTP Server for GHRSST data: ftp://aodaac2cbr.act.csiro.au/imos/GHRSST

IMOS Ship of Opportunity SST Data OPeNDAP site:

http://opendap-

tpac.arcs.org.au/thredds/dodsC/IMOS/SO OP/SOOP-SST/

IMOS Ship of Opportunity Meteorological, SST and Flux Data OPeNDAP site:

http://opendap-

tpac.arcs.org.au/thredds/dodsC/IMOS/SO OP/SOOP-ASF/catalog.html

IMOS Southern Ocean Flux Station Web Page:

http://imos.org.au/sofs.html 
GHRSST XII -

Proceedings, Edinburgh

GHRSST Project Office

NOAA/NESDIS SST Quality Monitor Site (SQUAM):

http://www.star.nesdis.noaa.gov/sod/sst/s

quam/index.html

\subsection{Journals/Reports}

Andreu-Burillo, Isabel, Gary Brassington, Peter Oke and Helen Beggs (2010) Including a new data stream in in BLUElink Ocean Data Assimilation System, Australian Meteorological and Oceanographic Journal, 59:77-86.

Beggs, Helen (2007) A High-Resolution Blended Sea Surface Temperature Analysis over the Australian Region, BMRC Research Report, Bureau of Meteorology, Melbourne, Australia, 43 pp.

http://www.bom.gov.au/bmrc/pubs/rese archreports/RR130.pdf

Beggs, Helen (2008) GAMSSA - A New Global Australian Multi-Sensor SST Analysis, Submitted to Proceedings of the 9th GHRSST-PP Science Team Meeting, Perros-Guirec, France, 9-13 June 2008.

Beggs, H., R. Verein, H. Kippo, $M$. Underwood, I. Barton, C. Steinberg, E. Schulz, R. Hibbins, A. Thomas and G. Ball (2009a). Enhancing Ship of Opportunity Sea Surface Temperature Observations in the Australian Region, GHRSST 2009 International Users Symposium Conference Proceedings, Santa Rosa, USA, pp. 147-150. http://imos.org.au/soopdoc.html

Beggs, Helen, Chelle Gentemann and Peter Steinle (2009b) Real-time skin sea surface temperature analyses for quality control of data assimilated into NWP models, extended abstract, The Fifth WMO International Symposium on Data Assimilation of Observations in Meteorology, Oceanography and Hydrology, Melbourne, Australia, 5-9 October 2009.

http://cawcr.gov.au/projects/SST/5WM O DASymp Extended Abstract Begg s Sep2009.pdf

Beggs, H., R. Verein and G. Paltoglou (2010a) Calibration and validation of
Issue: Final

Date: September 27, 2011

AVHRR sea surface temperatures using observations from ships of opportunity, In: Proceedings "Oceans from Space" Venice 2010, 26-30 April, 2010, p. 37-38.

Beggs H., L. Majewski, G. Paltoglou, E. Schulz, I. Barton and R. Verein (2010b) Report to GHRSST11 from Australia - BLUElink and IMOS, In: Proceedings of the 11th GHRSST Science Team Meeting, Lima, 21-25 June 2010, p. 21-31.

https://www.ghrsst.org/documents/q/cat egory/ghrsst-science-teammeetings/ghrsst-xi-lima/

Beggs, H., A. Zhong, G. Warrren, O. Alves, G. Brassington and T. Pugh (2011a) RAMSSA - An Operational, High-Resolution, Multi-Sensor Sea Surface Temperature Analysis over the Australian Region. Australian Meteorological and Oceanographic Journal, 61, 1-22.

http://www.bom.gov.au/amoj/papers.ph p?year $=2011$

Beggs H., R. Verein, G. Paltoglou, $H$. Kippo and M. Underwood (2011b) Enhancing ship of opportunity sea surface temperature observations in the Australian region, Journal of Operational Oceanography, (in prep.)

Brassington, G. B., Tim Pugh, Claire Spillman, Eric Schulz, Helen Beggs, Andreas Schiller and Peter R. Oke (2007) BLUElink> Development of Operational Oceanography and Servicing in Australia, J. Res. Practice in Information Technology, 39, 151164.

Casey, K, C. Donlon and the GHRSST Science Team (2010) The Recommended GHRSST Data Specification (GDS) Revision 2.0 [online], 1 October 2010, 120 pp. http://www.ghrsst.org/documents.htm? parent $=708$

Gentemann, C. L., C. J. Donlon, A. StuartMenteth and F. J. Wentz, Diurnal signals in satellite sea surface temperature measurements, Geophysical Research Letters, 30(3), 2003. 
Maturi E., A. Harris, C. Merchant, J.Mittaz, R. Potash, W. Meng and J. Sapper (2008) NOAA's Sea Surface Temperature Products from Operational Geostationary Satellites, Bull. American Met. Soc., Dec 2008, 1877-1888.

OSI-SAF Project Team (2006) Ocean \& Sea Ice SAF Atlantic Sea Surface Temperature Product Manual, Version 1.6, October 2006, MétéoFrance, http://www.osi-

saf.org/biblio/docs/ss1 pmatlsst 1 6.p df

Paltoglou, G., H. Beggs and L. Majewski (2010) New Australian High Resolution AVHRR SST Products from the Integrated Marine Observing System, In: Extended Abstracts of the $15^{\text {th }}$ Australasian Remote Sensing and Photogrammetry Conference, Alice Springs, 13-17 September, 2010. http://imos.org.au/srsdoc.html

Puri, K., Dietachmayer, G., Mills, G.A., Davidson, N.E., Bowen, R.A. and Logan, L.W. 1998. The new BMRC Limited Area Prediction System, LAPS. Aust. Met. Mag., 47, 203-33.

Puri, K. et al. (2010) Preliminary results from Numerical Weather Prediction implementation of ACCESS. CAWCR
Research Letters 5, 15-22. http://www.cawcr.gov.au/publications/re searchletters/CAWCR Research Lette rs 5.pdf

Rea, Anthony (2004) Recent Improvements to the NOAA AVHRR SST Product at the Australian Bureau of Meteorology, Presented to the Fifth GODAE High Resolution SST Pilot Project Science Team Workshop, Townsville, Australia, 25-30th July, 2004, http://imos.org.au/srsdoc.html

Smith N. S., B. Ebert and G. Warren (1999) The Bureau of Meteorology SST Analysis System, An informal paper produced as background for the OOPCIAOPC Workshop on SST Analyses for Climate, International Research Institute, LDEO, Palisades NY USA, Nov 10-12, 1998. http://www.bom.gov.au/bmrc/ocean/BL UElink/SST/The Bureau of Meteorolo gy SST analysis system.doc

Zhong, Aihong and Helen Beggs (2008) Analysis and Prediction Operations Bulletin No. 77 - Operational Implementation of Global Australian Multi-Sensor Sea SurfaceTemperature Analysis, 2 October 2008.

http://cawcr.gov.au/projects/SST/GAM SSA BoM Operational Bulletin 77.pdf 


\title{
R08 - REPORT FROM THE NAVOCEANO RDAC
}

\author{
Bruce McKenzie $^{(1)}$, Doug May ${ }^{(1)}$, Dr. Jean-François Cayula ${ }^{(2)}$, Keith Willis ${ }^{(1)}$ \\ (1)Naval Oceanographic Office, Stennis Space Center, MS (US), \\ Email: bruce.mckenzie@navy.mil, doug.may@navy.mil, keith.d.willis@navy.mil \\ (2 QinetiQ North America/Technology Solutions, SSC, MS (US), \\ Email: j.cayula@ieee.org
}

\begin{abstract}
The Naval Oceanographic Office (NAVOCEANO) continues to provide GHRSST L2P products from NOAA-18 Global Area Coverage (GAC), NOAA-19 GAC, NOAA-19 Local Area Coverage (LAC), and MetOp-A GAC. NAVO also provides their $1 / 10^{\text {th }} \mathrm{km}$ composite as an L4 product. NAVO ocean models operationally ingest SST data from the real-time REMSS AMSRE L2P, Medspiration MSG L2P, and the UPA AATSR L2P. Future plans are to convert our L2P and L4 products to GDS V2.0.
\end{abstract}

\section{Introduction}

NAVO will present details on their ongoing L2P and L4 products and their planned modifications. The GHRSST data provided to the ocean analyses and forecasts include matchup statistics calculated using satellite specific drifting buoy matchup databases. Statistics from these matchup databases will be shown (Figure 1). Work preparing for the launch of the NPOESS Preparatory Project (NPP) and the processing of Visible Infrared Imaging Radiometer Suite (VIIRS) data will also be presented.

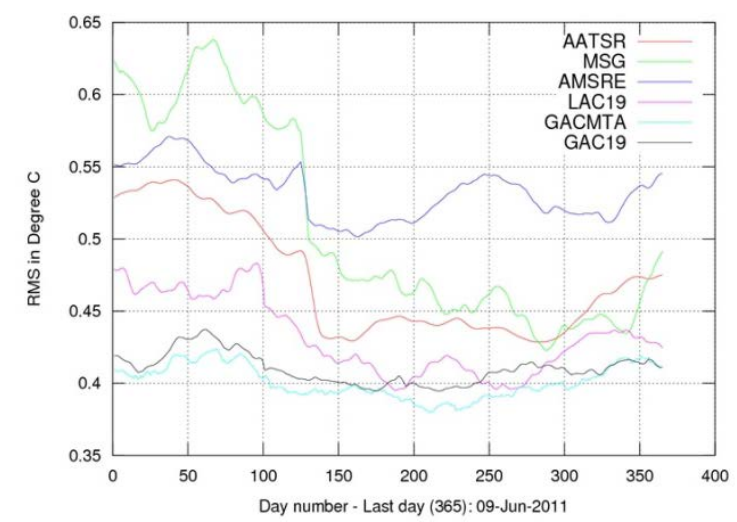

Figure 1. SST matchup statistics for best quality

\section{L2P and L4 products produced}

The current set of L2P products produced to GDS1.0 V1.5 specifications are NOAA18 GAC, NOAA-19 GAC, MetOp-A GAC, and NOAA-19 LAC. The L2P variables filled in by NAVO are lat, lon, sea_surface_temperature, sst_dtime, SSES_bias_error, SES_standard_deviation_error, DT_analysis, aerosol_optical_depth, aod_dtime_from_sst, sources_of_aod, satellite_zenith_angle, rejection_flag, confidence flag, proximity_confidence. NAVO also provides three additional variables and encourages other data providers to do the same. These are:

brightness_temperature_4um, brightness_temperature_11um, brightness_temperature_12um.

and

These values prove useful for the Navy Coupled Ocean Data Assimilation (NCODA) systems at NAVO and Fleet Numerical Meteorology and Oceanography Center (FNMOC). The JPL GDAC fills in the sea_ice_fraction and wind_speed variables in all NAVO L2P products.

The NAVOCEANO L4 product is a $1 / 10^{\text {th }}$ degree composite updated daily from operational SST retrievals generated at NAVO (NOAA-18 GAC, NOAA-19 GAC, MetOp-A GAC, NOAA-19 LAC, MetOp-A Full Resolution Area Coverage (FRAC), and GOES-11), Remote Sensing Systems daily gridded AMSRE, and the JPL Pentad Climo 1985 - 1999.

\section{L2P products used by NAVO}

NAVO operationally retrieves UPA AATSR L2P, Medspiration MSG L2P, and realtime REMSS AMSRE L2P from the JPL GDAC. These SST datasets are 
GHRSST XII -

Proceedings, Edinburgh

GHRSST Project Office

reformatted into flat binary files containing just the best quality retrievals which are then provided to the NAVO ocean models and the FNMOC meteorological models. The data is also put into a SST database from which a matchup database is created. Statistics from the MDB are included with the MSG and AATSR flat binary files. The AMSRE data retains its original statistics. NAVO would like to see
Issue: Final

Date: September 27, 2011

the brightness temperatures used in the MSG and AATSR SST generation included in the L2P files.

\section{Conclusion}

The GHRSST project is not only a valuable source of SST data for NAVOCEANO, but a great collaborative group to work on issues that impact all aspects of SST generation. 


\title{
R09 - REPORT FROM MULTI-SENSOR IMPROVED SEA-SURFACE TEMPERATURE (MISST) FOR IOOS
}

\author{
Chelle Gentemann $^{(1)}$, Ken Casey ${ }^{(2)}$, Peter Cornillon ${ }^{(3)}$ \\ (1)Remote Sensing Systems, Santa Rosa, CA, USA, Email: gentemann@remss.com \\ (2) NOAA/NODC, Silver Spring MD, USA, Email: Kenneth.Casey@noaa.gov \\ (3) University of Rhode Island, Graduate School of Oceanography, Narragansett, RI, \\ USA, Email: pcornillon@gso.uri.edu
}

This project was approved but remains unfunded at this date (June 17, 2011). The project outlined below is contingent on funding.

This project builds on the successful partnership developed for the Multi-sensor Improved Sea-Surface Temperature (MISST) for GODAE project (2004-2009). The proposed work picks up where MISST left off in 2009 with many of the same objectives; therefore we will refer to the new project as MISST also. The objectives of this project are to (1) improve and continue generation of satellite SST data and SST analyses in the IOOS DMAC and CF compliant Group for High Resolution Sea Surface Temperature (GHRSST) Data Specification GDS format; (2) distribute and archive these data; and (3) use this improved SST data in applications, many specifically targeted for the Integrated Ocean Observing System (IOOS).

The partnership consists of 28 scientists from industry, academia, and government with wide ranging experience spanning the initial calibration of satellite sensors, development of SST algorithms, assessment of SST uncertainties, production of NRT satellite data, research into data fusion methodologies and the production of blended data sets, research into diurnal warming and the cool skin effect which both affect satellite SST measurements, and applications that utilize SSTs.

The project research priorities are identified below.

(1) Research into satellite calibration and validation, specifically to improve sensor error characterization. The project partners have extensive experience in satellite SST algorithm development, assessment, and improvement. Estimates of SST uncertainty, currently part of the GHRSST data, will be further improved through satellite cross-calibration and in situ validation research.

(2) Research into diurnal warming and cool skin modeling, specifically to improve use of satellite data within analysis systems and our understanding of upper ocean physics. Project partners have produced significant advances in our understanding of diurnal warming and the cool skin effect, publishing 12 peer reviewed articles on this topic during the previous MISST project. Several models will be further advanced through satellite and in situ diurnal warming and cool skin research. The project will follow the guidance of the GHRSST diurnal warming working group toward developing a global diurnal model.

(3) Research into multi-satellite data fusion, accounting for skin and diurnal effects. Project partners produce 8 different multi-satellite SST analyses using various methodologies. Through this proposal, partners will utilize the research into SST errors, diurnal warming and cool skin effects to improve their utility of SST measurements and accuracy of their SST analyses.

(4) Routine production of satellite SST data conforming to the GHRSST Data Specification (GDS) Version 2, with data distribution and preservation through the GHRSST Global Data Assembly Center (GDAC) and Long Term Stewardship and Reanalysis Facility (LTSRF). Partners in the project currently produce satellite SST data from every US sensor currently flying, and a number of blended SST data analyses, in the GHRSST GDS version 1.6. In 2011, the GHRSST format 
GHRSST XII -

Proceedings, Edinburgh

GHRSST Project Office

changes to GDS version 2.0. Both data providers and data users must adapt their systems to account for this improvement in the data formatting. All partners will continue to distribute data through the GDAC and LTSRF.

(5) Use of these advanced products to increase understanding of our oceans and coasts, so decision makers can take action to improve safety, enhance the economy, and protect the environment. This partnership has members who produce the data as well as users to best ensure the rapid and accurate dissemination of the data. A number of application projects are partnered through this proposal, including several that will work directly with IOOS regional associations (RAs) to investigate coral health, fisheries, ocean fronts, and data availability of SST for the IOOS RAs.

Specific task for this proposal are given below:

Task 1. Improved, sustained generation of GHRSST data

Task 1.1. Continue production of existing data

Task 1.2. New GDS 2.0 products

Task 1.3. Improved lake temperatures
Issue: Final

Date: September 27, 2011

Task 1.4. Improved ancillary data

Task 1.4.1. Improved diurnal modeling

Task 1.4.2. Cool skin effect

Task 1.5. Improved error estimates

Task 1.6. L4 analysis

Task 1.6.1 Update existing data to GDS 2.0

Task 1.6.2. New L4 data to GDS 2.0

Task 1.6.2.1 Validation of highresolution features

Task 1.6.2.2 Formal analysis error estimates for hypothesis testing

Task 2. Distribution \& Long-term stewardship

Task 2.1. GDAC

Task 2.2. LTSRF

Task 2.3. Improved SST access for IOOS partners

Task 3. Applications within IOOS to improve decision making

Task 3.1. Coral health

Task 3.2. Outreach to IOOS Regional Associations and Users

Task 3.3. Ocean fronts

Task 3.4. Ocean Forecasts

Task 3.5. NWP

Task 3.6. Coupled Modeling and data impact studies 


\section{R10 - ESA RDAC REPORT}

\section{Nigel Houghton}

ESA/ESRIN, Frascati, Italy, Email: Nigel.Houghton@esa.int

ESA produces the GHRSST format L2P product for ATSR-1, ATSR-2 and AATSR data from the ERS-1, ERS-2 and Envisat satellites respectively. This data set is generated and archived at ESA's UK Multimission Processing and Archiving Centre (UK-MM-PAC) at Farnborough in UK. The output format is GDS1.7/ NetCDF3.

UK-MM-PAC produces the L2P product using the software developed by $a$ consortium of companies in 2008. This software has proved to be very reliable and resilient with Version 1.5 in operation since May 2009.

The data is generated in two flavours.

Firstly, for NRT data from AATSR, UKMM-PAC receives the data from the Envisat Ground Stations in Kiruna, Sweden and from ESRIN, Italy the latter acquiring data via the Artemis Data Relay Satellite. UK-MM-PAC receives the $\mathrm{L} 2$ $1 \mathrm{~km}$ product from the Ground Stations and generates L2P which is then transferred (pushed) to:

- PO.DAAC, JPL.

- Met. Office, Exeter.

- Ifremer (medspiration)

The data is also available via ftp from UKMM-PAC where is it held in rolling archive (although nothing has been deleted from this rolling archive to date). Once the input $\mathrm{L} 2$ has been received at UK-MM-PAC, the L2P is generated quickly and, notwithstanding problems at the Ground Stations themselves, the L2P is disseminated within three hours of the acquisition of the data on satellite.
Secondly, from the L2 archive quality product for ATSR-1, ATSR-2 and AATSR, the archive quality $\mathrm{L} 2 \mathrm{P}$ is generated. ATSR-1 and ATSR-2 are complete with AATSR data complete up to approximately four weeks after data acquisition and growing as new archive quality L2 data is received and generated to L2P. This data is also available via ftp and has now been delivered to the LTSRF on media. The total size of this archive is currently approximately 1.5TBytes.

Quality checks are made on the products by the ESA Support Teams to ensure the high quality of disseminated data.

Further information on the ESA L2P products can be found at http://earth.esa.int/object/index.cfm?fobjec $\underline{\mathrm{tid}=1451 \& S \quad|\mathrm{D}=14 \&| \quad \mid \mathrm{D}=64}$

Access to the data is granted on a successful Cat1 application through the EOPI website at http://eopi.esa.int/esa/esa. The application procedure is fast and access credentials are normally issued within a couple of days.

Future plans include the update to the software to GDS2/NetCDF4 expected to start 2011Q4. Furthermore, a new version of the ATSR processing software expected 2011Q4 will result in a change to the NRT product and a complete reprocessing of the Archive product. ESA is seeking ways to improve the end-to-end process that should also yield improvements in the timeliness of the L2P product. 


\section{R11 - REPORT FROM MYOCEAN RDAC}

\section{Hervé Roquet ${ }^{(1)}$, Jean-François Piollé(2) ${ }^{(2)}$ Bruno Buongiorno-Nardelli ${ }^{(3)}$, Jacob} Høyer $^{(4)}$, Hanne Heiberg ${ }^{(5)}$, Matthew Martin ${ }^{(6)}$, David Poulter ${ }^{(7)}$ and others

(1)Météo-France (Lannion, France), Email : Herve.Roquet@meteo.fr

(2) IFREMER (Brest, France), Email : Jean.Francois.Piolle@ifremer.fr

(3) CNR (Napoli, Italy), Email : Bruno.Buongiornonardelli@cnr.it

(4) DMI (Copenhagen, Denmark), Email : jlh@dmi.dk

(5) Met.no (Oslo, Norway), Email : h.heiberg@met.no

(6) UK Met Office (Exeter, United Kingdom), Email : Matthew.Martin@metoffice.gov.uk

(7) NERC/NOCS (Southampton, United Kingdom), Email : D.J.S.Poulter@soton.ac.uk

\section{ABSTRACT}

The MyOcean project, which is funded by the European Union for a 3 year period (2009-2012), includes the development and operations of a Thematic Assembly Centre for satellite SST products (SST TAC). The SST TAC provides in particular near real-time global and regional L3/L4 products, derived from GHRSST L2P SST sources, to MyOcean Monitoring and Forecast Centres (MFCs), but also to external MyOcean users. The main progress areas in the SST TAC since last year have been its successful operational acceptance as part of MyOcean V1 system, which was opened to external users in December 2010, and the production and release of a 23 year (1985-2007) global SST re-analysis, based on the OSTIA analysis system. The SST TAC activities should be continued and enhanced in the framework of the MyOcean-2 project (2012-2014), which is currently under negotiation with the European Union.

\section{Introduction}

MyOcean is a EU-funded project, which was kicked-off in April 2009 for a 3 year duration. Its main objective is to transition into operations and operate the first European Marine Core Services, which have been developed in the framework of the EU-funded MERSEA project (20042008). Its architecture is based on seven ocean Monitoring and Forecast Centres (MFCs), covering the Global Ocean, the Mediterranean Sea, the Black Sea, the Arctic Ocean, the Baltic Sea, the Atlantic North West Shelves and the Iberian plateau, on five data Thematic Assembly Centres (TACs) for in-situ observations and satellite SST, Ocean Colour, Surface Topography and Sea Ice products, and on a centralised information system and service desk for delivering products and services.

Several European Institutes (MétéoFrance, UK Met Office, IFREMER, Met.no, DMI, CNR, NERC/NOCS) are contributing to the development and the operation of SST TAC. The SST TAC is a significant European contribution to the GHRSST, in the following areas :

- near real-time processing and delivery of global and regional L3/L4 SST products,

- near real-time processing and delivery of GMPE products,

- global SST re-analysis (OSTIA),

- systematic building and/or collection of Match-up Data Bases, and continuous quality monitoring of input L2P SST sources.

In this paper we present the progress of MyOcean SST TAC activities since GHRSST XI, and give an overview of its plans during the last year of the current MyOcean project (up to March 2012), as well as in the framework of its follow-on project (MyOcean-2, 2012-2014).

\section{Main achievements since GHRSST XI}

The main achievements of SST TAC since GHRSST XI are: 
GHRSST XII -

Proceedings, Edinburgh

GHRSST Project Office

- the operational acceptance of SST TAC near real-time production, as part of MyOcean V1 system, which was opened to external users in December 2010

- the production, validation and delivery of a 23 year (1985-2007) global SST re-analysis, based on the OSTIA analysis system

- the implementation of a routine quality monitoring of input L2P SST sources. The results are displayed on the SST TAC cal/val home page :

- http://projets.ifremer.fr/cersat/Data/Qual ity-control/MyOcean-SST-QC/ControlValidation-MyOcean

- the implementation of common metrics for the quality monitoring and validation of all SST TAC near real-time L3/L4 products. The results are displayed on the SST TAC cal/val home page : http://projets.ifremer.fr/cersat/Data/Qual ity-control/MyOcean-SST-QC/ControlValidation-MyOcean

The current SST TAC near real-time L3/L4 products, which are distributed operationally as part of MyOcean V1 system, are :
Issue: Final

Date: September 27, 2011

- L3 : daily global multi-sensor (L3S) product at $0.1^{\circ}$ horizontal resolution, daily mono- (L3C) and multi-sensor (L3S) products at $0.02^{\circ}$ horizontal resolution over European Seas

- L4 : daily global SST analysis at $0.05^{\circ}$ horizontal resolution (OSTIA), daily regional SST analyses over the Mediterranean Sea and the Black Sea at both $0.0625^{\circ}(\mathrm{HR})$ and $0.01^{\circ}$ (UHR) horizontal resolutions, over the Baltic Sea at $0.03^{\circ}$ horizontal resolution, over the Arctic at $0.05^{\circ}$ horizontal resolution and over the European North West Shelves at $0.02^{\circ}$ horizontal resolution (ODYSSEA)

SST TAC products are available to MyOcean registered users, through FTP or OpenDAP/THREDDS servers. Registration requires downloading, filling and signing up a Service Level Agreement (SLA), which is available from the MyOcean web site (http //www.myocean.eu.org/ ).

The MyOcean Service Desk (servicedesk@myocean.eu.org ), operated currently by the UK Met Office, is the single point of contact for MyOcean Users for all issues related to MyOcean services.

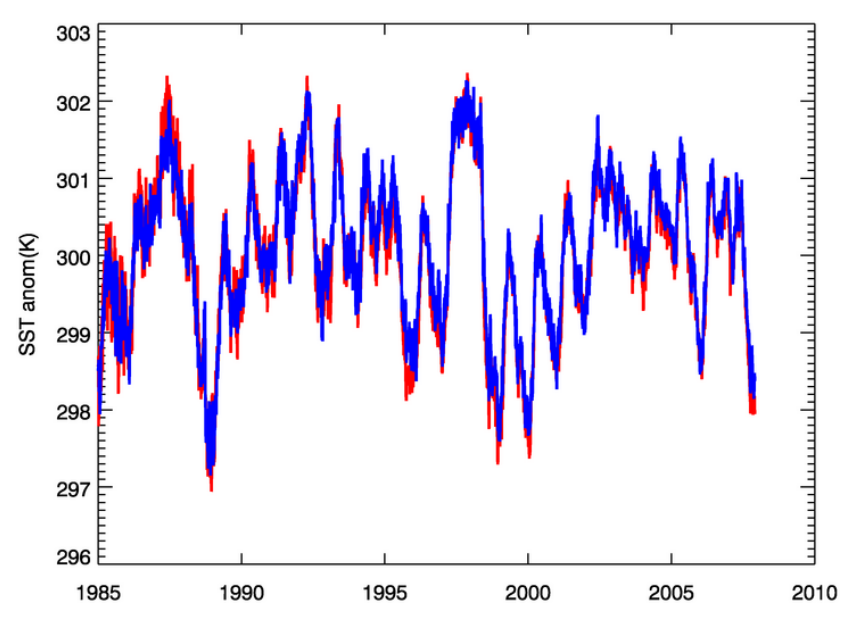

Figure 1: Nino3.4 climate index comparison for OSTIA (Red) and Reynolds (Blue) re-analyses. 

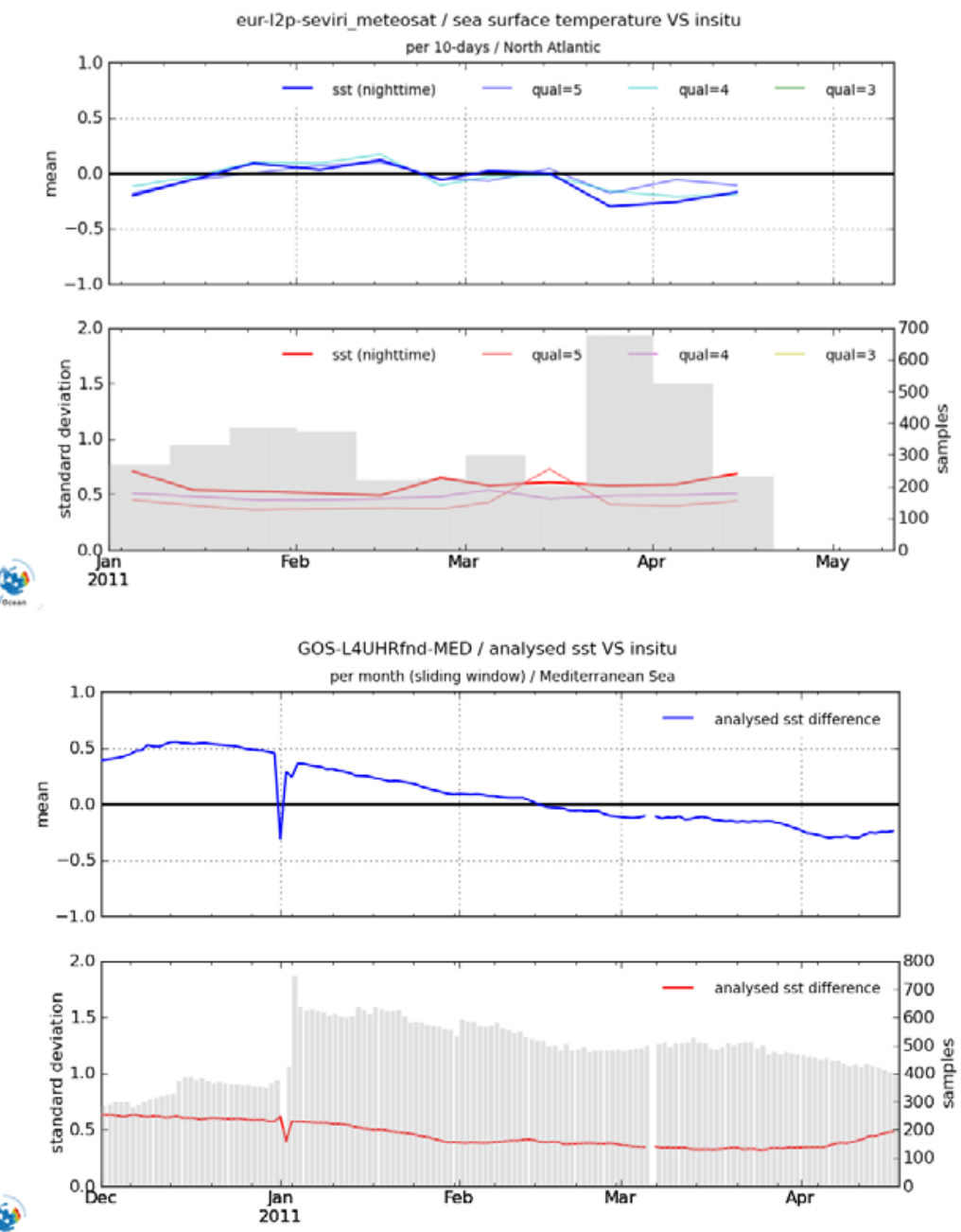

Figure 2 : examples of validation statistics computed against drifters SST measurements, as displayed on the MyOcean SST TAC cal/val web site. Top : SEVIRI L2P data over Northern Atlantic (input data for the SST TAC), Bottom : Ultra High Resolution SST analysis over the Mediterranean Sea (output product from the SST TAC).

\section{Future plans}

\subsection{Up to the end of the current MyOcean project (March 2012)}

The main SST TAC activities and products upgrades planned in the framework of the current MyOcean project are :

- transition to GDS V2

- preparation activities for the SST TAC operational acceptance as part of MyOcean V2 system (technical evolution of central systems : web site, OpenDAP/THREDDS servers, central catalogue etc...), which should be opened to external users in December 2011

- resolution increase of regional SST analyses over the Baltic Sea (from $0.03^{\circ}$ to $0.02^{\circ}$ ) and the Arctic Sea (from $0.05^{\circ}$ to $0.03^{\circ}$ )

- near real-time operational processing and delivery of new SST TAC products : daily multisensor (L3S) products over the Mediterranean Sea and the Black Sea at $0.01^{\circ}$ horizontal resolution, daily global SST analysis at $0.1^{\circ}$ horizontal resolution (ODYSSEA) 
GHRSST XII -

Proceedings, Edinburgh

GHRSST Project Office

3.2 After the current MyOcean project : MyOcean-2 (2012 - 2014)

During MyOcean-2, the SST activities will still focus on quality monitoring and inter-calibration of level 2 satellite SST products, and on operational processing and continuous improvement of L3/L4 global and regional satellite SST products. In addition, the following evolutions are planned:

- use of new input L2P SST sources, in particular Sentinel-3A SLSTR L2P SST products processed centrally at EUMETSAT, according to the agreement between EUMETSAT and ESA. The SLSTR data will provide continuity for current ENVISAT AATSR SST data, which are used as reference SST data by the SST TAC in the current satellite SST intercalibration approach.

- development of an Ice Surface Temperature analysis, which will
Issue: Final

Date: September 27, 2011

complement the SST analyses in the high latitude areas

- increase in the time sampling of L3/L4 satellite SST products, to provide information on the SST diurnal variations

- production of several SST reanalyses at global and regional scales

The MyOcean-2 proposal has been submitted in November 2010. It has been evaluated positively by the European Union, and is now in its negotiation phase.

\section{MyOcean products usage}

The access to MyOcean products and services was opened in November 2009 (V0 system). Figure 3 shows the number of registered external users at the end of April 2011, for the various MyOcean TACs and MFCs. At this date, the SST TAC had more than 180 registered users outside MyOcean.

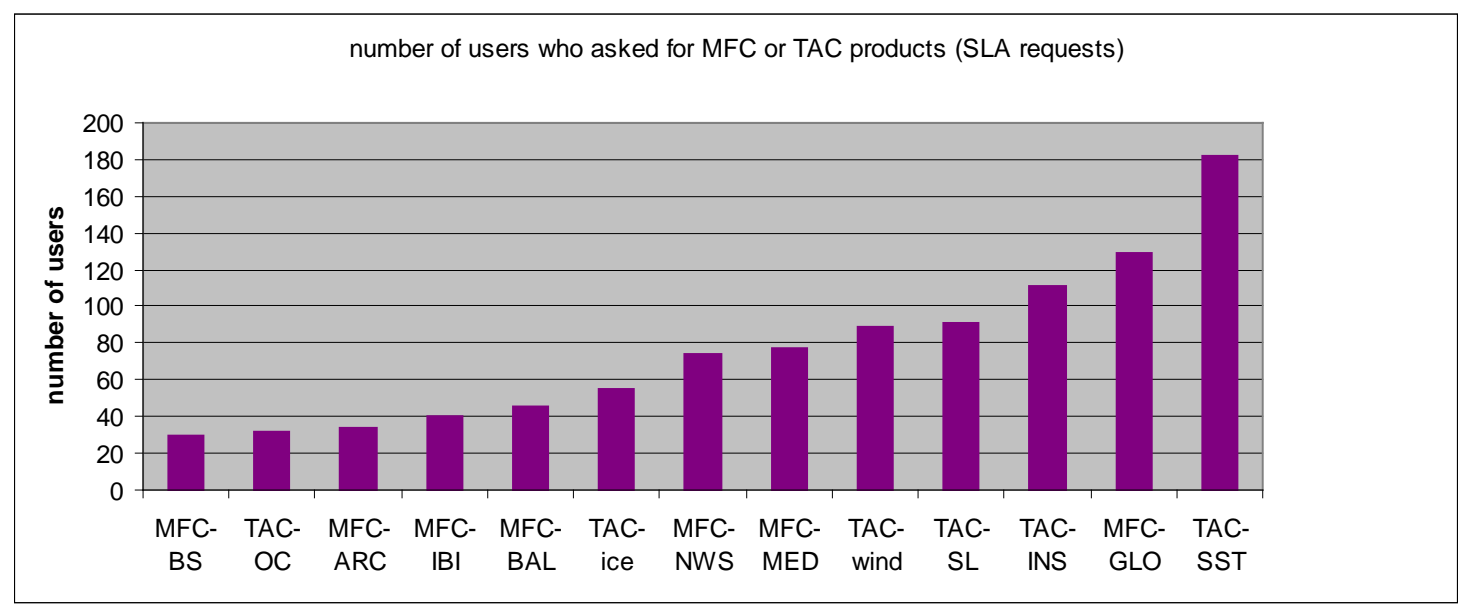

Figure 3 : number of registered external users at the end of April 2011, for the various MyOcean TACs and MFCs.

\section{References}

Buongiorno Nardelli, B., C. Tronconi and R. Santoleri, High and Ultra-High resolution processing of satellite Sea
Surface Temperature data over the Southern European Seas in the framework of MyOcean project, submitted to Remote Sens. of Env., 2010. 


\title{
R12 - REPORT FROM EUMETSAT AND OSI SAF
}

Anne O'Carroll ${ }^{(1)}$, Pierre Le Borgne ${ }^{(2)}$, Steinar Eastwood ${ }^{(3)}$

\author{
(1)EUMETSAT, Eumetsat-Allee 1, 64295 Darmstadt (Germany), \\ Email: Anne.Ocarroll@eumetsat.int \\ (2) Centre de Météorologie Spatiale,Météo-France, Lannion (France), \\ Email : Pierre.LeBorgne@meteo.fr \\ (3) Norwegian Meteorological Institute, Oslo (Norway), Email : S.Eastwood@met.no
}

\begin{abstract}
The present OSI SAF (EUMETSAT Ocean \& Sea Ice Satellite Application Facility) SST chains have been delivering operational products for more than 7 years now. They have been upgraded in 2011 , mainly because the product characteristics do no longer meet the users' needs as expressed by the Group for High Resolution Sea Surface Temperature. Users request better time (1 hour) and space $(5 \mathrm{~km})$ resolution and wish for distinct products for each platform. The new geostationary chain at Météo-France/Centre de Météorologie Spatiale (CMS) is now delivering hourly products at $0.05^{\circ}$ resolution in a regular grid, from $60^{\circ} \mathrm{S}$ to $60^{\circ} \mathrm{N}$ and from $135 \mathrm{~W}$ $15 \mathrm{~W}$ (GOES-E) or 60W- 60E (MSG), in GDS V2.0 format. Similarly the Norwegian Meteorological Institute (METNO) is now producing 12 hourly products on a polar stereographic grid derived from NOAA-18, -19 and Metop AVHRR. METNO products are delivered in HDF5 and GRIB, and soon also will be available in GDS V2.0 format.
\end{abstract}

EUMETSAT central facilities have produced, since March 2011, a demonstrational Metop IASI skin SST product in GDS V2.0 L2Pcore netCDF4 format. The SSTs are those contained within the operational EUMETSAT IASI L2 Product Processing Facility product, available from EUMETSAT since April 2008. The IASI SST L2Pcore contains skin SSTs, flags, quality information and SSES plus an auxiliary wind-speed field, but no further auxiliary data.

\section{Introduction}

The present Sea Surface Temperature (SST) products of the OSI SAF SST chain (as of January 2011) were defined in 1997 at the start of the OSI SAF development phase. They have been produced on a 0.1 ${ }^{\circ}$ grid covering the Atlantic from $100^{\circ} \mathrm{W}$ to $45^{\circ} \mathrm{E}$. A description of the present operational chain is presented in the Atlantic SST Product User Manual [1]. The "Low and Mid Latitude Atlantic" (LML) region has been covered since 2001 (GOES-E) or 2004 (MSG) by a joint use of GOES-E and MSG derived 3 hourly products, after synthesis of the original hourly fields.

In the 12 hourly "Merged Atlantic Products" (MAP), AVHRR SST products derived by METNO at high latitudes have been added to extend the area up to $90^{\circ} \mathrm{N}$

The OSI SAF team at MétéoFrance/Centre de Météorologie Spatiale (CMS) and at METNO decided to upgrade their SST chains, essentially because the product characteristics no longer meet the users' needs as expressed by the Group for High Resolution Sea Surface Temperature. Users request better time (1 hour) and space $(5 \mathrm{~km})$ resolution and wish for distinct products for each platform.

Aside to these operational developments, METNO and CMS have continued their study on diurnal warming at high latitudes, concluding that significant diurnal warming events of several Kelvin are commonly observed in the Arctic [2] and in the Antarctic [3]. 
GHRSST XII -

Proceedings, Edinburgh

GHRSST Project Office

Sea surface temperatures have been produced as part of the Infrared Atmospheric Sounding Interferometer (IASI) L2 Product Processing Facility (PPF) product at EUMETSAT since April 2008 [4]. The IASI L2 product [5,6] is available from the EUMETSAT data centre. There is a user requirement for SSTs to be provided in GHRSST L2P format. Therefore work has been done at EUMETSAT to provide the IASI SSTS in GDS V2.0 L2P format. Currently the products are provided in 'L2Pcore' format, containing the skin SSTs, SSES, flags and quality levels, plus ECMWF wind speed in order for users to understand whether observations may be influenced by diurnal surface temperature variations. It is likely that the OSI-SAF will add the extra auxiliary information required by GDS V2.0, such as sea ice fraction and aerosol information, in the 2013 time-frame to produce full IASI L2P products, and to incorporate the IASI matchup buoy validation into the OSI-SAF validation software. The IASI SST L2Pcore product has been available through ftp access since 24 ${ }^{\text {th }}$ March 2011 from the EUMETSAT EO portal:

http://www.eumetsat.int/Home/Main/DataP roducts/ProductNavigator/index.htm?l=en. Dissemination of the IASI SST L2Pcore through the PO.DAAC/GDAC will be considered following validation and feedback from the users.

In this presentation we focus on the new OSI SAF products that should become operational in June 2011. In section 2 we review the geostationary derived products (GOES-E and MSG); and in section 3, the new polar orbiter products (NOAA-18, NOAA-19 and Metop). Section 4 contains information on the IASI SST L2Pcore produced at EUMETSAT.

\section{New geostationary SST products at MFICMS}

The upgraded OSI SAF geostationary SST chain includes the following main steps [7]:
Issue: Final

Date: September 27, 2011

- Preprocessing: Every frame of MSG/SEVIRI or GOES-E imager radiometric data (MSG: every 15 minutes; GOES-E: every 30 minutes) is ingested in full space resolution.

- Cloud mask control.

- SST calculations.

- Quality level determination.

Operational products are then produced by remapping over a $0.05^{\circ}$ regular grid SST fields obtained by aggregating all SST data available within one hour, with priority given to the value the closest in time to the product nominal hour. Figure 1 shows an example of an hourly MSG derived SST product from $60^{\circ} \mathrm{S}$ to $60^{\circ} \mathrm{N}$ and $60^{\circ} \mathrm{W}$ to $60^{\circ} \mathrm{E}$ (the SEVIRI regular grid); this figure also shows the GOES-E SST product produced by the same chain on a $0.05^{\circ}$ grid from $60^{\circ} \mathrm{S}$ to $60^{\circ} \mathrm{N}$ and $135^{\circ} \mathrm{W}$ to $15^{\circ} \mathrm{W}$. The present GOES-E platform (GOES-13) does not have a 12 micron channel enabling daytime SST calculations: GOES-E derived SST is restricted to nighttime conditions. The final products result from collecting data from various slots within one hour. Consequently they are considered as "L3C" (remapped collated) in the GHRSST product definition. The final hourly product characteristics are the following:

Projection: linear scaling in latitude and longitude

Resolution: 0.05 degree in latitude and longitude

Size: $\quad 2400$ columns, 2400 lines

Longitude and latitude limits:

GOES-E SST:60S-60N; 135W-15W

MSG SST: $60 S-60 N ; 60$ W- 60E

The GOES-E and MSG hourly SST products are available in $\mathrm{L} 3 \mathrm{C}$ netcdf format through IFREMER and in GRIB ed.2 disseminated through EUMETCast. $\mathrm{L} 3 \mathrm{C}$ is the netcdf format recommended by the Group for High Resolution Sea Surface Temperature [8]. 

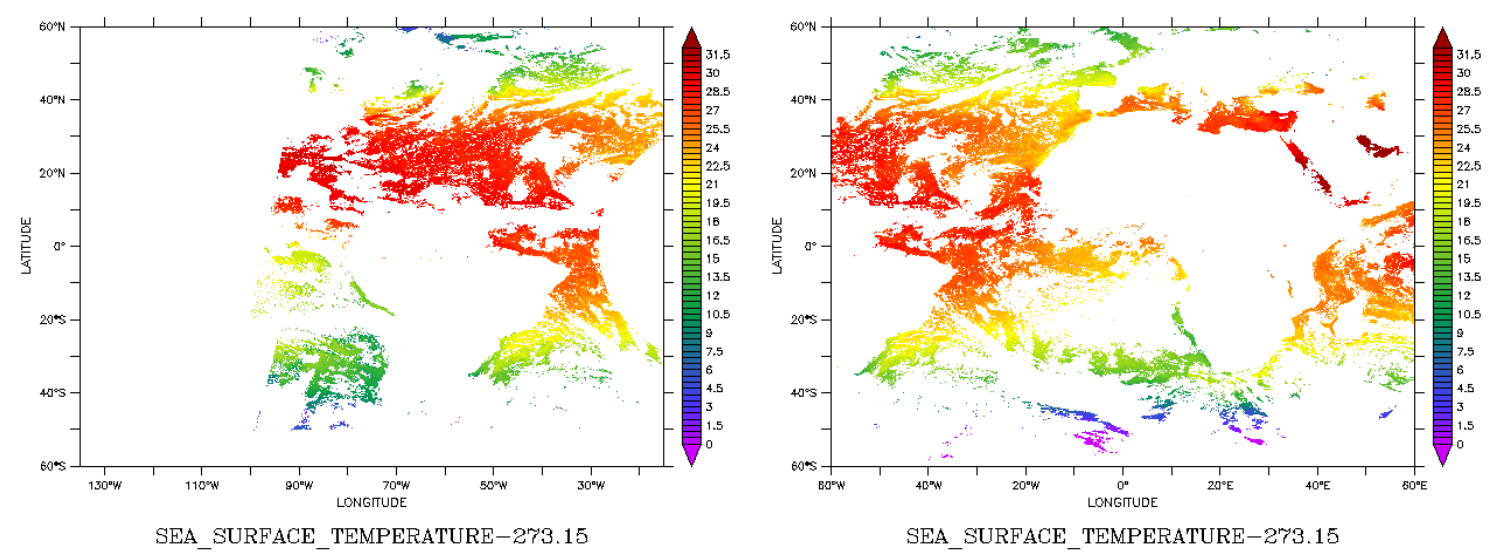

Figure 1: Upgraded geostationary chain SST products at 00:00 on the 10 $0^{\text {th }}$ of September 2010; left, GOES13 (with the most western part of the area masked due to daytime conditions), right: MSG

A detailed validation study has been conducted from the $1^{\text {st }}$ of November to the $31^{\text {st }}$ of January 2011, see the geostationary SST validation report
[9]. The validation results for quality levels 3 to 5 (recommended for quantitative use) are presented in Table 1.

\begin{tabular}{|c|c|c|c|}
\hline $\begin{array}{c}\text { Quality } \\
\text { levels: } \\
\text { 3 to 5 }\end{array}$ & MSG Night & MSG Day & $\begin{array}{c}\text { GOES-E } \\
\text { (night) }\end{array}$ \\
\hline nbc & $\mathbf{2 7 3 5 3}$ & $\mathbf{4 0 8 9 5}$ & $\mathbf{2 8 9 1 5}$ \\
\hline bias & $-\mathbf{0 . 0 5}$ & $\mathbf{0 . 0 2}$ & -0.12 \\
\hline stdev & $\mathbf{0 . 4 9}$ & $\mathbf{0 . 4 6}$ & $\mathbf{0 . 4 7}$ \\
\hline
\end{tabular}

Table 1: Meteosat-9 results (with algorithm bias correction): Number of cases, mean and standard deviation of the error, for quality levels from 3 to 5 , from the $1^{\text {st }}$ of November 2010 till the $31^{\text {st }}$ of January 2011.

The new geostationary chain uses the method described in [10] which consists in calculating a predicted algorithm bias from simulated brightness temperatures. Figure

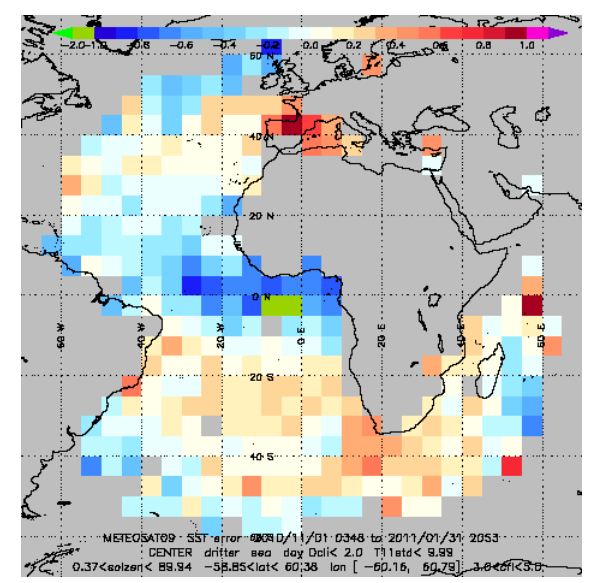

2 illustrates the impact of applying this algorithm bias correction on the regional biases.

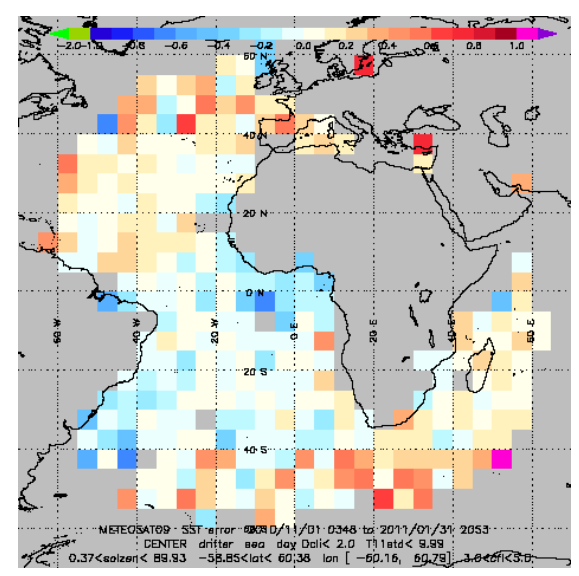

Figure 2: Meteosat-9: Nighttime biases against drifters binned in $5 \times 5^{\circ}$ boxes over the whole validation period. Left panel: without algorithm correction, right panel: with algorithm correction. 
GHRSST XII -

Proceedings, Edinburgh

GHRSST Project Office

\section{New polar orbiter products at METNO}

A specific L3 High Latitude SST product is produced at METNO covering the North Atlantic High Latitudes (AHL) north of $50 \mathrm{~N}$ [11]. This product used to be a part of the MAP SST products, but the MAP product is superseded by a Low and Mid Latitude product based on geostationary data (see above) and a High Latitude product based on AVHRR polar orbiting data. The AHL SST products are derived from AVHRR polar orbiter data received at the local receiving station at METNO together with data received through the EUMETCast ATOVS Retransmission Service (EARS).

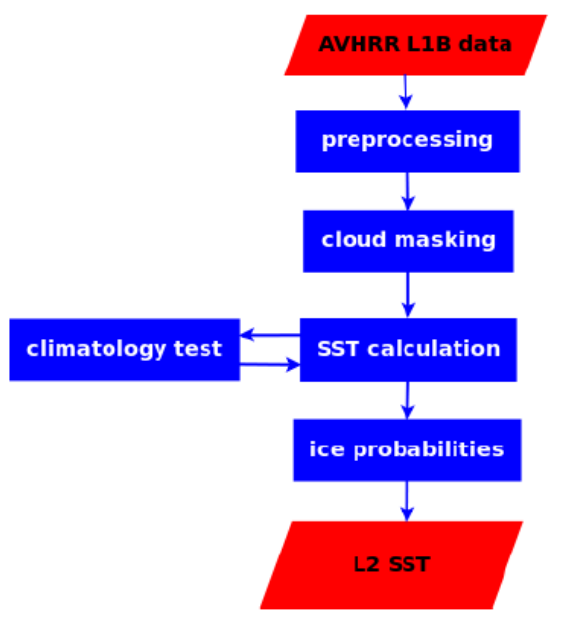

Issue: Final

Date: September 27, 2011

Intermediate L2 SST products for each pass are input to the AHL L3 SST product that is delivered every 12 hours. These 12-hourly products are available in HDF5 and GRIB format through the OSI SAF High Latitude FTP server (ftp://osisaf.met.no/prod). See also http://osisaf.met.no for product monitoring, validation, news messages and other information.

The primary SST calculations are at present made from each AVHRR pass received at METNO from NOAA-18, NOAA-19 and Metop-2. The main L2 processing steps are shown the left part of Figure 3.

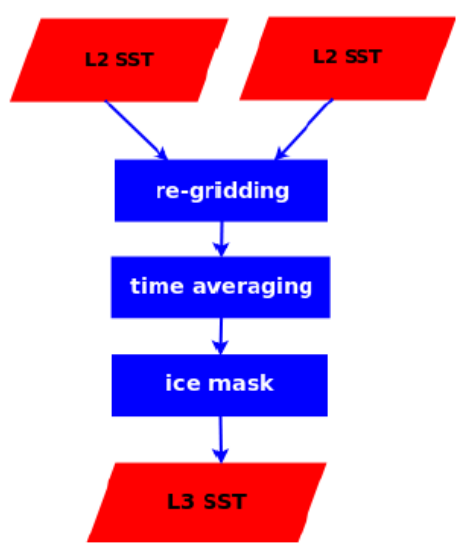

Figure 3: AHL OSI SAF SST calculation scheme

The final L3 12-hourly $5 \mathrm{~km}$ AHL SST product is produced by combining available L2 $1.5 \mathrm{~km}$ satellite pass SST products, following the processing steps shown in the right part of Figure 3.

From the matchup database various statistics are made to validate the accuracy and precision of the products, mainly by investigating the bias and standard deviation of the estimated SST compare to the in situ measurement. Initial validation results are presented in the $\mathrm{AHL}$ SST validation report [12] and in the quarterly reports from the OSI SAF. The final 12 hourly product characteristics are the following:

(Geographical definition for Northern Hemisphere Grid)
Projection: Polar stereographic projection true at $60^{\circ} \mathrm{N}$

Resolution: $5.0 \mathrm{~km}$

Size: $\quad 1260$ columns, 900 lines

Central Meridian: $0^{\circ}$

Lower left corner:

$37.39928^{\circ} \mathrm{N}, 40.16765^{\circ} \mathrm{W}$

Radius of Earth: 6371000 m

PROJ-4 string:

$$
+ \text { proj=stere }+a=6371000+b=6371000
$$$$
\text { +lat_0 }=90 \text { +lat_ts }=60+\text { lon_ } 0=0
$$

These products are available in HDF5 and GRIB format. They will be available in GDS V2.0 format in the beginning of 2012. The product coverage is shown in Figure 4. Actual data coverage depends on EARS coverage. 


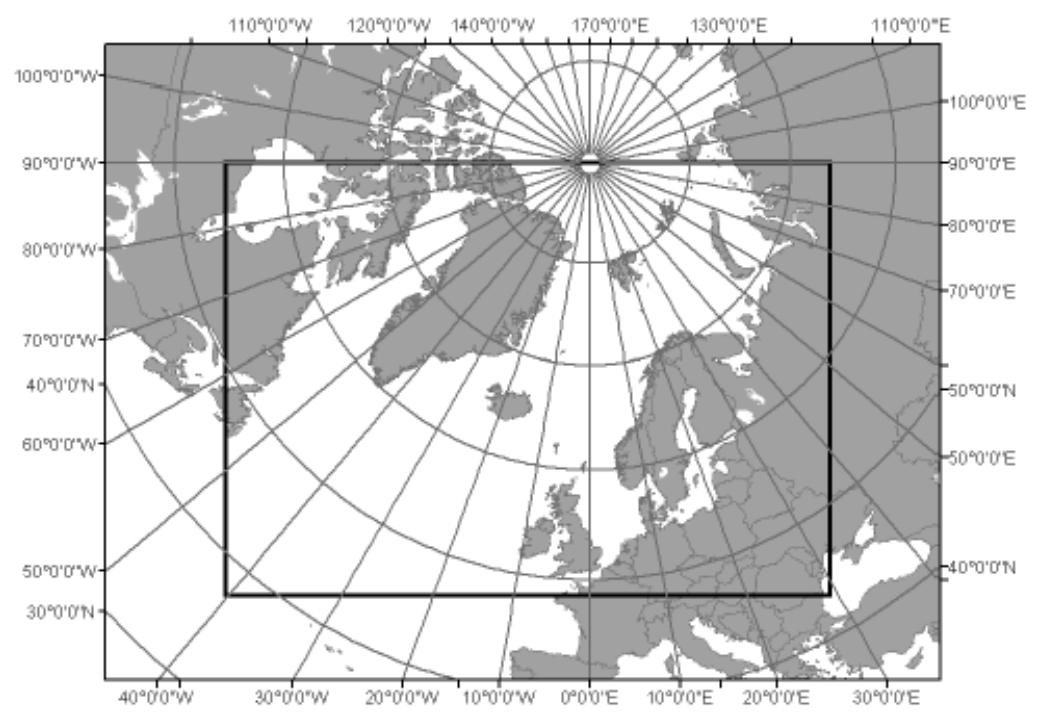

Figure 4: Product coverage of the AHL OSI SAF 12 hourly SST products (marked by the black rectangle).

\section{New SST products at EUMETSAT}

The IASI SST L2Pcore product contains the following fields following the GDS V2.0 specification: sea_surface_temperature, sst_dtime, sses_bias, sses_standard_deviation, I2p_flags, quality_level, wind_speed, satellite_zenith_angle, and solar_zenith_angle. The files are provided in netCDF4 format. The IASI L2Pcore is a swath product available in near-real time, and the resolution of the IASI IFOV is 0.01465 radians [13].

The IASI L2 PPF underwent an operational upgrade to version 5 in September 2010. Although, there were no changes to the retrieval of SST in this version, there were changes to the cloud detection and characterisation methods in the IASI PPF. Updates were made to the NWP cloud test, the AVHRR cloud fraction and the optical thickness test. Further upgrades to the cloud detection are planned for the second half of 2011 to include a new cloud detection method based on artificial neural networks. Further possible developments influencing the SST retrieval over the next few years [T. August, personal communication] include: improvements to address the slight angular dependency; the inclusion of band 3 (shorter wavelengths) in the retrieval at night-time; the use of OSI-SAF sea-ice edge information; and the detection of dust layers for flagging and possibly correction.

The SSES scheme developed for IASI SST observations uses integrated water vapour derived from the IASI water vapour profiles contained in the IASI L2 PPF. Further information on the method can be found in [14].

IASI SSTs are routinely compared to drifting buoy SSTs in order to compute bias statistics and to derive the SSES [14]. The OSI-SAF Metop/AVHRR matchup database is collocated with the IASI SSTs to produce a multi-matchup dataset of Metop AVHRR, IASI and drifting buoy SST observations. IASI SST minus buoy SST statistics for October 2010 to April 2011 covering quality levels 2 to 5 , give a bias of $-0.30 \mathrm{~K}$ and a standard deviation of $0.30 \mathrm{~K}$, for 509 collocations. These are 3sigma night-time statistics, with quality control performed [15], where the IASI skin SSTs have been converted to a subskin SST by the method described in [16]. The global standard deviation of errors, calculated using the method presented in [17] are 0.26K (IASI), 0.14K (AVHRR), and $0.19 \mathrm{~K}$ (drifting buoys). The bias and standard deviation of IASI SSTs compared to drifting buoys (at night-time) has reduced by $0.1 \mathrm{~K}$ compared to the 
results presented in [15], due to the upgrades to the IASI L2 PPF in September 2010. The overall global IASI SST uncertainty has decreased from
$0.34 \mathrm{~K}$ to $0.26 \mathrm{~K}$ over two consecutive 6 monthly periods, centred on the PPF upgrade.
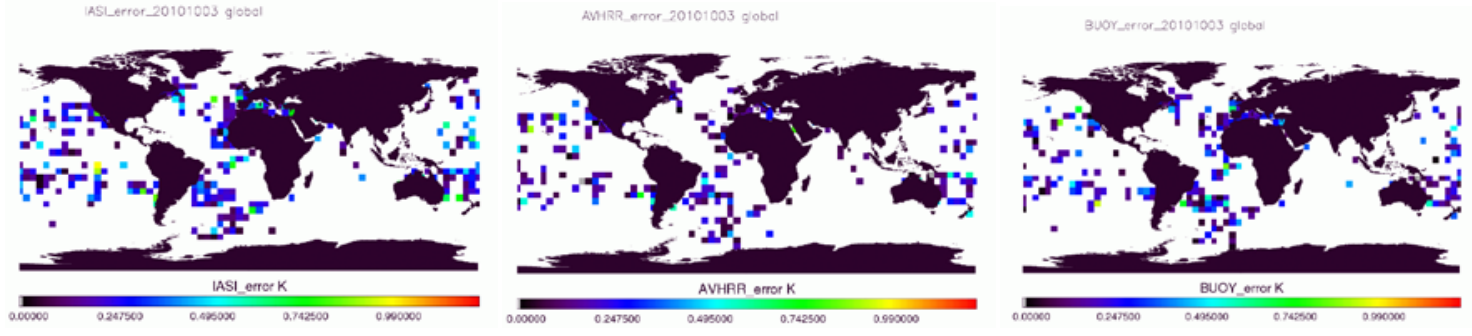

Figure 5. Standard deviation of error averaged in 5-degree boxes for IASI, AVHRR, and drifting buoy SSTs.

Figure 5 shows SST uncertainties, gridded in 5-degree boxes, for IASI, AVHRR and drifting buoy SST observations, derived from examining their couplet difference standard deviations and applying the method in [17]. These figures are using night-time observations, but with no extra quality control checks, in order to retain enough global spread. The global average uncertainties for this sample (with no 3sigma statistics to remove outliers) are $0.34 \mathrm{~K}$ (IASI), 0.15K (AVHRR), and 0.41K (drifting buoys). The larger buoy error is influenced by anomalous buoy observations as shown by high uncertainties in figure 5. Comparisons of the AVHRR and IASI maps, shows that IASI brings benefit in different regions to AVHRR SST observations and vice versa, therefore the two observation sources are complementary to each other.

SST from IASI is a long-term and stable contribution to the GHRSST array of products, due to the committed continuity of this instrument upon the Metop series of satellites up to 2023, and to the infrared sounding instruments planned for Post-EPS and MTG-S enabling data coverage up to the early 2030's and beyond. IASI SSTs provide an additional source of observations to the GHRSST range of products. The use of the additional IASI SSTs within level-4 analyses could benefit error characterisation, where the strengths of different instrument observations can be fully utilised. In addition, SST retrieved from IASI has the potential for improved atmospheric correction over other sensors using commonly used multi-spectral techniques.

\section{Conclusion}

New SST products have been defined for the OSI SAF operational production, offering distinct products per satellite platform and a better resolution and time sampling compared to the present ones. They have been produced and validated for several months and should be declared officially operational in June 2011. For more information, see http://www.osi-saf.org.

IASI L2Pcore SSTs have been produced as a demonstrational product at EUMETSAT since March 2011, and are available through ftp from the EUMETSAT EO portal:

http://www.eumetsat.int/Home/Main/DataP roducts/ProductNavigator/index.htm?l=en. The IASI SSTs have been validated to have a cool bias with respect to drifting buoys with a global mean difference of $0.3 \mathrm{~K}$ and standard deviation of $0.3 \mathrm{~K}$. Analyses of the global distribution of differences between IASI, AVHRR, and drifting buoys show that IASI and AVHRR SSTs are complementary observations to each other, often giving benefit in different regions.

\section{References}

EUMETSAT, 2006, Atlantic Sea Surface Temperature Product Manual, SAF/OSI/M-F/ TEC/MA/121, http://www.osi-saf.org 
GHRSST XII -

Proceedings, Edinburgh

GHRSST Project Office

Eastwood, S., P. Le Borgne, S. Péré and D. Poulter, 2011, Diurnal variability in Sea Surface Temperature in the Arctic, Accepted, Rem. Sens. Env.

Le Borgne, P., S. Péré, S. Eastwood, D. Poulter, and J.F. Piollé, 2010, Diurnal variability of Sea Surface Temperature at high latitudes, EUMETSAT conference, Cordoba, Spain.

IASI Level 2 Product Processing Facility, EUM.MET.REP.07.0224, http://www.eumetsat.int/Home/Main/Da taProducts/Resources/index.htm?|=en

IASI Level 2 Product Guide, EUM.OPSEPS.MAN.04.0033,

http://www.eumetsat.int/Home/Main/Da taProducts/Resources/index.htm?|=en

IASI L2 Surface Temperature: PPF v5 Validation

Results,

EUM.MET.TEN.10.0188, http://www.eumetsat.int/Home/Main/Da taProducts/Resources/index.htm?|=en

EUMETSAT, 2011A, Geostationary Sea Surface Temperature Product User Manual, http://www.osi-saf.org

GHRSST, 2010, GDS 2.0 Technical specifications, https://www.ghrsst.org

EUMETSAT, 2011B, SST retrieval from MSG/SEVIRI and GOES-E operational SST retrieval upgraded chain: validation report., http://www.osi-saf.org

Le Borgne, P., Roquet, $H$. and C.J. Merchant, 2011, Estimation of sea surface temperature from the Spinning Enhanced Visible and Infrared Imager, improved using numerical weather
Issue: Final

Date: September 27, 2011

prediction, Rem. Sens. Env., 115, 5566, DOI: 10.1016/j.rse.2010.08.004, 2011.

EUMETSAT, 2011C, Atlantic High Latitude L3 SST Product User Manual, http://www.osi-saf.org

EUMETSAT, 2011D, Validation Report for the Atlantic High Latitude L3 Sea Surface Temperature product, http://www.osi-saf.org

IASI Level2 Product Format Specification, EPS.MIS.SPE.980760, http://www.eumetsat.int/Home/Main/Da taProducts/Resources/index.htm?|=en

Single Sensor Error Statistics Scheme for IASI L2P sea surface temperature, EUM.MET.DOC.11.0142, http://www.eumetsat.int/Home/Main/Da taProducts/Resources/index.htm?|=en

Validation of IASI L2Pcore sea surface temperature, EUM.MET.DOC.10.0472, http://www.eumetsat.int/Home/Main/Da taProducts/Resources/index.htm?|=en

Donlon, C.J., P.J. Minnett, C. Gentemann, T.J. Nightingale, I.J. Barton, B. Ward, M.J. Murray, 2002: Toward improved validation of satellite sea surface skin temperature measurements for climate research, J. Climate, 15, 353-369

O'Carroll, A.G., J.R. Eyre, and R.W. Saunders, 2008: Three-way error analysis between AATSR, AMSR-E, and in situ sea surface temperature observations, J. Atmos. Oceanic. Tech., 25, 1197-1207 


\title{
R13 - REPORT FROM JAPAN (JAXA, JMA AND TOHOKU UNIVERSITY)
}

\author{
Misako Kachi ${ }^{(1)}$, Hiroshi Murakami ${ }^{(1)}$, Keiji Imaoka ${ }^{(1)}$, \\ Shiro Ishizaki ${ }^{(2)}$, Yukio Kurihara ${ }^{(2)}$, Udai Shimada ${ }^{(2)}$, Noriya Yoshioka ${ }^{(2)}$, \\ Hiroshi Kawamura ${ }^{(3)}$, Futoki Sakaida ${ }^{(3)}$, Kotaro Hosoda ${ }^{(3)}$ \\ (1) Japan Aerospace Exploration Agency (JAXA), Tsukuba (Japan), \\ Email : kachi.misako@jaxa.jp \\ (2)Japan Meteorological Agency (JMA), Tokyo (Japan), Email : s ishizaki@met.kishou.go.jp \\ (3)Tohoku University, Sendai (Japan), Email : kamu@ocean.caos.tohoku.ac.jp
}

\begin{abstract}
Recent Japanese activities, including Japan Aerospace Exploration Agency (JAXA), Japan Meteorological Agency (JMA), and Tohoku University are summarized and reported. JAXA operates its GHRSST server to distribute AMSR-E SST product in near-real-time, and is working on update of the GDS format version from 1.6 to 2.0 and addition of other satellite SST product. JAXA is also developing next generation satellite instruments to observe SST. After the 11th GHRSST-PP Science Team Meeting, several satellite SST observations were incorporated to global daily sea surface temperature (MGDSST) analysis. In order to improve MTSAT SST, Meteorological Satellite Center (MSC)/JMA is testing modified processing system for MTSAT SST. Tohoku University continues the generation of New Generation SST for Open Ocean (NGSST-O) since 2003.
\end{abstract}

\section{Introduction}

In this paper, we will report current status of Japanese activities to contribute to the GHRSST community, and their future plans. Three major players in Japan provide report to GHRSST XII.

In Section 2, Japan Aerospace Exploration Agency (JAXA) reported current status of JAXA satellite missions related to SST, and JAXA GHRSST server and its products.

In Section 3, Japan Meteorological Agency (JMA) reported their activities since GHRSST XI.

In Section 4, Tohoku University reported their activity regarding high-spatial (0.05- deg grid) merged SST product (L4) over the Western North Pacific region (13$\left.63^{\circ} \mathrm{N}, 116-166^{\circ} \mathrm{E}\right)$.

\section{Report from JAXA}

\subsection{Introduction}

JAXA develops series of two instruments; optical and microwave imagers.

As optical imagers to observe SST, JAXA developed the Ocean Color and Temperature Scanner (OCTS) onboard the Advanced Earth Observing Satellite (ADEOS) operated from 1996 to 1997, the Global Imager (GLI) onboard the Advanced Earth Observing Satellite-II (ADEOS-II) operated from 2002 to 2003, and is developing the Second generation Global Imager (SGLI), which will be carried by the first generation of the Global Change Observation Mission (GCOM) - Climate (GCOM-C1) scheduled to be launched in Japanese Fiscal Year (JFY) of 2014.

As passive microwave imagers to observe SST, JAXA developed the Advanced Microwave Scanning Radiometer (AMSR) onboard the ADEOS-II, AMSR for EOS (AMSR-E) onboard NASA's EOS Aqua satellite, which has been operating since 2002, and is developing AMSR2, which will be carried the first generation of the GCOM - Water (GCOM-W1) scheduled to be launched in JFY 2011.

C-band $(6.9 \mathrm{GHz})$ channels on AMSR, AMSR-E and AMSR2 are indispensable for retrieving global sea surface temperature and soil moisture. All-weather and frequent measurements enables analyses of rapid changes of SST. Timeproven infrared measurement and 
GHRSST XII -

Proceedings, Edinburgh

GHRSST Project Office

microwave observations are in complementary situation in terms of spatial resolution and error sources. AMSR-E SST data is used to obtain cloud-free high resolution SST products by merging with various infrared sensor data. JAXA has provided AMSR-E brightness temperature (TB) and geophysical parameters including SST to wide user community to serve their interest, such as weather forecast, fisheries, etc.. Since AMSR2 has almost same channel sets to AMSR-E, it is highly expected to overlap and succeed AMSR-E observation record.

JAXA Earth Observation Research Center (EORC) has been operating the JAXA GHRSST server to distribute GLI, AMSR and AMSR-E SST data to GHRSST community. To access the server, please send a request e-mail to ghrsst@jaxa.jp. Current data format is GDS v1.6, but will be updated to GDS v2.0 soon.

\subsection{Current status of JAXA missions}

\subsubsection{AMSR-E on NASA's Aqua satellite}

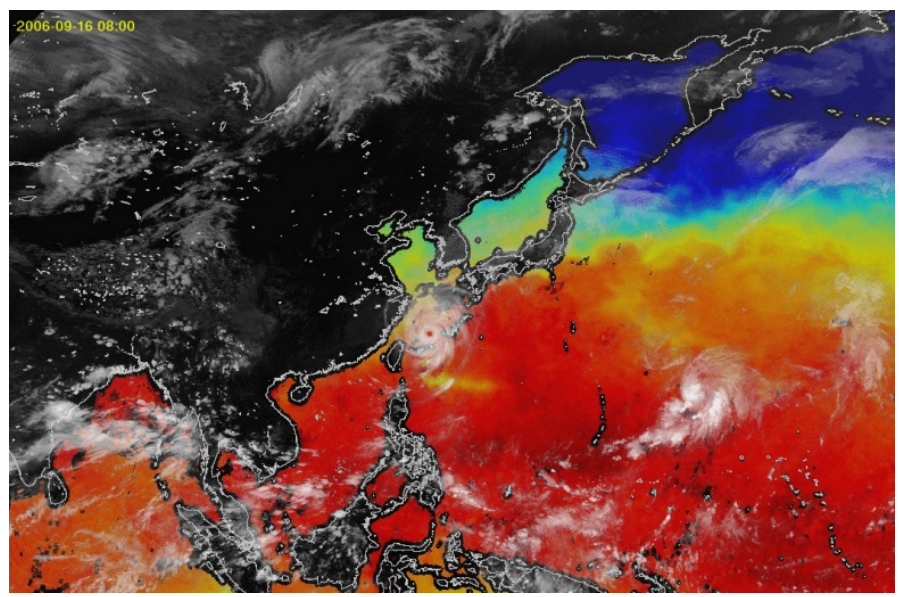

Figure 1. Sea Surface Temperature estimate by AMSR-E for 14-18 September 2006, onto which picture of JMA MTSAT-1 cloud image was superimposed. SST decrease (yellow) along with typhoon track in southwest of Japan is clearly seen.

\subsubsection{Overview of GCOM mission}

GCOM is planned as the comprehensive observation system of the Earth System's essential variables of atmosphere, ocean, land, cryosphere, and ecosystem. Most of these observations are expected to provide data commonly useful to the
Issue: Final

Date: September 27, 2011

AMSR-E still continues its observation over 9-years after the launch on May 4, 2002. Since microwave measurement can estimate SST through clouds, it can provide cloud-through frequent SST mapping compared to SST estimated by Infrared (IR) instruments. AMSR-E can provide information regarding SST under typhoon (Figure 1). As typhoons go over the ocean, their footprints of colder SST are left. This is due to the stirring and upwelling effects caused by the strong winds of typhoon. Microwave technique is capable of observing this kind of phenomenon, which is usually difficult for IR observations due to the extensive coverage of clouds by typhoon.

Minor version up of AMSR-E SST algorithm has been applied to standard product since 17 February 2010. At JAXA GHRSST server, we updated the processing system since 25 March 2010 and provided both new and old version data until 16 April 2010. Current version number is v5.13. 
GHRSST XII -

Proceedings, Edinburgh

GHRSST Project Office

sized satellites; GCOM-W (water) will carry AMSR2, which is being developed based on the experience of the AMSR-E on EOS Aqua satellite; and GCOM-C (climate) will carry SGLI. Three consecutive generations of GCOM satellites with one year overlap in orbit enables over 13 years observation in total. About GCOM missions, see details in (2).

\subsubsection{AMSR2 on GCOM-W1 satellite}

AMSR2 is multi-frequency, total-power microwave radiometer system with dual polarization channels for all frequency bands. The instrument is a successor of AMSR and AMSR-E. The frequency bands include $6.925,7.3,10.65,18.7$, 23.8, 36.5, and 89.0-GHz. The 6.925-GHz band is not primarily allocated for Earth observation, but is defined as the primary band for ground-to-ground and ground-tosatellite radio communications. Due to this, we frequently find radio-frequency interference (RFI) signals in $6.925-\mathrm{GHz}$ brightness temperatures observed by AMSR-E, particularly over land. The 7.3$\mathrm{GHz}$ channels were added to help mitigate RFI influences based on limited observational evidence such as aircraft measurements. Since the 6.925-GHz channels have been successful and promising for retrieving Sea Surface Temperature (SST) over the ocean, we will keep those channels unchanged. RFI identification methods will be investigated by comparing brightness temperatures from those two frequency bands. The $2.0 \mathrm{~m}$ diameter antenna, which is larger than that of AMSR-E $(1.6 \mathrm{~m})$, provides better spatial resolution at the same orbital altitude. Although we have had experience with the 2.0m-diameter dish itself for AMSR on ADEOS-II, a deployable antenna system with such a large aperture has been a new engineering development.

GCOM-W1 will be launched in JFY2011 (end of 2011 or early 2012), and will be placed in orbit at an altitude of about $700 \mathrm{~km}$ and with a local time of ascending node of 1330 . This orbit is desirable for AMSR2 to maintain consistency with AMSR-E. GCOM-W1 will participate in the A-Train satellite constellation. Participating
Issue: Final

Date: September 27, 2011

agencies approved the JAXA proposal that the GCOM-W1 position in the A-Train be in front of the Aqua satellite.

In December 2010, Dr. Shibata Akira is selected as algorithm developer for AMSR2 SST standard product. He is also algorithm developer for AMSR-E SST standard product (see details in (1)). Prelaunch algorithms for AMSR2 were installed to the GCOM-W1 mission operation system in March 2011, and test and evaluation activities are in progress.

JAXA plans to deliver AMSR2 data to operational/research organizations, which implement joint study with JAXA, for calibration/validation purposes about 3month after launch, when initial check out phase is completed. To general researchers, we will distribute AMSR2 data about 1-year after the launch, when calibration/validation phase is completed. Standard products of AMSR2 will be distributed through new GCOM-W1 Data Distribution Service system as well as AMSR-E and AMSR standard products. In addition to capabilities to search, order, and download data in various formats (HDF, netCDF, geoTIFF) through Web site, direct access to HDF data by sftp access is available to registered users.

\subsubsection{SGLI on GCOM-C1 satellite}

SGLI is a versatile, general purpose optical and infrared radiometer system covering the wavelength region from near ultraviolet to infrared. SGLI system consists of two components; SGLI-VNR (Visible \& Near infrared push-broom Radiometer); and SGLI-IRS (shortwave \& thermal InfraRed Scanner) to optimize optics for each wavelength range. Two major new features are added to SGLI, they are $250 \mathrm{~m}$ spatial resolution for 11 channels and polarization/multidirectional observation capabilities. The $250 \mathrm{~m}$ spatial resolution will provide enhanced observation capability over land and coastal areas where the influences of human activity are most obvious. The polarization and multidirectional observations will enable us to retrieve aerosol information over land. Precise observation of global aerosols is a key for 
GHRSST XII -

Proceedings, Edinburgh

GHRSST Project Office

improving climate-prediction models. Although GLI and the Moderate Resolution Imaging Spectroradiometer (MODIS) are also equipped with $250 \mathrm{~m}$ spatial resolution channels, the number of channels is limited to six and two for GLI and MODIS, respectively. Also, this is the first opportunity for the medium resolution wide-swath spectrometers to have the polarization/multidirectional observing capabilities.

The $250 \mathrm{~m}$ resolution data of SGLI-VNR will enable to detect more fine structure in the coastal area such as river outflows, regional blooms, and small currents SST and ocean color products derived from SGLI will provide additional information to AMSR2 SST.

\subsection{Current status of JAXA GHRSST server}

Issue: Final

Date: September 27, 2011

\subsubsection{Transfer to GDS2.0}

JAXA GHRSST server currently distributes AMSR-E near-real-time L2P data in GDS v1.6 format following instruction by GHRSST Data Processing Specification.

Since autumn 2010, we have been working on update of GDS format version to v2.0 following GHRSST instructions. Some new fields are introduced in GDS v2.0 format. For AMSR-E SST data, we will chose following data sources shown in Table 2. For GDS v2.0 format, we will distribute AMSR-E SST data both in L2P and L3C formats. Modification of program is almost completed, but we still need to apply it to automatic processing system. We will start to distribute new format data for AMSR-E SST in near future.

Table 1: List of new fields for GDS v2.0 and data source for AMSR-E SST

\begin{tabular}{|l|l|}
\hline field & data source \\
\hline dt_analysis & OISST provided by NCDC \\
\hline wind_speed & AMSR-E L2 Sea Surface Wind Speed product at same orbit \\
\hline sea_ice_fraction & AMSR-E L2 Sea Ice Concentration product at same orbit \\
\hline
\end{tabular}

\subsubsection{Addition of VIRS SST}

Upon request from GHRSST IX science team meeting, we're also working on addition of Visible Infrared Scanner (VIRS) SST products on JAXA GHRSST server with GDS format. VIRS is visible infrared imager, which has been carried by the Tropical Rainfall Measuring Mission (TRMM) satellite since 1997. Currently, VIRS SST data from December 1997 to

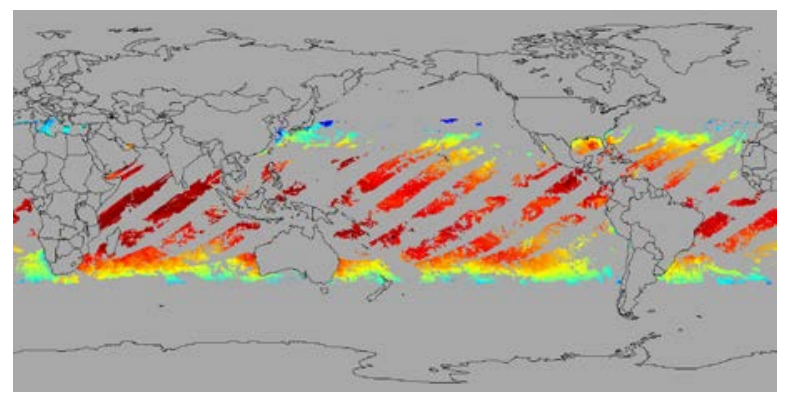

present is produced and distributed in plain binary format through JAXA's TRMM ftp server.

As same as AMSR-E SST, VIRS SST L2P and L3C data in GDS v2.0 format will be processed in near-real-time (3-to-6 hours after observation), and distributed through JAXA GHRSST server in near future. Figure 3 is sample images of VIRS SST for L3C format.

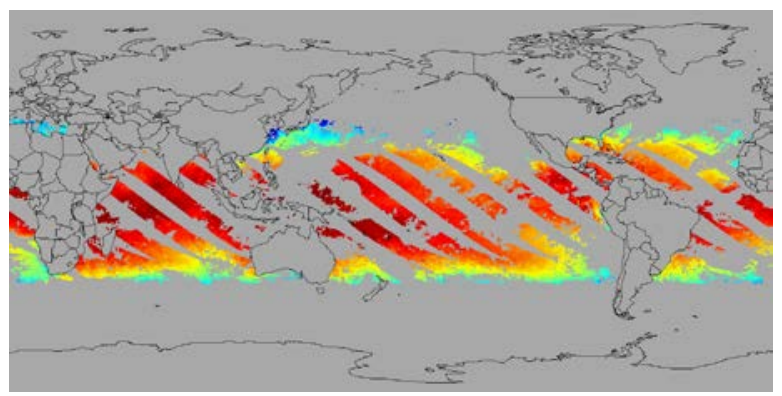

Figure 2. Sample images of VIRS SST L3C data for ascending orbits (left panel) and for descending orbits (right panel).

\subsection{Activities and Plan for $\mathbf{2 0 1 1 - 2 0 1 6}$}

Currently, we're planning following activities during 2011 and 2016 as shown in Table 2. 
Table 2: List of JAXA activities and plans

\begin{tabular}{|l|l|}
\hline Year & Activities and plans \\
\hline 2011 & $\begin{array}{l}\text { Release of AMSR-E SST in GDS v2.0 format. } \\
\text { Addition of VIRS SST to JAXA GHRST server. } \\
\text { Start new GCOM-W1 Data Distribution Service for test operation (AMSR-E } \\
\text { and AMSR standard products are available). }\end{array}$ \\
\hline $\begin{array}{l}\text { Late 2011 or } \\
\text { Early 2012 }\end{array}$ & $\begin{array}{l}\text { Launch of GCOM-W1 satellite. } \\
\text { Release of AMSR2 products to collaborative organizations (L+3month) and } \\
\text { Update of AMSR2 SST algorithm and apply it to AMSR-E data to produce } \\
\text { continuous data set. } \\
\text { Addition of AMSR2 SST to JAXA GHRSST server. }\end{array}$ \\
\hline 2012 & $\begin{array}{l}\text { Launch of Global Precipitation Measurement (GPM) core satellite. } \\
\text { Consideration of extension of AMSR2 SST algorithm to other satellite } \\
\text { microwave imagers (TBD). }\end{array}$ \\
\hline 2014 & Launch of GCOM-C1 satellite (TBD). \\
\hline 2015 & $\begin{array}{l}\text { Release of SGLI data products (TBD). } \\
\text { Addition of SGLI SST to JAXA GHRSST server (TBD). }\end{array}$ \\
\hline 2016 or later & Launch of GCOM-W2 satellite (TBD). \\
\hline
\end{tabular}

\section{Report from JMA}

\subsection{Introduction}

Office of Marine Prediction (OMP)/ JMA developed a SST analysis system to generate global daily SST data (Merged satellite and in-situ data Global Daily Sea Surface Temperature: MGDSST) in 2004. This SST analysis system produces $1 / 4^{\circ}$ resolution, daily global SST analysis, using both satellite and in-situ SST observation. As an analysis scheme, the MGDSST analysis adopts optimal interpolation (OI) method which considered not only spatial correlation but also temporal correlation. JMA started to implement operational (real-time) analysis of the MGDSST in 2005 using GAC AVHRR SST (NOAA-15 and NOAA16) provided by NOAA, and AQUA/AMSR-E SST by JAXA. By 03UTC each day, the operational analysis of the previous day's (real-time analysis) is available through the NEAR-GOOS Regional Real Time Data Base (RRTDB: http://goos.kishou.go.jp/ registration is required prior to use). The MGDSST analysis contributes to the GHRSST MultiProduct Ensemble (GMPE) median SST.
The MGDSST is used for the regional ocean data assimilation system (Multivariate Ocean Variational Estimation system / Meteorological Research Institute Community Ocean Model for the Western North Pacific: MOVE/MRI.COM-WNP ; see details in (3)), in which the MGDSST is used as observation data. MOVE/MRI.COM-WNP well reproduces the ocean states in the seas around Japan and provides better prediction of current and temperature field for one month. The MGDSST is also used as a lower boundary condition in the numerical weather prediction models.

Because the Ol method applied in the MGDSST analysis considers temporal correlation, this method requires the observation data after the target day in order to produce the more appropriate analysis. On the other hand, long term, consistent time series of the SST analysis is needed for climate research. For these reason, OMP/JMA implemented reanalysis of the MGDSST from 1985 to 2004 using AVHRR Pathfinder Version 5 SST. After 2005, OMP/JMA reprocesses the observation data to generate the MGDSST data (delayed analysis) in 
GHRSST XII -

Proceedings, Edinburgh

GHRSST Project Office

operation with about 5-month delay using GAC AVHRR SST and AQUA/AMSR-E SST. The MGDSST reproduces global SST field well, although high-frequency SST variation is underestimated (see details in (4)).

After geostationary satellite MTSAT-1R was launched, Meteorological Satellite Center (MSC) /JMA had generated several types of products, including SST, using observation of MTSAT-1R. In 2009, in order to reduce biases of the MTSAT1R SST, MSC and OMP/JMA developed new processing system for MTSAT-1R SST based on a method of Maturi et al., (2008) (see details in (5)). These SST products are included in Monthly Report of Meteorological Satellite Center (CD-ROM; see,

http://mscweb.kishou.go.jp/product/library/ report/index.htm). After MTSAT-2 was in operation, MSC/JMA started to generate SST product using MTSAT-2 observations instead of MTSAT-1R.

\subsection{Current Status of the MGDSST Analysis}

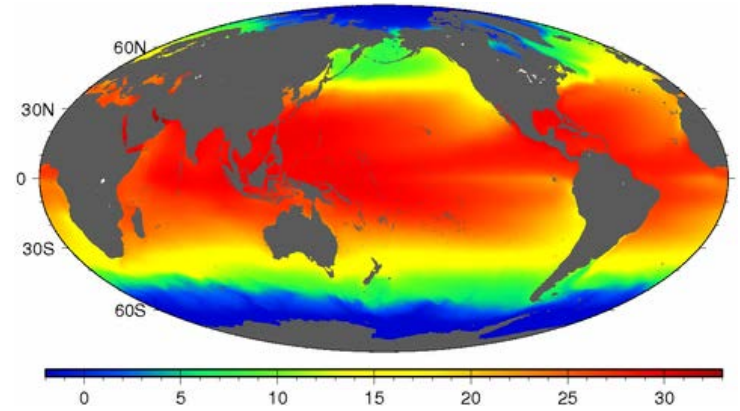

Issue: Final

Date: September 27, 2011

In February 2011, SST observed by NOAA-19/AVHRR (both GAC and HRPT data) was incorporated to the operational (real-time) analysis of MGDSST. On the other hand, NOAA-17/AVHRR SST was excluded from MGDSST analysis since December 2010. Currently, OMP/JMA uses GAC AVHRR SST (NOAA-18, NOAA-19 and MeTop-A) provided by NOAA/NESDIS, HRPT AVHRR SST (NOAA-18 and NOAA-19) locally received by MSC/JMA and AQUA/AMSR-E SST produced by JAXA in order to generate operational MGDSST data.

In May 2011, JMA has developed new climatological normals of atmospheric variables for the period from 1981 to 2010 . At the same time, climatologies of SST have been updated using the normals for the period from 1981 to 2010 instead of one from 1971 to 2000. Since reanalysis of the MGDSST covers the period only after 1985, we took account into another SST analysis (COBE-SST), which is based on in-situ observations, before1985.

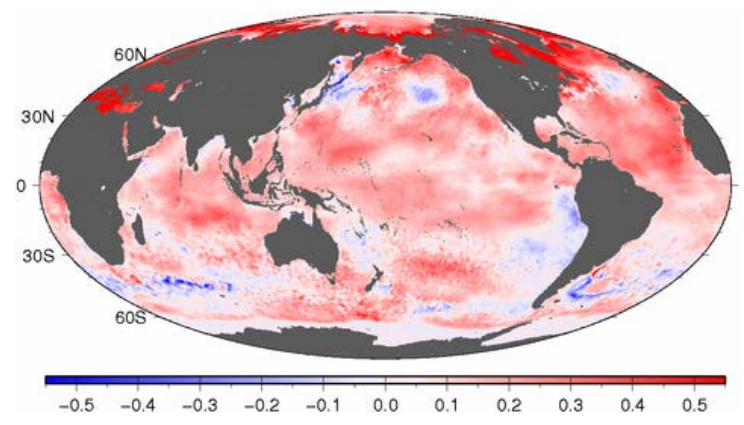

Figure 3. (left panel) New monthly climatologies of MGDSST for July. (right panel) Differences between new and old monthly SST climatologies for July (New - Old).

\subsection{Current Status of the MTSAT SST Product}

SSTs from MTSAT-1R and MTSAT-2 observations show a good performance for monitoring ocean states. But additional efforts to reduce biases are required for incorporating to SST analysis, since the current method produces MTSAT SSTs with large negative biases in the areas where satellite zenith angles are larger than 50 degrees. JMA plans to develop a new method for producing MTSAT SSTS by the end of 2011 . The improvements will be made for the calculation of water vapor absorption and sea surface emissivity and for a cloud screening method. 


\section{GHRSST Project Office}
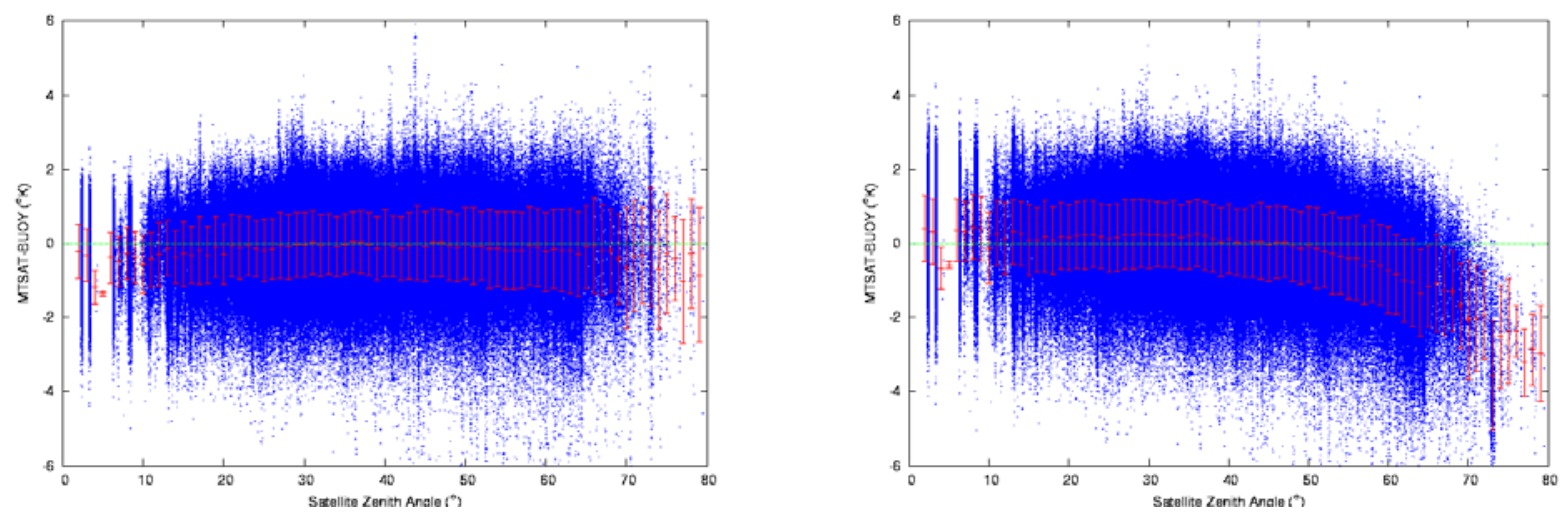

Figure 4. Departures of MTSAT-2 SSTs from BUOY observations as a function of satellite zenith angle. (top panel) for SST produced with new method. (bottom panel) for current SST products.
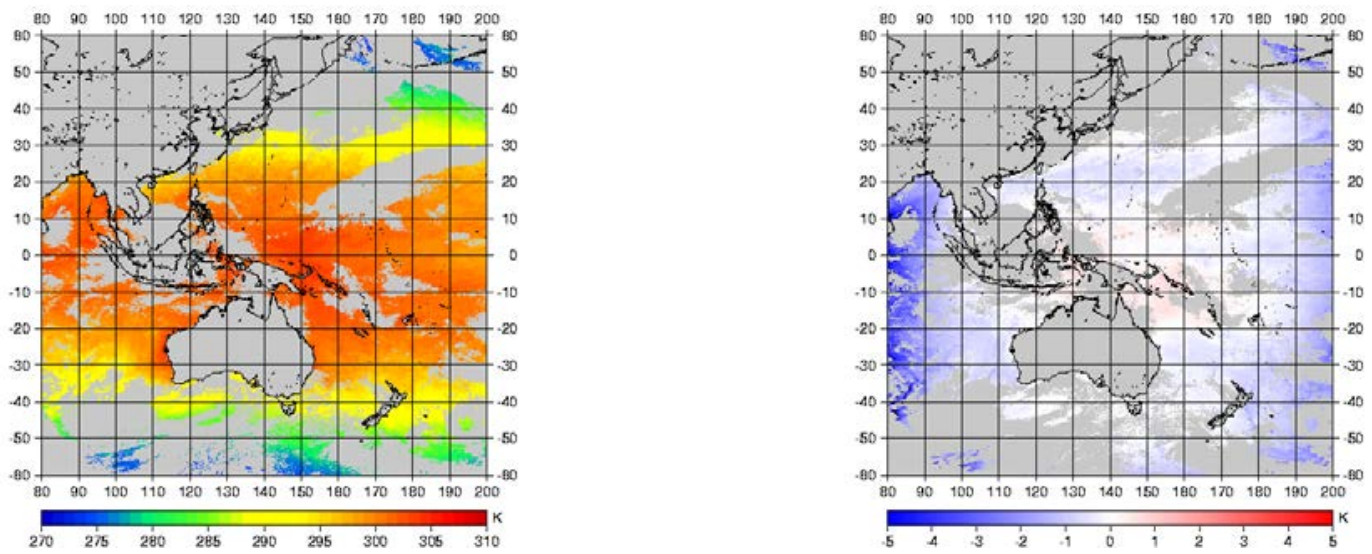

Figure 5. (top panel) Example of daily composite map of the new MTSAT-2 SST for 1 March, 2011 and (bottom panel) its difference from the current MTSAT-2 SST (current minus new).

\subsection{Future Plan}

OMP/JMA is implementing reanalysis of MGDSST for the period from 1981 to 1984 using AVHRR Pathfinder Version 5.2 SST. OMP/JMA will replace climatologies of SST for the period of 1981 to 2010 after the implementation.

Developing a system to create and deliver MGDSST files of NetCDF version based on GDS-2.0 format is one of the issues to be discussed in these years.

The new method which improves SST retrieved from MTSAT observation will be applied to operational systems by the end of 2011. How to incorporate new version of the MTSAT SST into SST analysis will be discussed.

In collaboration with NWP division of JMA, OMP/JMA discusses about a design of a next generation SST analysis system. In this discussion, requirements from SST users (e.g. NWP group), new satellites which will be launched (e.g. GCOM-W2, HIMAWARI-8) and progresses in developing analysis scheme will be considered in order to develop the new analysis system.

\section{Report from Tohoku University}

\subsection{Demonstration Operation of New Generation SST for Open Ocean (NGSST-O)}

Real-time generation and distribution of the New Generation Sea Surface Temperature for Open Ocean (NGSST-O) product began in September 2003 as a demonstration operation of the Global Ocean Data Assimilation Experiment (GODAE) High-Resolution Sea Surface Temperature Pilot Project (see details in (6)). Satellite sea surface temperature (SST) observations from infrared radiometers (AVHRR, MODIS) and a microwave radiometer (AMSR-E) are objectively merged to generate the 
NGSST-O product, which is a qualitycontrolled, cloud-free, high-spatialresolution $\left(0.05^{\circ}\right.$ gridded), widecoverage $\left(13-63^{\circ} \mathrm{N}, 116-166^{\circ} \mathrm{E}\right)$, daily SST digital map. The NGSST-O demonstration operation system has been developed in cooperation with the Japanese Space
Agency (JAXA) and has produced six years of continuous data without gaps. Comparison to in situ SSTs measured by drifting buoys indicates that the root mean-square error of NGSST-O has been kept at approximately $0.9^{\circ} \mathrm{C}$ (Figure 6).

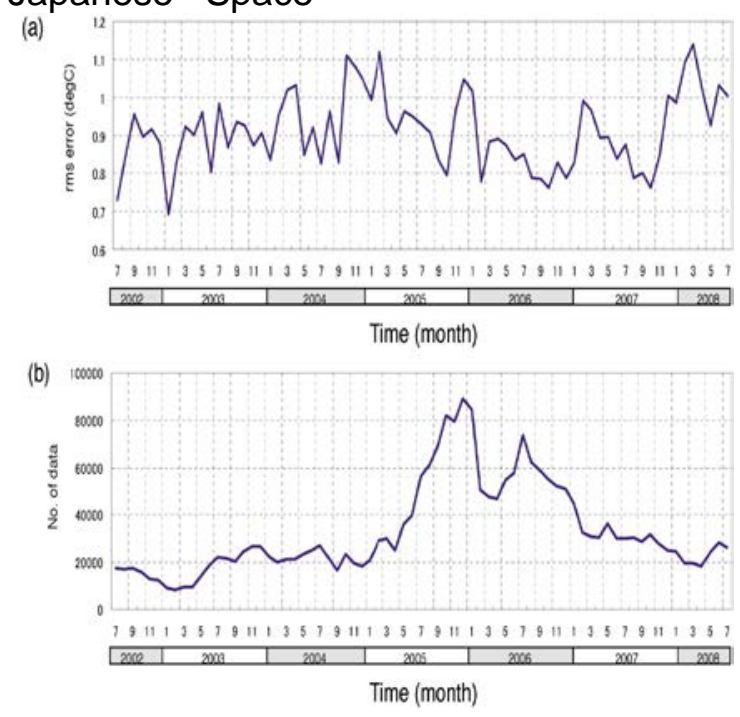

Figure 6. Time series of (a) root mean-square (rms) error and (b) match-up numbers. In situ SSTs measured by drifting buoys were used as reference SSTs. The rms error was calculated every month.

\section{Conclusion}

Activities and plans of JAXA, JMA and Tohoku University are described. They are working closely and sharing information regarding satellite instruments and SST data each other.

JAXA GHRSST server has been operating, and continues updating in order to meet requirements from the GHRSST science team. Satellite-based SST data from future missions such as AMSR2 on GCOM-W1 and SGLI on GCOM-C1 will also be distributed to the community.

JMA incorporated several satellite SST observations to their MGDSST analysis. In order to improve MTSAT SST, they are testing modified processing system for MTSAT SST.

Tohoku University continues the generation of New Generation SST for Open Ocean (NGSST-O) since 2003.

\section{References}

A Shibata, AMSR/AMSR-E SST algorithm development : removal of ocean wind effect", Italian J. Remote Sensing, Vol.30/31, pp131-142, 2004.

K Imaoka, M Kachi, H Fujii, H Murakami, $M$ Hori, A Ono, $\mathrm{T}$ Igarashi, $\mathrm{K}$ Nakagawa, T Oki, Y Honda and $\mathrm{H}$ Shimoda, Global Change Observation Mission (GCOM) for monitoring carbon, water cycles, and climate change", Proc. of the IEEE, Vol.98(5), pp.717734, May2010.

N Usui, S Ishizaki, Y Fujii, $\mathrm{H}$ Tsujino, T Yasuda and $M$ Kamachi, Meteorological Research Institute multivariate ocean variational estimation (MOVE) system : Some early results", Advances in Space Research, Vol.37, pp806-822, 2006.

S Iwasaki, M Kubota and $\mathrm{H}$ Tomita, Inter-comparison and evaluation of global sea surface temperature products", Int. J. Remote Sensing, Vol.29 (21), pp6263-6280, 2008.

E Maturi, A Harris, C Merchant, J Mittaz, R Potash, W Meng and J Sapper, NOAA's Sea Surface Temperature Products from operational Geostationary Satellites", Bull. 
GHRSST XII -

Proceedings, Edinburgh

GHRSST Project Office

American Met. Soc., Vol.90(12), pp1877-1888, Dec2008.

F Sakaida, H Kawamura, S Takahashi, T Shimada, Y Kawai, K Hosoda and $\mathrm{L}$ Guan, Research and development of
Issue: Final

Date: September 27, 2011

the New Generation Sea Surface Temperature for Open Ocean (NGSSTO) product and its demonstration operation", J. Oceanogr., Vol.65(6), pp859-870, 2009. 


\title{
R14 - REPORT FROM THE GLOBAL DATA ASSEMBLY CENTER (GDAC) AND FROM THE DATA ASSEMBLY AND SYSTEMS TECHNICAL ADVISORY GROUP (DAS-TAG)
}

\author{
Edward M. Armstrong (1), Andrew Bingham, Jorge Vazquez, Charles \\ Thompson, Thomas Huang, Chris Finch
}

(1) Jet Propulsion Laboratory, California Institute of Technology, 4800 Oak Grove Dr, Pasadena, CA (USA), Email: Edward.m.armstrong@ipl.nasa.gov

\section{ABSTRACT}

In 2010/2011 the Global Data Assembly Center (GDAC) at NASA's Physical Oceanography Distributed Active Archive Center (PO.DAAC) continued its role as the primary clearinghouse and access node for operational Group for High Resolution Sea Surface Temperature (GHRSST) datastreams, as well as its collaborative role with the NOAA Long Term Stewardship and Reanalysis Facility (LTSRF) for archiving. Here we report on our data management activities and infrastructure improvements since the last science team meeting in June 2010. These include the implementation of all GHRSST datastreams in the new PO.DAAC Data Management and Archive System (DMAS) for more reliable and timely data access. GHRSST dataset metadata are now stored in a new database that has made the maintenance and quality improvement of metadata fields more straightforward. A content management system for a revised suite of PO.DAAC web pages allows dynamic access to a subset of these metadata fields for enhanced dataset description as well as discovery through a faceted search mechanism from the perspective of the user. From the discovery and metadata standpoint the GDAC has also implemented the NASA version of the OpenSearch protocol for searching for GHRSST granules and developed a web service to generate ISO 19115-2 compliant metadata records. Furthermore, the GDAC has continued to implement a new suite of tools and services for GHRSST datastreams including a Level 2 subsetter known as Dataminer, a revised POET Level 3/4 subsetter and visualization tool, a Google Earth interface to selected daily global Level 2 and Level 4 data, and experimented with a THREDDS catalog of GHRSST data collections. Finally we will summarize the expanding user and data statistics, and other metrics that we have collected over the last year demonstrating the broad user community and applications that the GHRSST project continues to serve via the GDAC distribution mechanisms. This report also serves by extension to summarize the activities of the GHRSST Data Assembly and Systems Technical Advisory Group (DAS-TAG).

\section{Introduction}

The GDAC serves as the key operational component for access and utility of GHRSST data products worldwide. Its primary mission is to ensure timely and transparent access to GHRSST datasets using a number of access protocols including FTP and OPeNDAP.

In this report we first describe key new improvements to the overall GDAC architecture. This includes implementation of the new PO.DAAC DMAS (data management and archiving system) that was reported on at the last meeting. Further sections are devoted to new products, and the development of tools and services for GHRSST dataset subsetting and access. The metadata section documents how the GDAC has been actively improving metadata and fostering discovery of GHRSST products, and helping to guide the development of an ISO-based metadata model. The last section details the GHRSST data usage statistics since 2010.

2. GDAC integration and evolution 
GHRSST XII -

Proceedings, Edinburgh

GHRSST Project Office

The original GDAC data interfaces to GHRSST data producers, data consumers and data archive (LTSRF) were designed and implemented over 6 years ago, and as reported at the last Science Team meeting, a new PO.DAAC data management architecture, DMAS has now been implemented for all GHRSST data streams. This new architecture has several improvements including scalability to handle increasing volumes of data ingest and dissemination.

In addition to aforementioned ingest and dissemination capabilities, further DMAS
Issue: Final

Date: September 27, 2011

functions included metadata registry into an upgraded Master Metadata Repository (MMR) in an Oracle database in conjunction with its web-based search and discover interface, FGDC metadata generation and implementation of the NODC interfaces for GHRSST data transfer for archiving, ingest data latency tracking and distribution metric capturing, and other enhanced operator functions. DMAS also assumes data management roles of the MODIS L2P RDAC including L2P ancillary filling. As shown in Figure 1, DMAS is a multi-mission data system that offers data ingestion, validation, catalog, archive, and distribution capabilities. All GHRSST data have being handled operationally by DMAS since June 2009.

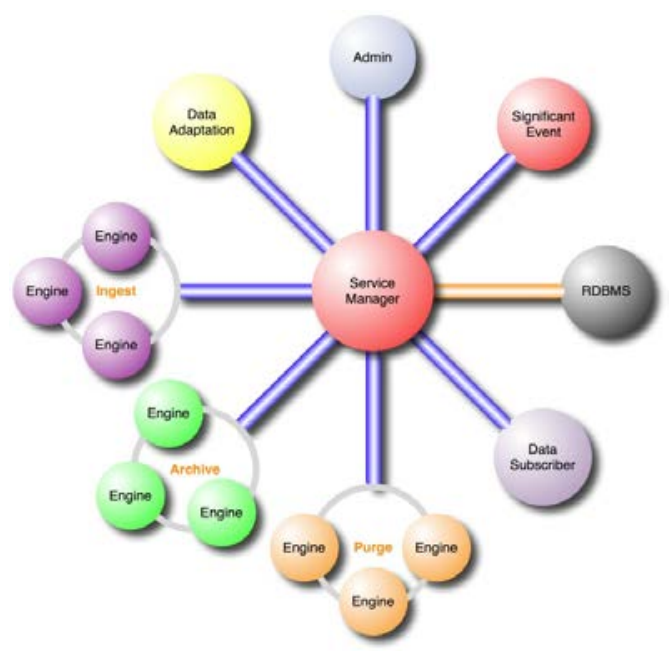

Figure 3. The top-level DMAS system architecture view.

\section{New Products}

The GDAC has continued to support the ingestion of new $\mathrm{L} 2 \mathrm{P}$ and $\mathrm{L} 4$ products. These include:

- A global version of the Level 4 MEaSURES Multisensor (MUR) $1 \mathrm{~km}$ dataset

- GOES-13 L2P

- MTSAT2 L2P

Forthcoming products:

- Windsat L2P

- Global Level 4 DMI_OI

\section{Tools and Services}

The PO.DAAC has made operational the existing beta version of the Dataminer Level 2 subsetter (Figure 2, http://podaactools.jpl.nasa.gov/dataminer/aegina/src/da taminer.php). This technology is an adaption of the Ifremer NAIAD (Enhanced Satellite Archive Dataminer) tool described in detail in last years report. The core of the NAIAD system is the "virtual tile" database whereby each swath data granule is tiled, or divided into regions (typically representing $500 \mathrm{~km} \times 500 \mathrm{~km}$ ). The spatial and temporal metadata associated with each data region is stored inside of a tile, as well as that region's statistical properties. Using this approach 
a user can quickly perform a space/time query and download only the granules meeting the search criteria via OPeNDAP connections to either the GDAC or LTSRF. Currently AMSRE, and Aqua and Terra MODIS L2P granules are accessible via Dataminer for subsetting.

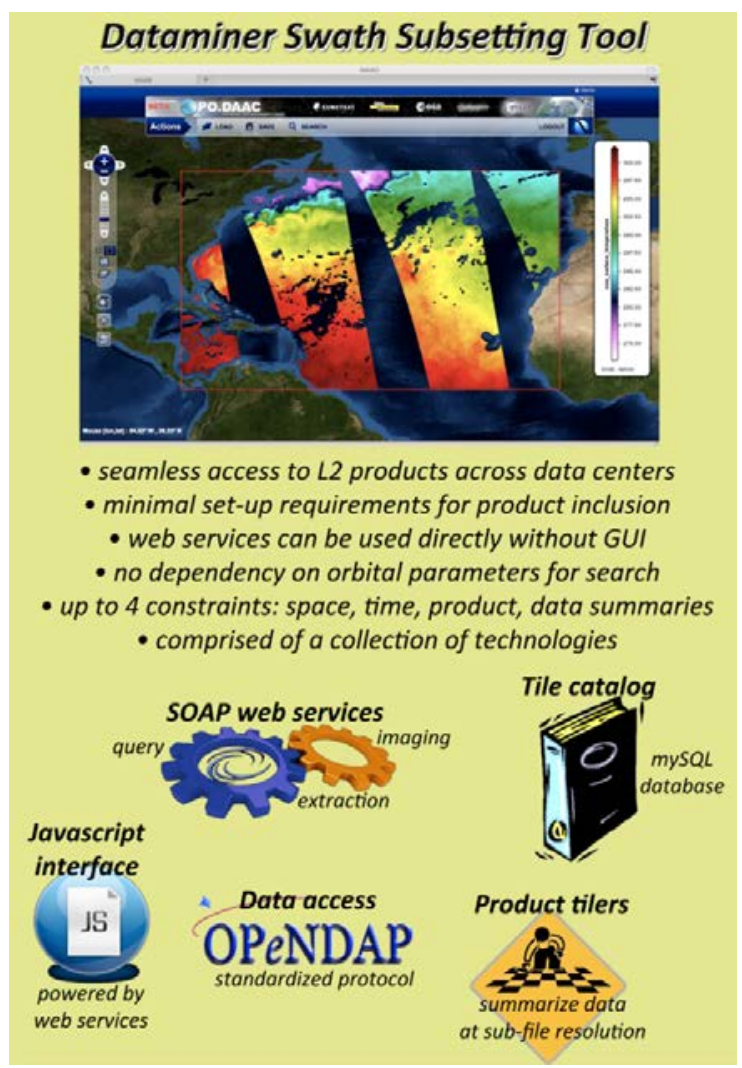

Figure 2. The features and components of the NAIAD/Dataminer system.

A Google Earth-based interface called State Of The Ocean (SOTO) has been implemented as a core visualization tool for the physical disciplines supported by the PO.DAAC including sea surface temperature. As shown in Figure 3, a user can globally visualize SST fields from the previous five days using GHRSST MODIS L2P, AMSRE L2P, or G1SST L4 or some combination thereof including SST anomaly data. Other parameters including wind, SSH and ocean chlorophyll are also available. No specialized software other than a web browser and the Google Earth plug-in is required to run this system. SOTO can be accessed from:

http://podaac-tools.jpl.nasa.gov/soto/

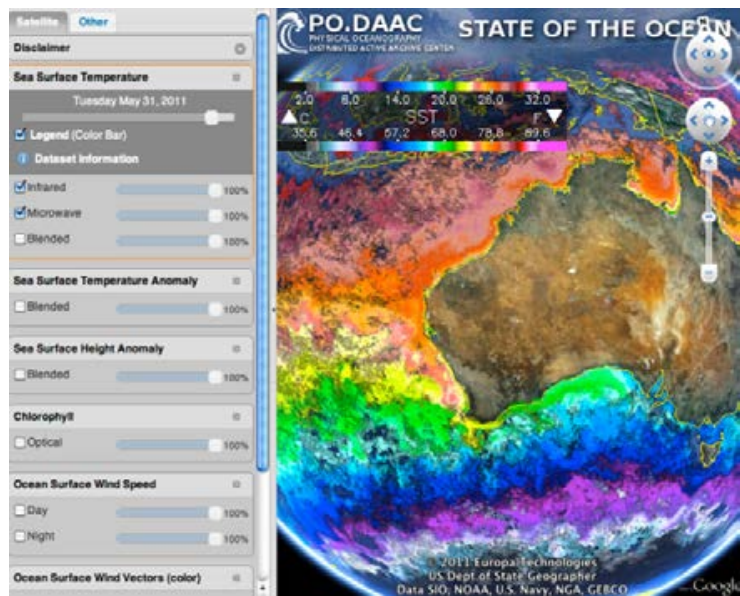

Figure 3. The SOTO Google Earth interface for GHRSST SST (and others).

The POET Level 3/4 subsetter and visualization tool has also received an upgrade to the user interface and has improved speed and functionality. It can be found at http://newpoet.jpl.nasa.gov . A number of GHRSST global Level 4 products are accessible via POET.

PO.DAAC has also experimented with an implementation of a THREDDS (Thematic Realtime Environmental Distributed Data Services) server, and will eventually deploy a version to aggregate GHRSST datasets into yearly and annual catalogs.

A GHRSST specific user forum has been established and will be managed at the PO.DAAC

(http://podaac.jpl.nasa.gov/forum/forum/4). This forum will allow a single location for future collaborations among science team members, technical advisory groups and eventually even users.

\section{Metadata and Discovery}

In the area of data discovery and metadata the GDAC has made significant progress as part of new strategies adopted in the PO.DAAC DMAS infrastructure. First, GHRSST datasets are now discoverable via a faceted search mechanism directly from the PO.DAAC website. An example of this interface is shown in Figure 4, where one option is to browse the entire "collection" of GHRSST datasets. Users are presented with a metadata summary page for each GHRSST dataset including an interface to access individual granules. Examples of 
GHRSST XII -

Proceedings, Edinburgh

GHRSST Project Office

other facets that can be browsed are "sensor", "platform" and "resolution." This interface is extendable and can be modified to suit the requirements and preferences of the user community.

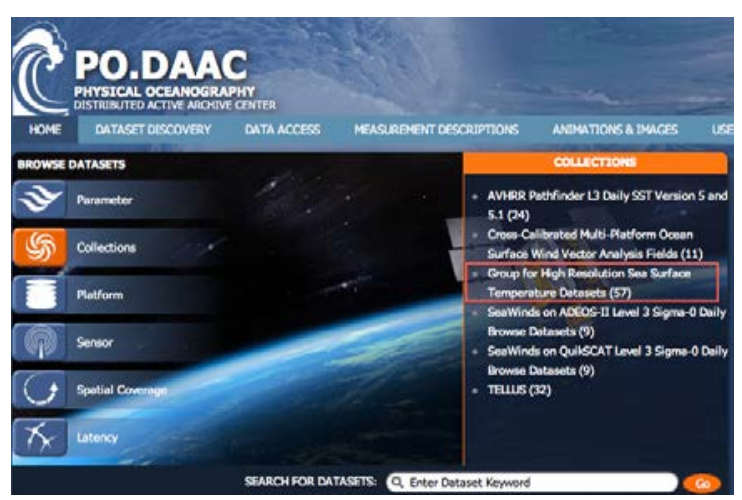

Figure 4. Faceted search capability for all PO.DAAC datasets including GHRSST. In this example the entire "collection" of metadata of all 57 GHRSST datasets will be browsed.

For dataset and granule discovery web services, the PO.DAAC has implemented a new data discover system: the Oceanographic Common Search Interface (OCSI) (Figure 5). In addition to serving as the backend infrastructure of PO.DAAC's faceted web search capability, OCSI is designed to support discovery of PO.DAAC data according to various metadata standards. Currently the system supports the ESIP Discovery Specification, the NASA extensions of the standard OpenSearch protocol (http://www.opensearch.org). This protocol specifies a way to discover data and return XML (atom/rss) structured search results based on a pre-defined user query. Initial search constraints are limited to keyword, space/time queries but can be eventually extended to other attributes one richer metadata are indexed at the granule level. OCSI has also implemented prototype support of ISO 19115-2 metadata records for GHRSST datasets following the metadata model specifications in GDS 2 (Figure 6).
Issue: Final

Date: September 27, 2011

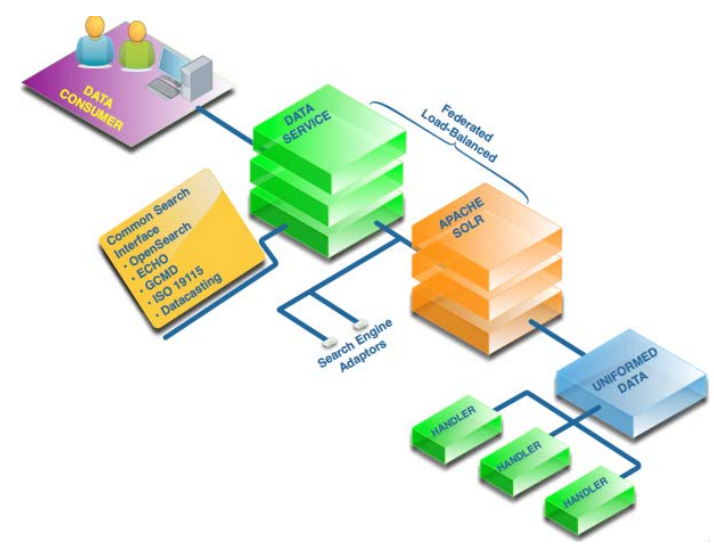

Figure 5. The web services of the Oceanographic Common Search Interface (OCSI) in yellow that interact with the PO.DAAC dataset inventory.

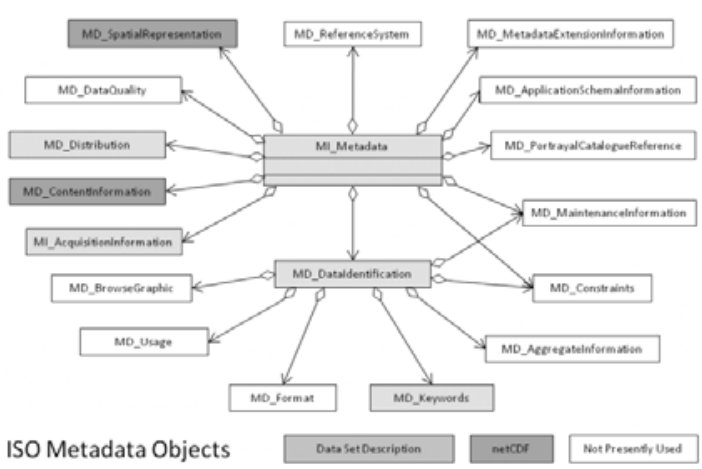

Figure 6. ISO 19115-2 objects for the GDS 2.0 metadata specification

From the perspective of the dataset metadata, the PO.DAAC has embarked on a major metadata quality improvement project. This effort is focused on improving the accuracy and completeness of the metadata attributes including the quality of the dataset description or abstract. A database interface and maintenance tool developed by the PO.DAAC makes this task much more straightforward and ensures consistency across all GHRSST datasets. Once this quality effort is complete by Fall 2011 all GHRSST datasets will be exported in the Directory Interchange Format (using OCSI) to the NASA Global Change Master Directory. This should significantly enhance the exposure of GHRSST 
products to a broader community of potential users.

The PO.DAAC continues to be active in providing GHRSST metadata to the NASA's Earth Observing System Clearinghouse (ECHO), a metadata search interface to all NASA earth science data holdings at the granule level. Currently, ECHO contains 32 GHRSST data sets with more than 270,000 granules. These data sets and granules are available for search through the new ECHO Reverb interface.

\section{GDAC data metrics}

The following figures (Figure 7 and 8) are representative summaries for the data volume (compressed) and number of users of GHRSST data from the GDAC since early 2006. One metric to note is that the GDAC has distributed 30 million granules representing about 50 TB of compressed volume since June 2010 (around 4.0 TB/month). More enhanced statistics will be reported at the June 2011 Science Team meeting.
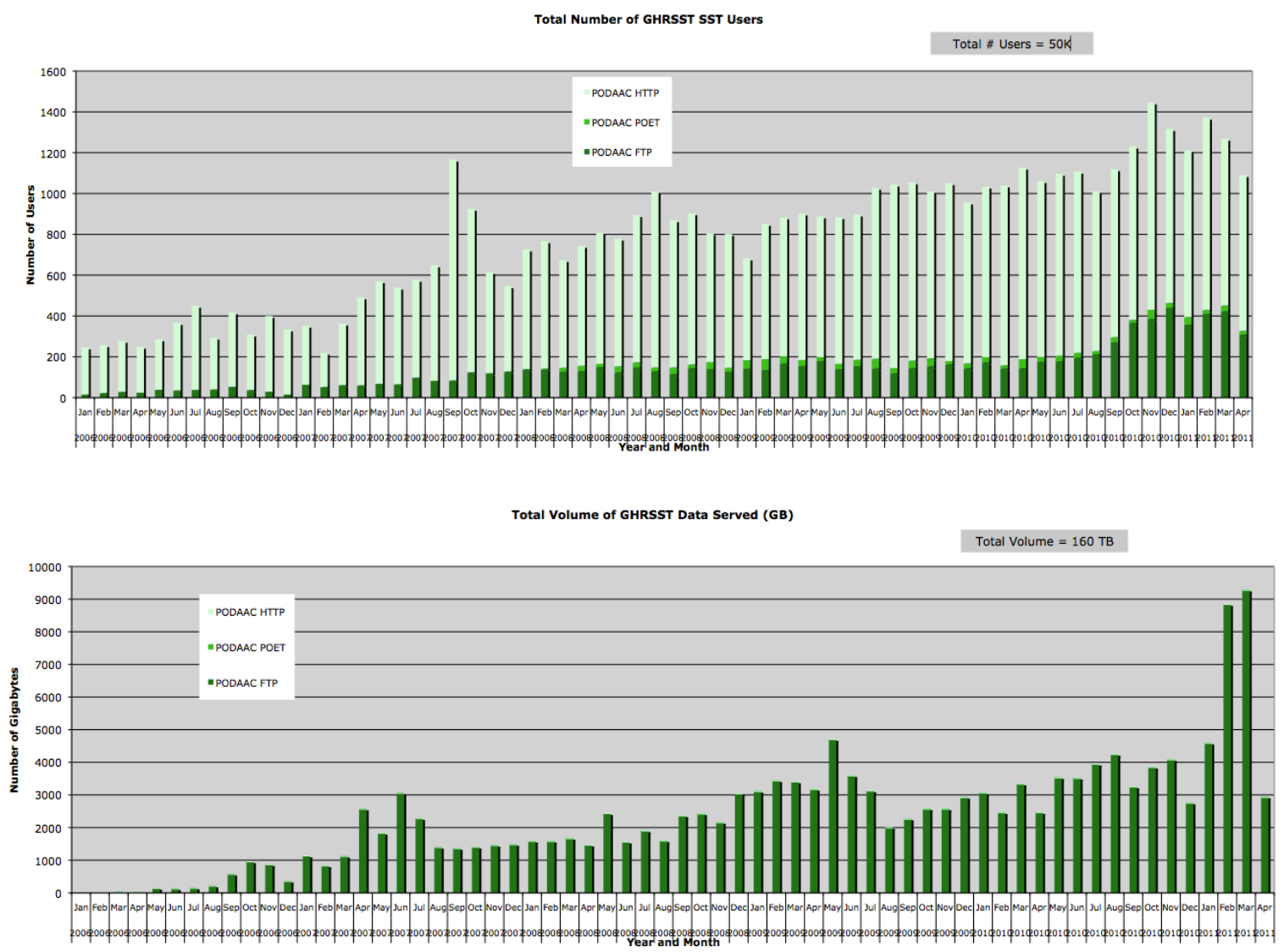

Figures 7 and 8. GDAC user and data distribution (compressed volume) summaries.

\section{Conclusion}

The Global Data Assembly Center (GDAC) continues to meet its requirements to distribute increasing numbers of GHRSST datasets and volumes, foster data discovery, maintain meaningful metadata records, implement robust data stewardship practices, and build new data utilization tools and services. GHRSST datastreams can now leverage off an improved and scalable data management system that has recently be put into place at the PO.DAAC as well as new subsetting, visualization, data discovery, web services and metadata tools. NASA has recognized the importance of GHRSST data (with the recent 2011 NASA Physical Oceanography proposal call emphasizing these products) while supporting the concept that leading edge research cannot be fostered without strong data management principles and infrastructure. The GDAC is committed to maintaining 
GHRSST XII -

Proceedings, Edinburgh

GHRSST Project Office

GHRSST data for all users in conjunction with the NOAA Longterm Stewardship and Reanalysis Facility well into the future.

This work was carried out at the Jet Propulsion Laboratory, California Institute of Technology, under a contract with the
Issue: Final

Date: September 27, 2011

National Aeronautics and Space Administration.

(C) 2011 California Institute of Technology. Government sponsorship acknowledged. 


\title{
R15 - REPORT FROM THE REANALYSIS TECHNICAL ADVISORY GROUP (RAN-TAG) AND THE LONG TERM STEWARDSHIP AND REANALYSIS FACILITY (LTSRF)
}

\author{
Kenneth S. Casey ${ }^{1}$, Tess B. Brandon ${ }^{2}$
}

(1) Chair, GHRSST Reanalysis Technical Advisory Group, NOAA National Oceanographic Data Center, US, Email: Kenneth.Casey@noaa.gov

(2) NOAA National Oceanographic Data Center, US, Email: Tess.Brandon@noaa.gov

\section{Introduction}

\subsection{Background}

Since the inception of the GODAE High Resolution Sea Surface Temperature (SST) Pilot Project (GHRSST-PP), it has been widely appreciated that satellite data sets produced in near-real time operational settings generally fail to provide the most accurate and consistent time series information possible. With this knowledge, the GHRSST-PP Science Team initiated the Reanalysis (RAN) program whose goals are: 1 ) to produce delayed-mode products of higher accuracy and consistency than the realtime SSTs by taking advantage of additional delayed mode data that cannot be used by the operational real time system; 2) to link the RAN products to existing longer-term SST analyses and 3) to enable a reprocessing capability so that future users of the data can reprocess or utilize the data. As such, the GHRSST RAN is as much about establishing a data processing and management system as it is about creating SST products.

In 2008 the GHRSST-PP evolved into the Group for High Resolution SST (GHRSST) program, taking on the new name as the overall GODAE project came to a close. In this context, the GHRSST RAN Technical Advisory Group (RANTAG) remains the formal GHRSST body that is responsible for the scientific and operational methods and algorithms used to generate delayed-mode GHRSST data products. The delayed mode products will be suitable for use as essential climate variables (ECVs) or climate data records, an important concept in environmental data management, which dictates longterm accuracy and consistency (e.g. NRC,
2000). Target accuracies for GHRSST reanalysis products are on the order of 0.3 $\mathrm{K}$ absolute and $0.1 \mathrm{~K}$ relative, with $\mathrm{a}$ temporal stability requirement of 0.01 K/decade. These ambitious targets are being used to help update Global Climate Observing System (GCOS) requirements and may not be strictly achievable given current satellite sensor technologies. However, they provide demanding and rigorous goals which push the RAN-TAG to continually search for and implement improvements to the data sets. They also help set the framework for the Committee on Earth Observing Satellites (CEOS) Virtual Constellation for SST, which is in the process of being approved and will utilize GHRSST and the RAN-TAG as its implementation mechanism to deliver ECVs to the Global Earth Observing System of Systems (GEOSS).

\subsection{Period of Report and Document Organization}

This document describes the current status of the GHRSST RAN-TAG with a focus on its activities since the $11^{\text {th }}$ GHRSST Science Team meeting, held in Lima, Peru from 21 -25 June, 2010. The year since that meeting has been a productive one for both GHRSST and the RAN-TAG as well. The remainder of this document covers four key areas of activity:

- GHRSST Long Term Stewardship and Reanalysis Facility (LTSRF) archive operations

- GHRSST/Global Climate Observing System (GCOS) intercomparison facility enhancements

- Active Archive Efforts at the NODC LTSRF 
GHRSST XII -

Proceedings, Edinburgh

GHRSST Project Office

- Reanalysis product developments

Several appendices close off the report with listing of LTSRF Operational Messages (Annex 1), LTSRF News Messages (Annex 2), LTSRF Automated Status Messages (Annex 3 ), and current members of the RAN-TAG (Annex 4).

2. Operations of the Long Term Stewardship and Reanalysis Facility

\subsection{Operational Reliability}

The LTSRF (http://ghrsst.nodc.noaa.gov) at NOAA's National Oceanographic Data Center (NODC) has successfully continued operations over the last year.
Issue: Final Date: September 27, 2011

Table 1: Summary of GHRSST data products in the NODC LTSRF. The ODYSSEA data sets indicated by an asterisk (*) remain in the archive but their ongoing production remains on hold. The European RDAC (EUR) expected this stoppage to last only a few months but it has continued for more than a year. Current expectation is for ODYSSEA products to resume production in GDS2-compliant format in December of 2011.

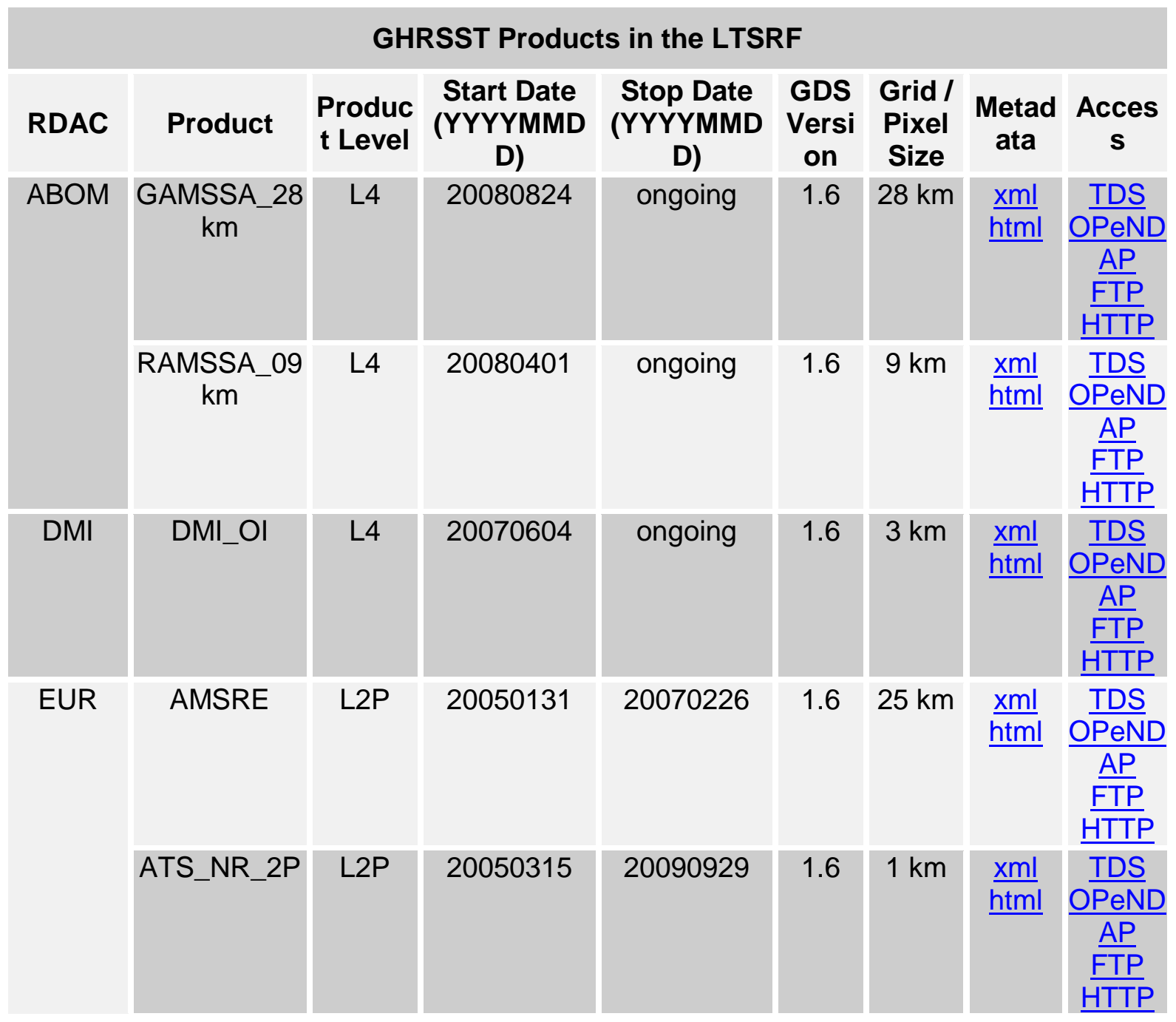


GHRSST XII -

Issue: Final

Proceedings, Edinburgh

GHRSST Project Office

Date: September 27, 2011

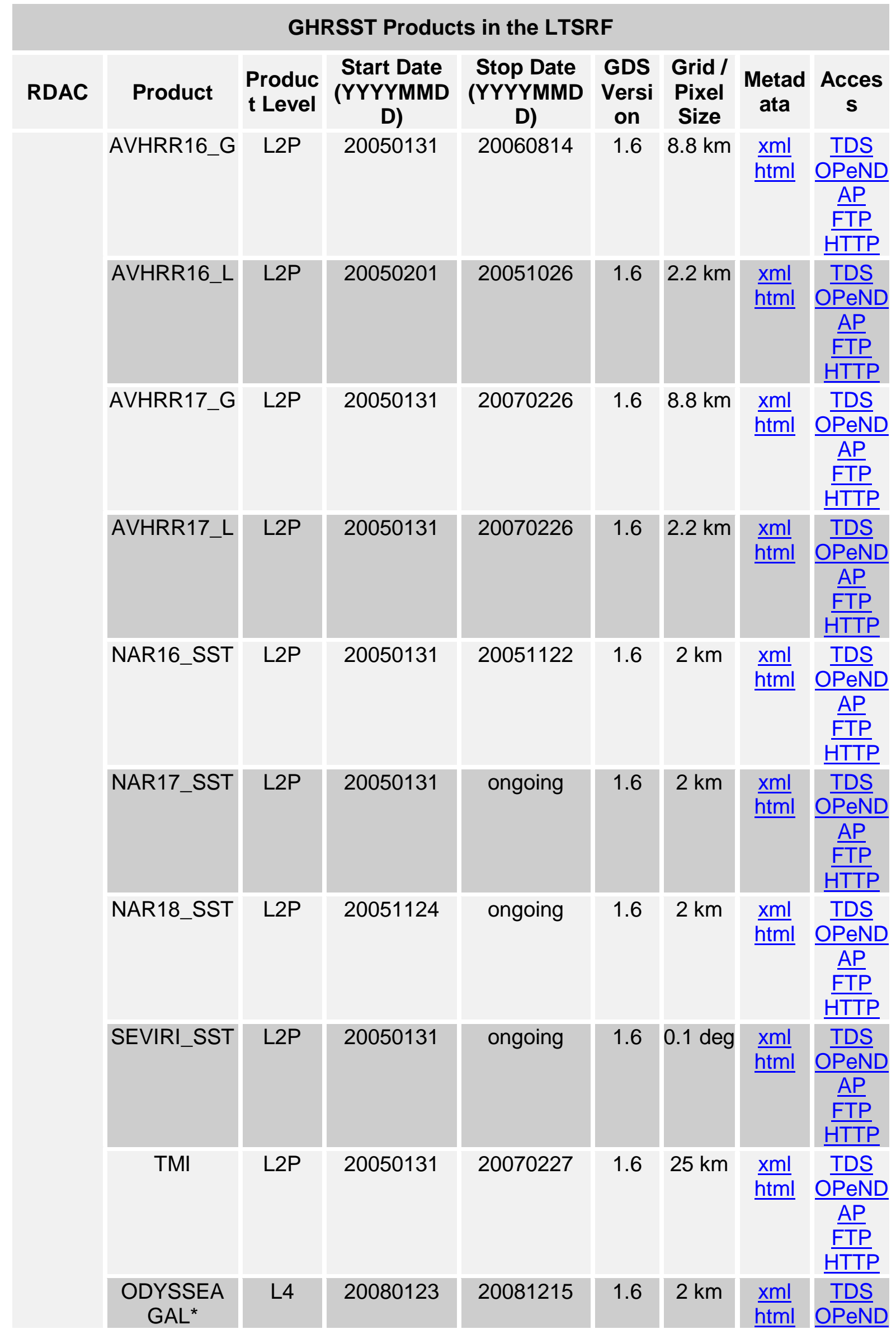




\section{GHRSST Products in the LTSRF}
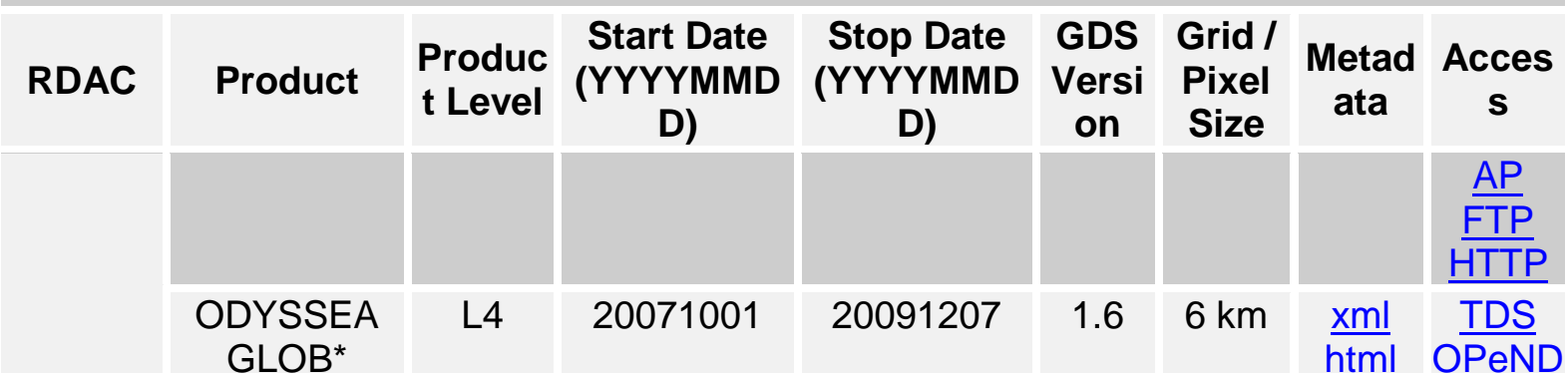

GLOB*

$\underline{\mathrm{html}} \underline{\mathrm{OPeND}}$

AP

$\frac{\text { FTP }}{\text { HTTP }}$

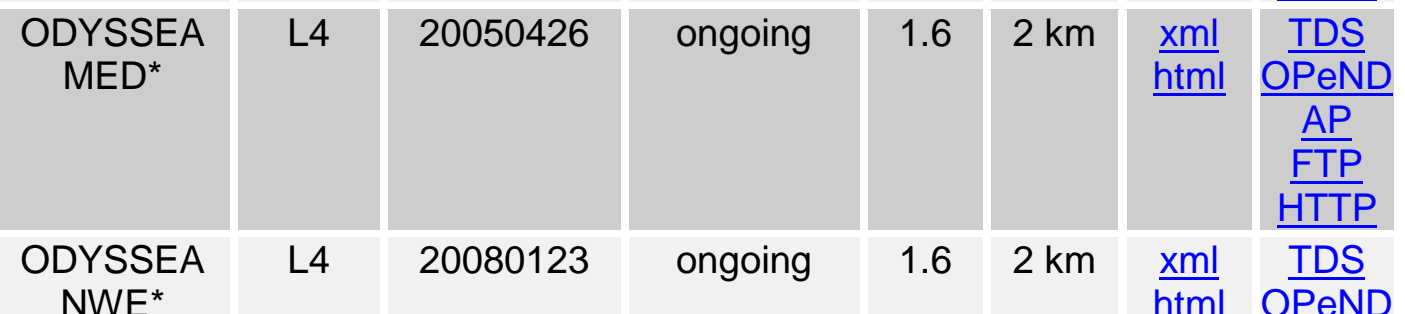

NWE*

$\underline{\text { html }} \underline{\text { OPeND }}$

AP

FTP

HTTP

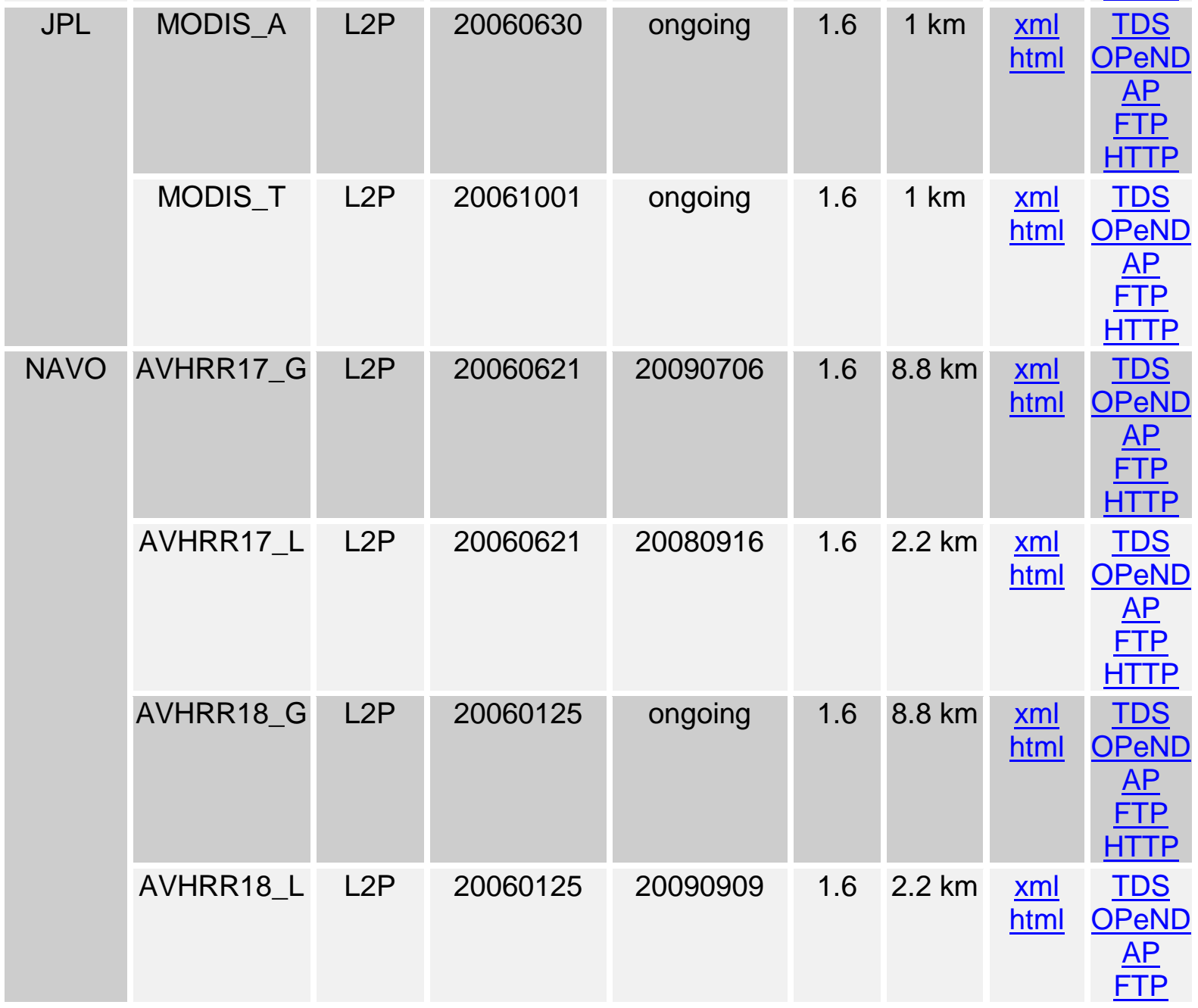




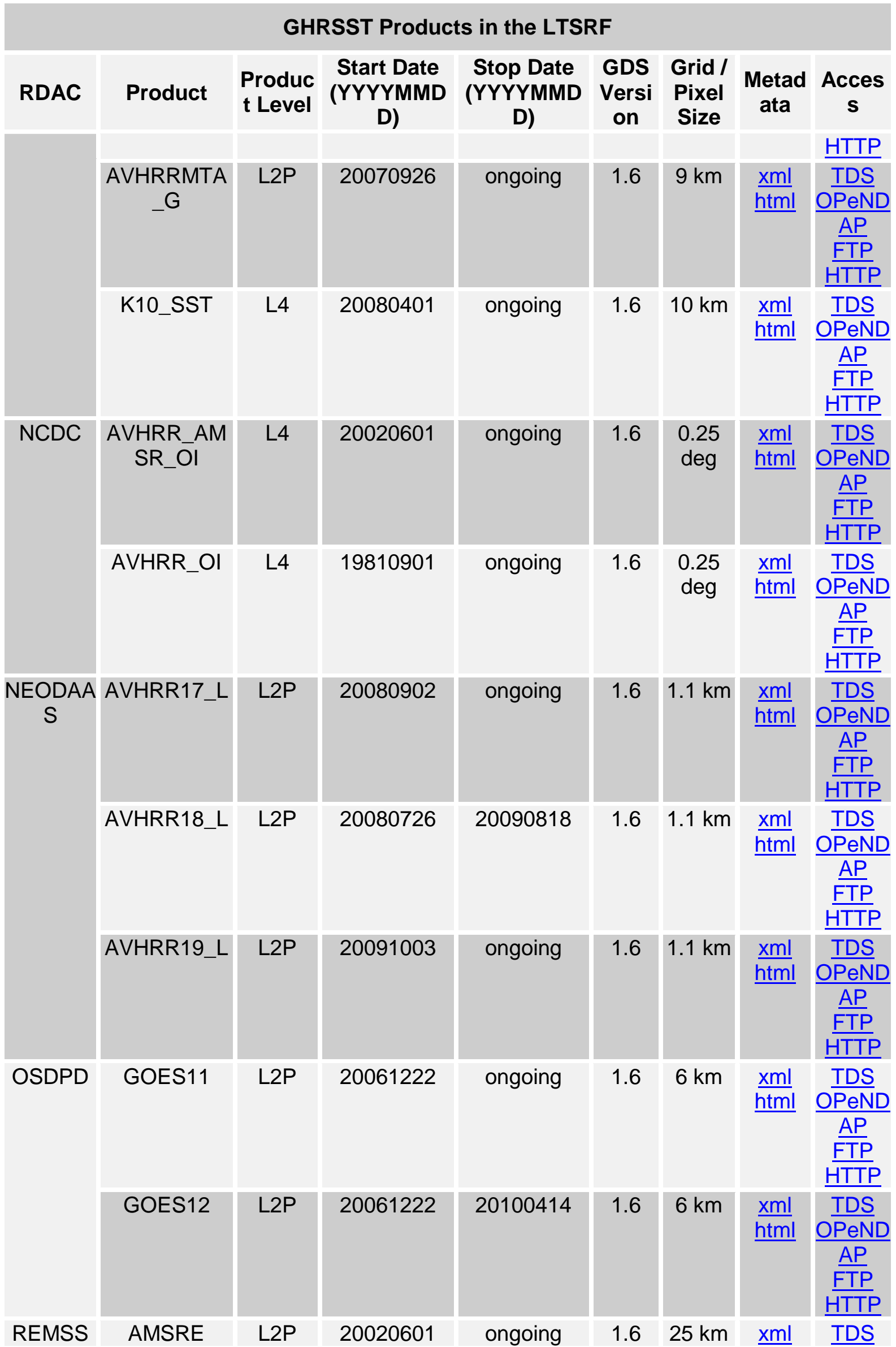




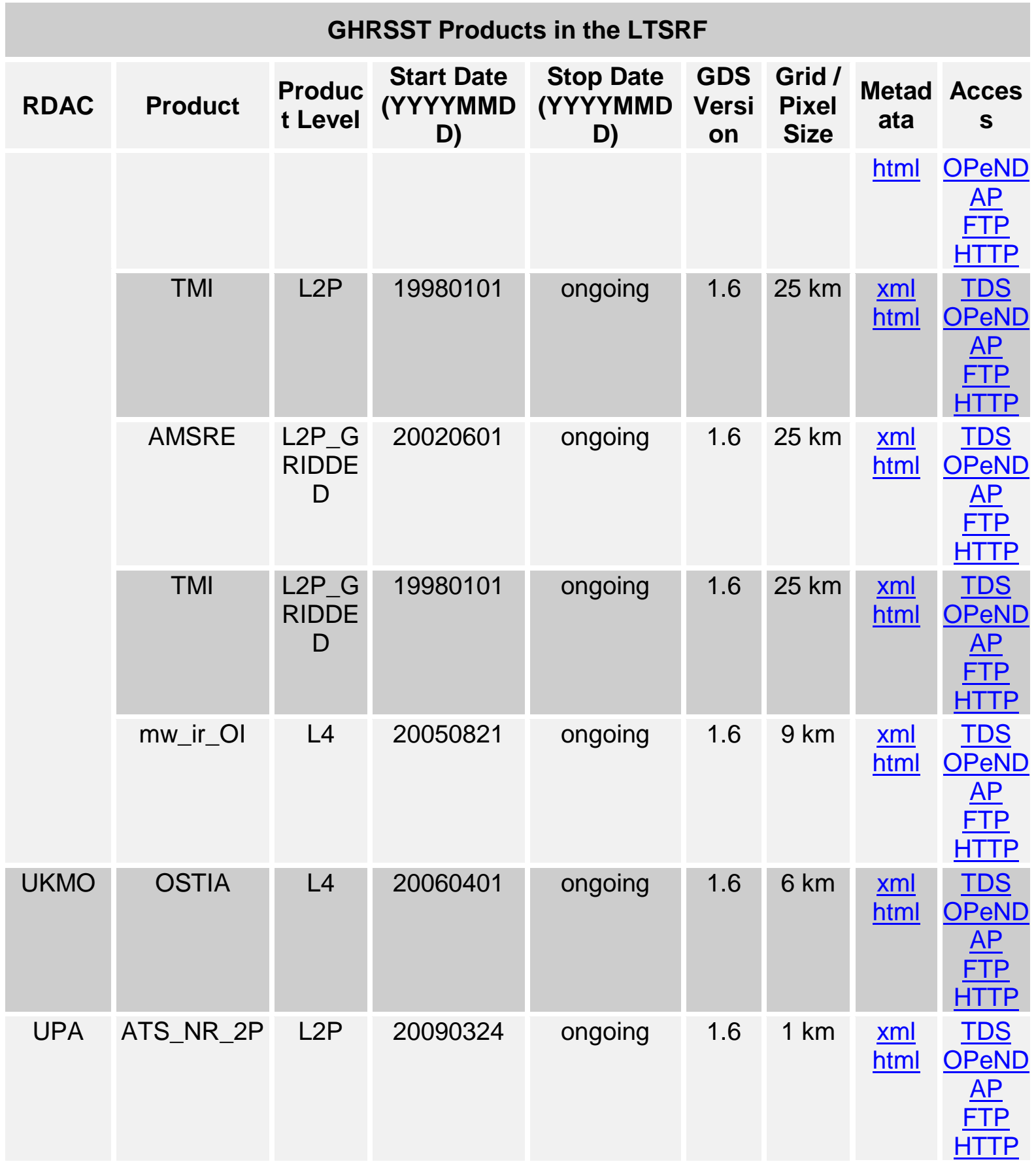

Table 2: RDAC codes and acknowledgements.

\section{RDAC Codes and Acknowledgements}

ABOM Australian Bureau of Meteorology, Australia

DMI Danish Meteorological Institute, Denmark

EUR Medspiration Project, Europe

JPL NASA Jet Propulsion Laboratory, Ocean Biology Processing Group and University of Miami, USA

NAVO Naval Oceanographic Office, USA

NCDC NOAA National Climatic Data Center, USA

NEODAAS NERC Earth Observation Data Acquisition and Analysis Service, United 
Kingdom

OSDPD NOAA Office of Satellite Data Processing and Distribution, USA

REMSS Remote Sensing Systems, Inc., USA

UKMO UK Meteorological Office, United Kingdom

UPA UK Multi-Mission Product Archive Facility, United Kingdom

\subsection{Archive Metrics}

Together, these L2P, L2P_GRIDDED, and L4 files occupy over 34 terabytes (compressed, estimated 68 terabytes uncompressed) of disk space, and consist of approximately 1,650,000 netCDF data files, an increase from 28 terabytes and $1,350,000$ files at the time of last year's report. Current temporal coverage varies for each product line, with the earliest data set available back to the beginning of 1981 (though the majority do not begin until 2005-2007).

The following four figures illustrate the

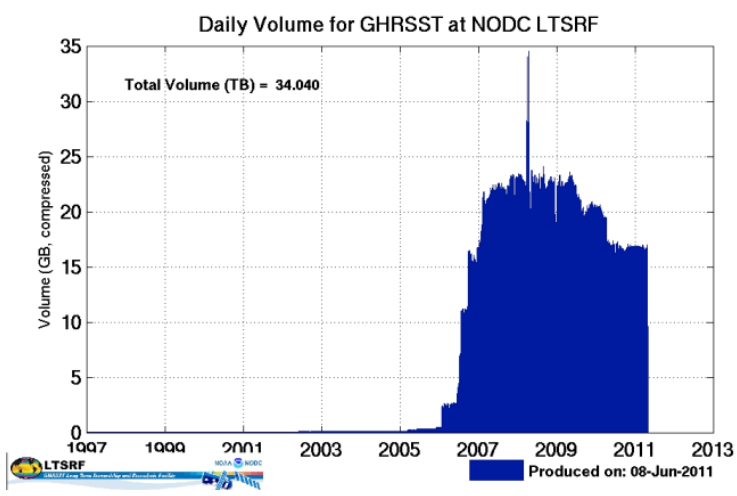

Figure 1: Volume in gigabytes arriving in the LTSRF each day.

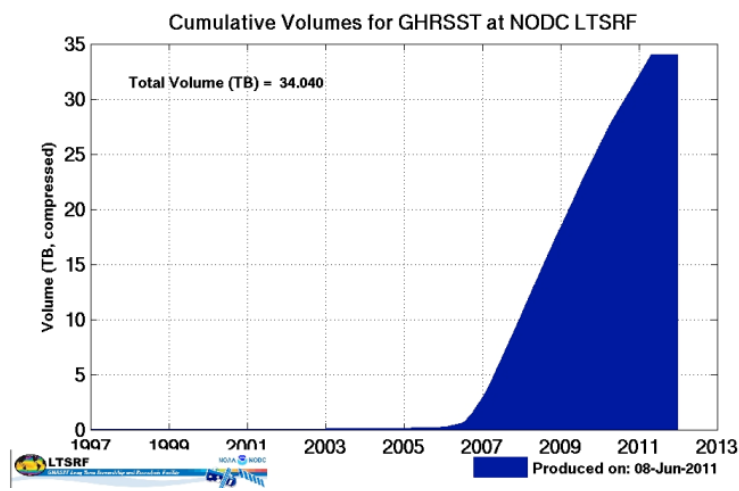

Figure 3: Cumulative volume in gigabytes in the LTSRF.

The data are grouped in the archive system in Archival Information Packages (AIPs, also known as NODC growth of the LTSRF archive. Figures 1 and 2 show the daily rates of GHRSST data in terms of volumes and numbers of netCDF files, respectively. Figures 3 and 4 show the cumulative growth of the archive in terms of volumes and numbers of netCDF files, respectively. These graphics are generated automatically each day and posted to the LTSRF web site. These graphs indicate that about 800 netCDF files occupying around 17 gigabytes (compressed, about 34 gigabytes uncompressed) arrive at the NODC LTSRF each day.

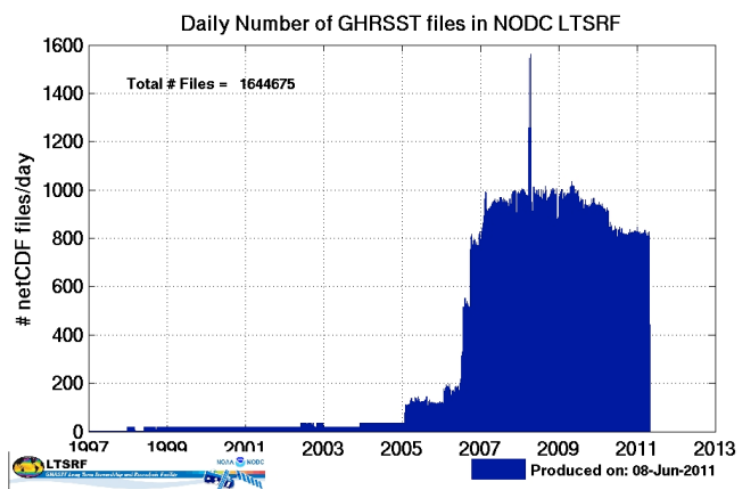

Figure 2: Number of netCDF files arriving in the LTSRF each day.

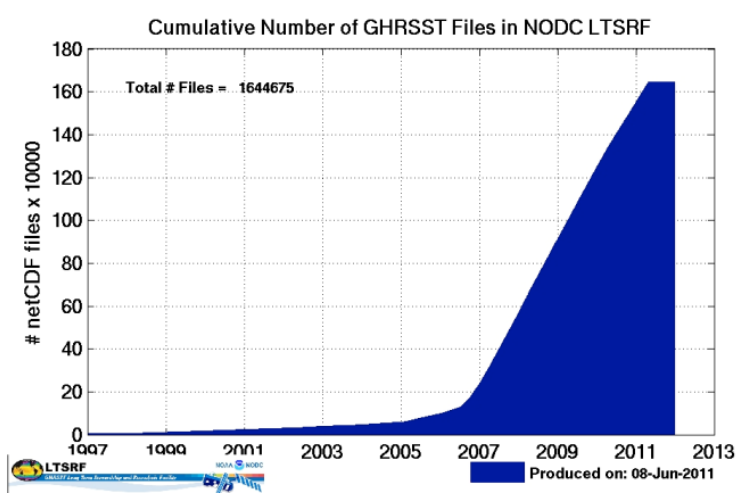

Figure 4: Cumulative number of netCDF files in the LTSRF.

"accessions"), or logical groupings of data. For GHRSST, an AIP is defined as the data from a single sensor (or analysis 
GHRSST XII -

Proceedings, Edinburgh

GHRSST Project Office

system and region), from a given RDAC, for a particular date. For example, all of the approximately 288 netCDF data files (and corresponding metadata files) from MODIS Aqua, produced by the JPL RDAC for 01 January 2007 are grouped into a single NODC accession. As of 15 June
Issue: Final

Date: September 27, 2011

2010, there were 59,982 GHRSST AIPs in the formal NODC archive systems. As of 09 June 2011, there were 67,511. The growth of the number of AIPs in the GHRSST archive is shown below in Figure 5. Like the previous four figures, this graphic is also updated automatically on a daily basis and posted to the LTSRF web site.

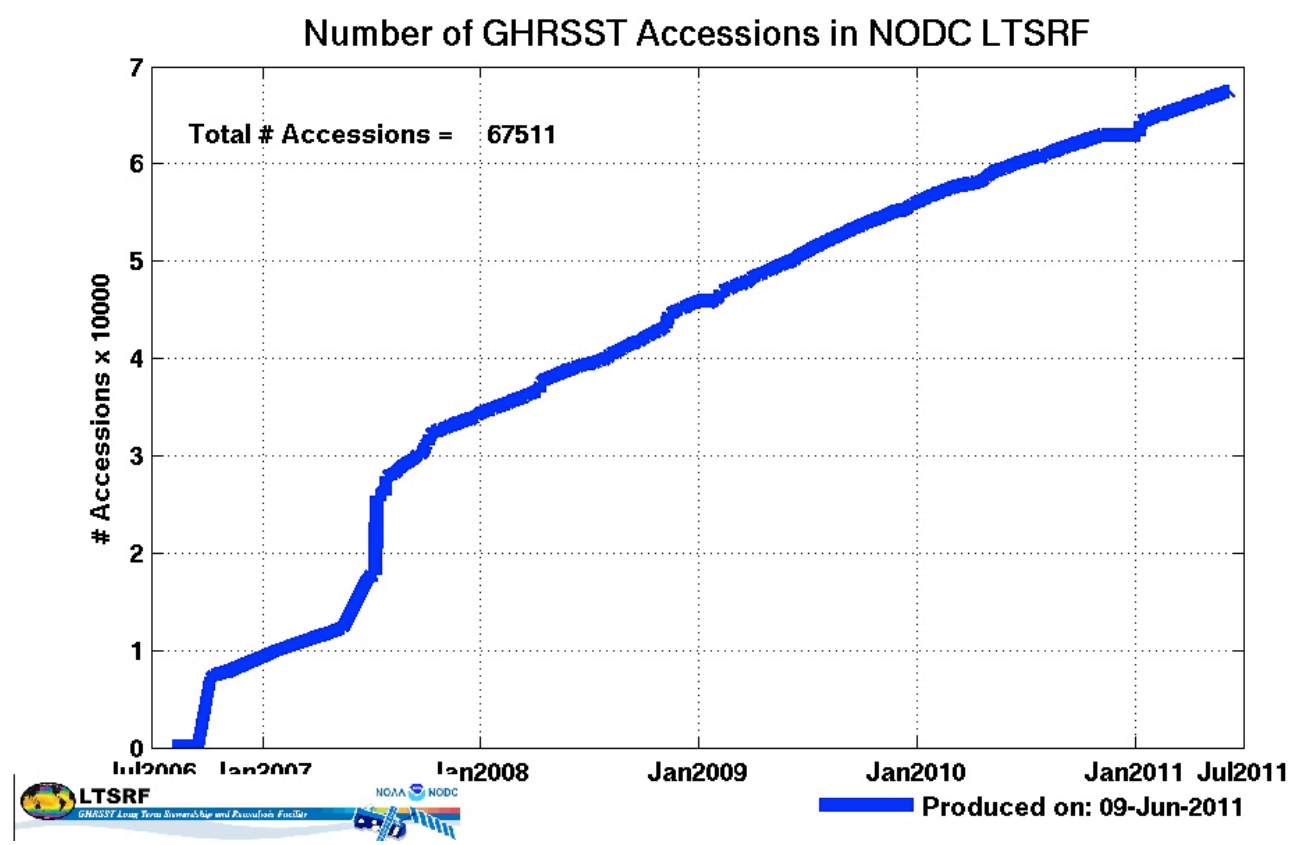

Figure 5: Growth in the number of accessions, or Archival Information Packages, in the LTSRF.

\subsection{Operational Reporting}

In addition to the automated graphics that are generated and posted to the LTSRF site on a daily basis, the LTSRF also maintains 3 Really Simple Syndication (RSS) feeds. The first feed is manually updated as necessary, providing LTSRF Operational Messages (http://ghrsst.nodc.noaa.gov/LTSRF OpM essages.xml) as demonstrated in Annex 1. The second syndication is a news feed for noteworthy items of interest to users of the

LTSRF

(http://ghrsst.nodc.noaa.gov/ghrsst news rss.xml). This feed is updated manually as needed, and Annex 2 lists the news items posted since the $11^{\text {th }}$ Science Team meeting. The third feed conforms to the
GHRSST draft specification on automated status reporting. This syndication provides automatically generated messages on a daily basis, which assess the current state of the LTSRF-GDAC connection based on the number of AIPS generated each day (http://ghrsst.nodc.noaa.gov/LTSRF OpSt atus.xml). Annex 3 displays a recent set of these automated messages.

\subsection{Archive Access Metrics}

The number of users accessing GHRSST data from the LTSRF continues to increase. Figure 6 (a, b, and c) summarizes the statistics since logs have been recorded at the LTSRF in June 2006. 
GHRSST XII -

Proceedings, Edinburgh

GHRSST Project Office

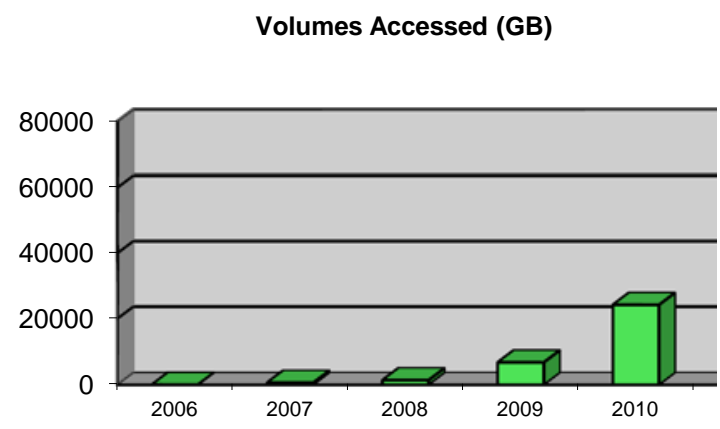

Number of Users

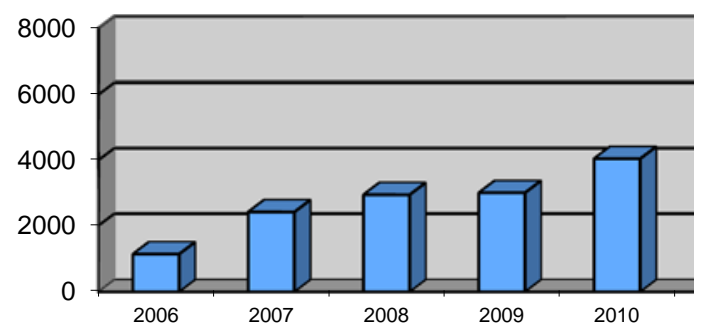

Number of Files

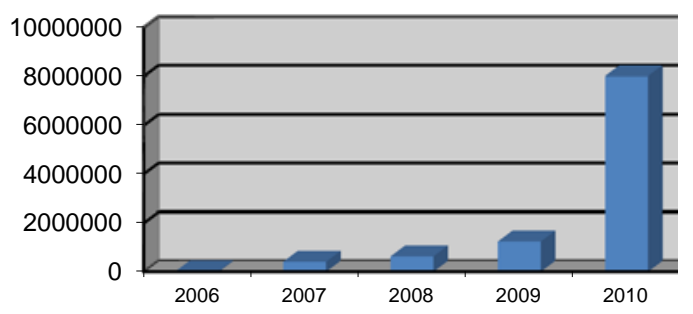

The general trend toward increasing numbers of netCDF files being accessed from the LTSRF continued. In 2006, 85 files were transferred per day on average over FTP, HTTP, and OPeNDAP services. That number grew to 1130 per day in 2007, 1734 in 2008, and 3413 in 2009. For 2010, 21,956 files per day were transferred with a huge spike in the
Issue: Final

Date: September 27, 2011

Figure 6a: Volumes of GHRSST data accessed at the NODC LTSRF (in gigabytes). 2011 numbers are projected based on Jan-May numbers.

Figure 6b: Number of users of GHRSST data at the NODC LTSRF (based on unique IP addresses). 2011 numbers are projected based on Jan-May numbers.

Figure 6c: Number of GHRSST data files accessed at the NODC LTSRF. 2011 numbers are projected based on Jan-May numbers.

number of files being accessed via OPeNDAP. The volume rates continued to increase as well, going from $0.2 \mathrm{~GB}$ per day in 2006 to 1.8 GB per day in 2007, 3.9 GB per day in 2008, and 19 GB per day in 2009. For 2010, 66 GB/day were transferred and for Jan - Jun 2011, 166 $\mathrm{GB} /$ day were accessed. 


\subsection{Adjudication of Anomalous Data Packages}

The NODC LTSRF has received over the course of the years of GHRSST operation a fairly large number of data packages that did not exactly meet the documented processes and specifications. A major effort was conducted this year to rectify the lingering issues with these anomalous collections of GHRSST data acquired from the GDAC. These anomalies can generally be placed into three categories: (1) data files received with incorrect or ambiguous associated metadata; (2) data files received for a previously-received archive information package for which there is no clear indication of whether they should replace the previous package, be added to it, replace only some portion of the existing package, or some combination of these; and (3) data files received that used the GDS 1 file name convention incorrectly, typically using the GDS version portion of the file name inappropriately as a file version.

This effort involved extensive collaboration with the GDAC and individual RDACs. The process resulted in enhancements to the LTSRF procedures to account for these anomalous data packages and to date has processed and archived approximately $60 \%$ of them by volume.

\section{Updates to the GHRSST/GCOS SST Intercomparison Facility}

In 2008, in conjunction with the Global Climate Observing System (GCOS) SST/Sea Ice Working Group, the LTSRF established an intercomparison facility for different SST analysis products and historical SST reconstruction data sets. Data cubes, intercomparison diagnostics, and browse graphics are available for all of these data sets in standard formats, including GDS1-compliant netCDF. A complete list of products currently included in the intercomparison framework appears in Table 1 below. Satellite era products are available on a one-degree weekly basis, while historical area data sets are available on monthly, five-degree grids.

Table 3: GHRSST/GCOS SST Intercomparison Products available at the intercomparison facility.

\begin{tabular}{cccc}
\hline Data Set Name & $\begin{array}{c}\text { Satellite Era } \\
(\mathbf{1 9 8 1}-\mathbf{2 0 0 7 )})\end{array}$ & $\begin{array}{c}\text { Historical Era } \\
(\mathbf{1 8 5 0}-\mathbf{2 0 0 8 )}\end{array}$ & In LAS \\
\hline AVHRR Pathfinder Version 5 & $\mathbf{X}$ & & $\mathrm{Y}$ \\
\hline Operational AVHRR & $\mathbf{X}$ & & $\mathbf{Y}$ \\
\hline NOAA Optimum Interpolation (OI) Version 2 & $\mathbf{X}$ & & $\mathbf{Y}$ \\
\hline NOAA Daily 1/4-degree OI Version 1 & $\mathbf{X}$ & & $\mathbf{Y}$ \\
\hline Hadley Centre SST V2 & $\mathbf{X}$ & $\mathbf{X}$ & $\mathbf{Y}$ \\
\hline Hadley Centre Sea Ice and SST (HadISST) V1 & $\mathbf{X}$ & $\mathbf{X}$ & $\mathbf{Y}$ \\
\hline NOAA Extended Reconstruction Version 3 & $\mathbf{X}$ & $\mathbf{X}$ & $\mathbf{Y}$ \\
\hline Kaplan Reconstructed & $\mathbf{X}$ & $\mathbf{X}$ & $\mathbf{Y}$ \\
\hline International COADS Version 2.4 & & $\mathbf{X}$ & $\mathbf{Y}$ \\
\hline COBE Analysis & & $\mathbf{X}$ & $\mathbf{Y}$ \\
\hline
\end{tabular}

Since its implementation, several additions and improvements have been made to the facility, which is available at http://ghrsst.nodc.noaa.gov/intercomp.htm l. This year, two new analysis products were received as part of a GCOS experiment testing several reanalysis systems using a common input data set (Table 4). In addition, the GCOS intercomparion data sets were added to 
the NODC Live Access Server (LAS). Figure 7 shows an example of a usergenerated Hovmoller diagram from the
GCOS SST LAS, showing the 1983 and 1987 El Niño events.

Table 4: Summary status on the progress of the GCOS common-input reanalysis experiment.

\begin{tabular}{cccc}
\hline Common-Input Reanalysis Product & Received & $\begin{array}{c}\text { Reformatted and } \\
\text { Metrics } \\
\text { Computed }\end{array}$ & In LAS \\
\hline Hadley Centre Sea Ice and SST (HadISST) & $\mathbf{N}$ & $\mathbf{N}$ & $\mathbf{N}$ \\
\hline Kaplan Reconstructed & $\mathbf{N}$ & $\mathbf{N}$ & $\mathbf{N}$ \\
\hline National Oceanography Centre Reanalysis & $\mathrm{N}$ & $\mathbf{N}$ & $\mathbf{N}$ \\
\hline COBE Analysis & $\mathrm{Y}$ & $\mathbf{N}$ & $\mathbf{N}$ \\
\hline NOAA Extended Reconstruction SST & $\mathrm{Y}$ & $\mathbf{N}$ & $\mathbf{N}$ \\
\hline
\end{tabular}

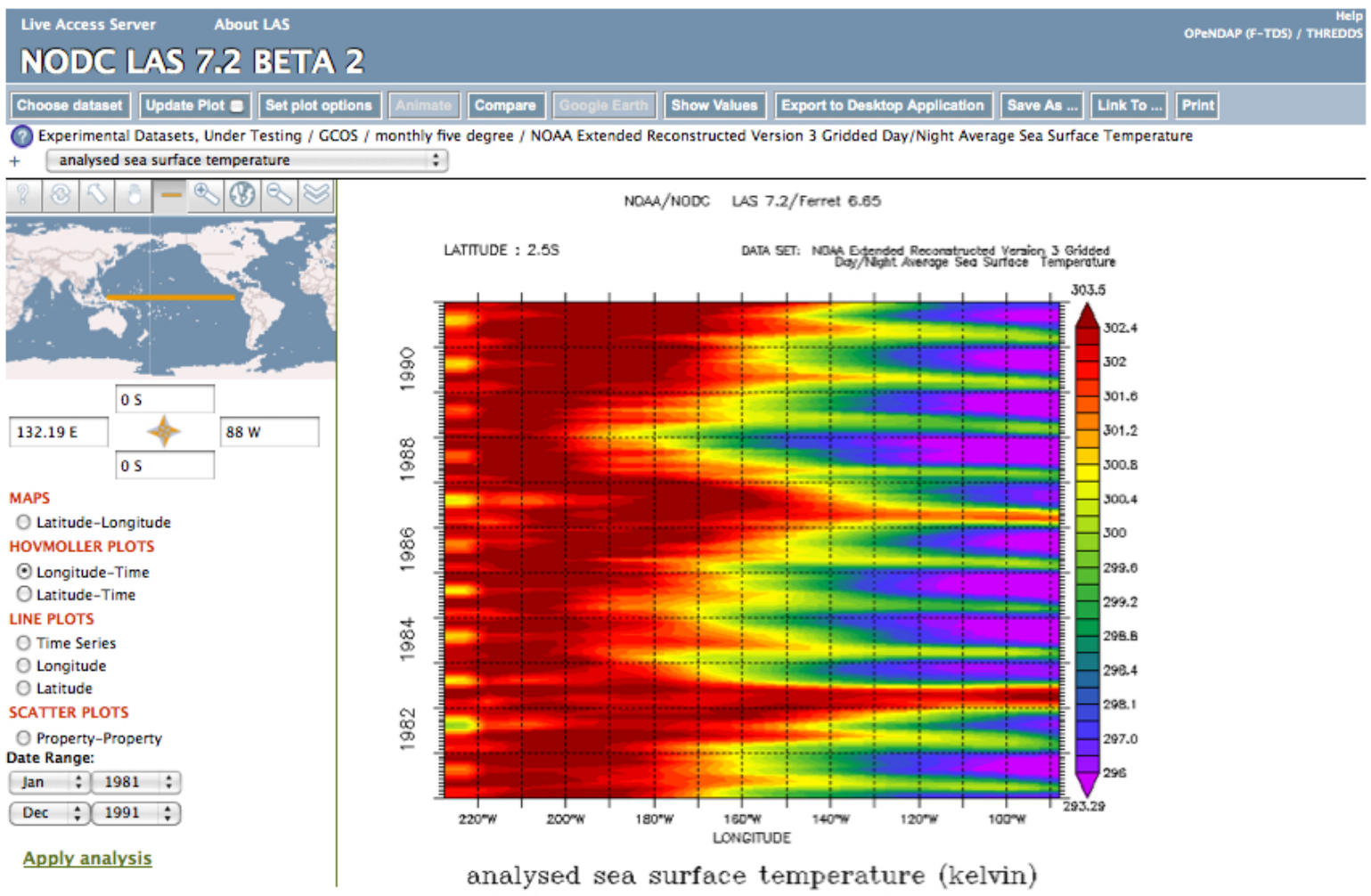

Figure 7: Example Hovmoller plot across the equator for 1981-1991, generated using the NODC LAS for the ERSST product. The 1983 and 1987 EI Nino periods are evident.

\section{Active Archive Efforts at NODC LTSRF}

Active archive efforts that commenced in 2008 continued this year, with annual reviews of GHRSST version 1 product metadata and feedback provided to RDACs on inconsistencies and issues with their products. With the final approval of the new GHRSST Data Specification
Version 2 (GDS2) in the fall of 2010, NODC staff are also actively working to update automated ingest procedures and prepare ISO 19115-2 metadata for all GHRSST products.

Browse graphic images continue to be created and have been extended to include every GHRSST netCDF file regardless of processing level. The 
process of generating a browse graphic forces the LTSRF archive to confirm and verify the contents of every package of data arriving into the archive. Currently, a PNG browse graphic is automatically generated for every L2P, L2P_GRIDDED and L4 data file arriving at the LTSRF. An accompanying $\mathrm{KML}$ wrapper is also generated, which allows the PNG graphic to be viewable in Google Earth. An example for the L2P AMSR-E product from Remote Sensing Systems is shown below in Figure 8.

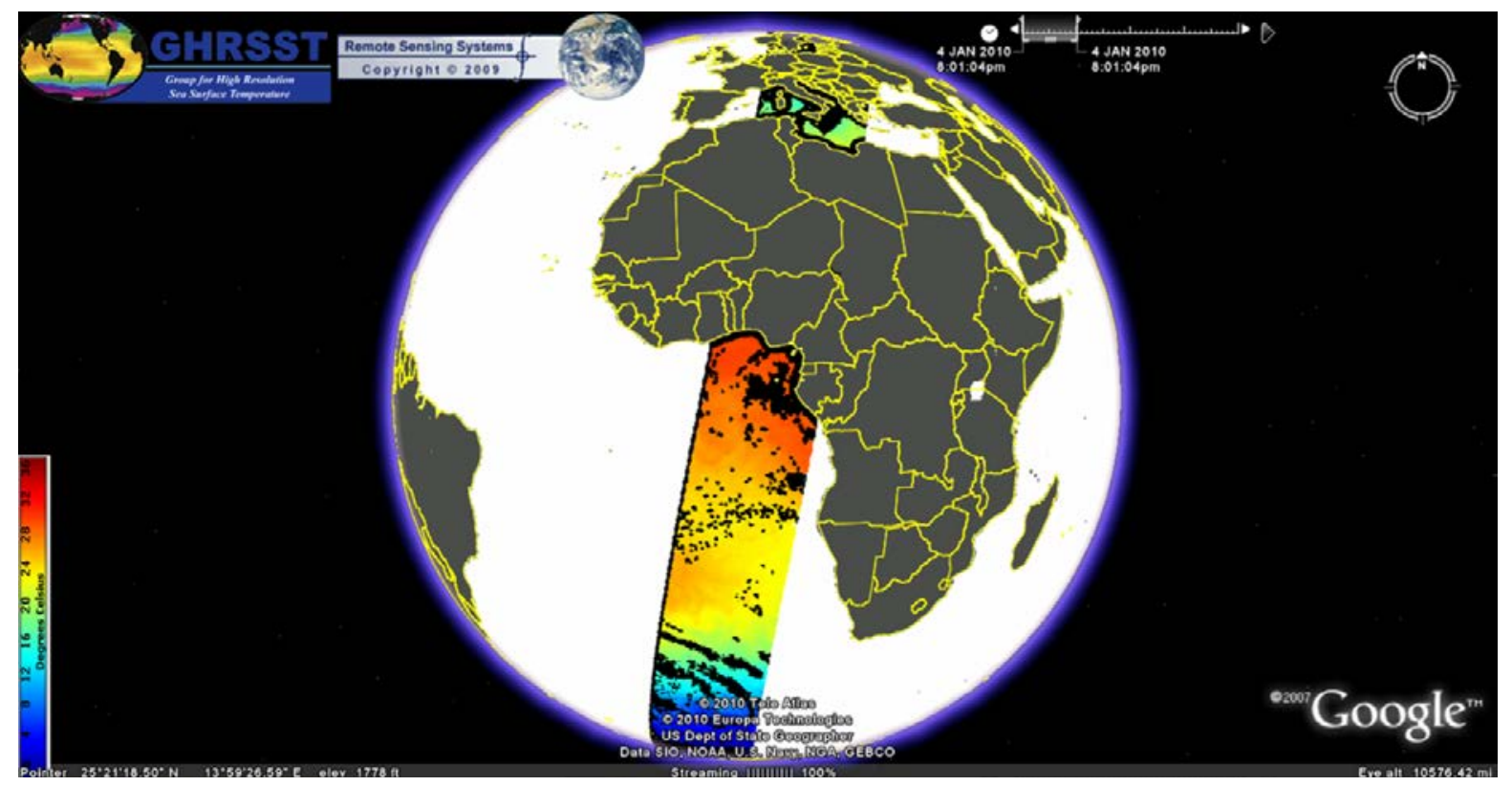

Figure 8: Example L2P REMSS AMSR-E browse graphic in Google Earth browser.

In addition to quality assurance, browse graphics increase the discoverability of GHRSST data holdings at the LTSRF. Users can quickly see the differences in spatiotemporal coverage and resolution among the various GHRSST products, helping them to choose which product is the best for their particular application.

Increasing the discoverability of and access to GHRSST data continues to be a major focus of the NODC LTSRF. In addition to HTTP and FTP, GHRSST data continue to be made available online from the LTSRF using the Data Access Protocol (DAP) via OPeNDAP's Hyrax server, and through the DAP, Web Coverage Service (WCS), and Web Mapping Service (WMS) via Unidata's THREDDS Data Server (TDS). Virtual aggregations making the gridded GHRSST products appear as single, 3dimensional "cubes" of data in space and time instead of a discrete collection of 2dimensional slices were made available through the NODC LAS this year (http://data.nodc.noaa.gov/las). The collection-level metadata records generated by NODC for each GHRSST product that are now accessible not only via Google searches but have also now been published to Geospatial One Stop (http://www.geodata.gov) and Data.gov (http://data.gov).

\section{Reanalysis Product Developments}

Siginificant progress continues to be made in the development of single-sensor and merged reanalysis SST products. Demand for these more accurate, consistent, and longer-term products continues to grow, with users ranging from fisheries scientists to numerical modelers interested in longer data sets than the GHRSST forward-mode operational data streams can provide. Requests for data continue to escalate rapidly at the LTSRF, providing clear evidence that users need more and longer SST data sets to achieve a range of societal benefits. Progress 
GHRSST XII -

Proceedings, Edinburgh

GHRSST Project Office

continues in the new ESA Climate Change Initiative $(\mathrm{CCl})$ project for SST to improve both the AVHRR and (A)ATSR sensor series data, and the (A)ATSR Reanalysis for Climate (ARC) project concluded its work on the ATSR series.

A major step forward for the AVHRR Pathfinder SST effort was just made with the public release of the AVHRR Pathfinder Version 5.2 (PFV5.2) data set in June of 2011 (http://pathfinder.nodc.noaa.gov). This new version of Pathfinder is available in L3C format and is nearly $100 \%$ compliant with the GDS2 specifications (it is missing only the pixel-by-pixel time specification and the SSES bias/standard deviations). It is a signifant stepping stone on the way to the future Pathfinder Version 6, which will include L2P, L3-uncollated (L3U), and L3collated (L3C) products generated using a new and improved coefficient scheme. Work to reprocess HRPT data from the AVHRR series is also underway at the Australian Bureau of Meteorology and the University of Rhode Island, and the $12^{\text {th }}$ GHRSST Science Team Meeting will include a breakout session for a new GHRSST Working Group focused on those data.

\section{Summary and Look Forward}

The past year has been a highly active one for GHRSST Reanalysis and the LTSRF. The large data management system has been maintained and improved, and progress made toward creating high resolution, multi-sensor reanalysis products. Preparations for the receipt of GDS2 data at the LTSRF have
Issue: Final

Date: September 27, 2011

been made, and it is anticipated that the new GHRSST standards will further enhance the usability of the GHRSST collection for climate-related applications. Growing numbers of users continue to access more and more GHRSST data every year. The GHRSST LTSRF archive continues to improve its level of quality assurance of the data as it flows into the archive and is working with RDACs to remedy problems that are found. The coming year looks even more promising, with longer time series of data being made available to a wider range of users in GHRSST GDS2 format from projects like Pathfinder and the ESA $\mathrm{CCl}$. Improvements to the SST intercomparison facilty for understanding key differences in the available data continue including dynamic subsetting and analysis through the NODC LAS. Next year, specifying a new Data Processing Framework (DPF) for the SST ECV will be a priority for the RAN-TAG, and efforts will continue to make GHRSST products more usable for the archive user community. As always, and above all else, international collaboration will continue to be the means by which the ambitious goals of GHRSST Reanalysis will be achieved.

\section{References}

NRC (2000). Ensuring the climate record from the NPP and NPOESS meteorological satellites, NAP, $71 \mathrm{pp}$.

Reynolds, R.W., T.M. Smith, C. Liu, D.B. Chelton, K.S. Casey, and M.G. Schlax (2007). Daily high-resolution blended analyses for sea surface temperature, Journal of Climate, vol. 20.

\section{Annex 1 : Listing of Operational Messages, May 2010 - May 2011}

\section{1-02-05: NODC Archives operational}

The NODC Archive system preventative maintenance has been completed. We hope this will result in improved archive performance. Thank you for your patience.

2011-02-05: NODC Archives
undergoing preventative maintenance

The NODC Archive system is undergoing preventative maintenance. Diagnostics revealed a problem with a disk in the ingest processing filesystem. The disk has been replaced and the filesystem was rebuilt and revalidated. However, in order to reduce stress on that filesystem and the underlying disks, it was decided to move 
the ingest processing to a new filesystem. That move is underway. Thank you for your patience.

\section{Annex 2 : Listing of LTSRF RSS News Messages, May 2010 - May 2011}

\section{1-05-24: AVHRR Pathfinder Version 5.2 Data Completes Public Review}

The GHRSST Long Term Stewardship and Reanalysis Facility at the NOAA National Oceanographic Data Center thanks everyone who participated in the public review and comment period for AVHRR Pathfinder Version 5.2 (PFV52). Numerous minor comments and questions were received but no major issues identified with the draft data set. The Pathfinder team at NODC and the University of Miami is making final adjustments based on these comments and plans to release the full PFV52 data set prior to the 12th GHRSST Science Team meeting, which is being held 27 June to 1 July 2011 in Edinburgh, Scotland. Thank you for helping us create the best possible AVHRR-based climate data record for SST!

\section{1-05-02: AVHRR Pathfinder Version 5.2 Data Released for Public Review}

The GHRSST Long Term Stewardship and Reanalysis Facility at the NOAA National Oceanographic Data Center announces a public review period for the AVHRR Pathfinder Version 5.2 (PFV52) sea surface temperature product. PFV52 was computed using an entirely modernized system, based on SeaDAS and incorporating several key changes as we prepare for the future Version 6 (PFV6) data set (no release has yet been established for PFV6). These changes include the use of an entirely new land mask, a modified grid, and the inclusion of sea ice and wind speed ancillary data to support the use of the SST data. Importantly, the new PFV52 data are provided in netCDF-4 (classic model, with internal compression and chunking) and are nearly 100\% compliant with the GHRSST Data Specification Version 2.0

(http://data.nodc.noaa.gov/pathfinder/PFV52 _PublicReview/GDS_TechSpecs_v2.0.pdf) for L3C products. These data deviate from that standard only in that the sses_bias, sses_standard_deviation, and sst_dtime variables are empty and the aerosol_dynamic_indicator variable is not yet present. We have made a collection of data spanning 2005-2010 (some days are presently missing) available for download via the following services:
HTTP:

http://data.nodc.noaa.gov/pathfinder/PFV52 PublicReview;

FTP:

ftp://ftp.nodc.noaa.gov/pub/data.nodc/pathfin der/PFV52_PublicReview; and THREDDS: http://data.nodc.noaa.gov/thredds/catalog/pat hfinder/PFV52_PublicReview. We ask you to review and examine these data, then send questions, comments, or concerns to Kenneth.Casey@noaa.gov by 11:59 PM EST on Monday, 23 May 2011. We will address as many problems identified through this public review as we can, prior to production of the full PFV52 data set. We expect to release the full PFV52 data set prior to the 12th GHRSST Science Team meeting, which is being held 27 June to 1 July 2011 in Edinburgh, Scotland. Thank you for helping us create the best possible AVHRR-based climate data record for SST!

2011-04-13: GHRSST Newsletter No. 3 for April 2011 available

The third monthly GHRSST newsletter is now available through the GHRSST Project Office website

at http://www.ghrsst.org/news/q/date/2011/04/1 3/ghrsst-newsletter-no-3/. News items featured include the continuation of Medspiration, a new SST product from IASI, global lake surface water temperatures from ATSR, and several others.

\section{1-03-25: EUMETSAT Launching IASI SST L2PCore}

GHRSST is pleased to announce the launch of the new Infrared Atmospheric Sounding Interferometer (IASI) Sea Surface Temperature (SST) product, identified as IASI SST L2PCore. The product has been designed in accordance with the specifications defined by GHRSST, and will initially contain SST, Single Sensor Error Statistics (SSES), quality levels, flags and collocated model surface winds. Quality levels for this product have been defined by stratifying against integrated water vapor, calculated by integrating IASI water vapor profiles from the IASI Level 2 products with pressure level information. SSES for each quality level have been derived using a matchup database of IASI SSTs collocated 
GHRSST XII -

Proceedings, Edinburgh

GHRSST Project Office

with in situ buoy SST observations (built up following GHRSST guidelines) spanning from 1 April to 28 September 2010. EUMETSAT plans to recalculate the SSES every 6 months. The IASI SST L2PCore product will be available via the EUMETSAT Data Centre and the FTP server, for which registration is possible on the EO Portal. Upon registration users will receive information on accessing the data on the FTP server. Test data of this product is currently available via FTP at: ftp://ftp.eumetsat.int/pub/EUM/out/OPS/User/ IASI_SST_L2PCORE/.

2011-02-16: Several updates to GHRSST products to be ingested into LTSRF

Beginning today, the GHRSST LTSRF at NODC will be processing a large volume of backlogged GHRSST data, ingesting it into the archive, and making it available online. This backlog contains updates to data from several GHRSST products, and may include the addition of new data files, removal of old data files, one-to-one replacement of existing data files, or some combination of these. Many of these updates have already been available via the GHRSST GDAC, but have remained offline at the LTSRF while we determined the best way to clearly capture and document such updates. If you have downloaded GHRSST data and would like to ensure that you have the most recent version of the data, please check the following: For users accessing data through the various LTSRF web services (HTTP, FTP, OPeNDAP, THREDDS) there will be an "accession update log" text file in the root GHRSST directory that contains a list of daily directories affected by the updates. This list will also contain corresponding NODC accession numbers and the data of the update, and will capture all changes to the GHRSST LTSRF collection moving forward. For users accessing data through the NODC Ocean Archive System, information about each update will be captured in the journal.txt file in the "about" directory of the accession. Note: ALL GHRSST data, old and new, will still be available through the NODC Ocean Archive System. For questions or concerns about this process, please contact the LTSRF. For more information about updates to individual products, please contact the Technical Contact for the relevant GHRSST RDAC.

2011-02-14: GHRSST Newsletter No. 2 for February 2011 available
Issue: Final

Date: September 27, 2011

The second monthly GHRSST newsletter is now available through the GHRSST Project Office website

at http://www.ghrsst.org/news.htm. News items featured include the GHRSST proposal to serve as the CEOS SST Virtual Constellation, a new ESA Climate Change Initiative RDAC, Brazilian SST activities, and many others.

2011-01-17: New GDSv2.0 AVHRR HRPT SSTskin product available for Australian region

HRPT AVHRR SSTskin data from NOAA-17, 18 and 19, covering the Australian region, are now available in real-time (within 3 hours of observation) in the formally released GDS v2.007 formats for swath and composite products. You can find SSTskin L2P (single swath) and $0.02 \times 0.02$ degree gridded SSTskin L3U (single sensor, single swath) and single day/single night L3C (single sensor, multiple swath) files at ftp://aodaac2cbr.act.csiro.au/imos. These data will be available via the GHRSST GDAC and LTSRF beginning in spring of 2011. For more information on the processing method used for these data, see Paltoglou et al (2010) from http://imos.org.au/srsdoc.html.

2010-11-29: GHRSST Newsletter No. 1 for November 2010 available

The first monthly GHRSST newsletter is now available through the GHRSST Project Office website at http://www.ghrsst.org/news.htm. News items featured include the announcement of a new Canadian Regional Data Assembly Center, a drifting buoys pilot project, a report from the NASA SST science team meeting held earlier this month in Seattle, and many others.

2010-11-16: GHRSST XII Science Team meeting 27 June to 1 July, 2011 in Edinburgh

The 12th Annual Science Team Meeting of the Group for High Resolution Sea Surface Temperature (GHRSST) will be hosted by the University of Edinburgh at the John McIntyre Conference Centre, Pollock Hall, 8 Holyrood Park Road, Edinburgh. The meeting will be held from 27 June to 1 July, 2011. Please indicate your interest by sending an email to ghrsst-edinburgh2011@reading.ac.uk with subject: attendance planned. For more information, see the GHRSST XII web page at http://www.ghrsst.org/GHRSST-XII.html. 
GHRSST XII -

Proceedings, Edinburgh

GHRSST Project Office

2010-11-15: New Global 1km Level 4 product from NASA JPL now available through GHRSST

The Multiscale Ultrahigh Resolution (MUR) blended sea surface temperature (SST) product has been updated to version 1 . This is a daily $1 \mathrm{~km}$ resolution product funded by the NASA MEaSUREs program to meet the goals of an accurate and consistent retrospective Level 4 SST record, for US coastal regions initially. The output data sets are made to GDS 2.0 specifications for Level 4 netCDF. Some of the improvements and differences of version in comparison to the previous version 0 (beta) are: 1 ) uses only nighttime input data from MODIS Aqua, Terra and AMSRE; 2) includes nighttime AVHRR data; 3) improves sensor bias corrections; and 4) improves spatial dependence of interpolation parameters. This product is produced at the NASA Jet Propulsion Laboratory by Dr. Mike Chin. The data for 2008-2010 are available from the GDAC at ftp://podaac.jpl.nasa.gov/GHRSST2/data/L4/ NCAMERICA/JPL/MUR/, and will be available from the LTSRF soon. Any questions concerning this product can be addressed to ghrsst@podaac.jpl.nasa.gov.

2010-10-28: Envisat mission extension update on AATSR data availability AATSR data are now available again in L2P format following the Envisat orbit lowering maneuver. Initial verification of the products has not highlighted any change in data quality. The product team will continue to monitor product quality and should have the first validation results in a week or so. Should you observe any issues with the data products or suspect any degradation in data quality, please contact Gary Corlett at gkc1@le.ac.uk.

2010-10-27: Joint DVWG, HL-TAG and STVAL Workshop

A joint workshop of the Data Variability Working Group, High Latitude Technical Advisory Group, and Sea Surface Temperature Validation Working Group will be held from 28 February to 2 March 2011 at the University of Colorado in Boulder, CO, USA. Further details can be found on the meeting webpage at http://www.ghrsst.org/Joint-DVWG,-HL-TAGand-ST-VAL-Workshop-2011.html.
Issue: Final

Date: September 27, 2011

\section{0-10-13: Envisat mission extension to cause gap in AATSR data}

The Envisat mission extension scenario will be implemented later this month, which will lower the spacecraft altitude and allow the orbit inclination to drift. The fuel saved from the drifting orbit should allow the mission to continue for another three years. Due to this maneuver, all data distribution from Envisat will stop completely between October 22nd and November 2nd, 2010. Once data flow is resumed, there should be no change in AATSR data quality and GHRSST users should continue to use AATSR data as normal. A statement confirming the quality of AATSR data after the change in orbit will be made at the beginning of December, 2010. Updates on data quality or changes to the implementation schedule will be announced via the GHRSST Program Office website as well as the LTSRF RSS News Feed as soon as they are known. For more information, refer to the full article on the GHRSST Program Office website at http://www.ghrsst.org/Envisat-missionextension-\%E2\%80\%93-implications-forAATSR-data-News.htm.

\section{0-10-01: Release of the GDS 2.0 Technical Specifications}

The GHRSST Data Specification 2.0 Technical Specifications document has been formally released today, and can be downloaded from the GHRSST Program Office at http://www.ghrsst.org/modules/documents/do cuments/GDS2.0_TechnicalSpecifications_v 2.0.pdf. This document represents a consensus on data format and content for the next generation of GHRSST products, and has been approved and endorsed by the GDS 2.0 Internal and External Review Boards as well as the GHRSST Advisory Council and the GHRSST Program Office. The document has been developed for data providers who wish to produce GHRSST data products, and for all users wishing to fully understand GHRSST product conventions, data file contents, and definitions. Your feedback on the GDS 2.0 is welcome. Please report it to the GHRSST Program Office at http://www.ghrsst.org. 


\section{Annex 3: Recent LTSRF RSS Automated Operational Status Messages}

2011-06-01: Ingested 13 new AIPs into NODC LTSRF - Status Yellow

May 31, 2011 9:37 PM

GHRSST archival status is "Yellow": 13 out of an expected 29 new Archival Information Packages (AIPS) were added today to the LTSRF archive at NODC. An AIP contains one day of data from one RDAC for one sensor or blended product. An AIP is also known as an NODC "accession".

2011-06-01: Ingested 4 updated AIPs into NODC LTSRF

June 1, 2011 3:44 AM

4 Archival Information Packages (AIPS) were updated today at the LTSRF archive at NODC. An AIP contains one day of data from one RDAC for one sensor or blended product. An AIP is also known as an NODC "accession".

2011-05-31: Ingested 49 new AIPs into NODC LTSRF - Status Green

May 31, 2011 10:31 AM

GHRSST archival status is "Green": 49 out of an expected 29 new Archival Information Packages (AIPS) were added today to the LTSRF archive at NODC. An AIP contains one day of data from one RDAC for one

sensor or blended product. An AIP is also known as an NODC "accession".
2011-05-31: Ingested 8 updated AIPs into NODC LTSRF

May 31, 2011 4:46 PM

8 Archival Information Packages (AIPs) were updated today at the LTSRF archive at NODC. An AIP contains one day of data from one RDAC for one sensor or blended product. An AIP is also known as an NODC "accession".

2011-05-30: Ingested 0 new AIPs into NODC LTSRF - Status Red

May 30, 2011 7:59 PM

GHRSST archival status is "Red": 0 out of an expected 29 new Archival Information Packages (AIPs) were added today to the LTSRF archive at NODC. An AIP contains one day of data from one RDAC for one sensor or blended product. An AIP is also known as an NODC "accession".

2011-05-30: Ingested 8 updated AIPs into NODC LTSRF

May 30, 2011 4:42 PM

8 Archival Information Packages (AIPs) were updated today at the LTSRF archive at NODC. An AIP contains one day of data from one RDAC for one sensor or blended product. An AIP is also known as an NODC "accession".

\section{Annex 4: RAN-TAG Membership}

Casey, Kenneth S. (Chairperson)

NOAA National Oceanographic Data Center

1315 East-West Highway

Silver Spring, MD 20910, USA

Tel: +01 301-713-3272 x133

Email: Kenneth.Casey@noaa.gov

Armstrong, Edward

NASA Jet Propulsion Laboratory

300/323 4800 Oak Grove Drive

Pasadena, CA 91109, USA

Tel: +01 818-393-6710

Email: edward.armstrong@jpl.nasa.gov
Emery, William

University of Colorado

CB 431

Boulder, CO 80309-0431, USA

Tel: +01 303-492-8591

Email: emery@frodo.colorado.edu

Reynolds, Richard W.

NOAA National Climatic Data Center

151 Patton Avenue

Asheville, NC 20881, USA

Tel: +01: (828) 271-4302

Email: Richard.W.Reynolds@noaa.gov 
GHRSST XII -

Proceedings, Edinburgh

GHRSST Project Office
Issue: Final

Date: September 27, 2011
Rayner, Nick

Met Office, Fitzroy Road, Exeter, EX3 1PB

United Kingdom

Tel: +44 1344854063

Email: Nick.Rayner@metoffice.gov.uk

Vazquez, Jorge

NASA Jet Propulsion Laboratory

300/323 4800 Oak Grove Drive

Pasadena, CA 91109, USA

Tel: +01 818-354-6980

Email: jorge.vazquez@jpl.nasa.gov

Larnicol, Gilles

CLS, Space Oceanography Division

8-10 rue Hermes, Parc Technologique du Canal,

Ramonville 31526, France

Tel: +33 561394753

Email: gilles.larnicol@cls.fr

Kawamura, Hiroshi

Center for Atmospheric and Oceanic Studies

Graduate School of Science

Tohoku University, Sendai 980-8578 Japan

Tel:+81 22-217-6745

Email: kamu@ocean.caos.tohoku.ac.jp

Beggs, Helen

BMRC, GPO Box 1289, Melbourne, Vic 3001

Australia

Tel: +61 396694394

Email: H.Beggs@bom.gov.au

Eileen Maturi

NOAA Office of Satellite Applications and

Research

Tel: +01 (301) 763-8102 x172

Email: Eileen.Maturi@noaa.gov

Jonah Roberts-Jones

Met Office, Fitzroy Road, Exeter, EX1 3PB

United Kingdom

Tel: +44 (0)1392 886441

Email: jonah.roberts-jones@metoffice.gov.uk

Christopher Merchant

School of GeoSciences, University of Edinburgh

Crew Building/King's Buildings

Edinburgh EH9 3JN, UK

Tel: +44 (0)131 6505097

Email: c.merchant@ed.ac.uk

Bruno Buongiorno Nardelli

Gruppo di Oceanografia da Satellite

CNR-Istituto per l'Ambiente Marino Costiero

Calata Porta di Massa - 80133 Napoli

Tel: +39-06-49934280

Email: bruno.buongiornonardelli@cnr.it
Wick, Gary A.

NOAA ESRL, R/ET6 325 Broadway

Boulder, CO 80305, USA

Tel: +01 303-497-6322

Email: Gary.A.Wick@noaa.gov

Donlon, Craig J.

European Space Agency/ESTEC (EOP-SME)

Keplerlaan 1, 2201 AZ

Noordwijk The Netherlands

Tel: +31 (0)715 653687

Email: Craig.Donlon@esa.int

Corlett, Gary

Space Research Centre, University of Leicester

University Road, Leicester, LE1 7RH

United Kingdom

Tel: +44(0)116-221-7757

Email: gkc1@leicester.ac.uk

Llewellyn-Jones, David

Space Research Centre, University of Leicester

University Road, Leicester LE1 7RH

United Kingdom

Tel: +44(0)116-252-5238

Email: dlj1@leicester.ac.uk

Toshio Michael Chin

Jet Propulsion Laboratory

M/S 238-600, 4800 Oak Grove Dr.

Pasadena, CA 91109

Tel: +1 (818) 393-2510

Email: Mike.Chin@jpl.nasa.gov

\section{Matthew Martin}

Met Office, Fitzroy Road, Exeter, EX1 3PB

United Kingdom

Tel: +44 (0)1392 886465

Email: matthew.martin@metoffice.gov.uk

Alexey Kaplan

Lamont-Doherty Earth Observatory

Columbia University, P.O. Box 1000 / 61 Route

9W

Palisades, NY 10964-8000, USA

Tel: +01 (845) 365-8689

Email: alexeyk@ldeo.columbia.edu

Steinar Eastwood

Norwegian Meteorological Institute

P.O. Box 43

Blindern, N-0313 Oslo, Norway

Tel: +47 22963354

Email: s.eastwood@met.no

Tess Brandon

NOAA National Oceanographic Data Center

1315 East-West Highway

Silver Spring, MD 20910, USA

Tel: +01 301-713-3272 x181

Email: Tess.Brandon@noaa.gov 


\title{
R16 - REPORT FROM THE INTER-COMPARISON TECHNICAL ADVISORY GROUP (IC-TAG)
}

\author{
Matt Martin $^{(1)}$, Sasha Ignatov ${ }^{(2)}$ \\ (1) Met Office, Fitzroy Rd, Exeter,( United Kingdom), \\ Email: matthew.martin@metoffice.gov.uk \\ (2) NOAA/NESDIS/STAR, 5200 Auth Road, Camp Springs, MD. 20746, USA; \\ Email: alex.ignatov@noaa.gov
}

\section{Introduction}

A large number of level 4 (L4) sea surface temperature (SST) analyses are produced by various institutes around the world, making use of the SST observations provided by the Global High Resolution SST (GHRSST) project. These are used by a number of groups including: numerical weather prediction centres; ocean forecasting groups; seasonal forecasting systems; climate monitoring and research groups. There is a requirement to develop international collaboration in this field in order to assess and inter-compare the different analyses, and to provide uncertainty estimates on both the analyses and observational products.

The GHRSST Inter-comparison Technical Advisory Group (IC-TAG) has been set-up in order to coordinate inter-comparison activities of L4 products within GHRSST, develop inter-comparison systems, improve the documentation of those systems and promote the use of intercomparison tools for use by other TAGs where appropriate.

There are currently three systems contributing to the IC-TAG:

- The GHRSST Multi-Product Ensemble (GMPE) system (http://ghrsstpp.metoffice.com/pages/latest analysis Isst monitor/daily/ens/index.html) which is run on a daily basis at the UK Met Office.

- The High Resolution Diagnostic Dataset (HRDDS) system (http://www.hrdds.net) which runs at the National Oceanography Centre, Southampton.
- The SST Quality Monitor (SQUAM) system

(http://www.star.nesdis.noaa.gov/sod/s st/squam/) which runs at NOAA NESDIS.

The IC-TAG includes representatives from each of the L4 analyses producers which are contributing to GMPE, HRDDS and SQUAM, plus technical experts from the GMPE, HRDDS and SQUAM systems. The IC-TAG is chaired by Matt Martin, with Vice Chair Alexey Kaplan. Current members of the IC-TAG include:

Chelle Gentemann (RSS, USA), Helen Beggs (BoM, Australia), Dave Poulter (NOCS, UK), Eileen Maturi (NOAA/OSPD, USA), Bruce McKenzie (NAVOCEANO, USA), Jim Cummings (NRL, USA), Emmanuelle Autret (IFREMER, France), Shiro Ishizaki (JMA, Japan), Craig Donlon (ESA), Alexander Ignatov (NOAA/NESDIS, USA), Prasanjit Dash (NOAA/NESDIS/CIRA, USA), Nick Rayner (Met Office Hadley Centre, UK), Viva Banzon (NOAA/NCDC, USA), Robert Grumbine (NOAA/NWS, USA), Richard Reynolds (NOAA/NCDC, USA), Martin Rutherford (Royal Australian Navy).

\section{Progress since the last GHRSST meeting}

It was suggested at the previous GHRSST meeting that effort be put into producing summary information about the various $L 4$ products in GHRSST to inform users which product may be suitable for their application. A template for this summary information was sent round to the various L4 producers, some of which have been completed and returned. 
GHRSST XII -

Proceedings, Edinburgh

GHRSST Project Office

A two-part paper is being prepared for submission to a special edition of DeepSea Research II. The first part of the paper provides an overview of the L4 systems contributing to GMPE and SQUAM (based on the input provided by the L4 producers), and provides an intercomparison of the various systems in GMPE. The second part provides an overview of the SQUAM system and shows some inter-comparison and validation results.

An overview of progress this year with the IC-TAG systems is provided below, with some sample results from the paper in preparation.

\section{GMPE (Matt Martin)}

The GMPE system (run on a daily basis at the UK Met Office) takes inputs from various analysis production centres on a routine basis and produces ensemble products. The analysis systems currently contributing to the GMPE system are:

1. OSTIA (Met Office, UK);

2. NAVO K10 (Naval Oceanographic Office, USA);

3. MGDSST (Japan Meteorological Agency, Japan);

4. RSS MW (Remote Sensing Systems, USA);

5. RSS MW+IR (Remote Sensing Systems, USA);

6. FNMOC (Fleet Numerical Meteorology and Oceanography Centre, USA);

7. NOAA AVHRR OI (National Oceanic and Atmospheric Administration, USA);

8. NOAA AVHRR/AMSR OI (National Oceanic and Atmospheric Administration, USA);

9. CMC (Meteorological Service of Canada);

10. GAMSSA (Bureau of Meteorology, Australia).

During the past year, the GMPE system has been contributing to the European
Issue: Final

Date: September 27, 2011

MyOcean

project

(http://www.myocean.eu.org). Access to the GMPE data can be obtained by emailing the MyOcean service desk (servicedesk@myocean.eu.org). The data can be viewed interactively using a Web Map Service at http://data.ncof.co.uk:8080/ncWMS/godiva 2.html. The anomalies of products from the ensemble median are used as a monitoring tool to highlight when particular analyses are outliers. For instance, the GMPE is used on a daily basis to monitor the OSTIA system. The GMPE product disseminated through MyOcean currently has 94 registered users.

Inter-comparisons have been carried out using the various contributing $L 4$ analyses to the GMPE, and the GMPE median. The main aims were to assess the accuracy of the GMPE median compared to each of the individual $L 4$ analyses, to assess other aspects of the usefulness of these analyses including the horizontal resolution of features within each analysis, and to assess the possibility of using the standard deviation in the GMPE as a measure of uncertainty in the GMPE median estimate.

Near-surface Argo data have been shown (Merchant and Corlett, pers. comm.) to provide a good estimate of foundation SST using a triple-collocation of Argo data with surface drifters and AATSR data. None of the contributing L4 analyses to the GMPE use Argo as a data-source, so this data provide the basis for an independent validation of the accuracy and bias in the SST products

An example of the comparison with nearsurface Argo data is shown in figure 1. It is clear from this that the GMPE median provides a more accurate estimate of foundation SST than any of the input L4 products with an overall standard deviation of $0.40 \mathrm{~K}$ compared to the nearsurface Argo data and a bias of $0.03 \mathrm{~K}$. 
GHRSST XII -

Proceedings, Edinburgh

GHRSST Project Office

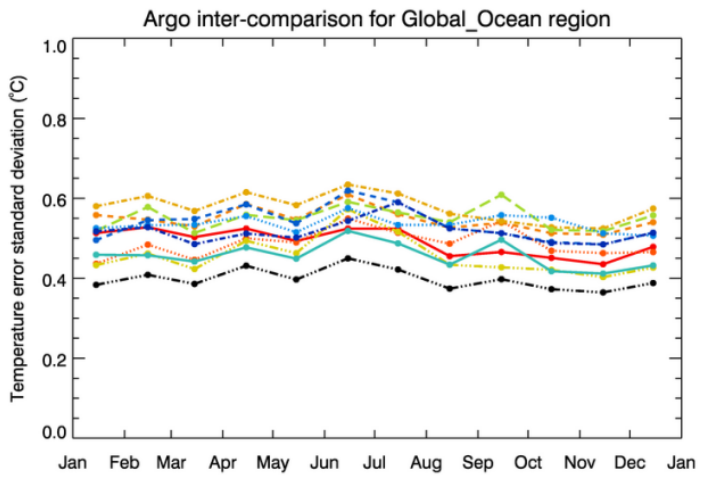

2010
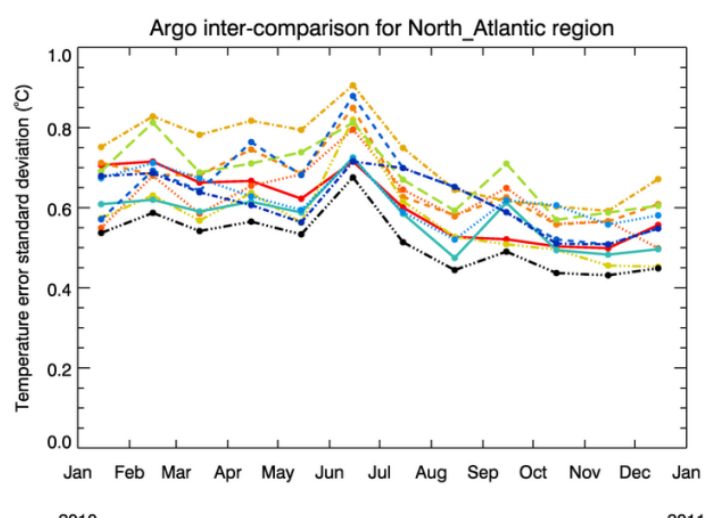

2010 Argo inter-comparison for Tropical_Pacific region 2011
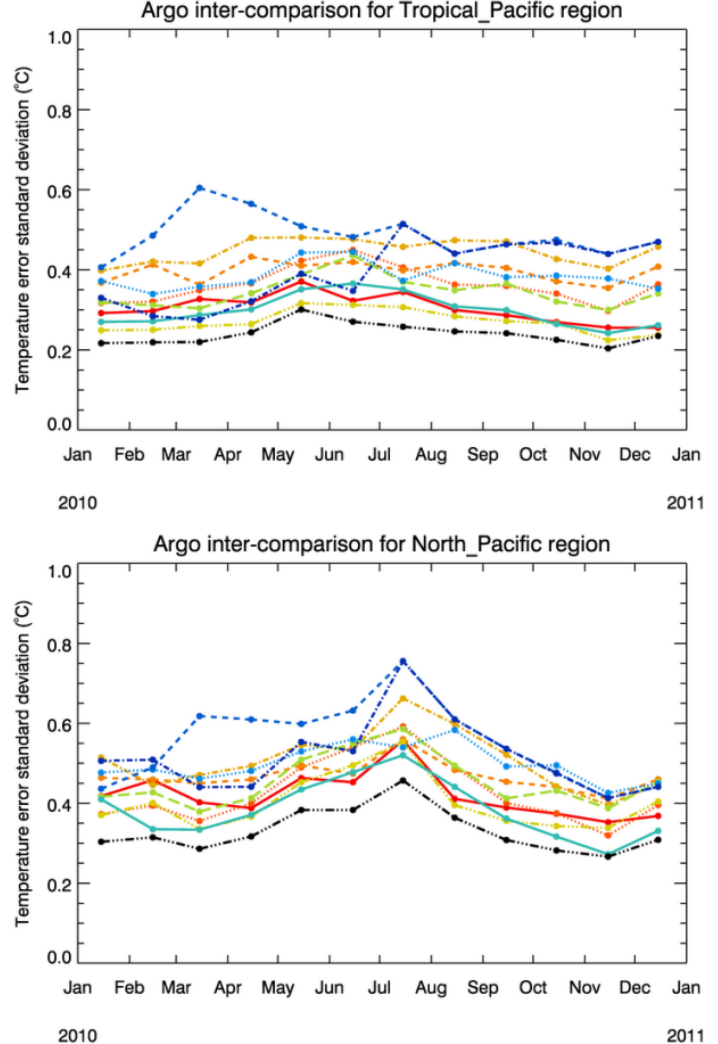

Issue: Final

Date: September 27, 2011
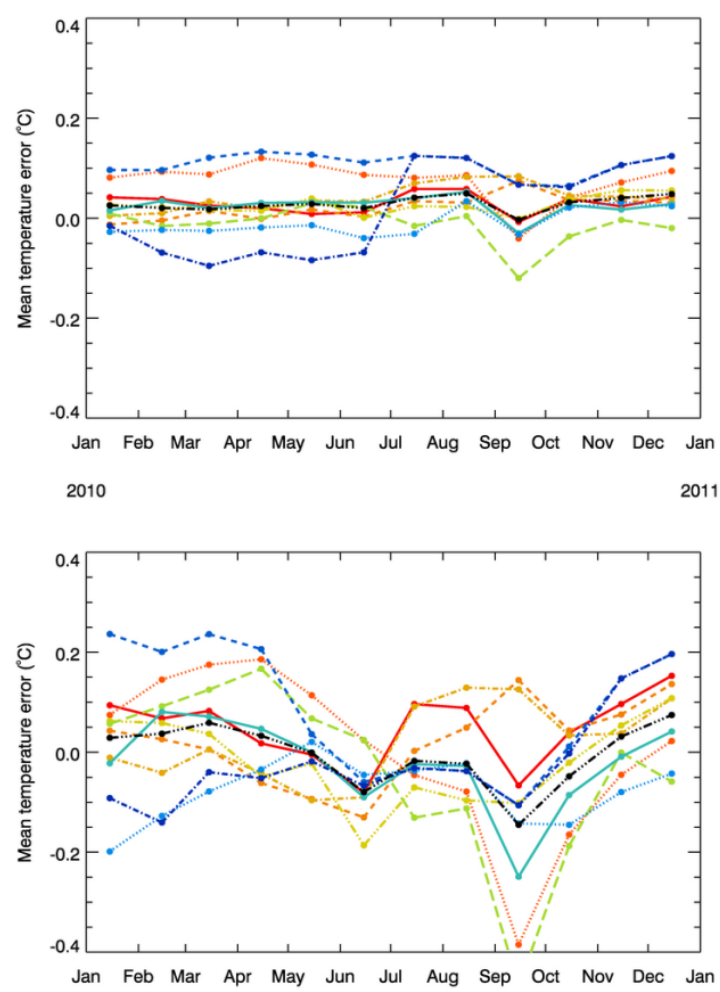

2010

2011
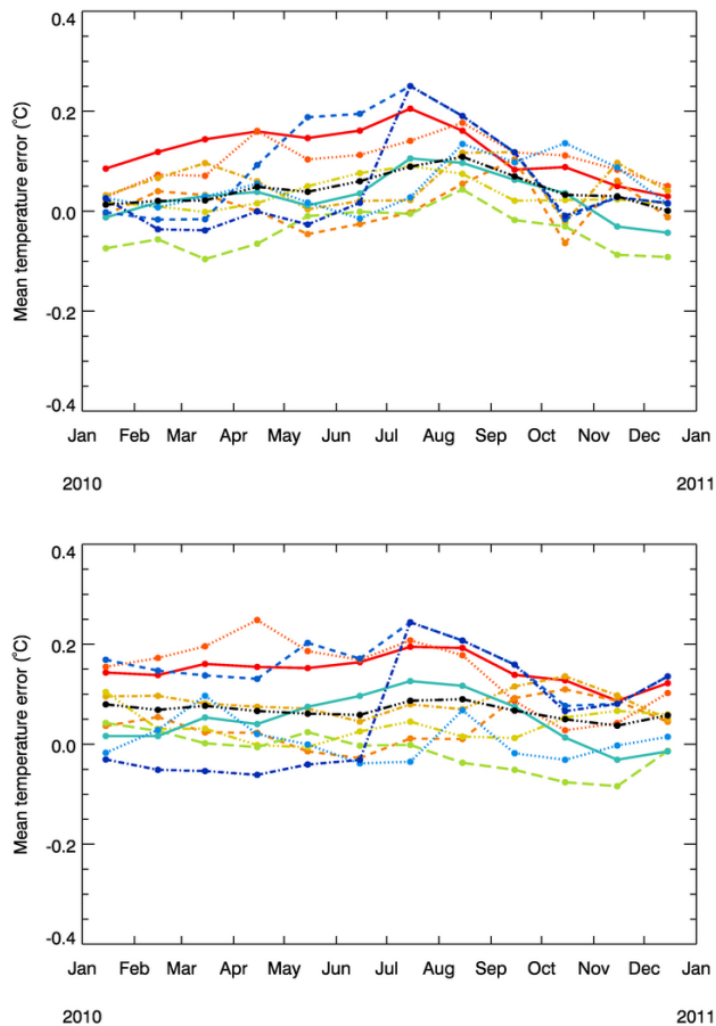
Figure 1. Standard deviation (left) and mean (right) differences between each L4 analysis (including the GMPE median) and near-surface Argo data calculated monthly during 2010. The statistics are shown for three regions: Globally (top), the North Atlantic (second row), the Tropical Pacific (third row) and the North Pacific (bottom). A legend showing which line corresponds with each analysis is shown to the right.

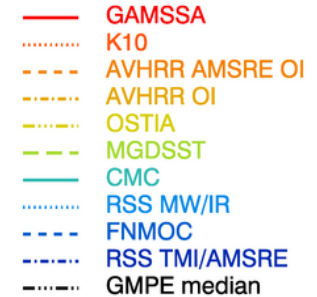

HRDDS (David Poulter)

SQUAM (Alexander Ignatov)

The SQUAM system is run on a daily basis at NOAA/NESDIS. It comprises three main modules: L2, L3, and L4. Only L4-SQUAM progress is documented in this report.

L4-SQUAM takes inputs from various analysis production centres on a routine basis and cross-compares various L4 products and consistently validates them against quality controlled (QCed) in situ data, including drifters, mooring buoys, and ships. The QCed in situ data are available from another online near-real time NESDIS syste, in situ Quality Monitor (iQuam,

http://www.star.nesdis.noaa.gov/sod/sst/iq uam/).

The analysis systems currently contributing to L4-SQUAM are (all are daily products):

1. Reynolds AVHRR (NOAA/NCDC, USA);

2. Reynolds AVHRR/AMSRE (NOAA/NCDC, USA);

3. RTG Low-Resolution (NOAA/NCEP, USA);

4. RTG High-Resolution (NOAA/NCEP, USA);

5. NAVO K10 (Naval Oceanographic Office, USA)

6. NESDIS POES-GOES Blended (NOAA/NESDIS, USA)

7. G1SST (NASA/JPL, USA);

8. OSTIA Operational (UK Met Office, UK);

9. OSTIA Reanalysis (UK Met Office, UK);

10. CMC (Meteorological Service of Canada);
11. GAMSSA (Bureau of Meteorology, Australia);

12. ODYSSEA (MERSEA, France);

13. GMPE (UKMO, UK).

During the past year, the L4-SQUAM system has been contributing to the Joint Center for Satellite Data Assimilation SST Project. Access to the SQUAM page is freely available to all users and does not require registration.

Inter-comparisons have been carried out using the various contributing $L 4$ analyses to the L4-SQUAM. The main objective of the L4-SQUAM web page is to facilitate feedback to L4 data producers about product performance, foster collaboration between various L4 groups towards products improvements and reconciliation, and assist L4 data users in identifying the most appropriate products for their specific needs. Differences between various combinations of L4 products, and their validation against iQuam in situ data, are reported as global maps, histograms, time series, and Hovmoller diagrams.

Specific L4-SQUAM progress since GHRSST-XI meeting include

- Adding 7 new L4 products to monitoring: NAVO K10, GMPE, POES-GOES blended, CMC02, G1SST, GAMSSA, and OSTIA Reanalysis

- Adding consistent validation against QCed in situ data (drifters, moored buoys, and ships) from iQuam (see Figs. 2-3)

- Adding sea ice analyses (see Figs. 23)

Effect of accounting for ice bits is shown in Figure 2 

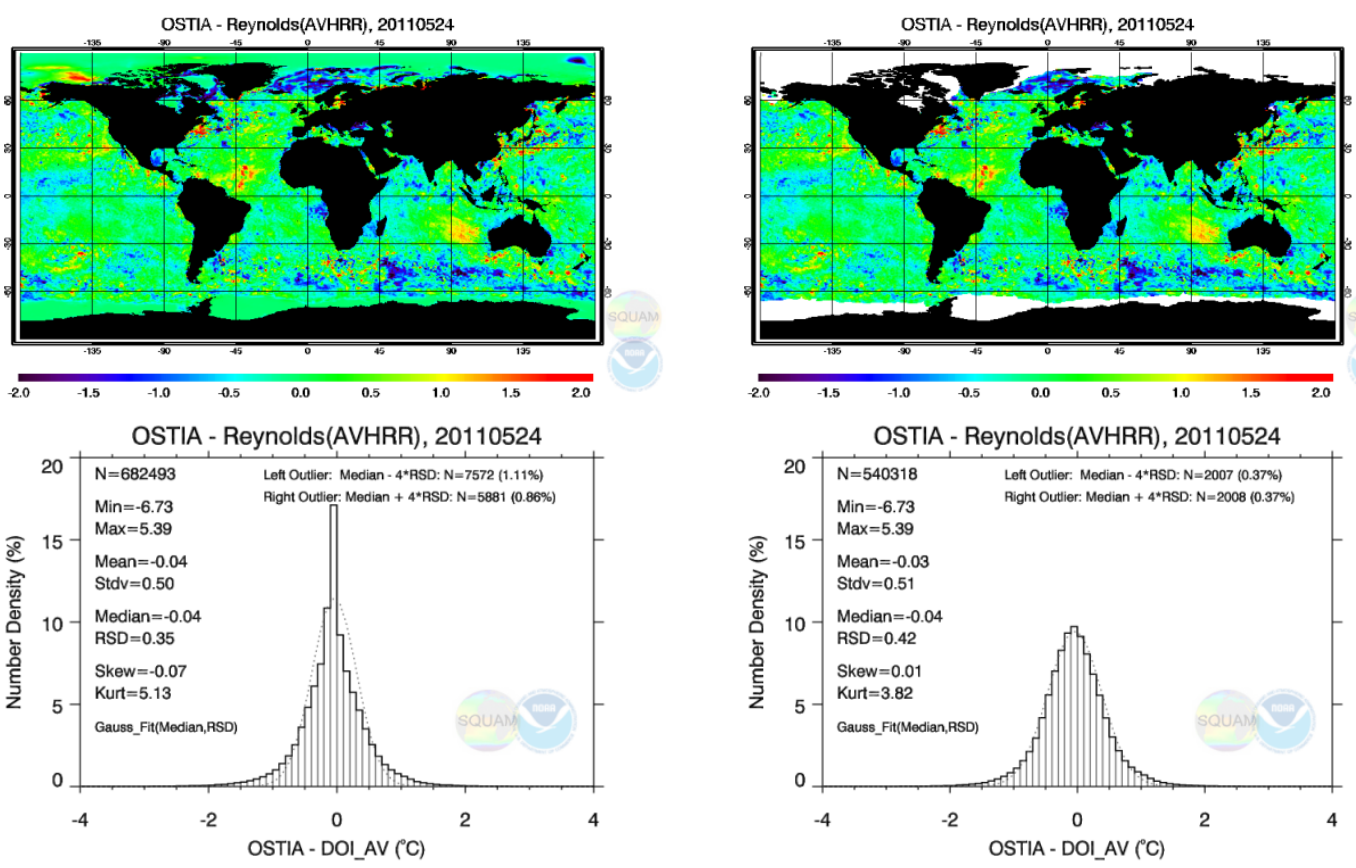

Figure 2. Maps and histograms of "OSTIA minus Reynolds" SST. Left panels: grids with ice mask set included in the comparisons. Right panels: ice pixels excluded. Note that histogram of difference becomes more Gaussian, because icy pixels with default SST value are excluded from comparisons.

Validation of various L4 data wrt drifters and comparison with GMPE is shown in
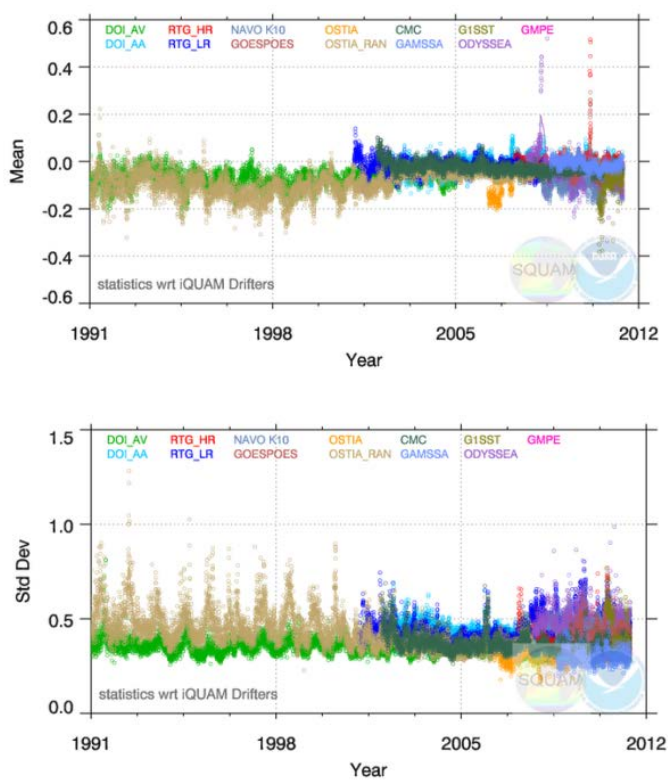

Figure 3
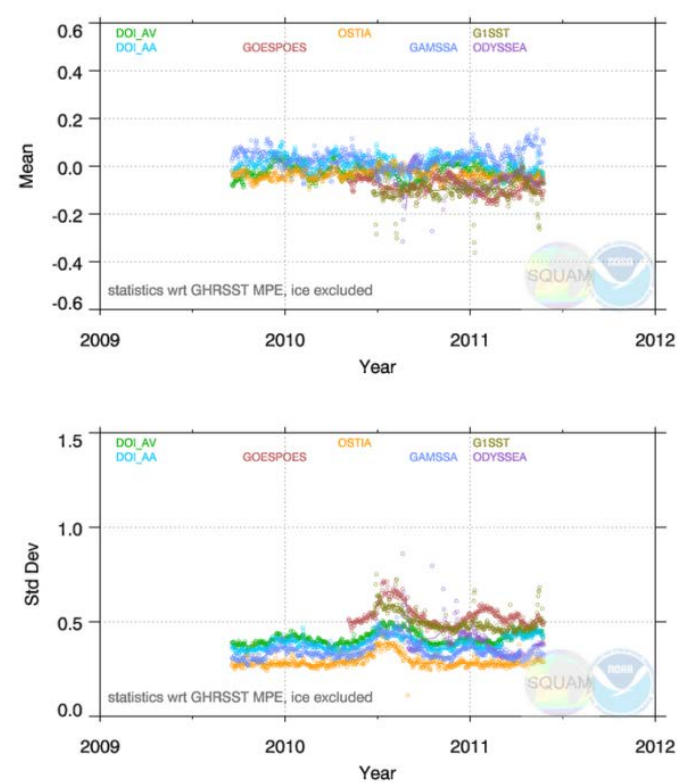

Figure 3. Left panels: Validation of various L4 fields against uniformly QCed in situ data (drifters) from iQuam. Right panels The same but wrt GMPE. 
GHRSST XII -

Proceedings, Edinburgh

GHRSST Project Office

\section{Activities and Plans 2011-2016}

\section{Short-term plans}

In the short-term, a number of activities are planned including:

- Obtaining the remainder of the summary information on the L4 systems and writing an overview for the GHRSST web-site, aimed at users;

- completing the two-part paper on GMPE and SQUAM;

- transferring the GMPE web-pages across to a new site, with a re-design of the pages;

- producing GMPE files which include anomaly and gradient information for each analysis;

- introducing new L4 products into GMPE and SQUAM;

- adding consistent validation against QCed in situ data for all products in SQUAM;

- adding remaining L2 SST products, including geostationary (GOES, MSG, MTSAT) and polar (MODIS, VIIRS, (A)ATSR, AMSR-E)

\section{Longer-term plans}

A number of issues have been raised over the past year with regard to the estimation of analysis error in the L4 products. A project should be started within the ICTAG aimed at investigating the way error estimation is currently done with a view to improving and potentially attempting to standardise it.

The GMPE system will contribute to the ESA SST Climate Change Initiative project. In this context, available long-term
Issue: Final

Date: September 27, 2011
L4 SST products will be combined into a version of GMPE.

Analyses will be conducted in SQUAM towards reconciliation of various SST products monitored in this system, through accounting for diurnal cycle and improving sensor calibration and characterization from which L2 SST products are derived.

Adding ARGO floats to iQuam, and extending time series from current 1991 to at least 1981 (AVHRR/2 era) are considered. Once accomplished, validation in SQUAM will be extended back in time for those products available prior to 1991 (Reynolds, OSTIA Reanalysis), and validation against QCed ARGO floats will be consistently added for all products.

\section{Funding}

The work for GMPE is currently funded by the MyOcean European project and by UK funding. It is hoped that this funding will continue in the follow-on project to MyOcean (called MyOcean2) which is currently under negotiation. Funding from ESA will also be available to begin work on a longer-term GMPE product.

The work for L2 SQUAM is currently funded under US GOES-R and JPSS Projects. L3-SQUAM work was not funded but was accomplished "at will". L4SQUAM was partially supported by the US Joint Center for satellite data Assimilation in FY11, and this support continues into FY12. Long-term sustained funding for L4SQUAM support is yet to be identified. 


\section{R18 - REPORT FROM THE SATELLITE SEA SURFACE TEMPERATURE VALIDATION WORKING GROUP (ST-VAL WG) \\ Prepared on behalf of ST-VAL by Gary Corlett}

University of Leicester, UK, Email: gkc1@le.ac.uk

\section{Introduction}

The Sea Surface Temperature Validation Technical Advisory Group (ST-VAL TAG) is responsible for defining and providing the Single Sensor Error Statistics (SSES) for GHRSST L2P products. In addition the group looks at all aspects of the validation process.

The current challenges for the group are to:

- Ensure the SSES Common Principles are implemented across all L2P data sets without compromising the data quality of any one data set.

- Better understand all elements of the SST validation process is required, including reference data quality, matchups limitations and the provision of meaningful uncertainty estimates to users.

Members of the ST-VAL group and others from the GHRSST community participated in a Joint Workshop of the DVWG, HLTAG and ST-VAL groups, which was hosted by the University of Colorado in Boulder on 28 February to $2^{\text {nd }}$ March 2011. The joint workshop was to recognise the common scientific issues of the three groups. Highlights from the validation part of the workshop are presented in Section 0.

The main issue that require the attention of the GHRSST Science Team at GHRSST XII is:

1. The use of Argo a reference data set: Much work has been carried out over the last year in demonstrating the capability of Argo sub-surface measurements (3-5 $\mathrm{m}$ depth) under favourable conditions to be used solely for validation of L2P and higher data products.
The science team needs to adopt Argo as a reference dataset for GHRSST work. Further details are given in Section 0.

The ST-VAL breakout during GHRSST XII will focus on two specific topics:

1. QC of drifting buoy data (see Section $0)$

2. GHRSST requirements for ship measurements of SST (see Section 0)

In addition the breakout will start the process of developing a work plan for the ST-VAL group for the period 2011 to 2016 (see Section 0).

\section{Main activities since GHRSST XI}

\subsection{Boulder Workshop}

This section contains a brief overview of what was discussed and presented at the workshop. Copies of presentations given during the workshop are available through the GHRSST website at https://www.ghrsst.org/documents/q/categ ory/ghrsst-science-team-meetings/dv-hlstval-workshop-2011-boulderl.

The current GHRSST reference source for providing Single Sensor Error Statistics (SSES) are drifting buoys, and all groups who provide SSES (or do any type of validation with drifting buoys) use a method of quality control that is unique to their processing system. Good quality control of drifting buoys is vital as this can have an effect on calculated difference to satellites of up to $0.2 \mathrm{~K}$. Several issues were discussed by Pierre Le Borgne (CMS) and Sasha Ignatov (NOAA), including their current blacklist approach, should blacklisted buoys be reused, and should a master blacklist be maintained by GHRSST. Clearly more work is needed on this issue and so it will be on one of the main topics discussed at the ST-VAL 
breakout at the next GHRSST science team meeting.

The subject of future validation requirements and opportunities was discussed in some detail. There were presentation on current and future radiometer deployments from Werenfrid Wimmer (NOCS), Bill Emery (UoC) and Peter Minnett (UoM), including an aircraft based radiometer that will be able to 'map' the spatial variability of the ocean skin within the satellite footprint as well as a report on the testing of the next generation M-AERI instrument. As well as radiometers, Helen Beggs (BoM) demonstrated the improved accuracy of the bureau's IMOS hull-mounted sensors compared to other VOS observations; and interesting point noted was the convergence of all non-radiometric ship observations below $50{ }^{\circ} \mathrm{S}$.

Presentations on AVHRR algorithm improvements and validation were given by George Paltoglu and Helen Beggs (BoM), whose new retrieval scheme focusing on the Australian region is achieving $\sim 0.25 \mathrm{~K} \mathrm{SD}$ to drifters at night time, as well as Bob Evans (UoM) on improvements in the next generation Pathfinder dataset, and Igor Tomazic (Rudjer Boskovic Institute, Zagreb) on a regional AVHRR algorithm for the Adriatic Sea using AATSR as a reference sensor. The issue of the validation uncertainty budget was discussed by Sandra Castro (UoC) and by the VS, who estimated the uncertainty in comparing a point measurement to a satellite pixel to be $\leq$ $0.1 \mathrm{~K}$ from two different approaches. Reports were also given by Owen Embury (UoE) on ARC validation results and by Anne O'Carroll on the latest IASI L2P product using 3-way statistics.

The afternoon was spent in two parallel breakout sessions:

1. Exploitation of future SST cal/val activiites by GHRSST (see Section 0)

2. Discussion of ESA SST_CCI project multi-sensor match-up dataset (MMD) and round robin algorithm selection process (RRDP). Further details on the MMD and the RRDP will be provided at their official launch as part of the EarWiG breakout session at GHRSST XII.

\subsection{The use of Argo as a reference dataset}

A key aim of the ST-VAL group is to create a global reference dataset that is solely used for validation and is not used in producing the L2P or higher products offered by GHRSST. The current choice for SSES is drifting buoys, but drifting buoy data is also used in retrievals and in bias correction for the analysis products. Other possible reference datasets include ship-borne radiometers, which are traceable to national temperature standards such as SI and are therefore essential for constructing climate data records, and the moored buoy network. However, the extremely limited number of ship-borne radiometers and limited coverage of moorings means neither is really suitable for validation of daily L2P and higher level products.

Initial work instigated by Chris Merchant (University of Edinburgh) has shown that Argo near-surface measurements offer great potential to provide this data; this initial work was promoted by a DWWG talk from Steven Riser during GHRSST IX who demonstrated high accuracy before and after deployment from several recovered floats. In addition, recent work by Castro et al. (2010) has demonstrated the use of research grade sub-surface measurement sin providing improved retrievals.

Initial comparisons to the AATSR sensor on Envisat has shown the intrinsic high accuracy of an Argo float (Riser reported $0.0005 \mathrm{~K}$ at GHRSST IX) means the total match-up uncertainty from comparing Argo SST-depth to AATSR SST-skin is comparable to that of ship-borne radiometers (which is a SST-skin to SSTskin comparison) under night time conditions (i.e. once any residual thermal stratification of the ocean surface has been mixed away) and is considerably 
during the day. A summary of the match-

Table 0-1

\begin{tabular}{|l|c|cc|c|cc|}
\hline \multirow{2}{*}{ Retrieval } & \multicolumn{3}{|c|}{ Drifters } & \multicolumn{3}{c|}{ Argo } \\
\cline { 2 - 6 } & Number & Median (K) & RSD(K) & Number & Median (K) & RSD (K) \\
\hline Day time & 40074 & +0.02 & 0.32 & 822 & +0.03 & 0.28 \\
Night time & 50790 & +0.00 & 0.35 & 701 & +0.00 & 0.29 \\
\hline
\end{tabular}

Table 0-1: Comparison of AATSR operational SSTs versus drifting buoys and Argo near-surface matchup results. Drifting buoy data from ICOADS; Argo data from Met Office EN3; match-ups limited to wind speeds $>6 \mathrm{~ms}^{-1}$; robust statistics; match-ups are nearest pixel within $+/-2 \mathrm{hrs}$

The results in Table 0-1 show consistent median differences using both drifters and Argo, but a significant reduction is seen for the RSD for both day and night matchups. This reduction is equivalent to removing $\sim 0.2 \mathrm{~K}$ from the match-up process, which is currently our best estimate of the drifting buoy uncertainty (the Argo uncertainty is $-0.005 \mathrm{~K}$ as reported by Riser at GHRSST IX).

The high accuracy Argo data allows us to better understand the geophysical uncertainty of comparing a single point in situ measurement to a 1-km satellite pixel. Although the above match-ups are not exactly coincident, their relatively similar global distribution and median differences allows us to adopt the approach of approach to O'Carroll et al. (2008) and use multi-way statistics to estimate the uncertainty of comparing a single point in situ measurement to a 1-km AATSR pixel to be $\sim 0.1 \mathrm{~K}$.

Further work looking at validating other sensors and products is of course required but the GHRSST Science Team needs to agree on adopting Argo as a reference dataset sooner rather than later.

\subsection{Ship observations}

A further useful dataset for ST-VAL are in situ ship measurements. Currently the GHRSST community has a very limited requirement for ship-borne SST data due to their geographical coverage and usually high measurement uncertainty. However, recent advances led by the Australian Bureau of Meteorology (see Helen Beggs presentation at Boulder) have shown that in situ ship measurements with comparable accuracy to current drifting buoys can be fairly easily obtained.

The ST-VAL group is coordinating with the JCOMM Ship Observations Team (SOT) to better define the GHRSST requirements for ship SSTs and lan Barton gave a presentation on behalf of GHRSST at the recent SOT meeting in Hobart. Ian will report on the SOT meeting as part of the ST-VAL breakout and an open discussion will be held to discuss and better define our future requirements for in situ SSTs from ships.

\subsection{Report from the GHRSST ST-VAL Breakout Session on Future Cal/Val Activities}

Prepared on behalf of ST-VAL by Helen Beggs and Peter Minnett

Participants: Peter Minnett, Anne O'Carroll, Yi Chao, Helen Beggs, Andrea Kaiser-Weiss, Werenfrid Wimmer, Xiaofang Zhu.

The following categories of future SST $\mathrm{cal} / \mathrm{val}$ activities were discussed in the context of GHRSST needs:

1. Ship-borne SST Radiometers 
More countries should be persuaded to operate SST radiometers on ships.

Peter Minnett said that without SST radiometers on ships we cannot obtain climate data records because you need radiometric ship skin SST to maintain long-term consistency between satellite SST sensors. MAERI has been logging SSTskin since 1995. Therefore prior to the mid-90's we cannot provide the basis to ensure the satellite SST form a consistent climate record with SI traceability. As a minimum, each satellite sensor should be validated once in an ocean region during its lifetime, although this is not optimal.

\section{Argo}

A detailed study of the utility of Argo floats for validating satellite SSTs should be carried out within GHRSST. $\mathrm{Yi}$ Chao said that the "thermal recharging floats" which are under development, and which have a potential indefinite lifetime for relatively rapidly repeating profiles near the surface, require a few degrees temperature differential to operate, so will not likely achieve this poleward of $50^{\circ}$ so may not be useful at high latitudes. But they do have a potential to provide useful data over much of the oceans. The cost per thermal recharging float would be around \$40k to \$50k.

\section{Drifters}

Thermistors should be better calibrated as currently data buoy thermistors are not individually calibrated prior to deployment. The data transmission format should also be changed to allow SST measurements to be reported at $0.01 \mathrm{~K}$ resolution. The addition of thermistors chains to measure diurnal heating would be of use to relate the surface measurements to temperatures at depth.

4. Ships
Peter Minnett suggests the possibility of using hull-contact sensors on VOS-Clim ships installed with automatic weather stations. We should also push for the periodic recalibration of engine intake sensors on volunteer observing ships.

5. Animals

Increasing numbers of good thermometers that transmit measurements by satellite are being attached to animals, including large marine mammals and birds, Evidence is accumulating that these measurements are of sufficient quality to be used for the validation of satellite-derived SST. At present these data are not readily available. Yi Chao said most of the CTD sea lion data is not available in real-time on the GTS.

\section{Existing campaigns}

There are often opportunities for SST validation cruises. In the coming year there are:

- Aquarius Mission - Salinity Validation Cruises

Yi Chao is a project scientist on the Aquarius mission, which is scheduled for launch in June 2011. Aquarius SSS derivation requires SST to an accuracy of $0.5 \mathrm{~K}$. Therefore we could write a proposal to obtain support to operate an $\mathrm{M}$ AERI on cruises to validate Aquarius SST. The Salinity Processes in the Upper Ocean Regional Study (SPURS) Field Experiment is planned for 2012 in the sub-tropical North Atlantic $\left(25^{\circ} \mathrm{N}, 28^{\circ} \mathrm{W}\right)$ and will have a seven day repeat. They will collect subsurface and surface observations. See http://spurs.jpl.nasa.gov

- March 2012 cruises: WHOI flux buoy (Bob Weller) PMEL mooring $(x 2)$ Seagliders (x3) 
Wave Gliders (x2)

STS floats (x25) - Steve Riser profiling floats

SSS drifters (x75)

ML floats (x2)

Microstructure Profiler

ASIP (used to be called SkinDeEP)

Flux bow mast

- July 2012: French cruise

- Fall 2012: UNOLS cruise

- Spring 2013: UNOLS cruise

Yi Chao suggested that an SST radiometer could go on one of these cruises. All cruise data will be available in near realtime to the public.

7. Dynamo Campaign (Indian Ocean)

- US ship may be Revelle.

- Japanese ship will be the Mirai.

- Peter Minnett may install a MAERI or ISAR on the US ships.

8. Repeating cruises

- PIRATA and TRITON: Peter Minnett will contact PI's to have an ISAR or M-AERI to be installed on these vessels.

- Helen Beggs suggested the possibility of having an ISAR or M-AERI installed on the MV Whana Bhum or Xutra Bhum.

- Peter Minnett can provide a document summarising the ship requirements for a M-AERI. Werenfrid Wimmer can provide a similar document for the ISAR.

9. How do we deal with combined skin/bulk SSTs?
- Ancillary surface forcing variables can be obtained from the following:

- AWSs on ships that are wellmaintained

- Scatterometer winds

- NWP assimilation fields

- Met flux moorings (see OceanSites web site)

\section{References}

Castro, S.L., Wick, G.A., Minnett, P.J., Jessup, A.T., Emery, W.J., 2010. The impact of measurement uncertainty and spatial variability on the accuracy of skin and subsurface regression-based sea surface temperature algorithms, Remote Sensing of Environment, 114, 2666-2678.

O'Carroll, A. G., Eyre, J.R., Saunders, R.W., (2008). Three-way error analysis between AATSR, AMSR-E, and in situ sea surface temperature observations. Journal of Atmospheric and Oceanic Technology, 25, 1197-1207

\section{Membership of STVAL}

The current members of the STVAL group are:

Sandra Castro, Jean-Francois Cayula, Gary Corlett, Prasanjit Dash, Steinar Eastwood, Bill Emery, Bob Evans, Chelle Gentemann, Andy Harris, Jacob Hoeyer, Alexander Ignatov, Alexy Kaplan, Pierre Le Borgne, Leon Majewski, Bruce McKenzie, Peter Minnett, Jon Mittaz, Chris Mutlow, Tim Nightingale, Anne O'Carroll, Jean-Francois Piollé, Igor Tomazic, Werenfrid Wimmer and Feng Xu.

Members of the in situ QC sub-group are:

Emmanuelle Autret, Steinar Eastwood, Jacob Hoeyer, Pierre Le Borgne, Alexander Ignatov, Leon Majewski, Matt Martin, Bruce McKenzie and Sonia Péré. 


\title{
R20 - REPORT FROM THE DIURNAL VARIABILITY WORKING GROUP (DVWG)
}

\author{
Gary A. Wick (Chair) \\ NOAA/ESRL/PSD, Boulder, CO 80305, US, Email : gary.a.wick@noaa.gov
}

\begin{abstract}
This report summarizes the recent activities of the Diurnal Variability Working Group (DWWG) in the period between the GHRSST XI and XII meetings and highlights our priority activities and goals. The overall focus of the DVWG is to provide the GHRSST community with critical science to enable the best possible characterization and estimation of diurnal warming in support of the creation of improved sea surface temperature (SST) products. Activities range from basic research to generation of analyses of diurnal warming.
\end{abstract}

\section{Achievements}

A key focus of the activities of the DVWG during the past year was the joint workshop of the DVWG, HL-TAG, and STVAL held at the University of Colorado, Boulder, USA February $28^{\text {th }}-$ March $2^{\text {nd }}$. The workshop enabled members of the DVWG to share their recent progress, coordinate ongoing activities, and interact with other groups analyzing critical data for the study of diurnal warming events and important applications for which information on diurnal warming is needed. This was the first joint meeting of the groups and the interactions proved extremely beneficial. DVWG group members were also involved in meetings and activities associated with the NASA SST Science Team and European
Research Network for Estimation from Space of Surface Temperature (ERNESST).

Recent scientific activities of the group can be broadly grouped into the areas of characterization of diurnal warming events, model development, evaluation and intercomparison of the models, and application of models and observations to the generation of diurnal warming analyses. The following briefly highlights progress in each of these areas. Presentations on many of the topics are available online in the documentation from the Boulder workshop.

Work led by S. Eastwood continues to explore the existence of significant diurnal warming in high-latitude regions. Events observed in multiple satellite datasets have been further compared with available in situ observations and evaluated for their relationship to factors such as wind speed, water column depth, and optical properties. Preliminary modeling studies have been undertaken to evaluate how well the observed warming can be predicted. The results suggest that, while diurnal warming can be simulated in conditions representative of the high latitudes, the amplitudes are still not equal to what is observed (Figure 1). A paper on this work is to be submitted. 


\section{Modeling Arctic DW}

\section{Observed SST 21.06.2010 Modeled SST}
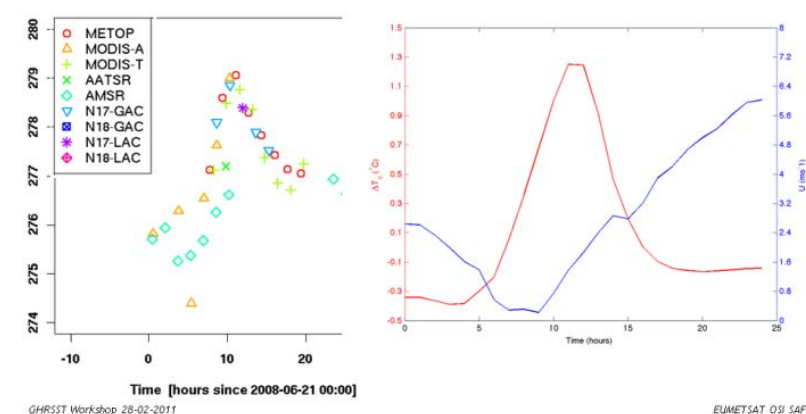

Figure 1. Example of observed and modelled diurnal warming in the arctic. The observed amplitude is 2.5-3K while the modelled amplitude is $1.6 \mathrm{~K}$. Courtesy S. Eastwood.

A new project by $X$. Zhu and $P$. Minnett has begun to study in detail diurnal variability in shallow coastal waters. The work incorporates extensive new observations, analyses of correlations to key forcing parameters, and preliminary simulations with parameterizations and 1$D$ models. Under the unique conditions, the relationship between warming and the wind speed was found to be complex suggesting challenges in accurately predicting its magnitude.

An important ongoing activity that cuts across the areas of diurnal warming characterization and model evaluation is the Tropical Warm Pool diurnal variability experiment (TWP+). Led by $\mathrm{H}$. Beggs, this effort has compiled SST observations and model fields of parameters such as winds and fluxes for detailed study of diurnal variability in tropical regions. The domain for the study is $25^{\circ} \mathrm{S}$ to $15^{\circ} \mathrm{N}, 90^{\circ} \mathrm{E}$ to $170^{\circ} \mathrm{E}$ over the periods 1 January to 30 April 2009 and 1 January to 30 April 2010. Compilation of the data was completed this year and plans were developed for multiple analyses including event characterization, model validation, and impact assessments. The domain and key elements of the data set are shown in Figure 2. The experiment should be a major topic of discussion at GHRSST XII.

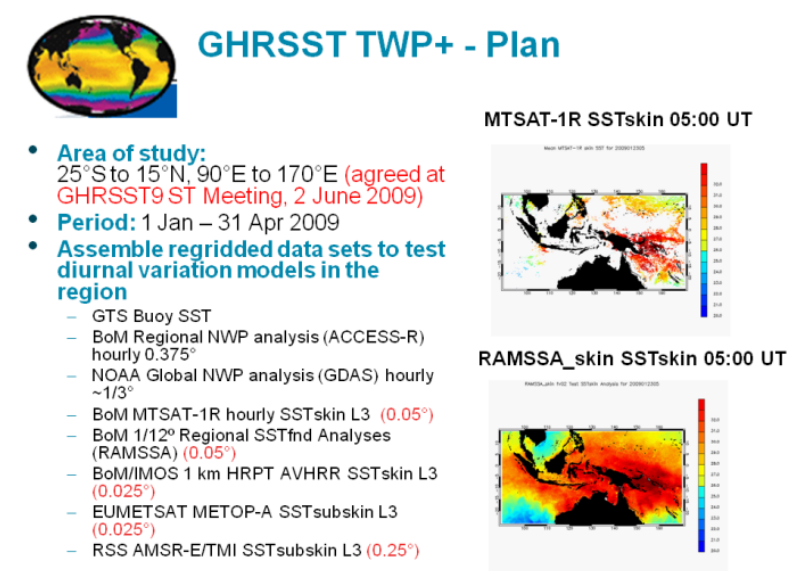

Figure 2. Domain and components of the TWP+ experiment. Courtesy H. Beggs.

Model development and evaluation efforts are ongoing by multiple members of the
DVWG. C. A. Clayson conducted new testing of the performance and stability of 
the Kantha-Clayson diffusion model applied to near-surface warming simulations and used the results to construct a new simplified parameterization that better captures large amplitude events. R. Weihs has been working extensively with the POSH model and has identified several modifications in cooperation with C. Gentemann. A new release of the model is planned for the near future. Access will require registration so that the users can be informed of any future changes to the model. G. Wick is continuing to lead an effort to intercompare the performance of several diurnal warming models using both idealized forcing and cruise observations. This work, to be completed this year is significant for its provision of additional information on expected uncertainties in estimates of diurnal warming.

A high priority goal of the DVWG (see also below) is to facilitate production of diurnal warming analyses providing regional and/or global fields of the warming at specified depths and temporal intervals to complement current daily SST analyses. While more work is still required in this area, important progress is being made. A. Harris is continuing work to explore use of a reduced resolution version of the Kantha-Clayson model for producing global estimates of diurnal warming. $\mathrm{R}$. Weihs has run the POSH model with forcing fields from the MERRA reanalysis at 0.25 degree resolution to create global diurnal warming fields. Peak modeled warming varied from day-to-day but was generally less than $4 \mathrm{~K}$. The results were also used to demonstrate the impact of inclusion of the diurnal cycle in estimates of the latent and sensible heat fluxes. Over the period studied, failure to include diurnal variations resulted in the underestimate of the latent heat flux by 2 $4 \mathrm{~W} / \mathrm{m}^{2}$ on average with peak differences of $6-7 \mathrm{~W} / \mathrm{m}^{2}$ (see Figure $\mathrm{X}$ ). Work to utilize SEVIRI data to create operational diurnal warming analysis in the Atlantic and Mediterranean regions is underway and $P$ LeBorgne will report in more detail at GHRSST XII.

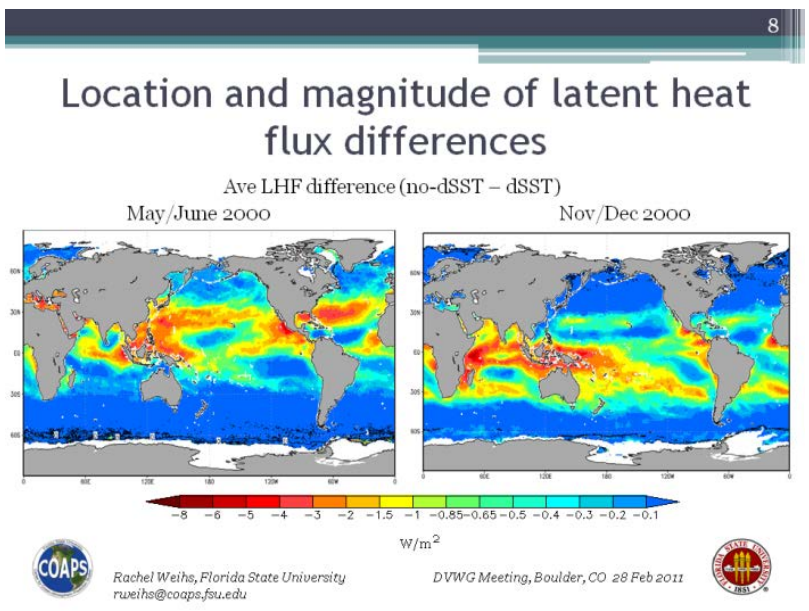

Figure 3. Illustration of impact of diurnal variations on estimates of the latent heat flux. Courtesy R. Weihs.

Important discussions took place between those developing models for diurnal warming and producers of Level 4 analyses who could utilize these tools in the generation of their analyses. Presented material highlighted some of the limitations and corresponding accuracies of current diurnal warming models when applied to global operational forcing fields. While better incorporation of estimated diurnal warming in analysis generation is highly desired, many of the producers were still uncomfortable with the current uncertainty levels. More work is required to conclusively demonstrate the positive impact of modeled warming estimates across a broad range of analysis approaches. 


\section{Priorities and Plans}

The DWWG priorities are centered on providing the GHRSST community and users with improved estimates of diurnal warming both for individual satellite retrievals and on global grids to complement current foundation SST analyses. Significant progress has been made in improving the understanding and modeling of diurnal warming, and now it is important to apply these results to better convey diurnal warming estimates and associated uncertainties in a cohesive manner. Specific priorities include:

- Development, validation, and production of diurnal warming analyses

- Continued model development and evaluation

- Provision of guidance on recommended/consensus approaches

- Improved web presence facilitating access to diurnal warming models, estimates, and uncertainties

A key product missing from the GHRSST suite is global/regional fields of estimated diurnal warming at specified times and depths for users requiring information on diurnal warming in their applications. While some information is present in individual retrievals, our analyzed fields focus on providing daily average or foundation values. Provision of either initial operational fields or recommended methodologies is perhaps the single greatest priority of the group. Further user input on the ideal information content of these products is still required.

To support these analyses, as well as provision of information on the amount of diurnal warming present in individual L2P products, model development and evaluation activities should continue. A necessary component of this work is to develop better estimates of uncertainties in predictions of diurnal warming.

As model development continues, it is important for the DVWG group to convey information on the applicability and capabilities of the different available models. While it is unlikely there is a single one-size-fits-all diurnal warming model that GHRSST should endorse, we should utilize our collective experience to highlight our recommendations on what the models can and cannot do and what models work best under different circumstances.

One way to better achieve this objective is to improve the information content of the DWWG web pages. Through these pages it is desirable to facilitate improved access to the different available models, test scripts for their application, information on their associated uncertainties, and available validation data. Activities have begun in this area but should be a continuing process as new models and results become available.

Application of the TWP+ data will be a key focus of DWWG activities in the coming year to pursue these objectives.

A common theme that has emerged from the various model development and testing activities is that there is still relatively limited direct observational data of large diurnal warming events with which the models can be validated. Several group members have cited the need for additional accurate observations accompanied by a full set of coincident environmental data with which the different models can be forced. The possibility of calling for or endorsing a dedicated experiment to collect the required observations has been discussed. The group is planning to pursue preparation of a white paper to document our requirements, provide justification for our needs, and explore possible experimental concepts. As this is completed, the DWWG will work to circulate the document and look for opportunities to include the concepts in ongoing or upcoming field programs.

The activities of the DVWG are largely performed on a best-effort basis under broader support for SST related research activities. Few within the group have funding dedicated specifically to diurnal warming research. The group remains highly dedicated, but progress will remain 
GHRSST XII -

Proceedings, Edinburgh

GHRSST Project Office

constrained by available time and resources.

\section{Our Users}

Overall, the DWWG seeks to serve the entire GHRSST team and associated user community through provision of enhanced information on diurnal variability in the SST. In the short term, however, specific subsets of the user group have demonstrated the most immediate needs for diurnal warming estimates. These groups include those producing the L4 analyses and those conducting research on air-sea interactions and climate variability where failure to include modulation of the SST by diurnal processes can result in significant biases in their results. To include daytime data influenced by diurnal warming in L4 analyses, estimates of the amount of warming present in each retrieval and its associated uncertainty are required. Improved models providing more accurate warming estimates and more detailed uncertainties should enable inclusion of additional data in the analysis providing
Issue: Final

Date: September 27, 2011

improved coverage and better accuracy. An increasing body of research is demonstrating the value of incorporating resolution of diurnal signals for applications such as improved forecasting of the Madden-Julian Oscillation. Improved models can continue to be incorporated into weather and climate models to improve their forecasts, and diurnal warming analyses will facilitate better inclusion of diurnal variability in observationally based analyses. As applications of SST continue to increase in complexity, it is anticipated that a growing segment of the user community will seek more detailed information on diurnal variability.

A complete understanding of the detailed requirements of the external science and user community for information on diurnal warming is still being solicited. As this information is gathered, the DVWG will work to support the needs of the broad user base. 


\title{
R21 - REPORT FROM THE APPLICATIONS AND USERS SERVICES TECHNICAL ADVISORY GROUP (AUS-TAG)
}

\author{
Jorge Vazquez $^{(1)}$, Edward Armstrong ${ }^{(1)}$, Toshio M. Chin ${ }^{(1)}$
}

(1) Jet Propulsion Laboratory, California Institute of Technology, 2800 Oak Grove, Pasadena, CA 91109, Email: jorge.vazquez@jpl.nasa.gov

\section{Version 1.1}

\section{ABSTRACT}

Major accomplishments for the year 2010 were final revision of the User's Manual, along with the implementation of Tools and Services that included the Dataminer which allows for subsetting of MODIS Aqua, MODIS Terra, and AMSR-E GHRSST Level 2 Preprocessed data, implementation of the GHRSST forum, consolidation of AUS-TAG membership, and implementation of the "State of the Ocean" (SOTO) tool. A GHRSST forum has also been implemented. Each of these will be discussed below in the context of contributions to the GHRSST project. All the tools have been implemented at the GDAC.

\section{GHRSST Forum}

A GHRSST Users forum has been setup. Individual groups have been setup based on the Technical Advisory Group and Science Team function. The forum and registration may be accessed at:

\section{http://podaac.jpl.nasa.gov/forum}

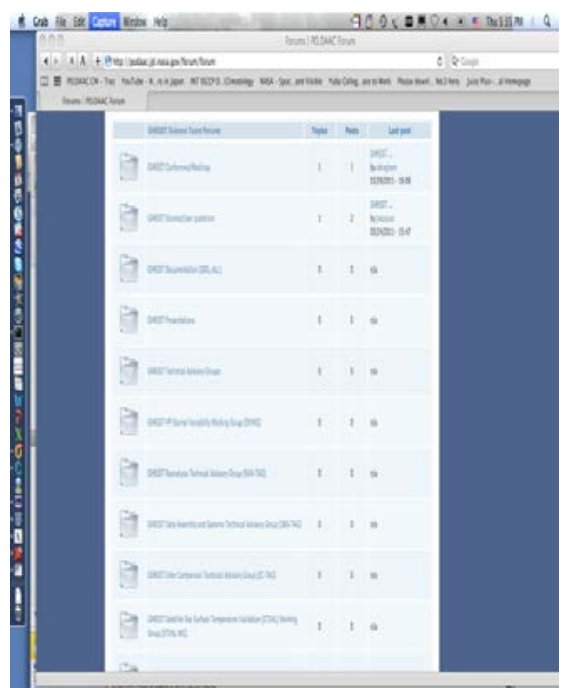

2. Accessing data: data mining tool
New technology has been implemented at the PO.DAAC that allows for spatial and temporal subsetting of GHRSST L2P data. MODIS Aqua and MODIS Terra data have been added to the available data sets. Thus, three GHRSST data sets are now available through the Dataminer and include MODIS Terra and Aqua and AMSR-E. A beta version is available for testing at:

http://podaac-tools.jpl.nasa.gov/dataminer095/

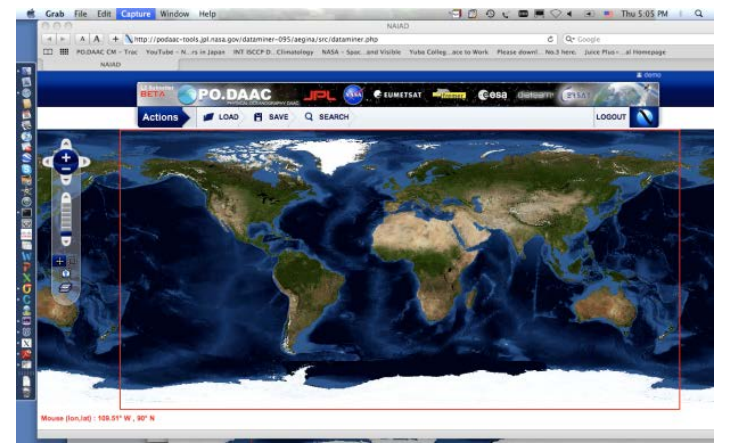

Dataminer is a web tool for searching and subsetting Level 2 (swath) data. It was developed originally by the French agency IFREMER (an upcoming collaboration is in the works), and modified at PO.DAAC.

An overview of the capabilities of this tool:

- Easily search for Level 2 (swath) data based on a spatial bounding box and time range

- Additionally filter your searches using basic statistical metadata collected from the original data ( $\min , \max$, etc)

- Get an image preview of your search results before downloading the raw data, with a colorbar for reference 
The PO.DAAC implemented a new visualization tool for viewing data in near real-time. The tool may be accessed at:

http://podaac-tools.jpl.nasa.gov/soto/

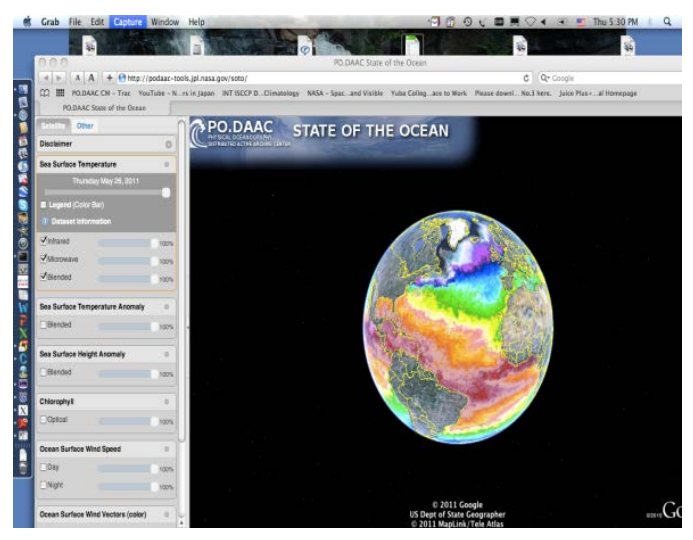

Current data sets included AMSR-E, MODIS, and blended product.

The tool allows for overlaying of data sets. Additionally in-situ is incorporated which can be overlaid with other SST data sets and/or winds and current data.

\section{User's manual}

A revised draft of the User's Manual has been reviewed. Changes have been implemented. The Users Manual, once approved, will be accessed through the GHRSST web site and the GDAC.

\section{Facilitation for New Users}

GHRSST now supports a wide range of products and services, but this can be confusing for users just "dropping by" the GHRSST website only to download SST data for their particular needs. For example, a marine biologist may sometimes not have the same computer resources as well as background in spacebased remote sensing that the traditional users/supporters of GHRSST typically possess. To channel their needs/questions, the AUS-TAG has started planning an introductory webpage (currently code-named "one-pager") for the new GHRSST users

that can be accessed easily from the main www.ghrsst.org webpage. It packages the information that a new user may need to choose, download, and read the GHRSST data as quickly as possible. 
The one-pager contains short descriptions of the following topics along with the pointers to theexisting GHRSST webpages for more details:

1) What GHRSST offers: We currently focus on core products including L2P, L4, and L3.

2) What the SST data are: There are two parts: one is an educational component on how space-based SST measurements varies due to boundary layering and diurnal warming (depth and diurnal issues); the other one is a list of resolutions, time periods, variable lists, etc for each product.

3) Where/how to download the data sets: links to GDAC and LTSRF and the tools that these distribution centers offer

to shorten the download time/effort.

4) How to read the GHRSST data sets: netCDF is not simple for uninitiated; we have so far provided template codes to read $L 2 P$ and $L 4$ product files in $C$, FORTRAN, MATLAB, IDL.

\section{Conclusions}

Users of GHRSST data continue to increase. With the emergence of the historically reanalyzed products, there has been a significant increase in using GHRSST data for scientific research. Near-real time capabilities continue to emerge, specifically in numerical weather forecasting and fisheries. New technologies, such as the dataminer, have been implemented which allow for temporal and spatial subsetting.

Challenges still remain which must be addressed for maximizing the use of GHRSST data. These include how to collect metrics on usage from such tools as the dataminer and THREDDS. Implementation of netcdf4 for use in THREDDS and OPenDAP. Additionally a dashboard concept needs to be developed where users can get near real time information on the status of data streams. Plans need to be formulated for both of the above.

\section{APPENDIX I: Terms of Reference}

Terms of Reference

Applications and User Services Technical Advisory Group (AUS-TAG) for the Group for High Resolution Sea Surface Temperature

\section{Version 0.5}

The Applications and User Services Technical Advisory Group (AUS-TAG) of the Group for High Resolution Sea Surface Temperature (GHRSST) was formed at the 10th meeting of the GHRSST Science Team (GHRSST-ST) in Santa Rosa California, June 1-5, 2009. The GHRSST-ST voted to form the group as a result of the need to consolidate and facilitate better communications for user's and application's support within the GHRSST science and user community.

\section{RESPONSIBILITIES}

- Manage all aspects of the GHRSST User Manual.

o This includes overseeing all new versions, providing periodic reviews, as deemed necessary by the science team, and maintaining the latest version to the user community

- Maintain and develop methods for data discovery within the GHRSST R/GTS.

o This includes making recommendations to the science team on new technologies that could improve data access and usability. Will also work closely with the data management technical advisory group in the 
GHRSST Project Office

implementation of these new technologies.

- Actively solicit (using acceptable outreach tools, workshops, symposia, brochures etc) catalog and publish GHRSST user feedback in order that the groups within GHRSST may act on feedback.

- Help coordinate and facilitate GHRSST users symposium, as deemed appropriate by the GHRSSTST. This does not include chairing the event. Such chairs will be appointed in coordination with the GHRSST-ST Science Chair.

- Develop new methods of user documentation

o This will include working closely with and facilitating communication between the different technical advisory groups, such as the Diurnal Variability, Inter-comparisons technical advisory group and others.

\section{MEMBERSHIP}

Chair and Vice-Chair of the AUS-TAG shall be appointed by the GHRSST-ST. Term of chairmanship is at the discretion of the GHRSST-ST and will be reviewed periodically by the GHRSST-ST and science chair. Membership of the AUSTAG shall be done on volunteer basis, appointed by the chair and co-chair, or by the GHRSST-ST. There will be no limits on the term of membership 


\title{
R22 - REPORT FROM THE GHRSST HIGH LATITUDE - TECHNICAL ADVISORY GROUP
}

\author{
Prepared on behalf of GHRSST HL-TAG by Jacob L. Høyer
}

Danish Meteorological Institute, Email: jlh@dmi.dk

\section{Introduction}

The HL-TAG was formed at the GHRSST 10 science team meeting in Santa Rosa in 2009.

The main focus of the HL-TAG group is:

- The validation of existing surface temperature and sea ice products in the high latitude

- The development of new products, e.g. in the Marginal Ice Zone (MIZ).

- Follow the diurnal warming at high latitudes and the development of SST and Sea ice in the high latitudes.

The first HL-TAG meeting outside the science team meetings was help in Copenhagen, March 2010. During the last year, a second HL-TAG meeting was held in Boulder in Feb-March 2011 in collaboration the Diurnal Variability Working Group (DV-WG)and the SST validation (ST-VAL) working group.

\section{Progress during the last year}

Most of the progress in the HL-TAG group was reported at the meeting in Boulder. The report is therefore based upon the minutes from the meeting.

From the Boulder meeting:

Validation studies have shown that the Metop_A SST data processed by CMS had a positive bias of up to 0.4 degrees in the summer. Pierre LeBorgne has looked into the split window algorithm performance in relation to atmospheric profiles. The positive errors of the split window algorithms have been analysed with the HIRLAM atmospheric profiles. They are likely induced by anomalous profiles and the use of RTTOV indicate that using the radiative transfer models can correct for the bias. CMS has plans of implementing the use of NWP and RTTOV on their operational chains in 2012 for the European Seas and 2014 for global ocean.
Peter Minnett presented three approaches towards developing alternative algorithm for SST IR retrievals:

- Genetic Algorithm (GA) based equation discovery - to derive alternative forms of the correction algorithm

- Regression tree - to identify geographic regions with related characteristics

- Support Vector Machines - to minimize error using state-of-the-art non-linear regression

The GA approach used a Darwinian approach to mutate among different operators and equations to reach a new algorithm that performed better that the old one. The method was applied to the MODIS matchup database and the success criteria was the RMS error when compared to buoy data. The derived algorithm was very similar to the NLSST algorithm, but also identified a scan mirror effect, which is an instrument effect that should have been corrected for.

The regression tree algorithm identified different regions with similar error characteristics. The regions looked in general as expected with latitudinal zones, except some regions, which had a more longitudinal structure. The SVM results do not out-perform GA and Regression Tree algorithms

Jacob Hoeyer has performed validation activities in the Arctic Ocean including 6 different GHRSST L2P satellite products for the Arctic Ocean (AATSR, METOP_A, MODIS AQUA, MODIS TERRA, AMSRE, NAVOCEANO-GAC), north of 60 Deg $N$. The results showed some interesting bias variations in AMSRE and METOP_A and generally concluded that the AATSR and NAVOCEANO-GAC data were the best quality data set for the Arctic. Satellite Error scales were calculated to be about 1- 
GHRSST XII -

Proceedings, Edinburgh

GHRSST Project Office

2 days and $300-500 \mathrm{~km}$, for spatial and temporal scales, respectively.

Florence Fetterer from NSIDC presented their current ice products and plans to do a sea ice reanalysis. NSIDC has a new Multisensor analyzed sea ice extent product called MASIE (Nsidc.org/data/masie), which has a spatial resolution of $4 \mathrm{~km}$ and may be an alternative to the ice products currently used in GHRSST.

Rasmus Tonboe from DMI presented the simulations results from a combined thermodynamic model and microwave emissivity model that simulates the microwave emission from multiyear sea ice. The results indicated that the $6 \mathrm{GHz}$ emissivity is by far the most constant and that the penetration depth was a function of the salinity and the temperature of the sea ice.

Jonah Robert Jones from UK Metoffice looked at how sea ice was treated in the OSTIA level 4 reanalyis and a few corrections to the operational algorithms has been made. The OSTIA ice extent was compared to other sea ice products and was found to have to large a sea ice extent. It was commented that the use of 1.8 as the freezing temperature for Sea ice is not representative for all regions in the Arctic Ocean and that a the use of climatological salinity maybe would give better a ice extent.

Mike Steel showed results from the analysis of the unusual ice melting and the oceanographic conditions in the Beaufort Gyre, where a near surface temperature maximum was identified under the ice, due to solar penetration through a thinner ice. Mike also presented his situ observations plans in the Arctic Ocean this summer. They included Three Metocean Uptempo buoys, profiling floats and wave gliders to be deployed north of Alaska. All data will be available on the Cadis AON (http://aoncadis.ucar.edu/).

Helen Beggs analyzed different satellite products covering the Southern Ocean and found persistent and systematic biases in may of the products. In particular, the level
Issue: Final

Date: September 27, 2011

4 products, which were only based upon Infrared observations showed a systematic bias in the vicinity of the ice edge, due to the persistent cloud cover in this region.

The NOAA/NESDIS SST Quality Monitor tool (SQUAM) was presented by Prasanjit Dash. The tool is a near-real time tool to monitor the performance of the global SST products and is very efficient in comparing the satellite level 2,3 and 4 products to in situ observations, such as drifting buoys, moored buoys and ship observations. In addition, the different products can be compared against each other and double differences can be calculated. The different statistics can be displayed in several ways, such as time series, histograms, Hovmüller diagrams etc.

\section{Activity plan for 2011-2016}

The HL-TAG group has not made specific activity plans for the year 2011-2016. This will be discussed during the science team meeting in Edinburgh.

The development within the high latitudes will be linked to projects, which have already been funded. A few of these projects are mentioned below:

ESA Climate Change Initiative (CCl) project on SST. Within this project, research and development will include: high latitude SST development and testing, Marginal Ice Zone SST and Improved high latitude cloud and ice detection

Within the Ocean and Sea Ice Satellite Application Facility (OSI-SAF) the next 5 years will focus upon improving the existing global sea surface temperature products and to develop the ice surface temperature algorithms for the Metop satellites.

A small MyOcean R\&D project on the Multisensor bias adjustment methods in the Arctic ocean will be carried out within the next year. The project is administrated by $\mathrm{J} L$ L. Høyer and include $\mathrm{P}$. LeBorgne and S. Eastwood. The work will focus upon using several satellite sensors for improving the operational satellite L2P products in the Arctic Ocean. The method 
GHRSST Project Office

will be used to improve the Level 4 SST production within MyOcean.

A research network for surface temperature in the UK has recently been funded. The activities in the second year of the project will focus upon quantifying surface temperature (land, sea and ice) across the Arctic. The network is coordinated by C. J. Merchant, J.J.Remedios and N. A. Rayner.

In the Southern Ocean, the Bureau of Meteorology will continue their algorithm development and validation activities within the Integrated Marine Observing System
(IMOS) project to reprocess and improve the $1 \mathrm{~km}$ HRPT observations.

Within Sea ice, The National Snow and Ice data Center has planned a sea ice reprocessing effort, which will require input from the HL-TAG group. The reprocessing project will therefore be followed closely and the HL-TAG group will provide input as a user group of the products.

\section{Main users}

The main users of the products and results from the HL-TAG are the operational forecasting institutions (ocean and atmosphere) and climate scientists. 
R23 - REPORT FROM THE ESTIMATION AND RETRIEVALS WORKING GROUP TO THE $12^{\text {TH }}$ GHRSST SCIENCE TEAM MEETING Prepared on behalf of EARWiG by Andy Harris

NOAA-CICS, University of Maryland, Cooperative Institute for Climate Studies, College Park, Maryland, USA, Email:Andy.Harris@noaa.gov

\section{Introduction}

The Estimation And Retrievals Working Group is tasked with investigating all aspects of retrieving sea surface temperature from satellite-observed radiances. This is certainly an innovation since the original focus of GHRSST was to characterize uncertainties in existing SST products. However, recent advances in the field mean that it is time to revisit the main issues and explore various techniques with the hope of at least increasing physical understanding and, sooner or later, being in a position to provide best practice recommendations for data providers.

Major aspects under consideration include:

- Instrument calibration

- Cloud detection (IR)

- The inverse problem

- Effects of aerosols (IR)

- Side-lobe \& precipitation contamination (MW)

Characterization of uncertainties in the above processes is a key element of the EARWiG remit, with natural impact on errors reported in the GHRSST products (primarily $\mathrm{L} 2 \mathrm{P}$ ). There are natural linkages with the ST-VAL and HL TAGs, and also the DV-WG. Equally as important will be linkages to related external groups, primarily ERNESST and the NASA SST Science Team.

GHRSST-XII will be the first opportunity for EARWiG to meet. Since there will only be two hours available, and part of the time will be allocated to discussion of an algorithm intercomparison exercise for the ESA SST_cci project, it is proposed that the group devotes the rest of the break-out session to the following:

1. Overview of current status for the main issues, including short $(\sim 10$ minute + questions) presentations of current work from various members to spark the creative process.

2. Discussion of the desired modus operandi of EARWiG. For example, given the existence of ERNESST and the NASA SSTST, should EARWiG simply function as a reporting mechanism? How formal a role should EARWiG play in enabling science via these external groups?

3. In the light of $1 \& 2$, proposed way forward, e.g. planned EARWiG meeting, possibly in collaboration with afore-mentioned GHRSST and/or external groups.

4. Confirmation of membership/interest (can be ongoing throughout the meeting) and suggestions for contacting other potentially interested parties. 


\title{
NIGHTTIME-ONLY OPTIMUM INTERPOLATION SST FIELDS
}

\author{
Patria Viva Banzon ${ }^{(1)}$, Richard W. Reynolds ${ }^{(2)}$
}

(1)National Climatic Data Center NOAA/NESDIS), (USA), Email : viva.banzon@noaa.gov

(2) Cooperative Institute for Climate and Satellites,(USA), Email :

\section{richard.w.reynolds@noaa.gov}

A climate data record (CDR) is defined by the National Research Council as "a time series of measurements of sufficient length, consistency, and continuity to determine climate variability and change". Due to multi-year and multi-decadal oscillations that can mask out the climate change signal, 30 years is often cited as the nominal minimum length required for a CDR. AVHRR-derived sea surface temperatures (SSTs) are available from Nov 1981 to the present. It is a continuous dataset from a single instrument series that is rapidly approaching the CDR record length. To meet the consistency requirement of a $C D R$, it is useful to minimize the bias effects of diurnal variability in the SST satellite record. One way to achieve this is to use nighttime-only in situ and AVHRR data. Preliminary work is presented here comparing nighttime- and daytime-only SST analyses. Both are also compared against the current operational NOAA daily $1 / 4$ deg OISST product, where data for daytime and nighttime are combined. The methodology applied is similar to that for the daily OISST except that separate analyses are made for the night and day. Bias correction is a very important aspect of the NOAA OISST methodology, and is examined in detail. 


\title{
AUSTRALIAN BUREAU OF METEOROLOGY SST REQUIREMENTS FOR NWP AND ATMOSPHERE-OCEAN COUPLED MODELS
}

\author{
Helen Beggs, Paul Sandery, Vaughan Barras and Peter Steinle \\ Centre for Australian Weather and Climate Research, Bureau of Meteorology, Melbourne \\ (Australia), Email: H.Beggs@bom.gov.au
}

\begin{abstract}
The Australian Bureau of Meteorology has an increasing requirement for higher spatial resolution sea surface temperature (SST) products and analyses at both foundation (pre-dawn) and skin $(-10 \mu \mathrm{m})$ depths for model ingest and verification. This paper will describe current and future SST requirements for operational Numerical Weather Prediction (NWP) and atmosphereocean coupled model systems.
\end{abstract}

\section{Introduction}

The Australian Bureau of Meteorology (Bureau) runs global $(-100 \mathrm{~km})$ and regional $(5-37 \mathrm{~km})$ NWP models and eddy-resolving $(10 \mathrm{~km})$ ocean forecast models. These systems have recently been combined to form an experimental regional, $2-10 \mathrm{~km}$ horizontal resolution, atmosphere-ocean coupled model. As elsewhere, the trend at the Bureau is to produce higher and higher resolution models, requiring not just higher spatial resolution satellite sea surface temperature (SST) products and analyses, but also resolution of the diurnal cycle of SST in the surface ocean. In this paper we present current and future requirements of the Bureau's suite of NWP and atmosphereocean coupled models.

\section{NWP SST Requirements}

Numerical weather prediction uses observed current weather and ocean conditions as input into mathematical models of the atmosphere to predict the weather. SST and sea-ice affect the behaviour of the overlying atmosphere and vice versa. Consequently, NWP systems need to be regularly updated with the latest
SST and sea-ice observations to ensure an accurate forecast. Daily analyses of both SST and sea-ice extent and concentration are required by many operational NWP systems. SST affects the formation and subsequent evolution of tropical cyclones, convection and thunderstorms, cyclogenesis, sea fog and sea breezes.

\subsection{Boundary Condition}

NWP models commonly use gap-free, optimally interpolated analyses of in situ and satellite SST observations as a boundary condition over the ocean. The analysis may be either produced from a blend of SSTs at different depths (SSTblend), where the observations have not been corrected for variations with depth, or a "foundation" SST (SSTfnd), where SST observations have been corrected for the surface ocean coolskin or diurnal warm layer either by filtering or use of a diurnal variation (DV) model.

Currently, The Bureau of Meteorology (Bureau) uses the RAMSSA daily, $1 / 12^{\circ}$ resolution, SSTfnd analyses ${ }^{1}$ as the boundary condition for its operational regional NWP models $\left(0.375^{\circ}\right.$ ACCESS-R, $0.11^{\circ}$ ACCESS-A, $0.11^{\circ}$ ACCESS-TC and $0.05^{\circ}$ ACCESS-C) $)^{2}$ and the GAMSSA daily, $1 / 4^{\circ}$ resolution, SSTfnd analyses ${ }^{3}$ as the boundary condition for its global, $\sim 1^{\circ}$ horizontal resolution, NWP model (ACCESS-G). All the NWP forecast systems persist the SSTfnd throughout the forecast period (up to 10 days).

RAMSSA SSTfnd analyses have a standard deviation when compared with independent buoy SSTfnd estimates from the subsequent day of around $0.4^{\circ} \mathrm{C}^{1}$ and GAMSSA of around $0.5^{\circ} \mathrm{C}^{4}$. The impact of 


\section{GHRSST Project Office}

the accuracy of the SSTfnd analyses on the ACCESS NWP models is yet to be determined, but the World Meteorological Organisation (WMO) goals for accuracy of SST analyses used as a boundary condition for global NWP models is $0.3^{\circ} \mathrm{C}^{6}$.

Higher spatial resolution regional SSTfnd analyses will be required within two years for the planned $0.0135^{\circ}$ resolution "ACCESS-C" NWP models over the major Australian cities. At these high spatial resolutions, $1 \mathrm{~km}$ resolution, operational, real-time (within 3 hours) SST data streams from METOP, NOAA and Sentinel-3 polarorbiting satellites will be critical.

The Bureau also plans to investigate whether using forecast SSTskin $(-10 \mu \mathrm{m}$ depth) analyses, rather than using a persisted SSTfnd as the boundary condition, might improve the skill of NWP forecasts.

\subsection{Quality Control of Satellite Sounder} Observations

Another use of SST analyses in NWP is for the quality control of satellite atmospheric sounder channels that peak near the ocean surface. Currently, the RAMSSA and GAMSSA SSTfnd analyses are used as a proxy for SSTskin, but in 2011/2012 the Bureau plans to trial the use of regional skin SST analyses ${ }^{6}$ produced from a combination of RAMSSA SSTfnd analyses and a diurnal warming model using NWP forecast surface winds as inputs (Figure 1). In regions of the ocean experiencing high insolation and low winds, SSTskin daily variations as high as $6^{\circ} \mathrm{C}$ have been observed (e.g. Figure 2). The presence of cloud can also result in anomalously cool SSTskin measurements from infrared sensors on satellites, and therefore a realtime estimate of SSTskin can aid in determining if atmospheric sounder profile data close to the ocean surface are affected by cloud.

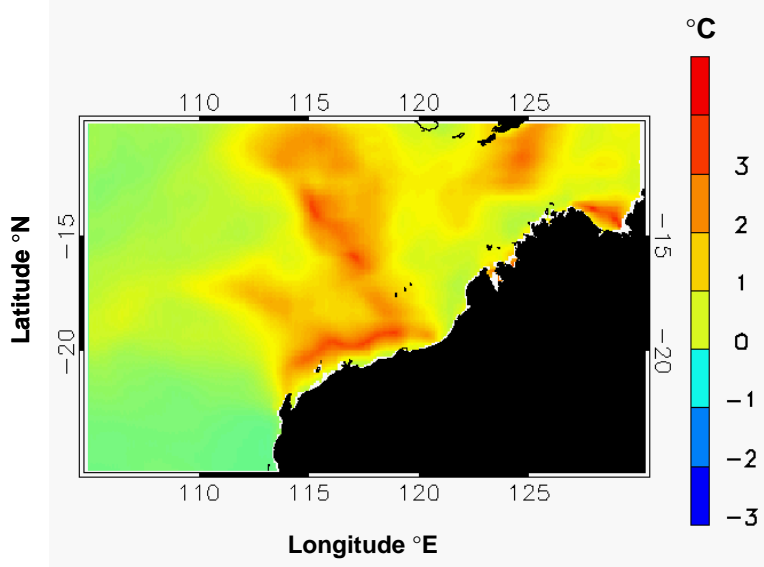

Figure 1. RAMSSA_skin SSTskin minus RAMSSA SSTfnd for 12 March 2009 at 0600 UTC ( 2 pm LT) over the region $105^{\circ} \mathrm{E}$ to $130^{\circ} \mathrm{E}, 10^{\circ} \mathrm{S}$ to $25^{\circ} \mathrm{S}$.

The Bureau's NWP data assimilation systems require SSTskin estimates within 2 hours of the nominal NWP analysis time, so an efficient DV model is required. The required accuracy of the SSTskin estimates is yet to be determined. However, the estimates of SSTskin using a DV model and a foundation SST needs to be closer to the true SSTskin than the current assumption that RAMSSA or GAMSSA SSTfnd equates to SSTskin.

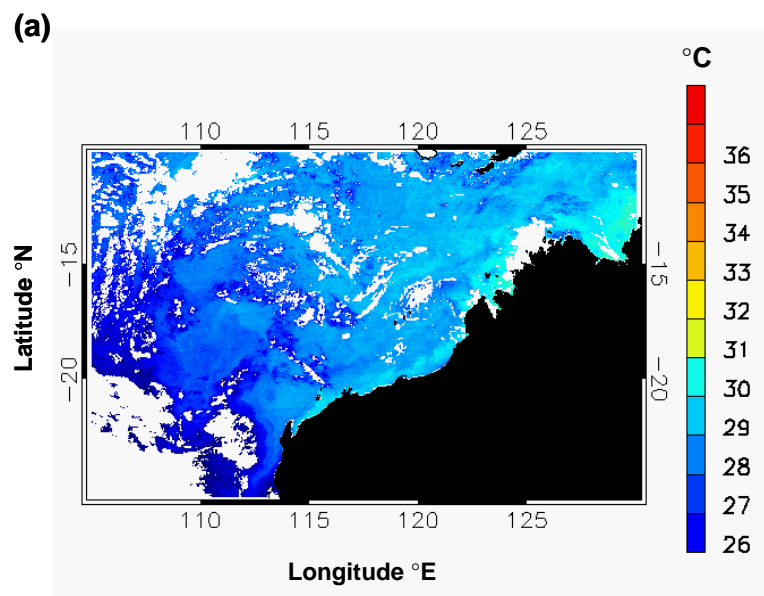




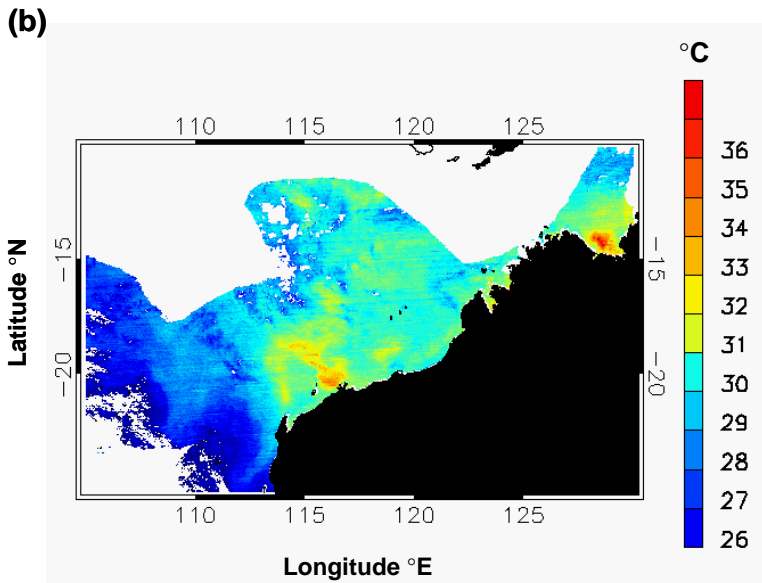

Figure 2. Bureau-IMOS MTSAT-1R SSTskin for (a) 11 March 2009 at 1730 UT ( 1:30 am LT) and (b) 12 March 2009 at 0630 UT ( 2:30 pm LT) over the region $105^{\circ} \mathrm{E}$ to $130^{\circ} \mathrm{E}, 10^{\circ} \mathrm{S}$ to $25^{\circ} \mathrm{S}$.

\section{Atmosphere-Ocean Coupled Model SST Requirements}

Air-sea interaction influences the state of the ocean and atmosphere. A coupled atmosphere-ocean model is required to resolve this interaction, which can be important in extreme weather such as tropical cyclones. Through the BLUElink Ocean Forecasting Australia Project (http://www.bom.gov.au/bluelink) the Bureau has developed a mesoscale ocean eddy resolving $\left(0.025^{\circ}-0.1^{\circ}\right)$ re-locatable coupled limited area modelling system (CLAM) based on the ACCESS NWP system $^{2}$, OASIS4 coupling software and MOM4p1 ocean general circulation model, extending the work of Sandery et al. $(2010)^{7}$. CLAM is one-way nested inside operational OceanMAPS (Ocean Modelling, Analysis and Prediction System $)^{8}$ and has versions using ACCESS-R $\left(0.375^{\circ}\right)$ and ACCESS-TC $\left(0.15^{\circ}\right)$. At present it is used as a research system for both testing the impact of coupling on ocean and weather forecasting and developing new scientific methods and capabilities. CLAM provides a dynamically evolving SST as a boundary condition for the ACCESS NWP models and more physically representative air-sea surface fluxes in both models. Since CLAM uses a 3D ocean model, the SST is influenced by various factors in addition to heat and momentum surface fluxes such as the underlying heat content and structure of the mixed layer and thermocline. It is therefore an important requirement that the ocean component of CLAM is accurately initialised using data assimilation of both remotely sensed and in situ data. High spatial and temporal resolution SSTfnd and SSTskin analyses products are therefore important for assimilation, validation and improving understanding of model representativeness.

Since the CLAM ocean model is nested inside and initialised using OceanMAPS, the accuracy of SST in the latter is also important. OceanMAPS assimilates satellite SSTfnd data (AMSR-E and GAC AVHRR), satellite altimetery and in situ profiles of temperature and salinity. At present OceanMAPS is run twice-weekly at the Bureau at $0.1^{\circ}$ resolution over the Australian region ${ }^{8}$. This system is about to be replaced by OceanMAPS2, which will run a daily forecast using a staggered four member ensemble.

\subsection{SST Data Assimilation}

OceanMAPS uses the BLUElink Ocean Data Assimilation System (BODAS) ${ }^{9}$ as its analysis component. The operational BODAS system currently assimilates AMSR-E SSTsubskin retrievals from the Aqua satellite and NAVOCEANO GAC AVHRR L2P SSTblend retrievals from METOP-A and operational NOAA satellites, converted by filtering to a foundation $\mathrm{SST}^{10}$.

Ocean models require satellite SST for assimilation that is reliable, timely and accurate. Spatial resolution of the IR SST assimilated into ocean models $(9 \mathrm{~km})$ is not currently an issue due to the relatively coarser horizontal resolution of the global and regional ocean models. However, future regional ocean models may require higher resolution $(1 \mathrm{~km})$ SST, particularly within $50 \mathrm{~km}$ of coastlines. 
GHRSST XII -

Proceedings, Edinburgh

GHRSST Project Office

\subsection{Validation}

In addition to assimilation, the Bureau's new downscaled regional atmosphere-ocean coupled models in particular will require accurate $\left(<0.3^{\circ} \mathrm{C}\right)$, ultra high-resolution (1 $\mathrm{km}$ ), level 3 (composite) or level 4 (analysis) SST products for validation.

\subsection{Diurnal Variation Model}

SST in coastal and inland regions has large variability due to the diurnal cycle of solar radiation, which enhances surface characteristics of the land and sea and forces land-air-sea interactions, i.e. landsea breezes. Furthermore, under certain conditions the diurnal cycle of SST can be pronounced (Figure 2). There will be situations where this scale of variability will have a significant influence on the atmosphere. In order to facilitate research in this area CLAM will require SSTskin estimates preferably at an hourly frequency. CLAM at present uses SST $(2.5 \mathrm{~m})$ as the atmospheric boundary condition. A DV model will be included in CLAM to estimate SSTskin and the impact of using this as a boundary condition. The atmospheric model in CLAM will provide inputs to the DV model to produce SSTskin minus SST $(2.5 \mathrm{~m})$ (DSST). The required accuracy of the SSTskin estimates is yet to be determined. However, the estimates of SSTskin using SST $(2.5 \mathrm{~m})$ plus a modelled DSST should be closer to the true SSTskin than the current assumption that the SST $(2.5 \mathrm{~m})$ output of the ocean model equates to SSTskin.

There is evidence to suggest that incorporation of diurnally varying SSTskin has a significant effect upon time integrated heat fluxes supplied to the boundary layer ${ }^{11}$. The effect of the addition of diurnal variations of SSTskin upon the fluxes of sensible and latent heat is of particular interest, particularly in regions of low wind speed and in the tropics. It is also important to consider the effect of fractional cloud
Issue: Final

Date: September 27, 2011

cover upon the radiative forcing estimate derived from SSTskin.

\section{Conclusion}

Higher spatial resolution NWP and atmosphere-ocean coupled models at the Bureau of Meteorology will require increasingly higher resolution and more accurate satellite SST products and analyses at both skin and foundation depths. In the short term, there is a need for $\sim 0.02^{\circ}$ resolution regional SSTfnd analyses as a boundary condition for the planned $0.0135^{\circ}$ resolution regional NWP models and to validate $0.025^{\circ}$ resolution regional atmosphere-ocean coupled models. A fast, accurate, diurnal variation model is required that can use the forecast surface fields from the Bureau's ACCESS NWP models to predict the difference between SSTskin and either the analysis SSTfnd or the coupled model $2.5 \mathrm{~m}$ SST.

\section{References}

Beggs, H, A Zhong, G Warren, O Ives, G Brassington and $T$ Pugh (2011) RAMSSA - An Operational, HighResolution, Multi-Sensor Sea Surface Temperature Analysis over the Australian Region, Australian Meteorological and Oceanographic Journal, 61, 122. (http://www.bom.gov.au/amoj/papers .php?year=2011)

Puri , K et al. (2010) Preliminary results from Numerical Weather Prediction implementation of ACCESS. CAWCR Research Letters 5, 15-22. (http://www.cawcr.gov.au/publications/re searchletters/CAWCR Research Letter s 5.pdf)

Zhong, A and H Beggs (2008) Analysis and Prediction Operations Bulletin No. 77 - Operational Implementation of Global Australian Multi-Sensor Sea SurfaceTemperature Analysis, 2 October 2008. (http://www.bom.gov.au/bmrc/ocean/BL 
GHRSST XII -

Proceedings, Edinburgh

GHRSST Project Office

UElink/SST/GAMSSA BoM Operational Bulletin 77.pdf)

Beggs, H, L Majewski, G Paltoglou, E Schulz, I Barton and R Verein (2010) Report to GHRSST11 from Australia BLUElink and IMOS, In: Proceedings of the 11th GHRSST Science Team Meeting, Lima, 21-25 June 2010, p. 2131.

(https://www.ghrsst.org/documents/q/cat egory/ghrsst-science-teammeetings/ghrsst-xi-lima/)

Eyre, J, E Anderson, E Charpentier, L Ferrantier, L Ferranti, J Lafeuille, M Ondras, J Pailleux, $F$ Rabier, $L P$ Riishojgaard (2009) Requirements of numerical weather prediction for observations of the ocean. Community whitepaper to OceanObs09, Venice, Italy, 21-25 September, 2009.

(http://www.oceanobs09.net/blog/)

Beggs, H, C Gentemann and P Steinle (2009) Real-time skin sea surface temperature analyses for quality control of data assimilated into NWP models, extended abstract, The Fifth WMO International Symposium on Data Assimilation of Observations in Meteorology, Oceanography and Hydrology, Melbourne, Australia, 5-9 October
Issue: Final

Date: September 27, 2011

(http://cawcr.gov.au/bmrc/ocean/BLUElin k/SST/5WMO DASymp Extended Abstr act Beggs Sep2009.pdf)

Sandery, P A, G B Brassington, A Craig and $T$ Pugh, 2010. Impacts of oceanatmosphere coupling on tropical cyclone intensity change and ocean prediction in the Australian region. Monthly Weather Review, 138(6), 2074-2091.

Brassington, G B, T Pugh, C Spillman, E Schulz, H Beggs, A Schiller and P R Oke (2007) BLUElink> Development of Operational Oceanography and Servicing in Australia, J. Res. Practice in Information Technology, 39, 151-164.

Oke, P R, A Schiller, D A Griffin and G B Brassington (2005) Ensemble data assimilation for an eddy-resolving ocean model of the Australian region, Q. J. R. Meteorol. Soc., 131, 3301-3311.

Andreu-Burillo, I, G Brassington, P Oke and H Beggs (2010) Including a new data stream in in BLUElink Ocean Data Assimilation System, Australian Meteorological and Oceanographic Journal, 59:77-86.

Webster, P. J., C. A. Clayson, and J. A. Curry (1996), Clouds, radiation, and the diurnal cycle of sea surface temperature in the tropical western Pacific, J. Clim., 9, 1712-1730. 


\title{
CLASSIFICATION OF SEA-ICE IN HIGH LATITUDE RETRIEVALS FROM AATSR
}

\author{
Claire Bulgin $^{(1)}$, Chris Merchant ${ }^{(2)}$
}

(1) University of Edinburgh, Edinburgh, EH7 3JN (UK), Email : cbulgin@staffmail.ed.ac.uk (2) University of Edinburgh, Edinburgh, EH7 3JN (UK), Email : c.merchant@ed.ac.uk

\section{ABSTRACT}

SST records developed from satellite data are dependent on the effective screening of cloud-contaminated observations from clear-sky observations. At high latitudes this screening process is further complicated by the presence of sea-ice, adding a third class of observations. The Generalized Bayesian Cloud Screening (GBCS) algorithm developed within the ATSR Reprocessing for Climate (ARC) project [Embury and Merchant, 2011] uses Bayes' theorem to calculate the probability of clear-sky or cloud for a given scene, enabling the user to tailor the severity of the cloud screening specifically to their application rather than using a predefined threshold. Here we develop this into a three-way classifier capable of identifying sea-ice. We develop ice classification firstly using the 11 and $12 \mu \mathrm{m}$ channels only, compatible with data from ATSR1, ATSR2 and AATSR, which is important in making long-term records of SST. We also explore the added value of using additional channels where available - the 3.7 $\mu \mathrm{m}$ channel during the night and the 0.6 and $1.6 \mu \mathrm{m}$ channels during the day, to enhance ice detection in AATSR data.

\section{Introduction}

Continuity in the design of space-borne instruments means that current SST algorithms can be applied to archived data to generate long-term global records of SST essential for climate applications. Cloud detection is a fundamental preprocessing step in generating SST estimates as even thin and patchy undetected cloud can distort the retrieved SST by several Kelvin [Zavody, 1999]. Cloud detection from satellite imagery is inherently difficult (particularly when limited to thermal channels) with patchy cirrus, low-lying warm stratus, sub-pixel cloud and cloud edges being the most difficult to identify. In Arctic regions, identifying areas of clear-sky over ocean is further complicated by the presence of sea-ice. Ice detection from satellite data is difficult at both infrared and visible wavelengths as the ice surface temperature can be close to the freezing temperature of water, particularly in regions where melt ponds form on top on the ice surface. In the visible channels ice or snow reflectance is dependent on the surface texture, grain size and liquid water content [Painter, 2004].

Arctic regions are arguably one of the most important regions in which to obtain long-term records of SST. Projected changes in future climate as a result of anthropogenic emissions are closely linked to a reduction in sea-ice extent with concerns over changes in the global energy balance and sea-level rise [Johannessen et al., 1999; Levitus et al., 2001; Johannessen et al., 2004; Overpeck et al., 2006]. In this paper we add a third class to the GBCS Bayesian two-way clear and cloud classifier, subdividing the 'clear' pixels into 'clear-over-ocean' and 'clear-over-ice'. We initially use only data from the 11 and $12 \mu \mathrm{m}$ channels in an algorithm that can be applied to both day and nighttime observations, and to all of the ATSR instruments. We demonstrate the ability of this algorithm to improve detection of ocean pixels in regions of sea-ice and then explore the added value of including the $3.7 \mu \mathrm{m}$ channel during the night and the 0.6 and $1.6 \mu \mathrm{m}$ channels during the day, for ice detection.

\section{11 and $12 \mu \mathrm{m}$ Bayesian Cloud and Ice Detection}

The probabilistic cloud-screening algorithm derived from Bayes' theorem gives the probability of a given class of 
observation (in this case ocean, cloud or ice) based on observations and background knowledge of the observed state [Merchant et al., 2005; English et al., 1999]. In its general form this can be written as:

$$
P\left(\operatorname{class}_{x} \mid \mathbf{y}^{o}, \mathbf{x}^{b}\right)=\frac{P\left(\mathbf{y}^{o} \mid \mathbf{x}^{b}, \operatorname{class}_{x}\right) P\left(\mathbf{x}^{b} \mid \text { class }_{x}\right) P\left(\text { class }_{x}\right)}{P\left(\mathbf{y}^{o} \mid \mathbf{x}^{b}\right) P\left(\mathbf{x}^{b}\right)}
$$

Where $\mathrm{P}$ denotes probability, $\mathbf{y}$ is the observation vector and $\mathbf{x}$ is the state vector. Superscripts ' 0 ' and ' $b$ ' denote observed and background respectively. The probability density function (PDF) of the observations given the background state, $P\left(\mathbf{y}^{o} \mid \mathbf{x}^{b}\right)$, can be expressed as a function of each of the possible classes assuming that these are independent. For the three classes, clear-sky over ocean (c), cloud $(\bar{c})$ and clear- sky over ice (i), this can be written as:

$$
P\left(\mathbf{y}^{o} \mid \mathbf{x}^{b}\right)=P(c) P\left(\mathbf{y}^{o} \mid \mathbf{x}^{b}, c\right)+P(\bar{c}) P\left(\mathbf{y}^{o} \mid \mathbf{x}^{b}, \bar{c}\right)+P(i) P\left(\mathbf{y}^{o} \mid \mathbf{x}^{b}, i\right)
$$

and in its general form, with respect to ' $n$ ' classes as:

$$
P\left(\mathbf{y}^{o} \mid \mathbf{x}^{b}\right)=\sum_{n} P\left(\operatorname{class}_{x}\right) P\left(\mathbf{y}^{o} \mid \mathbf{x}^{b}, \operatorname{class}_{x}\right)
$$

It can be assumed that the background state (prior information) is independent of the class probability: $\left(P\left(\mathbf{x}^{b} \mid\right.\right.$ class $\left.)=P\left(\mathbf{x}^{b}\right)\right) \quad[$ Merchant et al.,
2005]. Therefore, by substituting (3) into (1) we obtain an equation for probability of a given classification $(x)$ given any number of classes (n) [Mackie, 2009]:

$$
P\left(\operatorname{class}_{x} \mid \mathbf{y}^{o}, \mathbf{x}^{b}\right)=\frac{P\left(\operatorname{class}_{x}\right) P\left(\mathbf{y}^{o} \mid \mathbf{x}^{b}, \operatorname{class}_{x}\right)}{\sum_{n} P\left(\operatorname{class}_{n}\right) P\left(\mathbf{y}^{o} \mid \mathbf{x}^{b}, \operatorname{class}_{n}\right)}
$$

For each class we decompose the probability $\left(P\left(\right.\right.$ class $\left.\left._{x} \mid \mathbf{y}^{o}, \mathbf{x}^{b}\right)\right)$ into a spectral and textural component, which we assume are independent. We approach the calculation of the clear-sky over ice probability in a similar way to the clear-sky over ocean, using the fast-forward radiative transfer models RTTOV-10 [Hocking et al., 2011] and VisRTM [Merchant et al., 2008] to simulate infrared brightness temperatures and top of atmosphere (TOA) reflectance. For all clear-sky observations the spectral component of the probability is forward modelled driven using numerical weather prediction data from the ECMWF [Embury et al., 2011]. The calculated PDF is assumed to be Gaussian and is derived using a reduced state vector consisting of surface temperature (ST) and total column water vapour, the two dominant terms in determining brightness temperature
[Merchant et al., 2005]. A more detailed description of the retrieval scheme and forward modelling can be found in Embury and Merchant (2011).

Over ice we use the ECMWF reanalysis skin temperature as ST prior, and assume an error of $5 \mathrm{~K}$. In the 11 and $12 \mu \mathrm{m}$ channels we define the surface emissivity as a function of viewing zenith angle using laboratory measurements of fine dendritic snow [Hori et al., 2006; Cheng et al., 2010]. We use snow rather than bare-ice emissivity as Arctic sea-ice is likely to be covered by overlying snow. For cloud, the joint PDFs for the 11 and $12 \mu \mathrm{m}$ channels are calculated empirically [Merchant et al., 2005]. The textural PDF for each class is defined as the local standard deviation in the $11 \mu \mathrm{m}$ brightness temperature over a $3 \times 3$ pixel domain, and is calculated empirically for all classes. We construct the ice textural PDF using the normalised 
difference snow index (NDSI) to identify ice pixels, which will include both surface ice and ice clouds. Figure 1 shows the textural PDFs for ocean, cloud and ice in the nadir and forward view used during the day. At night we use the same textural
PDFs for ice as we cannot use the NDSI to identify ice. The ice texture PDF is broad, similar to the cloud PDF indicating that the ice surface is less homogeneous than the ocean.
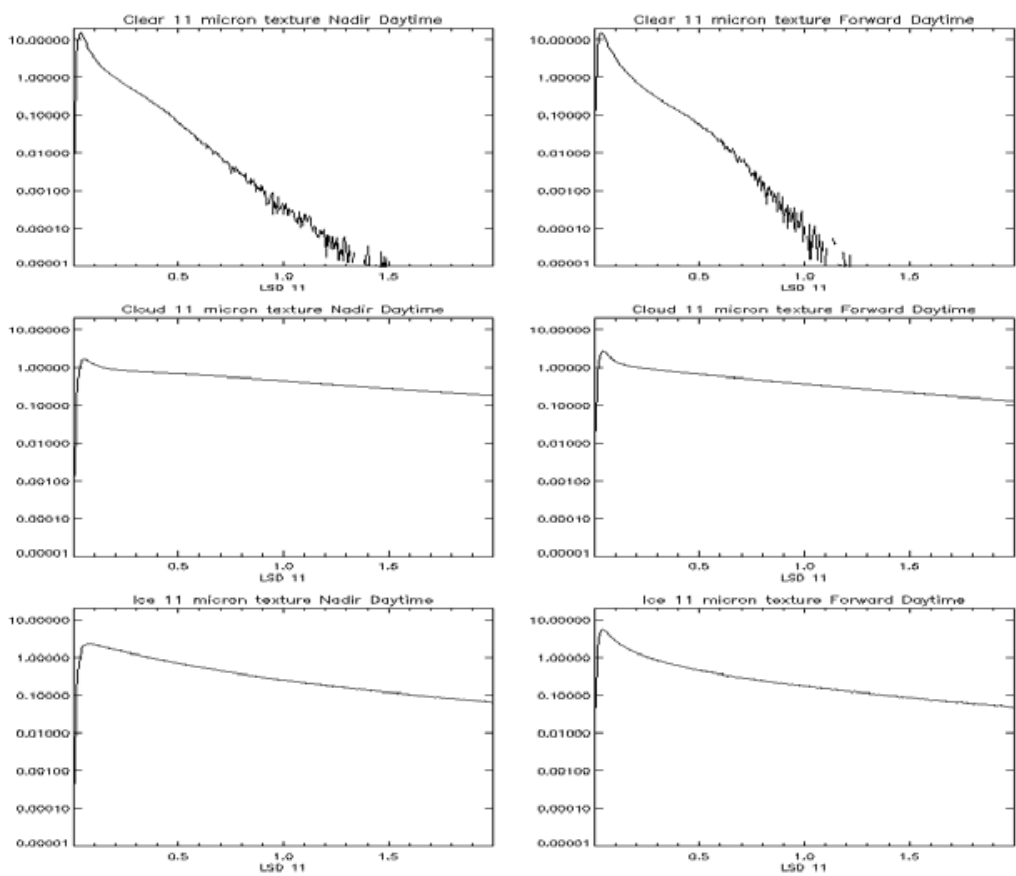

Figure 1. Empirical textural PDFs for AATSR for the nadir and forward views for clear sky over ocean (top), cloud (middle) and clear-sky over ice (bottom).

We demonstrate the performance of the 11 and $12 \mu \mathrm{m}$ only three-way classification using one nighttime and one daytime test scene. We then use the same scenes in the following section to demonstrate the benefits of including additional channels in the ice detection when available. Figure 2 shows a $11 \mu \mathrm{m}$ nighttime image over the Kara Sea north of Russia from the $9^{\text {th }}$
February 2007 and the corresponding clear-sky probability from the GBCS twoway classifier [Embury and Merchant, 2011]. The two-way classifier flags an area of clear-sky in the top right-hand corner at a 95\% confidence level, which corresponds well with a small clear-sky feature that can be identified in the $11 \mu \mathrm{m}$ image. 

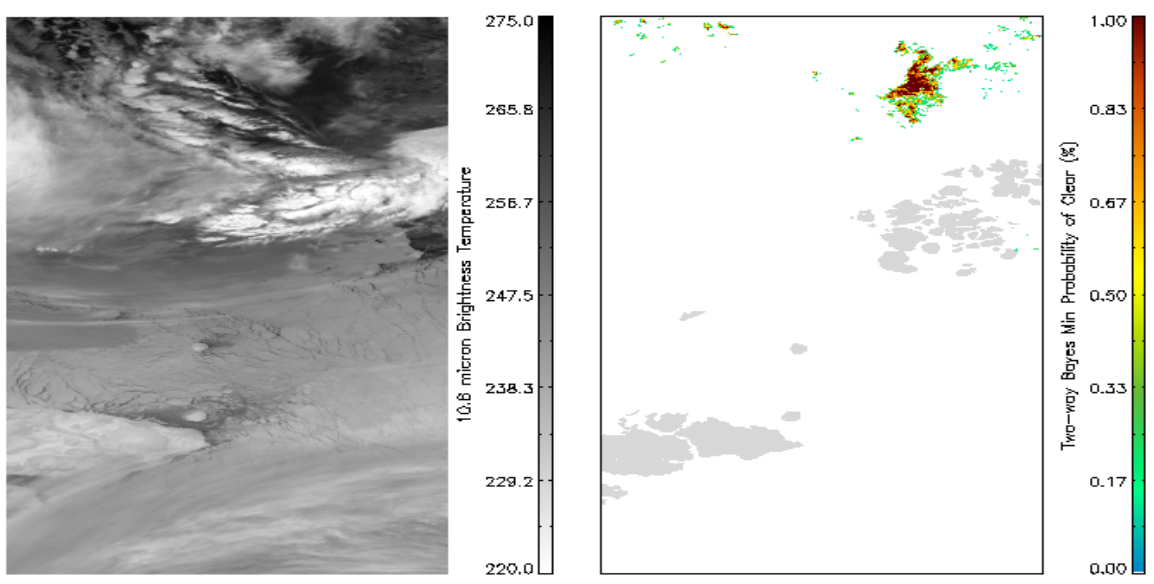

Figure 2. AATSR $10.8 \mu \mathrm{m}$ brightness temperature over the Kara Sea north of Russia on 09/02/2007 (left) and dual-view probability of clear, using the two-way classifier and the 11 and $12 \mu \mathrm{m}$ channels only (right).

In Figure 3 we compare the clear-sky probability from the two-way classifier (on the left), with the clear-sky over ocean and clear-sky over ice outputs from the threeway classifier. We find that both identify the same area of clear-sky in the top right of the image, but that the three-way classifier is more conservative in the number of pixels that it would pass for SST estimation at the $95 \%$ confidence level. This more conservative estimate may exclude thin or partially submerged ice near the edges of ice floes, which is preferential for SST estimation. The qualitative spatial distribution of pixels with a classification of clear-sky over ice looks reasonably consistent with what can be identified in the $11 \mu \mathrm{m}$ imagery. The swath of ice in the centre of the image and in the top right corner (away from the clear sky area) both look plausible, although the ice in the top right corner does seem to extend too far into the 'clear-over-ocean' region. In the top left corner it is more difficult to discern by visual inspection alone whether there is any ice present, and this is one of the difficulties in validating ice detection algorithms at night. Quantitatively, this ice probability field would not be useful in flagging ice for use in other applications such as ice surface temperature retrieval.
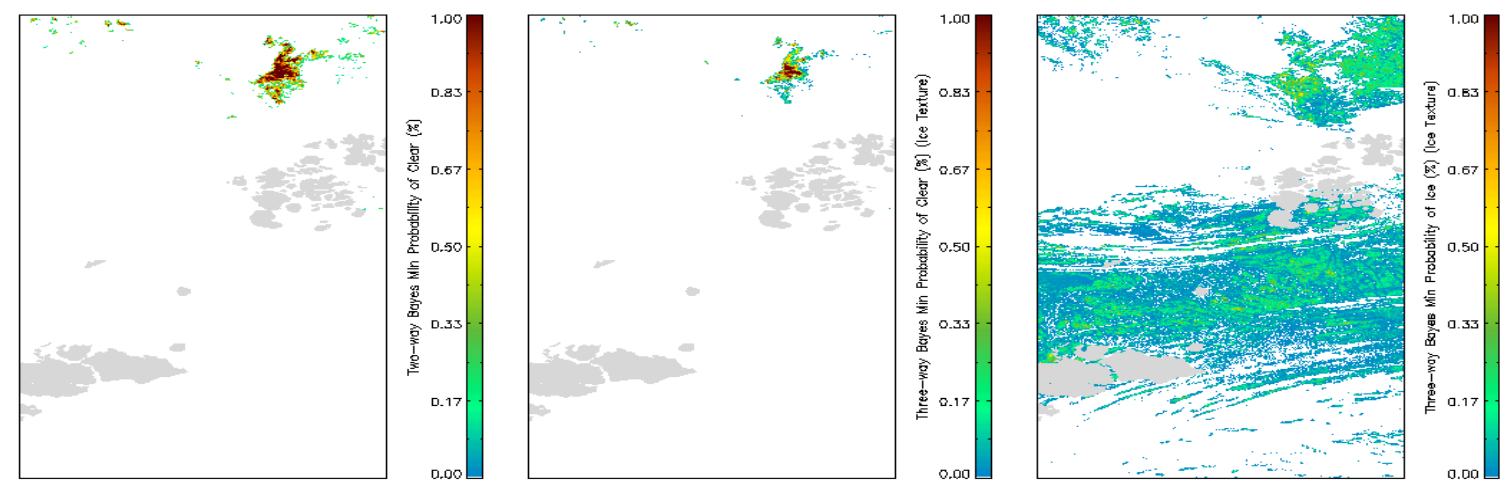

Figure 3. Dual-view probability of clear using the 11 and $12 \mu \mathrm{m}$ channels and the two-way classifier (left) and dual-view probability of clear-sky over ocean (centre) and clear-sky over ice (right) using the threeway classification with the 11 and $12 \mu \mathrm{m}$ channels only over the Kara Sea.

Figure 4 shows the second example, a daytime image over Svalbard on $22^{\text {nd }}$ April
2007. Reflectance data in the visible channels are also available in daytime 
imagery, which aids visual identification of ice and cloud features. In the two-way classification of clear-sky pixels, those close to the land appear to be ice-free in the satellite imagery. The pixels towards the centre and right-hand side of the image that would also pass as 'clear-sky' using the $95 \%$ threshold are an example of where the two-way classification erroneously identifies sea-ice as ocean, and would introduce errors in the SST estimate.
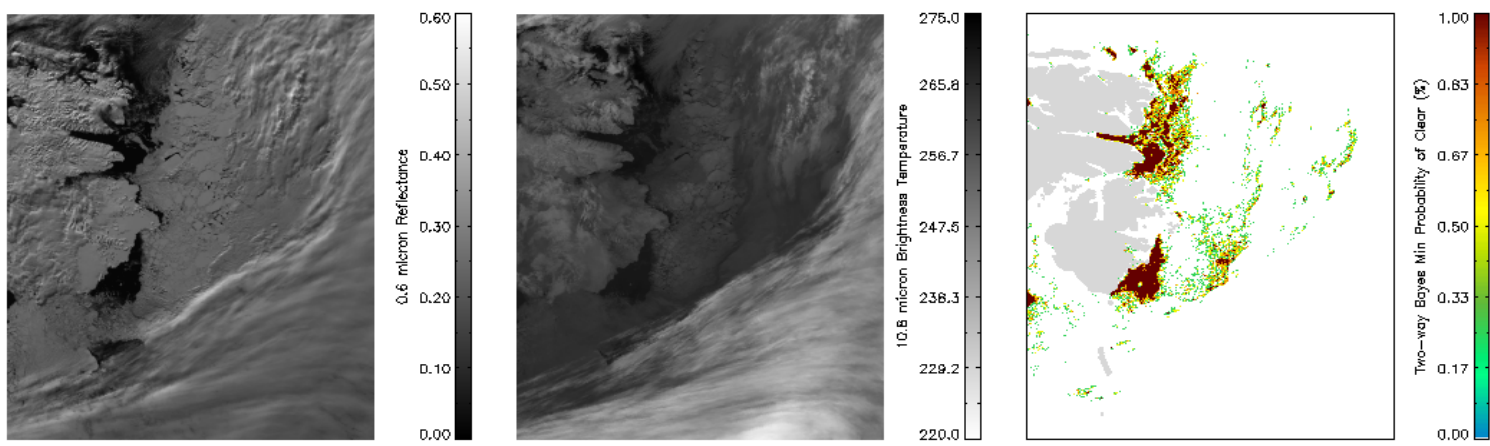

Figure 4. AATSR $0.6 \mu \mathrm{m}$ reflectance (left) and $10.8 \mu \mathrm{m}$ brightness temperature over Svalbard (centre) on the 22/04/2007 and dual-view probability of clear using the two-way classifier and the 11 and $12 \mu \mathrm{m}$ channels only (right).

When we examine the three-way classification (Figure 5), we find that the regions close to the land still have a high probability of being clear-sky over ocean, whilst the probability of those pixels over the ice floe being 'clear-over-ocean' has decreased significantly, and these would be excluded at the $95 \%$ threshold. The ice probability field is similar to that of the
Kara Sea image in that applying a $95 \%$ confidence threshold would not result in any positive ice detection. The spatial extent of pixels identified as having a low probability of being ice does capture the ice floes, but also wrongly identifies some low-lying cloud just to the right of the image centre and the ocean pixels close to the coast.
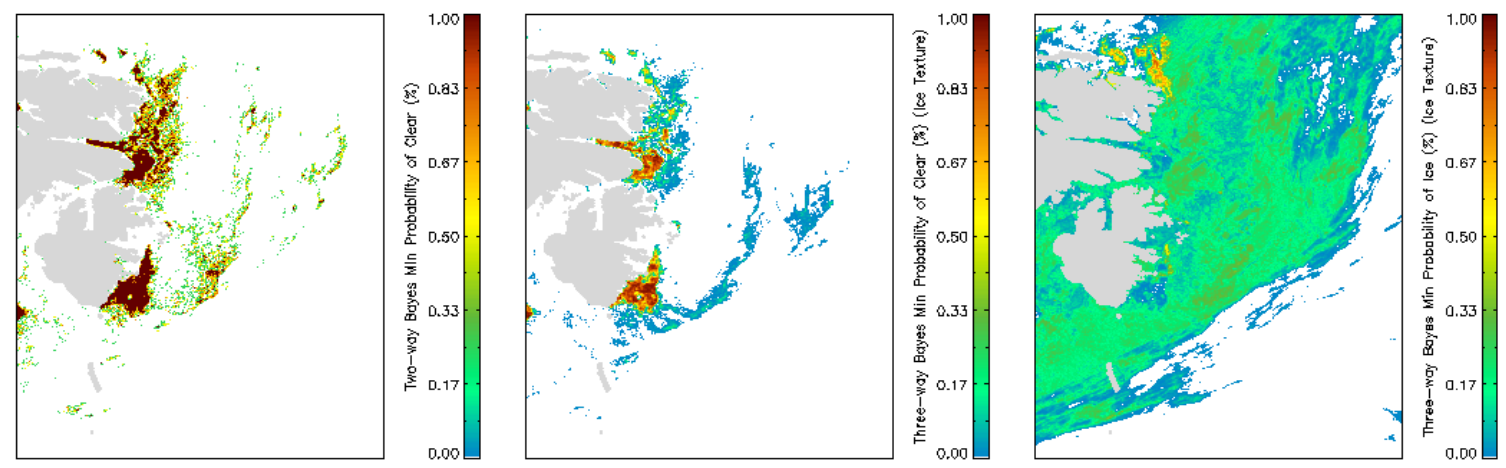

Figure 5. Dual-view probability of clear using the 11 and $12 \mu \mathrm{m}$ channels and the two-way classifier (left) and dual-view probability of clear-sky over ocean (centre) and clear-sky over ice (right) using the threeway classification with the 11 and $12 \mu \mathrm{m}$ channels only over Svalbard.

\section{Enhanced Bayesian Ice Detection}

In this section we evaluate the benefit of including additional channels in the threeway classifier. We consider the Kara Sea image first, and include the $3.7 \mu \mathrm{m}$ channel defining the emissivity angular dependence using measurements made by Dozier and Warren (1982). Figure 6 shows the clear-sky probability from the two-way classifier using three channels 
(left) and the 'clear-over-ocean' probability from the three-way classification in the centre. Both probability fields look identical to those derived using the 11 and $12 \mu \mathrm{m}$ channels only, and there is no further benefit in terms of identifying valid pixels for SST retrieval. In the ice probability field we see a marked improvement in the ability of the classifier to identify ice, and a sensible threshold could now be set to flag ice for other applications. The spatial extent of the ice has also been refined, and the ice edges are distinct from both the cloud edges across the central swath and the edges of the open water in top right corner of the image.
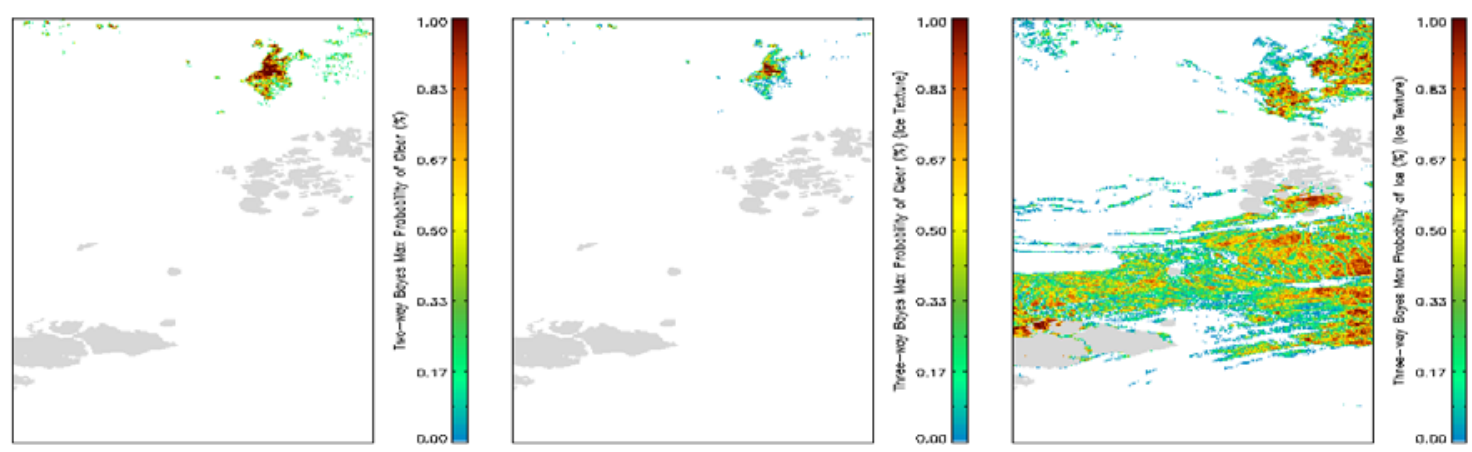

Figure 6. Dual-view probability of clear using the 3.7, 11 and $12 \mu \mathrm{m}$ channels and the two-way classifier (left) and dual-view probability of clear-sky over ocean (centre) and clear-sky over ice (right) using the three-way classification with the 3.7, 11 and $12 \mu \mathrm{m}$ channels only over the Kara Sea.

During the day we cannot use the $3.7 \mu \mathrm{m}$ channel as it is contaminated by solar radiation and instead use a joint PDF of the 0.6 and $1.6 \mu \mathrm{m}$ to distinguish between water, ice and cloud. For cloud this is calculated empirically, and for ice and ocean conditions this is modeled using VisRTM. VisRTM includes a model of ocean reflectance [Embury et al, 2011] and for ice we assume a Lambertian surface reflectance using values from literature as our prior albedo [Nakamura et al., 2001]. At present we have not developed the dual-view joint PDF for cloud so Figure 7 shows the results for the nadir only view. We find here that using the additional channels increases the probability of clear-sky over ocean close to the coast, giving a similar spatial distribution of clear-sky pixels as the dualview two-way classifier, which uses the 1.6, 11 and $12 \mu \mathrm{m}$ channels. We also find when using the maximum channel combination that the ice classification shows excellent agreement with what can be seen in the satellite imagery. The ice floes are more clearly defined and distinguished from the open water close to the coast, and the area of low-lying cloud overlying the ice in the centre right of the image has now been correctly flagged as cloud. 

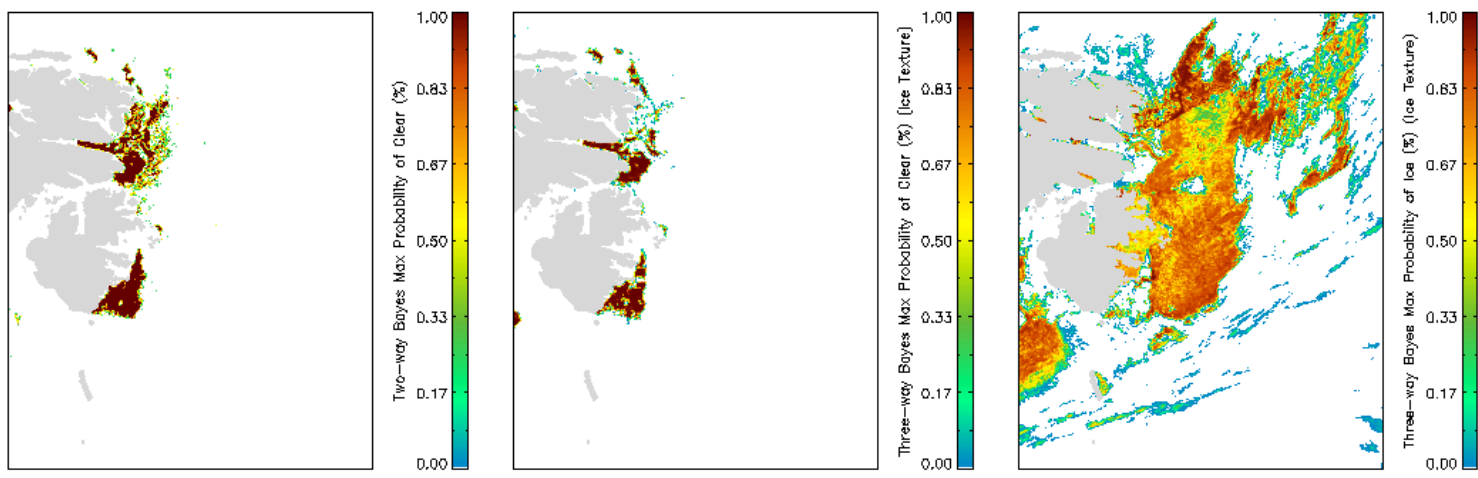

Figure 7. Dual-view probability of clear using the 1.6, 11 and $12 \mu \mathrm{m}$ channels and the two-way classifier (left) and nadir view probability of clear-sky over ocean (centre) and clear-sky over ice (right) using the three-way classification with the 0.6, 1.6, 11 and $12 \mu \mathrm{m}$ channels only over the Kara Sea.

\section{Conclusion}

Identifying ocean pixels suitable for SST retrieval from remote sensing data in Arctic regions requires a pre-processing algorithm capable of identifying and discarding both cloud and sea-ice affected observations. We show here that the GBCS Bayesian cloud detection scheme can usefully be developed into a threeway classifier with the capability of detecting both cloud and ice. We find promising results when using a minimum channel combination that can be applied to data across the whole ATSR archive. In our test cases, the three-way classifier reduces sea-ice contamination in the clear-sky pixels identified for use in SST estimation, which would be beneficial in studying long-term records of SST in the Arctic. We also find that by adding additional channels to both the day and nighttime Bayesian algorithms we have a useful tool for ice detection distinguishing ice floes well from ocean and cloud. At present this scheme has only been evaluated using a small number of test cases but will undergo rigorous validation in the algorithm selection process within the SST climate change initiative project.

\section{References}

Cheng, J. and S. Liang, F. Weng, J. Wang, and X. $\mathrm{Li}$, Comparison of radiative transfer models for simulating snow surface thermal infrared emissivity, IEEE Journal of Selected Topics in Applied Earth Observations and Remote Sensing, 3(3), 323-336, 2010.
Dozier, J. and S. G. Warren, Effect of viewing angle on the infrared brightness temperature of snow, Water Resources Research, 18(5),1424,1434, 1982.

Embury, O. and C. J. Merchant, A reprocessing for climate of sea surface temperature from the along-track scanning radiometers: A new retrieval scheme, Remote Sensing of Environment, 2011 (in press).

Embury, O. and C. J. Merchant, and M. J. Filipiak, A reprocessing for climate sea surface temperature from the along-track scanning radiometers: Basis in radiative transfer, Remote Sensing of Enironment, 2011 (in press).

English, S. J. and J. R. Eyre, and J. A. Smith, A cloud-detection scheme for use with satellite sounding radiances in the context of data assimilation for numerical weather prediction, Quarterly Journal of the Royal Meteorological Society, 125, 2359-2378, 1999.

Hocking, J. and P. Rayer, and R. Saunders, RTTOV v10 users guide, Technical report, EUMETSAT Satellite Application Facility on Numerical Weather Prediction (NWP SAF), 2011. Doc ID: NWPSAF-NO-UD-023.

Hori, M. and T. Aoki, T. Tanikawa, H. Motoyoshi, A. Hachikubo, K. Sugiura, T. J. Yasunari, H. Eide, R. Storvold, Y. Nakajima, and F. Takahashi, Insitu measured spectral directional emisisivity fo snow and ice in the 8-14 $\mu \mathrm{m}$ atmospheric window, Remote Sensing of Enviromnent, 100, 486-502, 2006.

Johannessen, O. M and E. V. Shalina, and M. W. Miles, Satellite evidence for an arctic sea ice cover in transformation, Science, 286,19371939, 1999.

Johannessen, O. M. and L. Bengtsson, M. W. Miles, S. I. Kuzmina, V. A. Se- menov, G. H. Alekseev, A. P. Nagurnyi, V. F. Zakharov, L. P. Boblev, L. 
GHRSST Project Office

H. Pettersson, K. Hasselmann, and H. P. Cattle, Arctic climate change: observed and modelled temperature and sea-ice variability, Tellus $A, \mathbf{5 6}$ (4), 328-341, 2004.

Levitus, S. and J. I. Antonov, J. Wang, T. L. Delworth, K. W. Dixon, and A. J. Broccoli, Anthropogenic warming of the earth's climate system, Science, 292, 267-270, 2001.

Mackie, S. Exploiting Weather Forecast Data for Cloud Detection, PhD thesis, University of Edinburgh, School of Geosciences, 2009.

Merchant, C. J. and A. R. Harris, E. Maturi, and S. MacCallum, Probabilistic physically based cloud screening of satellite infrared imagery for operational sea surface temperature retrieval, Quarterly Journal of the Royal Meteorological Society, 131, 2735-2755, 2005.

Merchant, C. J. and C. P. Old, O. Embury, and S. N. MacCallum, Generalized Bayesian cloud screening algorithm theoretical basis, Technical report, School of Geosciences, University of Edinburgh, 2008. Version 2.1.
Nakamura, T. and O. Abe, T. Hasegawa, R. Tamura, and T. Ohta, Spectral reflectance of snow with a known grain-size distribution in successive metamorphism, Cold Regions Science and Technology, 32, 13-26, 2001.

Overpeck, J. T. and B. L. Otto-Bliesner, G. H. Miller, D. R. Muhs, R. B. Alley, and J. T. Kiehl, Paleoclimatic evidence for future ice-sheet instability and rapid sea-level rise, Science, 311, 1747-1750, 2006.

Painter, T. H. and Dozier, J., Measurements of the hemispherical-directional reflectance of snow at fine spectral and angular resolution, Journal of Geophysical Research, 109, D18115, 2004.

Zavody, A. M. and C. T. Mutlow, and D. T. Llewellyn-Jones, Cloud clearing over the ocean in the processing of data from the along-track scanning radiometer (ATSR), Journal of Atmospheric and Oceanic Technology, 17, 595615, 2000. 


\title{
GLOBAL 1-KM SEA SURFACE TEMPERATURE (G1SST) FOR RESEARCH AND APPLICATIONS
}

\author{
Yi Chao, Zhijin Li, Peggy Li, Benyang Tang
}

Jet Propulsion Laboratory, USA, Email : Yi.Chao@jpl.nasa.gov

Global 1-km Sea Surface Temperature (G1SST) data are described. G1SST combines the SST measurements from multiple satellites and in situ platforms. The satellite SST data are from the Level 2 preprocessed (L2P) data products, including infrared (IR) sensors on both polar-orbiting satellites (e.g., AVHRR, METOP, MODIS, AATSR) with a spatial resolution of $1 \sim 2 \mathrm{~km}$ and geostationary satellites (e.g., GOES, MTSAT, SEVIRI) with a spatial resolution of 5 6 km, as well as microwave sensors (e.g., AMSR-E, TMI) with a spatial resolution of $25 \mathrm{~km}$. In-situ SST data are obtained from the Global Ocean Data Assimilation Experiment (GODAE) server consisting of thousands of daily ship and buoy measurements. All these SST observations from sixteen satellite sensors and in-situ platforms are used to produce a merged SST product at 1-km using a 2dimensional variational (2DVAR) method (Chao, Y., Z. Li, J. D. Farrara, and P. Huang: Blended sea surface temperatures from multiple satellites and in-situ observations for coastal oceans, Journal of Atmospheric and Oceanic Technology, Vol. 26, No.7, 1435-1446, 2009).

The G1SST data are produced daily in near real-time with yesterday's blended product usually available in the morning. Images are published at the JPL OurOcean portal (http://ourocean.jpl.nasa.gov/SST) with subsetting capabilities. The digital data can be freely downloaded from the JPL OurOcean portal as well as from the GHRSST Global Data Assembly Center (GDAC) as a Level 4 (L4) data product. A number of improvements are being added during the past year including an improved high-resolution land-mask, bias corrections, adding the HRPT data from Australian Bureau of Meteorology. Plans to further improve G1SST will be discussed including a diurnal correction and multi-scale data processing. 


\title{
COUPLED OCEAN-ATMOSPHERE INTERACTION AT OCEANIC MESOSCALES \\ Dudley Chelton
}

\author{
College of Oceanic and Atmospheric Sciences, Oregon State University, Corvallis, OR \\ 97331-5503, U.S.A, Email: chelton@coas.oregonstate.edu
}

Satellite observations have revealed a remarkably strong positive correlation between sea-surface temperature (SST) and surface winds on oceanic mesoscales of 10-1000 km. The widespread existence of this SST influence on surface winds in regions of strong SST gradients throughout the World Ocean, and the detailed structure of the surface wind response to SST, have only become evident over the past decade from simultaneous all-weather microwave satellite measurements of SST and surface winds.

These satellite observations have stimulated considerable scientific interest in the implications of this air-sea interaction to the large-scale and mesoscale circulation of both the atmosphere and ocean. Convergence and divergence of surface winds in regions of spatially varying SST generate vertical motion that can penetrate deep into the atmosphere. Spatial variability of the SST field also results in a curl of the wind stress and its associated upwelling and downwelling that feeds back on the ocean and alters the SST itself.

The air-sea interaction revealed in the satellite observations of SST and surface winds is thus a full 2-way coupling between the ocean and atmosphere that likely influences winds in the troposphere and may therefore be important to the general circulation of the atmosphere. Since state-of-the-art global models used for numerical weather prediction and climate research all appear to underestimate the surface wind response to SST by at least a factor of 2, any indirect influence of SST on tropospheric winds is considerably underestimated in these models. Accurate, high-resolution SST analyses are crucially important for these models to be able to reproduce the observed surface wind response to SST. Improvements are also needed in the technical aspects of the models (e.g., the parameterization of vertical mixing and/or the vertical grid spacing in the marine atmospheric boundary layer).

\section{Reference}

Chelton, D B and Xie, S-P: Coupled ocean-atmosphre interaction at oceanic mesoscales, Oceanography, 23, 52-69, 2010.

http://tos.org/oceanography/archive/234 chelton.html 


\section{WHAT'S IN A GHRSST L4 OR L2P FILE?}

\section{T. Mike Chin}

Jet Propulsion Laboratory (Pasadena, California, USA), Email: mike.chin@jpl.nasa.gov

\begin{abstract}
Variables contained in each of L4 and L2P files at GDAC were listed. As of March 2011, there were eleven L4 products and sixteen L2P products for the date of 2011/060 (March 1, 2011). For the L4 products, the variable list was identical except that nearly half of the products were missing the "sea ice fraction" variable. For the L2P products, there was a much wider variation in the list; however, 10 variables were common to all products examined. While this exercise was originally motivated by writing a semiuniversal software to read each of the L4 and L2P files, the lists examined may provide some samples of "current practices" in implementation of the GHRSST Data Processing Specification (GDS). Examination of the values in some of the variables, such as flags and masks, may yield deeper insights into such practices and interoperability of these files.
\end{abstract}

\section{Introduction}

GHRSST has made a great stride in developing common data formats for the level-2 and level-4 (and other) SST data products. So, what is "common" right now? This question has emerged while writing software routines to read GHRSST compliant L2P and L4 data files

(https://www.ghrsst.org/data/ghrsstdata-tools/). Lists of the variables in the $\mathrm{L} 2 \mathrm{P}$ and $\mathrm{L} 4$ files are thus made from the files obtained from GDAC (ftp://podaac-

ftp.jpl.nasa.gov/allData/ghrsst/data/), for the date of 2011/060 (March 1, 2011). This exercise was done in early March 2011; the list of files and their contents may be different by now and in the future.

\section{Variables in the L4 products}

The following $11 \mathrm{~L} 4$ products were found: ABOM/GAMSSA_28km,

ABOM/RAMSSA_09km, DMI/DMI_OI, JPL/MUR, JPL/RTO_SST, JPL_OUROCEAN/G1SST, NAVO/K10_SST, NCDC/AVHRR_AMSR_OI, NCDC/AVHRR_OI, REMSS/mw_ir_OI, UKMO/OSTIA.

Total of 7 variables were found in these L4 files, as shown in the table below. All 11 files contained the variables lat, lon, time, analysed_sst, analysis_error, mask; however, only 6 of 11 files contained the variable sea_ice_fraction.

\begin{tabular}{|l|c|}
\hline variable name & fraction of products using it \\
\hline Lat & $11 / 11$ \\
Lon & $11 / 11$ \\
time & $11 / 11$ \\
analysed_sst & $11 / 11$ \\
analysis_error & $11 / 11$ \\
mask & $11 / 11$ \\
sea_ice_fraction & $6 / 11$ \\
\hline
\end{tabular}


GHRSST Project Office

\section{Variables in the L2P products}

The following $16 \mathrm{~L} 2 \mathrm{P}$ products were found: AMSRE/REMSS, ATS NR 2P/UPA, AVHRR18_G/NAVO, AVHRR19_G/NAVO, AVHRR19_L/NAVO,

AVHRR19_L/NEODAAS,

AVHRR_METOP_A/EUR,

AVHRRMTA_G/GOES11/OSDPD,

GOES13/OSDPD, MODIS_A/JPL, MODIS_T/JPL, MSG02/OSDPD,
NAR18_SST/EUR, SEVIRI_SST/EUR, TMI/REMSS.

A product directory can contain multiple products. In order to list the variables contained in a file, only the first granule file encountered in each directory was examined.

The following 10 variables were found in all of the L2P products

\begin{tabular}{|l|c|}
\hline variable name & fraction of products using it \\
\hline lat & $16 / 16$ \\
lon & $16 / 16$ \\
time & $16 / 16$ \\
sst_dtime & $16 / 16$ \\
sea_surface_temperature & $16 / 16$ \\
SSES_bias_error & $16 / 16$ \\
SSES_standard_deviation_error & $16 / 16$ \\
confidence_flag & $16 / 16$ \\
proximity_confidence & $16 / 16$ \\
rejection_flag & $16 / 16$ \\
\hline
\end{tabular}

The following variables were found in the majority of the L2P products

\begin{tabular}{|l|c|}
\hline variable name & fraction of products using it \\
\hline brightness_temperature_11um & $4 / 16$ \\
brightness_temperature_12um & $4 / 16$ \\
brightness_temperature_4um & $4 / 16$ \\
probability_of_clear_sky & $4 / 16$ \\
cool_skin & $4 / 16$ \\
diurnal_amplitude & $4 / 16$ \\
polar_stereographic & $4 / 16$ \\
sea_surface_temperature4 & $4 / 16$ \\
solar_zenith_angle & $4 / 16$ \\
chlorophyll_a & $4 / 16$ \\
SSES_bias_error4 & $4 / 16$ \\
SSES_standard_deviation_error4 & $4 / 16$ \\
proximity_confidence4 & $4 / 16$ \\
atsr_dual_nadir_sst_difference & $4 / 16$ \\
K_490 & $4 / 16$ \\
\hline
\end{tabular}


GHRSST Project Office

\section{Summary}

The L4 files have identical variable contents, except for the sea ice fraction variable missing in nearly half of the products.

The L2P files have a much wider variety in variable contents. Of the 38 different variables found,

10 were reported by all L2P products. As many as 24 variables were found in a single L2P file. Some of these variables may be sensor specific ("experimental variables").
Some high-volume products (e.g., MODIS) have nearly 300 granule files per day. The large data volume can affect usage (e.g., downloading); possible ways to address this may be: (i) variablewise subsetting (e.g., OPeNDAP), (ii) formation of a higher level (i.e., L3) product.

This brief and superficial exercise does not include comparison of the uses of (values in) some of the variables, such as flags and masks, that are under GDS control. 


\title{
SOURCES OF ERROR IN SATELLITE-DERIVED FLUXES
}

\section{Carol Anne Clayson}

Florida State University, USA, Email : cclayson@fsu.edu

In this talk I will discuss the current state of the art satellite-derived air-sea turbulent flux methodologies, with a highlight on the main sources of errors. A particular focus will be on uncertainties in SST analyses and spatial/temporal resolution and their impacts on the overall flux budgets. These uncertainties will be compared with uncertainties from the other retrieved parameters needed for the turbulent fluxes. I will conclude with a discussion of needed developments in satellite SST products in order to improve flux estimates.

\section{THE IMPACT OF DIURNAL WARMING ON SST FRONTS}

\author{
Peter Cornillon $^{(1)}$, John Salter ${ }^{(1)}$, Pierre Le Borgne ${ }^{(2)}$
}

(1) University of Rhode Island, GSO, Narragansett, RI 02882 USA), Email : pcornillon@me.com isalter@gso.uri.edu

(2) Meteo-France, CMS, BP 50747, 22307, Lannion France, Email: pierre.leborgne@meteo.fr

Diurnal warming tends to "cap" the upper ocean with a very thin $(<1 \mathrm{~m})$ layer of relatively warmer water. The spatial distribution of these warm patches is in general determined by that of the atmosphere; i.e., diurnal warming regions are imprinted on the ocean surface by the atmosphere determined primarily by cloud cover and wind speed. This means that sea surface temperature (SST) gradients in these regions may be influenced by the atmosphere. In this study, we examine the degree to which SST fronts and gradients that exist at 500 local sun time (LST) are obliterated by diurnal warming and the degree to which SST fronts and gradients are created by diurnal warming. In both cases, the generated as a function of time of day and of the degree of warming. The analysis was based on July MSG data for the Mediterranean Sea. All Julys from 2003-2008 were used. Diurnal warming induced fronts were seen to peak with a probability of approximately $10 \%$ at 1400 LST and for diurnal warming events of 2 to $3.5^{\circ} \mathrm{K}$. In contrast, preliminary results suggest that persistent fronts, with a front probability of approximately 5\%, were eroded over the course of the day by more than $20 \%$; i.e., down to values on the order of $4 \%$ when averaged over all diurnal warming bins. Similar results are obtained for the gradient fields. 


\section{GLOBAL SEA SURFACE TEMPERATURE RETREIVAL USING AN OPTIMAL ESTIMATION ALGORITHM}

\section{${\text { Caroline } \text { Cox }^{(1)} \text {, Haiyan Huang }}^{(2)}$, Don Grainger ${ }^{(2)}$, Richard Siddans ${ }^{(1)}$, Caroline Poulsen $^{(1)}$, Gareth Thomas ${ }^{(2)}$, Elisa Carboni ${ }^{(2)}$ Tim Nightingale $^{(1)}$}

(1)RAL Space, STFC, Harwell Oxford (UK), Email: caroline.cox@stfc.ac.uk

(2) AOPP, University of Oxford (UK)

An optimal estimation algorithm has been applied to top of atmospheric radiances measured with the Advanced Along Track Scanning Radiometer (AATSR) in order to retrieve the sea surface temperature (SST) under cloud-free conditions. The algorithm used in this work is the Oxford-RAL Retrieval of Aerosol and Cloud (ORAC) model which has recently been extended to calculate SSTs in addition to cloud and aerosol properties. The retrieved SSTs are compared with global buoy data and the operational AATSR SST product for the months of January, April, July and October of 2008. The results of this comparison will be used to assess biases in the model and hence any improvements that may be made to the retrieval algorithm. In particular, the effect of sea surface emissivity, wind speed and viewing geometry are investigated. 


\title{
RECENT ADDITIONS TO THE SST QUALITY MONITOR (SQUAM)
}

\author{
Prasanjit Dash $^{(1,2)}$, Alexander Ignatov ${ }^{(1)}$ \\ (1)NOAA NESDIS / CSU CIRA, 5200 Auth Rd, MD 20746 (USA), \\ Email: prasanjit.dash@noaa.gov \\ (2) NOAA NESDIS STAR OSB, 5200 Auth Rd, MD 20746 (USA), \\ Email: alex.ignatov@noaa.gov
}

For a few decades, satellite-based sea surface temperature (SST) products have been operationally generated by a number of agencies from data onboard Low Earth Orbit (LEO) and Geostationary (GEO) satellites. NOAA AVHRR based SST products have the longest record, and the newer advanced sensors are employed to generate more accurate products. The GEO satellites also have long history tracing back to GOES imager as well as newer improved sensors such as SEVIRI onboard Meteosat Second Generation. These past and present satellite missions have been providing data to the meteorological and remote sensing communities and will be continued by follow-up missions, such as the JPSS, GOES-R and Meteosat Third Generation. Different Level-2 (L2) and Level-3 (L3) SST products will continue to be generated by different agencies and employing different SST algorithms, cloud removal and quality control techniques, which may come in different formats, and with different ice-sea and data quality flags. A number of uniformly gridded model SST data have also been generated to provide gap-free coverage of the world oceans, known as Level-4 or L4 analysis fields.

With all these data in hand, the major challenge is to (cross)-evaluate performance and validate these products against in situ data, using a consistent and uniform metric. Towards this end, the GHRSST (http://www.ghrsst.org) established a concerted effort and placed a framework for pursuing these activities, via international exchange and assigning specific tasks to different working and technical advisory groups. As a contribution to the GHRSST and to meet the NESDIS requirements, the SST Quality Monitor (SQUAM) was developed in the last years for ensuring quality, stability, and cross-platform consistency of a number of SST products. The SQUAM is designed to operate in near real-time (NRT) and provides diagnostics through a web-interface for both the SST developers and the researchers at: http://www.star.nesdis.noaa.gov/sod/sst/s quam/.

Initially, the SQUAM was meant to monitor only the NESDIS L2 GAC SST products, namely the SSTs from the heritage Main Unit Task (MUT) system and the newer Advanced Clear-Sky Processor over Oceans (ACSPO) system, currently operational at NESDIS. Gradually other L2, L3, and L4 products were also added, in collaboration with research partners from other agencies. The current major modules in SQUAM are listed below (all accessible from SQUAM main page):

- NESDIS MUT GAC (L2): http://www.star.nesdis.noaa.gov/sod/ss t/squam/MUTI

- NESDIS ACSPO GAC (L2): http://www.star.nesdis.noaa.gov/sod/ss t/squam/ACSPO/

- NAVOCEANO GAC (L2) http://www.star.nesdis.noaa.gov/sod/ss t/squam/NAVO/

- NESDIS and O\&SI SAF FRAC (L2): http://www.star.nesdis.noaa.gov/sod/ss t/squam/FRACl

- PathFinder v5.0 GAC (L3): http://www.star.nesdis.noaa.gov/sod/ss t/squam/PFl. L4 products: http://www.star.nesdis.noaa.gov/sod/ss t/squam/L4/

The L2 and L3 diagnostics provided by SQUAM are based on statistical analyses of differences between satellite SST $\left(T_{S}\right)$ and a number of global level-4 (L4) and in 
GHRSST Project Office

situ reference SST fields $\left(T_{R}\right)$. The underlying assumption is that the probability density function of global SST differences $\left(\Delta T_{S}\right)$ is close to a Gaussian distribution. Consequently, the analyses are based on $\Delta T_{S}$ histograms, time series of the statistical moments (conventional and robust), global maps, dependence plots $\left(\Delta T_{S} \quad\right.$ vs. observational and atmospheric parameters such as view zenith angle, water content etc.), and Hovmöller diagrams. Median and a robust standard deviation are used to identify and remove outliers for quality control. Double differencing (DD) technique was implemented to quantitatively measure the "cross-platform" and "day-night" consistencies. Hovmöller-like diagrams are used to visualize time-series of the dependences. Cross-evaluation of various L4 SSTs was also included in SQUAM, to serve the needs of $L 4$ producers and users, and validation of L4 SSTs against in situ SST has been recently added to the L4-SQUAM. Work is underway to add in situ validation for the L2 and L3 products, too, in a consistent way.

The SQUAM was first presented at GHRSST-X in Santa Rosa. Based on the initial results and diagnostics, considerable effort was made to extend the capabilities of SQUAM and to further include a number of operational SST products, including GEO product (SEVIRI). Here we report the recent additions and developments. 


\title{
THE GLOBAL MONITORING FOR ENVIRONMENT AND SECURITY (GMES) SENTINEL-3 MISSION
}

\author{
C. Donlon ${ }^{(1)}$, B. Berruti, A. Buongiorno, M-H. Ferraria, J. Frerick, P. Goryl, U. Klein, \\ H. Laur, C. Mavrocordatos, J. Nieke, H. Rebhan, B. Seitz, J. Stroede, and R. \\ Sciarra \\ (1) ESA/ESTEC, Keplerlaan 1, 2201 AZ, Noordwijk, The Netherlands. \\ ESA/ESRIN, via Galileo Galilei, Frascati Rome, Italy, Email: craig.donlon@esa.int
}

Global Monitoring for Environment and Security (GMES) has been established to address the needs of European policy makers to access accurate and timely information to better manage the environment, understand and mitigate the effects of climate change and ensure civil security. Sentinel-3 is a European Earth Observation satellite mission designed to ensure the long-term collection of uniform quality data products generated and delivered in an operational manner to GMES services in the marine environment with contributions to land, atmospheric, emergency, security and cryospheric applications. The mission includes a series of satellites over a 20 year period starting with the launch of Sentinel-3a in 2013 and during full operations two identical satellites will be maintained in the same orbit with a phase delay of $180^{\circ}$. Sentinel-3 measurement requirements have been derived from operational user needs to insure data continuity for the observation and monitoring of:

- Sea surface topography (SSH), significant wave height $(\mathrm{Hs})$ and surface wind speed derived over the global ocean to an equivalent accuracy and precision as that presently achieved by ENVISAT Radar Altimeter-2 (RA-2).

- Enhanced surface topography measurements in the coastal zone, sea ice regions and over inland rivers, their tributaries and lakes.

- Sea surface temperature (SST) determined for oceanic and coastal waters globally to an equivalent accuracy and precision as that presently achieved by the ENVISAT Advanced Along Track Scanning Radiometer (AATSR) over the ocean (i.e. $<0.3 \mathrm{~K}$ ), at a spatial resolution of $1 \mathrm{~km}$.

- Visible, Near Infrared, Short-Wave Infrared, and Thermal Infrared radiances for oceanic, inland and coastal waters, land surfaces including sea ice and ice sheets determined to an equivalent level of accuracy and precision as ENVISAT Medium Resolution Imaging Spectrometer (MERIS), AATSR and SPOT Vegetation data with complete ocean coverage in 2-3 days, complete land coverage in 1-2 days, a spatial resolution of $\leq 0.3 \mathrm{~km}$ and simultaneously and co-registered with SST measurements.

The GMES Sentinel-3 mission addresses these requirements by implementing and operating the following components:

- A Synthetic Aperture Radar Altimeter (SRAL) instrument, a passive microwave radiometer (MWR) a GPS receiver and laser retro-reflector for precise orbit determination providing continuing the legacy of ENVISAT RA-2 and Cryosat.

- An Ocean and Land Colour Imager (OLCl) delivering multi-channel optical measurements for ocean and land providing continuity to ENVISAT MERIS.

- A Sea and Land Surface Temperature Radiometer (SLSTR) delivering accurate surface ocean, land and ice temperature providing continuity of ENVISAT AATSR. 
GHRSST XII -

Proceedings, Edinburgh

GHRSST Project Office

- An operational ground segment providing access to core data product in a near
Issue: Final

Date: September 27, 2011

real time mode and a collaborative ground segment providing additional data products for specific users and purposes in collaboration with other entities.

This poster provides an overview of the GMES Sentinel-3 mission. 


\title{
SEA SURFACE TEMPERATURE FROM THE ALONG-TRACK SCANNING RADIOMETER REPROCESSING FOR CLIMATE PROJECT.
}

\author{
Owen Embury $^{1}$, Christopher J. Merchant ${ }^{2}$
}

(1) University of Edinburgh, Edinburgh (UK), Email : owen.embury@ed.ac.uk

(2) University of Edinburgh, Edinburgh (UK), Email: c.merchant@ed.ac.uk

\begin{abstract}
Based on Along-Track Scanning Radiometer (ATSR) data the ATSR Reanalysis for Climate (ARC) dataset provides daily Sea Surface Temperature (SST) observations from mid-1991 through to end-2009. Compared to operational ATSR SSTs the ARC data use improved retrieval techniques, cloud and dust detection, and inter-satellite homogenisation. Comparison against in situ indicates regional biases are $<0.1 \mathrm{~K}$, inter-satellite differences are $<0.05 \mathrm{~K}$, while maintaining maximum stability and independence from the in situ record.
\end{abstract}

ARC data are available in NetCDF format in daily average fields at 0.1 degree resolution including: skin SST, sub-skin SST, and depth SST (0.2m and $1.0 \mathrm{~m})$.

\section{Introduction}

The (A)ATSR Reprocessing for Climate (ARC) project has produced a new, high quality record of sea surface temperature (SST) from the Along Track Scanning Radiometer (ATSR) series of instruments intended for climate change research. In order for the SST record to be suitable for climate the ARC project aims were (Merchant et al. 2008):

- Independence from other records

- At least 15 years global coverage

- Regional biases $<0.1 \mathrm{~K}$

- Stability of $0.05 \mathrm{~K}$ per decade

- Both skin and bulk SSTs

- Comprehensive error characterization

ARC SSTs have been generated by reprocessing the ATSR Level $1 \mathrm{~b}$ dataset through to end 2009 from the (A)ATSR multi-mission archive held at NEODC.
This paper presents a brief overview of the ARC data set. With a description of the SST retrieval scheme in Section 2, the available data in Section 3, and a summary of the comparison with in situ measurements in Section 4.

\section{Retrieval Scheme}

ARC SSTs are estimated using a coefficient-based retrieval scheme (Embury and Merchant 2011) which is robust to the presence of stratospheric aerosol from the Mount Pinatubo eruption in 1991. The coefficients are banded by: total column water vapour (TCWV) to reduce the effects of atmospheric variability on the nadir and day-time retrievals; satellite zenith angle to reduce viewing angle dependent biases from the dual-view geometry; and year to account for changes in trace gas concentrations during the ATSR missions.

The accurate calibration and characterisation of the ATSR instruments (for most channels) mean it is possible to derive the retrieval coefficients from radiative transfer (RT) model outputs without use of in situ SST measurements (Embury et al. 2011). However, small uncertainties in the characterisation of the instruments result in $-0.1 \mathrm{~K}$ differences in the retrieved SSTs. These have been eliminated by cross-calibrating between the three ATSR instruments. The homogenised SSTs therefore remain independent of the in situ record.

The cross-calibration process compares the observed AATSR-ATSR2 differences against the differences predicted by simulation. These inter-satellite differences are used to adjust the RT simulations for the earlier satellite bringing the retrieved SSTs into alignment with the later instrument. The same process is then 
GHRSST XII -

Proceedings, Edinburgh

GHRSST Project Office

repeated for the ATSR2-ATSR1 overlap accounting for the increased detector temperature at the end of the ATSR1 mission. As the calibration of the ATSR1 instrument is known to have varied with the 12 micron detector temperature, the adjustment applied to ATSR1 is interpolated from zero at start-of-mission to that found from the overlap analysis at the end-of-mission.

Finally, being based on RT simulations the $A R C$ retrieval coefficients are sensitive to the skin temperature of the ocean. In order to produce a depth-SST product which can be compared to in situ measurements, ARC data includes estimates of SST0.2m and SST1.0m which are estimated using the Fairall and Kantha-Clayson models to account for skin and thermal stratification effects (Embury et al. 2011).

\section{Data Available}

Due to the various channel and view combinations available with the ATSR instrument, there are several different retrieval "algorithms" possible. Firstly, there are dual-view retrievals (indicated by the letter D) which use both the nadir and forward views from the ATSR instrument and nadir-only retrievals (indicated by the letter $\mathrm{N}$ ) which only use the nadir view. The dual-view retrievals are the recommended SSTs as they are much more robust to atmospheric variability. The nadir-only retrievals, for instance, are not robust to stratospheric aerosol and will be negatively biased during the years following the Mount Pinatubo eruption in 1991.

Secondly there are both three-channel retrievals (indicated by the number 3 ) using the 3.7, 11, and 12 micron channels and two-channel retrievals (indicated by the number 2) which only use the 11 and 12 micron channels. The three-channel retrievals are more accurate than the twochannel retrievals, but they are only valid at night as the 3.7 micron channel is strongly affected by reflected solar radiance.

From the above, the recommended "algorithm" is the D3 retrieval at night, and the D2 for day. However, there are cases
Issue: Final

Date: September 27, 2011

where users may wish to use the others. For instance, when the consistency of the retrieval method throughout the complete time period, both day and night, is the primary requirement then the D2 SSTs should be used. Alternatively, if very lownoise retrievals are required at the expense of aerosol robustness and daytime capability then the N3 SSTs could be considered.

All SSTs are available as skin estimates (this is the SST which the satellite observes), and depth and time adjusted SSTs for $20 \mathrm{~cm}$ and $1.0 \mathrm{~m}$ below the sea surface. The depth SSTs have been adjusted to a common Local Equatorial Crossing Time (LECT) of 10:30 to account for the change in orbit between the ATSR1/2 (10:30 LECT) and the AATSR instrument (10:00 LECT).

\section{Comparison with in situ}

Global statistics comparing the ARC SSTs with in situ drifter measurements are shown in Tables 1 (median difference) and 2 (robust standard deviation), the AATSRin situ differences are shown as a function of latitude in Figure 1 and total column water vapour in Figure 2. The median differences from Table 1 show that the ARC SSTs are meeting the criteria for biases $<0.1 \mathrm{~K}$ on a global scale and highlight the consistency between different sensors, channel combinations, and day/night retrievals.

The robust standard deviations (RSD) in Table 2 are representative of the combination "retrieval noise" and the error in the in situ measurement. For instance, the lowest RSD of $0.180 \mathrm{~K}$ for the AATSR $\mathrm{N} 3$ retrieval is effectively the error in the in situ measurements and is comparable to the $\sim 0.2 \mathrm{~K}$ drifter error found by O'Carroll et al. (2008). The D3 retrieval, while it is more robust to atmospheric conditions and aerosol, does suffer from slightly elevated noise levels. This is due to the use of the forward view which introduces additional noise through the radiometric noise in the second view, and the slight mismatch between the nadir and forward views (the increased zenith angle of the forward view means the pixels are larger and do not 
GHRSST XII -

Proceedings, Edinburgh

GHRSST Project Office

have a one-to-one correspondence with nadir view pixels). However, the increase is small and does not represent any regional or systematic issues. The RSD for the D2 retrieval is in turn slightly higher than the D3 retrieval. This is because the D2 retrieval does not use the 3.7 micron channel and is therefore more sensitive to atmospheric variability, particularly for extremely high values of TCWV where the RSD increases further (Figure 2). However, as shown in Figure 1, the effects are still small and zonal biases are within the $0.1 \mathrm{~K}$ target. Finally, the RSD for the $\mathrm{N} 2$ retrieval is the highest, this is related to systematic errors in the retrieval itself with zonal biases of $\sim 0.1 \mathrm{~K}$ seen in Figure 1 .

Comparing the statistics for the AATSR to the earlier instruments there are only small changes in the bias relative to drifters (Table 1) but there is an increase in RSD for ATSR2 and ATSR1. This is due to differences between the instruments and changes in the in situ observing network over time. The length of ATSR2 and AATSR data periods is roughly the same, but the ATSR2 period contains only $\sim 30 \%$ the number of matches found for AATSR as the drifter network has been expanded over the years. In addition, most of the drifter measurements during the AATSR period are with 1 hour of the satellite observation; however, during the ATSR2 period the satellite-drifter time differences are larger increasing the noise due to geophysical variability between the two observations. The ATSR1 period contains even fewer matches $(-30 \%$ of the ATSR2 count) and even higher RSDs. During this time the drifter network was even smaller and had restricted geographical coverage. In the case of ATSR1 we see that the RSD is higher for the D2 retrieval than the $\mathrm{N} 2$, this is due to increased radiometric noise and pickup from the on board cooler in the satellite data from the elevated detector temperatures. While the ATSR1 single view N2 retrieval is less affected by instrument noise the D2 is still the recommended SST for any climate applications. This is because the N2 retrieval will be subject to the zonal biases discussed above and it is not robust to stratospheric aerosol so the retrievals are
Issue: Final

Date: September 27, 2011

biased cold by up to $0.5 \mathrm{~K}$ after the Mount Pinatubo eruption (this is not clear in the statistics of Table 1 as the majority of the drifter observations are from the end of the ATSR1 mission after the aerosol effects had dissipated).

\begin{tabular}{|c|l|l|l|l|}
\hline Matches & N2 & N3 & D2 & D3 \\
\hline ATSR1 & & & & \\
\hline $\begin{array}{l}\text { Day: } \\
15362\end{array}$ & -0.028 & & 0.045 & \\
\hline $\begin{array}{c}\text { Ngt: } \\
11741\end{array}$ & -0.068 & & 0.032 & \\
\hline ATSR2 & & & & \\
\hline $\begin{array}{c}\text { Day: } \\
52786\end{array}$ & 0.012 & & 0.053 & \\
\hline $\begin{array}{c}\text { Ngt: } \\
\text { 41780 }\end{array}$ & 0.034 & 0.045 & 0.052 & 0.046 \\
\hline AATSR & & & & \\
\hline $\begin{array}{c}\text { Day: } \\
\text { 187865 }\end{array}$ & 0.007 & & 0.047 & \\
\hline $\begin{array}{c}\text { Ngt: } \\
\text { 149260 }\end{array}$ & -0.012 & 0.006 & 0.020 & 0.017 \\
\hline
\end{tabular}

Table 1. Median of ARC SST0.2m - in situ drifter measurements.

\begin{tabular}{|c|c|c|c|c|}
\hline Matches & N2 & N3 & D2 & D3 \\
\hline \multicolumn{5}{|l|}{ ATSR1 } \\
\hline $\begin{array}{l}\text { Day: } \\
15362\end{array}$ & 0.472 & & 0.501 & \\
\hline $\begin{array}{l}\text { Ngt: } \\
11741\end{array}$ & 0.469 & & 0.502 & \\
\hline \multicolumn{5}{|l|}{ ATSR2 } \\
\hline $\begin{array}{c}\text { Day: } \\
52786\end{array}$ & 0.347 & & 0.306 & \\
\hline $\begin{array}{c}\text { Ngt: } \\
41780\end{array}$ & 0.340 & 0.228 & 0.282 & 0.243 \\
\hline \multicolumn{5}{|l|}{ AATSR } \\
\hline $\begin{array}{c}\text { Day: } \\
187865\end{array}$ & 0.307 & & 0.218 & \\
\hline $\begin{array}{c}\text { Ngt: } \\
149260\end{array}$ & 0.315 & 0.180 & 0.216 & 0.197 \\
\hline
\end{tabular}

Table 2. Robust standard deviation of ARC SST0.2m - in situ drifter measurements. 


\section{GHRSST Project Office}

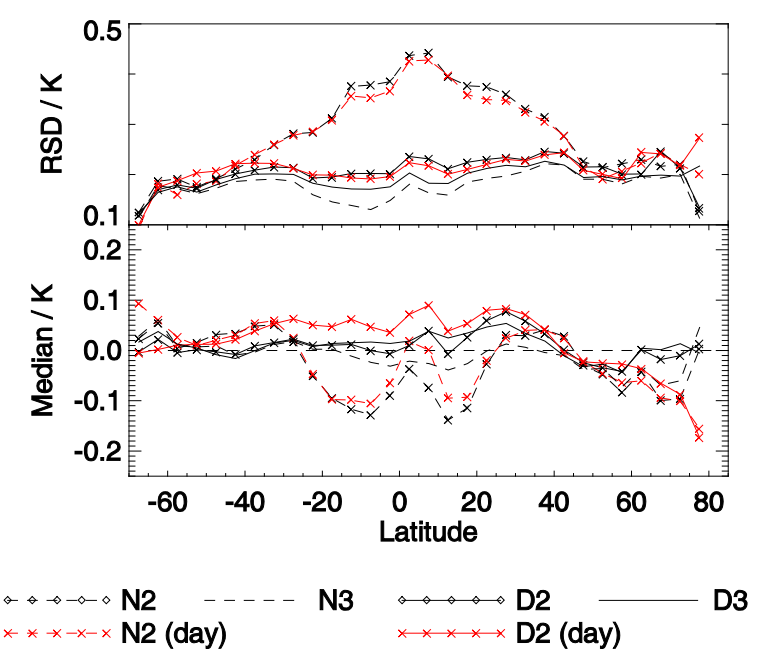

Figure 1. AATSR retrieved SST-0.2m - in situ drifter measurements as a function of latitude.

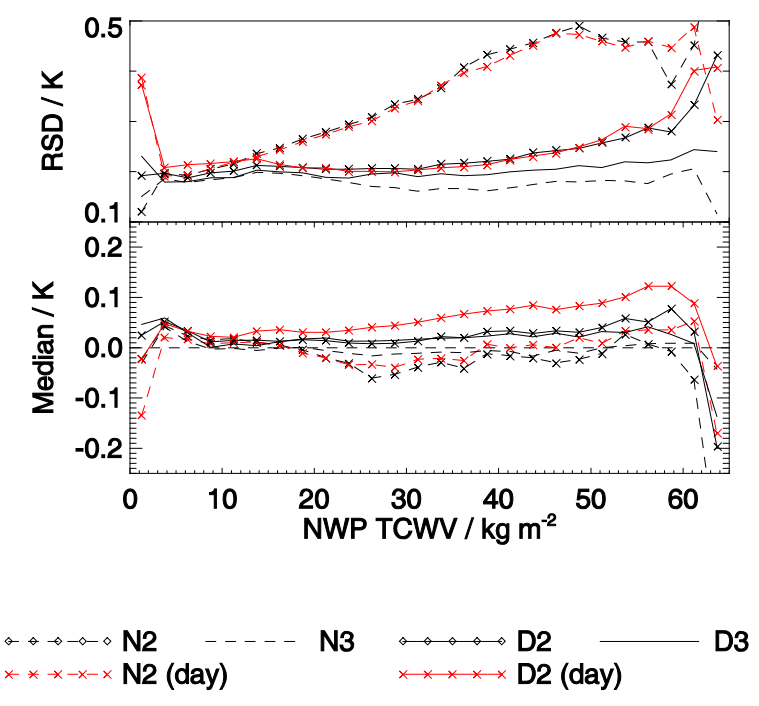

Figure 2. AATSR retrieved SST-0.2m - in situ drifter measurements as a function of total column water vapour.

\section{Conclusion}

The ARC SST products described here are represent a consistent reprocessing of the (A)ATSR multi-mission archive to produce data suitable for climate applications. The SSTs are generated independently of in situ measurements and show low regional biases and good stability.

The ARC SST products are currently being prepared for release and will be available from the NERC Earth Observation Data Centre (NEODC).

\section{References}

Embury, O., C.J. Merchant and M.J. Filipiak, A reprocessing for climate of sea surface temperature from the along-track scanning radiometers: Basis in radiative transfer, Rem. Sens. Env., In Press, Corrected Proof, 2011, DOI: $10.1016 /$ j.rse.2010.10.016.

Embury, O., C.J. Merchant, M.J. Filipiak, A reprocessing for climate of sea surface temperature from the along-track scanning radiometers: A New Retrieval Scheme, Rem. Sens. Env., In Press, 2011.

Embury, O., C.J. Merchant, G.K. Corlett, A reprocessing for climate of sea surface temperature from the along-track scanning radiometers: Preliminary validation, accounting for skin and diurnal variability, Rem. Sens. Env., In Press, 2011.

Good, E.J., X. Kong, O. Embury and C.J. Merchant, An Infrared Desert Dust Index for the AlongTrack Scanning Radiometers, Rem. Sens. Env., In Press, 2011.

Merchant, C.J., D. Llewellyn-Jones, R.W. Saunders, N.A. Rayner, E.C. Kent, C.P. Old, D. Berry, A.R. Birks, T. Blackmore, G.K. Corlett, O. Embury, V.L. Jay, J. Kennedy, C.T. Mutlow, T.J. Nightingale, A.G. O'Carroll, M.J. Pritchard, J.J. Remedios and S. Tett, Deriving a sea surface temperature record suitable for climate change research from the along-track scanning radiometers, Adv. Sp. Res., 41 (1), 1-11, 2008, DOI: 10.1016/j.asr.2007.07.041.

O'Carroll, A.G., J.R. Eyre, R.W. Saunders, ThreeWay error analysis between AATSR, AMSR-E, and in situ sea surface temperature observations. J. Atmos. Oceanic Technol., 25 (7), 1197-1207, 2008, DOI: 10.1175/2007JTECHO542.1 


\title{
OSTIA REANALYSIS: A HIGH RESOLUTION SST AND SEA-ICE REANALYSIS.
}

\author{
Emma Fiedler ${ }^{(1)}$, Jonah Roberts-Jones ${ }^{(2)}$, Matthew Martin ${ }^{(3)}$. \\ (1) Met Office, Fitzroy Rd, Exeter,( United Kingdom), emma.fiedler@metoffice.gov.uk \\ (2) Met Office, Fitzroy Rd, Exeter,( United Kingdom), jonah.roberts-jones@metoffice.gov.uk \\ (3) Met Office, Fitzroy Rd, Exeter,( United Kingdom), matthew.martin@metoffice.gov.uk
}

A high resolution SST and sea-ice reanalysis has been produced using the Operational SST and sea Ice Analysis (OSTIA) system which runs at the UK Met Office. The output is a combined foundation SST and sea ice concentration product on a $1 / 20^{\circ}(\sim 6 \mathrm{~km})$ grid. The OSTIA reanalysis is a global, daily product running from $1^{\text {st }}$ Jan 1985 to $31^{\text {st }}$ Dec 2007. Observational data sources used in the reanalysis are the AVHRR Pathfinder archive, (A)ATSR multimission archive, ICOADS in-situ archive and sea-ice data from the EUMETSAT OSISAF archive. Input data is passed through an automatic quality control system and a bias correction on selected satellites is carried out. OSTIA then uses a multi-scale optimal interpolation scheme to assimilate in-situ and satellite SST observations onto a first guess field provided by the previous analysis with a relaxation to climatology.

An overview of the OSTIA reanalysis system and the data used will be presented. Results of the assessment of version 1.0 of the OSTIA reanalysis will be shown including validation statistics, comparisons to other reanalysis products and to the operational OSTIA system where there is an overlap with the reanalysis period.

\section{OPTIMAL ESTIMATION OF SST FROM AVHRR 1991-2010 Mark Filipiak $^{(1)}$ and Christopher Merchant ${ }^{(1)}$ \\ (1)University of Edinburgh, Scotland (UK), Email : mif@staffmail.ed.ac.uk}

The whole series of AVHRR measurements (1978-2010) is being used in a physical retrieval (optimal estimation) of SST to derive a consistent SST record over this period. The physical retrieval uses ECMWF ERA Interim/ ERA 40 results for the atmospheric parameters, OI SST v2 (in ERA Interim/ERA 40) for the background SST, RTTOV 8 for the radiative transfer, CLAVR-x for the cloud masking, and a modified version of ARC (ATSR Reanalysis for
Climate) for the optimal estimation. Estimates are based on two IR channels $(11+12$ micron, day and night) or three IR channels $(11+12+3.7$ micron, night only), for Maximum A Priori (MAP) and Maximum Likelihood (ML) retrievals.

Results (currently not bias-corrected with respect to in situ measurements) for 1991-2010 are presented and compared with Pathfinder V5/5.1 SSTs for the same period. 


\title{
APPLYING BARNE'S TECHNIQUE FOR DAILY SST (AVHRR AND TRMM I MICROWAVE) IMAGES COMPOSITION
}

\author{
Gutemberg Borges França ${ }^{(1)}$, Antônio do Nascimento Oliveira ${ }^{(1)}$ \\ Bianca Couto Ruivo $^{(1)}$, Rosa Cristhyna de Oliveira Vieira Paes ${ }^{(1)}$
}

(1) Federal University of Rio de Janeiro, Department of Meteorology, Laboratory of Applied Meteorology, University City Campus, CEP: 21.941-916 Rio de Janeiro, RJ - Brazil., Email: gutemberg, antonio, bianca.ruivo, rosa@lma.ufri.br

\section{ABSTRACT}

The Oceanographic Modeling and Observation Research (REMO) is network composed of four Brazilian universities, National Petroleum Agency and Petrobras which his main goals are: a) to develop an assimilative ocean forecast system for the Brazilian continental shelf and slope regions, and b) to supply information to the environmental authorities in case of oil disasters. In this work, a system for daily cloud free sea surface temperature (SST) composition based on thermal AVHRR (level 2) and microwave TMI (level 2) data from the region in $45^{\circ} \mathrm{S} 15^{\circ} \mathrm{N}-70^{\circ} \mathrm{W} 15^{\circ} \mathrm{W}$ is presented. Barnes's objective analysis (Barnes, 1964) is utilized as interpolator to merge these two data sources, which have different spatial and temporal resolutions in a daily SST composition in a regular $\left(0.05^{\circ} \sim 5.5 \mathrm{~km}\right)$ grid product. Comparison with in situ SST (daily average) measurements from 11 PIRATA's buoys (moored) and 23 drift buoys from National Buoys Program (PNBOIA) from Brazilian Navy were carried out and the values of RMSE and correlation are 0.19 and 0.25 and 0.98 and 0.94 , respectively. Summarily, the validation results are quite consistent (with SST composition accuracy less than $1.0^{\circ} \mathrm{C}$ ). The SST product - generated by REMO - has been used in the assimilation procedure by numerical oceanographic model.

\section{Introduction}

Sea surface temperature (SST) plays an important role to model the surface energy flux and to understand the exchange of energy and moisture between the ocean and the atmosphere. This parameter is one of the main indicators of climate variability (Barton, 1995). It is also crucial to determine weather and global climate conditions. The SST, moreover, is of great importance in the initialization of numerical models of weather, climate and ocean circulation prediction once it not only allows better refinement of forecasting results but also contributes to the understanding of ocean-atmosphere dynamics (Carvalheiro et al., 2008). This paper present a composition daily cloudfree SST (L4) based on infrared AVHRR (level 2) and microwave TMI (level 2) data by applying the sub-optimal interpolation technique. In addition, some comparison results with in situ SST measurements from moored and driffiting buoys.

\section{SST Composition}

\subsection{Applied Data}

Thermal infrared data from satellites NOAA 18 and 19 were used to produce the daily SST fields. Originated from NAVOCEANO MCSST data set and provided by PODAAC (Physical Oceanography Distributed Active Archive Center), product level 2 was selected. These data have a spatial 
resolution of $0.081^{\circ}(\sim 9 \mathrm{~km})$, with images acquired only twice daily (PODAAC, 2010). The microwave SST data generated by TRMM/TMI was also used here. These data are daily made available, have global coverage and are separated in ascending and descending orbit segments with spatial resolution of $0.25^{\circ}(\sim 25 \mathrm{~km})$ (REMSS, 2010).

\subsection{Methodology}

Figure 1 schematically shows the methodology applied to generate daily analysis cloud-free SST (approximately 5.5 $\mathrm{km})$. The first step refers to AVHRR and TMI data input. The second one is the interpolation using Barnes (1964) objective analysis technique and finally the comparison with in situ SST measurements from moored and driffiting buoys

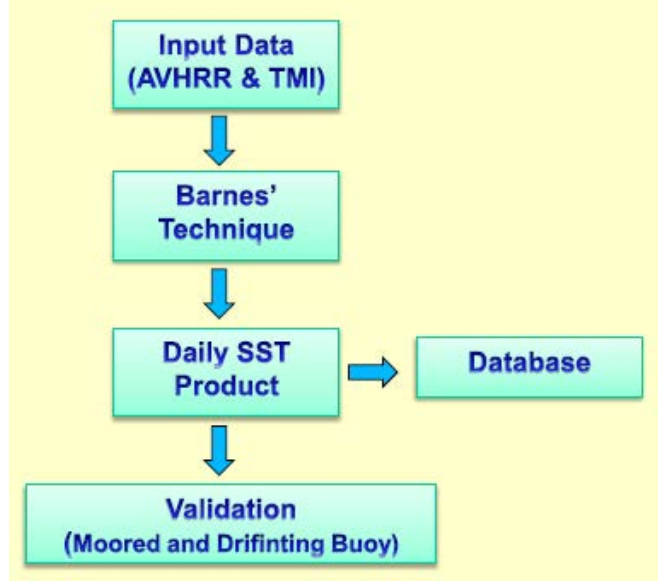

Figure 1. Daily SST composition scheme plus validation.

\section{Validation}

Two databases in situ SST measurements were used in the validation of the SST compositions, been collected 11 buoys (approximately 1 meter deep) of the PIRATA project and 23 drift buoys from National Buoys Program (PNBOIA) from Brazilian Navy for the period from September 2002 to December, 2010 (see Fig. 2).

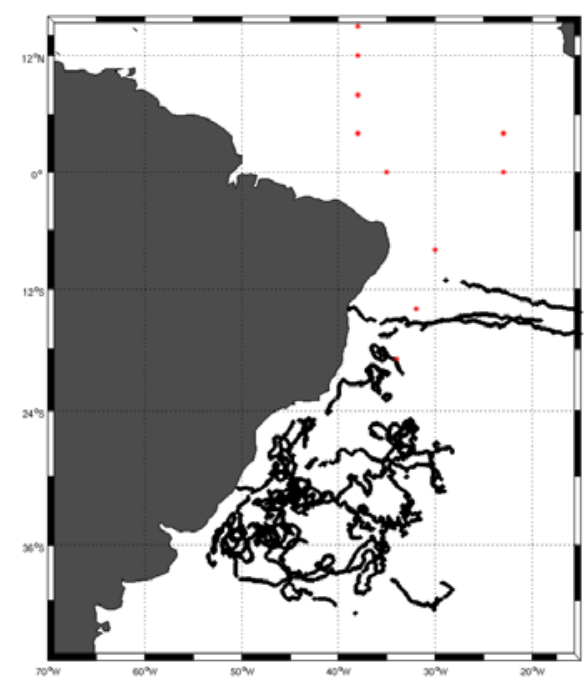

Figure 2. Map the spatial distribution of buoys.

Table 1 and 2 shows a statistics summary of the results of the comparison between the daily analysis cloud-free SST and in situ SST measurements, through the values of RMSE, MAE, MBE and the correlation coefficient. The correlation coefficients for all moored buoys (see Table 1) are higher than 0.85 and the values of RMSE, MAE and MBE have not exceeded 0.6 for all in situ measurements, which corroborate the usual accuracy of SST estimation process by remotely sensed data (França \& Carvalho, 2004). In addition, Table 2 the drifting buoys showed very consistent results, with values of RMSE and correlation of 0.250 and 0.944. Summarily, the validation results are quite consistent with SST composition accuracy less than $1.0^{\circ} \mathrm{C}$. 
GHRSST Project Office

Table 1. Comparison statistics between daily SST composition and average daily in situ SST collected from eleven buoys (moored) of PIRATA's project.

\begin{tabular}{|c|c|c|c|c|c|}
\hline $\begin{array}{c}\text { Moored } \\
\text { Buoy }\end{array}$ & Average & RMSE & MAE & MBE & Correlation \\
\hline $4^{\circ} \mathrm{N}-23^{\circ} \mathrm{W}$ & $\begin{array}{l}\text { Buoy: } 28.06 \\
\text { REMO: } 27.94\end{array}$ & 0.329 & 0.248 & -0.089 & 0.927 \\
\hline $0^{\circ} \mathrm{N}-23^{\circ} \mathrm{W}$ & $\begin{array}{l}\text { Buoy: } 26.58 \\
\text { REMO: } 26.42\end{array}$ & 0.301 & 0.241 & -0.163 & 0.978 \\
\hline $0^{\circ} \mathrm{N}-35^{\circ} \mathrm{W}$ & $\begin{array}{l}\text { Buoy: } 27.51 \\
\text { REMO: } 27.48\end{array}$ & 0.244 & 0.187 & -0.036 & 0.928 \\
\hline $\begin{array}{l}19^{\circ} \mathrm{S}- \\
34^{\circ} \mathrm{W}\end{array}$ & $\begin{array}{l}\text { Buoy: } 26.36 \\
\text { REMO: } 26.27\end{array}$ & 0.288 & 0.228 & -0.098 & 0.989 \\
\hline $\begin{array}{l}15^{\circ} \mathrm{N}- \\
38^{\circ} \mathrm{W}\end{array}$ & $\begin{array}{l}\text { Buoy: } 26.18 \\
\text { REMO: } 26.04\end{array}$ & 0.321 & 0.238 & -0.131 & 0.975 \\
\hline $\begin{array}{l}12^{\circ} \mathrm{N}- \\
38^{\circ} \mathrm{W}\end{array}$ & $\begin{array}{l}\text { Buoy: } 26.96 \\
\text { REMO: } 26.72\end{array}$ & 0.410 & 0.314 & -0.240 & 0.963 \\
\hline $8^{\circ} \mathrm{N}-38^{\circ} \mathrm{W}$ & $\begin{array}{l}\text { Buoy: } 27.83 \\
\text { REMO: } 27.73\end{array}$ & 0.275 & 0.210 & -0.101 & 0.965 \\
\hline $4^{\circ} \mathrm{N}-38^{\circ} \mathrm{W}$ & $\begin{array}{l}\text { Buoy: } 27.99 \\
\text { REMO: } 27.94\end{array}$ & 0.244 & 0.188 & 0.185 & 0.892 \\
\hline $8^{\circ} \mathrm{S}-30^{\circ} \mathrm{W}$ & $\begin{array}{l}\text { Buoy: } 27.59 \\
\text { REMO: } 27.53\end{array}$ & 0.193 & 0.153 & -0.064 & 0.983 \\
\hline $\begin{array}{l}14^{\circ} \mathrm{S}- \\
32^{\circ} \mathrm{W}\end{array}$ & $\begin{array}{l}\text { Buoy: } 26.97 \\
\text { REMO: } 26.81\end{array}$ & 0.317 & 0.253 & -0.158 & 0.973 \\
\hline $\begin{array}{l}12^{\circ} \mathrm{N}- \\
23^{\circ} \mathrm{W}\end{array}$ & $\begin{array}{l}\text { Buoy: } 26.76 \\
\text { REMO: } 26.43\end{array}$ & 0.573 & 0.439 & -0.334 & 0.966 \\
\hline
\end{tabular}

Table 2. Comparison statistics between daily SST composition and average daily in situ SST collected from twenty-three buoys (drifting) of PNBOIA.

\begin{tabular}{|l|l|l|l|c|}
\hline \multicolumn{1}{|c|}{ Average } & RMSE & MAE & MBE & Correlation \\
\hline $\begin{array}{l}\text { Buoy: } 19.628 \\
\text { REMO: } 19.627\end{array}$ & 0.250 & 0.191 & -0.002 & 0.944 \\
\hline
\end{tabular}


GHRSST XII -

Proceedings, Edinburgh

GHRSST Project Office

\section{Conclusion and way forward}

The SST product - generated by REMO has been used in the assimilation procedure by numerical oceanographic model in order to model more precisely ocean circulation. The results have showed that the method to obtain cloud free SST as proposed is quite consistent one. The RMSE, MAE, MBE and CORR have demonstrated ted the good performance over all when daily comparing between estimated SST and in situ SST (from eight PIRATA 's buoys) were made for during the period from September 2002 to December, 2010 as showed in table 1 and 2.

\section{References}

Barnes, S., -1964- A technique for maximizing details in numerical weather map analysis, Journal Applied Meteorology. 3: 395-409.

Carvalheiro, L. C. O., Bernardo, S., Costa, P. D., Orgaz, M. D. J. M. \& Yamasaki, Y.
Issue: Final

Date: September 27, 2011

-2008- Validação da temperatura da superfície do mar a partir de medições de satélite, Livro de Resumos das XXX Jornadas Científicas da Associação Meteorológica Espanhola.

França, G. B. \& Carvalho, W. S. -2004- Sea surface temperature GOES-8 estimation approach for the brazilian coast, vol. 25, International Journal of Remote Sensing, 17: 3439-3450.

Physical Oceanography Distributed Active Archive Center (PODAAC) -2010- Dados de temperatura da superfície do mar, Available at:<ftp://podaac.jpl.nasa.gov/pub/sea_sur face_temperature/avhrr/navoceano_mcs st/doc/avhrr_navoceano.html>, Accessed 20 September 2010.

Remote Sensing Systems (REMSS) -2010Dados de temperatura da superfície do mar, Available at: <http://www.remss.com/tmi/tmi_descripti on.html>, Accessed 20 September 2010. 


\title{
MULTI-SENSOR IMPROVED SEA-SURFACE TEMPERATURE
} (MISST) FOR IOOS

\author{
Chelle Gentemann ${ }^{(1)}$, Ken Casey ${ }^{(2)}$, Peter Cornillon ${ }^{(3)}$
}

(1)Remote Sensing Systems, Santa Rosa, CA, USA, Email :gentemann@remss.com

(2) NOAA/NODC, Silver Spring MD, USA, Email: Kenneth.Casey@noaa.gov

(3) University of Rhode Island, Graduate School of Oceanography, Narragansett, RI, USA, Email: pcornillon@gso.uri.edu

This project builds on the successful partnership developed for the Multi-sensor Improved Sea-Surface Temperature (MISST) for GODAE project (2004-2009). The proposed work picks up where MISST left off in 2009 with many of the same objectives; therefore we will refer to the new project as MISST also. The objectives of this project are to (1) improve and continue generation of satellite SST data and SST analyses in the IOOS DMAC and CF compliant Group for High Resolution Sea Surface Temperature (GHRSST) Data Specification GDS format; (2) distribute and archive these data; and (3) use this improved SST data in applications, many specifically targeted for the Integrated Ocean Observing System (IOOS). Details of the project status and plans will be presented 


\title{
CONSISTENCY OF SEA SURFACE TEMPERATURE ANALYSES IN DEPICTING ENSO BEHAVIOR
}

\author{
Michelle M. Gierach(1), Tong Lee ${ }^{(1)}$
}

(1) Jet Propulsion Laboratory/California Institute of Technology, 4800 Oak Grove Drive, Pasadena, CA 91109 (USA), Email: michelle.gierach@jpl.nasa.gov

El Niño-Southern Oscillation (ENSO), a dominant mode of climate variability on interannual time scales, influences circulations of the atmosphere and ocean and biogeochemical cycles significantly. Because of the associated impacts on flood and drought, agriculture, and fishery, etc., it has important socio-economic consequences. Characterizing ENSO behavior using sea surface temperature (SST) products is important to the understanding and prediction of ENSO. A number of SST products exist today, some of which extended back to the 1800s. However, their consistency in depicting ENSO behavior has not been investigated systematically. Here we use four SST analyses to examine their consistency in describing ENSO conditions in the past three decades (the satellite era). The datasets include the NOAA $1 / 4^{\circ}$ Daily Optimum Interpolation Group for High Resolution SST (GHRSST), NOAA Extended Reconstructed SST version 3 (ERSSTV3b), NOAA Optimum Interpolation
Sea Surface Temperature version 2 (OISSTV2), and Hadley Centre SST version 1 (HadISST1) products. We analyzed conventional Niño indices computed from SST anomalies (referenced to the seasonal climatology for a common period), the amplitudes of $\mathrm{El}$ Niño and La Niña determined from these indices, lowfrequency change in these amplitudes, and the spatial structure of large El Nino events such as the 1997-98 eastern-Pacific El Niño and the 2009-10 central-Pacific El Niño. The SST products have different smoothing or de-correlation scales (ranging from a couple to several hundred kilometers) during their gridding procedures in order to fill data gaps or suppress spatial noise. The impact of smoothing on the representation of ENSO characteristics is investigated. Our results highlight the importance of resolution in characterizing ENSO characteristics and have important implications to the reliability of using historical reconstruction of SST to infer low-frequency change in ENSO behavior. 


\title{
OCEAN HEAT BUDGET FOR THE FLORIDA REEF TRACT: THE ROLE OF SMALL-SCALE PROCESSES IN MAINTAINING PERSISTENT COASTAL SEA TEMPERATURE GRADIENTS.
}

\author{
Lewis J. Gramer ${ }^{(1)}$, Arthur J. Mariano ${ }^{(2)}$, James C. Hendee (3) \\ (1) Cooperative Institute for Marine and Atmospheric Studies, University of Miami, Miami, \\ Florida, USA, Email : lew.gramer@noaa.gov \\ (2) Rosenstiel School of Marine and Atmospheric Sciences, University of Miami, Miami, \\ Florida, USA, Email : amariano@rsmas.miami.edu \\ (3) Atlantic Oceanographic and Meteorological Laboratories (AOML), NOAA, Miami, Florida, \\ USA, Email: jim.hendee@noaa.gov
}

\section{ABSTRACT}

Effective management and conservation of coral reefs and related ecosystems depends critically on a detailed, quantitative understanding of the physical environment (Keller et al. 2009). Thermal and photothermal stresses are major considerations in managing coral reef ecosystems. Physical insights into sea temperature variability offer a way to identify reefs or larger coastal areas that may be more or less prone to temperature extremes under varying light and weather conditions (Hoegh-Guldberg et al. 2005; Yee et al. 2008). An approximately 20 year record of hourly mean in situ sea temperature at seven shallow-water coral reef sites in the Florida reef tract (FRT), has been analyzed for variability at periods from multi-day to interannual. Variability is dominated by annual, semi-annual, and diurnal periods. At sites furthest offshore, variability at nearinertial and "mesoscale" bands (28h and 3-6 days, resp.) is also found, while at the shallowest sites variability in a broad "weather band" of 7-40 days is observed. An apparently robust interannual warming trend is seen at one site only. An oceanic heat budget is estimated based on these and coincident in situ meteorological data, atmospheric reanalysis, numerical ocean model outputs, and estimates of heat exchange with the sea floor substrate. The dominant balance at most sites is between insolation and latent cooling, with benthic heat exchange also being significant at the shallowest site. However at sites near the reef crest bordering the forereef slope, terms for sub-kilometer scale ocean processes, including both enhanced lateral mixing (Park and Chu 2008), and horizontal convection - the so-called thermal siphon (Monismith et al. 2006, Hughes and Griffiths 2008) - are found to be necessary to balance the heat budget. The impact of these processes on the physical oceanography and ecology of these coral reefs is potentially significant (e.g., Monismith et al. 2010). The resulting heat budget closely predicts the amplitude of sea temperature variability at periods from "mesoscale" up to annual (3-365 days). This result encompasses sites where a long record of in situ sea temperature data is available, but which are otherwise diverse in their local topography and the thermal forcing and dynamics that are expected to dominate at each. Preliminary results are presented from applying the heat budget to high-resolution remote sensing data at other sites within the FRT and the Caribbean Sea where in situ data on sea temperature are sparse or wholly lacking. Implications for remote sensing of sea temperature and sea temperature variability within coral reefs and other ecologically sensitive coastal areas are discussed.

\section{References:}

Hoegh-Guldberg O, Fine $M$, Skirving $W$, Johnstone R, Dove S, Strong A, Coral bleaching following wintry weather. 
Limnology and Oceanography 50:265271, 2005

Hughes GO, Griffiths RW, Horizontal convection. Annual Review of Fluid Mechanics 40:185-208, 2008

Keller BD, Gleason DF, McLeod E, Woodley $\mathrm{CM}$, Airame S, Causey BD, Friedlander AM, Grober-Dunsmore R, Johnson JE, Miller SL, Steneck RS, Climate Change, Coral Reef Ecosystems, and Management Options for Marine Protected Areas. Environmental Management 44:1069-1088, 2009

Monismith SG, Genin A, Reidenbach MA, Yahel G, Koseff JR, Thermally driven exchanges between a coral reef and the adjoining ocean. Journal of Physical Oceanography 36:1332-1347, 2006

Monismith SG, Davis KA, Shellenbarger GG, Hench JL, Nidzieko NJ, Santoro AE, Reidenbach MA, Rosman JH, Holtzman $\mathrm{R}$, Martens CS, Lindquist NL, Southwell MW, Genin A, Flow effects on benthic grazing on phytoplankton by a Caribbean reef. Limnology and Oceanography 55:1881-1892, 2010

Park S, Chu PC, Thermal fronts and crossfrontal heat flux in the southern Huanghai Sea and the East China Sea. Acta Oceanologica Sinica 27:1-20, 2008

Yee SH, Santavy DL, Barron MG, Comparing environmental influences on coral bleaching across and within species using clustered binomial regression. Ecological Modelling 218:162-174, 2008 


\title{
COMPARISON OF SATELLITE SST PRODUCTS WITH IN SITU SKIN SST MEASUREMENTS
}

\author{
Lei Guan, Kailin Zhang, and Weicheng Teng \\ Ocean Remotes Sensing Institute, Department of Marine Technology, College of \\ Information Science and Engineering, Ocean University of China, 238 Songling Road, \\ Qingdao, 266100, China, Email: leiguan@ouc.edu.cn
}

\begin{abstract}
Sea Surface temperature (SST) is an essential indicator for climate change. Nowadays there are a number of sensors in orbit with the capacity of SST observations, including mid and thermal infrared as well as microwave observations from space. High accuracy and stability of the satellite SST products are required for long-term climate data record of global SST. It is important to routinely collect in situ SST measurements for evaluation of the quality of the satellite SST products. The infrared SST autonomous radiometer (ISAR), made by the University of Southampton, has been deployed on the research vessel Dong Fang Hong II of Ocean University of China. The ISAR designed with an accuracy of $0.1 \mathrm{~K}$ was calibrated at National Physical Laboratory, UK, in June 2009, through the Committee on Earth Observation Satellites (CEOS) comparison of Infrared radiometry in support of satellite calibration and validation for measuring SST for studies of climate change. The R/V Dong Fang Hong II operates mainly in the China Seas, for about 300 days per year. The skin SST measurements have been collected in the China Seas since September 2009. The in situ measurements are used to evaluate the satellite SST products.
\end{abstract}

\section{Introduction}

Sea Surface temperature (SST) is an essential indicator for climate change. It requires high accuracy and stability of satellite data for long-term climate data record of global SST. Nowadays there are a number of sensors in orbit with the capacity of SST observations, including mid and thermal infrared as well as microwave observations from space. The infrared and microwave radiometers measure the radiation of the skin layer of sea surface. The vertical temperature structure of the upper ocean is both complex and variable depending on the level of shear-driven ocean turbulence and the air-sea fluxes of heat, moisture, and momentum [Donlon et al., 2002]. Surface temperature deviations greater than $3.0 \mathrm{~K}$, referenced to subsurface temperatures below the extent of surface heating are not uncommon and may persist for hours [Gentemann \& Minnett, 2008; Kawai \& Wada, 2007]. To meeting the requirement of the climate data record of SST, it's very important to collect high-quality in situ skin SST measurements for the validation of the satellite SST products. The infrared SST autonomous radiometer (ISAR) is designed as a self-calibrating instrument capable of measuring in situ sea surface skin temperature to an accuracy of $0.1 \mathrm{~K}$ and operating autonomously without service up to 3 months [Donlon et. al., 2008]. An ISAR made by the University of Southampton, has been deployed on the research vessel Dong Fang Hong II of Ocean University of China since September 2009. The R/V Dong Fang Hong II operates mainly in the China Seas, for about 300 days per year. In this paper, the shipboard measurements of skin SST by the ISAR is described and the preliminary analysis of the comparison between satellite products and in situ skin SST data is presented.

\section{ISAR Measurements of the Skin SST}

The ISAR was calibrated at National Physical Laboratory, UK, in June 2009, through the Committee on Earth Observation Satellites (CEOS) comparison of Infrared radiometry in support of satellite calibration and validation for measuring SST for studies of 
climate change [Theocharous et al., 2010]. The results demonstrate the designed accuracy of the instrument. It was deployed on the compass deck of R/V Dong Fang Hong II, around $13 \mathrm{~m}$ above the sea surface (See figure 1). An optical rain gauge, MiniORG made by Optical Scientific, Inc., was used to control the shutter of the ISAR [Donlon et. al., 2008]. Two video cameras were deployed, one used to monitor the sea state and the other used to monitor the shutter of the radiometer. Besides the original data logging system of the instrument, a backup data logging system was developed. The backup data were automatically recorded in the flash disks. The ISAR operated in the China Seas for one cruise starting in September 2009 and nine cruises during the period from March to December in 2010. The tracks of the valid skin SST measurements by ISAR during the 10 cruises are shown in figure 2. The measurements were located in Bohai Sea, Yellow Sea, the East China Sea and the northern South China Sea.

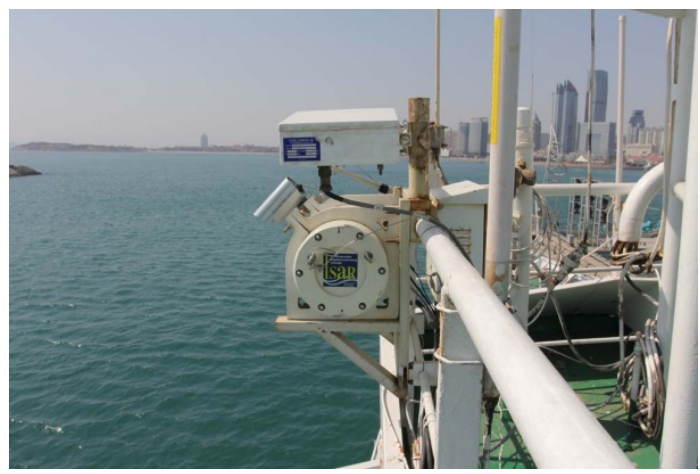

Figure 1. ISAR deployed on R/V Dong Fang Hong II.

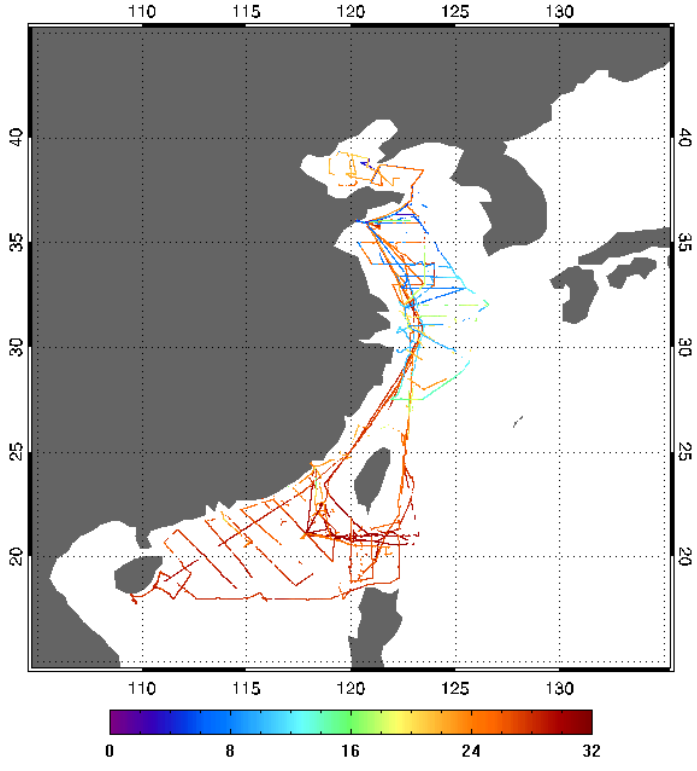

Figure 2. The skin SST measurements by ISAR.

\section{Comparison of the Satellite SST Products with in situ Skin SST Measurements}

The collected ISAR SST measurements were used to evaluate the accuracy of satellite SST products in the China Seas. The preliminary analyses of the comparison of NOAA AVHRR and Aqua AMSR-E SST products against in situ SST were carried out. The NOAA HRPT data have been received and processed by the TeraScan system of the Ground Station at Ocean University of China since 2000. More than 40,000 passes have been archived. The SST products were projected to equal-angle maps with spatial resolution of $0.01^{\circ}$ in the coverage from $10^{\circ} \mathrm{N}$ to $50^{\circ} \mathrm{N}$ and $105^{\circ} \mathrm{E}$ to $145^{\circ} \mathrm{E}$. The matchups between NOAA-18, 19 AVHRR SST products and ISAR skin SST measurements were produced with the temporal window of 1 hour and spatial window of $0.01^{\circ}$. The ISAR SST measurements within the temporal and spatial windows were averaged. The number of matchups is limited, totally 475 , mainly due to the influence of weather conditions. The comparison of AVHRR and ISAR SST show negative bias of 0.16 $\mathrm{K}$ and standard deviation of $0.67 \mathrm{~K}$. The Aqua AMSR-E SST products were processed and delivered by Remote 
GHRSST XII -

Proceedings, Edinburgh

GHRSST Project Office

Sensing Systems. The spatial resolution of the products is $0.25^{\circ}$. The matchups were produced with the temporal window of 1 hour and spatial window of $0.25^{\circ}$. The ISAR SST measurements within the temporal and spatial windows were also averaged. The number of matchups is 109. The initial result of AMSR-E SST against ISAR SST shows positive bias of $0.30 \mathrm{~K}$ and standard deviation of $0.83 \mathrm{~K}$. The possible error sources include some cloud detection failures of AVHRR data at nighttime, and the sub-pixel variation of. The spatial difference may cause large error especially in the area with strong SST gradient due to the interaction of coastal cold water and warm currents. Further investigation with more in situ measurements will be carried out.

\section{Summary}

The ISAR has been deployed on R/V Dong Fang Hong II, continually collecting the in situ measurements of sea surface skin temperature in the China Seas. The data collected during September 2009 to December 2010 were used to evaluate the accuracy of NOAA AVHRR and Aqua
Issue: Final

Date: September 27, 2011

AMSR-E SST products. More satellite products will be investigated in the future with increasing measurements of in situ skin SST.

\section{References}

Donlon, C. J., P. J. Minnett, C. Gentemann, T. J. Nightingale, I. J. Barton, B. Ward, M. J. Murray, Toward Improved Validation of Satellite Sea Surface Skin Temperature Measurements for Climate Research. J. Climate, 15, 353-369, 2002.

Donlon, C., I. S. Robinson, M. Reynolds, W. Wimmer, G. Fisher, R. Edwards, T. J. Nightingale, An Infrared Sea Surface Temperature Autonomous Radiometer (ISAR) for Deployment aboard Volunteer Observing Ships (VOS). J. Atmos. Oceanic Technol., 25, 93-113, 2008.

Gentemann, C. L., and P. J. Minnett, Radiometric measurements of ocean surface thermal variability, J. Geophys. Res., 113, C08017, doi:10.1029/2007JC004540, 2008.

Kawai, Y., and A. Wada, Diurnal sea surface temperature variation and its impact on the atmosphere and ocean: A review, J. Oceanogr., 63(5), 721-877, 2007.

Theocharous, E, E . Usadi , N. P. Fox,: CEOS comparison of IR brightness temperature measurements in support of satellite validation. Part I: Laboratory and Ocean surface temperature comparison of radiation thermometers. NPL Report OP 3, July 2010. 


\title{
NASA ACTIVITIES AND PERSPECTIVES.
}

\author{
Peter Hacker $^{(1)}$, Eric Lindstrom ${ }^{(2)}$
}

(1) NASA Headquarters, Washington, DC (USA), Email: peter.w.hacker@nasa.gov

(2) NASA Headquarters, Washington, DC (USA), Email: eric.j.lindstrom@nasa.gov

NASA supports a range of activities addressing satellite-derived sea surface temperature (SST) data products including: studies with a mission-focus; work on product generation and improvement; support for data product management and delivery; and funding for research and applications. Studies with a mission-focus include a broad range of activities related to sensors currently providing data, as well as studies for missions in the planning and prelaunch stages. Product generation and improvement activities are ongoing and are in direct support of the Group for High Resolution Sea Surface Temperature (GHRSST). A focal point for data product management and delivery is at the NASAsupported PODAAC/GDAC at the Jet Propulsion Laboratory. With regard to research and applications, NASA has organized its sea surface temperature Science Team (SST-ST) in 2010 around the measurement/parameter rather than around a particular mission. A basis for recent activity over the past two years has been the white paper produced by the Interim
Sea Surface Temperature Science Team (ISSTST) on 18 June 2010. The white paper addressed three areas: requirements placed on satellite derived SST products; a framework for the characterization of the error budget for satellite-derived SST products; and recommendations for tasks that need to be undertaken to improve satellite-derived SST products. Building on this framework, the recent ROSES-2011 call for proposals in Physical Oceanography contains two research themes, one of which focuses on the "exploitation of sea-surface temperature products" for physical oceanography science rather than for development or improvement of data products. This program is intended to utilize and exploit the GHRSST data products for research over the next three years. In addition, NASA participates in the National Oceanographic Partnership Program for joint funding of SST projects, and intends to use the SST-ST to help coordinate activities between federal agencies and internationally. 


\section{MULTI SENSOR VALIDATION AND ERROR CHARACTERISTICS OF ARCTIC SATELLITE SEA SURFACE TEMPERATURE OBSERVATIONS}

Jacob L. Hoeyer ${ }^{(1)}$, loanna Karagali ${ }^{(2)}$, Rasmus Tonbo ${ }^{(1)}$ Gorm Dybkjær $^{(1)}$

(1)Center for Ocean and Ice, Danish Meteorological Institute, Lyngbyvej 100, DK-2100, Denmark,Email : jlh@dmi.dk

(2) Risø DTU-Technical University of Denmark, Frederiksborgvej 399, Roskilde, 4000

(Denmark), Email: ioka@risoe.dtu,dk

Six of the operational global satellite sea surface temperature products from Infrared and microwave sensors are validated in a consistent way for open waters north of 60 degrees North including the Arctic Ocean in summer. The 15 months of validation using drifting buoy in situ observations show that the ENVISAT AATSR $1 \mathrm{~km}$ data and the 9 $\mathrm{km}$ NAVOCEANO NOAA 18 data to be superior, while the microwave AMSR-E data has superior coverage because of the microwave's penetration of cloud cover.

The performance of the different satellite products is examined for seasonal variations, cold waters, solar elevation angles and near the Marginal Ice Zone. The validation results identify errors in the satellite products related to the observations techniques, data processing and cloud masking. The temporal and spatial scales of the errors are derived for all satellite products using the satellite versus in situ match-up dataset. The typical temporal scales of the errors are 1-2 days and the characteristic spatial scales of the errors are on the order 300 to 500 kilometres. The error cross correlation between the different satellite products reveal that the infrared sensors show significant correlation in the errors, whereas the errors on the microwave AMSR-E products has a low correlation with all the infrared sensors. Finally, preliminary results will be shown from multisensor bias correction study, which aims at using the differences in the error characteristics to generate a merged reference field with lower noise than the individual satellite products. 


\title{
JOINT RETRIEVALS OF AEROSOL AND SST FROM AATSR
}

\author{
Haiyan Huang ${ }^{(1)}$, Don Grainger ${ }^{(1)}$, Andrew Smith ${ }^{(1)}$, Gareth Thomas ${ }^{(1)}$, Elisa \\ Carboni $^{(1)}$, Richard Siddans ${ }^{(2)}$, Caroline Cox ${ }^{(2)}$, Caroline Poulsen ${ }^{(2)}$ \\ (1)AOPP, Oxford University, UK, Emails: huang@atm.ox.ac.uk, grainger@atm.ox.ac.uk, \\ smith@atm.ox.ac.uk,gthomas@atm.ox.ac.uk, elisa@atm.ox.ac.uk \\ (2)Rutherford Appleton Laboratory, U.K, Emails: richard.siddans@stfc.ac.uk, \\ caroline.cox@stfc.ac.uk, caroline.poulsen@stfc.ac.uk
}

The Oxford-RAL Retrieval of Aerosol and Cloud (ORAC) algorithm is an optimal estimation scheme that has been extended to generate joint retrievals of aerosol and sea surface properties from AATSR. In particular the infrared emissions by aerosol and trace gases are included in the retrieval of sea surface temperature which uses both the nadir and forward view. The SSTs are compared with the ship-based (ISAR) measurements and buoy-based (MEDSPIRATION) measurements. The comparisons with ISAR for the year 2006 and with buoys for the April and July in 2008 are shown. The aerosol retrievals are compared with the values from the MAN campaign.

\section{ET-OOFS REQUIREMENTS}

\section{Shiro Ishizaki}

Japan Meteorological Agency, Otemachi Chiyodaku, Tokyo, Japan Email : s_ishizaki@met.kishou.go.jp

After the GODAE project was ended, Expert Team for Operational Ocean Forecasting Systems (ET-OOFS) was organized as one of the expert teams of the JCOMM Service and Forecasting System Program Area (SFSPA). ET-OOFS aims to coordinate activities relating to operational ocean forecasting systems across all international operators. One of the major tasks of the team is to contribute observational requirements for operational ocean forecasting systems to the "Statement of Guidance" and the WMO Rolling Requirements Review process. In this presentation, overview of ET-OOFS activity and current status of producing observational requirements will be presented. 


\title{
REQUIREMENTS ON SST FOR NWP, AIR-SEA INTERACTION AND OCEAN MODELLING
}

\author{
Peter A.E.M. Janssen ${ }^{(1)}$ \\ (1)E.C.M.W.F., Reading, U.K., Email : p.janssen@ecmwf.int
}

At ECMWF SST and Sea Ice products are used in a number of forecasting and analysis systems. These data play an important role in the 10-day deterministic forecast and the ensemble prediction system, the monthly forecast and in the seasonal forecast. Examples of their use will be highlighted during the talk, while also some of the problems, such as inconsistencies between sea ice cover and SST, will be discussed.

During the past decades we have seen a massive increase in spatial resolution of our forecasting system. At the moment the deterministic global system has a resolution of $16 \mathrm{~km}$. As consequence, the impact of lakes on the local weather has now become a serious issue for us. Simple lake models have been developed and they will be shortly introduced in our operational system. There is no need to emphasize that the performance of such a lake model will benefit tremendously when surface temperature and ice cover data would be available.

\section{OCEAN WAVE EFFECTS ON THE DAILY CYCLE IN SST}

\section{Peter A.E.M. Janssen}

\author{
E.C.M.W.F., Reading, U.K., Email : p.janssen@ecmwf.int
}

Ocean waves represent the interface between the ocean and the atmosphere,and, therefore, a wave model is needed to compute not only the wave spectrum, but is also required to determine the processes at the air-sea interface that govern the fluxes across the interface.

Here, starting from earlier results with the Turbulent Kinetic Energy Equation, a simple model is developed that allows for the inclusion of wave dissipation effects, effects of Langmuir turbulence and buoyancy on

the simulation of the daily cycle in SST. This model is applied to a simulation of the daily cycle in SST and it is shown by a comparison with observations of SST that ocean wave dissipation plays an important role in the amplitude of the daily cycle. 


\title{
THE ROLE OF SMALL-SCALE AND SHORT-TERM SST VARIABILITY IN THE ERROR OF GRIDDED OBSERVATIONS
}

\author{
Alexey Kaplan \\ Lamont-Doherty Earth Observatory of Columbia University, Palisades, NY 10964 (United \\ States), Email : alexeyk@ldeo.columbia.edu
}

Optimal analyses of climate fields require a priori error estimates for the input data. To be truly optimal, high-resolution gridded analyses of sea surface temperature (SST) that blend together various kinds of satellite and in situ data have to specify for all input data sets their error variances and, ideally, covariances. Satellite observations of physical fields represent averages over the satellite sensor's footprint, which might be smaller or larger than the size of the analysis grid box. The difference between the true gridded values, i.e., grid box averages, and the individual observations is thus affected by the physical variability on the scales in between the observational footprint and the grid size. Short-term temporal variability within the averaging limits of the spatiotemporal grid box creates similar problems. In situ SST data may enter analysis procedures either individually, as zero footprint observations, or as averages over some space-time bins (a.k.a. "superobservations"). Their error is due to both the incomplete sampling of the bin volume and a measurement error of individual data reports. This approach to observational error modeling in gridded data sets of in situ and satellite observations was tested here. Pathfinder v.5 AVHRR SST data set as well as other L3 data sets were used to produce maps of spatial and temporal variability of SST within monthly $1^{\circ} \times 1^{\circ}$ bins. These maps, together with the data on the number of available observations, were used to construct theoretical error estimates for data sets of gridded observations and to predict the magnitude of the differences between them. Comparisons of such predictions with standard deviations of the actual differences between data sets showed a good skill of the proposed error model. 


\title{
DIURNAL WARMING IN THE NORTHERN EUROPEAN SHELF SEAS:
} OBSERVATIONS VS. MODELLING.

\author{
Ioanna Karagali ${ }^{(1)}$, Jacob L. Hoeyer ${ }^{(2)}$ \\ (1)Risø-DTU, Technical University of Denmark, Frederiksborgvej 399, Roskilde, 4000 \\ (Denmark), Email : ioka@risoe.dtu.dk \\ (2) Centre for Ocean and Ice, Danish Meteorological Institute, Copenhagen $\varnothing$ \\ (Denmark), Email : jlh@dmi.dk
}

\begin{abstract}
Diurnal variability has been identified from five years of hourly SEVIRI SST observations. Quantification of diurnal warming indicated frequent occurrences during spring and summer with maximum anomalies exceeding $4.5 \mathrm{~K}$, mostly occurring between 14:00 and 16:00 LT. Given the importance of diurnal warming and its occurrence as satellite observations prove, modelling the diurnal variability of SST is of high importance. For this purpose, two models are evaluated; the prognostic scheme from Zeng and Beljaars (2005) and the statistical model described in Filipiak et al. (2010). Model runs were performed for one year, February 2009 until January 2010 (inclusive), using HIRLAM outputs. Evaluation of the spatial extend of diurnal warming from SEVIRI and the models, indicated areas where the models consistently missed diurnal variability. Merchant et al. (2008) noted influential $\mathrm{K}_{\mathrm{d}(490)}$ variability in the North Sea and Baltic Sea that could explain up to $20 \%$ of the mean peak dSST spatial variance. Based on such findings, we proceed to correlate observations and model outputs with $\mathrm{K}_{\mathrm{d}(490)}$ and to evaluate any possible dependence. A successful representation of the daily variability from models can be accommodated, amongst others, in blended SST fields representative of foundation temperatures.
\end{abstract}

\section{Introduction}

Diurnal warming in the Northern European Shelf Seas from SEVIRI observations has been formerly investigated by Karagali and Hoeyer (2010). Using five years of hourly SST retrievals (2004-2009), it has been shown that anomalies higher than 2 $\mathrm{K}$ occurred during the late spring and summer months of every year. Duration did not exceed 3 hours for $75 \%$ of the cases, even though events of 7 hours or more were identified. Maximum anomalies in most cases were between 2 and $3 \mathrm{~K}$ but cases of more than $4.2 \mathrm{~K}$ were recorded, usually occurring between 1400 and 1700 LT.

Within this context, the ability to model the diurnal variability is of high importance for various applications. In the present paper, two different models that use NWP outputs for wind and surface fluxes are implemented for a test period of 1 year. modelled and observed anomalies are compared in terms of amplitude and spatial extend. The aim is to evaluate the performance of the models and to assess the potential impact of various physical parameters on diurnal variability. Especially, the impact of the mean attenuation coefficient $K_{d(490)}$ is assessed and the potential

In this paper we present some preliminary results on the comparisons between modelled and observed anomalies in the North Sea and the Baltic Sea. A short description of the data and the models can be found in Section 2. Results are presented in Section 3 and conclusions are drawn in Section 4.

\section{Data and Methods}

SEVIRI SST observations from 2004 until 2009 were utilized for an evaluation of diurnal variability in the North Sea/Baltic Sea area. Night time reference fields were generated from SEVIRI observations between 0000 and 0300 LST and averaged over an interval of 7 days. Validation against in situ observations from various locations around Denmark was performed for both the hourly SEVIRI observations and the reference fields. 
GHRSST XII -

Proceedings, Edinburgh

GHRSST Project Office

The diffuse attenuation coefficient at 490 $\mathrm{nm}$ is a measure of the optical properties of the water column. Higher $\mathrm{K}_{\mathrm{d}(490)}$ values indicate higher water turbidity and as shown in Merchant et al. 2008, tend to promote diurnal warming. For the purposes of the present study, fields from two different sources were obtained. The GlobColour project produces daily $\mathrm{K}_{\mathrm{d}(490)}$ fields based on the merged chlorophyll-A concentration for case 1 water. The DMI $\mathrm{K}_{\mathrm{d}(490)}$ product is based on case 2 water, especially produced for the Baltic Sea.

$$
\begin{gathered}
D(t)=Q|t| \frac{a(t)}{1+b(t) W^{2}(t)}+c|t\rangle \\
D(t)=f(t) \frac{|Q| t\rangle)}{\left(\varrho c_{p} d\right)}
\end{gathered}
$$

Cooling periods are defined by $\mathrm{Q}<0$ and require the density $\rho$ and specific heat of water $c_{p}$ along with the climatological mixed layer depth (d). For the climatological mixed layer depth (d) the entire water column depth was used as the area is characterized by very small depths.

Moreover, the prognostic scheme of Zeng and Beljaars (2005), referred as ZB, was implemented. This model also requires

$$
\begin{array}{cc}
T_{s}-T_{-\delta}=\frac{\delta}{\varrho_{w} c_{w} k_{w}}\left(Q+R_{s} f_{s}\right) & \text { Equation 3. } \\
\frac{\partial\left(T_{-\delta}-T_{-d}\right)}{\partial t}=\frac{Q+R_{s}-R(-d)}{d \varrho_{w} c_{w} v /(v+1)}-\frac{(v+1) k u_{w}}{d \varphi_{t}(d / L)}\left(T_{-\delta}-T_{-d}\right) & \text { Equation 4. }
\end{array}
$$

wind and surface fluxes, along with an a priori knowledge of foundation temperatures. In this case, the SEVIRI reference fields were utilized as representative of night-time conditions. The scheme also allows for an option of the surface layer depth within which warming occurs (d). Two different thresholds were set, namely $d_{1}=3 \mathrm{~m}$ and $\mathrm{d}_{2}=6 \mathrm{~m}$.$$
\text { 3. Results }
$$

Both models were forced with NWP outputs from HIRLAM, for wind, shortwave and longwave radiation, sensible and latent heat fluxes. The net heat flux $q$ was estimated and all fields were re-gridded to match the SEVIRI grid. A test period from February 2009 to January 2010 was selected.
SEVIRI anomalies from 1300 to 1600 were collocated with corresponding wind from HIRLAM, SSI from SEVIRI, $\mathrm{K}_{\mathrm{d}(490)}$ from $\mathrm{DMI}$ and the GlobColour project, dSST from the FMKLB and Z-B models and integrated heat flux Q' from 0300 LT until the time of SEVIRI anomalies. The correlation matrix for all the parameters is shown in Figure 1. 


\section{GHRSST Project Office}

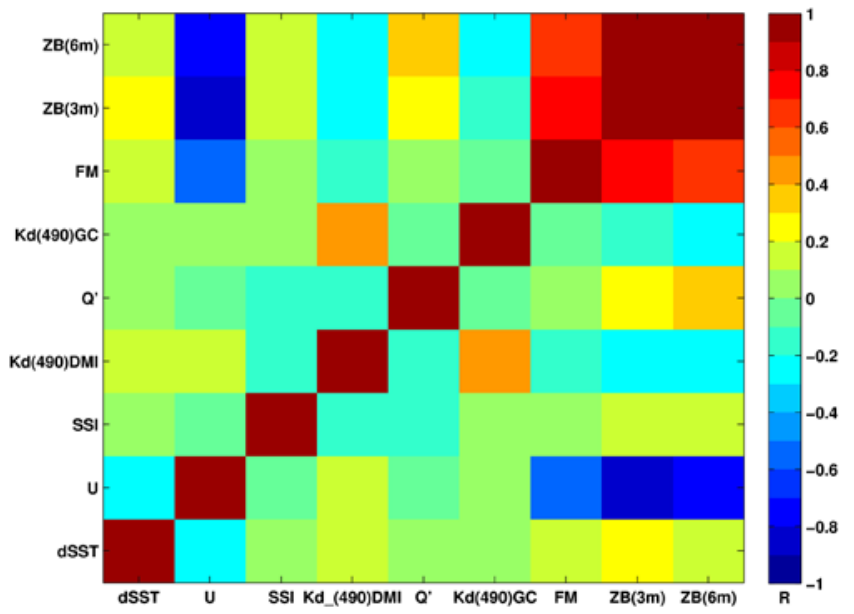

Figure 1. Correlation matrix for all the parameters included in the analysis.

In general, correlation between SEVIRI dSST and other physical parameters is not very good even though the correct trends are found. Especially for SSI and Q', correlation is ranging between 0.1 and 0.2 . Also, correlation with the $\mathrm{DMI} \mathrm{K}_{\mathrm{d}(490)}$ is slightly better than with the GlobCol product. There is a slightly higher negative correlation with $U$, in the order of 0.3 but no strong supportive evidence for the dependence of dSST on $U$ and $Q^{\prime}$ is found.

Correlation between the two $\mathrm{K}_{\mathrm{d}(490)}$ products does not exceed 0.5 , highlighting the difference between case-I and case-II waters. As far as the two models are concerned, dSST is negatively correlated with $U$ for all three model set-ups, with lower correlation value for the FMKLB model, followed by the Z-B $6 \mathrm{~m}$, while the $Z-B \quad 3 \mathrm{~m}$ shows the highest correlation. This reverses when correlation with $Q^{\prime}$ is considered. Lowest correlation is found again for the FMKLB model, followed by the Z-B $3 m$ while the Z-B $6 m$ has the highest positive correlation ( $r=0.4-0.5)$.

If one examines individual events, the differences between models and observations are highlighted in the amplitude and shape of the diurnal cycle. Figure 3 shows an example for July $3^{\text {rd }}$ 2009, including SEVIRI and modelled dSST from 0400 to 2300 LST for a single grid cell with co-ordinates $56^{\circ} 0 " \mathrm{~N}$ and $7^{\circ}$ 27" E. Unfortunately, SEVIRI anomalies are available from 1000 LT, so no clear conclusion on the pre-warming period can be drawn. The ZB 3m model, even though slightly overestimating, captures best the maximum anomaly in terms of amplitude and time.

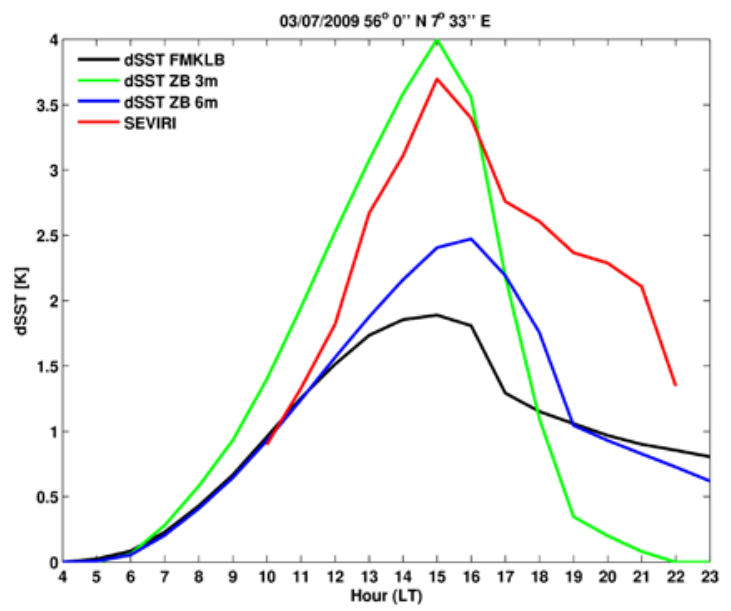

Figure 2. Example of dSST from SEVIRI observations and FMKLB and ZB models for a single grid cell on July $3^{\text {rd }} 2009$.

The spatial extend of modelled and observed anomalies more than $2 \mathrm{~K}$ is shown in Figure 3. Results are presented as hours of anomalies for the test period. All models seem to overestimate the spatial extend of warming, especially the ZB 3m scheme.

\section{Conclusion}

The low SEVIRI biases found when compared with in situ observations ensure that the observed anomalies are representative of the true state of SST warming. Thus, any possible 
GHRSST XII -

Proceedings, Edinburgh

GHRSST Project Office

overestimation of diurnal warming from SEVIRI is rather unlikely. Nonetheless, correlation between SEVIRI observed anomalies and other parameters known to promote diurnal variability does not show satisfying results. To the contrary, correlation was found generally low but correct trends can be identified.

When model anomalies are considered, there seems to be an overestimation in terms of frequency. That is not the case for the magnitude, as in the example of one single cycle for one location the magnitude of SEVIRI anomalies is either

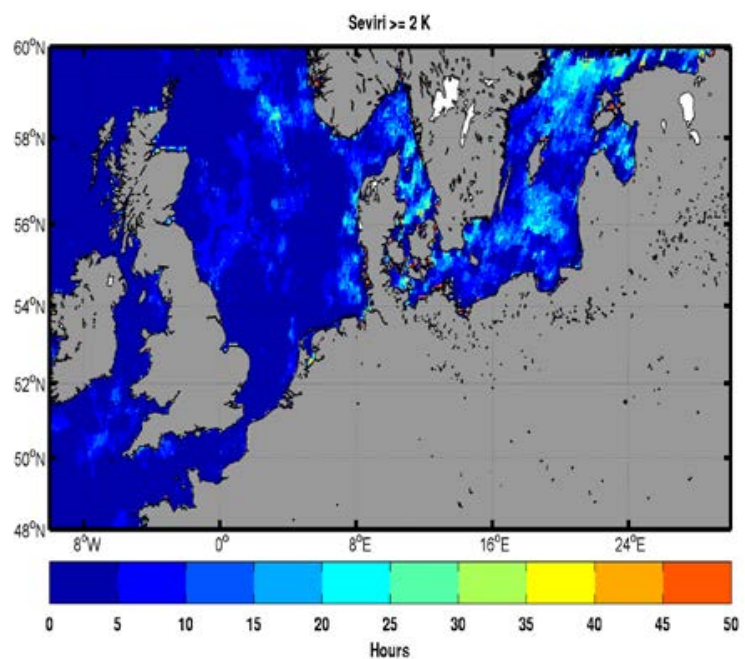

(a) SEVIRI

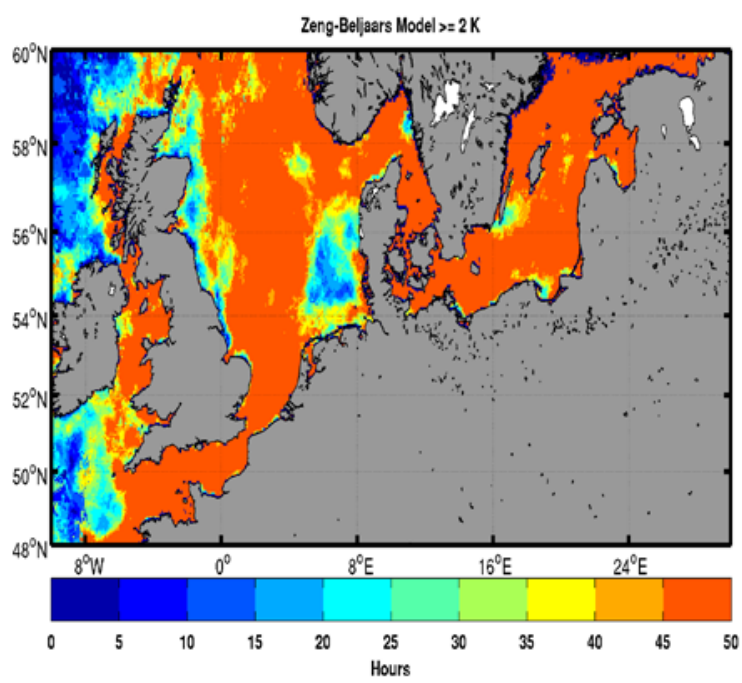

(c) $Z B$ model with $z_{1}=3 m$.
Issue: Final

Date: September 27, 2011

slightly overestimated (ZB-3m) or highly underestimated (ZB-6m, FMKLB). There does seem to be a dependence on the optical properties, but the correlation between model dSST and $\mathrm{K}_{\mathrm{d}(490)}$ is found negative. Models do not capture the spatial extend of warming as they consistently miss warming in some areas while they overestimate it in others. The Zeng-Beljaars scheme seems to have the skill to accommodate the optical properties of the water in an indirect way, as it includes a parameter for the depth of the surface layer where warming occurs.

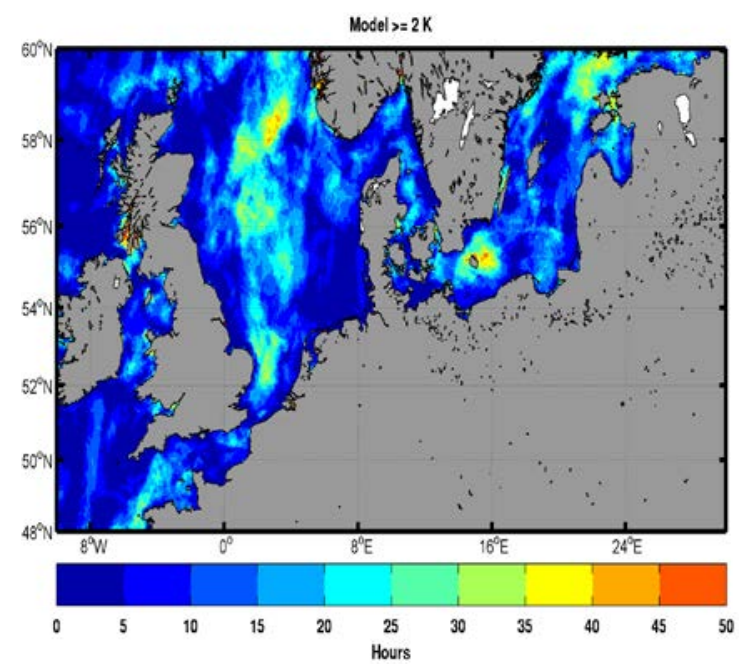

(b) FMKLB model

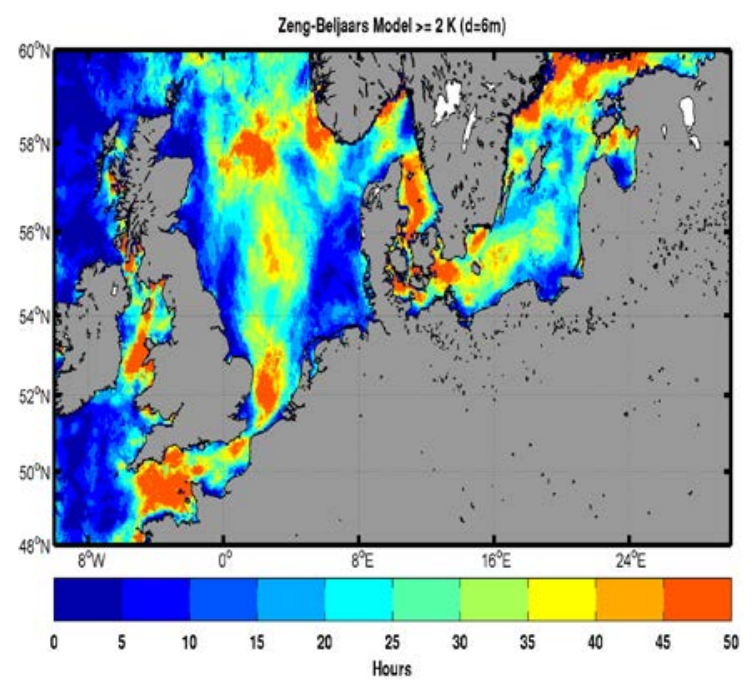

(d) ZB model with $z_{1}=6 \mathrm{~m}$.

Figure 3. Hours of observed anomalies > 2K, from February 2009 to January 2010 (all inclusive). 
GHRSST Project Office

\section{References}

Zeng, X. and A. Beljaars, A prognostic scheme of sea surface skin temperature for modelling and data assimilation, Geophysical Research Letters 32 (L14605), 2005.

Merchant C.J., Filipiak, M.J., Le Borgne, P., Roquet, H., Autret, E., Piollé, J.-F. and S. Lavender, Diurnal warm-layer events in the western Mediterranean and European shelf seas, Geophysical Research Letters 35, L04601, 2008.
Filipiak, M.J., Merchant, C.J., Kettle, H. and $P$. Le Borgne, A statistical model for sea surface diurnal warming driven by numerical weather prediction fluxes and winds, Ocean Sci. Discuss. 7, pp. 1497-1532, 2010.

Karagali, I. and J.L. Hoeyer, Diurnal warming in the North Sea and Baltic Sea, Proceeding of GHRSST XI Science Team Meeting (G11)-Lima 2010 G11, pp. 82-83, 2010. 


\title{
ANALYSES OF SATELLITE AND IN SITUSST FOR CLIMATE APPLICATIONS
}

\author{
Elizabeth C. Kent ${ }^{(1)}$, David I. Berry ${ }^{(2)}$
}

(1) National Oceanography Centre, Southampton, UK, Email : eck@noc.ac.uk

(2) National Oceanography Centre, Southampton, UK, Email: $\underline{d y b @ n o c . a c . u k}$

The challenges and benefits of combining satellite and in situ SST records for climate applications will be briefly outlined, focussing in particular on the assessment of uncertainty in the climate record. Some of the most popular long term SST datasets, for example HadISST from the Met Office and ERSST from NOAA, already combine in situ and satellite observations. Typically the satellite observations are used to provide coverage, either directly or through the projection of modes of variability on to sparse in situ observations. The in situ record is used to provide stability, for example removing the effects of aerosol contamination on infrared measurements of SST from satellites.

Recent results from an assessment of SST from the Along Track Scanning Radiometer (ATSR) series of satellites against requirements for consistency will be presented. Along with other recent assessments of SST data quality by joint analyses of satellite and in situ data, these studies suggest the potential for improved long term SST records and the characterisation of uncertainty in those records. 


\title{
STUDY ON PRODUCING THE MULTI-SENSOR SST WITH HIGH RESOULTION OVER THE EAST ASIA AT KMA/NIMR
}

\author{
Mi-Ja Kim ${ }^{(1)}$, Mi-Lim Ou${ }^{(2)}$, Youngmi Kim ${ }^{(3)}$ \\ National Institute of Meteorological Research (NIMR), KMA, Seoul (Korea), \\ Email : (1) kimmee74@korea.kr, (2) milim@korea.kr, (3) kymi19@korea.kr
}

\begin{abstract}
Korea Meteorological Administration has produced the MCSST from geostationary and polar orbit satellite data over the East Asia. In this study, we investigated the merging method using the SSTs derived from infrared and microwave sensor data and evaluated the accuracy of merged SST.

In comparison with buoy SSTs for each satellite-derived SST, the RMSE of MTSAT1R, AVHRR and AMSR-E are $0.75^{\circ} \mathrm{C}, 0.62$ ${ }^{\circ} \mathrm{C}$ and $0.9{ }^{\circ} \mathrm{C}$, respectively. The error characteristics were applied for the merging process. The objective analysis approach based on the Gauss-Markov theory is used for merging, and the merged SST is produced in daily basis with $5 \mathrm{~km}$ in space from 2007 to 2009 over the East Asia. In comparison with OSTIA which is daily mean data with same spatial resolution, the bias and RMSE of merged SST are $-0.12{ }^{\circ} \mathrm{C}$ and $0.94{ }^{\circ} \mathrm{C}$. And the results comparing with buoy SST are $0.12{ }^{\circ} \mathrm{C}$ and $1.34{ }^{\circ} \mathrm{C}$.
\end{abstract}

\section{Introduction}

The multi-sensor satellite derived SSTs show different characteristics in time and space. The SSTs retrieved from different satellite sensors were first produced MCSST from MTSAT-1R, NOAA/AVHRR data over the East Asian region. The SST from AMSR-E data was collected via ftp L2 products from NSIDC (National Snow and Ice Data Center). The RMSEs of the SSTs derived from MTSAT-1R, AVHRR and AMSR-E are $0.75{ }^{\circ} \mathrm{C}, 0.62{ }^{\circ} \mathrm{C}$ and $0.9^{\circ} \mathrm{C}$ in East Asian region from March 2006 to February 2008, respectively. The error characteristics were applied for the merging process.
The purpose of this study is to retrieve merged infrared and microwave SST product to make the best use of characteristics of each sensor types. Thus, the merged SST with $5 \mathrm{~km}$ resolution was derived by the objective analysis method based on the Gauss-Markov theory (Guan and Kawamura, 2003).

\section{Retrieval and validation of merged sst}

In 2009, it was used objective analysis approach to merge multi-sensor SSTs in daily basis with $25 \mathrm{~km}$ spatial resolution for 2007. The accuracy of the merged SST was about $0.81{ }^{\circ} \mathrm{C}$ against the buoy SST. In comparison with OISST, RMSE was about $0.83^{\circ} \mathrm{C}$.

In 2010, the spatial resolution of the merged SST was improved about $5 \mathrm{~km}$ using the same objective method for the period from 2007 to 2009. To validate the merged SST, the drifting buoys and OSTIA (Operational SST and Sea Ice Analysis) data are used.

In comparison with buoy SST, bias and RMSE are $0.12{ }^{\circ} \mathrm{C}$ and $1.34{ }^{\circ} \mathrm{C}$, respectively. Figure 1 shows the scatter plot between the merged SST and the buoy SST.

The OSTIA SST with about $6 \mathrm{~km}\left(1 / 20^{\circ}\right)$ was remapped to have the same grid point as merged SST. In comparison with the OSTIA SST, bias and RMSE are $-0.12{ }^{\circ} \mathrm{C}$ and $0.94{ }^{\circ} \mathrm{C}$. The mean distributions of the merged SST and the OSTIA SST for 20072009 are shown in Figure 2 with their difference. 


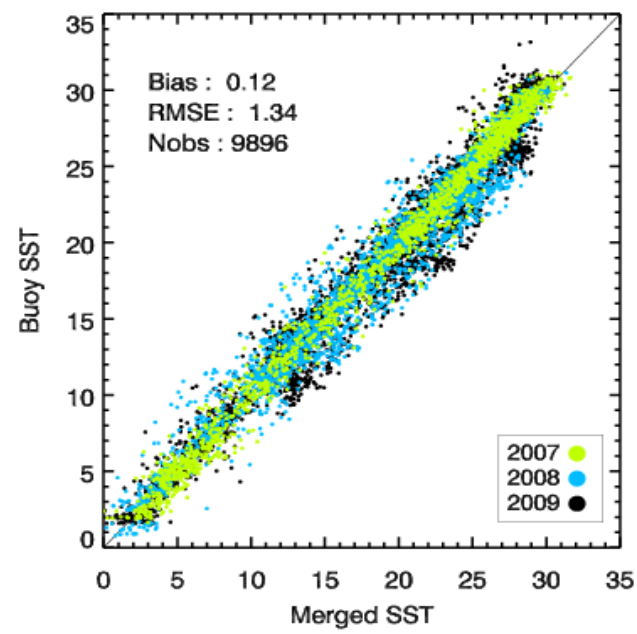

Figure 1. The scatter plot between the buoy and the merged SST during 2007-2009.

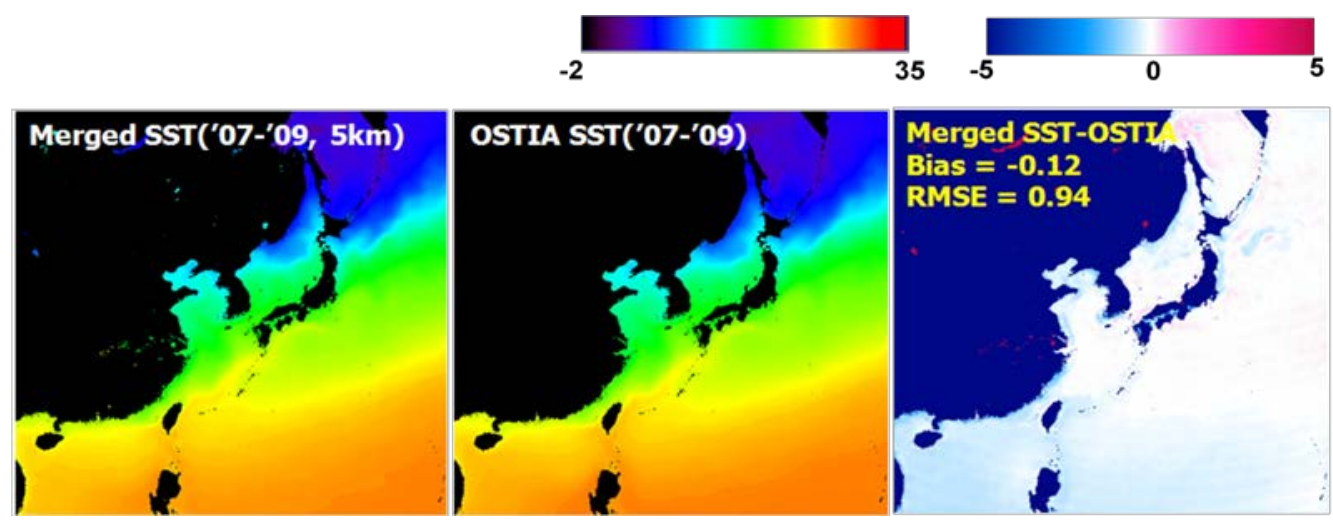

Figure 2. The merged SST at NIMR (left), the OSTIA SST at UK Met Office (middle) averaged for 2007-2009, and the difference between the merged SST and the OSTIA SST (right).

\section{Summary}

In comparison with buoy SST, the RMSE of the merged SST with higher resolution has been increased compared to the merged SST with lower resolution. However, the detailed features of the sea current were better expressed.

To improve the accuracy of the high resolution SST, it should be considered the different characteristics of multi-sensor SSTs in time and space such as diurnal variation and satellite zenith angle.

\section{Acknowledgement}

This study was supported by the project "Research for the Meteorological Observation Technology and Its Application" at National Institute of Meteorological Research, KMA.

\section{References}

E. F. Carter and A. R. Robinson, Analysis Models for the Estimation of Oceanic Fields, Journal of Atmospheric Oceanic Technology, 4, 49-74, 1987.

Lei Guan and Hiroshi Kawamura: SST Availability of Satellite Infrared and Microwave Measurements, J. Oceanogr., 59, 201-209, 2003.

McClain, E. P.: Global sea surface temperatures and cloud clearing for aerosol optical depth estimates. Int. J. Remote Sens., 10, 763-769, 1989. 
GHRSST XII -

Proceedings, Edinburgh

GHRSST Project Office

Richard W. Reynolds, Thomas M. Smith, Chunying Liu, Dudley B. Chelton,
Issue: Final

Date: September 27, 2011

Kenneth S. Casey and Michael G. Schlax, Daily High-Resolution Blended Analyses for Sea Surface Temperature, J. Climate, in Press Revised, 2007. 


\title{
MERMAID: PROPOSED EXTENSION TO FURTHER SENSORS I PRODUCTS INCLUDING THE (A)ATSR SERIES AND SENTINEL-3 OPTICAL INSTRUMENTS.
}

\author{
Samantha Lavender ${ }^{(1)}$, Kathryn Barker ${ }^{(1)}$, Constant Mazeran ${ }^{(2)}$, Christophe \\ Lerebourg $^{(2)}$ Chris Kent $^{(1)}$, and Odile Fanton D'Andon
}

(1) ARGANS Limited. 19 Research Way, Tamar Science Park, Derriford, Plymouth, Devon, PL6 8BT. Email: slavender@argans.co.uk

(2) ACRI-ST. 260 route du Pin Montard, BP 23406904 Sophia Antipolis Cedex, France.

\begin{abstract}
The MEris MAtchup In-situ Database (MERMAID) currently provides matchups between Reduced Resolution (RR) MERIS imagery and in-situ data provided by contributing Principle Investigators (PIs). A proposed extension to the activity is the inclusion of the current (A)ATSR series of instruments and future Sentinel-3 (S-3) optical instruments; Ocean Land Color Instrument $(\mathrm{OLCl})$ and Sea Land Surface Temperature Radiometer (SLSTR). An outline of MERMAID is provided here to demonstrate the mature tool presently available for MERIS and to highlight the potential to extend beyond MERIS to future radiometric products and sensors.
\end{abstract}

\section{Introduction}

In the framework of the Medium Resolution Imaging Spectrometer (MERIS) Quality Working Group (QWG) activities, a database centralising in-situ optical measurements has been set up and funded by the European Space Agency (ESA), and is a joint effort between by ACRI-ST (France) and ARGANS Ltd (UK). MERMAID (Barker et al., 2008; http://hermes.acri.fr/mermaid: see Figure 1) has the long-term objectives of:

a) Enabling the assessment of the MERIS Level 2 (L2) products delivered by the ENVISAT ground segment;

b) Supporting the monitoring of these MERIS products over the lifetime of the mission by providing a complete temporal coverage of the mission; c) Supporting vicarious adjustment of the instrument and atmospheric correction.

d) Providing a centralised validation resource to the ESA Optical Data Processor, ODESA.

MERMAID currently contains in-situ fully normalised water reflectances with concurrent and comparable extractions of the MERIS L2 products (including flags and auxiliary data), and intermediate products from the L1 to L2 processing. Recent activities have linked MERMAID to the Optical Data processor of the European Space Agency (ODESA; http://earth.eo.esa.int/odesa), so that MERIS matchups can be processed and then compared with new algorithms / processing techniques. ODESA supplies the user community with the MERIS Ground Segment development platform MEGS, including source code, embedded in an efficient framework for testing and for validation activities. Such facilities include match-up processing \& analysis, and so for this, ongoing work has extended the MERMAID database to include the Inherent Optical Properties (IOPs), bio-geophysical parameters and atmospheric parameters e.g. inclusion of the AERONET-OC (Ocean Colour) data.

Data supplied to MERMAID undergoes quality control procedures improve the quality of the products provided, and the MERMAID team work closely with the PIs (i.e. the MERIS Validation Team, MVT) to enable cooperation and collaboration and ensure quality of service. An in-situ measurements protocols document is provided on the website, produced in collaboration with the PIs, to accompany 
the database and describes the in-situ datasets in MERMAID, the quality control flags, and the procedures of each $\mathrm{PI}$ whose dataset is included in MERMAID.

The first S-3 satellite is scheduled to launch in 2013 as part of the European Global Monitoring for Environment and
Security (GMES) programme. The mission aims to produce consistent longterm datasets with both an improved accuracy and reliability, and carries both an advanced radar altimeter (SLSTR) and visible-infrared optical imaging (OLCl) instruments. MERMAID could provide the basis for extension to validation activities for these sensors, and we outline here what is presently done for MERIS.

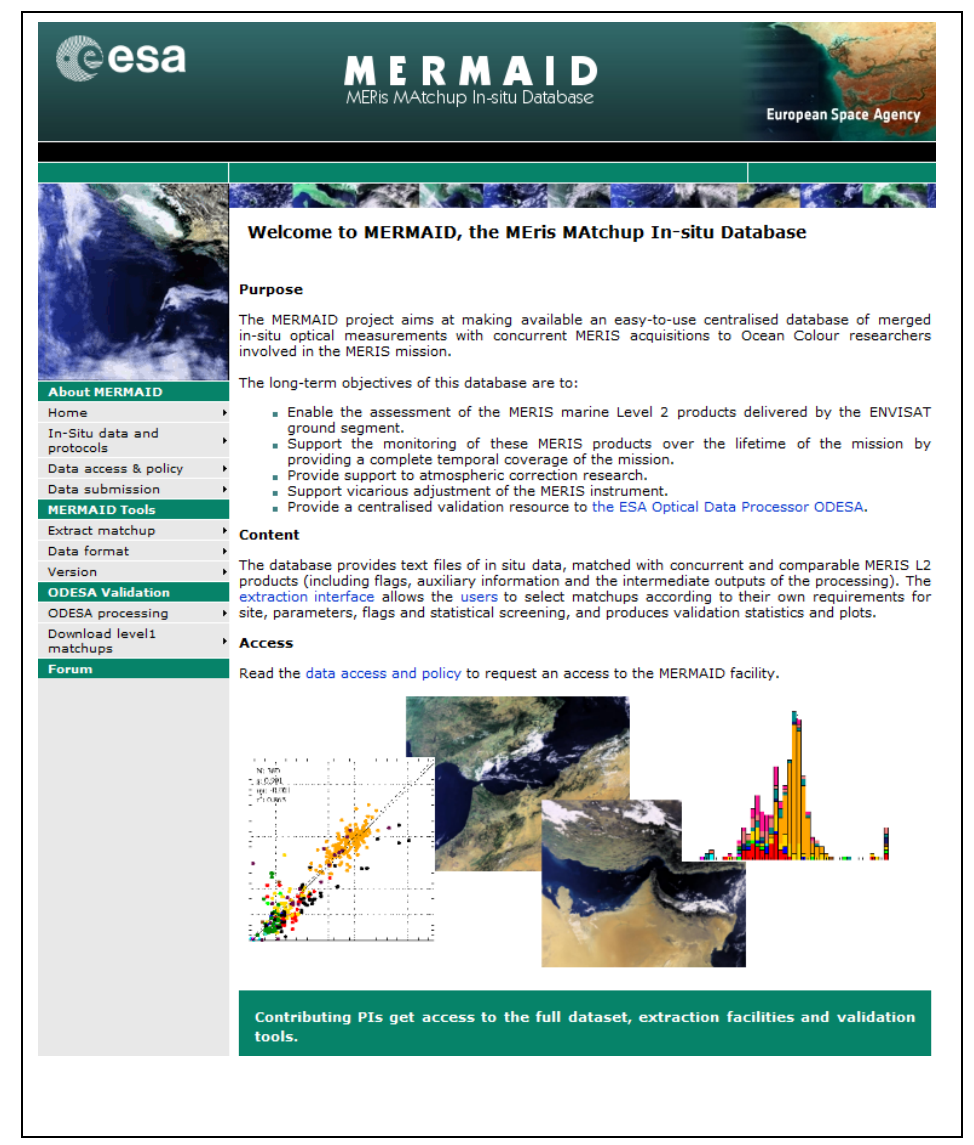

Figure 1: mermaid home page (HTTP://HERMES.ACRI.FR/MERMAID)

\section{MERMAID Matchup Extraction}

MERMAID extractions are accessed through a user-friendly versatile web interface; the 'Extract Matchup' option of the website menu (Figure 2). A password for access is provided to contributing PIs but non-PIs can also gain access through a Service Level Agreement (requested from mermaid@acri.fr). A range of options are available, all allowing for adaptable and flexible matchup selection.

The interface allows users to specify their own extraction criteria from a wide range of options. At the top of the page, a user can firstly choose whether matchups are derived from online data processed with different versions of the MERIS Ground Segment data processing prototype (MEGS), currently version 8.0 or 7.5.1 where:

- $\quad 7.5 .1$ is identical to $2^{\text {nd }}$ reprocessing of the MERIS dataset, but with updated geolocation;

- 8.0 is the $3^{\text {rd }}$ reprocessing. 


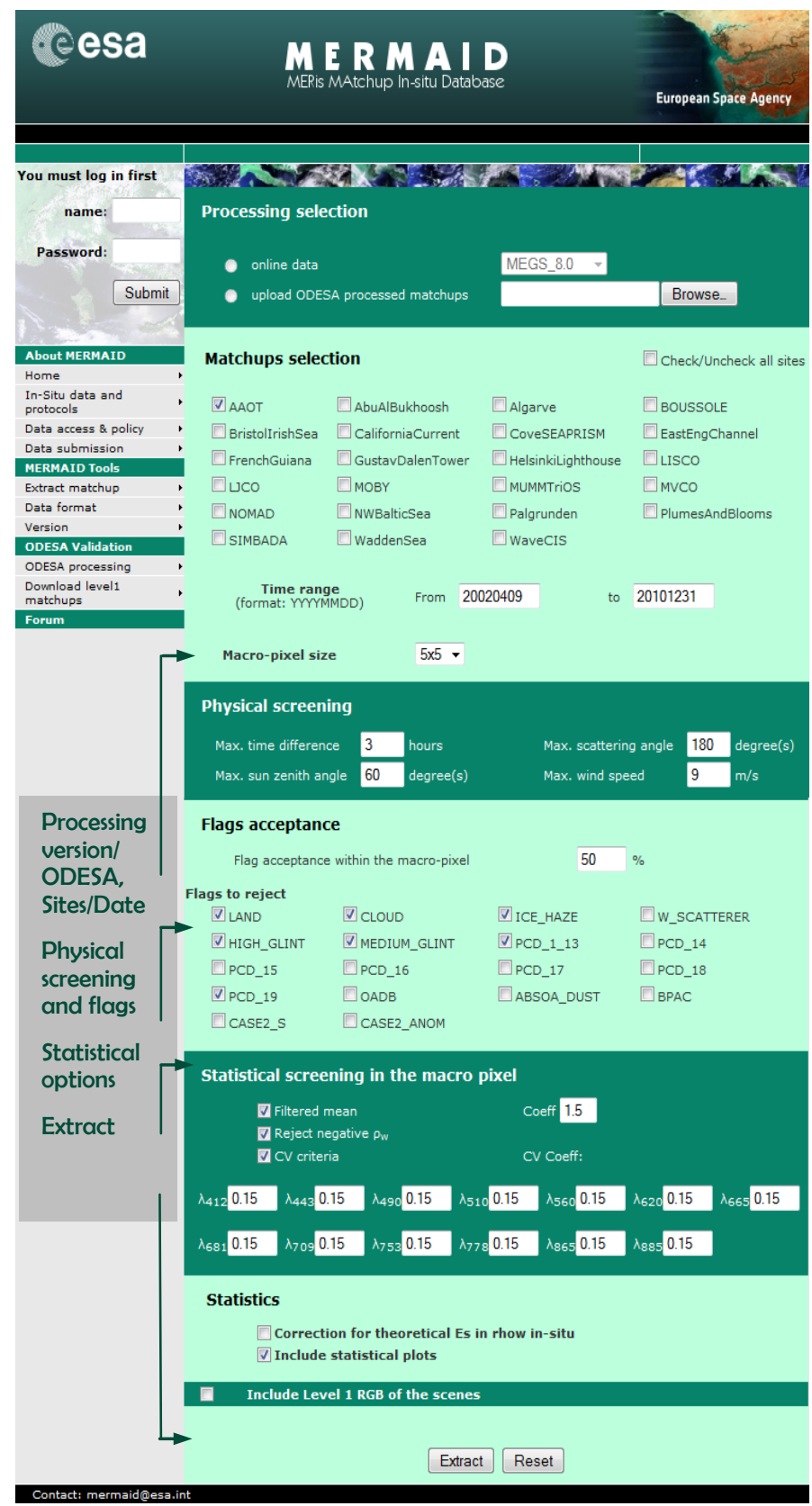

Figure 2: MERMAID website matchup selection page

The other option is for users to upload their own ODESA processed matchups e.g. when the Level 1 (L1) data, downloadable http://hermes.acri.fr/mermaid/download/do wnload.php, has been processed with a customised version of the ODESA code e.g. a new algorithm:

\section{L1 extraction $\rightarrow$ ODESA processing $\rightarrow$ L2 output $\rightarrow$ MERMAID tools $\rightarrow$ validation results}

Next, users can select from a range of matchup sites and date ranges (starting in April 2002). The lower half of the webpage provides the user with the ability to refine the selection criteria depending on physical variables (e.g. maximum time between the in-situ measurement and MERIS pixel, geometry), flagging and macro pixel size $(1,3 \times 3,5 \times 5$ pixels $)$ and statistical screening options. The output options include RBG quick-look and statistical plot inclusion, and the option to 
GHRSST XII -

Proceedings, Edinburgh

GHRSST Project Office

correct the in-situ measurements for theoretical downwelling solar irradiance $\left(E_{s}\right)$; this is useful when the in-situ sensor may be tilted and therefore potentially measuring a lower $E_{s}$ than would be expected for a vertical measurement (e.g. Barker et al., 2010).

MERMAID data is protected with a Data Policy (viewable at http://hermes.acri.fr/mermaid/policy/policy php), and users are notified of this upon request of an extraction. Before extraction is delivered, users must accept the conditions that include:

- Consult the PI(s) via e-mail to get approval of in-situ data use, inform him/her/them of his/her/their data use and offer co-authorship.

- Acknowledge the PIs and associated projects, e.g.: We thank (the Project/PI) for the (cruise/experiment) data.
Issue: Final

Date: September 27, 2011

- Acknowledge the MERMAID facility and services, e.g.: We thank ACRIST, ARGANS and ESA for access to the MERMAID system. (http://hermes.acri.fr/mermaid)

Upon extraction, the user is presented with the zipped ASCII text file of extractions (in comma separated value, csv, format) to download plus plots \& histograms of matchups; see Figures 3 and 4 . The extracted file contains a series of mandatory data fields (metadata, PI name for traceability, water reflectances at the MERIS bands or within $5 \mathrm{~nm}$, quality control flags) and additional parameters where available at the user-chosen site(s). The website provides a document explaining in more detail the extraction file format.

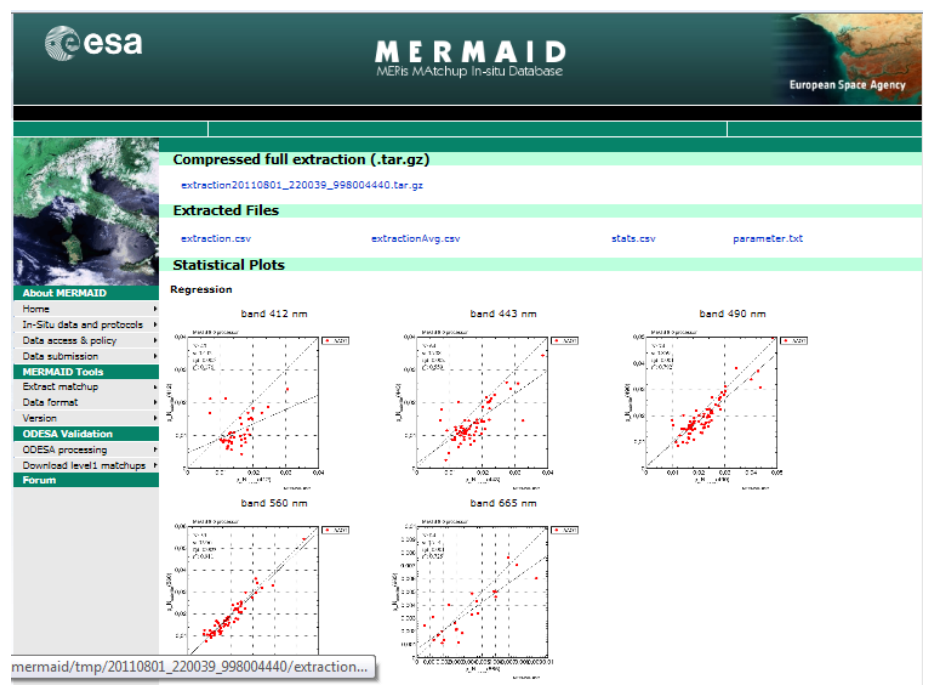

Figure 3: MERMAID website extraction provision page, and sample quick-look plots. 


\section{GHRSST Project Office}

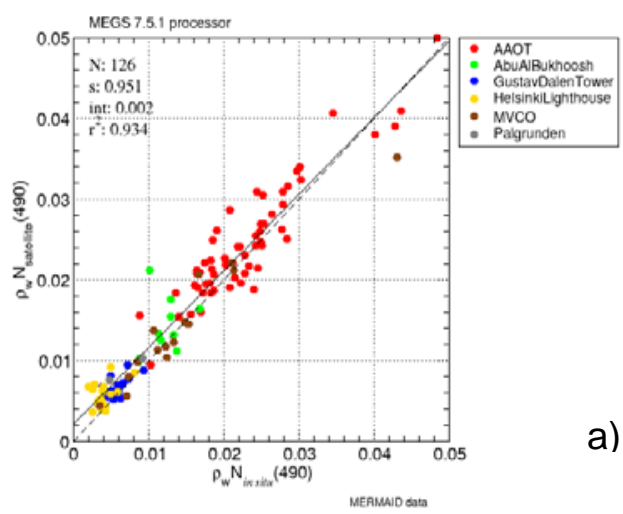

a)

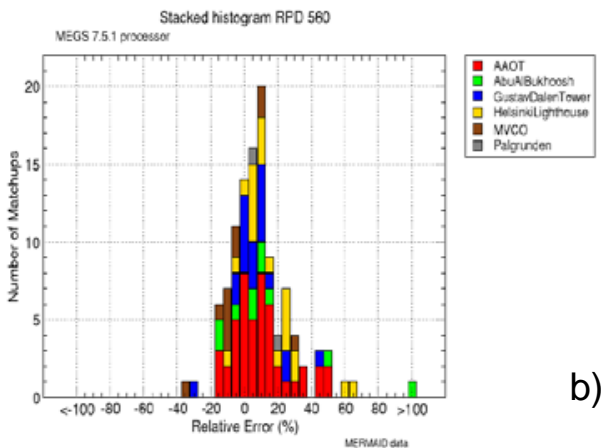

Figure 4. Example MERMAID plots of a) in-situ normalised water reflectance ( $x$ axis) versus satellite normalised water reflectance ( $y$ axis) for a selection of AERONET-OC sites and b) a stacked histogram of normalised in-situ reflectance error for the same sites.

\section{Discussion and Conclusion}

ARGANS is jointly developing the SLSTR prototype processor with Rutherford Appleton Laboratory (RAL SPACE); coding the Fire Radiative Power and Land Surface Temperature algorithms. Also, ARGANS is co-developing the OLCl processor (an extension of MEGS) with ACRI-ST. Therefore, a natural extension to MERMAID would be to include the reflectance channels for both OLCl and SLSTR. In the shorter term, before the launch of S-3, the inclusion of the (A)ATSR series of instruments (ATSR-1, ATSR-2 and AATSR) that are now available as a 15 year multi-mission archive is also a possibility.

Furthermore, there are potential synergies beyond validation; the concept of a Mission Performance Centre could be initiated with MERMAID as the foundation and taken forward with a wider scope for algorithm development and calibration.
ODESA, for instance, with its link to MERMAID, has potential for extension beyond its current capabilities for algorithm testing and validation for MERIS (and in the future SLSTR/OLCI). In the wider context, the Database for Imaging Multi-spectral Instruments and Tools for Radiometric Intercomparison (DIMITRI), which ARGANS is also developing on behalf of ESA, is a medium resolution optical sensor L1 intercomparison and recalibration tool (Bouvet 2006), incorporating (A)ATSR and ATSR-2 among others. Intercomparison and recalibrated products from DIMITRI could feasibly be linked to MERMAID and ODESA. This particular project extends to collaboration with CEOS/IVOS (Committee on Earth Observing Satellites I Infrared and Visible Observation Satellite group) for intercomparison with other calibration methodologies operated by different agencies. The CalVal Portal (http://calvalportal.ceos.org) is a central 
GHRSST Project Office

location for CEOS calibration and validation activities such as these and would welcome initiatives as proposed here for bringing communities and agencies together.

Therefore, there is a strong potential for MERMAID and its heritage to service and benefit the sea surface temperature (SST) community; the facilities and collaboration that are already in place are readily extendable. The poster presentation at the GHRSST XII Edinburgh meeting provided a forum for initial discussions for how such a facility could benefit the SST community. These will now be considered by the MERMAID team and activities taken forward.

\section{References}

Barker, K., C. Mazeran, C. Lerebourg, M. Bouvet, D. Antoine, M. Ondrusek, G. Zibordi, S. Lavender, MERMAID: MERis MAtchup In-situ Database. Proceedings of the 2 nd (A)ATSR and
MERIS Workshop, Frascati, Italy, September 2008

Barker, K., Zagolski, F., Huot, J-P., Mazeran, C., Lerebourg, C. In-situ radiometric measurement protocol and data analysis, including the effects of tilt on downwelling solar irradiance. Proceedings of Ocean Optics XX, Anchorage, Alaska, September 2010.

Bouvet, M. Intercomparison of imaging spectrometers over the Salar de Uyuni (Bolivia), Proceedings of the MERIS AATSR Validation Team Workshop, 2006

\section{Acknowledgement}

The MERMAID activity is primarily funded as part of ESA-ESRIN contract $\mathrm{N}^{\circ} 21091 / 07 / \mathrm{I}-\mathrm{OL}$ (primed by ACRI-ST) with ARGANS also undertaking MERIS research activities within ESA-ESRIN contract $\quad N^{\circ} 21652 / 08 / I-O L$. DIMITRI activities are funded within ESA-ESTEC contract N4000101605/10/NL/CBi. 


\title{
A POSSIBLE CONNECTION BETWEEN EL NINO AND SST ALGORITHM ERRORS IN THE TROPICAL ATLANTIC IN SPRING 2010
}

\author{
Pierre Le Borgne, Anne Marsouin, Sonia Péré, Hervé Roquet \\ Météo-France/Centre de Météorologie Spatiale, Lannion, France, Email : \\ Pierre.LeBorgne@meteo.fr
}

\begin{abstract}
EUMETSAT Ocean \& Sea Ice Satellite Application facility (OSI SAF) SST products have been extensively validated at Centre de Météorologie Spatiale (CMS) in 2010. This validation revealed that the Meteosat Second Generation Spinning Enhanced Visible and Infra-Red Imager (MSG/SEVIRI) derived SST errors have been exceptional in spring 2010, because of their amplitude, and because they happened earlier in spring than usual. They were associated but not geographically correlated to abnormally high SST values in this region. Positive anomalies are sometimes present in tropical Atlantic in post El Niño springs (such as spring 2010). According to Enfield and
\end{abstract}

Mayer (1997), this abnormal warming of the tropical Atlantic waters would be due to a reduction of trade wind speeds leading to a northward displacement of the Intertropical Convergence Zone (ITCZ) and the associated large atmospheric water vapor content. The present study shows that this displacement affects mainly atmospheric layers between 900 and $700 \mathrm{hPa}$. The CMS operational algorithm is particularly vulnerable to any increase of humidity at these levels. In consequence there could be a link between El Niño, the Tropical Atlantic positive SST anomalies and the MSG/SEVIRI negative algorithmic errors between 0 and $20^{\circ} \mathrm{N}$.

\section{OPERATIONAL USE OF NWP MODEL OUTPUTS IN SATELLITE SST CALCULATIONS}

\author{
Pierre Le Borgne, Gérard Legendre, Hervé Roquet \\ Météo-France/Centre de Météorologie Spatiale, Lannion, France, Email :
}

Pierre.LeBorgne@meteo.fr

The use of simulated brightness temperatures (BTS) derived from Numerical Weather Prediction model profiles has been proposed as a solution to eliminate regional biases from satellite derived Sea Surface Temperatures. The methods adopted in practice are either a full Optimal Estimation (OE) Technique (Merchant et al. 2008 and 2009) or a simple algorithm bias correction method (LeBorgne et al 2011). In the frame of the EUMETSAT/OSI-SAF, CMS has used
ECMWF profiles in an operational mode to correct for MSG/SEVIRI derived SST biases since November 2010. This presentation will review:

- the practical issues raised by adjusting simulated to observed BTs

- the respective performances and limitations of the OE and algorithm bias correction, based on the preliminary validation results observed at CMS. 


\title{
INCREASING AMPLITUDE OF EL NIŇO IN THE CENTRAL EQUATORIAL PACIFIC AND RECORD WARMING IN THE SOUTH PACIFIC AND WESTERN ANTARCTICA
}

\author{
Tong Lee ${ }^{(1)}$, Micheal McPhaden ${ }^{(2)}$ \\ (1) Jet Propulsion Laboratory/NASA, Pasadena, California (USA), \\ Email : Tong.Lee@jpl.nasa.gov \\ (2) Pacific Marine Environmental Laboratory/NOAA, Seattle, WA (USA), \\ Email: Michael.J.McPhaden@noaa.gov
}

El Niño events can have widespread impacts around the world. Knowledge about changes of El Niño characteristics is important to the understanding and prediction of El Niño, its impacts, and its relation to climate variability and change. Classical El Niño events have their warmest anomalies in the eastern-equatorial Pacific (EP). In the past two decades, there have been frequent occurrences of a new type of El Niño that have the warmest anomalies in the centralequatorial Pacific (CP). In this study, we use the NOAA $1 \%$ GHRSST product (1982 onward) to show that the amplitude of El Niño in the CP region has almost doubled in the past three decades, with the strongest warming occurring in 2009-10. This is related to the increasing magnitude as well as occurrence frequency of $\mathrm{CP}$ El Niño events. While SST in the CP region during El Niño years has been increasing, those during neutral and La Niña years have not. Therefore, the well-documented warming trend of the warm pool in the CP region, attributed by some to the effects of global warming on background SST, is primarily a result of progressively stronger El Niño events rather than a general rise of background SST (at least for the past three decades). Associated with the 2009-10 El Niño (the strongest El Niño) is a record warming event in the South Pacific and western Antarctica. The implications of the changing El Niño characteristics on extratropical regions are discussed. 


\title{
RELATING SEA SURFACE TEMPERATURE TO WATER AND CARBON DIOXIDE FLUXES
}

\author{
W. Timothy Liu and Xiaosu Xie
}

Jet Propulsion Laboratory, California Institute of Technology, MS 300-323, Pasadena, CA

91109, U.S.A. Email: w.t.liu@jpl.nasa.gov

Current spacebased estimations of ocean surface evaporation and carbon dioxide fluxes, the major surface sources and sink of greenhouse gases, rely on the bulk parameterization of turbulent flux. We have pursued spacebased estimate of these two fluxes, both using the traditional bulk parameterization and also from the conservation principle based on transport in the atmosphere. We have examined the physics behind heir inherent differences, so as to characterize and understand their roles in global water and carbon cycles. The bulk parameterization of the carbon dioxide and water fluxes requires the partial pressure (fugacity) of carbon dioxide at sea and the specific humidity at the interface. The interfacial specific humidity is the saturation humidity of sea water at surface (skin) temperature, and we have developed and validated a statistical model to estimate carbon dioxide fugacity at sea using surface temperature as a input factor. Sea surface temperature is also the surface signature of oceanic responses to forcing by the surface fluxes. The quality of sea surface temperature from diurnal to decadal scales, from coastal to open ocean is examined as a critical factor in the estimation of these fluxes and the understanding of oceanic response. 


\title{
GLOBAL LAKE SURFACE WATER TEMPERATURES FROM ATSR.
}

\author{
Stuart N MacCallum ${ }^{(1)}$, Christopher J Merchant ${ }^{(2)}$ and Aisling Layden ${ }^{(3)}$
}

(1) University of Edinburgh, UK, Email : s.maccallum@ed.ac.uk

(2) University of Edinburgh, UK, Email: c.merchant@ed.ac.uk

(3) University of Edinburgh, UK, Email: a.layden@sms.ed.ac.uk

\section{ABSTRACT}

The ATSR Reprocessing for Climate - Lake (ARC-Lake) project applies optimal estimation (OE) retrievals and probabilistic cloud screening methods to provide LSWT estimates from the series of (Advanced) Along-Track Scanning Radiometers. Variations in physical properties such as elevation, salinity, and atmospheric conditions are accounted for through the forward modelling of observed radiances. Therefore, the OE retrieval scheme developed is generic - i.e., applicable to all lakes. Observations of Lake Surface Water Temperature (LSWT) have been obtained for 258 of Earth's largest lakes from ATSR-2 and AATSR imagery from 1995 to 2009. Empirical orthogonal function (EOF) techniques have been applied to the LSWT retrievals (which contain gaps due to cloud cover) to reconstruct spatially and temporally complete time series of LSWT. Observations of Lake Ice Cover (LIC) are also made, during the day only, using threshold tests on the reflectance channels. LSWT observations have been validated against in situ data, yielding satellite-in situ differences of $0.3 \mathrm{~K} \pm 0.9 \mathrm{~K}$ for day-time and $-0.3 \pm 0.8 \mathrm{~K}$ for night-time observations. LIC products also show good agreement with in situ observations, with the same ice classification given in over $75 \%$ of the match-ups. Following validation, a variety of data products have been generated for potential use in numerous science applications.

\section{Introduction}

Lakes are a vital component of the Earth's fresh water resources, and are of fundamental importance for terrestrial life. Lake water temperature is one of the key parameters determining ecological conditions within a lake, as it influences both chemical and biological processes. In addition to the impact on lake ecology, lake water temperatures determine air-water heat and moisture exchanges, and are therefore vital for understanding the hydrological cycle. Lake surface water temperature (LSWT) and lake ice cover (LIC) observations therefore have potential environmental and meteorological applications for inland water management and numerical weather prediction (NWP).

In this paper we present: an overview of the methods used to derive observations of LSWT and LIC, a summary of validation results, and an insight into potential applications of the data.

\section{Methods}

The methodology of this study (MacCallum and Merchant, 2011) can be broken down into five components: lake definition, LSWT retrieval algorithms, LSWT validation techniques, ice detection and validation, and data product generation. An outline of each of these components is given in the following sections.

\section{Lake Definition}

This study considers the world's "large" natural lakes, conventionally taken to be those in excess of $500 \mathrm{~km}^{2}$ in surface area (Herdendorf, 1982 and Lehner and Döll, 2004). In addition, some lakes slightly smaller have been included because they are of scientific interest and/or have validation data available. Three reservoirs have also been included at the request of a member of the ARC-Lake User Group (Environment Canada). 
Figure 1 shows the locations of the 263 lakes considered in this study and their size distribution (note that an adequate number of successful LSWT retrievals were obtained for only 258 of these lakes). The distribution can be interpreted as a joint consequence of hydrological factors (availability of surface water) and geological factors (glaciation, rifting, etc). Lakes where in situ observations are freely available are also marked in Figure 1. The number of such lakes is small and their geographic coverage limited. However, in situ observation campaigns have been identified for additional lakes and efforts to obtain further in situ data are ongoing.

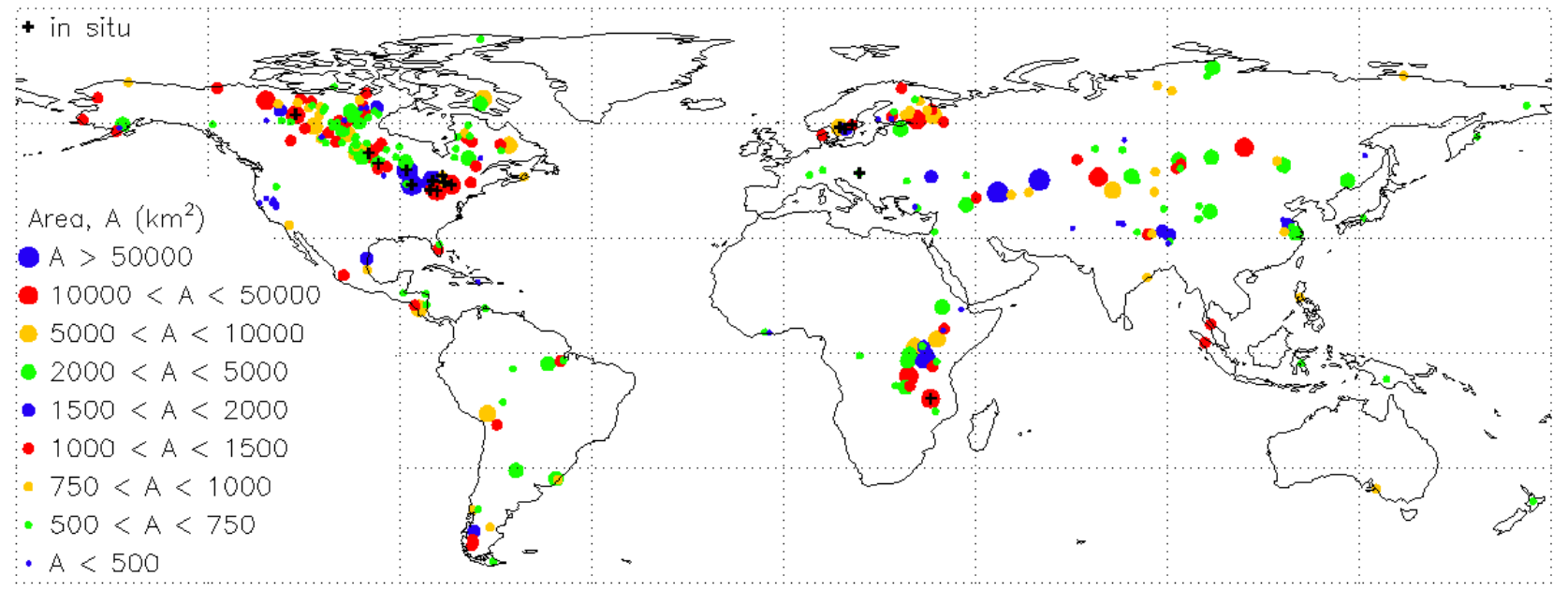

Figure 1: Location and area of 263 large target lakes considered in this study. Lakes where in situ observations are available to the study are marked with the + symbol.

Following the identification of the target lakes (the full list of which is available at www.geos.ed.ac.uk/arclake) a lake mask was developed, to enable satellite observations at a given location to be correctly attributed to an individual lake. This is a non-trivial problem due to complexities in lake shape and ambiguities in defining boundaries with inflows and lakes with connecting filaments of water.

A lake mask was derived through consolidation of the NAVOCEANO gridded land/water mask (from https://www.ghrsst.org/data/ghrsst-datatools/navo-ghrsst-pp-land-sea-mask/) and level-1 polygons from the Global Lakes and Wetlands Database, GLWD (Lehner and Döll, 2004). The resulting lake mask contains unique lake IDs (from the GLWD) on the $1 / 120^{\circ} \times 1 / 120^{\circ}$ NAVOCEANO grid. It is hierarchically structured and is available from www.geos.ed.ac.uk/arclake.

The use of the GLWD polygons to provide unique lake IDs enables lakes with highly complex shapes to be represented, as demonstrated in Figure 2. Here the mask is able to correctly associate multiple separate groups of water cells as belonging to the same lake, Lake Astray, Canada. 


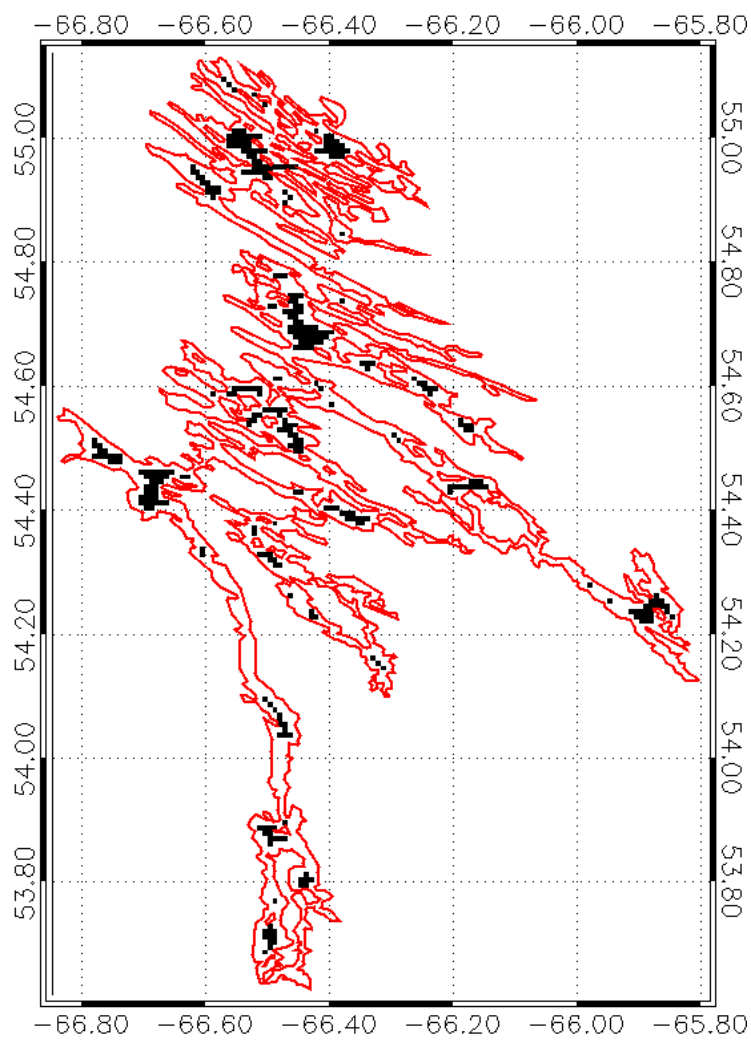

Figure 2: Lake Astray (Canada) in the consolidated NAVOCEANO/GLWD land/water mask. White: land cells in mask. Black: water cells in mask. Red: polygon from GLWD. White cells within the red polygon contain both water and land, so are masked as land.

\section{LSWT Retrieval Algorithms}

LSWT retrievals are performed using combinations of the three infra-red channels available on the ATSRs: 3.7, 10.8, and $12 \mu \mathrm{m}$. The ATSR infra-red radiometer is calibrated to high accuracy, achieved through: (a) on-board calibration using two reference targets, (b) a Stirling-cycle cooler (Závody et al, 1994), and (c) a dual-view geometry that enables robust atmospheric correction (Merchant et al, 1999). Global coverage is provided every three days in the tropics, with more frequent observation possible at higher latitudes. All ATSRs have flown on platforms with stable late-morning orbits, yielding consistent overlap periods to support their application to global climate monitoring. Spatial resolution is $\sim 1 \mathrm{~km}$ at the nadir, and restricts the size of lake surface features (and indeed the minimum size of lakes) that can be observed. However, this is still fine enough to enable some spatial variations in temperature to be resolved, particularly in the larger lakes.
The LSWT retrieval algorithm consists of a cloud detection and temperature retrieval component, both of which depend upon forward modelling of clear-sky infra-red observations of the ATSRs. The radiative transfer model used is RTTOV8.7 (Saunders et al, 2006), driven by the nearest numerical prediction (NWP) profile for the state of the atmosphere from the European Centre for Medium Range Weather Forecasting (ECMWF).

A major motivation for this study is the relatively inadequate observational information available to NWP systems on lake water temperature, which is becoming more important as lake dynamics are increasingly included in landatmosphere interactions schemes. A corollary of this is that NWP is not a good source of surface temperature for forward modelling of the infrared satellite observations. Following initialisation using a combination of monthly climatology from MODIS observations (Brown et al, 1999) and lake-mean temperature climatology simulations from the lake model FLake (Mironov, 2008), empirical orthogonal function (EOF) techniques 
GHRSST XII -

Proceedings, Edinburgh

GHRSST Project Office

(Alvera-Azcárate, 2005) are used to reconstruct a spatially complete time-series of LSWT from the sparse ATSR observations. An iteratively updated version of this is used as the source of prior LSWT in the forward modelling.

Brightness temperatures (BTs) seen for lakes by imagers such as the ATSRs are generally less than the true surface temperature. This deficit (known as the "atmospheric correction") is caused by net absorption of IR radiance by the atmosphere (partially dependent on the total column water vapour) and by the surface emissivity being less than unity. The BT-LSWT relationships therefore depend on the altitude (affecting atmospheric impact) and salinity (affecting emissivity) of the lakes, both of which are highly variable across the target lakes. These variables are captured in the RTTOV8.7 forward model, providing strong motivation for employing forward modelling-based cloud detection and LSWT retrieval.

Inadequacies in cloud detection are linked to significant uncertainties. Typical threshold based cloud detection schemes for SST (Závody et al, 1994) distinguish clear and cloudy skies through predefined tests on ranges for BTs and interchannel BT differences. These threshold tests should ideally be dependent on parameters such as surface temperature, atmospheric profiles and satellite zenith angle. Pre-specifying thresholds that are successful across a wide set of circumstances is challenging, particularly so for lakes, where the range of circumstances is greater than for ocean surfaces (because of the range of elevations and the differences between maritime and continental air masses). Spatial coherence information is also used to distinguish clouds and water, and similar comments apply to determining these thresholds also.

Applying cloud detection for SST to lake bodies gives a useful result in some cases - particularly for the largest lakes with altitudes near sea level. It also helps if they are saline and are at a temperature no too different from SSTs for their latitude. Thus, SST schemes often give sensible results for the Great Lakes, Caspian Sea, etc, at least for some seasons However, more modest lakes can display greater spatial variability than is typical for the ocean, because of the effects of depth variations and thermal barring. This can trip tests for spatial coherence, leading to false
Issue: Final

Date: September 27, 2011

detection of cloud. The BT-LSWT relationships are also changed by high elevation (less intervening atmosphere to affect IR radiance) and by continentality of air-mass. This can lead to false detection, and also failure to detect. Failures to detect cloud can cause large errors in retrieved LSWT.

For cloud detection in this study, we use a Bayesian approach (Merchant et al, 2005, Mackie et al 2010a, and Mackie et al 2010b) informed by the forward modelling discussed above. This compares the expected (modelled) and observed BTs, and calculates in the context of various relevant uncertainties the probability the observation being clear-sky. The only threshold in the scheme is the threshold in the probability of clear sky above which LSWT retrievals are made.

Although the Bayesian approach adapts to the atmospheric conditions automatically (to the degree these are represented in NWP), the spatial coherence statistics currently used are still those developed for ARC SST (Merchant et al, 2008a).

Earlier work (Marsham, 2003) established that LSWT retrieval using standard ATSR SST retrieval coefficients is prone, for some lakes, to retrieval biases of $0.5 \mathrm{~K}$. (By retrieval bias, we mean the systematic offset between satellite and true LSWT that arises from imperfection in the retrieval algorithm. Occasional "biases" from failures in cloud detection can be larger.) This contrasts with a level of SST retrieval bias for ATSR that is generally $<0.2 \mathrm{~K}$. One solution could be to specify lake-specific retrieval coefficients. But this is not really a scalable solution as we look forward to later phases of the project, where more lakes will be tackled.

The LSWT retrieval is therefore done by optimal estimation (OE). We use a simplified formulation of the inverse problem originally developed for SST observations from the Advanced Very High Resolution Radiometer (AVHRR) (Merchant et al, 2008b). This formulation includes only LSWT and total column water vapour as retrieved (state) variables (all though full profile forward modelling is of course used). No radiance bias correction is yet derived for ATSR BTs, so the RTTOV8.7-simulated BTs are used "as is". 
GHRSST XII -

Proceedings, Edinburgh

GHRSST Project Office

\section{LSWT Validation Techniques}

In situ observations from moored buoys located in 16 of the target lakes are used as reference temperatures for validation of the LSWT retrievals. Within this set of 16 lakes there are 52 observation sites. This dataset of in situ observations comprises those data that were freely available at the outset of this study and is relatively limited in terms of number of lakes and geographic coverage (most of the available validation data are from the Great Lakes). The locations of the 16 lakes with available in situ data are shown in Figure 1.

Most sites provide hourly observations over time periods of years; however in some cases observations are only daily and/or more short lived or sporadic in their temporal coverage. At sites where the lake is frozen for considerable lengths of time, in situ observations are unavailable during the frozen period.

Clear-sky LSWT retrievals are averaged over a $5 \times 5$ pixel box, equivalent to the resolution of the ARC-Lake data products $\left({ }^{1} / 20^{\circ}\right)$, centred on the buoy location. Matching against in situ observations is performed spatially (within $1 \mathrm{~km}$ ) and temporally (within 3 hours). In total there are $\sim 15500$ match-ups for ATSR-2 and $\sim 17500$ for AATSR.

Retrieved OE LSWTS are validated against the in situ observations and also compared to equivalent validation of LSWTs from the operational ATSR cloud screening and retrieval scheme (designed principally for SST and labelled "ATS"). Assessment has been carried out for retrievals using the various standard channel combinations for both day and night time observations. Results for day time D2 (dual view, $11 \mu \mathrm{m}$ and $12 \mu \mathrm{m}$ channels) and night time D3 (dual view, $3.7 \mu \mathrm{m}, 11 \mu \mathrm{m}$ and $12 \mu \mathrm{m}$ channels), using the Bayesian maximum channel-set cloud screening, are presented for AATSR in the Results section.
Issue: Final

Date: September 27, 2011

\section{Ice Detection}

The ARC-Lake project also provides observations of lake ice cover (LIC). This is based on the Normalized Snow Difference Index (NSDI) (Hall et al, 1995) and is limited to daytime observations (as it uses visible reflectance channels). Preliminary assessment of the LIC product has been carried out through qualitative analysis of case study imagery and quantitative comparison with ice charts from the NOAA Great Lakes Ice Atlas (Assel, 1993) and the National Ice Center (www.natice.noaa.gov). Ice chart data are averaged to the $1 / 20 \mathrm{x}$ $1 / 20^{\circ}$ grid on which the ARC-Lake LIC product is output, and the fractional ice cover in each grid cell compared across the winter period. As for temperature observations, the availability of in situ ice cover observations is also limited to a small number of lakes and only cover the Great Lakes. Results of this preliminary validation exercise are presented in the Results section.

\section{Data Product Generation}

A key aim of the ARC-Lake project is to provide spatially and temporally resolved data products of LSWT and LIC. Clear-sky LSWT retrievals and LIC estimates are averaged over $1 / 20^{\circ} \times 1 / 20^{\circ}$ longitude/latitude grid cells for each day/night of observations. These averaged observations are stored (along with ancillary information) in NetCDF files on both a per-lake basis (covering all years of observations) and a global basis (covering all lakes for a single day).

Further spatial and temporal averaging is also applied, to generate spatially-resolved $\left({ }^{1} / 20^{\circ} \times 1 / 20^{\circ}\right)$ and lake-mean climatology and time-series over a range of averaging intervals (daily, monthly, twice-monthly, and seasonal). Equivalent data products are also generated from the spatially complete EOF-based LSWT reconstructions. All data products are freely available via the ARCLake project website 
GHRSST Project Office

(www.geos.ed.ac.uk/arclake). A summary of the possible variants of data products is

given in Table .

\begin{tabular}{|c|c|}
\hline Attribute & Variants \\
\hline Source & Observations / Reconstructions \\
\hline Coverage & Per-lake / Global \\
\hline Time & Day / Night \\
\hline Spatial Resolution & $0.05^{\circ}$ grid / Lake-mean \\
\hline Temporal Averaging Type & Climatology / Time-series \\
\hline Temporal Averaging Period & Seasonal / Monthly / Twice-monthly / Daily \\
\hline
\end{tabular}

Table 1: Overview of the types of data product available through ARC-Lake.

\section{Results}

\section{LSWT Validation}

The results of the validation study, where OE LSWT retrievals are compared with in situ observations and operational retrievals,
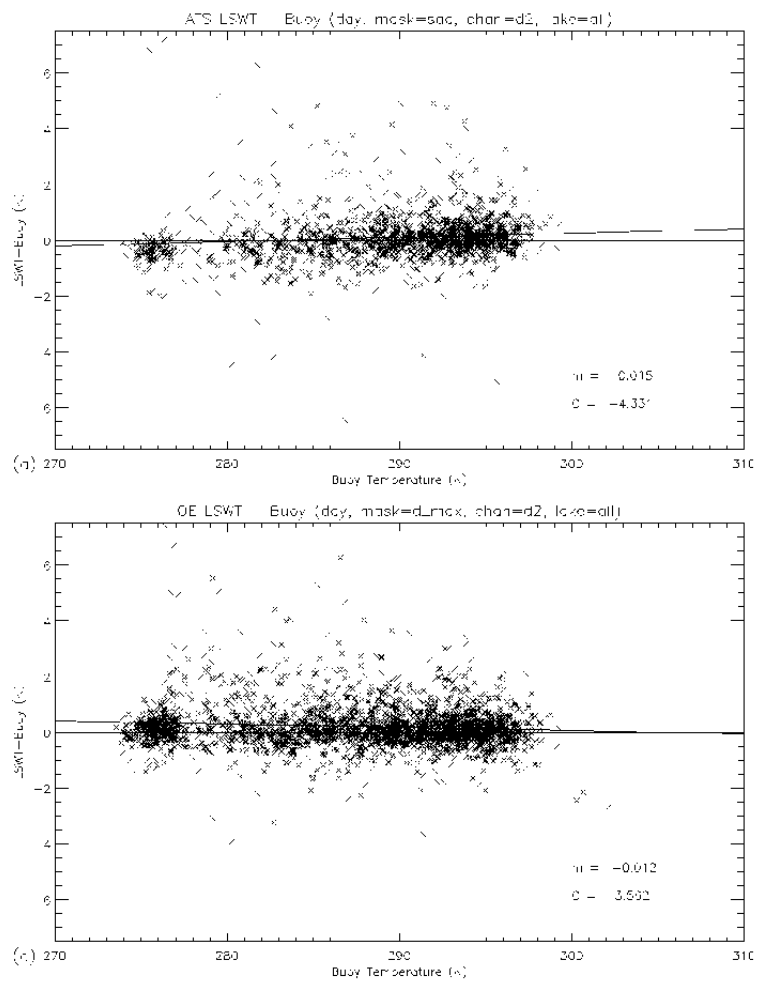

are presented in Figure 3 and Tables 1 and 2 and for AATSR. Statistics for the ATSR-2 validation are also provided in Tables 1 and 2. All results are for direct comparison of satellite and buoy observations: no adjustment is made for the skin-bulk effect.
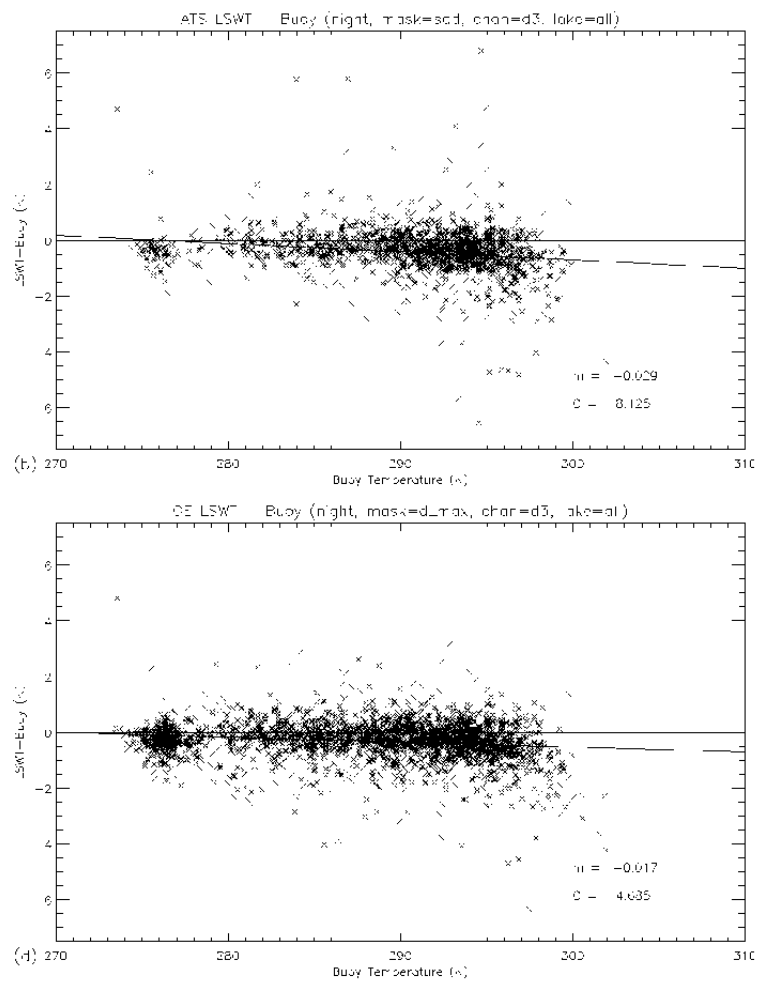

Figure 3: LSWT-Buoy differences against buoy temperature for AATSR. (a) and (b) operational day and night, (c) and (d) OE scheme, day and night. Trend lines are marked on the plots and their gradient, $m\left(K K^{1}\right)$, and intercept, $C(K)$ are given. 
Biases from the operational and $\mathrm{OE}$ retrievals are of the order expected for skinbulk comparisons. RSDs are also comparable across the retrieval schemes. However, the Bayesian cloud screening used in ARC-Lake returns a greater number of observations than the operational method (44-69\% more match-ups). The increased number of match-ups mainly arises from the lower end of the temperature range, where the operational threshold tests seem most likely to return false detection of clouds. This increased number of observations coupled with no increase in retrieval uncertainty gives us confidence that the Bayesian cloud screening offers consistently improved cloud masking.

For the operational retrievals using different channel combinations, the mean satellite-in situ differences range from $0.12 \mathrm{~K}$ to $0.88 \mathrm{~K}$ (day) and $-0.52 \mathrm{~K}$ to $0.18 \mathrm{~K}$ (night) for AATSR, with robust standard deviations (RSDs) of the order $0.5-0.6 \mathrm{~K}$. The spread of mean differences is reduced to $0.18 \mathrm{~K}$ to $0.23 \mathrm{~K}$ (day) and $-0.46 \mathrm{~K}$ to $-0.31 \mathrm{~K}$ (night) when the OE retrievals and Bayesian cloud mask are used. RSDs are similar to or lower than those from the ATS retrieval for all retrieval types, with the RSD for the N2 daytime case $\sim 0.15 \mathrm{~K}$ lower. SDs from the $\mathrm{OE}$ scheme are also lower for all retrieval types, indicating a reduction in outliers compared to the operational scheme, despite passing a greater fraction of matches as clear. The consistency of biases and RSDs across retrieval schemes is of particular importance for extending the new LSWT retrievals to include ATSR-1, in the light of to the early failure of the $3.7 \mu \mathrm{m}$ channel on this instrument.

\begin{tabular}{|c|c|c|c|c|c|c|c|c|c|}
\hline \multirow{2}{*}{$\begin{array}{l}\text { Day I } \\
\text { Night }\end{array}$} & \multirow{2}{*}{$\begin{array}{l}\text { View I } \\
\text { Channels }\end{array}$} & \multicolumn{4}{|c|}{ ATSR-2 } & \multicolumn{4}{|c|}{ AATSR } \\
\hline & & $\mathbf{N}$ & $\begin{array}{l}\text { Mean } \\
\text { Diff. }\end{array}$ & SD & RSD & $\mathbf{N}$ & $\begin{array}{l}\text { Mean } \\
\text { Diff. }\end{array}$ & SD & RSD \\
\hline Day & D2 & 812 & 0.24 & 1.09 & 0.56 & 1522 & 0.12 & 1.03 & 0.55 \\
\hline Night & D3 & 1529 & -0.32 & 0.73 & 0.53 & 1496 & -0.41 & 1.20 & 0.53 \\
\hline
\end{tabular}

Table 2: Validation statistics for LSWT retrievals performed using the operational cloud-masking and LSWT retrieval scheme (ATS). View/channels indicates the views (D=dual) and the number of channels used in the retrieval (e.g. D2 is a dual-view, twin-channel retrieval).

\begin{tabular}{|l|l|l|l|l|l|l|l|l|l|}
\hline Day I $\begin{array}{l}\text { View Iight } \\
\text { Channels }\end{array}$ & & \multicolumn{4}{|l|}{ ATSR-2 } & \multicolumn{2}{l|}{ AATSR } \\
\cline { 3 - 10 } & $\mathbf{N}$ & $\begin{array}{l}\text { Mean } \\
\text { Diff. }\end{array}$ & SD & RSD & $\mathbf{N}$ & $\begin{array}{l}\text { Mean } \\
\text { Diff. }\end{array}$ & SD & RSD \\
\hline Day & D2 & 1179 & 0.42 & 0.96 & 0.53 & 2570 & 0.23 & 0.86 & 0.49 \\
\hline Night & D3 & 2184 & -0.18 & 0.74 & 0.52 & 2536 & -0.31 & 0.75 & 0.53 \\
\hline
\end{tabular}

Table 3: Validation statistics for LSWT retrievals performed using ARC-Lake Bayesian (maximum channel set) cloud masking and ARC-Lake OE LSWT retrieval scheme. View/channels indicates the views (D=dual) and the number of channels used in the retrieval (e.g. D2 is a dual-view, twin-channel retrieval).

\section{LIC Validation}

Reflectance channel imagery and the ARCLake ice mask are shown in Figure 4 for Lakes Erie and Huron. Ice is clearly visible in the false colour image as mid-blue regions (darker than the land) with adjacent black areas being open water. The ice mask (red in the right-hand image of Figure 4) captures most of the clearly visible ice cover, with only a region (with partial cloud 
cover) on Lake Erie not detected. Good correspondence between the ARC-Lake ice

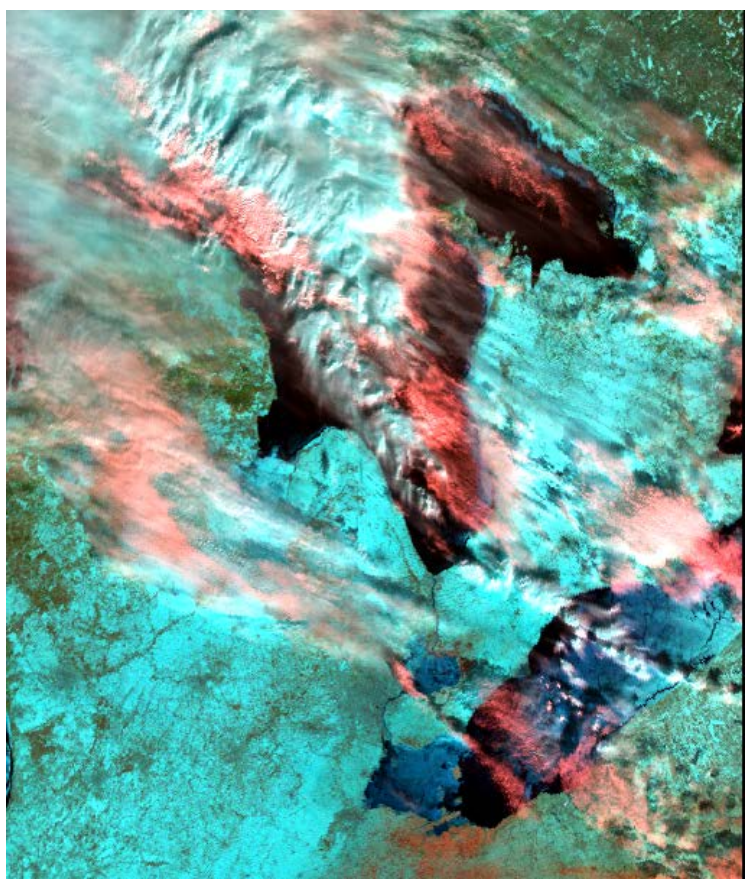

mask and the ice chart data is also observed for this case study.

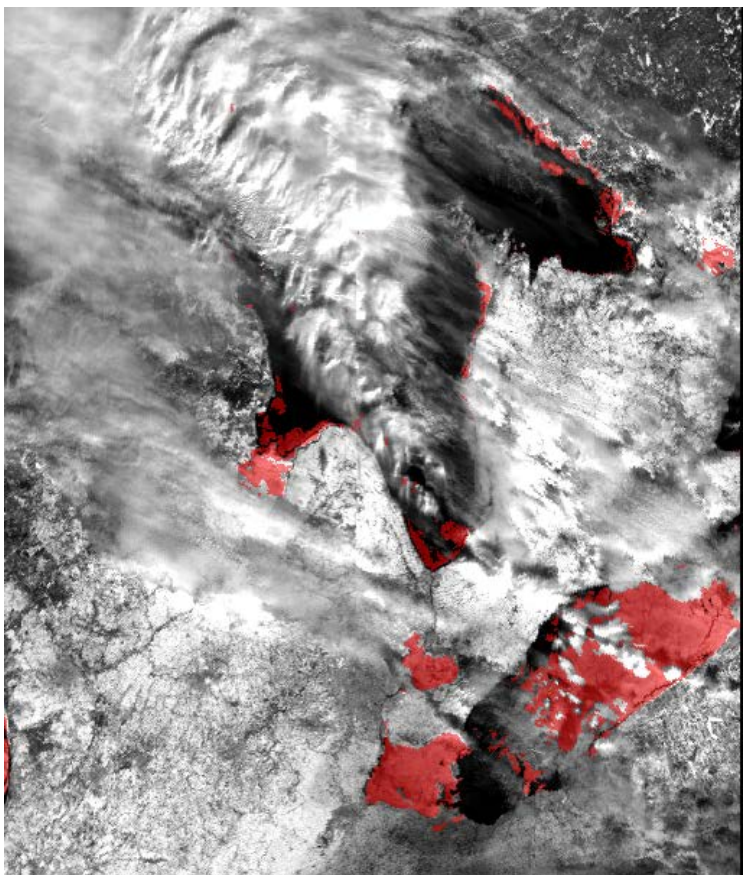

Figure 4: Example of ice detection for Lakes Erie and Huron on 21/01/97. (left) False colour image from ATSR-2 reflectance channels $(0.66 \mu \mathrm{m}, 0.87 \mu \mathrm{m}$ and $1.6 \mu \mathrm{m})$ for nadir view. (right) $0.66 \mu \mathrm{m}$ reflectance in nadir view with ARC-Lake ice mask overlain in red.

Overall results of the quantitative comparison between the LIC product and ice charts for ATSR-2 and AATSR combined are presented in Table. Ideally the diagonal elements of this table should be large, particularly so for open water (0\%) and ice-covered (>85\%), where there should be less ambiguity about the surface type. Reasonable levels of agreement are observed between the LIC product and the ice charts, with the same classification given in over $75 \%$ of the matches, and agreement to within one class exceeding $90 \%$.

\begin{tabular}{|c|c|c|c|c|}
\hline $\begin{array}{ll}\text { Ice Charts } & \text { ARC-Lake }\end{array}$ & $0 \%$ & $1-15 \%$ & $15-85 \%$ & $>85 \%$ \\
\hline $0 \%$ & 64.0 & 0.84 & 0.77 & 0.19 \\
\hline $1-15 \%$ & 8.84 & 0.62 & 0.65 & 0.65 \\
\hline $15-85 \%$ & 2.48 & 0.88 & 2.25 & 2.56 \\
\hline$>85 \%$ & 2.47 & 1.02 & 3.25 & 8.64 \\
\hline
\end{tabular}

Table 4: Results of comparison of ARC-Lake LIC product from ATSR-2 and AATSR with ice charts over all the Great Lakes. Values are the percentage of cells matching each surface classification pair between ARC-Lake LIC and the ice charts. 


\section{Science Applications}

Perhaps the most obvious application of the ARC-Lake data products is in improving our knowledge of basic lake climatological information. As shown in Figure 1, most lakes are poorly monitored in situ, therefore ATSR observations offer a potentially far more globally complete picture of lake climatology, since 1991. Figure 2 and Figure 3 provide examples of the type of climatological information that can be determined from the ARC-Lake data products. Figure 2 shows the lake-mean seasonal LSWT range (from reconstructions) for the 258 target lakes, where enough valid LSWT observations were made to enable the reconstruction to be derived, and illustrates the global nature of the coverage provided. Low temperature ranges are observed in the tropics, with the peak temperature ranges occurring at around $45^{\circ} \mathrm{N}$. Moving to higher latitudes the temperature range generally decreases again as the lakes do not receive sufficient heating following the frozen period to reach such high temperatures. Figure 3 shows the lake-mean seasonal trend in LSWT for Lake Balaton (Hungary) and compares ARC-Lake climatology to that from MODIS and from the online lake model, FLake (Mironov, 2008). Broadly good agreement is observed for this case but for some other lakes ARCLake provides a more reasonable seasonal climatology than MODIS.

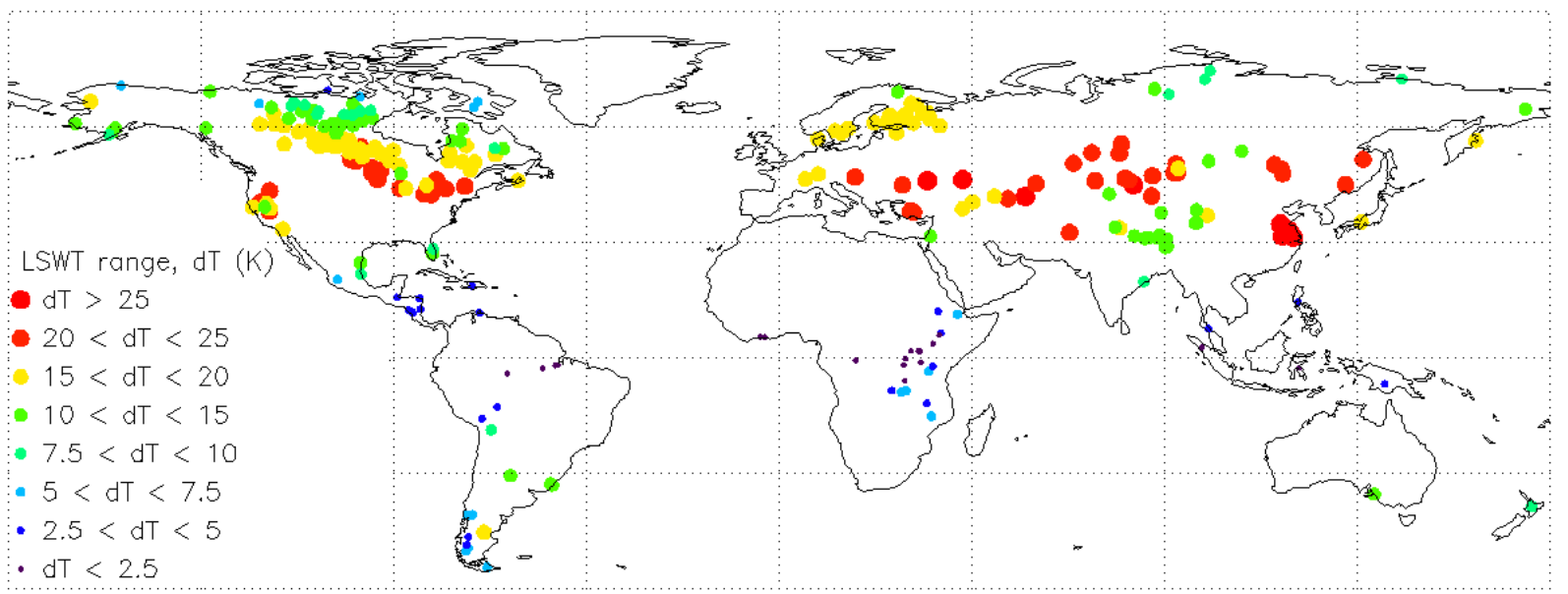

Figure 5: Example of basic climatological information available from ARC-Lake. Mean max.-min. LSWT over the ATSR2/AATSR lifetime (1995-2009), derived from EOF-based reconstructions.

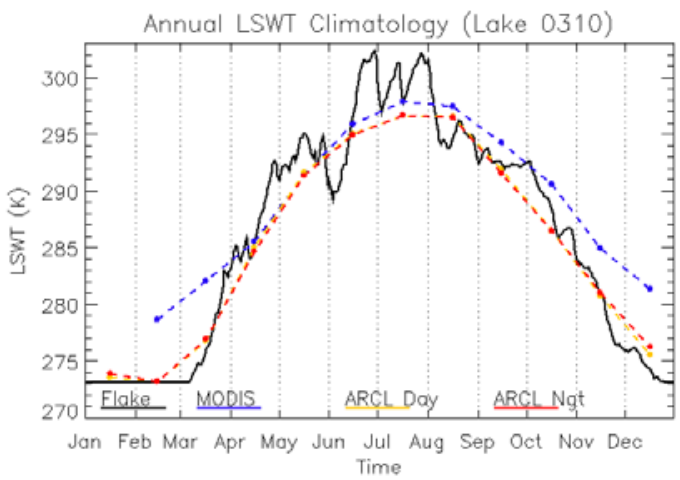

Figure 6: Example of seasonal LSWT climatology derived from ARC-Lake EOF-based reconstructions for Lake Balaton, Hungary. 
The LSWT observations have potential application in the verification of lake physics models. As a basic example, the LSWT from OE retrievals are compared with an empirical LSWT model (Straskraba, 1980). LSWT observations for lakes at elevations < $2000 \mathrm{~m}$ above sea level, in the northern hemisphere, are averaged in $10^{\circ}$ latitude bands and compared to model output at equivalent latitude. Results of this comparison are presented in Figure 7. Good agreement is observed between model and observations, with broadly similar seasonal cycles in LSWT across latitudes. Although broadly similar, the observations do provide a more realistic representation of the seasonal LSWT cycle through better characterisation of the frozen period at high latitudes and the time-lag in warming with increasing latitude.

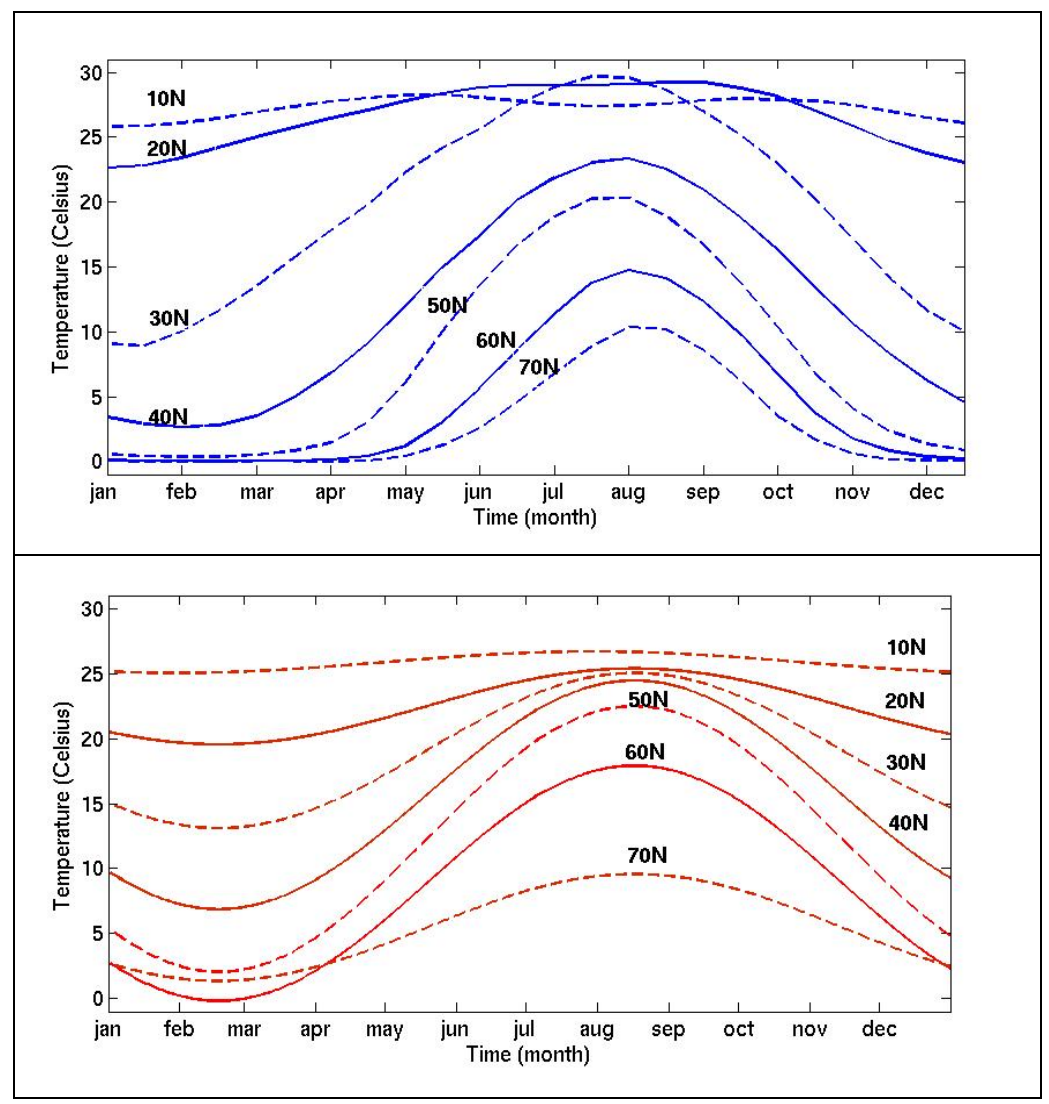

Figure 7: Comparison of observed annual LSWT cycle in $10^{\circ}$ latitude bands (top) with modeled LSWT cycle (bottom) from Straskraba (1980).

Recent work (Schneider et al, 2009) has found dramatic warming trends in Lake surface temperatures over a number of North American lakes. For Lake Tahoe, warming trends of $>0.1 \mathrm{~K}$ decade $^{-1}$ are shown over the ATSR lifetime. Unfortunately the data available to Schneider et al (2009) did not include the overlap periods between ATSR instruments and was also missing ATSR-2 data in the late 90s. ARC-Lake observations for ATSR2 and AATSR (Figure ) do not yield the same dramatic warming trend as found by Schneider et al (2009) when all available data are considered. The difference between "AL" and "SC" warming trends in Figure suggests that much of the $0.1 \mathrm{~K} \mathrm{yr}^{-1}$ difference in warming trends between Schneider et al (2009) and this study may be accounted for by the relatively warm 
years (1996-1998) not available to Schneider et al (2009). ATSR-1 has not yet been processed in ARC-Lake and relatively low LSWT observations for these years (1991-1994) are also expected to contribute to the difference in observed trends.
Differences between the ATSR sensors have been accounted for in the ARC-Lake results but due to the limited time period of analysis, these results should be interpreted with caution.

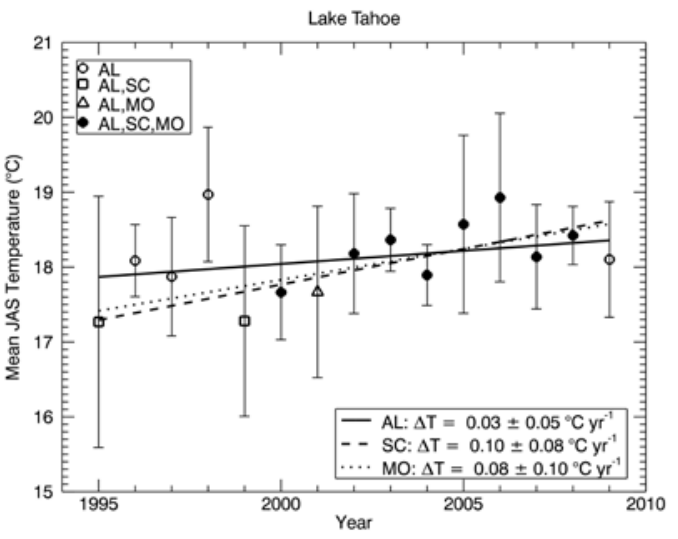

Figure 8: LSWT warming trends for Lake Tahoe (USA). AL, SC, and MO indicate years where ARC-Lake data are available (AL), ATSR data were available in Schneider et al (2009) (SC), and where MODIS data are available (MO). Trends are shown for each of these sets of years and the years for which each of these sets applies are indicated by the plot symbols.

LSWT observations of the form available from ARC-Lake, if made operational, have potential to improve NWP, through assimilation. This is demonstrated in Figure 9, where ARC-Lake observations are compared with NWP data and in situ observations for Lake Nyasa. A climatological cycle is represented in the
NWP data but the magnitude of the NWP temperatures are significantly different to those observed in ARC-Lake. There is good agreement between ARC-Lake and in situ observations, giving confidence that the ARC-Lake observation provide accurate LSWTS

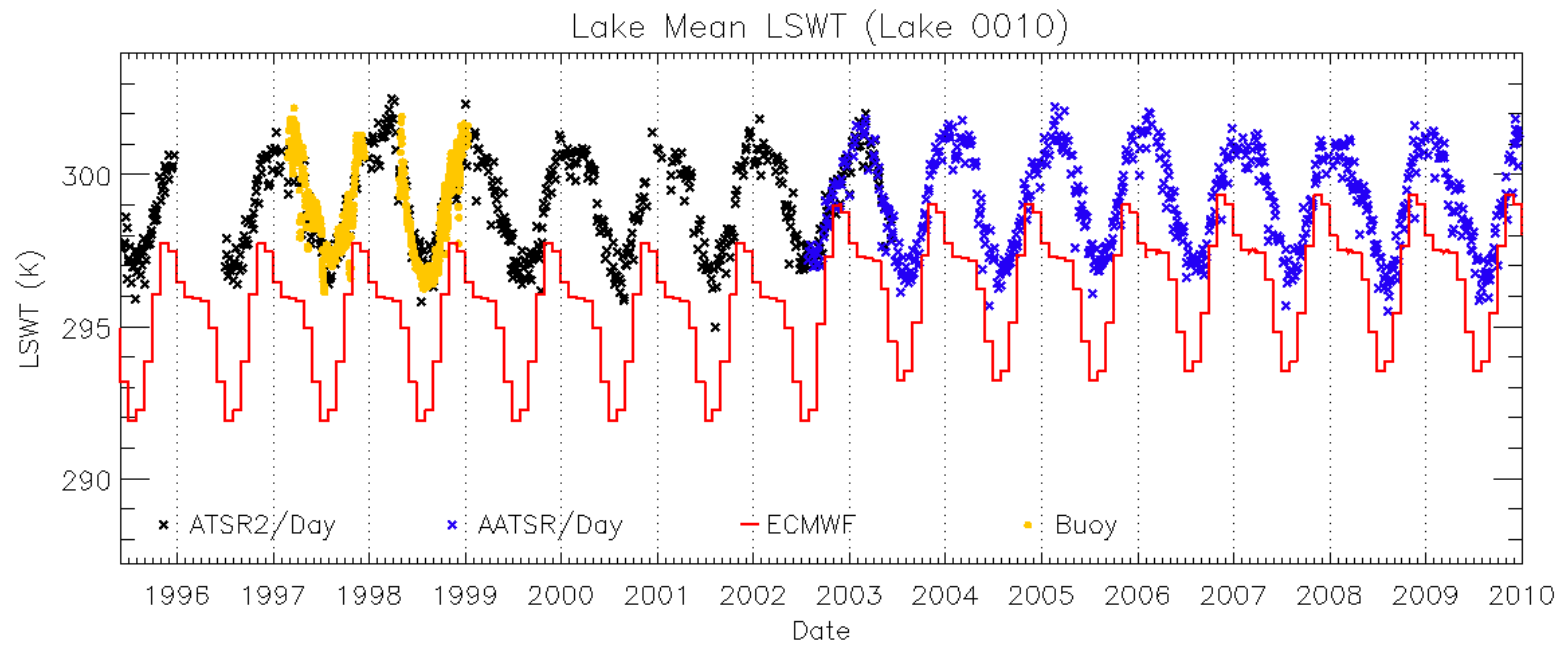

Figure 9: Comparison of surface temperatures from NWP and ARC-Lake observations for Lake Nyasa. NWP data are ECMWF ERA-40 from 1995-2002 and EXMWF operational from 2002-2010. In situ observations are shown in orange. 
GHRSST XII -

Proceedings, Edinburgh

GHRSST Project Office

\section{Conclusion}

Optimal estimation (OE) LSWT temperature retrievals and Bayesian cloud screening have been applied successfully to 258 of the 263 target large lakes. No empirical tuning of retrievals has been used, so that the new satellite LSWTs are independent of in situ observations, being based on the physics of radiative transfer. The new LSWT retrievals have been validated against in situ observations, and, relative to operational retrievals using techniques designed principally for SST observation, provide improved coverage (by $\sim 50 \%$ overall), reduced biases (within $\sim 0.2 \mathrm{~K}$ of the expected mean difference from in situ at night), and reasonable precision $(\sim 0.8 \mathrm{~K})$.

Validation of the LSWT product against in situ observations shows good consistency across different channel/view combinations, with satellite-in situ mean differences agreeing to within $\sim 0.15 \mathrm{~K}$ across all combinations (considering day and night, and each ATSR separately). Uncertainties are also consistent across retrievals, with RSDs within $0.1 \mathrm{~K}$. The statistics of difference from in situ observations across both ATSR-2 and AATSR are $0.3 \pm 0.9 \mathrm{~K}$ for daytime and $-0.3 \pm 0.8 \mathrm{~K}$ for night-time. The night time mean difference of $-0.3 \mathrm{~K}$ needs to be interpreted in the light of the cool skin effect that makes the radiometric temperature of a water body a few tenths of kelvin cooler that the temperature below the surface. The robust SD of differences is of the order $0.5 \mathrm{~K}$ for both day and night.

The problem of inaccurate NWP for lake surface temperatures has been illustrated for Lake Nyasa. In order to provide a reasonable prior LSWT for cloud detection and retrieval, an EOF technique has been used to fill data gaps to create a spatially complete LSWT field from the ATSR observations. A by-product of applying these techniques is the creation of spatially and temporally complete time-series for $>$ $50 \%$ of the target lakes (equivalent climatologically averaged products are
Issue: Final

Date: September 27, 2011 derived for the remaining lakes). These data have potential use in NWP systems that include lake temperature in their landatmosphere interaction scheme, and for lake model validation. Moreover, the new LSWT time series will be useful in quantifying the recent climatology and variability of many lakes where other data are sparse or absent. Trends in LSWT in the data need to be treated with caution until LSWT data are available for all ATSR sensors and until differences between the ATSR sensor calibration and observation times (which differ by 30 minutes) are accounted for.

An ice detection algorithm has also been implemented. This has been demonstrated to provide good correspondence $(>75 \%$ agreement) to ice chart data for the Great Lakes. The ice detection algorithm uses visible reflectance imagery and is therefore only available for daytime observations.

LSWT and LIC data products are now available from the ARC-Lake project (www.geos.ed.ac.uk/arclake), covering a variety of spatial and temporal averaging scenarios. Several improvements are planned for future data releases. Look-up tables used in cloud detection that are based on ocean observations will be replaced with lake-specific tables. Provision for lakes with boundaries evolving over time will be made via an automated water detection algorithm. Methods for lake icedetection and discrimination from ice-clouds will be improved. Overlaps between sensors will be used to inter-calibrate the LSWTs for different ATSRs. Most fundamentally, the time series will be extended back to 1991 by including ATSR-1.

\section{Acknowledgements}

The authors gratefully acknowledge that this work is funded by the European Space Agency under contract 22184/09/I-OL. The lake processor is a modification of the processor built during the ATSR Reprocessing for Climate (ARC) project, that was funded by the UK Natural 
Environment Research Council (NE/D001129/1) and the Department of Energy and Climate Change (CPEG 31).

\section{References}

Alvera-Azcárate, A., A. Barth, M. Rixen, and J. Beckers, Reconstruction of incomplete oceanographic data sets using empirical orthogonal functions: application to the Adriatic Sea surface temperature, Ocean Modelling 9(4), 325-346, 2005.

Assel, R. A., NOAA Data Report ERL GLERL-24: A Computerized Ice Concentration Data Base For The Great Lakes, NOAA, Great Lakes Environmental Research Laboratory, 1983

Brown, O. B., P. J. Minnett, R. Evans, E. Kearns, K. Kilpatrick, A. Kumar, and R. Sikorski, MODIS Infrared Sea Surface Temperature Algorithm - Algorithm Theoretical Basis Document Version 2.0. University of Miami, Miami, FL., 1999.

Hall, D., G. A. Riggs, and V. V. Salomonson, Development of methods for mapping global snow cover using moderate resolution imaging spectroradiometer data. Remote Sensing Environment, 54(2), 127-140, 1995

Herdendorf, Charles E., Large Lakes of the World, Journal of Great Lakes Research 8(3), 379-412, 1982.

Lehner, B. and P. Döll, Development and validation of a global database of lakes, reservoirs and wetlands, Journal of Hydrology 296(1-4), 1-22, 2004

MacCallum, S. N. and C. J., Merchant, (submitted). Surface Water Temperature Observations of Large Lakes by Optimal Estimation. Canadian Journal of Remote Sensing.

Mackie, S., C. J. Merchant, C. Old, O. Embury, and P. Francis, Generalised Bayesian cloud detection for satellite imagery. Part 1: Technique and validation for night-time imagery over land and sea, International Journal of
Remote Sensing, 31(10): 2573-2594, 2010 a.

Mackie, S., C. J. Merchant, C. Old, O. Embury, and P. Francis, Generalised Bayesian cloud detection for satellite imagery. Part 2: Technique and validation for day-time imagery, International Journal of Remote Sensing, 31(10), 2595-2621, 2010b

Marsham, J. H. Lake temperatures - thermal remote sensing and assimilation into a lake model, PhD thesis, The University of Edinburgh, 2003.

Merchant, C. J., A. R. Harris, M. J. Murray, and A. M. Zavody. Toward the elimination of bias in satellite retrievals of sea surface temperature, 1, Theory, modeling and interalgorithm comparison, Journal of Geophysical Research 104(C10), 23,565-23,578, 1999.

Merchant C. J., A. R. Harris, E. Maturi, and S. N. MacCallum, Probabilistic physically-based cloud screening of satellite infra-red imagery for operational sea surface temperature retrieval, Quarterly. Journal of the Royal Meteorological Society, 131(611), 27352755, 2005.

Merchant, C., D. Llewellyn Jones, R. Saunders, N. Rayner, E. Kent, C. Old, D. Berry, A. Birks, T. Blackmore, and G. Corlett, Deriving a sea surface temperature record suitable for climate change research from the along-track scanning radiometers. Advances in Space Research 41(1), 1-11, 2008a.

Merchant, C. J., P. Le Borgne, A. Marsouin, and $\mathrm{H}$. Roquet, Optimal estimation of sea surface temperature from split-window observations, Remote Sensing of Environment, 112, 2469-2484, 2008b

Mironov, D., Parameterization of lakes in numerical weather prediction. Description of a lake model. COSMO Technical Report. Deutscher Wetterdienst, Offenbach am Main, Germany. Report No. 11, 2008. 
Saunders, R., P. Brunel, S. English, P. Bauer, U. O Keeffe, and P. Francis, RTTOV8 - Science and Validation Report, U.K. Met. Office, NWP SAF, 2006.

Straskraba, M., The effects of physical variables on freshwater production: analyses based on models, Functioning of Freshwater Ecosystems, edited by
E.D. Le Gren and R.H McConnell, 13-84. Cambridge University Press, 1980.

Závody, A. M., M. R. Gorman, D. J. Lee, D. Eccles, C. T. Mutlow, and D. T. Llewellyn-Jones. The ATSR data processing scheme developed for the EODC. International Journal of Remote Sensing 15(4), 827-843, 1994. 


\title{
NOAA SEA SURFACE TEMPERATURE PRODUCTS GEOSTATIONARY SST L2P AND BLENDED SST L4 PRODUCTS
}

\author{
Eileen Maria Maturi ${ }^{(1)}$, Andrew Harris ${ }^{(2)}$ Jonathan Mittaz $^{(2)}$ John Sapper ${ }^{(3)}$, Robert \\ Potash $^{(3)}$, Krystal Repoff ${ }^{(3)}$, Prasanjit Dash ${ }^{(4)}$
}

(1) NOAA/NESDIS/STAR, Camp Springs, MD (USA), Email: eileen.maturi@noaa.gov

(2) University of Maryland, CICS, College Park, MD (USA), Emails: andy.harris@noaa.gov, and Jon.mittaz@noaa.gov

(3) NOAA/NESDIS/OSPO, Camp Springs, MD (USA), Emails: john.sapper@noaa.gov, bob.potash@noaa.gov and krystal.repoff@noaa.gov

(4) Colorado State University, CIRA, Fort Collins, CO (USA), Email:

Prasaniit.Dash@noaa.gov

\section{ABSTRACT}

The Group for High-Resolution Sea Surface Temperature (GHRSST) is an international effort focused on the task of producing bestpractice sea surface temperature (SST) products in a common data format. Formats and content are specified for level 2 (L2; data at observed pixels), level 3 (L3; gridded but with gaps) and level 4 (L4; gridded gap-free analysis) products. The National Oceanic and Atmospheric Administration's (NOAA) Office of National Environmental Satellite Data and Information Services (NESDIS) generates operational geostationary SST products in GHRSST L2P format and a daily global SST analysis in GHRSST L4 format. NOAA provides L2P SST products for all operational geostationary satellites GOESEast (E) and West (W), MTSAT-2 and MSG-2 (Meteosat-9). The GOES E and W L2P products are derived from $1 / 2$-hourly GOES-East \& West North \& South sectors, the MTSAT-2 product is produced every hour and the MSG-2 product is produced every 15 minutes. Both the MTSAT-2 and MSG-2 L2P SSTs are derived from full disk images. All products (GOES, MTSAT and MSG) are in satellite projection and also contain the full L2P ancillary fields as specified in the GHRSST format GDS 1.7.

Operational SST retrievals from GOES E and W, MTSAT-2, MSG-2, NOAA-19 and MetOp-A satellites are then used to produce an operational daily global, high resolution
SST GHRSST L4 Analysis at $11 \mathrm{~km}$. In the near future we will also be producing an analysis at $5 \mathrm{~km}$ resolution.

In 2012, NOAA /NESDIS is scheduled to generate an operational AMSR-E SST GHRSST L2P product which will also be included in the operational daily global SST GHRSST L4 analyses. In addition, an operational Geostationary Global 24-Hour SST GHRSST L3 product is scheduled to be generated.

\section{Introduction}

The GHRSST L2, L3 and L4 SST products provide important information to the wide variety of NOAA users on the quality of the SST retrievals. For example, the NWS/Ocean Modelling Branch requires bias free SST products with corresponding error estimates for each observation before these products can be assimilated into their operational forecast models. The NOAA Climate Project Office requires improved SST accuracy (bias and error estimation) for their climate data sets. The National Marine Fisheries Service needs good temporal resolution and accurate SSTs for the location of critical temperature fronts for their mandated management of fisheries and mammals.

Geostationary data have a dominant influence where they are available due to the high temporal sampling. This paper will provide a brief description of the geostationary NOAA/NESDIS GHRSST 
GHRSST XII -

Proceedings, Edinburgh

GHRSST Project Office

L2P, L3 and L4 products, examples of these products and future GHRSST products.

\section{NOAA/NESDIS Geostationary SST L2P Products}

This section describes the scientific basis behind the approach taken by NOAANESDIS to satisfy the requirements of the most widely used of the three levels of products, which is Level 2P (L2P - "P" stands for preprocessed) for the operational GOES-SST. The contents of the L2P products are described extensively in the GHRSST documentation, specifically the GDS V1.7, which can be found on the GHRSST web site (www.ghrsst-pp.org) and the techniques used to derive the SST and cloud mask are described in Maturi et al. (2008). The L2P format requires the appending of a number of ancillary data records to the standard SST value at each pixel, including latitude, longitude, time, cloud proximity, aerosol optical depth, wind speed, surface solar irradiance, sea ice fraction and single-sensor error statistics (SSES). The latter parameters are the primary means of providing end-users with quantitative information on data accuracy. The methodology used for their derivation in the NOAA GOES-SST L2P product is provided later.

While a number of data providers have provided a subset of the full L2P record (referred to as L2P-Core), NOAA has decided to implement the full data product at the onset. The dataset includes the full L2P ancillary field, but, as is common with
Issue: Final

Date: September 27, 2011

other data providers, a few important features need to be noted. The aerosol field is derived from the NESDIS operational daily aerosol optical depth analysis, and values are sampled to each retrieval on a nearest-neighbor basis. The "age of observation" value that is available with the analysis is also included - in this case the range may extend to several days rather than the 24 hours specified in GDS v1.7. Meteorological fields are derived from the NCEP Global Forecast System forecast fields and bi-linearly interpolated to the SST retrieval location. Surface solar irradiance is derived from the 3-hr average for the period in which the SST observation was made. Wind speeds are additionally interpolated in time. Finally, the "proximity confidence" is actually derived from the Bayesian probability of clear-sky (see Merchant et al., 2005), with probability ranges of 0.8-0.95, 0.950-0.99, 0.99-0.999, 0.999-1.0 mapping to proximity confidence values of $2,3,4 \& 5$, respectively. Our L2P products also contain an optional field with the actual probability values, with a range from 0.95 to 1 in steps 0.0002 . It should be noted that the GDS documentation describes the infrared Proximity Confidence value as a means of determining the probability of a particular SST observation being cloud-contaminated. Since this is exactly what the Bayesian clear-sky probability estimate already calculates, it was deemed appropriate to include this in the GOES-L2P record as well. Some example fields are shown in Figure 1. 


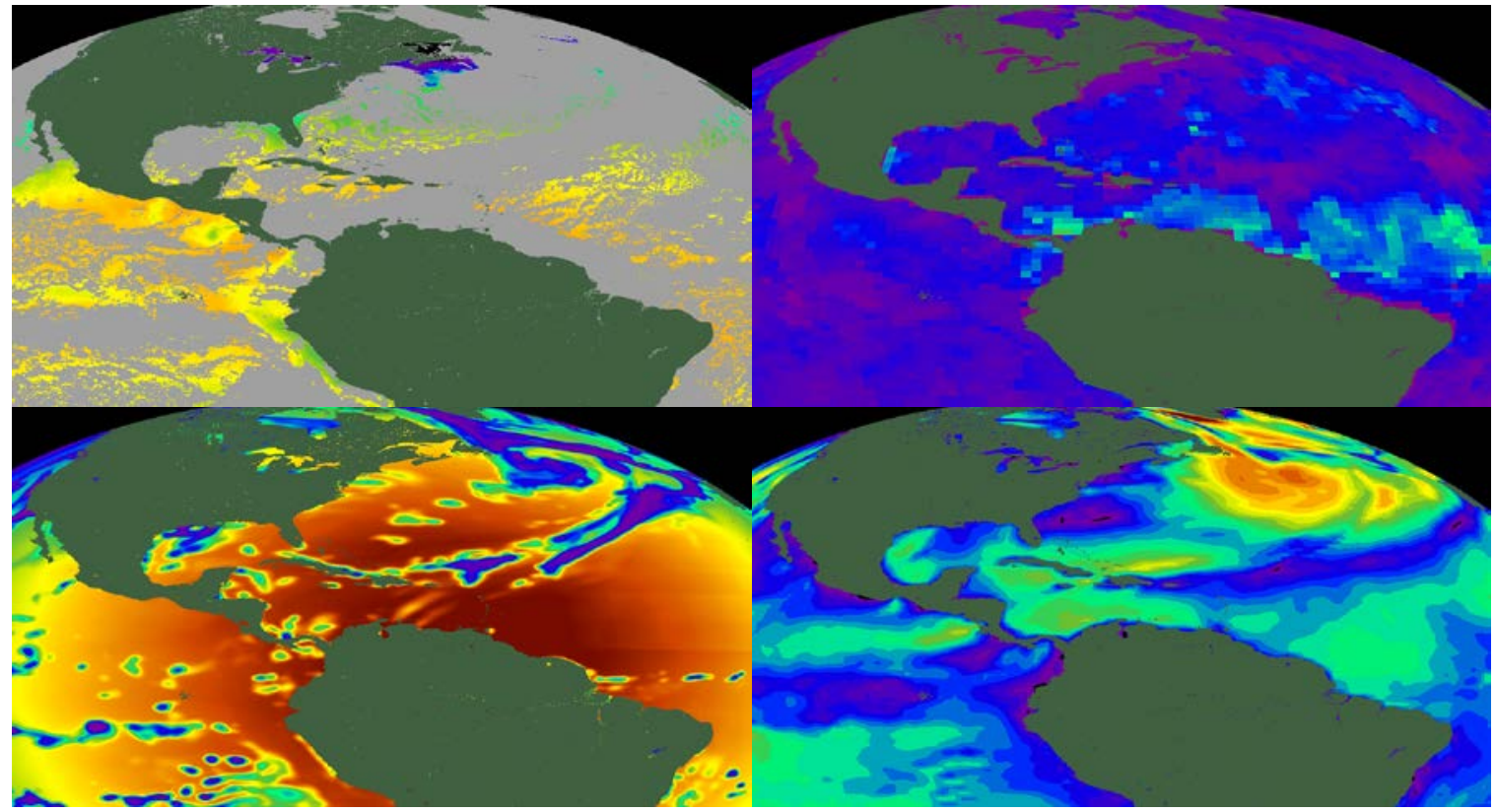

Figure 1. Example fields taken from GOES-E N Hemisphere sector L2P file for 28 March, 2011, 1515

UTC. Clockwise from top-left are SST, aerosol optical depth, wind speed and solar surface irradiance respectively. Note the $1^{\circ}$ resolution of the aerosol optical depth field, and the fact that the insolation is the average from 1500 - 1800 UTC (1000 - 1300 local time at the center of the image). Actual product size is $3460 \times 1827$ pixels.
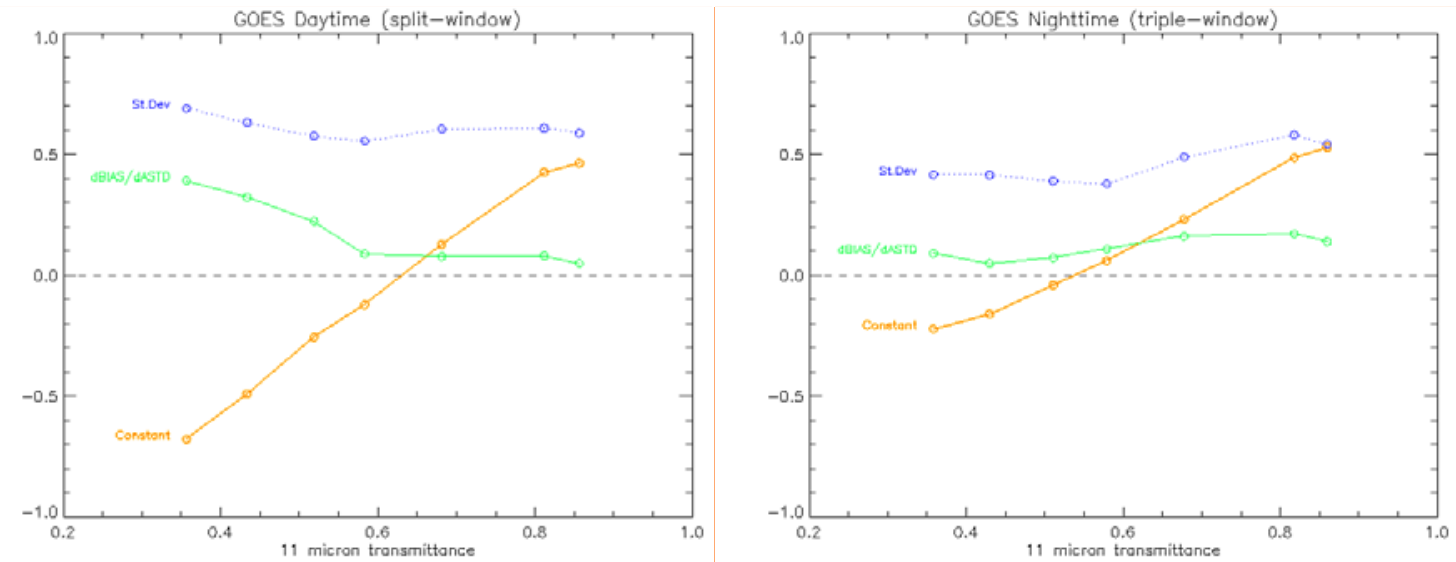

Figure 2. Dependence of bias and $\sigma$ estimation on $\tau_{11 \mu m}$ for GOES-10 daytime and nighttime SST retrievals. The "constant" term indicates systematic disagreement between radiative-transfermodeled and satellite-observed brightness temperatures, and indicates the need for radiance bias adjustment. Note also the increase in sensitivity to ASTD at lower transmittances for the daytime (split-window) retrieval, whereas the nighttime (triple-window) retrieval displays much less sensitivity, as expected.

\section{Single-Sensor Error Statistics (SSES)}

The GDS documentation is in a process of continual development. NOAA has recognized that there are certain limitations in the already somewhat unwieldy L2P data record (e.g. there is no entry for water vapor - a significant source of SST retrieval error in the infrared). Thus the decision was taken to use the SSES to encapsulate information that could not be derived from 
the ancillary data already contained within the $\mathrm{L} 2 \mathrm{P}$ record. Retrieval errors have been characterized for the GOES SST retrievals using NCEP forecast fields and fast radiative transfer modeling. Since the OPTRAN fast model is already run for the Bayesian probabilistic cloud detection as part of the GOES SST processing, the results were written out to intermediate files for use in calculation of retrieval bias and standard deviation. The retrieval errors are assumed to depend on clear-sky transmittance (the main cause of nonlinearity in linear retrieval methods) and airsea temperature difference (ASTD). The dependence of bias on ASTD itself is assumed to be a function of clear-sky transmittance, since the sensor receives less signal from the surface in low transmittance atmospheres and the success of the retrieval is therefore more dependent on being close to the mean state used to derive the linear retrieval coefficients in such cases.

The practical approach taken to obtain the retrieval error is to derive coefficients of dependence on ASTD for different bins of clear-sky transmittance at $11 \mu \mathrm{m}\left(\tau_{11 \mu \mathrm{m}}\right)$ using matches to in situ SSTs from buoys. The coefficients are derived separately for each sensor and algorithm (i.e. day and night). Post-correction values of $\sigma$ are then obtained for each $\tau_{11 \mu \mathrm{m}}$ bin. Coefficients to calculate bias and $\sigma$ are then estimated by interpolation with respect to $\tau_{11 \mu \mathrm{m}}$ from the individual bin mean $\tau_{11 \mu \mathrm{m}}$ values. Examples of derived SSES values for GOES-10 SST (day and night) are shown in Figure 2. At present, the ASTD value that is applied to derive the correction is derived solely from the NCEP analysis fields, although there is the scope for including the effect of sub-grid SST variability in the estimate (e.g. from the actual SST value at each point within the image c.f. $1^{\circ} \times 1^{\circ}$ averages that coincide with the NCEP grid).

\section{SST L2P Parameters}

The NOAA/ NESDIS operational geostationary SST L2P products are in GDS 1.7 format while the pre-operational geostationary SSTs are in GDS2.0. These L2P netCDF product files have 22 parameters for each pixel: SST, Time, Latitude, Longitude, Satellite Zenith Angle, Aerosol Optical Depth, Surface Solar Irradiance, Wind Speed, Uncertainty estimates (bias and S.D.), Proximity Confidence Value, QC flags (including cloud and land), Ice concentration, Deviation from analysis SST, Temporal coincidences of ancillary data c.f. SST observation, Source codes for ancillary data, Probability of clearsky (optional field).

The data sets for GOES-E and W, MTSAT2, and MSG-2 are derived from their specific SST area files (satellite projection). For the GOES-E and W North and South sectors, the data set is derived every $1 / 2$ hour (4 files per $1 / 2$ hour). The MTSAT-2 data set is derived every hour on full disk and the MSG-2 is derived every 15 minutes on full disk. This is done to ensure that the validation and error information are consistent with each other.

\section{NOAA/NESDIS SST L4 Products}

\section{NOAA Global High Resolution SST Analysis 11-km/5-km}

NOAA/NESDIS High Resolution Global SST Analysis has been operational since 2007. This analysis combines geostationary and polar satellite SST retrievals to produce a single "best estimate" high resolution global SST analysis. The multi-scale OI procedure employs a recursive estimator which emulates the Kalman Filter and uses dataadaptive correlation length scales to provide a reasonable balance between noise reduction and detail preservation (Fieguth, 1998; Fieguth, et al., 2001). This allows for preservation of mesoscale features. Input datasets are individually bias-corrected against the operational NOAA/National Centers for Environmental Prediction 
(NCEP) operational Real-Time Global SST Analysis (RTG) High Resolution SST RTG_HR. The operational geostationary and POES SSTs and the RTG_HR are collected for the analysis period. The NCEP ice mask is applied to this analysis. Satellite SST data are bias-corrected and QC'ed against previous day's analysis. Next the analysis is run using all the valid SST data for the analysis day to generate a new analysis. Figure 3 shows an example of NOAA Global High Resolution SST Analysis $(11-\mathrm{km})$. The benefits of this analysis technique are: the 1) resolution of mesoscale oceanic features; 2) computational efficiency permitting rigorous treatment of many separate datasets; and 3) Data-adaptive correlation length scale preserves fine detail in data-rich environment (Khellah, et al., 2005).

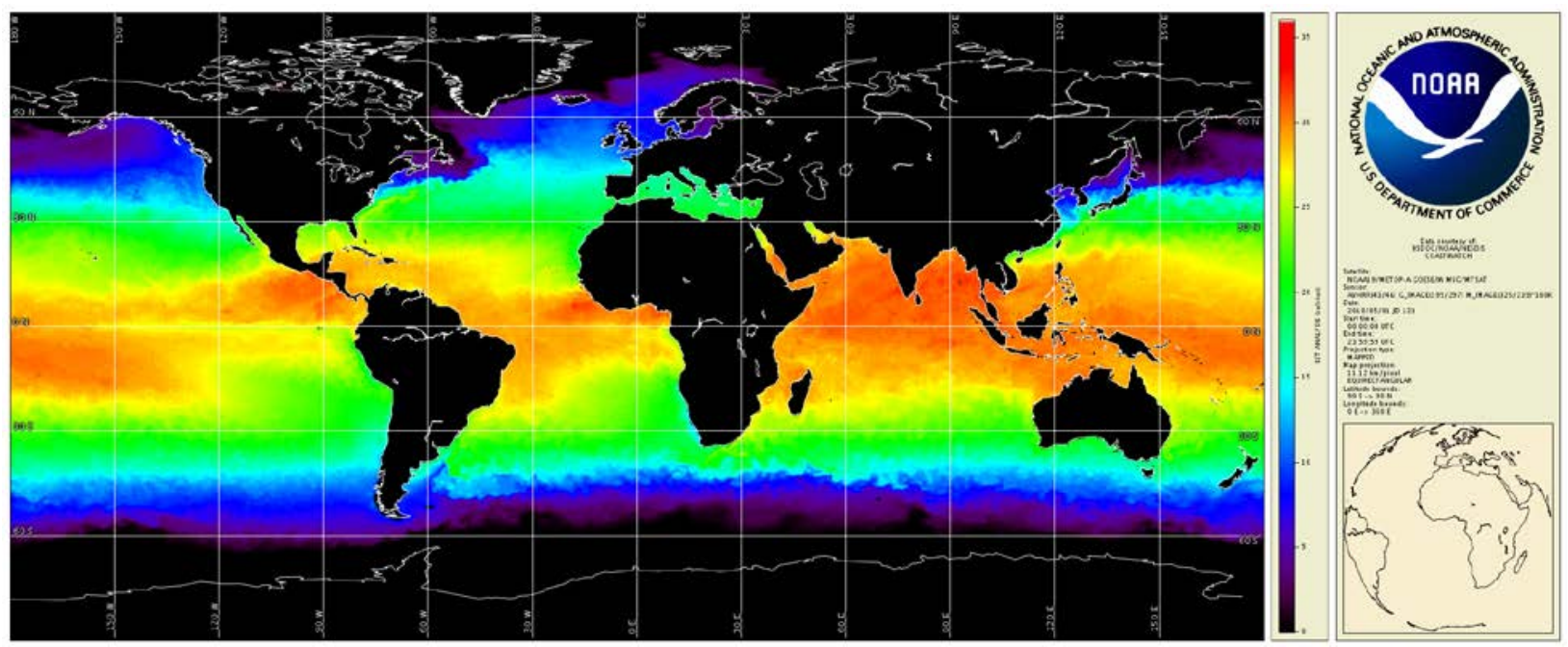

Figure 3: NOAA Global High Resolution SST analysis at 11-km. The satellite retrievals used in this analysis include: GOES-East and West, MTSAT-2, MSG-2, NOAA-19, and Metop-A.

\section{Future GHRSST Products}

NOAA/NESDIS is scheduled to generate an operational AMSR-E GHRSST L2P SST product and incorporate these retrievals into the NOAA operational Global High Resolution GHRSST L4 SST Analysis. The primary benefit will be the provisional SST retrievals in regions (e.g. high latitudes) where there is a lot of cloud cover. Upon the successful launch of GCOM-W, we intend to generate AMSR-2 GHRSST L2P products to replace the AMSRE-E L2P. In addition, GHRSST L3 Geostationary Global 1-hour and 24-hour SST products will be generated. However, there is no geostationary coverage over the Indian Ocean; we are looking into acquiring Elektro-L satellite data to generate Elektro-L GHRSST L2P SST product to alleviate the data-gap in geostationary SST retrievals. Figure 5 shows an example of the Global 24-hour Merged Geostationary product and illustrates the coverage of ELektro-L. 


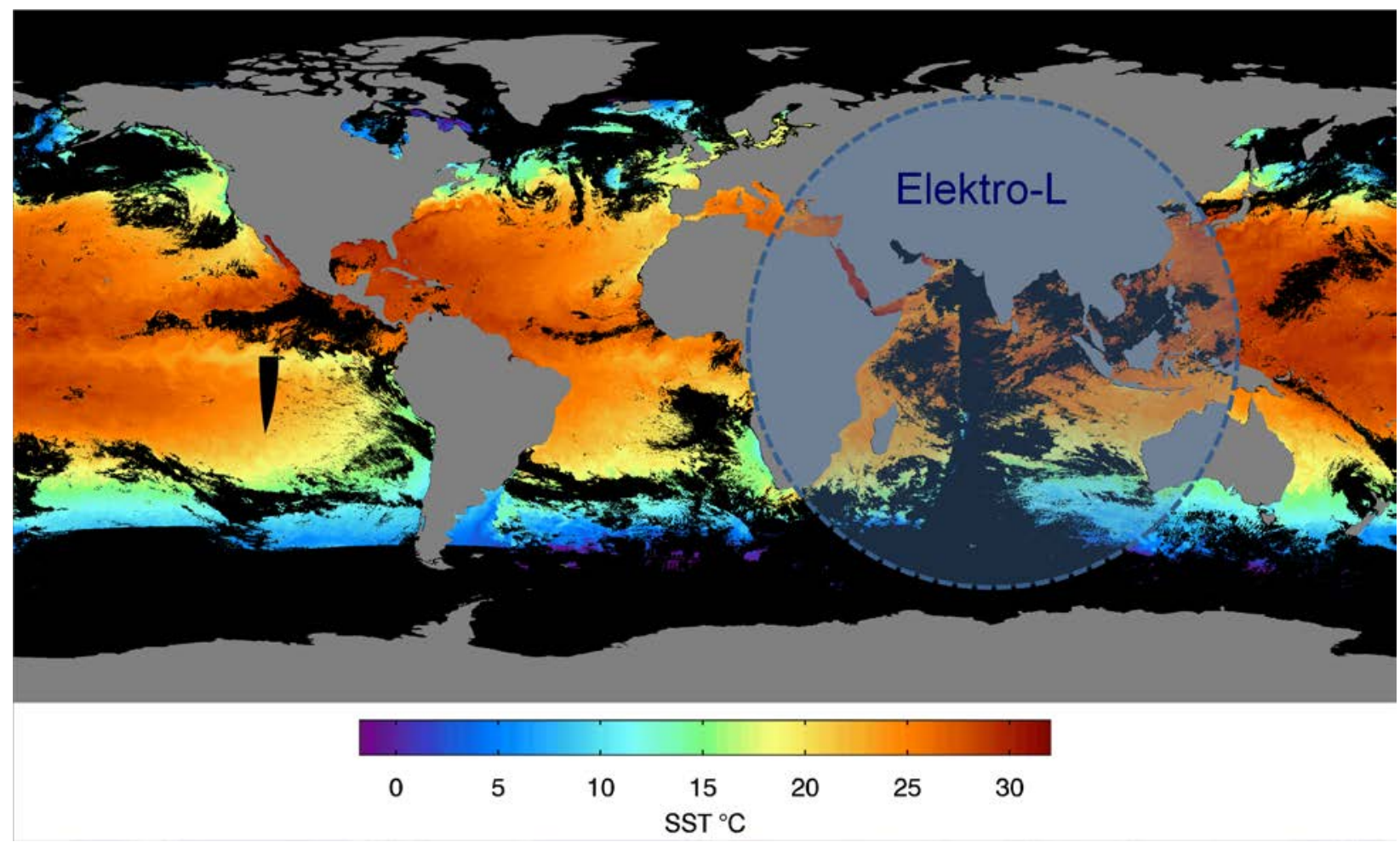

Figure 5: This Global 24 Merged Geostationary Product illustrates the full global coverage of geostationary satellites with Elektro-L.

\section{Conclusion}

The operational GHRSST L2P, L3 and L4 SST products that NOAA/NESDIS generates and plans to generate are/will be available to GHRSST and NOAA users. These GHRSST products provide a valuable improvement to the current NOAA operational geostationary and Global High Resolution SST Analysis.

\section{References}

Fieguth, P.W. et al., (1998) "Mapping Mediterranean altimeter data with a multiresolution optimal interpolation algorithm", J. Atmos. Ocean Tech, 15 (2): 535-546..

Fieguth, P., (2001) Multiply-Rooted Multiscale Models for Large-Scale
Estimation, IEEE Image Processing, 10 (11), 1676-1686.

Khellah, F., P.W. Fieguth, M.J. Murray and Allen, M. R. (2005) "Statistical Processing of Large Image Sequences", IEEE Transactions on Geoscience and Remote Sensing, 14 (1), 80-93

Maturi, E., A. R. Harris, C.J. Merchant, J. Mittaz, R. Potash, W. Meng and J. Sapper (2008), NOAA's Sea Surface Temperature Products from Operational Geostationary Satellites, Bull. Amer. Meteor. Soc., 89(12), 1877-1888.

Merchant C J, Harris A R, Maturi E and MacCallum S (2005), Probabilistic physically-based cloud screening of satellite infra-red imagery for operational sea surface temperature retrieval, Quart. J. Royal Met. Soc., 131, 2735-2755. 


\section{THE DBCP-GHRSST PILOT PROJECT}

\section{David Meldrum $^{(1)}$, Pierre Blouch ${ }^{(2)}$}

(1) SAMS, Oban, Scotland, Email: dtm@sams.ac.uk

(2) CNRM/CMM, Météo France, Brest, France, Email: pierre.blouch@meteo.fr

The Data Buoy Co-operation Panel (DBCP) was formed in the mid 80's to address the serious concerns surrounding the quality, quantity and timeliness of data being collected from the newly deployed fleets of satellite-tracked surface drifters. Over time these issues have largely been resolved and a global population of more than 1300 drifters now routinely reports good quality surface pressure and SST data via the GTS, overseen by a full-time Technical Coordinator based in Toulouse. In the last few years the DBCP has turned some of its attention to a number of pilot projects with the aim of evaluating new technologies that might in due course transition to operational use, and has tried to strengthen its links with other observing system groups, from both the in situ and remote sensing communities. In the case of GHRSST, the DBCP has worked with GHRSST participants to fully understand the needs for high resolution SST and to work with GHRSST in helping to equip the future drifter fleet with sensors that meet its requirements. Initial practical steps have been taken by Météo France over the past 12 months within the context of the DBCPGHRSST Pilot Project to equip a fleet of drifters with HRSST sensors. These now routinely report on the GTS in near real time. The aims and achievements to date of the Pilot Project will be described. 


\title{
SEA SURFACE TEMPERATURES FROM SATELLITE OBSERVATIONS AS CLIMATE DATA RECORDS
}

\author{
Christopher Merchant $^{(1)}$ \\ School of GeoSciences, University of Edinburgh (UK), Email : c.merchant@ed.ac.uk
}

The invitation for this presentation asked for a talk discussing "What users need to know about SST for the purpose of climate applications". The talk will try to stick to this brief, with the caveat that only satellite SST climate data records (CDRs) and analyses will be discussed.

A key thing that users need and want to know for climate applications is uncertainty information. Uncertainty information should be made "as simple as possible, but not simpler". The appropriate level of detail of probably varies significantly between different climate applications. Nonetheless, it appears that the present provision of uncertainty information with GHRSST SST products is insufficient for many, as will be shown.

The nature of the errors in SST observations will be discussed. Some errors are highly correlated, some are partially correlated, the simplest to handle are uncorrelated. In the context of the Along Track Scanning Radiometer (ARC) Reprocessing for Climate (ARC) one approach to estimating uncertainties at L2 and L3 has been developed, and will be described. In this view, the attached uncertainty is as much a product as the SST itself. The uncertainty itself requires validation, therefore, and some ideas on this will be presented.

Stability of observation and the degree of independence from other observations are particularly important for many climate applications. A major difficulty regarding stability is how it can be assessed, especially retrospectively. Another factor is the need to avoid aliasing of diurnal variability into SST CDRs based on observations whose local time varies systematically.

Getting uncertainty information attached to GHRSST SSTs as usefully as possible should arguably be a major area for development in GHRSST in the near future. Despite the brief given for the talk, the ultimate conclusion will be that SST producers alone can't define for climate users what they need to know. Conversely, climate users probably need to know some things about satellite CDRs in order to ask the questions that are right for them. It is hoped that this talk will contribute to the dialogue. 


\title{
THE EUROPEAN SPACE AGENCY CLIMATE CHANGE INITIATIVE FOR SEA SURFACE TEMPERATURE
}

\author{
Christopher Merchant
}

\author{
School of GeoSciences, University of Edinburgh (UK), Email : c.merchant@ed.ac.uk
}

In August 2010, the European Space Agency (ESA) kicked-off the Sea Surface Temperature (SST) project within its Climate Change Initiative $(\mathrm{CCl})$. The author is Science Leader on this three project, which involves many partners within the Group for High Resolution SST (GHRSST) and is designed to work closely with GHRSST (both as a community of scientists, and as a framework for SST product development).

The $\mathrm{CCl}$ overall in its present phase requires team to prototype systems for a dozen satellite-driven Essential Climate Variables (ECVs) and to generate products demonstrating their outputs. In the case of the SST CCl products, the approach is to build on (i) the Along Track Scanning Radiometer (ATSR) Reprocessing for Climate (ARC) project, (ii) a National Centre for Earth Observation (NCEO) project applying ARC techniques to Advanced Very High Resolution Radiometers (AVHRRs), and (iii) the ability to inferface to the Ocean and Sea Ice Satellite Application Facility (OSI-SAF) operations at Centre de Météorologie Spatiale (CMS).

The key data outputs from SST CCI will be:

- "climate-quality' time series of skin and depth SST estimates from ATSR and AVHRR observations, for 1991 - 2010

- demonstration of skin SST observation and analysis using ATSR, AVHRR, the Spinning Enhanced Visible and Infra-Red Imager (SEVIRI), and the Advanced Microwave Scanning Radiometer - E
(AMSRE-E), for a six month period in 2011/2012

- an extensive multi-sensor match-up dataset (MMD)

For SST as an ECV, accuracy, stability and consistency are key attributes of the products. The SST retrieval approach is to be defined in the light of an 'algorithm competition', referred to as the 'Round Robin'. The Round Robin will be based on data sets created for the purpose as appropriate extracts from the MMD, and will be designed to give a fair, blind comparison of the performance of different possible algorithms against several metrics for an SST climate data record. We strongly encourage scientists outside of the project to contribute competitor algorithms to the Round Robin. The details of this will be presented within the Estimations and Retrievals Working Group meeting at GHRSST 12 (Thursday).

The SST CCl project by June 2011 will have:

- Undertaken a significant User Requirements consultation

- Specified its planned products in detail

- Collected data for and defined the MMD and extracts for the Round Robin Data Package

- Started internal work on SST algorithm development

In the conference presentation, these activities will be reviewed in turn. 


\title{
DEFINITIONS OF SEA-SURFACE TEMPERATURE.
}

\author{
Peter J. Minnett \\ Meteorology \& Physical Oceanography, University of Miami (USA), Email: \\ pminnett@rsmas.miami.edu
}

The definitions of sea-surface temperature have been discussed within GHRSST several times, but it is clear that there is still some need for clarification. Without clear definitions, there is risk of miscommunication not only within GHRSST but also with the wider community. The purpose of this presentation is to revisit some of the aspects of what is meant by "sea-surface temperature" with the intention of resolving some questions that have arisen recently, and to stimulate a discussion that could lead to more refined, and robust, definitions.

\section{NEW APPROACHES TO THE INFRARED ATMOSPHERIC CORRECTION ALGORITHM.}

\author{
Peter J. Minnett ${ }^{(1)}$, Sareewan Dendamrongvit, ${ }^{(2)}$ Miroslav Kubat, ${ }^{(3)}$ \\ (1)Meteorology \& Physical Oceanography, University of Miami (USA), \\ Email: pminnett@rsmas.miami.edu \\ (2) Electrical and Computer Engineering, University of Miami (USA), \\ Email: s.dendamrongvit@umiami.edu
}

(3) Electrical and Computer Engineering, University of Miami (USA), Email: mkubat@miami.edu

The standard form of the algorithm for correcting the effect of the intervening atmosphere to derive sea-surface temperatures (SSTs) from brightness temperatures measured by spacecraft infrared radiometers in the 10-12 $\mu \mathrm{m}$ spectral interval is a weekly non-linear approximation to the radiative transfer equation and Planck's function. The widely used form of the algorithm, the Non-Linear SST algorithm, was first published in 1998 (Walton et al, 1998) and this has shown itself to be remarkably robust, in that subsequent attempt to improve it, by adding additional terms, have not resulted in SST retrievals that are more accurate when compared to independent measurements.

Given the limited degrees of freedom imposed by the small number of spectral channels used by imaging radiometers, the options for improving the NLSST are severely constrained. We have chosen to explore a different approach to generating atmospheric correction algorithms, and instead of starting from a linearization of the radiative transfer equation, we begin by exploiting numerical 'equation discovery' techniques on large data bases of satellite brightness temperatures, collocated and contemporaneous in suit measurements, and a broad selection of parameters that define the conditions the measurements. We used a Genetic Algorithm approach to derive a selection of formulations that produce accurate SST retrievals. One set of such algorithms has distinct similarities to the NLSST. These were then applied to a Regression Tree analysis to determine optimized coefficient sets that produce a minimum in the SST retrieval uncertainties, and the geographical regions where these are applicable. 
GHRSST XII -

Proceedings, Edinburgh

GHRSST Project Office

We will present the background to this approach and the results of a preliminary investigation using MODIS measurements.

\section{References}

Walton, C. C., W. G. Pichel, J. F. Sapper,
Issue: Final

Date: September 27, 2011

and D. A. May: The development and operational application of nonlinear algorithms for the measurement of sea surface temperatures with the NOAA polar-orbiting environmental satellites. Journal of Geophysical Research, 103, 27,999-928,012, 1998. 


\title{
SEVIRI SST AND SHIPBOARD SKIN SST COMPARISONS: INITIAL RESULTS.
}

\author{
Peter J. Minnett ${ }^{(1)}$, Pierre LeBorgne, ${ }^{(2)}$ Hervé Roquet, ${ }^{(3)}$ \\ (1)Meteorology \& Physical Oceanography, University of Miami (USA), Email: \\ pminnett@rsmas.miami.edu
}

(2) Météo-France/Centre de Météorologie Spatiale (France), Email: Pierre.LeBorgne@meteo.fr

(3) Météo-France/Centre de Météorologie Spatiale (France), Email: Herve.Roquet@meteo.fr

The Spinning Enhanced Visible and InfraRed Imager (SEVIRI) is mounted on the EUMETSAT MSG (Meteosat Second Generation) geosynchronous satellites that are positioned over the Gulf of Guinea at $0^{\circ} \mathrm{N}, 0^{\circ} \mathrm{E}$. SEVIRI has 12 spectral bands including two in the thermal infrared "atmospheric transmission window" at wavelengths of $10.8 \mu \mathrm{m}$ (Channel 9) and 12.0 $\mu \mathrm{m}$ (Channel 10). Under auspices of the EUMETSAT Ocean and Sea Ice Satellite Application Facility (OSI-SAF), the Centre de Météorologie Spatiale (CMS) has been deriving and distributing experimental hourly sea-surface temperature (SST) fields with a spatial resolution of $0.05^{\circ}$ latitude and longitude since June 2005. During this time there have been a number of research cruises in the Atlantic Ocean in which skin SSTs have been measured using M-AERIs (Marine-Atmospheric Emitted Radiance Interferometers). These comparisons provide traceability to the SI temperature standards and therefore fulfil an important requirement for considering SEVIRI SSTs to be a component of the SST Climate Data Record.

Here we report our initial findings resulting from comparisons between SEVIRI and MAERI SSTS. The uncertainty characteristics of the SEVIRI SSTs are presented and discussed. 


\title{
DIURNAL VARIABILITY IN SHALLOW COASTAL WATERS.
}

\author{
Xiaofang Zhu ${ }^{(1)}$, Peter J. Minnett ${ }^{(2)}$
}

(1) University of Miami (USA), Email: xiaofang.zhu@rsmas.miami.edu

(2) University of Miami (USA), Email: pminnett@rsmas.miami.edu

Understanding diurnal warming cycle and its vertical structure in the upper ocean is important for both the validation of satellitederived sea-surface temperature (SST) with in-situ measurements and the compilation of SST fields derived from satellite instruments at different times of day. In coastal regions, to study the diurnal variability is also important for understanding the survival of temperature sensitive species such as coral reefs.

The study methods include analysis of insitu data, empirical and physical modelling, and satellite data analysis. Two in-situ datasets where temperatures, along with local weather data, were recorded in 30-60 minute time resolution for a long period of time (5+ years) are analysed with the aim to relate diurnal variability in temperature to environmental forcing, and to illustrate the difference and similarity with the open ocean case. Present results show that for most cases, the diurnal variability in the shallow water is shaped by solar radiation, wind speed, tide as well as wind waves, but which mechanism is dominant is strongly location specific. For instance, tides play an important role in our Great Barrier Reef dataset while at Caribbean stations, the role is relative minor due to the small tidal elevation. A simple 1-dimensional model of a well-mixed water column works adequately well in moderate to strong wind cases $(>8 \mathrm{~m} / \mathrm{s})$, while for weaker winds, the determination is still to be made which of several 1-dimensional diurnal models can best simulate the profile of the shallow water diurnal variability. Future work will include analysis of the GHRSST Tropical Warm Pool Diurnal Variability Experiment (TWP+) data near Australia coast, and take advantage of the validated AVHRR, MTSAT data as well as regional NWP data. 


\title{
THE CALIBRATION OF THE BORADBAND INFRARED SENSORS ONBOARD NOAA SATELLITES.
}

\author{
Jonathan Mittaz ${ }^{(1)} \&$ Andrew Harris ${ }^{(2)}$ \\ (1)CICS,, University of Maryland. MD (USA), Email : Jon.Mittaz@noaa.govm \\ (2)CICS,, University of Maryland. MD (USA), Email : Andy.Harris@noaa.govm
}

\begin{abstract}
In this paper we look at the calibration of two of the major NOAA sensors used for SST retrievals with the aim of highlighting potential biases and errors in their calibration that can impact SSTs. We first show calibration problems and issues with the GOES-11 Imager starting with the fact that the GOES Imager has large (up to $0.7 \mathrm{~K}$ ) diurnally variable biases which peak around local midnight. We also show that the noise characteristics of the imager are time variable, both due to periodic gain changes as well as a slow degradation that is particularly evident in the $12 \mu \mathrm{m}$ channel. We then go on to discuss the far infrared sensors flown onboard the AVHRR and show two sources of bias - a scene temperature dependent bias which is seen in all AVHRR sensors as well as a large time dependent bias seen particularly in NOAA-16. We show that a new calibration scheme can, in theory, correct for both biases and should reduce SST biases from potentially large values to a reasonable level.
\end{abstract}

\section{Introduction}

Having well calibrated infrared sensors which are stable and have only negligible biases is central to providing accurate SSTs. Unfortunately, many of the standard infrared sensors used for SST generation are plagued with calibration problems and can show large biases. Recent work has shown that two of the NOAA infrared imagers, the GOES Imager and the AVHRR, both show significant problems. The GOES Imager seems to have a large, diurnally varying bias and the AVHRR has strong, scene temperature dependent trends together with large time dependent biases that are seen in some AVHRR sensors.

In this paper we present some of the work that is on-going to investigate the calibration issues for two particular sensors. In the case of the GOES Imager we discuss some of the initial studies regarding the diurnally variable bias which are based on comparisons with top-ofatmosphere radiance sources and also show the longterm variation of the GOES Imager $\mathrm{Ne} \Delta \mathrm{T}$ for the infrared channels. We also briefly discuss future work to try and ameliorate the problems. For the AVHRR we discuss the scene temperature bias seen with the operational calibration and discuss the impact of a new calibration methodology on this bias. We then move onto the AVHRR flown onboard NOAA-16 that is known to have serious problems in the current epoch (see for example its derived SST e.g. Dash et al. 2010). We show that by taking into account the changes in NOAA-16s thermal state and by using a new physically based calibration we can, at least in theory, remove much of the time variable biases seen in its derived SST and make the NOAA-16 AVHRR a usable instrument over its complete lifetime.

\section{The GOES Imager}

The first instrument we consider is the GOES-11 Imager. The GOES Imager is a 3 axis stabilized geostationary sensor and currently is located at $135^{\circ} \mathrm{W}$. It is calibrated using an on-board blackbody and the signal is clamped such that the recorded counts are electronically clamped to the signal seen when the detector is observing space. The gain is measured between observations (approximately every half hour) by observing the on-board blackbody, and the zeroth order bias term of the calibration is determined every pixel by interpolation. For full details of the standard calibration, see Weinreb et al. (1997). 
On top of the standard calibration discussed above, one other effect dominates the calibration of the GOES Imager. This is the so-called Midnight Blackbody Calibration Correction (MBCC), which is where the calibration system is contaminated by stray light (presumed to be from a heated sun shield) which is seen around the time of local midnight. This gives rise to an underestimate of the gain and leads (in the case of the $3.9 \mu \mathrm{m}$ channel) to an underestimate in the brightness temperature of order $1 \mathrm{~K}$. Operationally, there is a correction algorithm that is applied when the observed gain deviates from a prediction of the gain that is based on a simple model of the gain behavior that correlates the responsivity (defined as $\left(m+2 \gamma C_{B B}\right)^{-1}$ where $m$ is the gain, $\gamma$ is the non-linear coefficient and $C_{B B}$ is the blackbody counts) with the primary mirror temperature (see Weinreb \& Han 2003). Until recently it had been assumed that the MBCC only affected the $3.9 \mu \mathrm{m}$ channel and that the 11 and $12 \mu \mathrm{m}$ channels would not show any effect, but new results have shown that this is not the case and that, in fact, a similar problem exists in the longer wavelength channels (e.g. Mittaz \& Harris 2008). The bias introduced is of the order of (for the $11 \mu \mathrm{m}$ channel) approximately -0.7K. Recent work using GSICS (Global Space-based Inter-Comparison System) finds similar biases (see $\mathrm{Yu}$ et al. 2011 and Mundakkara et al. 2011) and also tentatively shows significant seasonal biases. From the point of view of retrieving SSTs the fact that the GOES Imager shows such large biases that have both a large diurnal signal as well as a proposed large seasonal effect must be taken into account for accurate SST retrievals. On the calibration side, studies indicate that at least the diurnal part of the bias may be reduced using a different MBCC scheme where the square of the gain $\left(\mathrm{m}^{2}\right)$ is related to the primary mirror temperature rather than the responsivity, but this has yet to be validated.

One other sensor effect has also been studied, which is the time behavior of the GOES Ne $\Delta \mathrm{T}$. In general the GOES Ne $\Delta \mathrm{T}$ has been determined near to launch and is not studied further. The actual $\mathrm{Ne} \Delta \mathrm{T}$, however, shows significant time variability. Figure 1 shows the $\mathrm{Ne} \Delta \mathrm{T}$ determined over a 3 year period for GOES-11 and shows two major effects. The first is the change in the $\mathrm{Ne} \Delta \mathrm{T}$ when the instrument gain is changed. Such gain changes are standard over a number of different geostationary satellites but as can be seen in Figure 1 have a big effect on the $\mathrm{Ne} \Delta \mathrm{T}$. The second effect is a clear degradation in the noise on the longer timescale that is especially obvious in the $12 \mu \mathrm{m}$ channel. Such gain variations need to be taken into account when retrieving SSTs particularly when using physical retrieval methods.

\section{The AVHRR}

Recent work has shown that the current calibration of the AVHRR is significantly biased. Comparisons with IASI have shown scene temperature dependencies of up to $0.5 \mathrm{~K}$ (see for example Wang \& Cao 2008) and a detailed study of the prelaunch calibration has shown that the prelaunch test data was severely compromised with straylight issues and 


\section{GHRSST Project Office}

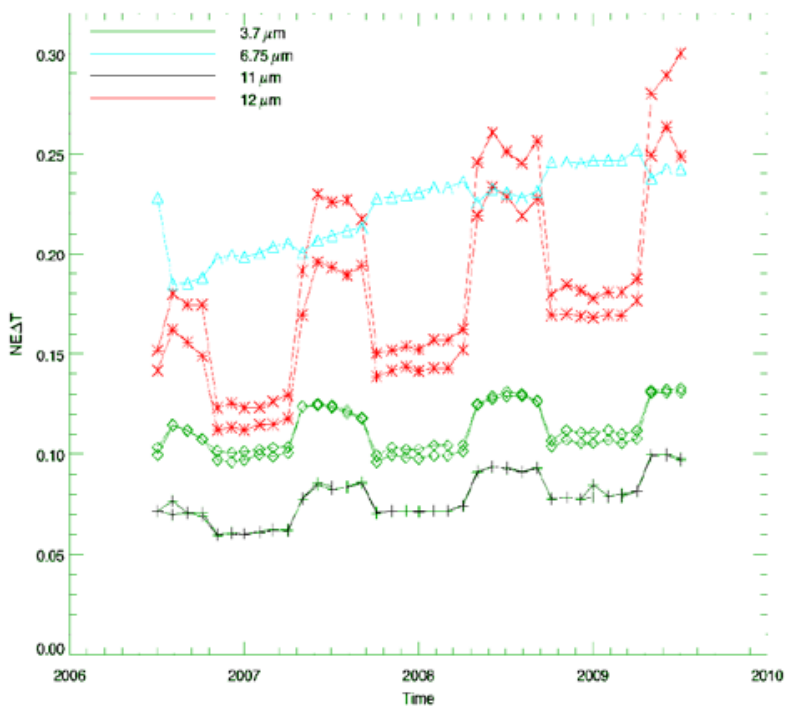

Figure 1. Variations in the NeAT for GOES-11 for a 3 year period showing significant Ne $\Delta T$ variations both due to gain changes in the instrument, as well as longer term degradations seen particularly in the 12 um channel

poor test procedures (Mittaz et al. 2009). A new calibration was derived in Mittaz et al. and this calibration has been used to study the MetOP-A AVHRR by comparing with IASI. This work has shown that in order to derive a good AVHRR calibration you must reparameterize the calibration to take into account the difference in the satellite environment between the prelaunch test chamber and the in-orbit conditions (see Mittaz \& Harris 2011 for details). This reparameterization must, of course, be done using accurate top of atmosphere radiance sources, which in this case was IASI. The Mittaz \& Harris work also shows that the AVHRR is capable of being an accurate instrument and can achieve an accuracy better than $0.1 \mathrm{~K}$ and a stability of better than $0.08 \mathrm{~K} /$ decade, at least in the case where the instrument temperature is also very stable.

While the case of the MetOp-A AVHRR shows the promise of a near climate ready AVHRR data record, the results found in the AVHRR/IASI comparison do not tell the whole story. It is, for example, known that the AVHRR flown on-board NOAA-16 show significant biases which give rise to large biases in the derived SST (see for example the SST comparisons shown on the SQUAM webpage, Dash et al. 2010). We have therefore studied in some detail the behavior of the NOAA-16 AVHRR by comparing the AVHRR with the AATSR, a satellite designed to provide radiances at a climate level of accuracy. What is found is that at the beginning of the mission (when the instrument temperature was stable) the AVHRR calibration is also stable and can be re-derived to give much improved radiances. When, however, we study times when the instrument temperature is variable, we start seeing biases in the radiances even though we are using the new calibration based on Mittaz et al. (2009) and Mittaz et al. (2011). In fact the size of the bias seems to correlate with the instrument temperature and Figure 2 shows just such a correlation for the 11 and $12 \mu \mathrm{m}$ channels. While there is a lot of noise shown in Figure 2 it is apparent that the average bias for temperatures between 288.0 and $288.5 \mathrm{~K}$ and for temperatures between 292 and 298K has changed. 


\section{GHRSST Project Office}
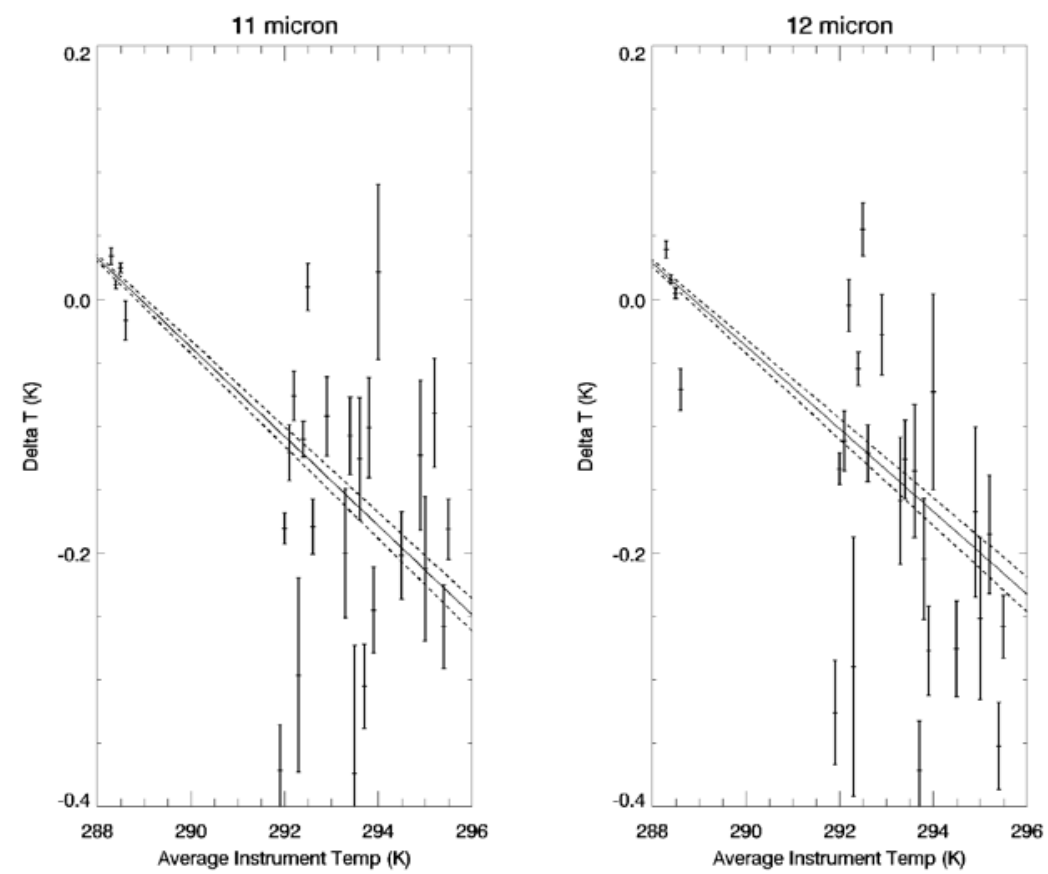

Figure 2. Shows the bias of the recalibrated NOAA-16 AVHRR relative to the AATSR plotted as a function of the AVHRR average orbital temperature. Also shown is a linear model fitted to the correlation.

As a first attempt we have fitted a simple linear model to the bias that is simply a function of instrument temperature (in this case taken to be the average orbital temperature) and have used this

$$
R=\left(a+b \times T_{\text {Instr }}\right)+\text { Gain } \times\left(C_{S}-C\right)+\gamma\left(C_{S}-C\right)^{2}
$$

where $T_{\text {Instr }}$ in the average orbital temperature, Gain is the derived gain and $\gamma$ is the non-linear term. As of now we have not been able to make a detailed assessment of any possible improvement that may be obtained by using Equation 1 but there is evidence of an improvement in calibration. Figure 3 shows a comparison of the new time variable calibration with MICROS data (for details on MICROS see Liang \& Ignatov 2010) for the $11 \mu \mathrm{m}$ channel. Since MICROS shows the difference between the AVHRR radiances and RTM modeled radiances, we have overplotted the estimated BT error derived by estimating what the effect of the instrument temperature dependence is correlation to try and correct the calibration. In essence we add a temperature dependent term to the calibration equation, giving (assuming a single scene temperature of 290K). As can be seen in Figure 3, the match is really quite good, and may of the features seen in the MICROS data can be explained by the bias in the radiances introduced by ignoring the time variable component of the calibration. More work, of course, needs to be done to work out what is occurring when the curves do not match and there are many possible sources of error beyond calibration ones including biases in the MICROS data itself such as RTM biases, NWP biases, effect of aerosol, interpolation errors and the fact that the current MICROS uses the operational calibration not the new Mittaz et al. calibration. 


\section{GHRSST Project Office}

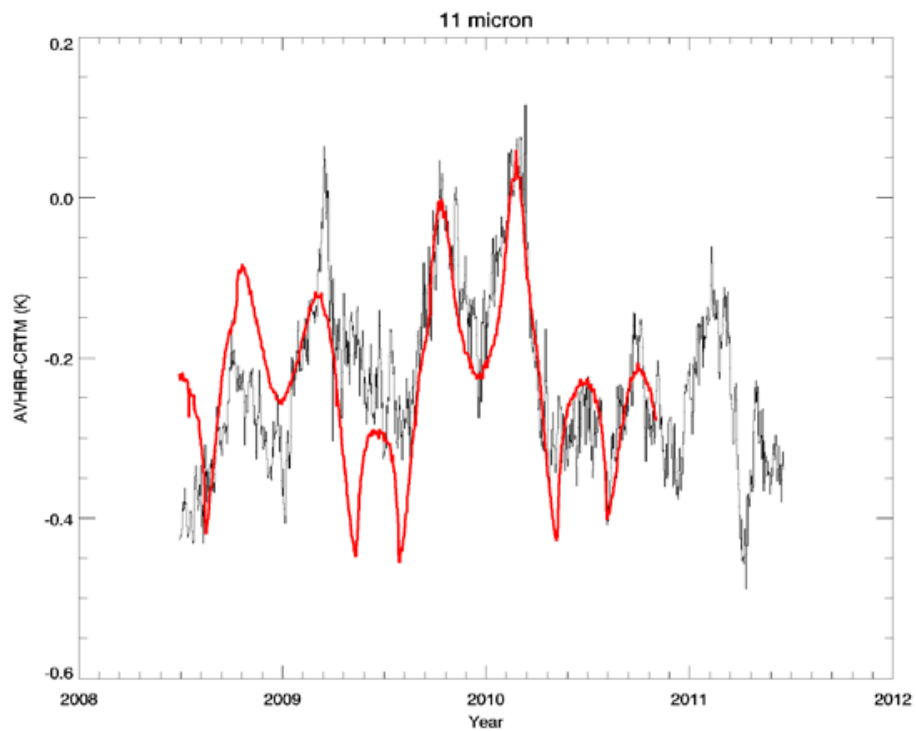

Figure 3. A comparison between MICROS AVHRR-RTM biases and the biases predicted from the new calibration. The black line is the data taken from the MICROS web site and the red line is the estimated bias based on the new calibration where the two curves have been renormalized to each other (it is expected that there are extra biases associated with the MICROS data). At certain times the agreement is very good with very similar time variability.

Finally, Figure 4 shows estimates of the 11 and $12 \mu \mathrm{m}$ channel biases as well as the $11-12 \mu \mathrm{m}$ bias that are introduced by not including a time variable component in the calibration. This plot can be used to estimate what impact such biases will have on SST retrieval. While it is not possible to show a direct comparison between SSTs derived using the old and new calibrations in this paper, work done by the Pathfinder team on Pathfinder V6 indicate that time variable SST biases closely match the channel biases shown in Figure 4. For an example of the NOAA-16 SST biases see Evans et al. (2011) and for an estimate of a comparison between the Evans et al. work and this new NOAA16 calibration see the talk associated with this paper.

\section{Conclusion}

We have shown that both Geostationary and Polar satellites currently have significant biases in their derived radiances. In the case of GOES-11 there is evidence for a $-0.7 \mathrm{~K}$ diurnally variable bias that is likely related to the Midnight Blackbody effect. GSICS work is also indicating that there are seasonal effects as well. Unless any SST retrieval algorithm takes such time variable biases into account (by, for example, using an hour by hour radiance bias scheme), these signals will persist into the SST data record. We have also shown that care must be taken when dealing with the GOES Ne $\Delta T$ since it is also time variable. In the case of the AVHRR sensors a new calibration is now becoming available which removes scene temperature dependencies but also, with the addition of a time dependent calibration, may also be able to remove apparent large time variable biases, at least in the case of NOAA-16. In terms of SST retrievals, the AVHRR the scene dependent bias in the current operational calibration may not be too much of an issue (regression will effectively remove the effect, and a simple radiance bias scheme can help with physical retrieval schemes). The time dependent error is more problematic, especially when the bias rapidly changes. The new time dependent AVHRR calibration will hopefully be able to remove this effect and enable the use of currently problematic AVHRR sensors for SST retrievals. 


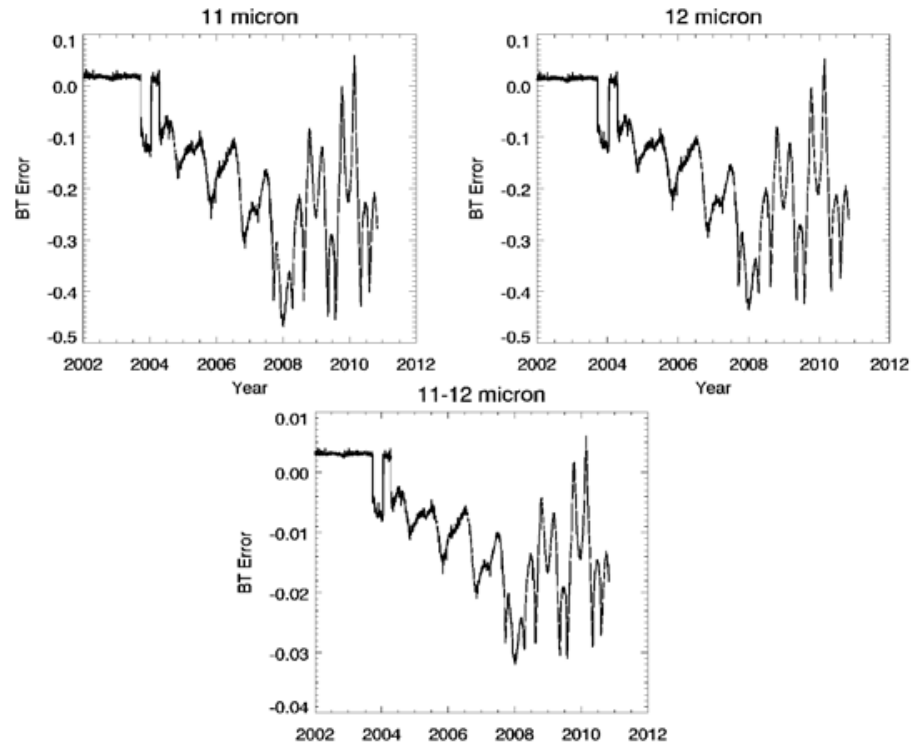

Figure 4. Estimated channel biases caused by not taking into account the time variable bias in the calibration for the 11 and $12 \mu \mathrm{m}$ channel.

\section{References}

Dash, P., Ignatov, A, Kihai, Y. and J. Sapper, 2010, The SST Quality Monitor (SQUAM). J. Atmos. Oceanic Technol., 27, 1899-1917

Evans, R \& G. Podesta, 2011, Characterizing and comparison of uncertainty in the AVHRR Pathfinder Versions 5 \& 6

SST field to various reference fields, DVHL-STVAL workshop 2011, Boulder, CO, 28 Feb. - 2 Mar 2011, https://www.ghrsst.org/files/download.ph $p ? m=$ documents \&f $=110407144753$ STVAL09EvansPathfinder.ppt

Liang, $X$ \& A. Ignatov, Monitoring of IR Clear-sky Radiances over Oceans for SST (MICROS), JTECH, 2011, doi: 10.1175/JTECH-D-10-05023.1

Mittaz, J. P. D. and A. R. Harris, 2008. Using AATSR as radiance calibration reference for NOAA satellites, Proceeding of $2^{\text {nd }}$ MERIS/(A)ATSR Users Workshop, H. Lacoste \& L. Ouwehand (Eds.) ESA SP-666, paper 859, Sept 2226 , in Frascati, Italy.
Mittaz, J.P.D. \& A. R. Harris, 2011. A Physical Calibration of the AVHRR/3 2: An in-orbit comparison of the AVHRR longwave thermal IR channels on-board MetOp-A with the IASI instrument, $J$. Atm.Ocean.Tech., in press.

Mittaz, J. P. D., Harris, A. R. \& J. T. Sullivan, 2009. A Physical Method for the Calibration of the AVHRR/3 Thermal IR Channels 1: The Prelaunch Calibration Data, J. Atm.Ocean.Tech., Vol. 26, pp 996-1019.

Mundakkara, R. X. Wu, F. Yu, L. Wang and G. Rancic, 2011. Properties of night time and day time calibration anomalies in infrared imaging channels of operational GOES-11 and GOES-12 satellite using Global Space Based Inter-Calibration System (GSICS), J. Atm. Ocean. Tech., to be submitted.

Weinreb, M., and D. Han, 2003. http://www.oso.noaa.gov/goes/goescalibration/blackbody/index.htm

Weinreb. M, M. Jamieson, N. Fulton, Y. Chen, J. Johnson, J. Bremer, C. Smith and J. Baucom, 1997. Operational calibration of Geostationary Operational Environmental Satellite-8 and -9 imagers 
GHRSST XII -

Proceedings, Edinburgh

GHRSST Project Office

and sounders, Applied Optics, vol 36(27), pp 6895-6904.

Yu, F., X. Wu, R. Mundakkara, L. Wang, Y. Li and M. Goldberg, 2011. Evaluation of
Issue: Final

Date: September 27, 2011

the diurnal and scan angle calibration variations of GOES Imager infrared data, IEEE on Geosciences and Remote Sensing, to be submitted. 
EUMETSAT SUPPORT TO GHRSST

\title{
Anne O'Carroll
}

\author{
EUMETSAT, Eumetsat-allee 1, 64295 Darmstadt (Germany), \\ Email : Anne.Ocarroll@eumetsat.int
}

The main purpose of the European Organisation for the Exploitation of Meteorological Satellites (EUMETSAT) is to deliver operational weather and climaterelated satellite data, images and products throughout all day and year. EUMETSAT also has commitments to operational oceanography and atmospheric composition monitoring. Activities over the next twenty years include the continuation of the Mandatory Programmes (MSG, EPS) and future (MTG, EPS-SG), which all include ocean observations of SST and sea surface winds. The continuation of the EUMETSAT Ocean Surface Topography Mapping optional programme (Jason-3 and beyond) will contribute an uninterrupted sea level rise monitoring data set. EUMETSAT is participating in other key ocean observation programmes such as GMES Sentinel-3, where EUMETSAT will operate the satellite and will serve the marine user community. Work towards access to relevant data from third-parties with the preparation of agreements with ISRO and SOA, will give EUMETSAT access to an enhanced ocean products catalogue. EUMETSAT supervises and coordinates its Satellite Application Facility (SAF) network. There are currently eight SAFs. The EUMETSAT Ocean and Sea-ice SAF is lead by Meteo-France with a consortium of institutes from EUMETSAT member states, and provides reliable and timely operational services related to meteorology, oceanography and the marine environment. EUMETSAT's Climate Monitoring Implementation Plan will also be discussed. 


\title{
DEVELOPMENT AND EVALUATION OF SEA SURFACE TEMPERATURE ALGORITHMS FOR GOES-R ABI USING MSG SEVIRI AS A PROXY
}

\author{
Boris Petrenko $^{(1)}$, Alex Ignatov ${ }^{(2)}$, Nikolay Shabanov ${ }^{(3)}$, Yury Kihai ${ }^{(4)}$ \\ ${ }^{(1)}$ NOAA/NESDIS/STAR/IMSG, 5200 Auth Road, Camp Springs, MD. 20746, USA; \\ boris.petrenko@noaa.gov \\ ${ }^{(2)}$ NOAA/NESDIS/STAR, 5200 Auth Road, Camp Springs, MD. 20746, USA; \\ alex.ignatov@noaa.gov \\ ${ }^{(3)}$ NOAA/NESDIS/STAR/IMSG, 5200 Auth Road, Camp Springs, MD. 20746, USA; \\ nikolay.shabanov@noaa.gov \\ ${ }^{(4)}$ NOAA/NESDIS/STAR/Dell Perot Systems, 5200 Auth Road, Camp Springs, MD. 20746, USA; \\ yury.kihai@noaa.gov
}

\begin{abstract}
Retrieval of sea surface temperature (SST) from geostationary platforms such as Meteosat Second Generation (MSG) and Geostationary Operational Environmental Satellites (GOES) benefits from continuous observations of a vast ocean area through the full diurnal cycle. SST will be one of the key products of the Advanced Baseline Imager (ABI) scheduled for launch in late 2014 onboard the new generation GOES-R series. Within the framework of preparations for the GOES-R ABI mission, a study was undertaken to select the SST algorithm, which provides the highest, and the most uniform SST accuracy and precision within the area observed by a geostationary sensor. Split-window channels of MSG Spinning Enhanced Visible and Infrared Imager (SEVIRI) were used in this study as a proxy for GOES-R ABI. The previously known algorithms, such as the Non-Linear Regression (NLR) and Optimal Estimation (OE) were implemented along with two newly developed algorithms, Incremental Regression (IR) and Corrected Non-Linear Regression (CNLR). OE, IR and CNLR adopted the first guesses for SST and brightness temperatures (BT) from,
\end{abstract}

respectively, Reynolds Daily HighResolution Blended SST and simulations with the Community Radiative transfer Model (CRTM). Consequently, the goal of these algorithms is to retrieve deviations of SST from the first guess (increments). OE retrieved SST increments with inversion of the radiative transfer model, whereas CNLR and IR used incremental form of NLR equation. The difference between CNLR and IR is that CNLR adopts NLR coefficients whereas IR uses coefficients optimized for incremental retrieval. Accuracy and precision of retrieved SST were evaluated by comparison to drifters' SST. The major observations from this study are as follows: 1) all algorithms adopting first guesses for SST and BTs are capable of improving SST accuracy and precision over NLR; and 2) Incremental Regression delivered the highest global SST precision and the most uniform distributions of regional SST accuracy and precision. Implementation and validation issues are addressed, including bias correction in simulated BT, preserving sensitivity of incremental SST algorithms to true SST variations, and selection of criteria for incremental algorithms' optimization and validation. 


\section{MEDSPIRATION LATEST DEVELOPMENTS} Jean-François Piollé ${ }^{(1)}$, Emmanuelle Autret ${ }^{(2)}$, Olivier Arino( ${ }^{(3)}$

(1) Ifremer / CERSAT, 29280 Plouzané (France), Email : jfpiolle@ifremer.fr (2) Ifremer / CERSAT, 29280 Plouzané (France), Email : emmanuelle.autret@ifremer.fr

(3)Olivier Arino, ESA / ESRIN, Frascati (Italy), Email: olivier.arino@esa.int

The Medspiration Project is a European initiative, funded by ESA (in the frame of DUE program), to combine sea surface temperature (SST) data measured independently by several different satellite systems into a set of data products that represent the best measure of SST, presented in a form that can be assimilated into ocean forecasting models or used for various kinds of application. It has pioneered the implementation of operational services for SST following GHRSST project recommendation and standards.

Medspiration is now releasing a new line of high resolution regional SST maps* over several predefined areas, namely Mediterranean sea, Brazil, South-Africa and Great Barrier Reef in Australia. These products are now available http://www.medspiration.org and updated on a daily basis, and a full reprocessing over Medspiration era (2005 to today) has been undertaken for the Mediterranean sea product. The presentation will cover these new achievements as well as some of the latest SST related activities ongoing at Ifremer/Cersat. 


\title{
SST PROFILES RECORDED BY UN-PUMPED NEAR-SURFACE ARGO MEASUREMENTS
}

\author{
Sarah Quinn $^{(1)}$, Justin Buck ${ }^{(2)}$, Jon Turton ${ }^{(3)}$, Andrea Kaiser-Weiss ${ }^{(4)}$
}

(1) University of Reading, Email: Sarah.Quinn@student.reading.ac.uk

(2) BODC , Email: ibuck@bodc.ac.uk

(3) Met Office, Email: jon.turton@metoffice.gov.uk

(4) GHRSST, Email: a.k.kaiserweiss@reading.ac.uk

\begin{abstract}
2,800 temperature profiles from nearsurface un-pumped Argo profiles by British Oceanographic Data Centre (BODC), Indian National Centre for Ocean Information Services (INCOIS) and University of Washington (UW)) were analysed for diurnal warming events. 62 cases were identified to belong to diurnal warming events, i.e., $6 \%$ of all Argo near surface measurements. The temperature is sampled every 6 seconds, i.e., giving typically 8 measurement points between $10 \mathrm{~m}$ depth and the surface. The pressure and depth error is analysed and thus the information content of the temperature profile is reviewed. Examples of interesting temperature Argo un-pumped profiles are discussed and compared to the respective satellite measurements.
\end{abstract}

\section{Introduction}

Accurate knowledge of the global sea surface temperature distribution and its temporal variation is a key input to forecasting and prediction systems to constrain the modelled upper-ocean circulation and thermal structure at daily, seasonal, decadal and climate timescales, for the exchange of energy between the ocean and atmosphere in coupled oceanatmosphere models and as boundary conditions for ocean forecasting models. The temperature gradients near the ocean surface are critical to heat exchange with the atmosphere and yet these gradients are very difficult to measure and are also very often poorly represented, even in the best ocean and climate models.
Argo near-surface measurements from British Oceanographic Data Centre (BODC), Indian National Centre for Ocean Information Services (INCOIS) and University of Washington (UW) were analysed. Temperature measurements are taken every 6 seconds for the upper part of the ocean i.e. between $10 \mathrm{dbar}$ and $0 \mathrm{dbar}$ difference to the surface, this corresponds to the first meter below the surface. Diurnal variability, for example in the Tropics, is very significant with up to several ${ }^{\circ} \mathrm{C}$ temperature change, which is delivered by solar irradiation and wind speed.

In this paper the initial study of the 2,800 profiles, paying particular attention to a few interesting profiles that drift from our normal expected results are presented. It will outline the procedure of locating the surface, the errors associated with the research, and initial summary of the results found.

\section{Procedure to find surface}

In order to find out when the float broke the surface we applied the following procedure. Then the pressure values were examined and we know the float ascends and takes readings every 6 seconds which approximately translates to $0.6 \mathrm{dbar}$. Therefore, it was identified where the pressure change between two points was less than $0.5 \mathrm{dbar}$ and in between these two would be the surface, as this would imply that the float has stopped ascending.

\section{Errors}

\subsection{Pressure errors}


The biggest uncertainty is the vertical location or the pressure measurements. All the floats of the near surface sampling type transmit the pressure recorded by the sensor at the surface before descending for a new profile, this pressure is usually used as the pressure bias for that profile but in this study we used a different method for the pressure bias. Having located the surface, all the remainder pressure readings after the surface were averaged and this was used as the new pressure offset for that profile. From the surface identification procedure, the profile error is $<0.6 \mathrm{dbar}$.

\subsection{Temperature errors}

The temperature accuracy requirement for Argo floats is $0.005{ }^{\circ} \mathrm{C}$ and from recalibration of the instruments after a period of a few months to a few years has shown that the instruments tend not to drift from this accuracy (Oka, 2004). Therefore, it seems that the main error with regards the temperature is to do with the time response of the thermometer rather than the actual accuracy of the thermometer. It depends on the model of the float but the time response may vary by $+/-10 \%$ and is approximately 0.6 seconds, which would relate to profile location error of 0.06 dbar, again significantly smaller than the pressure error (Johnson et al, 2006).

\section{Interesting profiles}

From Figure 1 it can be seen that the most significant temperature difference seems to be concentrated around southern hemisphere mid latitudes. It would have been expected to see more floats with large temperature difference around the Tropics, and in this graph it is not the case, although it must be noted that this is not due to there not being a large temperature difference in the last few meters but due to the lack of un-pumped profiles in these areas.

Furthermore, the comparison of maximum temperature difference at surface and local time (Fig 2) shows that the majority of the maximum temperature difference is seen in the afternoon. Although there are a few ascents that have show large temperature change late at night and early morning.

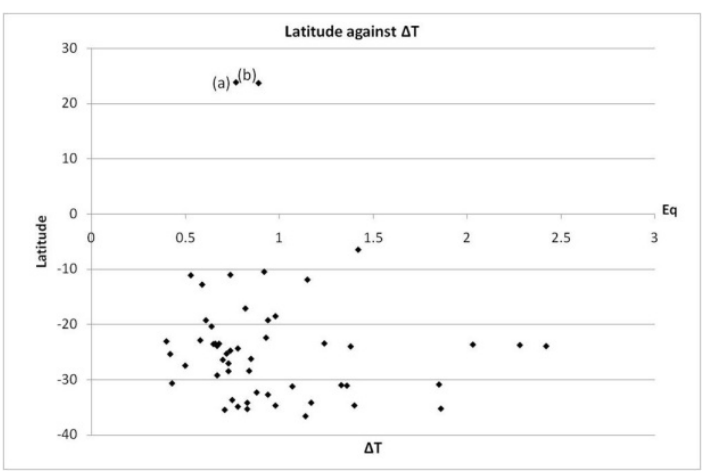

Figure 1. Temperature difference $\left({ }^{\circ} \mathrm{C}\right)$ of 62 profiles with reference to their location of latitude.

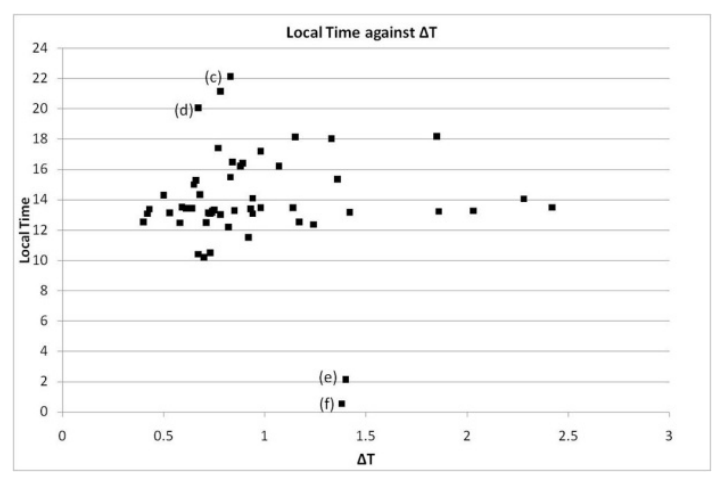

Figure 2. Temperature difference $\left({ }^{\circ} \mathrm{C}\right)$ of profiles with reference to the local time of their surfacing. 


\section{Conclusions}

From the 2,800 Argo near-surface profiles examined, recorded between October 2008 and May 2011, 62 of the profiles showed significant temperature difference in the top $1 \mathrm{~m}$ of the ocean and reach to a $2.4{ }^{\circ} \mathrm{C}$ difference for some profiles. There are some interesting cases identified of floats surfacing in the early hours of the morning and late at night showing a significant temperature difference which is unusual as the diurnal variability would be expected to be most significant or noticeable in the afternoon. Some interesting profiles have been identified illustrating that the mixed layer extends to depths between 2 and
8 dbar. An increase in coverage of Argo near-surface measurements would be desirable as the accuracy allows one to capture diurnal variability and adds valuable sub-surface information.

\section{References}

Johnson, $G$ et al. 'Sensor Corrections for Sea-Bird SBE-41CP and SBE-41 CTDs', American Meteorological Society. 28, 1117 - 1128, 2007.

Oka, E \& Kentaro, A. 'Stability of Temperature and Conductivity Sensors of Argo Profiling Floats', Journal of Oceanography. 60, 253-258, 2004. 


\title{
USER REQUIREMENTS FOR SST REANALYSIS.
}

\author{
Nick A Rayner ${ }^{(1)}$ and Simon A. Good ${ }^{(2)}$ \\ (1)Met Office Hadley Centre, Exeter (U.K.), Email : nick.rayner@metoffice.gov.uk \\ (2) Met Office Hadley Centre, Exeter (U.K.), Email : simon.good@metoffice.gov.uk
}

\begin{abstract}
Climate research comprises a diverse group of activities, but most of these need information on sea surface temperature (SST), either globally or regionally. However, the specific SST needs of these diverse activities can vary widely.

Before developing SST products, it is useful to perform an assessment of the requirements of the target user community to best ensure take-up of the resulting products, through appropriate product design.
\end{abstract}

\section{Introduction}

The European Space Agency (ESA) Climate Change Initiative (CCl) Sea Surface Temperature (SST) project, SST_cci, aims to improve SST satellite data records to meet the requirements of the climate research community. In preparation for the development of the SST_cci products, we asked several hundred climate scientists to think ahead five years to their future requirements for SST data.

Requirements were gathered from climate research users via four methods:

- a literature review of relevant documents from bodies such as the Global Climate Observing System (GCOS),

- review of lessons learned information provided by other projects,

- a questionnaire, which asked about currently available SST data, and future needs for SST data, five years from now,

- discussion sessions.

Based on the responses of over 100 of these scientists, we performed a user requirements analysis. This shows that their requirements are very varied. Even people performing similar research state different requirements. Nonetheless, we were able to analyse their responses for common themes and use this to specify products which we felt would meet the majority of needs, where possible.

Here, we show a selection of their stated requirements and explain some of the most stretching of these with examples of uses of SST data within climate research which push the boundaries of what is currently available.

For some of the questions, respondents were asked for three levels of requirements:

- Threshold: the limit at which the observation becomes ineffectual and is no use for the application.

- Breakthrough: the level at which a significant improvement in this application would be achieved.

- Objective: the maximum performance limit for the observation, beyond which no significant improvement in the application would be achieved.

We focus on five key areas: length of record; temporal resolution; frequency of data delivery; acceptable levels of drift; and provision of information about uncertainties.

The full user requirements document runs to 128 pages and the reader is referred to that for a full presentation of requirements (see http://www.esa-sst-cci.org ).

\section{Length of record}

We start by discussing the need for a multi-decadal record.

The bar charts in Figure 1 and subsequent figures show the multiple choice responses received to a particular question from the requirements questionnaire. They are not split by data level, but are colour-coded according to 
the primary research area of the

Threshold requirements
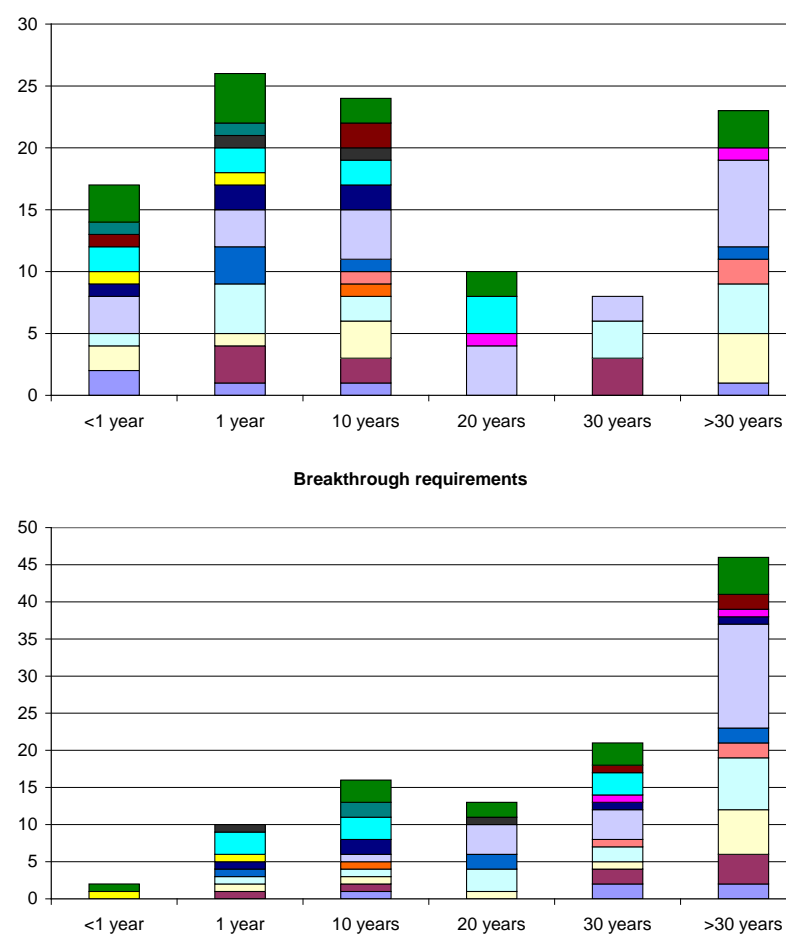

respondent.

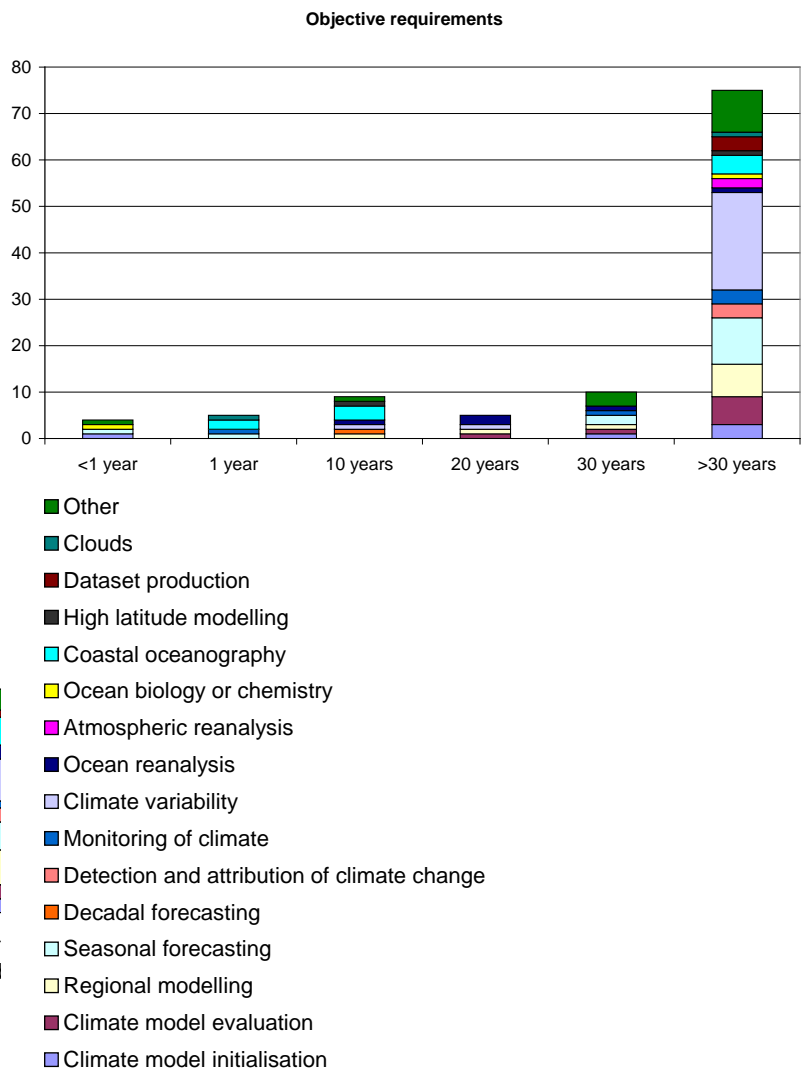

Figure 1. Threshold, breakthrough and objective requirements for temporal coverage (see User Requirements Document).

Values in tables come from an analysis of the results, often stratified by data level, to assess the level at which at least two thirds of the respondents would be satisfied. This analysis is written up separately as a technical note to the Product Specification Document (available on request from the authors).

\begin{tabular}{|l|l|l|l|}
\hline Data level & Threshold & Breakthrough & Objective \\
\hline 2 & 10 years & 20 years & $>30$ years \\
\hline 3 & 20 years & $>30$ years & $>30$ years \\
\hline 4 & 20 years & $>30$ years & $>30$ years \\
\hline
\end{tabular}

Table 1. Temporal coverage which would satisfy at least two thirds of the user group at the threshold, breakthrough and objective levels (see Technical Note to Product Specification Document).

Opinion is divided as to the length of record that is necessary to have a data set that is in any way useful (Figure 1). But when we examine the breakthrough and objective requirements, it is clear that the majority of users need records of greater than 30 years' length.

This is because the climate system comprises many interlinked oscillations, as well as being externally forced. For example, Knight et al. (2005) discuss the Atlantic Multi-decadal Oscillation, a surface manifestation of thermohaline circulation changes, which appears to have a period of about 70 years (Figure 2). Without multi-decadal climate records, these components of the climate system cannot be understood and used to inform our projections of decadal and longer-term changes. 

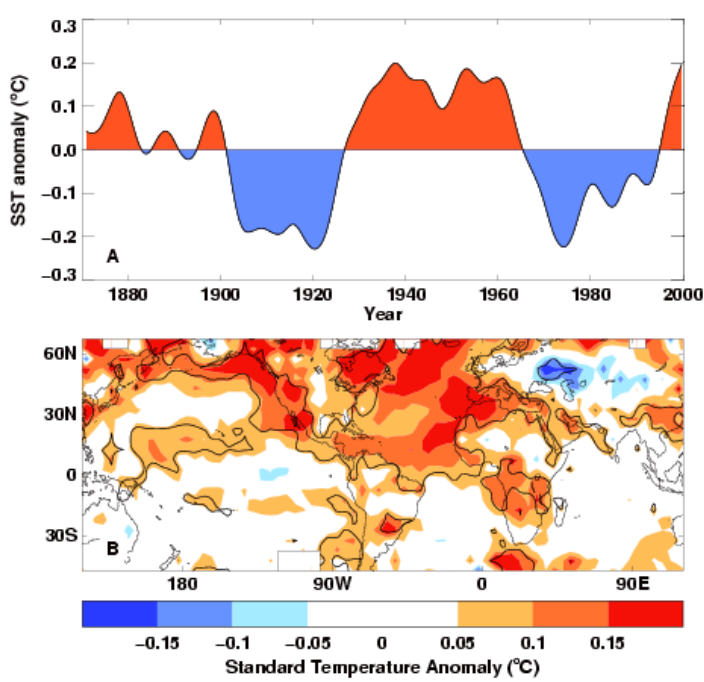

Figure 2. A. AMO index derived from detrended area-weighted mean North Atlantic SST anomalies by using a Chebyshev filter with a half-power period of 13.3 years. SST data are from the HadISST data set [Rayner et al., 2003]. B. Surface temperature anomaly associated with one positive standard deviation of the AMO index, calculated by regression of surface temperatures with the index and scaled by its standard deviation. Combined land and sea-surface temperature data are from an optimally interpolated version of the HadCRUTV data set [Jones et al., 2001]. The solid contour bounds regions significant at the $90 \%$ limit of a two-sided t-test accounting for auto-correlation using the method of Folland et al. [1991]. From Knight et al. (2005)

\section{Temporal resolution}

When we look at the threshold requirements (Figure 3), we get a fairly traditional picture of users' minimum

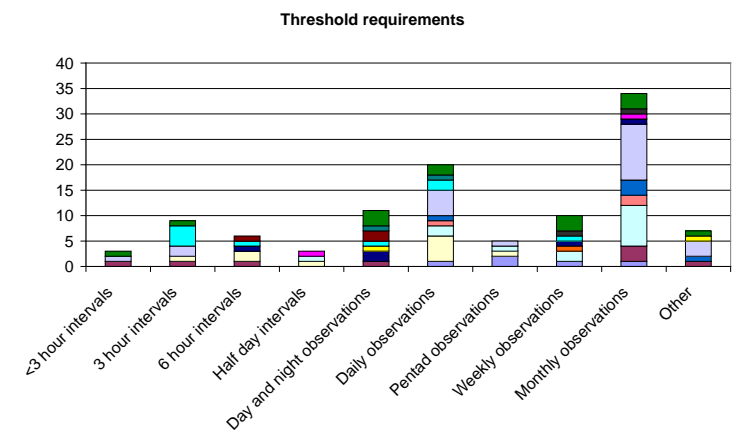

Breakthrough requirements

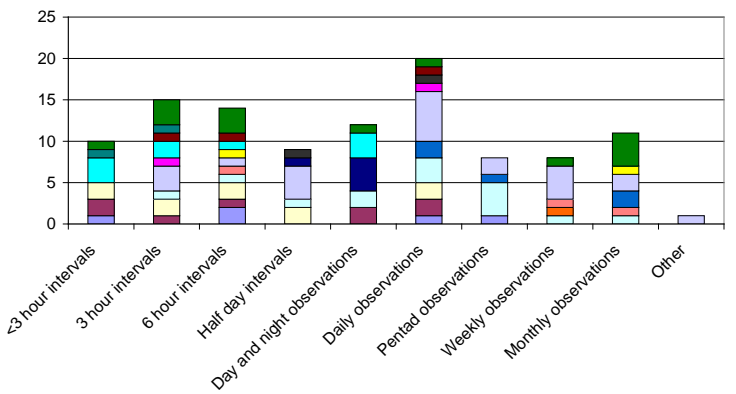

requirements for temporal resolution, with monthly data being the most commonly selected response, but with a significant minority needing daily data

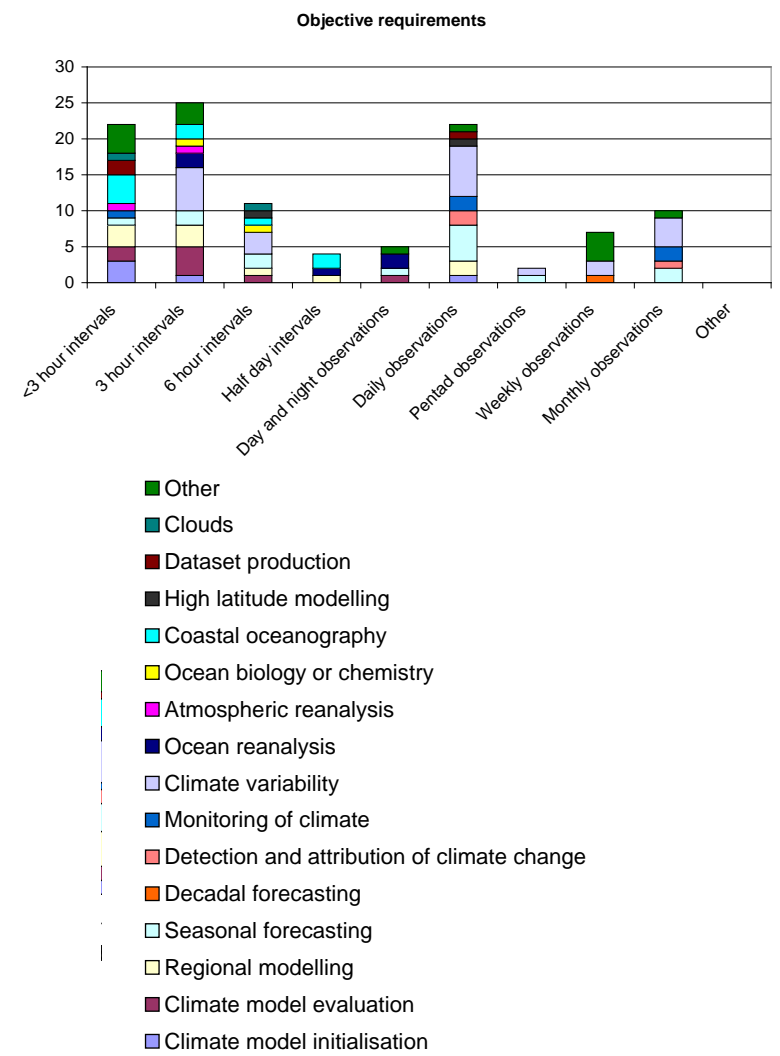

Figure 3. Threshold, breakthrough and objective requirements for frequency of SSTs at a location (see User Requirements Document). 
But those requiring daily data to gain a significant improvement from the SST become most common, with large numbers of potential users requiring subdaily resolution. Three-hourly becomes the most common response at the objective level.

But why does that matter to climate research?

In Figure 4, we show results from Minobe et al. (2008), who explored the effect of the Gulf Stream on tropospheric circulation in European Center for Medium-range Weather Forecasts (ECMWF) operational analyses. They proposed that the Gulf Stream results in deep convection during the day up to $300 \mathrm{hPa}$, which follows the warm edge of the Gulf Stream. This deep convection is proposed to then affect the circulation of the northern hemisphere through the generation of planetary waves. They found that better representation of the high SST gradients in this region improved the simulation of the deep circulation. However, in other areas of the world, deep convection is thought to be modulated by the diurnal cycle in SST, so requiring high temporal resolution as well as high spatial resolution information. Simulating the complex interaction between the ocean and the atmosphere correctly in a climate model, either driven by observed SST or coupled to an ocean model verified by high resolution SST, may be key to improving the accuracy of simulations of the climate.

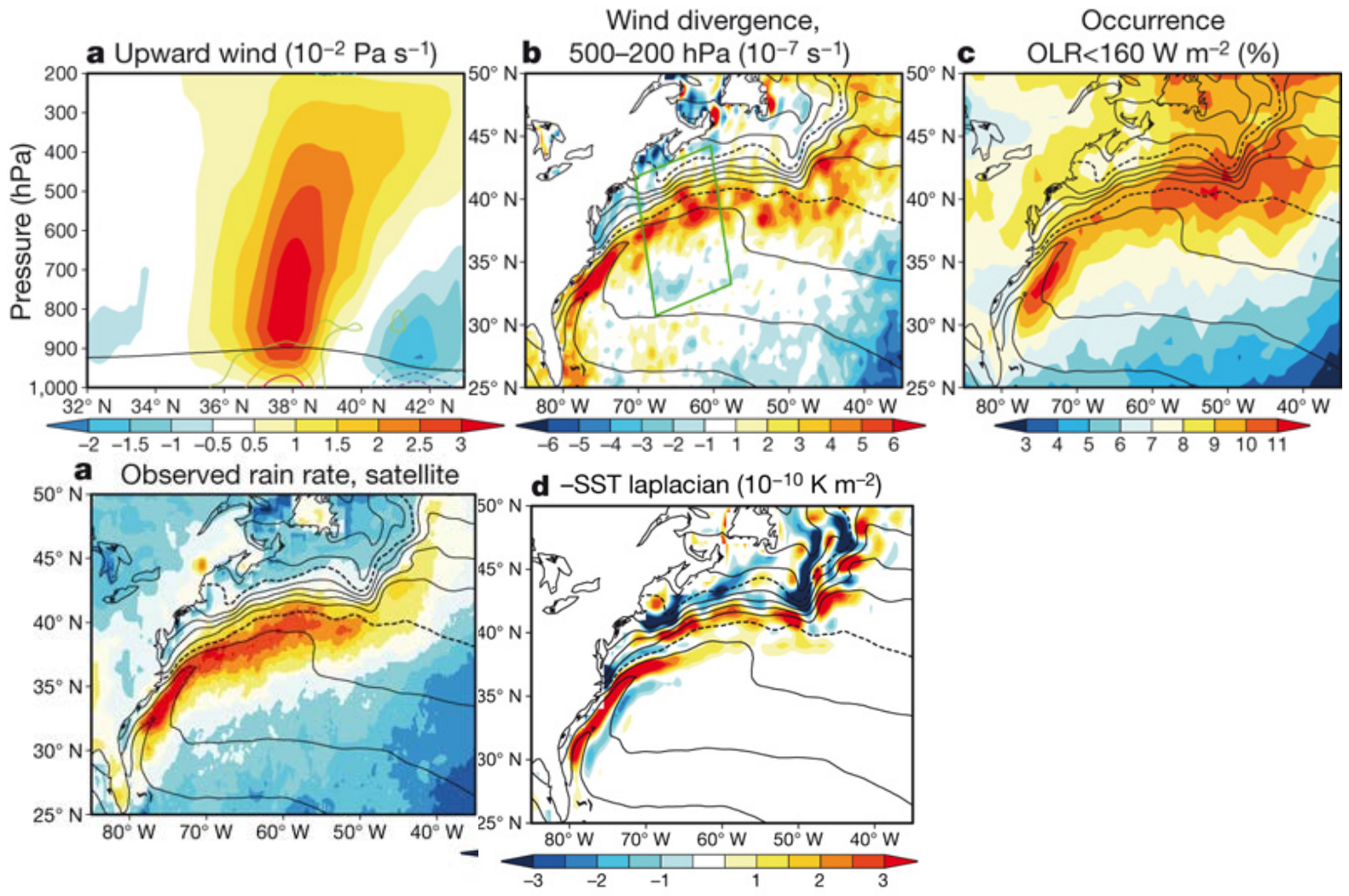

Figure 4. Figure after Minobe et al 2008 (combination of parts of their Figures 3, 2 and 1). Top: a.) Vertical wind velocity (upward positive; colour), boundary layer height (black curve) and wind convergence (contours for $\pm 1,2,3 \times 10^{-1} \mathrm{~s}^{-1}$ ) averages in the along front direction in the green box in b.), based on the ECWMF analysis. b.) Upper-tropospheric wind divergence averaged between 200 and $500 \mathrm{hPa}$ (colour) c.) Occurrence frequency of daytime satellite-derived OLR levels lower than $160 \mathrm{Wm}^{-2}$ (colour). Contours in b.) and c.) are for SST. Bottom: a.) Annual climatology of rain rate observed by satellites. Contours are for SST d.) sign-reversed SST laplacian in the ECMWF analysis. SST contours $\left(2^{\circ} \mathrm{C}\right.$ interval and dashed contours for $10^{\circ} \mathrm{C}$ and $20^{\circ} \mathrm{C}$ ) are shown. 
GHRSST Project Office

\section{Updating the record}

So, we have seen that a long record is needed, with sub-daily resolution. But, it is also important to update that record through time.

The survey respondents vary widely as to how frequently this should be done (Figure
5). Many are content with very infrequent updates to the record, but a significant number will ultimately need data within a day. This is a very stretching requirement because it is updating a climate record every day, rather than just adding an operational data set on to the end of $a$ reanalysis.
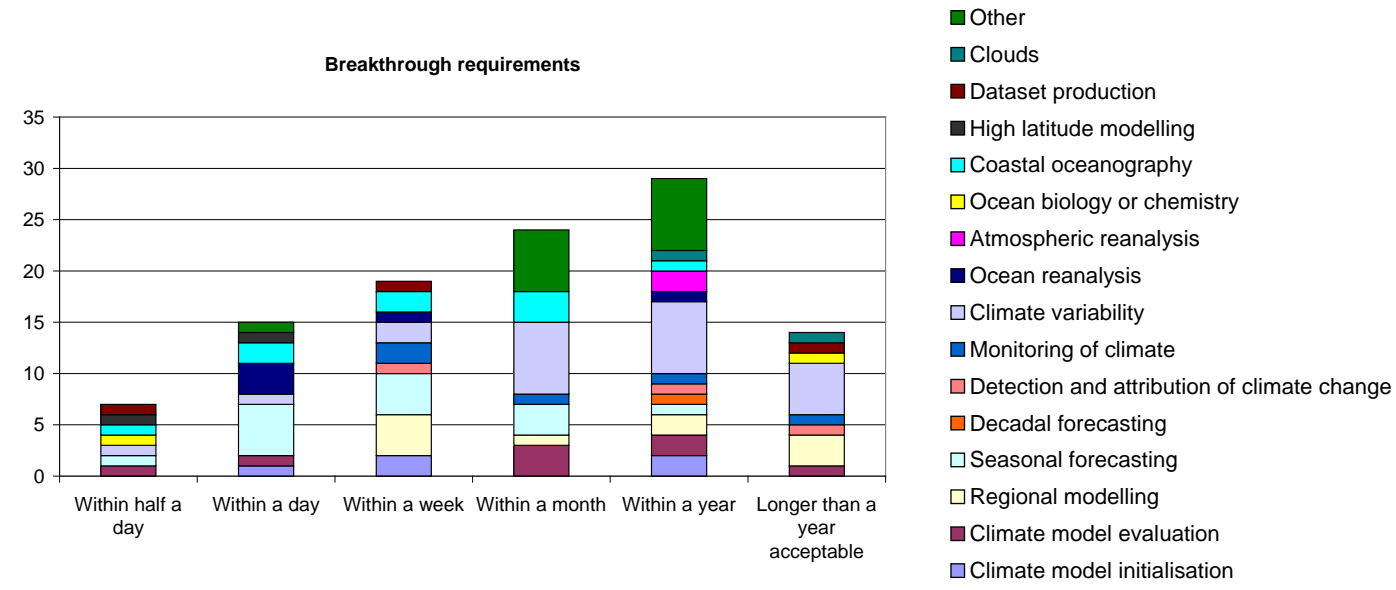

Figure 5. Breakthrough requirements for acceptable delay between data being recorded and their delivery (see User Requirements Document).

But why might you need a daily updating Climate Data Record?

The Met Office makes two seasonal forecasts every day for three weeks to generate an ensemble of forecasts for the coming season. SST measurements are assimilated into the high resolution operational ocean model to generate initial conditions and then two coupled forecasts are made using slightly different configurations of the model. Figure 6 shows the ensemble of experimental GloSea4 forecasts for Nino 3 average SST in June. Doing this every day provides sufficient ensemble spread and also allows the generation of an ensemble of monthly forecasts.

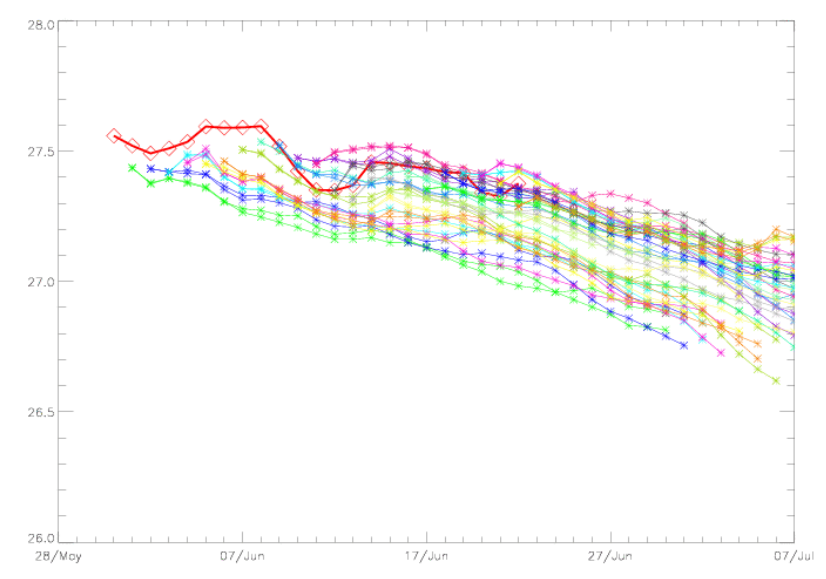

Figure 6. Evolution of SST in the Nino3 region in the GloSea4 analysis (thick red line with diamond symbols) with the associated first 30 days of the coupled ensemble forecast. There are two ensemble members per day, and each day is separately colour coded, starting with the 1st June -- which uses the (end of the) 31 May ocean analysis (thinner lines with ' $x$ ' symbols). (Figure courtesy of Drew Peterson.)

You might say that this is an operational and not a climate application, but the Met Office is working on producing seamless forecasts from daily to monthly to seasonal to decadal to multi-decadal. In addition, with every seasonal forecast 
comes a fifteen-year hindcast in order to provide validation statistics. It is very important for understanding the relationship between the hindcast and the forecast that consistent data are assimilated into both.

\section{How stable does the record have to be?}

In Tables 2 and 3 we have an analysis of the maximum acceptable level of drift that would satisfy two thirds of the potential users polled. If we look over all spatial scales, the breakthrough requirements are of the order a few hundredths of a degree per decade. This is also true of the requirements for stability over smaller spatial scales. Of course, it is questionable whether any of these are actually demonstrable - we need a highly stable reference data set and there are only a few locations around the world where that is available.

\begin{tabular}{|l|l|l|l|l|l|l|}
\hline & \multicolumn{3}{|c|}{ Grid cell } & \multicolumn{3}{c|}{$1000 \mathrm{~km}$} \\
\hline $\begin{array}{l}\text { Data } \\
\text { leve }\end{array}$ & Threshold & $\begin{array}{l}\text { Breakthroug } \\
\mathrm{h}\end{array}$ & Objective & Threshold & $\begin{array}{l}\text { Breakthroug } \\
\mathrm{h}\end{array}$ & Objective \\
\hline 2 & $\begin{array}{l}0.05^{\circ} \mathrm{C} / \mathrm{de} \\
\mathrm{C}\end{array}$ & $0.05^{\circ} \mathrm{C} / \mathrm{dec}$ & $\begin{array}{l}0.05^{\circ} \mathrm{C} / \mathrm{de} \\
\mathrm{C}\end{array}$ & $0.1^{\circ} \mathrm{C} / \mathrm{dec}$ & $0.05^{\circ} \mathrm{C} / \mathrm{dec}$ & $\begin{array}{l}0.05^{\circ} \mathrm{C} / \mathrm{de} \\
\mathrm{C}\end{array}$ \\
\hline 3 & $0.1^{\circ} \mathrm{C} / \mathrm{dec}$ & $0.1^{\circ} \mathrm{C} / \mathrm{dec}$ & $\begin{array}{l}0.02^{\circ} \mathrm{C} / \mathrm{de} \\
\mathrm{C}\end{array}$ & $\begin{array}{l}0.05^{\circ} \mathrm{C} / \mathrm{de} \\
\mathrm{C}\end{array}$ & $0.05^{\circ} \mathrm{C} / \mathrm{dec}$ & $\begin{array}{l}0.01^{\circ} \mathrm{C} / \mathrm{de} \\
\mathrm{C}\end{array}$ \\
\hline 4 & $0.1^{\circ} \mathrm{C} / \mathrm{dec}$ & $0.05^{\circ} \mathrm{C} / \mathrm{dec}$ & $\begin{array}{l}0.02^{\circ} \mathrm{C} / \mathrm{de} \\
\mathrm{C}\end{array}$ & $0.1^{\circ} \mathrm{C} / \mathrm{dec}$ & $0.01^{\circ} \mathrm{C} / \mathrm{dec}$ & $\begin{array}{l}<0.01^{\circ} \mathrm{C} / \\
\mathrm{dec}\end{array}$ \\
\hline
\end{tabular}

Table 2. Maximum acceptable level of drift, at grid cell and $1000 \mathrm{~km}$ scales, required to satisfy at least two thirds of the user group.

\begin{tabular}{|l|l|l|l|}
\hline & \multicolumn{3}{|l|}{ Aggregated over all spatial scales } \\
\hline $\begin{array}{l}\text { Data } \\
\text { level }\end{array}$ & $\begin{array}{l}\text { Threshol } \\
\mathbf{d}\end{array}$ & $\begin{array}{l}\text { Breakthroug } \\
\mathbf{h}\end{array}$ & Objective \\
\hline 2 & $\begin{array}{l}0.05^{\circ} \mathrm{C} / \mathrm{de} \\
\mathrm{C}\end{array}$ & $0.02^{\circ} \mathrm{C} / \mathrm{dec}$ & $\begin{array}{l}0.01^{\circ} \mathrm{C} / \mathrm{de} \\
\mathrm{C}\end{array}$ \\
\hline 3 & $0.1^{\circ} \mathrm{C} / \mathrm{dec}$ & $0.05^{\circ} \mathrm{C} / \mathrm{dec}$ & $\begin{array}{l}0.01^{\circ} \mathrm{C} / \mathrm{de} \\
\mathrm{C}\end{array}$ \\
\hline 4 & $0.1^{\circ} \mathrm{C} / \mathrm{dec}$ & $0.02^{\circ} \mathrm{C} / \mathrm{dec}$ & $\begin{array}{l}0.01^{\circ} \mathrm{C} / \mathrm{de} \\
\mathrm{C}\end{array}$ \\
\hline
\end{tabular}

Table 3. Maximum acceptable level of drift, aggregated over all spatial scales, required to satisfy at least two thirds of the user group.

But if decadal surface temperature change over the last few decades has been of the order one or two tenths of a degree (see Figure 7), then ideally the observing system we put in place to monitor that would have a drift of no more than $10 \%$ of that change. 


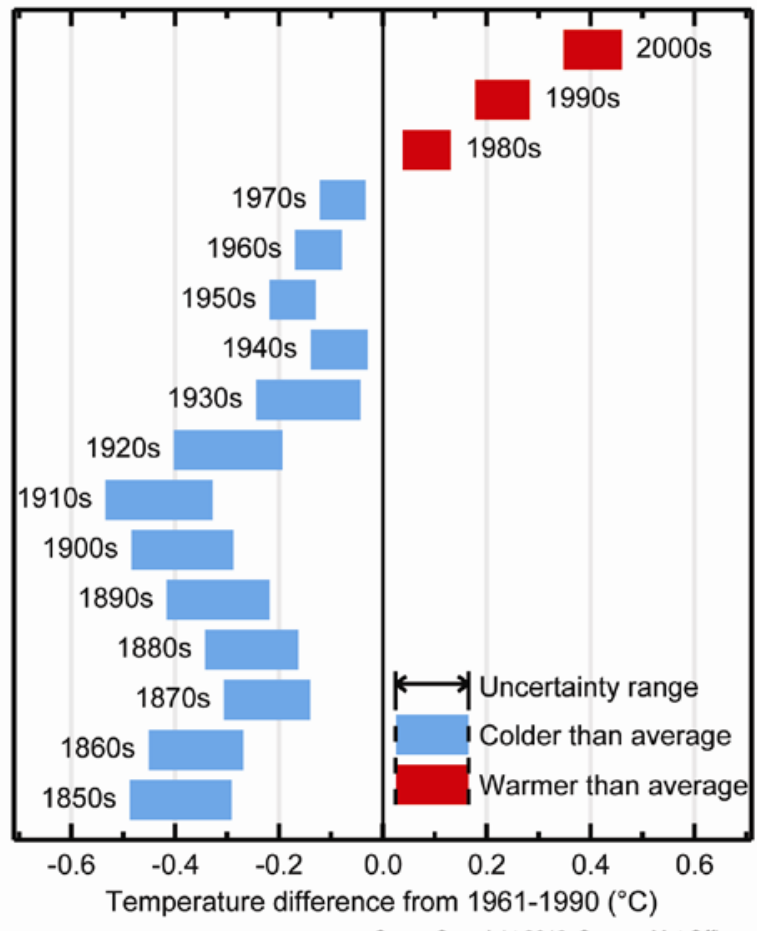

Crown Copyright 2010. Source: Met Office

Figure 7. Decadal, global average surface temperature anomaly in HadCRUT3 ( ${ }^{\circ} \mathrm{C}$, relative to 1961-90).

\section{Representation of uncertainties}

Users recognise that there are uncertainties in the measurements, but they need a clear understanding of how they behave. These are some of the statements in the SST_cci User Requirements Document on the subject of uncertainties:

"Uncertainties need to be characterised fully and improved relative to current datasets. This should include the full error budget of the translation from the input data to the products.

Confidence in uncertainty estimates needs to be stated. Uncertainty characteristics should be verified by comparison against independent observations.

Information about the correlation structure of errors is essential or desirable for most respondents."

It should be said that, as in other cases, many of the people polled stated simpler requirements than shown here. However, provision of information on uncertainties was a key area where potential users of the SST_cci products felt improvements needed to be made on what is currently available.

If users know the components of the uncertainties in the data and know how they are correlated, then they can start to explore the consequences of those uncertainties on their application. For example, Figure 8 shows a representation of the global mean SST anomaly over the last 160 years and its uncertainties, split according to uncertainty type. 


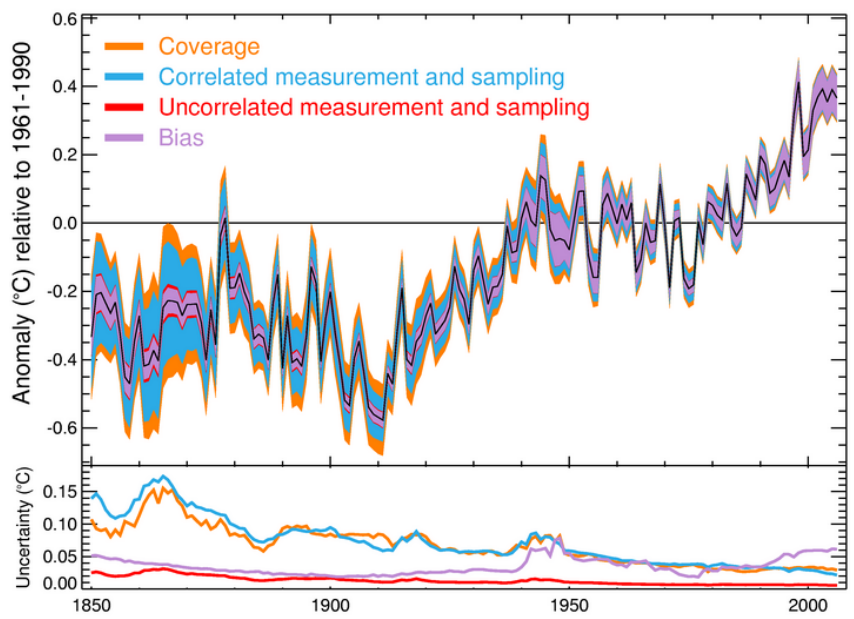

Figure 8. Annual, global average sea surface temperature anomaly $\left({ }^{\circ} \mathrm{C}\right.$, relative to $\left.1961-90\right)$ with its associated uncertainties separated according to coverage, bias-adjustment related, correlated measurement and sampling and uncorrelated measurement and sampling uncertainties (http://www.metoffice.gov.uk/hadobs/hadsst3/, Kennedy et al., 2011a and b).

The sizes of the uncertainties and their correlation structure can be used to generate an ensemble of equally-likely realisations of this time series, drawing samples from the relevant distributions.

An ensemble of surface temperature data sets has been used in Figure 9 in an

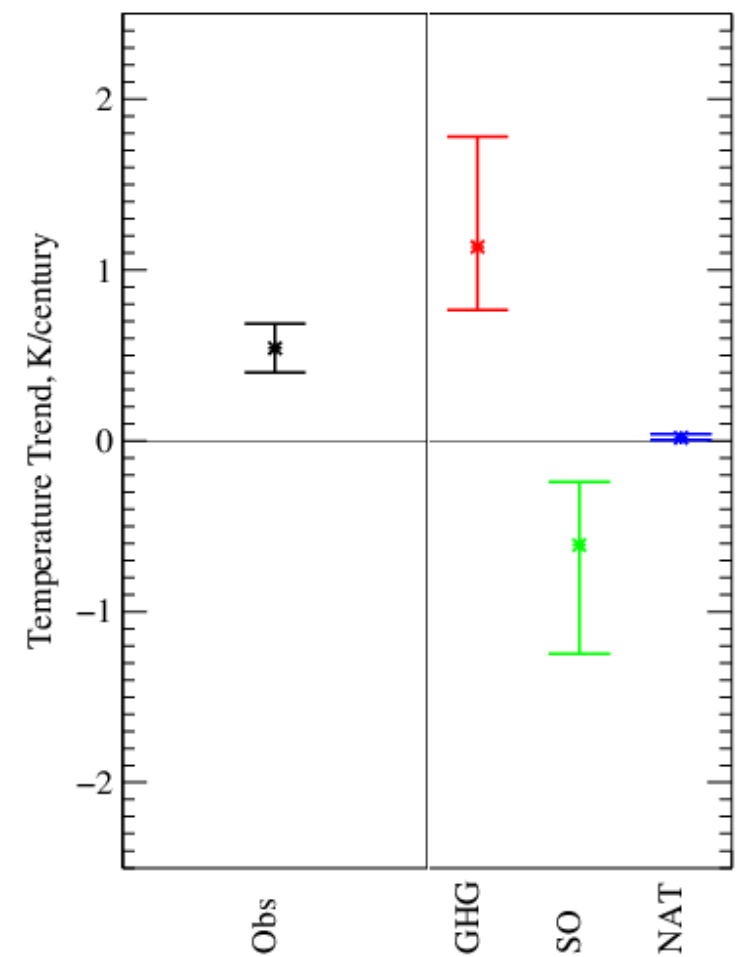

attribution study of land air temperature over Africa. (This figure represents the principle of the work only, and has since been superceded.) The temperature trends attributed to greenhouse gas forcing, sulphate aerosols and natural forcings have an associated uncertainty.

Figure 9. Observed Africa trends (Obs) compared to scaled simulated trends over the same region for GHG (greenhouse gas), SO (sulphate and ozone) and NAT (solar and volcanic) forcings that have been deduced in an attribution study for the 1900-1999 period. Time series comprise 10 year means and cover the three Giorgi regions making up Africa. The model used is HadCM3. Left: Includes uncertainty as assessed using internal 
climate variability from control simulations. Right: Additional boxes include the effect of the spread of an ensemble of equally-likely realisations of HadCRUT3 on the trends. (Figure courtesy of Gareth Jones.)

Originally the detection and attribution analysis only attempted to take into account uncertainty in the magnitude of the climate patterns being searched for and in internal variability, to deduce the contributions to the observed climate changes.

By using an ensemble of HadCRUT3, the analysis can also include observational uncertainties. Preliminary results suggest that the observational error has little influence on the attribution of greenhouse gas warming and other anthropogenic cooling in warming observed in large-scale regions of the world. But in Africa, including the HadCRUT3 ensemble increases the uncertainty ranges of the observed warming and the deduced contributions. So whilst uncertainty ranges are increased, the use of a HadCRUT3 ensemble better quantifies the attributions of the warming to different climate factors.

\section{Conclusion}

We have seen examples of climate applications of SST and other data that place stringent requirements on the reanalysis products that we are developing. Understanding why these are needed allows us to design our products to best meet these needs, even if at first they seem daunting and perhaps impossible. However, in doing so, we also need to recognise that other users' needs are different and sometimes simpler. We need to understand that designing products and systems that are flexible in terms of resolution and complexity of information will ensure that as many users as possible have access to the latest and best reanalysis products that we can produce.

\section{References}

Folland, C.K., Owen, J., Ward, M.N. and A. Colman (1991), Prediction of seasonal rainfall in the Sahel region using empirical and dynamical methods, J. Forecasting, 10, 21-56.

Jones P.D., Osborn, T.J., Briffa K.R., Folland C.K., Horton E.B.. Alexander L.V., Parker D.E. and N. Rayner (2001), Adjusting for sampling density in grid box land and ocean surface temperature time series, J. Geophys. Res., 106, 3371-3380.

Kennedy J.J., Rayner, N.A., Smith, R.O., Saunby, M. and Parker, D.E. (2011a), Reassessing biases and other uncertainties in sea-surface temperature observations since 1850 part 1: measurement and sampling errors, in press JGR Atmospheres

Kennedy J.J., Rayner, N.A., Smith, R.O., Saunby, M. and Parker, D.E. (2011b), Reassessing biases and other uncertainties in sea-surface temperature observations since 1850 part 2: biases and homogenisation. in press JGR Atmospheres

Knight, J. R., R. J. Allan, C. K. Folland, M. Vellinga, and M. E. Mann (2005), A signature of persistent natural thermohaline circulation cycles in observed climate, Geophys. Res. Lett., 32, L20708, doi:10.1029/2005GL024233.

Minobe, S., A. Kuwano-Yoshida, N. Komori, S.-PXie and R.J. Small (2008), Influence of the Gulf Stream on the troposphere, Nature, 452, 206209, 10.1038/nature06690

Rayner, N. A., Parker, D. E., Horton, E. B., Folland, C. K., Alexander, L. V., Rowell, D. P., Kent, E. C. and Kaplan, A (2003), Global analyses of sea surface temperature, sea ice, and night marine air temperature since the late nineteenth century, J. Geophys. Res.Vol. 108, No. D14, 4407, 10.1029/2002JD002670 


\title{
ESTIMATING AND PRESENTING UNCERTAINTIES IN AN HISTORICAL SEA-SURFACE TEMPERATURE ANALYSIS.
}

\author{
John J. Kennedy ${ }^{(1)}$, Nick A. Rayner ${ }^{(2)}$ and Michael Saunby ${ }^{(3)}$
}

(1)Met Office Hadley Centre, Exeter, U.K., Email : john.kennedy@metoffice.gov.uk

(2) Met Office Hadley Centre, Exeter, U.K., Email: nick.rayner@metoffice.gov.uk

(3) Met Office Hadley Centre, Exeter, U.K., Email: michael.saunby@metoffice.gov.uk

Historical sea-surface temperature analyses are used in a wide-variety of applications. For many users the ideal SST analysis would have very high temporal and spatial resolution and would look like the best fields that can currently be produced using highresolution satellite data. Unfortunately, given the sparsity of historical in situ observations, this is not possible.

Instead of this, analyses are often presented as a combination of a bestestimate together with an error range. This is a convenient way of expressing the magnitude of the uncertainty in the data, but it does not tell the user how the errors covary. What is their structure? Nor do the fields look like the high resolution fields derived from satellite data.

In developing the HadISST2 data set our aim has been to create an ensemble of equi-probable realisations of the data which are both consistent with the available data, span the range of uncertainty, and which contain 'realistic' temporal and spatial variability at the target resolution. 


\title{
OBJECTIVE DETERMINATION OF FEATURE RESOLUTION IN AN SST ANALYSIS
}

\author{
Richard W. Reynolds ${ }^{(1)}$, Dudley B. Chelton ${ }^{(2)}$ \\ (1) NOAA Cooperative Institute for Climate \& Satellites (CICS,) \\ Asheville, North Carolina (USA), Email: Richard.W.Reynolds@noaa.gov \\ (2) College of Oceanic and Atmospheric Sciences \\ and Cooperative Institute for Oceanographic Satellite Studies, \\ Oregon State University, Corvallis, Oregon (USA), Email: chelton@coas.oregonstate.edu
}

\begin{abstract}
Ocean model SST fields are used as 'true' SST data and subsampled based on actual satellite data coverage. The full and subsampled data are then compared as a function of spatial scales of variability using wavenumber auto- and cross-spectral analysis. The results showed that analyzed high-resolution features are generated with and without high resolution data. However, if only sparse high-resolution data are available because of the loss of infrared measurements because of cloud cover, the high-resolution features in an SST analysis are just noise. Not surprisingly, the actual data distributions are thus an accurate indicator of where the presence of smallscale features in an SST analysis can be considered valid. Based on a qualitative assessment, we suggest a threshold of at least $30 \%$ coverage by infrared measurements for an accurate SST analysis on wavelength scales of $25-50 \mathrm{~km}$.
\end{abstract}

\section{Introduction}

Many users and developers of sea surface temperature (SST) analysis products confuse analysis grid resolution with the scales of the SST features that can be resolved in the analysis. In any analysis procedure, irregularly spaced data are smoothed and interpolated onto a regular grid by some objective analysis procedure. As determined by the input data and smoothing parameters in the analysis, the feature resolution will in general vary in time and space. The purpose of this study is to show how analyses and actual space-time distributions of samples can degrade the feature resolution.

\section{Procedure}

The procedure used here begins with daily complete SST fields from a high-resolution global model, which are considered to be the "true" SST. These fields are then treated as data and subsampled based on actual satellite SST data distributions. The full and reduced SST data fields are then analyzed and compared to assess the influence of missing high-resolution measurements owing to data loss from cloud cover.

The "true" SST fields for this study were obtained from the ECCO2 model (Estimating the Circulation and Climate of the Ocean, Phase II, http://ecco2.jpl.nasa.gov/) and were then sampled to simulate actual infrared Advanced Very High Resolution Radiometer (AVHRR) and microwave Advanced Microwave Scanning Radiometer (AMSR) satellite measurement times and locations. The model SST fields were averaged over $4.8 \mathrm{~km}$ to simulate the AVHRR Pathfinder v5 grid. Similarly, the model SST fields were averaged over $50 \mathrm{~km}$ (the approximate footprint size for AMSR) on a $1 / 4^{\circ}$ grid. An analysis procedure is carried out on the complete model SST fields and on the $1 / 4^{\circ}$ and $4.8 \mathrm{~km}$ gridded fields, subsampled at the actual AMSR daily data locations for the low-resolution analysis and at the actual AVHRR daily data locations for the high resolution analysis. The subsampling procedure only kept the model SST if there were actual AMSR or AVHRR data at the same grid point at a given time. 
GHRSST XII -

Proceedings, Edinburgh

GHRSST Project Office

Both the low-resolution and high-resolution analyses use an optimum interpolation (OI) procedure. The first step consists of a lowresolution analysis that has been described (1). The second step produces a highresolution analysis that starts with the lowresolution analysis and a damped previous high-resolution analysis as a first guess as described in GHRSST-XI extended abstract. Both analyses were computed daily for 2 months, January and July 1993, using fully sampled SST fields from the model and the simulated satellite data coverage obtained by the above subsampling procedure. Satellite coverage was based on actual satellite sampling during January and July 2004 rather than during the 1993 time period of the ECCO-2 model simulation because AMSR data were not available before 2002. Use of data coverage from years that differ from the model years is not critical because only satellite observation times and locations were used, not the actual satellite SSTs. Although, both day and night satellite data were used, the daily coverage of the AVHRR was very sparse because of cloud cover, which is typically about $70 \%$ globally at any given time. To improve the coverage, 3 successive days of satellite observation times and locations were used to define the subsampled daily data. The subsampled data will henceforth be referred to as 'reduced' data; the complete data will be referred to as 'full' data.

\section{Results}

Zonal wavenumber spectra were computed for 6 regions for both January and July. However, only 2 are shown here: a Sargasso Sea region $\left(20 \mathrm{~N}^{\circ}-30^{\circ} \mathrm{N}, 60^{\circ} \mathrm{W}\right.$ $\left.30^{\circ} \mathrm{W}\right)$ and a Gulf Stream region $\left(37 \mathrm{~N}^{\circ}\right.$ $\left.44^{\circ} \mathrm{N}, 65^{\circ} \mathrm{W}-50^{\circ} \mathrm{W}\right)$.

Figure 1 shows the auto-spectra for July 1993 for the Gulf Stream region. The spectrum for the SST fields from the full high resolution 3-day data shows a smooth drop off from a spectral density of $10^{3}$ at the smallest wavenumbers to $10^{-3}$ at the largest.
Issue: Final

Date: September 27, 2011

The low-resolution OI analysis using the reduced data shows a drop relative to the full high resolution 3-day data by a factor of $10^{-4}$ between 0.01 and 0.03 cycles $\mathrm{km}^{-1}$. However, at larger wavenumbers the spectral density begins to oscillate. From simulations, we have determined that this is due to the use of linear interpolation from the low- to the high-resolution grid. (The low-resolution OI spectrum with full data, not shown, is almost identical to the lowresolution $\mathrm{Ol}$ with reduced data.) The highresolution Ols with both full and reduced data are very similar to the full high resolution 3-day data at wavenumbers below 0.06 cycles $\mathrm{km}^{-1}$. At higher wavenumbers (shorter wavelength scales), the high-resolution Ol with the full data actually has lower power than the full high resolution 3-day data and the highresolution OI based on reduced data.

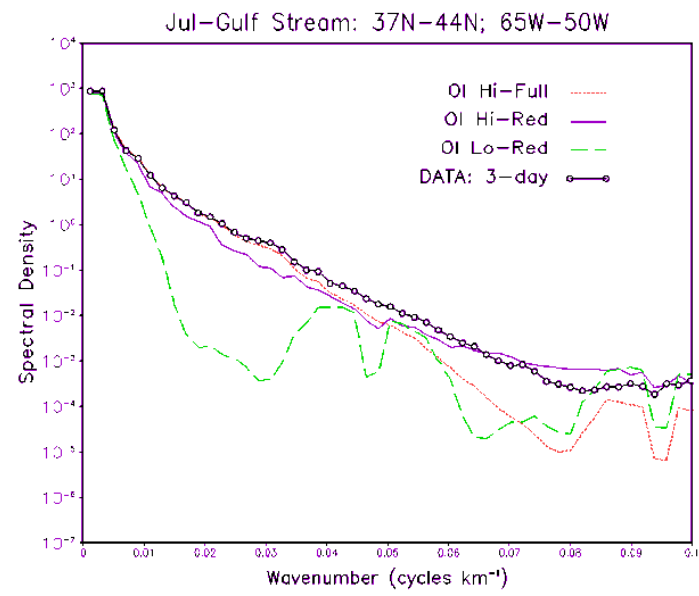

Figure 4. Auto-spectra of the $O I$ analyses and data for July 1993 for the Gulf Stream. 'Ol Hi-Full' is the high resolution $\mathrm{Ol}$ with the full data. 'OI HiRed' is the high resolution $\mathrm{OI}$ with the reduced data. 'OI Lo-Red' is the low resolution OI with the reduced data. 'DATA: 3-day' is the full high resolution 3-day data. The horizontal axis shows the wavenumber in cycles $\mathrm{km}^{-1}$.The vertical axis shows the spectral density $\left({ }^{\circ} \mathrm{C}^{2}\right.$ per cycle $\left.\mathrm{km}^{-1}\right)$.

Usually it is not possible to determine how much of the spectral density is noise and how much is signal. However, as 'truth' is defined here to be the full high resolution 3day model data, the co-spectra can be 
computed with respect to 3-days of highresolution full model SSTs. This is displayed in figure 2 as the squared coherence. The squared coherence can be viewed as the wavenumber distribution of the squared correlation. The squared coherence ranges between 0 for no coherence and 1 for perfect coherence.

Figure 2 shows the squared coherence for the Sargasso Sea region for July. For the low-resolution OI with reduced data, the squared coherence drops quickly with increasing wavenumber, reaching roughly 0.6 at 0.01 cycles $\mathrm{km}^{-1}$ and falling to near zero above 0.02 cycles $\mathrm{km}^{-1}$. The highresolution OI with full data remains above or near 0.9 until the wavenumber reaches 0.06 cycles $\mathrm{km}^{-1}$, above which it drops quickly with increasing wavenumber with some ringing at the highest wavenumbers. The high-resolution $\mathrm{OI}$ with reduced data decreases with increasing wavenumber more slowly than the low-resolution $\mathrm{OI}$, dropping below 0.5 at $0.03 \mathrm{~km}^{-1}$. Clearly the data reduction caused by clouds attenuates the high-resolution signals (i.e., the small scales of variability in the SST field). However, as shown in figure 1, it does not reduce the power at high wavenumbers. The variability at high wavenumbers in the high-resolution $\mathrm{OI}$ is therefore just noise!

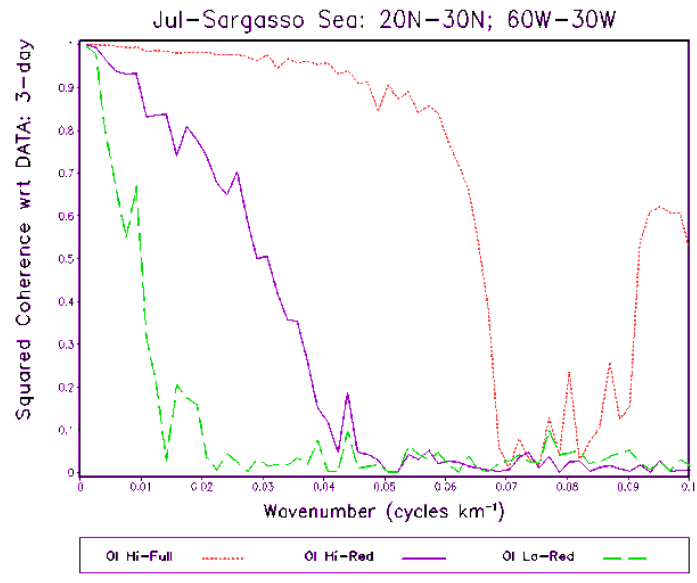

Figure 5. Squared coherence of the OI analyses for July 1993 for the Sargasso Sea with respect to the full high resolution 3-day data set. The vertical axis shows the squared coherence (range 0-1). Otherwise as in figure 1.

Figure 3 shows the squared coherence for the Gulf Stream region for July. The results are very similar to the squared coherence results for the Sargasso Sea (figure 2). In the Sargasso Sea region, however, the high-resolution OI with reduced data drops almost as quickly as the low-resolution OI. This shows that there are limited highresolution signals in the Gulf Stream region due to the lack of the high-resolution data because of more persistent cloud cover than in the Sargasso Sea.

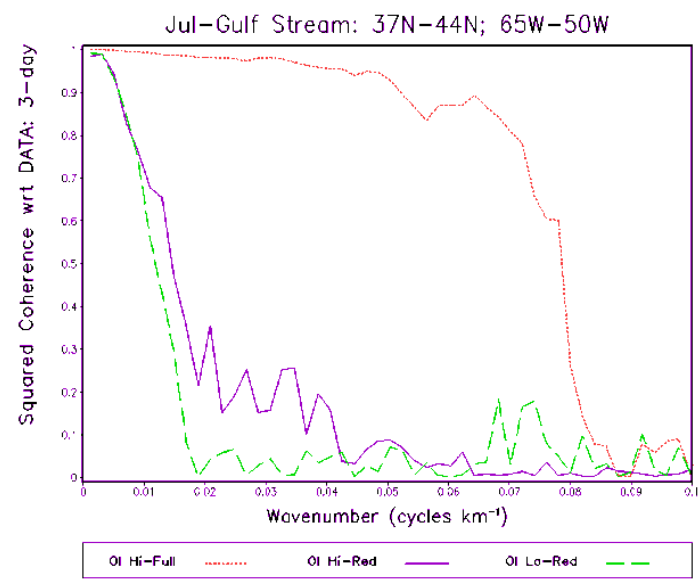

Figure 6. Squared coherence of the Ol analyses for July 1993 for the Gulf Stream with respect to the full 3-day data set. Otherwise as in figure 2.

The results were repeated for the Gulf Stream region on a daily basis (not shown). During periods of clear skies when reduced data were available (e.g., July 17), the results for the Gulf Stream are similar to the monthly results for the Sargasso Sea (figure 2). During periods of heavy cloud cover (e.g., July 1), the results are slightly worse than the monthly results for the Gulf Stream (figure 3).

Daily time series of the daily coherence averages between 0.02 and 0.04 cycles $\mathrm{km}^{-1}$ and the fractional coverage of the number of ocean grid points with data compared to the total. The time series are shown in figure 4 for the Gulf Stream for 
July. The two time series for this and other ocean regions showed remarkable similarity during each of the two months considered here. The comparisons indicated that at least $30 \%$ of ocean grid points must have data in each region for an accurate highresolution analysis at wavenumbers between 0.02 and 0.04 cycles $\mathrm{km}^{-1}$. This simple metric defines the regions of an SST analysis where small-scale features (wavelength scales of $25-50 \mathrm{~km}$ ) can be considered accurate in a high-resolution analysis.

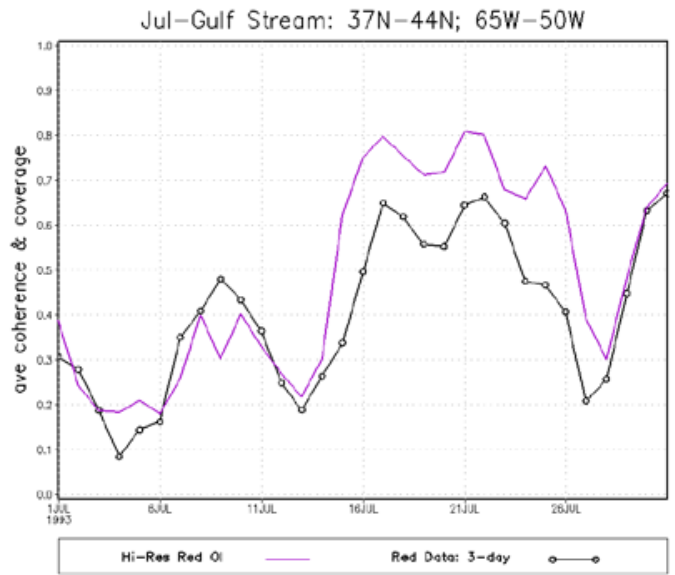

Figure 4. Daily time series of the July average coherence of the high resolution $\mathrm{OI}$ with respect to the full 3-day data set and the fractional coverage of the number of ocean grid points with data to the total. The coherence is averaged between 0.02 and 0.04 cycles $\mathrm{km}^{-1}$. The fractional coverage is computed over the Gulf Stream region $\left(37 \mathrm{~N}^{\circ}-44^{\circ} \mathrm{N}, 65^{\circ} \mathrm{W}-50^{\circ} \mathrm{W}\right)$. 'Hi-Res Red Ol' is the average coherence and 'Red Data: 3-day' is the fractional coverage. The horizontal axis is in days. The vertical axis is average coherence and fractional coverage.

Daily fields were computed for the fractional coverage of ocean grid points with highresolution data to the total number of grid points on a $1^{\circ}$ grid. The number of days then were calculated for each month for each $1^{\circ}$ grid point where the daily fractional coverage was at least $0.3(30 \%)$ in the $1^{\circ}$ grid box. Figures 5 and 6 show the number of days with coverage above $30 \%$ for January and July 2004, respectively. In the Sargasso Sea region, a high-resolution analysis is possible at least 18 days in
January and for more than 27 days in July. However, for the Gulf Stream region a highresolution analysis is not possible in January and is only possible for about 15 days during July. The difference between January and July is due to a strong seasonal cycle of cloud cover. Please also note the large seasonal differences in other regions, e.g., the northern Indian Ocean.

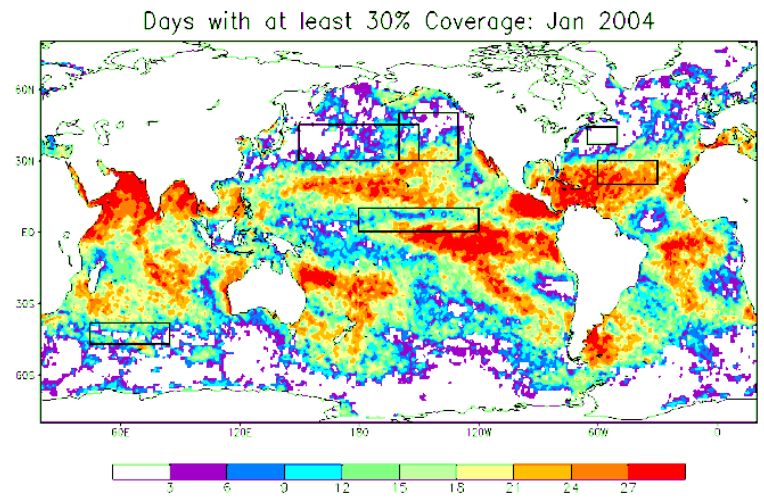

Figure 5. Number of days in January where the fractional coverage of the number of ocean grid points with data to the total exceeds $30 \%$. The fractional coverage was computed on a $1^{\circ}$ spatial grid.

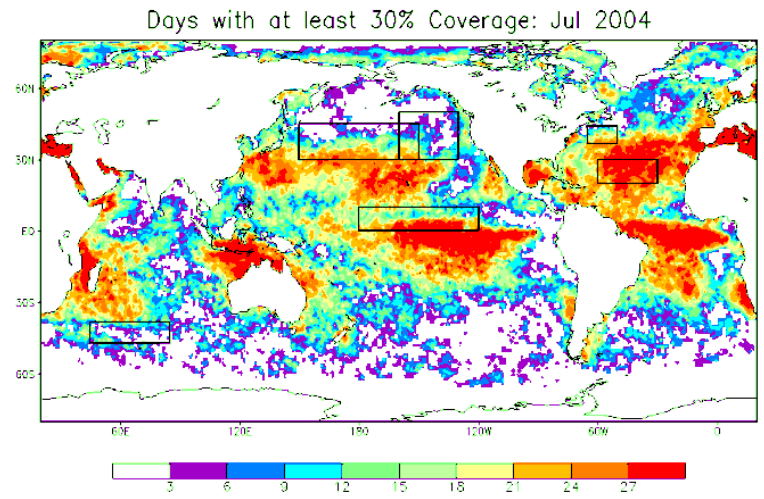

Figure 6. Number of days in July where the fractional coverage of the number of ocean grid points with data to the total exceeds $30 \%$. Otherwise as in figure 5.

\section{Conclusions}

Ocean model SST fields were used as 'true' SST and subsampled based on actual data 
coverage. The results show that the $\mathrm{Ol}$ procedure generated small-scale features in the SST analyses, both with and without high-resolution data. When the highresolution data are sparse, however, the small-scale features are only noise, not signal.

Fractional coverage of the number of ocean grid points with data to the total is a useful metric for assessment of when and where small-scale features in an SST analysis can be considered accurate. It is thus recommended that maps of fraction coverage be made available to users. We suggest a rough threshold of 30\% coverage on a $1^{\circ}$ spatial grid for accurate SST on scale of $25-50 \mathrm{~km}$.

\section{References}

- Reynolds, R. W., T. M. Smith, C. Liu, D. B. Chelton, K. S. Casey, and M. G. Schlax, "Daily high-resolution blended analyses for sea surface temperature", J. Climate, 20, 5473-5496, 2007. 


\title{
THE IMPACT OF NEW SOURCES OF SATELLITE DATA ON THE OSTIA SYSTEM.
}

\author{
Jonah Roberts-Jones ${ }^{(1)}$, Matthew Martin ${ }^{(2)}$.
}

(4) Met Office, Fitzroy Rd, Exeter,( United Kingdom), jonah.roberts-jones@metoffice.gov.uk (5) Met Office, Fitzroy Rd, Exeter,( United Kingdom), matthew.martin@metoffice.gov.uk

New satellite data streams have recently been tested in the Operational SST and sea Ice Analysis (OSTIA) system which runs at the UK Met Office. The operational system currently assimilates infrared radiometric observations from AATSR, GAC AVHRR, METOP AVHRR, NAR AVHRR and the SEVIRI satellite instruments, microwave radiometric observations from the AMSRE and TMI sensors and in-situ observations. All satellite data are provided through the GHRSST framework. Experiments have been carried out to assess the impact on the OSTIA system of the inclusion of new geo-stationary infrared data streams produced by the EUMETSAT OSI-SAF. A new processing chain is carried out in the production of SEVIRI data which has been assessed as a replacement for the current operational SEVIRI data. The additional use of GOES-EAST data has also been assessed. Experiments have been run to assess the impact of the addition of the IASI SST data produced by EUMETSAT.

All input data are passed through an automatic quality control system and a bias correction on selected satellites is carried out using the in-situ and AATSR data as a reference. OSTIA then uses a multi-scale optimal interpolation scheme to assimilate in-situ and satellite SST observations onto a first guess field provided by the previous analysis with a relaxation to climatology.

A brief overview of the OSTIA system and the new satellite data used will be presented. Results to assess the impact of the new data sources on the OSTIA system will be shown including validation statistics and comparisons of the SST output to those produced by the current operational OSTIA system. 


\section{GHRSST WINDSAT AND AMSR-E VERSION 7 SSTS}

\section{Chelle Gentemann ${ }^{(1)}$, Deborah K Smith ${ }^{(2)}$}

(1)Remote Sensing Systems, Santa Rosa, CA, USA, Email :gentemann@remss.com

(2) Remote Sensing Systems, Santa Rosa, CA, USA, Email: smith@remss.com

WindSat, a fully polarimetric passive microwave radiometer, was launched in January, 2003. It represents an important extension to the AQUA AMSR-E and TRMM TMI retrievals. Environmental variables, such as SST, wind speed, atmospheric water vapor, cloud water, and rain rate, are calculated using a multi-stage linear regression algorithm derived through comprehensive radiative transfer model simulations. SST retrieval is prevented only in regions with sun-glitter, rain, and close to land. In Polar Regions where cloud cover regularly prevents infrared observations of SSTs, the MW observations of SST provide a significant improvement. Although calibration problems complicated and delayed SST retrievals, WindSat SSTs are now routinely processed. The WindSAT SSTs were processed using an updated retrieval algorithm, RSS version 7 . The AMSR-E SSTs have also been reprocessed to version 7 . This new algorithm will be discussed and validation results for both datasets will be presented. 


\title{
USING GHRSST DATA CONSTRAINTS TO ESTIMATE THE CIRCULATION AND CLIMATE OF THE OCEAN
}

\author{
Jorge Vazquez ${ }^{(1)}$ Holger Brix ${ }^{(1,2)}$ Dimitris Menmenlis ${ }^{(1)}$ \\ (1) (Jet Propulsion Laboratory/California Institute of Technology) M/S 323, 8183546980 \\ Jorge.Vazquez@jpl.nasa.gov) \\ (2) UCLA and JPL/Caltech
}

Sea Surface Temperature (SST) from the Advanced Microwave Scanning Radiometer (AMSR-E) on board the Aqua Spacecraft is used to constrain a global, eddying, fulldepth-ocean, and sea-ice configuration of the Massachusetts Institute of Technology general circulation model (MITgcm). The model configuration is that used by NASA's Estimating the Circulation and Climate of the Ocean, Phase II (ECCO2) project and data constraints are applied using the adjoint method, which produces a solution that is everywhere consistent with model equations during the entire estimation period. Additional data constraints include Jason and Envisat sea level anomaly and ARGO temperature and salinity profiles. The control variables are initial temperature and salinity conditions and surface atmospheric boundary conditions, that it, 10-m wind velocity, 2-m air temperature and humidity, precipitation, and downward shortwave radiation. Sensor Specific Error Statistics (SSES) contained within the GHRSST Level 2 preprocessed AMSR-E data stream are used to characterize the error in the SST data. Model results are shown for both constrained (optimized) and unconstrained (baseline) solutions. These solutions are then directly compared with gridded AMSR-E SST data. The largest differences from these comparisons are located in coastal upwelling regions, namely the Eastern Boundary Upwelling Regions. Monthly averages for 2004 and 2009 indicate that AMSR-E SST data in these regions were colder than the unconstrained ECCO2 solution by up to $2^{\circ} \mathrm{C}$. After optimization, these biases were considerably reduced. We quantify the specific changes in the control parameters that lead to bias reduction. A case study off the African Coast, including the Benguela Current, indicates that the model underestimates the strength of the seasonal upwelling driven by the Ekman Transport near the coast. Further away from the coast, the shortwave radiation is a key driver of warm bias in the unconstrained solution. The results of this study show that GHRSST SST data products can provide effective dynamical constraints for ocean state estimation, especially in coastal regions that are associated with major upwelling events. 


\section{APPENDICES}




\section{APPENDIX 1 - PARTICIPANTS LIST}

Arino, Olivier

ESA-ESRIN

Via Galileo Galilei

00044 Frascati, Italy

留:

Email: olivier.arino@esa.int

Armstrong, Edward

NASA Jet Propulsion Laboratory

California Institute of Technology

M/S 300/323

4800 Oak Grove Drive

Pasadena, CA 91109, US

襍: +1-818-393-6710

Email: edward.armstrong@jpl.nasa.gov

Banzon, Viva Patria F

National Climatic Data Center (NOAANESDIS)

Remote Sensing \& Applications Division

151 Patton Avenue

Asheville, NC 28801-5001, US

湑: +1-828-271-4453

Email: Viva.Banzon@noaa.gov

Barton, Ian

CSIRO Marine Research

PO Box 1538

Hobart, Tasmania 7001, AU

蜀: +61 362325481

Email: ian.barton@ozemail.com.au

Beggs, Helen

Australian Bureau of Meteorology (BMRC)

CAWCR

GPO Box 1289

Melbourne, Victoria 3001, AU

的: +61396694394

Email: h.beggs@bom.gov.au

Brandon, Tess

NOAA National Oceanographic Data Center

1315 East-West Highway

Silver Spring, MD 20910, US

㥜: +1-301-713-3272 x 181

Email: Tess.Brandon@noaa.gov

Bulgin, Claire

The University of Edinburgh

School of GeoSciences

Crew Building

King's Buildings

Edinburgh, EH9 3JN, UK

的:

Email: cbulgin@staffmail.ed.ac.uk
Buongiorno Nardelli, Bruno

CNR/Istituto di Scienze dell'Atmosfera e del

Clima

Istituto per l'Ambiente Marino Costiero

Gruppo di Oceanografia da Satellite

Calata Porta di Massa80133 Napoli, Italy

留: +390649934280

Email: bruno.buongiornonardelli@cnr.it

Casey, Kenneth S

NOAA National Oceanographic Data Center

1315 East-West Highway

Silver Spring, MD 20910, US

留: +1-301-713-3272 ext 133

Email: Kenneth.Casey@noaa.gov

Chao, Yi

NASA Jet Propulsion Laboratory

California Institute of Technology

M/S 300/323

4800 Oak Grove Drive

Pasadena, CA 91109, US

留: +1-818-354-8168

Email: yi.chao@jpl.nasa.gov

Chelton, Dudley

Oregon State University

College of Oceanic and Atmospheric Sciences

104 COAS

Corvallis, OR 97331-5503, US

留: +1-541-737-4017

Email: chelton@coas.oregonstate.edu

Chin, Toshio Michael

NASA Jet Propulsion Laboratory

California Institute of Technology

M/S 300/323

4800 Oak Grove Drive

Pasadena, CA 91109, US

留: +1-818-393-2510

Email: mike.chin@jpl.nasa.gov

Clayson, Carol Anne

Florida State University

EOAS -- Meteorology

P.O. Box 3064520

Tallahassee, FL 32306-4520, US

淈: +1-850-644-0922

Email: cclayson@fsu.edu 
GHRSST XII -

Proceedings, Edinburgh

GHRSST Project Office

Corlett, Gary

Earth Observation Science

Space Research Centre

University of Leicester

University Road

Leicester, LE1 7RH, UK

退: +44 (0)116 2297712 +44 (0)789 4204135

Email: gkc1@le.ac.uk

Cornillon, Peter

University of Rhode Island

Graduate School of Oceanography

215 South Ferry Rd

Narragansett, RI 02882-1197, US

留: +1-401-742-2911

Email: pcornillon@gso.uri.edu

Cox, Caroline

STFC Rutherford Appleton Laboratory

RAL Space

Harwell Oxford

Didcot, OX11 0QX, UK

留: +44 (0)1235778282

Email: caroline.cox@stfc.ac.uk

Cummings, James

Naval Research Laboratory ( NRL)

7 Grace Hopper Ave

Monterey, CA 93943-5502, US

碿: +1-831-656-5021

Email: cummings@nrlmry.navy.mil

Dash, Prasanjit

NOAA/NESDIS/STAR

WWB Room 601-1

5200 Auth Road

Camp Springs, MD 20746, US

留: +1-301-763-8053 x168

Email: prasanjit.dash@noaa.gov

Donlon, Craig

ESA/ESTEC (EOP-SME)

Keplerlaan 1

2201 AZ Noordwijk, The Netherlands

淄: +31 (0)715 653687 +31 (0)627013244

$\left({ }^{*}\right.$ new*)

Email: Craig.Donlon@ESA.int

Eastwood, Steinar

The Norwegian Meteorological Institute

PO Box 43

Blindern N-0313 Oslo, Norway

留: +4798682042

Email: s.eastwood@met.no
Issue: Final

Date: September 27, 2011

Embury, Owen

The University of Edinburgh

School of GeoSciences

Crew Building

King's Buildings

Edinburgh, EH9 3JN, UK

留:

Email: owen.embury@ed.ac.uk

Evans, Robert

RSMAS/Umiami

Rosenstiel School of Marine and Atmospheric

Science

Meteorology and Physical Oceanography

4600 Rickenbacker Causeway

Miami, FL 33149-1098, US

留: +1-305-361-4799

Email: revans@rsmas.miami.edu

Fielder, Emma

Met Office

Fitzroy Road

Exeter, EX1 2SQ, UK

留: +44 (0)1392886043

Email: emma.fiedler@metoffice.gov.uk

Figa Saldana, Julia

EUMETSAT

Eumetsat Allee 1

64295 Darmstadt, Germany

通:

Email: julia.figa@eumetsat.int

Filipiak, Mark

The University of Edinburgh

School of GeoSciences

Crew Building

King's Buildings

Edinburgh, EH9 3JN, UK

留:

Email: mjf@staffmail.ed.ac.uk

Foley, Dave

NOAA Southwest Fisheries Science Center

Environmental Research Division

1352 Lighthouse Ave

Pacific Grove, CA 93950-2097, US

留: +1-831-648-0632

Email: dave.foley@noaa.gov

França, Gutemberg

Federal University of Rio de Janeiro

Depto Meteorologia/IGEO

Rua Athos da Silveira Ramos

274 Cidade Universitária - CCMN

22775-0462 Rio de Janeiro, Brazil

的:

Email: gutemberg@Ima.ufrj.br 
GHRSST XII -

Proceedings, Edinburgh

GHRSST Project Office

Gierach, Michelle

NASA Jet Propulsion Laboratory

California Institute of Technology

M/S 300/323

4800 Oak Grove Drive

Pasadena, CA 91109, US

留: +1-818-354-1933

Email: michelle.gierach@jpl.nasa.gov

Guan, Lei

Ocean Remote Sensing Institute

Ocean University of China

Department of Marine Technology

College of Information Science and

Engineering

238 Songling RoadQingdao, 266100, China

留: +86 (0)532 66782326; 66781802

Email: leiguan@ouc.edu.cn

Hacker, Peter

NASA

NASA Headquarters

NASA/SMD/ESD

300 E Street SW $3 B 74$

Washington DC, 20546-0001, US

留: +1-202-341-5442

Email: peter.w.hacker@nasa.gov

Harris, Andy

NOAA/Unif of MD

University of Maryland

Camp Springs, MD 20746, US

跑: +1 $3017638102 \times 169$

Email: andy.harris@noaa.gov

Hoeyer, Jacob

Danish Meteorological Institute (DMI)

Centre for Ocean and Ice

Lyngbyvej 100

2100 København $\varnothing$, Denmark

淈: +4539157203

Email: jlh@dmi.dk

Houghton, Nigel

ESA/ESRIN

Via Galileo Galilei

Casella Postale 64

00044 Frascati (RM), Italy

遍: +390694180667

Email: Nigel.Houghton@esa.int

Huang, Haiyan

University of Oxford

Atmospheric Oceanic and Planetary Physics

Clarendon Laboratory

Oxford, OX1 3PU, UK

㽞: +44 (0)7817971385

Email: huang@atm.ox.ac.uk
Issue: Final

Date: September 27, 2011

Ignatov, Alexander

NOAA/NESDIS/STAR

WWB Rm 601

5200 Auth Road

Camp Springs, MD 20746, US

留: +1-301-763-8102 x190

Email: Alex.Ignatov@noaa.gov

Ishizaki, Shiro

Japan Meteorological Agency (JMA)

Global Environment and Marine Department

Office of Marine Prediction

1-3-4 Otemachi

Chiyoda-kuTokyo, Japan

留: +81-3-3212-8341 ex5166

Email: s_ishizaki@met.kishou.go.jp

Janssen, Peter

ECMWF

Shinfield Park

Reading, RG2 9AX, UK

留: +44 (0)118-9499116

Email: p.janssen@ecmwf.int

Kachi, Misako

Earth Observation Research Center (EORC)

Japan Aerospace Exploration Agency (JAXA)

2-1-1 Sengen

Tsukuba-shi

Ibaraki 305-8505, Japan

墖: +81-50-3362-5027

Email: kachi.misako@jaxa.jp

Kaiser-Weiss, Andrea

National Centre for Earth Observation

The University of Reading

Dept of Meteorology

Earley Gate Bldg 58

Reading, RG6 6BB, UK

掘: +44 (0)118378 5579

Email: a.k.kaiserweiss@reading.ac.uk

Kaplan, Alexey

Lamont-Doherty Earth Observatory of

Columbia University

P.O. Box 1000

61 Route 9W

Palisades, NY 10964-8000, US

䘓: +1-845-365-8689

Email: alexeyk@Ideo.columbia.edu 
GHRSST XII -

Proceedings, Edinburgh

GHRSST Project Office

Karagali, Ioanna

Risø DTU-Technical University of Denmark

Wind Energy Division

Risø National Laboratory for Sustainable

Energy

Frederiksborgvej 399

Building 1254000 Roskilde, Denmark

留: +45 46775026

Email: ioka@risoe.dtu.dk

Kelliher, Hugh

Space ConneXions Ltd

8 Pondwick Road

Harpenden, AL5 2HG, UK

留: +44 (0)1582 712418

Email: hugh.kelliher@spaceconnexions.com

Kent, Elizabeth C

National Oceanography Centre, Southampton

University of Southampton

European WaySouthampton, SO14 3ZH, UK 留: +44 (0)2380596646

Email: eck@noc.ac.uk

Kim, Mi-Ja

NIMR/KMA

45 Gisangcheong-gil, Dongjak-gu

Seoul 156-720, KR

踏:

Email: kimmee74@korea.kr

Kim, Youngmi

NIMR/KMA

45 Gisangcheong-gil, Dongjak-gu

Seoul 156-720, KR

留: +82-2-6712-0392

Email:kymi19@korea.kr

Lavender, Samantha

ARGANS Ltd

Tamar Science Park

19 Research Way

Plymouth, PL6 8BT, UK

阿: +44 (0)1752 764298

Email: samantha.lavender@argans.co.uk

Le Borgne, Pierre

Meteo-France/CMS

Avenue de Lorraine

Lannion 22307, France

留: +33296056752

Email: pierre.leborgne@meteo.fr
Issue: Final

Date: September 27, 2011

Lee, Tong

NASA Jet Propulsion Laboratory

California Institute of Technology

M/S 300/323

4800 Oak Grove Drive

Pasadena, CA 91109, US

留: +1-818-354-1401

Email: Tong.Lee@jpl.nasa.gov

Liu, W. Timothy

NASA Jet Propulsion Laboratory

California Institute of Technology

M/S 300/323

4800 Oak Grove Drive

Pasadena, CA 91109, US

留: +1-818-354-2394

Email: w.t.liu@jpl.nasa.gov

Llewellyn-Jones, David

University of Leicester

Space Research Centre

University Road

Leicester, LE1 7RH, UK

淈: +44 (0)116 2525238

Email: dlj1@le.ac.uk

MacCallum, Stuart

The University of Edinburgh

School of GeoSciences

Crew Building

King's Buildings

Edinburgh, EH9 3JN, UK

留:

Email: s.maccallum@ed.ac.uk

Marsouin, Anne

Meteo-France

BP 50747

Lannion 22307, France

漹: +3310296056746

Email: anne.marsouin@meteo.fr

Martin, Matthew

Met Office

Fitzroy Road

Exeter, EX1 3PB, UK

溜: +44 (0)1392 886465

Email: matthew.martin@metoffice.gov.uk

Maturi, Eileen

NOAA/NESDIS/STAR

5200 Auth Road

Camp Springs, MD 20746, US

留: +1-301-763-8102 x172

Email: Eileen.Maturi@noaa.gov 
GHRSST XII -

Proceedings, Edinburgh

GHRSST Project Office

McKenzie, Bruce

Naval Oceanographic Office

Code NP32T NAVOCEANO

1002 Balch Blvd

Stennis Space Center, MS 39522, US

留: +1-228-688-4586

Email: bruce.mckenzie@navy.mil

Meldrum, David

Scottish Association for Marine Science

Scottish Marine Institute

Oban, PA37 1QA, Scotland, UK

迎: +44 (0)1631559273 +44 7774690630

Email: dtm@sams.ac.uk

Merchant, Christopher

The University of Edinburgh

School of GeoSciences

Crew Building

King's Buildings

Edinburgh, EH9 3JN, UK

呞: +44 (0)131 6505097

Email: c.merchant@ed.ac.uk

Minnett, Peter

RSMAS/Umiami

Rosenstiel School of Marine and Atmospheric

Science

Meteorology and Physical Oceanography

4600 Rickenbacker Causeway

Miami, FL 33149-1098, US

留: +1-305-421-4104

Email: pminnett@rsmas.miami.edu

Mittaz, Jonathan

NOAA/NESDIS/ORA

WWB Room 603-7

5200 Auth Road

Camp Springs, MD 20746, US

的: +1-301-763-8102 x189

Email: Jon.Mittaz@noaa.gov

Nightingale, Tim

STFC Rutherford Appleton Laboratory

RAL Space

Harwell Oxford

Didcot, OX11 0QX, UK

留: +44 (0)1235 445914

Email: tim.nightingale@stfc.ac.uk

O'Carroll, Anne

EUMETSAT

Eumetsat-Allee 1

64295 Darmstadt, Germany

留: +49 6151807676

Email: Anne.Ocarroll@eumetsat.int
Issue: Final

Date: September 27, 2011

Oliveira, Antônio

Federal University of Rio de Janeiro

Depto Meteorologia//GEO

Rua Athos da Silveira Ramos

274 Cidade Universitária - CCMN

22775-0462 Rio de Janeiro, Brazil

通:

Email: antonio@Ima.ufri.br

Orain, Françoise

Météo-France

Centre de Météorologie Spatiale

Avenue de Lorraine, BP 50747

22307 Lannion cedex, France

留: +33296056785

Email: francoise.orain@meteo.fr

Petrenko, Boris

NOAA/NESDIS/STAR/IMSG inc.

5200 Auth Rd

Camp Springs, MD 20746, US

很:

Email: boris.petrenko@noaa.gov

Piollé, Jean-François

CERSAT, Ifremer

29280 Plouzane

殿: +33-2-98224691

Email: jean.francois.piolle@ifremer.fr

Pisano, Andrea

CNR-ISAC Roma

Via Fosso del Cavaliere

100-133 Roma, Italy

留:

Email: andrea.pisano@artov.isac.cnr.it

Poulter, David

National Oceanography Centre, Southampton

University of Southampton

Waterfront Campus

European WaySouthampton, SO14 3ZH, UK

留: +44 (0) 238596107

Email: djsp@bodc.ac.uk

Quinn, Sarah

University of Reading

Department of Meteorology

Reading, RG6 6BB, UK

留:

Email: sarah.quinn@student.reading.ac.uk

Rayner, Nick

Met Office

Hadley Centre

Fitzroy Road

Exeter, EX1 3PB, UK

溜: +44 (0)1392 884291

Email: nick.rayner@metoffice.gov.uk 
GHRSST XII -

Proceedings, Edinburgh

GHRSST Project Office

Reynolds, Richard

NOAA Cooperative Institute for Climate\&

Satellites (CICS)

151 Patton Avenue

Asheville, NC 28801-5001, US

留: +1-828-271-4302

Email: richard.w.reynolds@noaa.gov

Roberts-Jones, Jonah

Met Office

Fitzroy Road

Exeter, EX1 3PB, UK

踏: +44 (0)1392 886441

Email: jonah.roberts-jones@metoffice.gov.uk

Robinson, lan

National Oceanography Centre, Southampton University of Southampton

Waterfront Campus

European WaySouthampton, SO14 3ZH, UK

留: $+44(0) 2380766267$

Email: isr@noc.soton.ac.uk

Roquet, Hervé

Météo-France

Centre de Météorologie Spatiale

Avenue de Lorraine, BP 50747

22307 Lannion cedex, France

留: +33296056764

Email: Herve.Roquet@meteo.fr

Rutherford, Martin

Navy METOC

Level 2 Building 89/90 Garden Island

Potts Point 2011 NSW, AU

跑: +61 409122778

Email: tdom@metoc.gov.au

Scott, Nicola

Met Office

S9 Saughton House,

Broomhouse Drive, Edinburgh EH11 3XQ, UK 踏: +44 (0)131528 7312

Email: nicola.scott@metoffice.gov.uk
Issue: Final

Date: September 27, 2011

Smith, Deborah

Remote Sensing Systems

444 Tenth St, Suite 200

Santa Rosa, CA 95401, US

届: +1-707-545-2904 Ext. 11

Email: smith@remss.com

Spinks, Paul

Space ConneXions Ltd

8 Pondwick Road

Harpenden, AL5 2HG, UK

留:

Email: paul.spinks@spaceconnexions.com

Vazquez, Jorge

NASA Jet Propulsion Laboratory

California Institute of Technology

$\mathrm{M} / \mathrm{S} 300 / 323$

4800 Oak Grove Drive

Pasadena, CA 91109, US

制: +1-8183546980/+1-626 8623771

Email: jorge.vazquez@jpl.nasa.gov

Veal, Karen

University of Leicester

Department of Astronomy

Leicester, LE1 7RH, UK

殿: +44 (0)116 2297705

Email: klv3@le.ac.uk

Wimmer, Werenfrid

National Oceanography Centre, Southampton

University of Southampton

School of Ocean on Earth Science

Laboratory of Satellite Oceanography

European WaySouthampton, SO14 3ZH, UK

留: +442380 597654

Email: w.wimmer@soton.ac.uk 


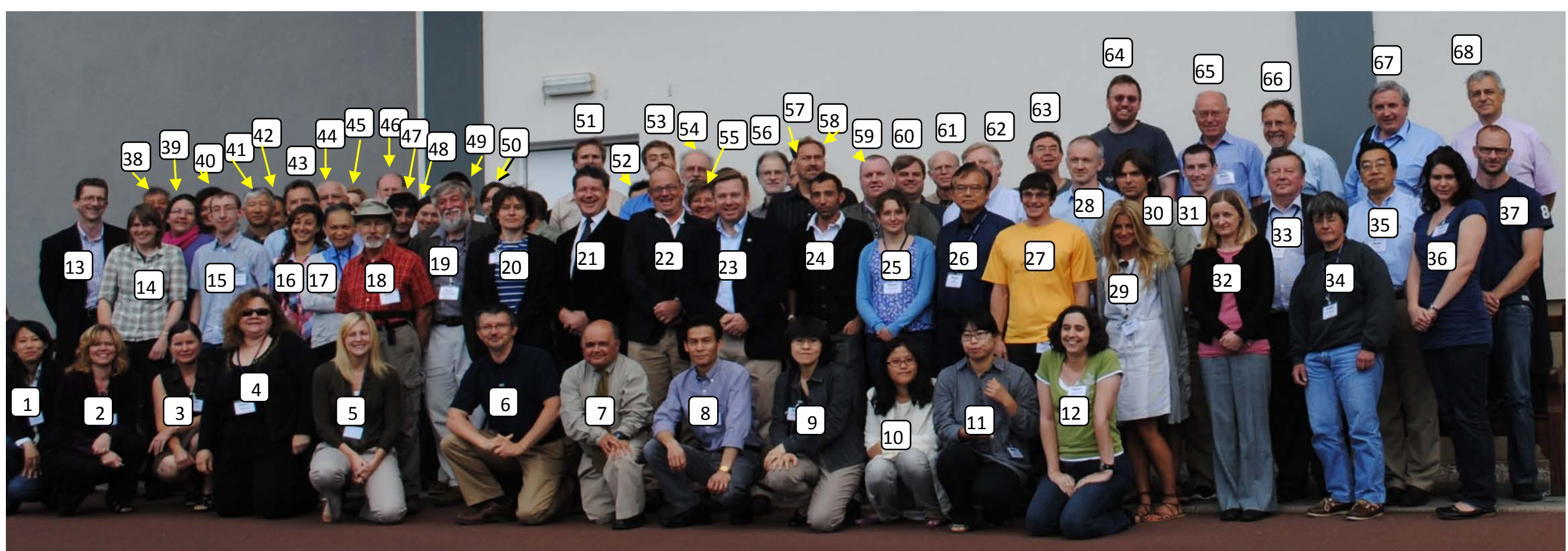

1. Lei Guan

2. Carol Anne Clayson

3. Helen Beggs

4. Eileen Mary Maturi

5. Michelle Gierach

6. Andy Harris

7. Jorge Vazquez

8. Tong Lee

9. Misako Kachi

10. Youngmi Kim

11. Mi-Ja Kim

12. Samantha Lavender

13. Chris Merchant

14. Claire Bulgin
15. Owen Embury

16. Ioanna Karagali

17. Viva Banzon

18. Bob Evans

19. Peter Minnett

20. Andrea Kaiser-Weiss

21. Craig Donlon

22. Olivier Arino

23. Kenneth Casey

24. Andrea Pisano

25. Emma Fiedler

26. Tim Liu

27. Jonah Roberts-Jones

28. Mark Filipiak
29. Nicky Scott

30. Jean-François Piollé

31. Stuart McCallum

32. Karen Veal

33. David Llewellyn-Jones

34. Anne Marsouin

35. Yi Chao

36. Caroline Cox

37. Jacob Hoeyer

38. Pierre Le Borgne

39. Françoise Orain

40. Anne O'Carroll

41. Mike Chin

42. Hervé Roquet
43. Peter Cornillon

44. Boris Petrenko

45. Mary Elizabeth Maturi?

46. Martin Rutherford

47. Bruno Buongiorno

Nardelli

48. Nick Rayner

49. Dick Reynolds

50. Tess Brandon

51. Steiner Eastwood

52. Shiro Ishizaki

53. David Poulter

54. Peter Janssen

55. Deborah Smith
56. Hugh Kelliher

57. Matt Martin

58. Ed Armstrong

59. Gary Corlett

60. Jon Mittaz

61. Dudley Chelton

62. Jim Cummings

63. Tim Nightingale

64. Werenfrid Wimmer

65. Ian Barton

66. Peter Hacker

67. Paul Spinks

68. Nigel Houghton 
GHRSST XII -

Proceedings, Edinburgh

GHRSST Project Office
Issue: Final

Date:September 27, 2011 


\section{APPENDIX 2 - SCIENCE TEAM 2011/12}

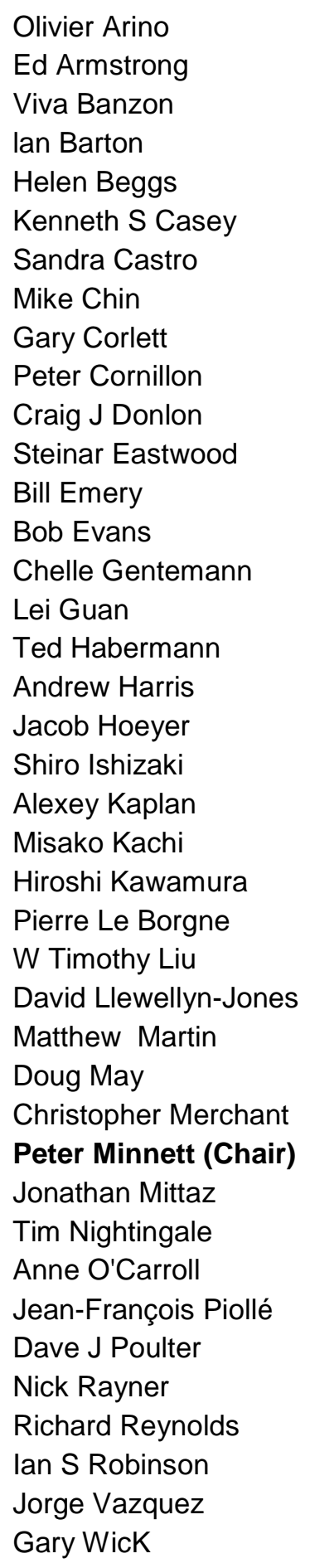

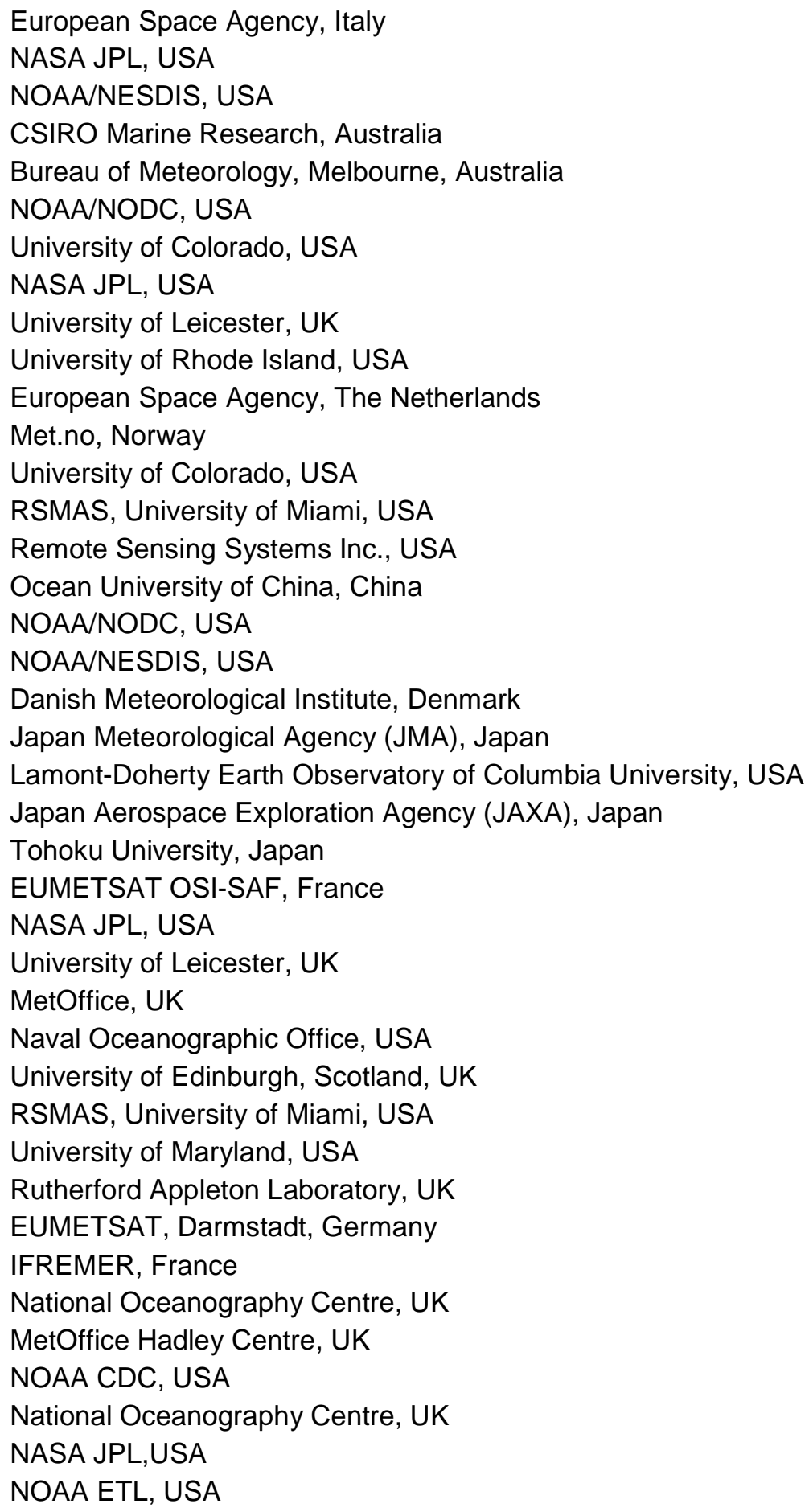

